\title{
BIODIVERSIDAD DE LA FLORA VASCULAR DE LA PROVINCIA DE MISIONES, REGIÓN PARANAENSE ARGENTINA
}

\author{
Christian A. Zanotti ${ }^{1}$, Héctor A. Keller² \& Fernando O. Zuloaga ${ }^{3}$
}

\author{
${ }^{1}$ Instituto de Limnología “Dr. Raúl A. Ringuelet” (CONICET), Bv. 120 y 62, La Plata, Buenos Aires, Argentina. \\ ${ }^{2}$ Instituto de Botánica del Nordeste, IBONE, Sargento Cabral 2139, Corrientes, Argentina. \\ ${ }^{3}$ Instituto de Botánica Darwinion, IBODA, San Isidro, Buenos Aires, Argentina; fzuloaga@darwin.edu.ar(autor corresponsal).
}

\begin{abstract}
Zanotti, C. A.; H. A. Keller \& F. O. Zuloaga. 2020. Vascular flora biodiversity of the province of Misiones, Paranaense region, Argentina. Darwiniana, nueva serie 8(1): 42-291.

We present an inventory of the vascular plants of the Misiones province, with a total of 3418 taxa, which accounts for more than $30 \%$ of the plants of Argentina. A total of 47 endemic taxa from Misiones province were analyzed and categorized following IUCN standards. We recognized 761 exclusive species for the province of Misiones within Argentina and species without records in recent times. Finally, a total of 16 native and introduced taxa are cited for the first time for Argentina.
\end{abstract}

Keywords. Argentina; Misiones; taxonomy; vascular plants.

Resumen. Zanotti, C. A.; H. A. Keller \& F. O. Zuloaga. 2020. Biodiversidad de la flora vascular de la provincia de Misiones, Región Paranaense, Argentina. Darwiniana, nueva serie 8(1): 42-291.

En este trabajo presentamos un inventario de las plantas vasculares de la provincia de Misiones, que incluye un total de 3418 taxones que representan más del 30\% del total de las plantas presentes en la Argentina. Se analizan los 47 taxones endémicos de Misiones y los categorizamos siguiendo los criterios de UICN. Identificamos a los 761 taxones que son exclusivos para la provincia de Misiones dentro de la Argentina y a las especies que no han sido colectadas en tiempos recientes. También se indican un total de 16 taxones que representan nuevas citas para la Argentina.

Palabras clave. Argentina; Misiones; plantas vasculares; taxonomía.

\section{INTRODUCCIÓN}

La provincia de Misiones es la que posee la mayor riqueza florística de la República Argentina (Zuloaga et al., 1999, 2019). Biogeográficamente, toda la provincia se enmarca dentro de la región Paranaense, la que se continúa en nuestro país en el nordeste de Corrientes, y en selvas en galería a lo largo de los ríos Paraná y Uruguay hasta el Delta del Río de La Plata, y áreas limítrofes en el este de Paraguay y el sur de Brasil. La selva Paranaense es, sin dudas, uno de los ecosistemas de mayor prioridad de conservación a nivel global debido a su alta biodiversidad, número de especies endémicas y grado de amenaza (Myers et al., 2000). Esta provincia biogeográfica ha recibido distintas denominaciones (ver Martínez Crovetto, 1963; Cabrera, 1976) y los tipos de vegetación dominante son selvas subtropicales en el norte y centro de la provincia y campos en el sur de la misma. Además, los ambientes de superficie limitada y de escasa representatividad como pastizales anegados, pastizales pasmófilos, rupícolas, en general incluidos en una matriz selvática, aportan a la flora provincial numerosas especies, e inclusive microendemismos. Entre estos 
ambientes poco representativos en superficie cabe señalar una ingresión de la provincia del Cerrado en áreas de areniscas del sur provincial (Chébez, 1996; Biganzoli \& Múlgura, 2004; Velazco et al., 2018).

La provincia cuenta con un Parque Nacional (Parque Nacional Iguazú), y otras tres áreas protegidas bajo administración nacional, 56 áreas naturales bajo jurisdicción provincial, 8 bajo jurisdicción municipal y otras 10 de jurisdicción privada (MERNR, 2020). Sin embargo, a pesar de la importancia de los inventarios de la biodiversidad para el desarrollo de planes de manejo de las áreas protegidas (Barzetti, 1993; Bertolini, 1999, 2005; Bertolini \& Gil, 1999; Bodrati et al. 2007a, b; Chebez, 2007; Gil \& Ciarmiello, 2007; Krauczuk, 2007; Soria, 1996), son pocos los estudios florísticos que se han realizado en su territorio (López Cristóbal et al., 1996; Tressens \& Revilla, 1996; Fontana, 1998, 2005; Daviña et al., 1999, Biganzoli \& Peralta, 2000; Biganzoli \& Múlgura de Romero, 2004; Freire et al., 2006) y no existen inventarios de las áreas protegidas de mayor superficie. Cabe destacar que en los últimos años se publicaron numerosas nuevas citas de registros para la flora de la provincia y de la Argentina (Deble et al., 2017; Delucchi \& Keller, 2010; Delucchi et al., 2016; Dematteis et al., 2012; Ezcurra \& Múlgura de Romero, 2001; Farinaccio \& Keller, 2014; Gatti, \& Keller, 2019; Hurrell et al., 2011, 2012, 2017; Judkevich et al., 2016; Keller \& Cáceres, 2018; Keller \& Crockett, 2015; Keller \& Kline, 2008; Keller \& Tressens, 2007, 2016; Keller et al., 2012, 2013; Keller, 2007, 2009, 2010a, b, 2011, 2015a, b, 2017; Marquez \& Keller, 2018; Meza Torres et al., 2010; Morillo \& Keller, 2016; Morillo et al., 2016; O’ Leary \& Keller, 2018; Peralta, 2001; Ponce, 2001; Queiroz \& Azevedo-Tozzi, 2013; Tressens \& Keller, 2006; Zanotti \& Keller, 2017).

El objetivo de la presente contribución es presentar un inventario actualizado de las plantas vasculares que crecen en la provincia, indicando taxones endémicos y su categorización siguiendo los criterios de UICN, verificar taxones que han sido citados y que ya no crecen en la provincia, o bien que no han sido colectados en un largo período, taxones exclusivos de la provincia y su relación con la flora vascular de la Argentina. Por último, se dan a conocer 16 nuevos registros para la provincia y para la Argentina.

\section{MATERIALES Y MÉTODOS}

Para el desarrollo de este trabajo se utilizó la base de datos DFA, Documenta Florae Australis (www. darwin.edu.ar/Iris, www.floraargentina.edu.ar). Esta base de datos incluye un inventario actualizado de la flora vascular de la Argentina y del Cono Sur. Además, se revisaron las colecciones de los herbarios CTES, LP y SI (Thiers, 2020) y se aportaron observaciones personales de los autores.

Para la evaluación del estado de conservación de los taxones endémicos de la provincia se utilizó la información extraída de las colecciones, a partir de la base de datos DFA. Se utilizaron las categorías y criterios de la lista roja IUCN versión 3.1 (2012), en la cual, los taxones a evaluar, se clasifican en 9 categorías según la amenaza de extinción en: Extinto (EX), Extinto en Estado Silvestre (EW), En Peligro Crítico (CR), En Peligro (EN), Vulnerable (VU), Cerca de la Amenaza (NT), Preocupación Menor (LC), Datos Deficientes (DD) y por último, No Evaluado (NE). A su vez, existen cinco criterios (AE) utilizados para evaluar a los taxones en una de las tres categorías de amenaza (CR, EN y VU) en: (A) Reducción del tamaño poblacional; (B) Distribución geográfica representada como extensión de presencia y/o área de ocupación; (C) Pequeño tamaño de la población y disminución;(D) Población muy pequeña o restringida y por último, (E) Análisis cuantitativo y que indique la probabilidad de extinción en estado silvestre. Para clasificar a un taxón en cualquier categoría de evaluación, se necesita sólo el apoyo de uno de los cinco criterios, aunque debería evaluarse bajo la mayor cantidad de criterios posibles. Para la mayoría de los taxones endémicos analizados en este trabajo, no se cuenta con información cuantitativa sobre las poblaciones, por ello sólo se pudo utilizar el criterio B para estimar el estatus de conservación, a excepción de Austrochthamalia teyucuarensis H.A. Keller, Oxypetalum teyucuarense Farinaccio \& H.A. Keller, Cypella suffusa Ravenna, los cuales se pudieron clasificar con el criterio D; en cambio, Topea micrantha (H.A. Keller) H.A. Keller y Hedeoma teyucuarensis H.A. Keller \& Tressens, además de ser clasificadas bajo el criterio B, también, se pudieron clasificar con el criterio D. Para realizar la evaluación mediante el criterio $\mathrm{B}$, se procedió al cálculo de la extensión de presencia (EOO), a través de la determinación del área $\left(\mathrm{km}^{2}\right)$ medida 
por un polígono convexo mínimo (el polígono de menor superficie que contenga todos los lugares de presencia, pero que ninguno de sus ángulos internos exceda los 180 grados). Los umbrales críticos para la EOO son $<100 \mathrm{~km}^{2}$ (CR), $<5.000 \mathrm{~km}^{2}$ (EN) y $<20.000 \mathrm{~km}^{2}$ (VU) (IUCN, 2012). Para el cálculo de la EOO de cada uno de los taxones, se utilizó la herramienta on-line GeoCat (Bachman et al., 2011). Para aplicar el criterio B, además del cálculo de la EOO, se debe tener en cuenta si los taxones cumplen por lo menos dos de las tres condiciones siguientes: a) severamente fragmentada o número de localidades $<10$; b) disminución continua observada, estimada, inferida o proyectada en cualesquiera de: (i) extensión de presencia, (ii) área de ocupación, (iii) área, extensión y/o calidad del hábitat, (iv) número de localidades o subpoblaciones, (v) número de individuos maduros; c) fluctuaciones extremas en cualesquiera de: (i) extensión de presencia, (ii) área de ocupación, (iii) número de localidades o subpoblaciones, (iv) número de individuos maduros. Además de tener en cuenta todos estos criterios y condiciones, se utilizaron datos de observaciones a campo, y datos extraídos de las etiquetas de herbario y de la literatura pertinente.

Para cada taxón del inventario se cita: hábito, ciclo de vida, estatus (si un taxón es nativo, endémico, introducido) para la provincia, altitud sobre el nivel del mar para la provincia, distribución (departamento/s de Misiones donde habita/n, o en el caso de no conocer el departamento, se presenta la región en donde es posible que habite dentro de la provincia, o en caso contrario se indica si es desconocida. Los departamentos se abrevian con el siguiente código: APO, Apóstoles; CAI, Cainguás; CAN, Candelaria; CAP, Capital; CON, Concepción; ELD, Eldorado; GMB, General Manuel Belgrano; GUA, Guaraní; IGU, Iguazú; LNA, Leandro N. Alem; GSM, Libertador General San Martín; MAY, 25 de Mayo; MON, Montecarlo; OBE, Oberá; SIG, San Ignacio; SJA, San Javier; SPE, San Pedro (Figura 1). Por último, se cita un ejemplar de referencia para cada taxón.

ÁREA DE ESTUDIO. La provincia de Misiones tiene una superficie de $29.081 \mathrm{~km}^{2}$, siendo la tercera de menor tamaño luego de C.A.B.A y la provincia de Tucumán, y está ubicada entre los paralelos $25^{\circ}$ y $28^{\circ} \mathrm{S}$ y los meridianos $53^{\circ}$ y $56^{\circ} \mathrm{O}$, en el nordeste de la República Argentina (Figura 1).
CLIMA Y SUELO. El clima es cálido y húmedo, con precipitaciones todo el año, llegando a superar los $2000 \mathrm{~mm}$ anuales; la temperatura media anual oscila entre $15-23{ }^{\circ} \mathrm{C}$ y la diferencia de temperatura media entre el mes más frío y el más cálido va desde $4-7^{\circ} \mathrm{C}$ a 10-12 ${ }^{\circ} \mathrm{C}$ (Cabrera, 1976; Rodríguez \& Silva, 2012); no obstante, la provincia no está exenta de heladas. En lo referente al relieve, Cabrera (1976) señala que es una provincia montañosa, con sierras de poca altura y con una elevación paulatina hacia el NE, llegando a los $800 \mathrm{~m}$ s.m. en Bernardo de Irigoyen (dpto. General Manuel Belgrano). Los suelos son lateríticos, arcillosos, ricos en hierro (rojos) y ácidos, profundos, pertenecientes mayoritariamente a Ultisoles, entre los que se destacan los Kandiudultes (Rodríguez \& Silva, 2012).

BIOGEOGRAFÍA. La provincia fitogeográfica Paranaense ocupa toda la provincia de Misiones y un sector del noreste de Corrientes, desde la desembocadura del Arroyo Itaembé en el río Paraná hacia el oeste, llegando al río Uruguay poco más al norte de Santo Tomé (Martínez Crovetto, 1963; Fontana, 2015). En la Argentina, la flora de esta provincia está relacionada a la de la provincia Chaqueña, con la que forma un ecotono hacia el sur y el oeste. También comparte numerosas especies con la provincia de las Yungas, la restante región selvática de Argentina, en particular con la Selva pluvial semicaducifolia pedemontana, presente en el noroeste argentino y además, presenta elementos en común con otros bosques secos estacionales neotropicales (Oyarzabal et al., 2018). Fuera de la Argentina, la provincia Paranaense, se encuentra en el este de Paraguay y sur del Brasil (Cabrera \& Willink 1973), y tiene afinidades con la provincia Atlántica. Cabe destacar la importancia de la provincia y su preservación en Misiones, considerando la enorme degradación, por la explotación madedera, cultivos y pastoreo, que ha sufrido tanto en Paraguay como en Brasil (Rodríguez \& Silva, 2012). Así, se considera que menos del $7 \%$ de la cobertura original de la provincia Paranaense persiste en Paraguay, Brasil y la Argentina (Galindo-Leal \& Câmara, 2003), estando mejor representada en la actualidad en la provincia de Misiones.

Se sigue aquí la clasificación de Cabrera (1976) de dos distritos en la provincia: el distrito de las Selvas Mixtas hacia el norte y centro y el distrito de 
los Campos en el sur de Misiones. El distrito de las Selvas Mixtas cubre la mayor parte de la provincia; predominan, entre otros, árboles de gran porte como Balfourodendron riedelianum (Engl.) Engl., Rutaceae, Nectandra angustifolia (Schrad.) Nees \& Mart., Lauraceae, Cabralea canjerana (Vell.) Mart. y Cedrela fissilis Vell., Meliaceae, entre otros. Hacia el noroeste de la provincia, en el área del Parque Nacional Iguazú, se destacan Aspidosperma polyneuron Müll. Arg., Apocynaceae, y el palmito, Euterpe edulis Mart., Arecaceae. Mientras que en los sectores más elevados de la provincia, en el sector noreste, se destaca la presencia del "pino paraná", Aracuaria angustifolia (Bertol.) Kuntze, Araucariaceae. Hacia el sur de la provincia, los campos se encuentran alternando con bosques de "urunday", Myracrodruon balansae (Engl.) Santin, Anacardiaceae, árbol mesófilo y pionero en suelos muy superficiales en lomadas, con roca aflorante. Por su parte, el Distrito de los Campos está constituido por sabanas de gramíneas y ciperáceas, pudiendo citarse entre los géneros más frecuentes de Poaceae a Andropogon, Aristida, Elionurus, Paspalum, Schizachyrium y Setaria, entre otros.

En lo que a conservación se refiere, los sectores más afectados de la provincia han sido, hasta el presente, los campos del sur y los corredores de las Rutas Nacionales 12 y 14, la primera sobre el río Paraná y la restante discurriendo a lo largo del centro de la provincia (Di Bitetti et al., 2003). En el sur, los cultivos principales, además de la actividad ganadera, son de yerba mate y té, mientras que hacia el norte, en las áreas antes citadas, se cultivan especies exóticas de pino y se ha extraído buena parte de las maderas más valiosas, como el cedro, guatambú, lapacho y el pino Paraná (Cabrera, 1976). Se considera que en la actualidad más del $50 \%$ de la vegetación natural ha sido reemplazada para desarrollar actividades agropecuarias (Biganzoli \& Múlgura de Romero, 2004).

\section{RESULTADOS Y DISCUSIÓN}

En la provincia de Misiones crecen un total de 3418 taxones, pertenecientes a 190 familias y 1095 géneros (Apéndice). Si comparamos este número de taxones con el total presente en la flora vascular Argentina, con 11050 taxones, se observa que en esta reducida provincia se halla el 30,92\% de toda la flora argentina. Este dato es aún más significativo considerando que Misiones tiene sólo el 0,8 \% de la superficie de la Argentina.

Entre las familias con mayor número de taxones representadas en la Flora de la provincia se pueden citara las Asteraceae (361), Poaceae (358), Fabaceae (289), Orchidaceae (151), Cyperaceae (141) y Malvaceae (126) (Tabla 1). Estos datos coinciden en general con los porcentajes que se hallan en la Flora Argentina en su totalidad (Zuloaga et al., 2019) y se destaca el elevado número de especies presente en la familia Orchidaceae.

Tabla 1. Familias con mayor número de taxones en la Flora de la provincia de Misiones.

\begin{tabular}{|c|c|}
\hline FAMILIA & $\mathbf{N}^{\circ}$ DE TAXONES \\
\hline ASTERACEAE & 361 \\
\hline POACEAE & 358 \\
\hline FABACEAE & 289 \\
\hline ORCHIDACEAE & 151 \\
\hline CYPERACEAE & 141 \\
\hline MALVACEAE & 126 \\
\hline EUPHORBIACEAE & 107 \\
\hline SOLANACEAE & 101 \\
\hline RUBIACEAE & 89 \\
\hline APOCYNACEAE & 89 \\
\hline
\end{tabular}

Entre los géneros con mayor número de taxones se pueden citar a Paspalum (50), Mimosa (49), Cyperus (48), Solanum (47) Baccharis (32) e Ipomoea (31) (Tabla 2).

Tabla 2. Géneros con mayor número de taxones en la Flora de la provincia

\begin{tabular}{|c|c|}
\hline GÉNERO & N $^{\circ}$ DE TAXONES \\
\hline PASPALUM & 50 \\
\hline MIMOSA & 49 \\
\hline CYPERUS & 48 \\
\hline SOLANUM & 47 \\
\hline BACCHARIS & 32 \\
\hline IPOMOEA & 31 \\
\hline OXYPETALUM & 29 \\
\hline OXALIS & 27 \\
\hline RHYNCHOSPORA & 28 \\
\hline SIDA & 28 \\
\hline
\end{tabular}




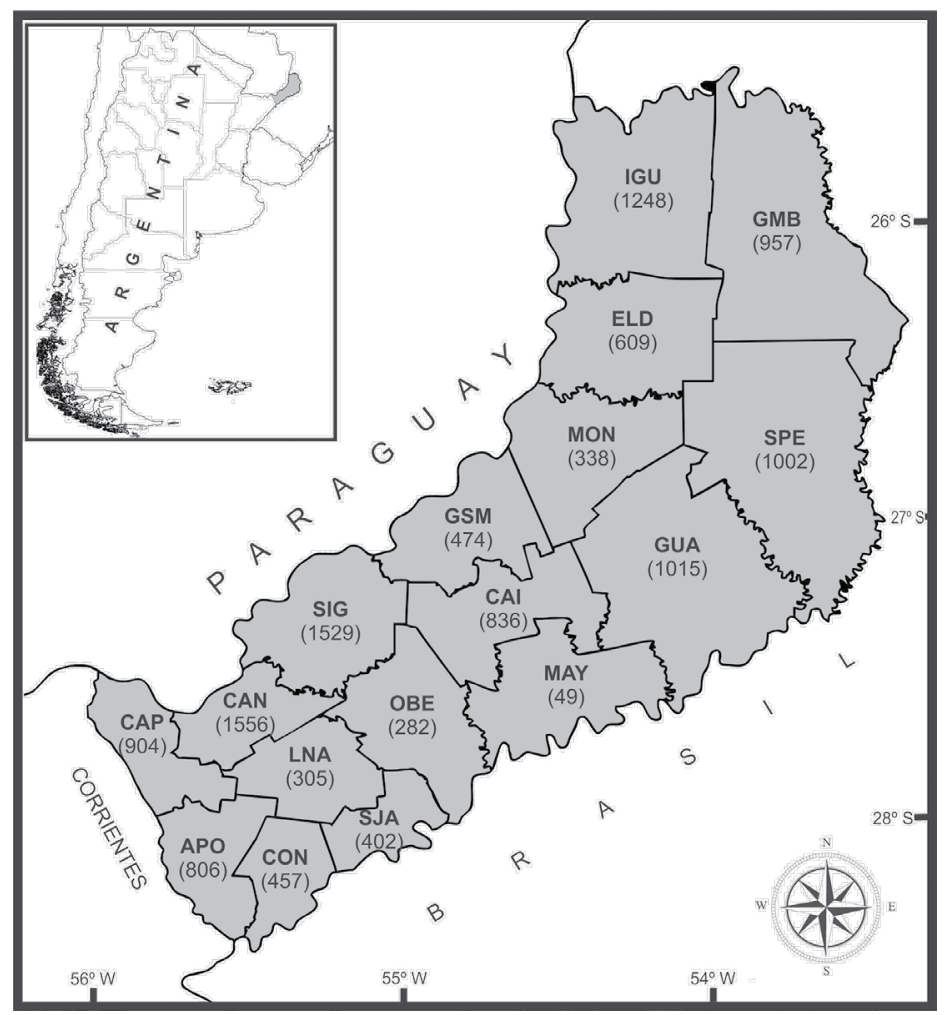

Fig. 1. Mapa de la provincia de Misiones. Entre paréntesis se indica el número total de taxones registrados en cada departamento. Abreviatura de los departamentos: APO, Apóstoles; CAI, Cainguás; CAN, Candelaria; CAP, Capital; CON, Concepción; ELD, Eldorado; GMB, General Manuel Belgrano; GUA, Guaraní; IGU, Iguazú; LNA, Leandro N. Alem; GSM, Libertador General San Martín; MAY, 25 de Mayo; MON, Montecarlo; OBE, Oberá; SIG, San Ignacio; SJA, San Javier; SPE, San Pedro.

Los departamentos políticos con mayor diversidad especifica e infraespecífica son Candelaria (1556 taxones) y San Ignacio (1529 taxones), lo que probablemente es debido a la suma de regiones diversas, como los campos y la presencia de bosques, en ambos departamentos (Figura 1). Estos son seguidos por Iguazú (1248), Guaraní (1015), San Pedro (1002), los restantes departamentos presentan menos de mil taxones. Cabe destacar, que este resultado también puede deberse al esfuerzo de colecta realizados en los diferentes departamentos de la provincia.

\section{Taxones endémicos}

En la provincia hay un total de 47 taxones endémicos; este bajo número se debe sin dudas a la continuidad de la Provincia Paranaense en el sur de Brasil y el este de Paraguay, compartiéndose numerosas especies entre Misiones y ambos países.
En la Tabla 3 se brinda información sobre familia, género, taxón y publicación original de estos endémicos y su categorización siguiendo los criterios de la UICN. El análisis de esta información permite concluir que la mayoría de los taxones endémicos, un total de 31, crecen en el Distrito de los Campos y ninguno de ellos se encuentra en un área protegida de la provincia, existiendo la posibilidad de que 11 de estos taxones ya se encuentren extintos. De los taxones endémicos siete crecen en las proximidades del Parque Provincial Teyú Cuaré. Cabe destacar que en este Parque, y en zonas aledañas, crecen otros 19 taxones que son exclusivos de esta área para la Argentina, hallándose también en Brasil y Paraguay (Biganzoli \& Múlgura de Romero, 2004). Por otra parte, en el Distrito de las Selvas Mixtas se registraron 15 taxones endémicos, la mayoría de ellos conocidos sólo fuera de áreas protegidas y 5 de ellos estarían probablemente extintos. 


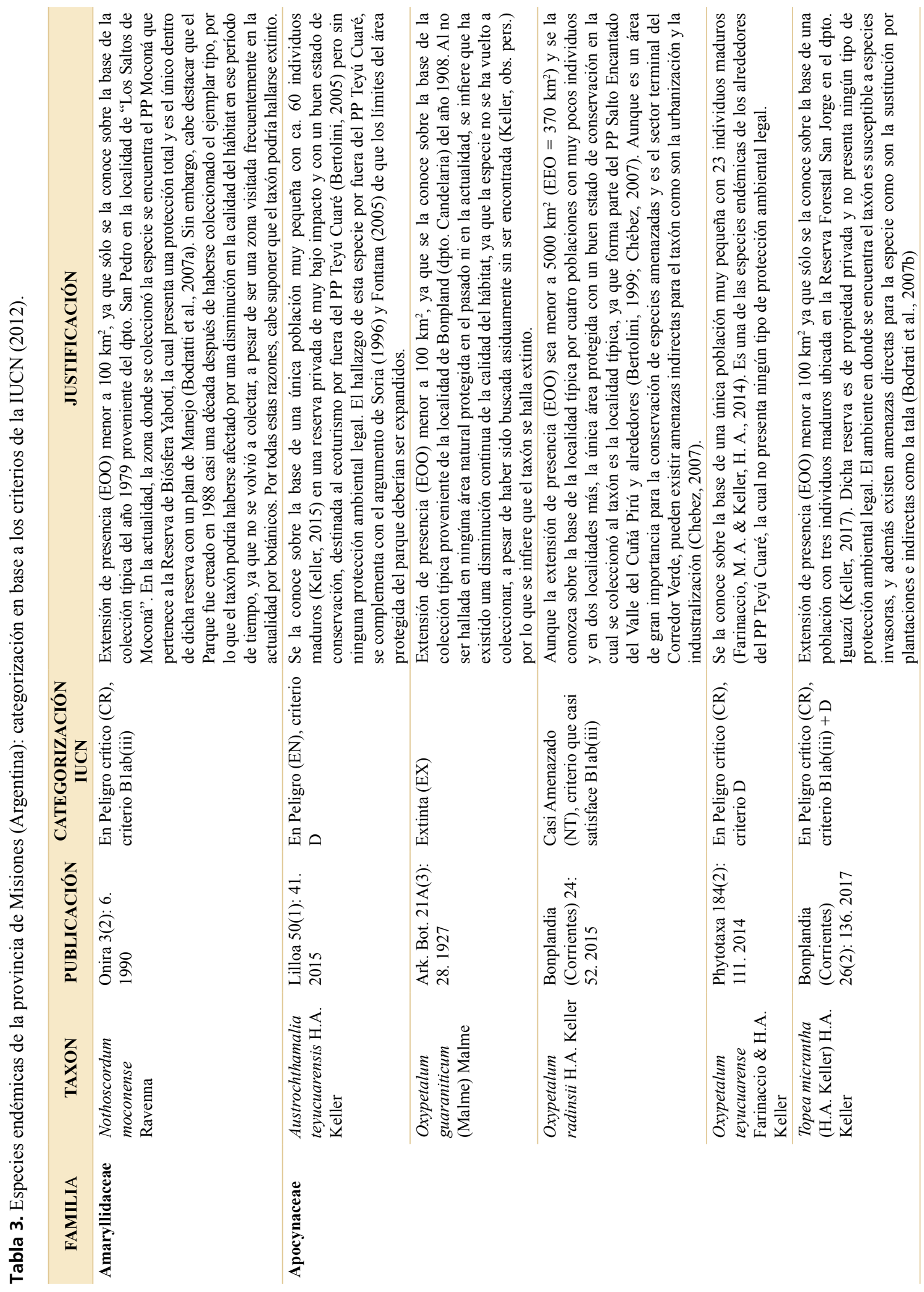




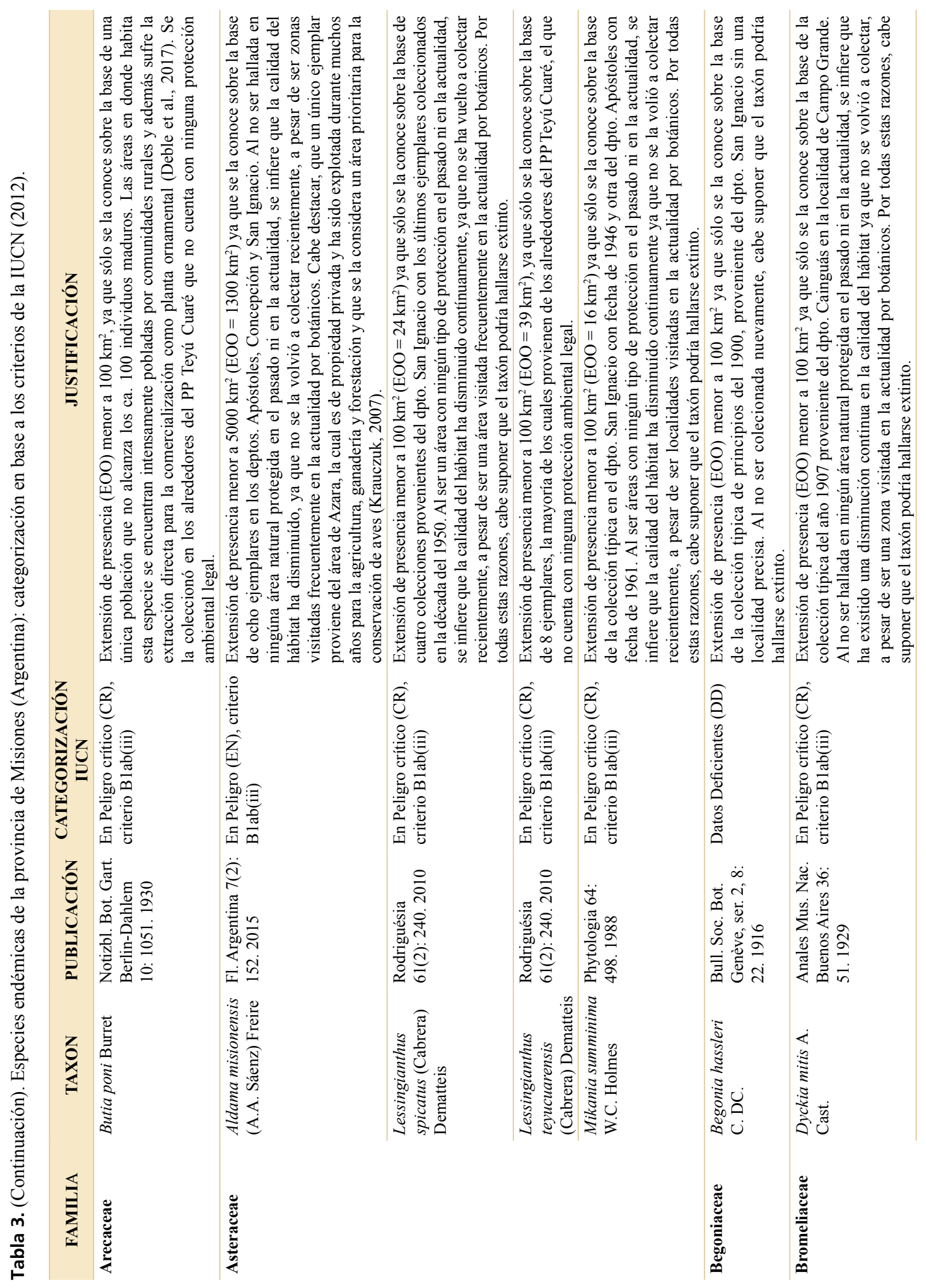




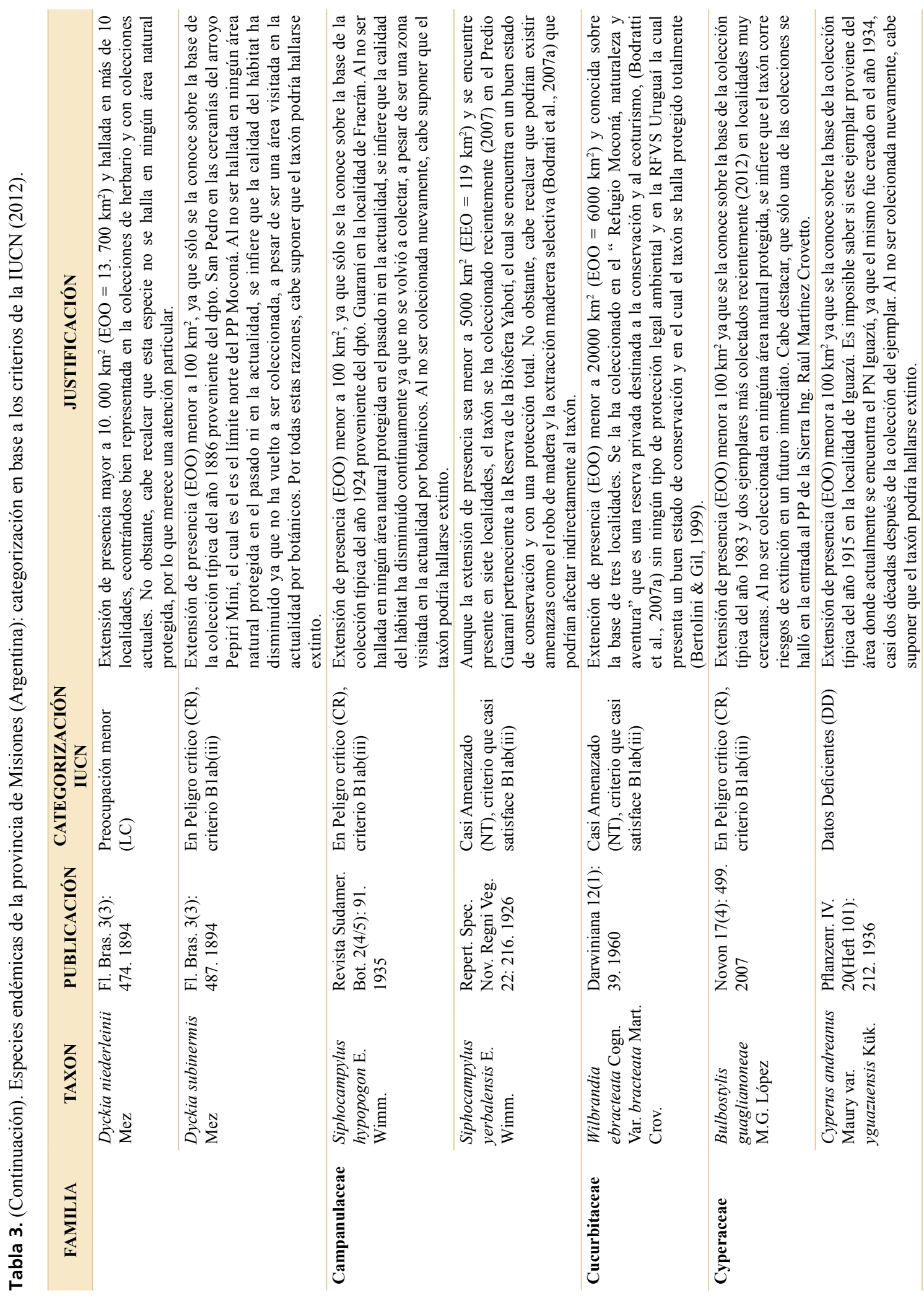




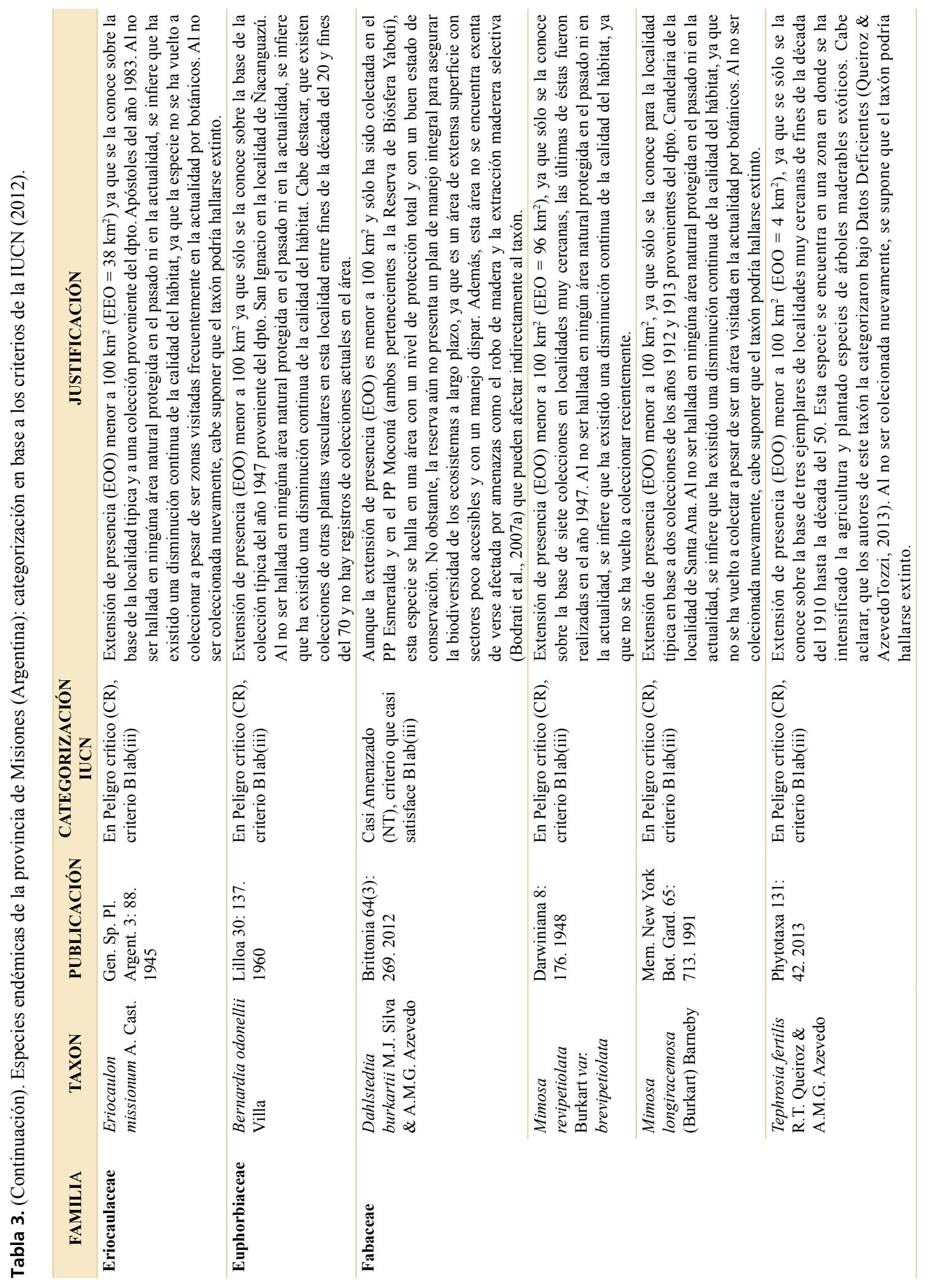




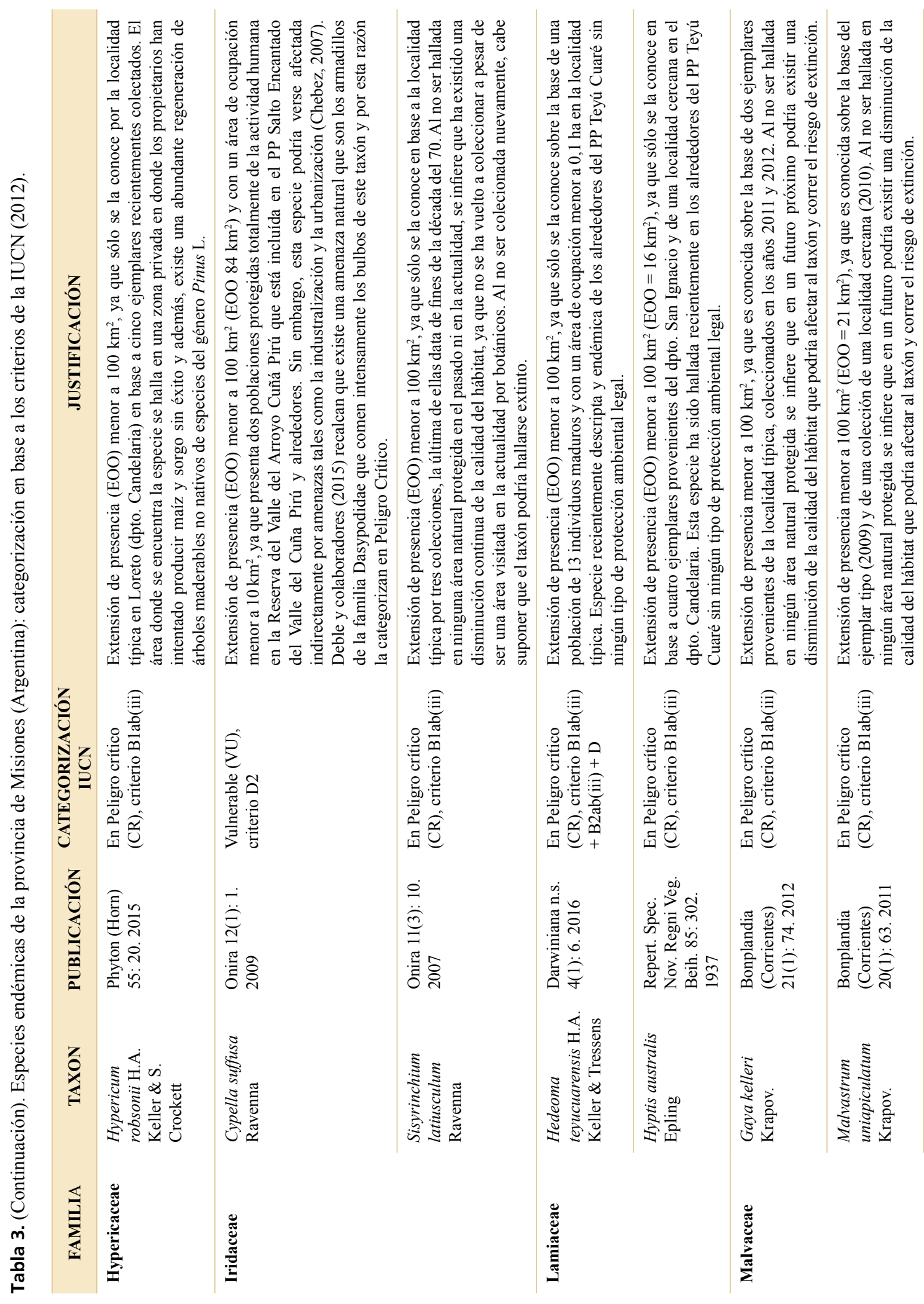




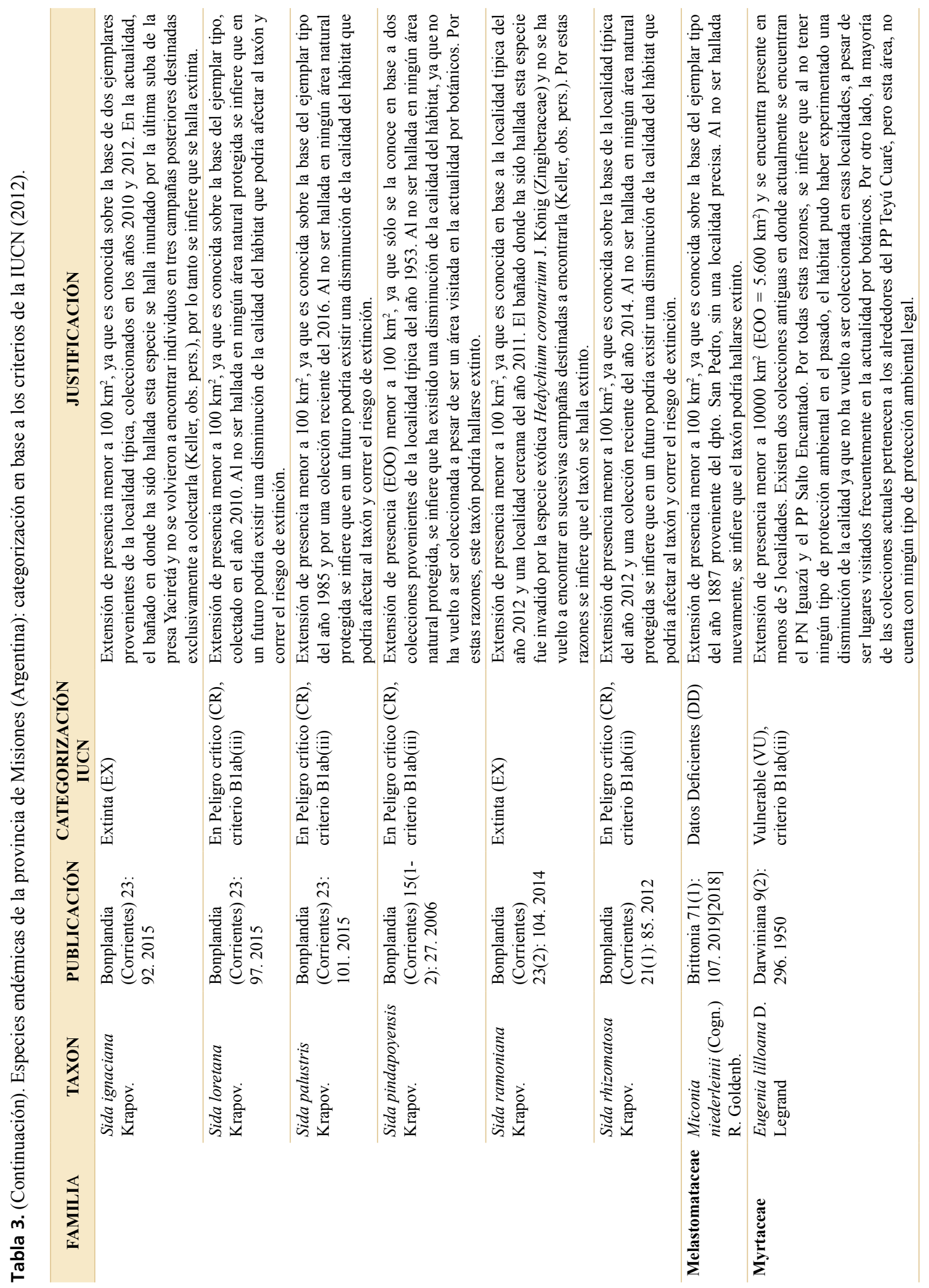




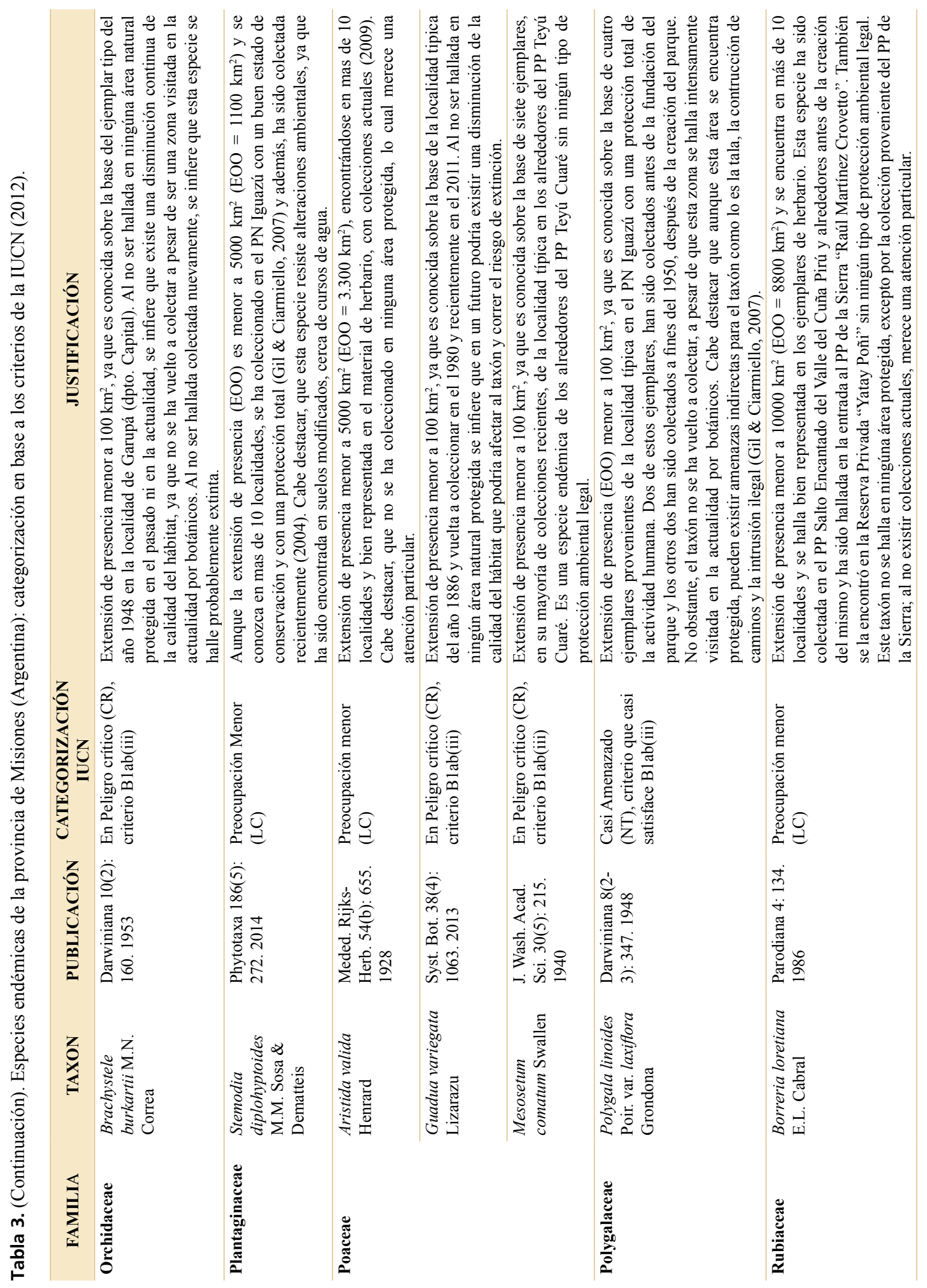




\section{Taxones exclusivos para la Argentina en la provincia}

Es interesante resaltar que un total de 761 taxones, lo que representa poco más de un $22 \%$ del total de los taxones para la provincia, crecen, en el contexto geográfico de la Argentina, sólo en Misiones (Apéndice). Estos taxones pertenecen mayormente a las Asteraceae (72), Orchidaceae (67), Fabaceae (65), Poaceae (38), Apocynaceae (37), entre otras. Además, las familias Vochysiaceae, Lindsaeaceae, Dilleniaceae son familias que en Argentina crecen exclusivamente en la provincia de Misiones, al igual que numerosos géneros, como Acanthostachys Klotzch (Bromeliaceae), Acosmium Schott (Fabaceae), Agarista G. Don ex D. Don (Ericaceae), Aiouea Aubl. (Lauraceae), Amorimia W.R. Anderson (Malpighiaceae), Ateleia (DC.) Benth. (Fabaceae), Austrochthamalia Morillo \& Fontella (Apocynaceae), Copaifera L. (Fabaceae), Cratylia Mart. ex Benth. (Fabaceae), Cryptocarya R. Br. (Lauraceae), Cyclolobium Benth. (Fabaceae), Dahlstedtia Malme (Fabaceae), Deparia Hook. \& Grev. (Athyriaceae), Dicranopteris Bernh. (Gleicheniaceae),
Elachyptera A.C. Sm. (Celastraceae), Euterpe Mart. (Arecaceae), Fischeria DC. (Apocynaceae), Grazielia R.M. King \& H. Rob. (Asteraceae), Gymnosiphon Blume (Burmanniaceae), Heliconia L. (Heliconiaceae), Hymenaea L. (Fabaceae), Kummerowia Schindl. (Fabaceae), Lafoensia Vand. (Lythraceae), Lastreopsis Ching (Dryopteridaceae), Lindsaea Dryand. ex Sm. (Lindsaeaceae), Macropodina R.M. King \& H. Rob. (Asteraceae), Metalepis Griseb. (Apocynaceae), Parapolystichum (Keyserl.) Ching (Dryopteridaceae), Phaeostemma E. Fourn. (Apocynaceae), Pueraria DC. (Fabaceae), Sloanea L. (Elaeocarpaceae), Sweetia Spreng. (Fabaceae), Tetracera L. (Dilleniaceae), Tetrorchidium Poepp. (Euphorbiaceae), Thaumatocaryon Baill. (Boraginaceae), Topea H.A. Keller (Apocynaceae), Trichomanes L. y Vandeboschia Copel. (Hymenophyllaceae), Voyria Aubl. (Gentianaceae).

\section{Nuevas citas para la Argentina}

En la presente contribución se brinda información sobre 16 nuevos registros para la Argentina de taxones nativos o introducidos registrados en la provincia de Misiones (Tabla 4).

Tabla 4. Lista de las nuevas citas para la Argentina. Marcados con $\left(^{*}\right)$ se muestran los taxones introducidos. Abreviatura de los colectores, HAK, Héctor A. Keller; FB, Fernando Biganzoli; OM, Osvaldo Morrone; FOZ, Fernando O. Zuloaga y MK, Mónika Kujawska.

\section{TAXÓN $\quad$ EJEMPLARES}

Acanthocladus brasiliensis (A. St.Hil. \& Moq.) Klotzsch ex Hassk HAK 13874 (Polygalaceae) (CTES)

Babiania angustifolia Sweet* (Iridaceae) (Arecaceae)

HAK 13726; HAK 13727 (CTES)

Calea kristineae Pruski (Asteraceae)
HAK 11713; HAK 13408 (CTES)

Especie considerada hasta el momento como endémica del centro-oeste, sudeste y sur del Brasil. En Misiones se la encontró en una reserva privada, en selva marginal del arroyo Urugua-i en el dpto. Iguazú con una única población de numerosos individuos.

Especie descripta originalmente para Sudáfrica. En Misiones se la encontró conformando poblaciones espontáneas como elemento ruderal en bordes de rutas y terrenos parquizados en Bernardo de Irigoyen y Campinas de Américo, en el dpto. General Manuel Belgrano.

Especie originalmente descripta para el sur del Brasil (Rio Grande do Sul). En Misiones se la encontró en un arbustal remanente de Acca sellowiana (O. Berg) Burret (Myrtaceae) muy deteriorado y reducido por la actividad agropecuaria (plantaciones de yerba mate), en la localidad de Campo Grande (dpto. Cainguás). Las poblaciones de Misiones cuentan con buena dotación de individuos, lo cual es destacable considerando que la localidad típica y única población hasta ahora conocida cuenta con muy pocos individuos.

Especie originalmente descripta para el sur del Brasil (Rio Grande do Sul). En Misiones se la encuentra en pastizales sobre afloramientos rocosos en el dpto. Candelaria, donde crece protegida del pastoreo gracias a que conforma poblaciones dentro de parches de Bromelia balansae Mez (Bromeliaceae). 


\section{A. ZANOTTI ET AL. Biodiversidad Flora Vascular de Misiones}

Tabla 4. (continuación).

\begin{tabular}{|c|c|c|}
\hline TAXÓN & EJEMPLARES & OBSERVACIONES \\
\hline $\begin{array}{l}\text { Crotalaria ochroleuca G. Don* } \\
\text { (Fabaceae) }\end{array}$ & $\begin{array}{l}\text { HAK } 14022 \\
\text { (CTES) }\end{array}$ & $\begin{array}{l}\text { Especie descripta originalmente para África. En el sudeste y sur del } \\
\text { Brasil se la registra como cultivada. En Misiones se la encuentra } \\
\text { naturalizada en bordes de rutas asfaltadas y caminos terrados, donde } \\
\text { forma densas poblaciones en el dpto. Eldorado. }\end{array}$ \\
\hline $\begin{array}{l}\text { Cypella herbertii (Lindl.) Herb. } \\
\text { subsp. brevicristata Ravenna } \\
\text { (Iridaceae) }\end{array}$ & $\begin{array}{l}\text { IAK } 13108 \\
\text { CTES) }\end{array}$ & $\begin{array}{l}\text { Subespecie originalmente descripta para el sur del Brasil (Rio Grande } \\
\text { do Sul) y para el noroeste del Uruguay (Artigas). En Misiones se la } \\
\text { encontró creciendo sobre pastizales anegados del dpto. Candelaria. }\end{array}$ \\
\hline $\begin{array}{l}\text { Dyckia choristaminea } \mathrm{Mez} \\
\text { (Bromeliaceae) }\end{array}$ & $\begin{array}{l}\text { HAK } 13416 \\
\text { (CTES) }\end{array}$ & $\begin{array}{l}\text { Especie originalmente descripta para el sur del Brasil (Rio Grande } \\
\text { do Sul). En Misiones se la encontró habitando en campos abiertos } \\
\text { rodeados de selva, sobre afloramientos basálticos, donde sus } \\
\text { poblaciones cuentan con numerosos individuos. El área donde se } \\
\text { encuentran dichos campos es propiedad de comunidades guaraníes } \\
\text { en el dpto. Cainguás. }\end{array}$ \\
\hline $\begin{array}{l}\text { Endlicheria paniculata (Spreng.) J.F. } \\
\text { Macbr. } \\
\text { (Lauraceae) }\end{array}$ & $\begin{array}{l}\text { HAK } 10420 \\
\text { (CTES) }\end{array}$ & $\begin{array}{l}\text { Especia nativa regitrada para Bolivia, Brasil, Ecuador, Panamá, } \\
\text { Paraguay, Perú y Venezuela. En Misiones se la encuentra en el interior } \\
\text { de la selva en todo el norte de Iguazú. }\end{array}$ \\
\hline $\begin{array}{l}\text { Ficus eximia } \text { Schott } \\
\text { (Moraceae) }\end{array}$ & $\begin{array}{l}\text { HAK } 570 \text { (CTES); } \\
\text { FB } 300 \text { (SI); OM } \\
1244 \text { (SI); OM } \\
1861(\mathrm{SI}) ; \mathrm{FOZ} \\
5065(\mathrm{SI})\end{array}$ & $\begin{array}{l}\text { Especie hasta el momento registrada como endémica para Brasil, en } \\
\text { donde se la encuentra en casi todos los estados. En Misiones crece en } \\
\text { bordes de selva y en la selva marginal de arroyos y ríos en los dptos. } \\
\text { Cainguás, Eldorado, Iguazú, San Ignacio, y San Pedro. }\end{array}$ \\
\hline $\begin{array}{l}\text { Gomphocarpus physocarpus E. Mey* } \\
\text { (Apocynaceae) }\end{array}$ & $\begin{array}{l}\text { HAK } 12280 \\
\text { (CTES, SI); HAK } \\
11996(\mathrm{CTES})\end{array}$ & $\begin{array}{l}\text { Especie nativa del sureste africano y se la halla ampliamente } \\
\text { naturalizada en diferentes países, en donde se la utiliza como planta } \\
\text { ornamental. En Misiones se la cultiva con el mismo fin y se la conoce } \\
\text { con el nombre de "bola de fraile", "bola de viejo". Esta especie forma } \\
\text { poblaciones espontáneas con abundante regeneración natural en bordes } \\
\text { de rutas, sectores domésticos abandonados y campos de pastoreo. }\end{array}$ \\
\hline $\begin{array}{l}\text { Lactuca virosa } \mathrm{L} . * \\
\text { (Asteraceae) }\end{array}$ & $\begin{array}{l}\text { HAK } 1673 \text { (CTES); } \\
\text { HAK } 2050 \text { (CTES); } \\
\text { HAK } 4164 \text { (CTES); } \\
\text { MK } 178 \text { (CTES) }\end{array}$ & $\begin{array}{l}\text { Especie muy difundida en el sur de Europa y también se la ha } \\
\text { registrado en Pakistán, India, Australia y EE. UU, donde crece } \\
\text { en forma silvestre. En Misiones crece de manera espontánea en } \\
\text { bordes de selva, bordes de rutas y caminos y en terrenos de cultivo } \\
\text { abandonados, siendo una especie muy frecuente y ampliamente } \\
\text { distribuida. Los lugareños de las áreas rurales de la provincia } \\
\text { emplean sus vástagos como forraje para cerdos y la llaman } \\
\text { "cerraja lisa". }\end{array}$ \\
\hline $\begin{array}{l}\text { Mimosa dolens Vell. subsp. acerba } \\
\text { (Benth.) Barneby } \\
\text { (Fabaceae) }\end{array}$ & $\begin{array}{l}\text { HAK } 3639 \text { (CTES, } \\
\text { SI) }\end{array}$ & $\begin{array}{l}\text { Subespecie nativa de Bolivia, Paraguay, centro-oeste, sudeste y sur } \\
\text { del Brasil. En Misiones se la encontró habitando en montes bajos } \\
\text { con Araucarias emergentes a más de } 800 \mathrm{~m} \text { s.m., en el Dpto. General } \\
\text { Manuel Belgrano. }\end{array}$ \\
\hline $\begin{array}{l}\text { Orthosia congesta Decne. } \\
\text { (Apocynaceae) }\end{array}$ & $\begin{array}{l}\text { HAK } 13903 \\
\text { (CTES); HAK } \\
12127 \text { (CTES); } \\
\text { HAK } 12129 \\
\text { (CTES) }\end{array}$ & $\begin{array}{l}\text { Especie nativa de Paraguay y nordeste, sudeste y sur del Brasil. En } \\
\text { Misiones se la encontró en el interior de la selva primaria y secundaria } \\
\text { en los dptos. Montecarlo y Eldorado. }\end{array}$ \\
\hline $\begin{array}{l}\text { Peltaea edouardii (Hochr.) Krapov. \& } \\
\text { Cristóbal } \\
\text { (Malvaceae) }\end{array}$ & HAK 3548 (CTES) & $\begin{array}{l}\text { Especie nativa del Paraguay y centro-oeste, sudeste y sur del Brasil. } \\
\text { En Misiones se la recolectó en Campinas de Américo, dpto. General } \\
\text { Manuel Belgrano a más de } 800 \text { m s.m., en un pequeño sector de pastizal } \\
\text { con presencia de elementos subarbustivos propios del Bioma Cerrado. }\end{array}$ \\
\hline $\begin{array}{l}\text { Podocarpus lambertii Klotzsch ex } \\
\text { Endl.* } \\
\text { (Podocarpaceae) }\end{array}$ & $\begin{array}{l}\text { HAK } 10336 \\
\text { (CTES) }\end{array}$ & $\begin{array}{l}\text { Especie de las selvas húmedas del sur y sudeste del Brasil. En } \\
\text { Misiones se halla introducida en varios departamentos de la provincia } \\
\text { donde regenera naturalmente, principalmente en bosques secundarios } \\
\text { próximos a las áreas donde se la cultiva. }\end{array}$ \\
\hline $\begin{array}{l}\text { Sida nemorensis Mart. ex Colla } \\
\text { (Malvaceae) }\end{array}$ & $\begin{array}{l}\text { HAK } 732 \text { (CTES); } \\
\text { HAK } 3040 \text { (CTES); } \\
\text { HAK } 4974 \text { (CTES) }\end{array}$ & $\begin{array}{l}\text { Descripta para el sur del Brasil (Paraná y Santa Catarina). En Misiones } \\
\text { se la encontró en pastizales de altura sobre afloramientos rocosos } \\
\text { a más de } 760 \mathrm{~m} \text { s.m., en vegetación ruderal resultante de cultivos } \\
\text { anuales en comunidades indígenas y en bordes de rutas asfaltadas. }\end{array}$ \\
\hline
\end{tabular}




\section{Taxones introducidos}

En la provincia hay un total de 200 taxones introducidos, pertenecientes a 55 familias. De éstas, la familia Poaceae es la mejor representada, con 42 taxones, seguida de las Asteraceae, con un total de 21. Tan sólo 18 taxones se encuentran introducidos y sólo presentes en la Argentina en la provincia de Misiones. Los géneros Babiania Ker Gawl., Gomphocarpus R. Br. y Plumeria (Apocynaceae), Deparia (Athyriaceae), Vernicia (Euphorbiaceae), Kummerovia (Fabaceae), Syzygium (Myrtaceae), Cymbopogon (Poaceae), Podocarpus (Podocarpaceae), Eriobotrya (Rosaceae) y Brugmansia (Solanaceae) son géneros introducidos sólo presentes en Misiones.

\section{AGRADECIMIENTOS}

Este trabajo fue parcialmente subsidiado con fondos de la Agencia Nacional de Promoción Científica y Tecnológica, PICT-2418-2016 y el Consejo Nacional de Investigaciones Científica y Técnicas, PIP-782. Los viajes de campo, de investigadores del IBODA a la provincia de Misiones, fueron realizados con subsidios de la National Geographic Society, \#6042-97, \#6698-00, \#7425-03. Deseamos expresar nuestro agradecimiento al personal del Instituto de Botánica del Nordeste (IBONE) y el Instituto de Botánica Darwinion (IBODA) por la colaboración recibida. También agradecemos a Agustín Abba (CEPAVE) por sus sugerencias en la caracterización de los endémicos. Por último, agradecemos a los editores de la revista y revisores por la valiosa contribución recibida en pos de la mejora de este artículo.

\section{BIBLIOGRAFÍA}

Bachman, S.; J. Moat, A. W. Hill, J. de la Torre \& B. Scott. 2011. Supporting Red List threat assessments with GeoCAT: geospatial conservation assessment tool. ZooKeys 150: 117126.

Barzetti, V. 1993. Parques y progreso. Áreas protegidas y desarrollo económico en América Latina y el Caribe. UICN

Bertolini, M. P. 1999. Plan de Manejo del Parque Provincial Salto Encantado del Valle del Cuñá Pirú. Ministerio de Ecología y Recursos Naturales Renovables de la provincia de Misiones. $99 \mathrm{pp}$.
Bertolini, M. P. 2005. Documento base para la discusión del Plan de Manejo del Parque Provincial Teyú Cuaré. Instituto Universitario Gastón Dachary. 105 pp.

Bertolini, M. P. \& G. Gil. 1999. Plan de manejo del Parque Provincial Urugua-í. Ministerio de Ecología y Recursos Naturales Renovables de la Provincia de Misiones y Administración de Parques Nacionales Delegación Regional Nordeste. 96 pp.

Biganzoli, F. \& P. Peralta. 2000. Informe preliminar de la flora del Valle del arroyo Cuña Pirú (Misiones, Argentina). Libro de Resúmenes, XII Reunión Anual de la Sociedad Botánica de Chile y XXVII Jornadas Argentinas de Botánica. Gayana, Botanica 57 (suplemento): 98.

Biganzoli, F. \& M. E. Múlgura de Romero. 2004. Inventario florístico del Parque Provincial Teyú Cuaré y alrededores (Misiones, Argentina). Darwiniana 42(1-4): 1-24.

Bodrati, A.; K. Cockle; V. Matuchaka \& C. Maders. 2007a. Reserva de la Biósfera Yabotí. En A. S. Di Giacomo, M. V. De Francesco \& E. G. Coconier (eds.). Áreas importantes para la conservación de las aves en Argentina. Sitios prioritarios para la conservación de la biodiversidad: 300-302.

Bodrati, A.; J. I. Areta, M. Codesido \& N. Rey. 2007b. Parque Provincial Uruzú y Reserva Forestal San Jorge. En Di Giacomo, A. S.; M. V. De Francesco \& E. G. Coconier (eds.). 2007. Áreas importantes para la conservación de las aves en Argentina. Sitios prioritarios para la conservación de la biodiversidad: 282-284.

Cabrera, A. L. 1976. Regiones fitogeográficas de la República Argentina, en Enciclopedia Argentina de Agricultura $y$ Jardinería 2(1). ACME, Buenos Aires.

Chébez, J. C. 1996. Misiones Ñu. Campos Misioneros, algo más que el confín de la selva. Nuestras aves, Asociación Ornitológica del Plata: 4-16.

Chebez, J. C. 2007. Parque Provincial Salta Encantado del Valle del Cuñá-Pirú. En Di Giacomo, A. S.; M. V. De Francesco \& E. G. Coconier (eds.). 2007. Áreas importantes para la conservación de las aves en Argentina. Sitios prioritarios para la conservación de la biodiversidad: 303-304.

Daviña, J. R.; M. E. Rodríguez, A. I. Honfi, G. J. Seijo, I. Insaurralde \& R. Guillen. 1999. Floristic studies of the Moconá Park, Misiones, Argentina. Candollea 54: 231-249.

Deble, L. P.; F. da S. Alves, A. González, A. S. de Oliveira-Deble. 2015. Three new species of Cypella (Iridaceae) from South America, and taxonomic delimitation of C. suffusa Ravenna. Phytotaxa 236(2): 101-120. DOI: 10.11646/phytotaxa.236.2.1

Deble, L. P.; H. A. Keller \& F. S. Alves. 2017. Resurrection and epitypification of Butia poni (Arecaceae), a neglected palm micro-endemic in the grasslands of Misiones, Argentina. Phytotaxa 316(2): 171-180. 


\section{A. ZANOTTI ET AL. Biodiversidad Flora Vascular de Misiones}

Delucchi, G. \& H. A. Keller. 2010. La naturalización del "níspero" Eriobotrya japónica (Rosaceae, Maloideae) en la Argentina. Bonplandia (Corrientes) 19(1): 71-77.

Delucchi, G., H. A. Keller \& J. A. Hurrell. 2016. Cinnamomum glanduliferum y $C$. verum (Lauraceae) naturalizadas en la Argentina. Bonplandia (Corrientes) 25(1): 33-41.

Dematteis, M.; H. A. Keller \& A. J. Vega. 2012. Piptocarpha angustifolia (Vernonieae, Asteraceae), nueva cita para la Flora Argentina. Boletín de la Sociedad Argentina de Botánica 47(3-4): 451-455.

Di Bitetti, M. S., G. Placci \& L. A. Dietz. 2003. Una vision de biodiversidad para la ecorregión del bosque Atlántico del Alto Paraná: diseño de un paisaje para la conservación y prioridades para las acciones de conservación. World Wildlife Fund, Washington DC.

Ezcurra, C. \& M. E. Múlgura de Romero. 2001. Sobre la presencia de Ditassa acerosa (Asclepiadaceae) en la Argentina. Hickenia 3: 95-97.

Farinaccio, M. A. \& H. A. Keller. 2014. Novelties in Oxypetalum (Apocynaceae-Asclepiadoideae) for the Argentine Flora. Phytotaxa 184: 109-114.

Fontana, J. L. 1998. Análisis sistemático-ecológico de la flora del sur de Misiones (Argentina). Candollea 53: 211-300.

Fontana, J. L. 2005. Una propuesta para la conservación de los pajonales del Diplothemio-Axonopodetum. San Ignacio, Provincia de Misiones (Argentina). Facena 21: 55-67.

Fontana, J. L. 2015. Flora y vegetación del nordeste de Corrientes y sur de Misiones. En Bauni, V., Homberg, M. \& V. Capmourteres (eds.). El Patrimonio Natural y Cultural en el área de influencia del Embalse de Yacyretá, Argentina. Capítuo 1: 9-27. Fundación de Historia Natural Félix de Azara, Buenos Aires.

Freire, S. E.; E. Urtubey, G. Sancho, N. D. Bayón, L. Katinas, G. Gutiérrez, D. A. Giuliano, A. A. Sáenz, L. Iharlegui \& G. Delucchi. 2006. Inventario de la biodiversidad vegetal de la provincia de Misiones: Asteraceae. Darwiniana 44(2): 375-452.

Galindo-Leal, C. \& I. G. Câmara. 2003. The Atlantic forest of South America: biodiversity status, threats and outlook. Island Press, Washington DC.

Gatti, F. E. \& H. A. Keller. 2019. Voyria aphylla (Gentianaceae), la primera eudicotiledónea micoheterótrofa registrada para la flora argentina. Bonplandia (Corrientes) 28(2): 159-162.

Gil, G. \& D. Ciarmiello. 2007. Parque Nacional Iguazú y alrededores. En Di Giacomo, A. S.; M. V. De Francesco \& E. G. Coconier (eds.). Áreas importantes para la conservación de las aves en Argentina. Sitios prioritarios para la conservación de la biodiversidad: 278-280. Aves Argentinas/ Asociación Ornitológica del Plata, Buenos Aires.
Hurrell, J. A.; G. Delucchi \& H. A. Keller. 2011. Carya illinoinensis (Juglandaceae) adventicia en la Argentina. Bonplandia (Corrientes) 20: 47-54.

Hurrell, J. A.; G. Delucchi \& H. A. Keller. 2017. Primer registro de Syzygium jambos (Myrtaceae) naturalizada en Misiones, Argentina. Bonplandia (Corrientes) 26(2): 125-132.

Hurrell, J. A.; G. Delucchi, H. A. Keller, P. Stampella \& E. L. Guerrero. 2012. Bryophyllum (Crassulaceae): especies ornamentales naturalizadas en la Argentina. Bonplandia (Corrientes) 21(2): 169-181.

IUCN. 2012. Guidelines for Application of IUCN Red List Criteria at Regional and National Levels: Version 4.0. IUCN Species Survival Commission, International Union for the Conservation of Nature. Gland, Switzerland and Cambridge, UK. iii +41 pp.

Judkevich, M. D.; R. M. Salas \& H. A. Keller. 2016. Randia brevituba (Rubiaceae), a New Species from the Southern Cone of America and Comments on Randia armata. Systematic Botany 41: 238-244.

Keller, H. A. 2007. Sobre la presencia en la Argentina de Lobelia reniformis (Campanulaceae) y Thaumatocaryon dasyanthum (Boraginaceae). Bonplandia (Corrientes) 16(34): 265-269.

Keller, H. A. 2009. La presencia en Argentina de Berberis laurina Billb. (Berberidaceae), una especie de uso múltiple. Kurtziana 35(1): 9-13.

Keller, H. A. 2010a. Phytolacca thyrsiflora (Phytolaccaceae) en la Argentina. Bonplandia (Corrientes) 19(1): 27-30.

Keller, H. A. 2010b. Aristolochia stomachoides (Aristolochiaceae), nueva cita para la Flora Argentina. Bonplandia (Corrientes) 19(2): 121-125.

Keller, H. A. 2011. Gymnosiphon divaricatus (Burmanniaceae), nuevo registro de una mico-heterótrofa para la Argentina. Darwiniana 49: 94-98.

Keller, H. A. 2015a. Austrochthamalia teyucuarensis (Apocynaceae: Asclepiadoideae), una nueva especie endémica de Misiones, Argentina. Lilloa 52(1): 40-45.

Keller, H. A. 2015b. Oxypetalum radinsii (Apocynaceae: Asclepiadoideae), una nueva especie endémica de Misiones, Argentina. Bonplandia (Corrientes) 24(1): 51-56.

Keller, H. A. 2017. Aenigma, un género sudamericano de Apocynaceae (Asclepiadoideae, Asclepiadeae) con tricomas vibrátiles en la corola. Lilloa 54: 58-73.

Keller, H. A. \& S. Cáceres. 2018. Oxypetalum chodatianum (Apocynaceae) en el Nordeste Argentino. Paraguay Biodiversidad 5(6): 49-51.

Keller, H. A. \& S. L. Crockett. 2015. Hypericum robsonii spec. nova sect. Trigynobrathys (Hypericaceae) from the Misiones province in Argentina. Phyton (Horn) 55(1): 17-29. 
Keller, H. A. \& G. J. Kline. 2008. Agrimonia villosa (Rosaceae), nuevo registro para la Argentina. Darwiniana 46: 356-359.

Keller, H. A. \& S. G. Tressens. 2007. Presencia en Argentina de dos especies de uso múltiple: Acca sellowiana (Myrtaceae) y Casearia lasiophylla (Flacourtiaceae). Darwiniana 45: 204-212.

Keller, H. A. \& S. G. Tressens. 2016. Hedeoma teyucuarensis (Lauraceae), una nueva especie de Misiones, Argentina. Darwiniana, nueva serie 4(1): 5-11.

Keller, H. A.; D. Sampaio \& S. G. Tressens. 2012. Primer registro de Sloanea (Elaeocarpaceae) para la Argentina. Darwiniana 50(1): 157-161.

Keller, H. A.; P. C. Stampella, G. Delucchi \& J. A. Hurrell. 2013. Vernicia fordii y Aleurites moluccanus (Euphorbiaceae) en la Argentina. Naturalización y etnobotánica. Boletín de la Sociedad Argentina de Botánica 48(3-4): 553-561.

Krauczuk, E. R. 2007. Azara. En Di Giacomo, A. S.; M. V. De Francesco \& E. G. Coconier (eds.). 2007. Áreas importantes para la conservación de las aves en Argentina. Sitios prioritarios para la conservación de la biodiversidad: 312315. Aves Argentinas/Asociación Ornitológica del Plata, Buenos Aires.

López Cristóbal, L.; L. A. Grance, D. C. Maiocco \& B. I. Eibl. 1996. Estructura y composición florística del bosque nativo, en el Predio Guaraní. Yvyraretá 7: 30-36.

Marquez, G. J. \& H. A. Keller. 2018. Primer registro del género Actinostachys (Schyzaceae) para la Argentina. Boletín de la Sociedad Argentina de Botánica 53 (3): 459-463.

Marquez, G. J.; A. Yañez \& D. Ocampo Terraza. 2020. Primer registro de Megalastrum oreocharis (Dryopteridaceae) para Argentina. Darwiniana, n.s. 8

Martínez Crovetto, R. 1963. Esquema fitogeográfico de la Provincia de Misiones (República Argentina). Bonplandia (Corrientes) 1: 171-223.

MERNR. 2020. https://ecologia.misiones.gob.ar/anp-listadodeareas-naturales-protegidas/ (Acceso: 13 Marzo 2020)

Meza Torres, E. I.; H. A. Keller \& C. Brussa. 2010. New Records of Ferns from Northeastern Argentina and Uruguay. American Fern Journal 100: 183-187.

Morillo, G. \& H. A. Keller. 2016. Un nuevo género y dos nuevas combinaciones en las Gonolobinae (Apocynaceae: Asclepiadoideae). Bonplandia (Corrientes) 25: 129-143.

Morillo, G.; S. A. Cáceres Moral \& H. A. Keller. 2016. Cristobalia, un nuevo género sudamericano de Gonolobinae (Apocynaceae, Asclepiadoideae, Asclepiadeae). Pittiera 40: 122-143.
Myers, M., R. A., Mittermeier, C. G. Mittermeier, G. A. B. Da Fonseca \& J. Kent. 2000. Biodiversity hotspots for conservation priorities. Nature 403: 853-858.

O'Leary, N. \& H. A. Keller. 2018. Primer registro de Citharexylum solanaceum (Verbenaceae) para la flora Argentina. Darwiniana, nueva serie 6(1): 108-112.

Oyarzabal, M.; J. Clavijo, L. Oakley, F. Biganzoli, P. Tognetti, I. Barberis, H. M. Maturo, R. Aragón, P. I. Campanello, D. Prado, M. Oesterheld \& R. J. C. León. 2018. Unidades de vegetación de la Argentina. Ecología Austral 28: 40-63.

Peralta, P. 2001. Clidemia (Melastomataceae) una nueva cita para la flora argentina. Hickenia 3: 115-117.

Ponce, M. M. 2001. Additions and corrections to the pteridophyte flora of northeastern Argentina. American Fern Journal 91: 70-72.

Queiroz, R. T. \& A. M. G. Azevedo-Tozzi. 2013. A new species of Tephrosia (Leguminosae-Papilionoideae-Millettieae) from Misiones, Argentina. Phytotaxa 131(1): 41-44.

Rodríguez, A. F \& Silva, M. 2012. Ecorregión Selva Paranaense. En Morello, J., Mateucci, S. \& Rodríguez, A. (eds.). Ecorregiones y complejos ecosistemicos argentinos. Capítulo 6: 225-245. Orientación Gráfica Editora, Buenos Aires.

Soria, A. 1996. Fundamentos Técnicos para la Ampliación del Parque Provincial Teyú Cuaré (Provincia de Misiones). Delegación Técnica Regional Nordeste Argentino. Administración de Parques Nacionales, Iguazú.

Thiers, B. [permanentemente actualizado, consulta 2020] Index Herbariorum: a global directory of public herbaria and associated staff. New York Botanical Garden's Virtual Herbarium, http://sweetgum.nybg.org/ih

Tressens, S. G. \& V. Revilla. 1996. Relevamiento florístico del Predio Guaraní (prov. Misiones). Libro de Resúmenes, XXV Jornadas Argentinas de Botánica: 465. Mendoza.

Tressens, S. G. \& H. A. Keller. 2006. Eugenia ramboi (Myrtaceae) en Argentina. Darwiniana 44(1): 294-297.

Velazco, S. J. E.; F. Galvão, H. A. Keller \& N. A. Bedrij. 2018. Cerrados in Argentina? Structure, diversity and biogeography of the woody component of a savanna in the Misiones Province. Rodriguésia 69(2): 335-349.

Zanotti, C. A. \& H. A. Keller. 2017. Nuevo registro y novedades taxonómicas de Piper miquelianum (Piperaceae secc. Ottonia) para la Flora Argentina. Bonplandia (Corrientes) 26(1): 51-56.

Zuloaga, F. O.; O. Morrone \& D. Rodríguez. 1999. Análisis de la biodiversidad en plantas vasculares de la Argentina. Kurtziana 27: 17-167.

Zuloaga, F. O.; M. J. Belgrano \& C. A. Zanotti. 2019. Actualización del catálogo de plantas vasculares del Cono Sur. Darwiniana, nueva serie 7(2): 208-278. 


\footnotetext{
APÉNDICE. INVENTARIO DE PLANTAS VASCULARES DE LA PROVINCIA DE MISIONES.

* Nuevas citas de taxones nativos o introducidos para la Argentina (16).

** Taxones endémicos de la provincia de Misiones (47).

*** Taxones nativos o introducidos solo presentes, para la Argentina, en la provincia de Misiones (696). Notar que las dos categorías anteriores también corresponden a taxones exclusivos de la provincia de Misiones.
}

\section{LYCOPHYTA Y MONILOPHYTA}

\section{ANEMIACEAE}

Anemia Sw., nom. cons.

Anemia phyllitidis (L.) Sw. var. phyllitidis

Hierba. Perenne. Nativa. 0-700 m.

Distribución: APO, CAI, ELD, GMB, GSM, GUA, LNA, OBE, SIG, SJA, SPE.

Ejemplar de referencia: Biganzoli, F. 1693 (SI).

Anemia phyllitidis (L.) Sw. var. tweedieana (Hook.) Hassl.

Hierba. Perenne. Nativa. 0-500 m.

Distribución: CAI, GMB, GUA.

Ejemplar de referencia: Deginani, N. B. 1133 (SI).

Anemia simplicior (H. Christ.) Mikel

Hierba. Perenne. Nativa. 0-500 m.

Distribución: CAN, GMB, MON.

Ejemplar de referencia: Keller, H. A. 4031 (CTES).

Anemia tomentosa (Savigny) Sw. var. anthriscifolia (Schrad.) Mickel

Hierba. Perenne. Nativa. 0-600 m.

Distribución: APO, CAI, GMB, LNA, OBE, SIG, SPE.

Ejemplar de referencia: Guillén, R. 130 (MNES, SI).

Anemia tomentosa (Savigny) Sw. var. tomentosa Hierba. Perenne. Nativa. 0-700 m.

Distribución: APO, CAN, ELD, GUA, IGU, OBE, SIG, SPE.

Ejemplar de referencia: Keller, H. A. 348 (CTES).

Anemia wettsteinii $\mathrm{H}$. Christ ${ }^{* * *}$ )

Hierba. Perenne. Nativa. 0-500 m.

Distribución: ELD, IGU.

Ejemplar de referencia: Keller, H. A. 3095 (CTES).

\section{ASPLENIACEAE}

\section{Asplenium L.}

Asplenium abscissum Willd.

Hierba. Perenne. Nativa. 0-500 m.

Distribución: CAI, CAN, GUA, SIG, SPE.

Ejemplar de referencia: Burkart, A. 1404 (SI).

Asplenium alatum Humb. \& Bonpl. ex Willd. $\left.{ }^{* * *}\right)$ Hierba. Perenne. Nativa. 0-700 m.

Distribución: SPE.

Ejemplar de referencia: Johnson, A. E. 153 (SI).

Asplenium argentinum Hieron.

Hierba terrestre. Perenne. Nativa. 0-700 m.

Distribución: CAI, GMB, GSM, GUA, SIG, SPE.

Ejemplar de referencia: Zuloaga, F. O. 5469 (SI).

Asplenium auriculatum $\left.\mathrm{Sw} .{ }^{* * *}\right)$

Hierba epífita. Perenne. Nativa. 0-500 m.

Distribución: IGU.

Ejemplar de referencia: Eskuche, U. G. 1700 (CTES, SI).

\section{Asplenium auritum $S w$.}

Hierba epífita o epipétrica. Perenne. Nativa. 0-500 m.

Distribución: GUA, IGU, SIG.

Ejemplar de referencia: Hunziker, J. H. 2636 (SI).

Asplenium balansae (Baker) L. Sylvestre $\left(^{* * *}\right)$

Hierba terrestre. Perenne. Nativa. 0-500 m.

Distribución: CAI, CON, IGU.

Ejemplar de referencia: Zuloaga, F. O. 5630 (SI).

\section{Asplenium brasiliense $\mathrm{Sw} .\left({ }^{* *}\right)$}

Hierba terrestre. Perenne. Nativa. 0-700 m.

Distribución: APO, CON, ELD, GMB, GSM, GUA, IGU.

Ejemplar de referencia: Guillén, R. 119 (MNES, SI). 
Asplenium claussenii Hieron.

Hierba. Perenne. Nativa. 0-700 m.

Distribución: APO, CAI, CAN, GMB, GUA, LNA, SIG, SPE.

Ejemplar de referencia: Morrone, O. 1531 (SI).

\section{Asplenium cuspidatum Lam.}

Hierba. Nativa. 0-500 m.

Distribución: SPE.

Ejemplar de referencia: Belgrano, M. J. 622 (SI).

\section{Asplenium depauperatum Fée}

Hierba terrestre o saxícola. Perenne. Nativa. 0-500 m.

Distribución: SIG.

Ejemplar de referencia: Diem, J. 1401 (SI).

\section{Asplenium formosum Willd.}

Hierba epífita o epipétrica. Perenne. Nativa. 0-500 m.

Distribución: GMB, SIG.

Ejemplar de referencia: Morrone, O. 3210 (SI).

\section{Asplenium gastonis Fée}

Hierba epífita. Perenne. Nativa. 0-800 m.

Distribución: CAI, CAN, GMB, GUA, IGU, OBE, SPE.

Ejemplar de referencia: Morrone, O. 1951 (SI).

\section{Asplenium inaequilaterale Willd.}

Hierba. Perenne. Nativa. 0-500 m.

Distribución: CAI, IGU, SIG.

Ejemplar de referencia: Riemel, R. 31 (SI).

Asplenium kunzeanum Klotzsch ex Rosenst. $\left({ }^{* *}\right)$ Hierba. Perenne. Nativa. 0-500 m.

Distribución: GUA.

Ejemplar de referencia: Keller, H. A. 1938 (CTES).

\section{Asplenium mucronatum $C$. Presl $\left(^{* * *}\right)$}

Hierba epífita. Perenne. Nativa. 0-800 m.

Distribución: IGU.

Ejemplar de referencia: Eskuche, U. G. 1761 (SI).

\section{Asplenium pulchellum Raddi $\left(^{* * *}\right)$}

Hierba. Perenne. Nativa. 0-500 m.

Distribución: CAI, CAN, GMB, IGU.

Ejemplar de referencia: Múlgura, M. E. 3893 (MNES, SI).
Asplenium radicans $\mathrm{L} .\left({ }^{* * *}\right)$

Hierba terrestre. Perenne. Nativa. 0-800 m.

Distribución: IGU.

Ejemplar de referencia: Riemel, R. 120 (SI).

Asplenium scandicinum Kaulf. $\left({ }^{* * *}\right)$

Hierba epífita. Perenne. Nativa. 0-800 m.

Distribución: GMB, GUA, IGU, SPE.

Ejemplar de referencia: Morrone, O. 1542 (SI).

Asplenium sellowianum $C$. Presl ex Hieron. Hierba. Perenne. Nativa. 0-600 m.

Distribución: CAN, GMB, SIG.

Ejemplar de referencia: Pérez, A. 180 (SI).

Asplenium serra Langsd. \& Fisch.

Hierba terrestre o epífita. Perenne. Nativa. 0-600 m.

Distribución: GUA, SIG.

Ejemplar de referencia: Keller, H. A. 52 (CTES, SI).

Asplenium serratum L. $\left.{ }^{* * *}\right)$

Hierba epífita. Perenne. Nativa. 0-800 m.

Distribución: ELD, GMB, IGU, SIG, SPE.

Ejemplar de referencia: Burkart, A. 14731 (SI).

Asplenium stuebelianum Hieron.

Hierba epífita. Perenne. Nativa. 0-800 m.

Distribución: IGU.

Ejemplar de referencia: Keller, H. A. 2330 (CTES).

Asplenium ulbrichtii Rosenst.

Hierba epífita o terrestre. Perenne. Nativa. 0-600 m.

Distribución: CAI, GMB, GUA, LNA.

Ejemplar de referencia: Deginani, N. B. 1376 (SI).

Asplenium uniseriale Raddi $\left({ }^{* *}\right)$

Hierba terrestre. Perenne. Nativa. 0-800 m.

Distribución: SPE.

Ejemplar de referencia: Marquez, G. J. 51 (LP).

\section{Hymenasplenium Hayata}

Hymenasplenium laetum (Sw.) L. Regalado \& Prada Hierba terrestre. Perenne. Nativa. 0-600 m. Distribución: CAN, GUA, IGU, SPE. Ejemplar de referencia: Pérez, A. 30 (G, LP, MNES). 
Hymenasplenium triquetrum (N. Murak. \& Moran) L. Regalado \& Prada ( $\left.{ }^{* * *}\right)$

Hierba. Perenne. Nativa. 100-800 m.

Distribución: CAI, CAN, GMB, GUA.

Ejemplar de referencia: Montes, J. E. s.n. (LP).

\section{ATHYRIACEAE}

Deparia Hook. \& Grev.

\section{Deparia petersenii (Kunze) M.Kato ${ }^{* * *}$ )}

Hierba rupícola. Perenne. Introducida. 0-500 m. Distribución: MON.

Ejemplar de referencia: Keller, H. A. 5943 (CTES).

\section{Diplazium Sw.}

Diplazium ambiguum Raddi $\left({ }^{* *}\right)$

Hierba. Perenne. Nativa. 0-500 m.

Distribución: IGU, SPE.

Ejemplar de referencia: Belgrano, M. J. 589 (SI).

Diplazium cristatum (Desr.) Alston

Hierba. Perenne. Nativa. 0-700 m.

Distribución: CAI, ELD, GMB, GSM, GUA, IGU, SPE.

Ejemplar de referencia: Guillén, R. 118 (MNES, SI).

\section{Diplazium herbaceum Fée $\left({ }^{* *}\right)$}

Hierba. Perenne. Nativa. 0-500 m.

Distribución: GMB, GUA, SIG.

Ejemplar de referencia: Meyer, T. 11710 (LIL).

\section{Diplazium lindbergii (Mett.) H. Christ (***)}

Hierba terrestre. Perenne. Nativa. 300-600 m.

Distribución: GMB, SPE.

Ejemplar de referencia: Morrone, O. 1362 (SI).

Diplazium moccennianum (Sodiro) C. Chr. ${ }^{* * *}$ )

Hierba. Perenne. Nativa. 0-500 m.

Distribución: IGU.

Ejemplar de referencia: Vanni, R. O. 3253 (CTES).

Diplazium turgidum Rosenst. ${ }^{* * *}$ )

Hierba terrestre. Perenne. Nativa. 0-500 m.

Distribución: SPE.

Ejemplardereferencia:Múlgura,M.E.4024(SI,MNES).

\section{BLECHNACEAE}

\section{Blechnum L.}

Blechnum asplenioides $\mathrm{S} w .\left({ }^{* * *}\right)$

Hierba terrestre o rupícola. Perenne. Nativa. 0-500 m.

Distribución: SIG.

Ejemplar de referencia: Morrone, O. 1849 (SI).

\section{Blechnum auriculatum $C$ av.}

Hierba terrestre o saxícola. Perenne. Nativa. 0-500 m.

Distribución: ELD, GUA.

Ejemplar de referencia: Burkart, A. 14605 (SI).

Blechnum austrobrasilianum de la Sota

Hierba. Perenne. Nativa. 0-500 m.

Distribución: GUA, SPE.

Ejemplar de referencia: Zuloaga, F. O. 5513 (SI).

\section{Blechnum gracile Kaulf. ( $\left.{ }^{* *}\right)$}

Hierba terrestre o saxícola. Perenne. Nativa. 0-700 m.

Distribución: ELD, GUA, IGU, SPE.

Ejemplar de referencia: Deginani, N. B. 1543 (SI).

\section{Blechnum laevigatum Cav.}

Hierba terrestre o saxícola. Perenne. Nativa. 0-600 m.

Distribución: GMB, IGU, SIG.

Ejemplar de referencia:,Pérez, A. 21 (MNES, SI).

\section{Blechnum lanceola $S w .\left({ }^{* * *}\right)$}

Hierba terrestre o saxícola. Perenne. Nativa. 0-700 m.

Distribución: CAN, ELD, GMB, GUA, IGU, SPE. Ejemplar de referencia: Tressens, S. G. 5796 (CTES, SI).

\section{Blechnum occidentale L.}

Hierba terrestre o saxícola. Perenne. Nativa. 0-500 m.

Distribución: GMB, GUA, IGU, SPE.

Ejemplar de referencia: Zuloaga, F. O. 5481 (SI).

Blechnum polypodioides Raddi $\left(^{* * *}\right)$

Hierba terrestre. Perenne. Nativa. 0-500 m. 
Distribución: SIG.

Ejemplar de referencia: Wagner, E. R. s.n. (SI).

\section{Lomaridium C. Presl}

Lomaridium acutum (Desv.) Gasper \& V.A.O. Dittrich $\left({ }^{* * *}\right)$

Hierba hemiepífita. Perenne. Nativa. 100-800 m. Distribución: GMB, GUA, IGU, SPE.

Ejemplar de referencia: Montes, J. E. 27451 (LP).

Lomariocycas (J. Sm.) Gasper \& V.A.O. Dittrich

Lomariocycas schomburghii (Klotzsch) Gasper \& A.R.Sm.

Hierba. Perenne. Nativa. 0-500 m.

Distribución: CAI.

Ejemplar de referencia: Schwindt, E. 4421 (LIL).

Neoblechnum Gasper \& V.A.O. Dittrich

Neoblechnum brasiliense (Desv.) Gasper \& V.A.O. Dittrich

Arborescente. Perenne. Nativa. 0-800 m.

Distribución: CAI, CAN, GMB, GUA, IGU, MON, SIG, SPE.

Ejemplar de referencia: Guillén, R. 25 (MNES, SI).

\section{Parablechnum C. Presl}

Parablechnum cordatum (Desv.) Gasper \& Salino Subarbusto. Nativa. 0-500 m.

Distribución: SIG.

Ejemplar de referencia: Keller, H. A. 13749 (CTES, LP).

Telmatoblechnum Perrie, D.J. Ohlsen \& Brownsey

Telmatoblechnum serrulatum (Rich.) Perrie, D.J. Ohlsen \& Brownsey

Hierba. Perenne. Nativa. 0-500 m.

Distribución: SIG.

Ejemplar de referencia: Keller, H. A. 10661 (CTES).

\section{CYATHEACEAE}

Alsophila R. Br.

Alsophila setosa Kaulf. ${ }^{* * *}$ )

Arborescente. Perenne. Nativa. 0-800 m.

Distribución: CAI, GMB, GUA, IGU, OBE, SPE.

Ejemplar de referencia: Morrone, O. 1453 (SI).

\section{Cyathea Sm.}

Cyathea atrovirens (Langsd. \& Fisch.) Domin Arborescente. Perenne. Nativa. 0-800 m.

Distribución: CAN, CON, GMB, GSM, GUA, IGU, SIG, SPE.

Ejemplar de referencia: de la Sota, E. 6077 (LP).

\section{DENNSTAEDTIACEAE}

\section{Dennstaedtia Bernh.}

Dennstaedtia cicutaria (Sw.) T. Moore

Hierba. Perenne. Nativa. 0-500 m.

Distribución: GMB, IGU, SPE.

Ejemplar de referencia: Guillén, R. 135 (CTES, G,MNES).

Dennstaedtia dissecta (Sw.) T. Moore ${ }^{* * *}$ )

Hierba. Perenne. Nativa. 0-500 m.

Distribución: GMB, SPE.

Ejemplar de referencia: Yañez, A. 103 (LP).

Dennstaedtia globulifera (Poir.) Hieron.

Hierba. Perenne. Nativa. 0-700 m.

Distribución: CAI, CAN, ELD, GMB, GSM, GUA, IGU, SPE.

Ejemplar de referencia: Morrone, O. 1450 (SI).

Hypolepis Bernh.

Hypolepis stolonifera Fée var. delasotae Arana, A. Yañez \& Schwartsb. $\left.{ }^{(* *}\right)$

Hierba. Perenne. Nativa. 400-700 m. 
Distribución: GMB, OBE, SPE.

Ejemplar de referencia: de la Sota, E. R. 6146 $(L P, B A)$.

\section{Hypolepis stolonifera Fée var. stolonifera $(* * *)$}

Hierba. Perenne. Nativa. 0-500 m.

Distribución: GMB, GUA, SPE.

Ejemplar de referencia: Zanotti, C. A. 540 (SI).

Pteridium Gled. ex Scop., nom. cons.

Pteridium esculentum (G. Forst.) Cockayne var. arachnoideum

Hierba. Perenne. Nativa. 0-800 m.

Distribución: CAI, ELD, GMB, GUA, IGU, SIG, SPE.

Ejemplar de referencia: Guillén, R. 122 (MNES, SI).

Pteridium esculentum (G. Forst.) Cockayne var. paedomorficum Schwartsb. \& J. Prado

Hierba. Perenne. Nativa. 0-700 m.

Distribución: CAI, IGU, MON, SIG, SPE.

Ejemplar de referencia: Montes, J. E. 3892 (LP).

\section{DICKSONIACEAE}

\section{Dicksonia L’Hér.}

Dicksonia sellowiana Hook. $\left.{ }^{* * *}\right)$

Arborescente. Perenne. Nativa. 0-600 m.

Distribución: GMB, GUA, SPE.

Ejemplar de referencia: Deginani, N. B. 1700 (SI).

\section{DIDYMOCHLAENACEAE}

\section{Didymochlaena Desv.}

Didymochlaena truncatula (Sw.) Sm.

Hierba. Perenne. Nativa. 200-700 m.

Distribución: CAI, GMB, GSM, GUA, IGU, SPE.

Ejemplar de referencia: Zuloaga, F. O. 6854 (SI).

\section{DRYOPTERIDACEAE}

Ctenitis (C. Chr.) C. Chr.

Ctenitis submarginalis (Langsd. \& Fisch.) Ching.

Hierba terrestre. Perenne. Nativa. 0-800 m.

Distribución: APO, CAI, GMB, GSM, GUA, IGU, LNA, SIG, SPE.

Ejemplar de referencia: Zuloaga, F. O. 5712 (SI).

Elaphoglossum Schott ex J. Sm., nom. cons.

Elaphoglossum pachydermum (Fée) T. Moore $(* *)$

Hierba terrestre. Perenne. Nativa. 0-500 m.

Distribución: CAN, SIG.

Ejemplar de referencia: Biganzoli, F. 394 (SI).

\section{Lastreopsis Ching}

Lastreopsis amplissima (C. Presl) Tindale $(* * *)$

Hierba terrestre. Perenne. Nativa. 0-500 m.

Distribución: SPE.

Ejemplar de referencia: Keller, H. A. 1894 (CTES).

\section{Megalastrum Holttum}

Megalastrum connexum (Kaulf.) A.R. Sm. \& R.C. Moran

Hierba terrestre. Perenne. Nativa. 0-700 m.

Distribución: CAI, GMB, GUA, IGU, LNA, SPE.

Ejemplar de referencia: Guillén, R. 310 (MNES, SI).

Megalastrum crenulans (Fée) A.R. Sm. \& R.C. Moran $\left({ }^{* *}\right)$

Hierba. Perenne. Nativa. 0-600 m.

Distribución: SPE.

Ejemplar de referencia: Zuloaga, F. O. 5551 (SI). 
Megalastrum oreocharis (Sehnem) Salino \& Ponce $\left({ }^{* * *}\right)$

Hierba. Perenne. Nativa. 0-600 m.

Distribución: SPE.

Ejemplar de referencia: Marquez, G. J. 409 (BA, $L P)$.

Nota: Nueva cita en este volumen de Darwiniana n.s., Marquez et al.

Megalastrum substrigosum R.C. Moran, J. Prado \& Labiak $\left.{ }^{* * *}\right)$

Hierba. Perenne. Nativa. 0-500 m.

Distribución: SPE.

Ejemplar de referencia: Kelly, S. 3 (LP).

\section{Parapolystichum (Keyserl.) Ching}

\section{Parapolystichum effusum (Sw.) Ching (***)}

Hierba terrestre. Perenne. Nativa. 300-800 m. Distribución: CAI, ELD, GMB, GSM, GUA, IGU. Ejemplar de referencia: Zuloaga, F. O. 5646 (SI).

Polystichum Roth, nom. cons.

Polystichum montevidense (Spreng.) Rosenst. Hierba. Perenne. Nativa. 0-700 m.

Distribución: CON, GMB.

Ejemplar de referencia: Múlgura, M. E. 4054 (SI,MNES).

\section{Polystichum platylepis Fée $\left({ }^{* *}\right)$}

Hierba. Perenne. Nativa. 0-500 m.

Distribución: GMB.

Ejemplar de referencia: Morrone, O. 1405 (SI).

\section{Polystichum platyphyllum (Willd.) C. Presl}

Hierba. Perenne. Nativa. 0-500 m.

Distribución: CAN.

Ejemplar de referencia: Burkart, A. 1536 (SI).

\section{Rumohra Raddi}

Rumohra adiantiformis (G. Forst.) Ching

Hierba. Perenne. Nativa. 0-500 m.

Distribución: CAN.

Ejemplar de referencia: Keller, H. A. 10514 (CTES).

\section{EQUISETACEAE}

\section{Equisetum L.}

Equisetum giganteum $\mathrm{L}$.

Hierba. Perenne. Nativa. 0-500 m.

Distribución: CAN, GUA, IGU, MON, SIG, SPE.

Ejemplar de referencia: Keller, H. A. 3282 (CTES).

\section{GLEICHENIACEAE}

\section{Dicranopteris Bernh.}

Dicranopteris flexuosa (Schrad.) Underw. $\left({ }^{* * *}\right)$ Hierba. Perenne. Nativa. 100-500 m.

Distribución: ELD, GMB, SIG.

Ejemplar de referencia: Biganzoli, F. 397 (SI).

\section{HYMENOPHYLLACEAE}

Crepidomanes (C. Presl) C. Presl

Crepidomanes pyxidiferum (L.) Dubuisson \& Ebihara var. australe Ponce \& Dubuisson $\left({ }^{* * *}\right)$

Hierba. Perenne. Nativa. 0-700 m.

Distribución: ELD, GMB, GUA, IGU, OBE, SPE.

Ejemplar de referencia: Zanotti, C. A. 568 (SI,P).

\section{Didymoglossum Desv.}

Didymoglossum hymenoides (Hedw.) Copel.

Hierba epífita. Perenne. Nativa. 0-500 m.

Distribución: CAN, GMB, GUA, IGU, SPE.

Ejemplar de referencia: Keller, H. A. 848 (CTES, SI).

Didymoglossum krausii (Hook. \& Grev.) C. Presl

Hierba saxícola. Perenne. Nativa. 0-500 m.

Distribución: GMB, IGU, OBE.

Ejemplar de referencia: Capurro, R. H. 1248 (BA). 
Didymoglossum reptans (Sw.) C. Presl

Hierba epífita. Perenne. Nativa. 0-500 m.

Distribución: GUA, IGU.

Ejemplar de referencia: Keller, H. A. 1119 (CTES).

\section{Polyphlebium Copel.}

Polyphlebium angustatum (Carmich.) Ebihara \& Dubuisson

Hierba epífita. Perenne. Nativa. 0-500 m.

Distribución: GMB, GUA, SPE.

Ejemplar de referencia: Tressens, S. G. 6850 (CTES, SI).

\section{Trichomanes L.}

Trichomanes anadromum Rosenst.

Hierba epífita. Perenne. Nativa. 0-500 m.

Distribución: SPE.

Ejemplar de referencia: Capurro, R. H. 971 (BA).

\section{Trichomanes cristatum Kaulf. $\left.{ }^{* * *}\right)$}

Hierba. Perenne. Nativa. 0-500 m.

Distribución: IGU.

Ejemplar de referencia: Keller, H. A. 13630 (CTES).

\section{Trichomanes pellucens Kunze $\left.{ }^{(* *}\right)$}

Hierba terrestre o epífita. Perenne. Nativa. 0-500 m.

Distribución: IGU.

Ejemplar de referencia: Rodríguez, F. M. 392 $(S I, B A)$.

\section{Trichomanes pilosum Raddi ${ }^{* * *}$ )}

Hierba saxícola. Perenne. Nativa. 0-500 m.

Distribución: CAN, SIG.

Ejemplar de referencia: Zuloaga, F. O. 6614 (SI).

\section{Vandenboschia Copel.}

Vandenboschia radicans (Sw.) Copel. $\left(^{* * *}\right)$

Hierba saxícola. Perenne. Nativa. 0-500 m.

Distribución: IGU.

Ejemplar de referencia: Keller, H. A. 53 (CTES, SI).

\section{ISOETACEAE}

\section{Isoetes L.}

Isoetes ekmanii U. Weber

Hierba. Anual. Nativa. 300-500 m.

Distribución: APO, CAN, GUA. (SI).

Ejemplar de referencia: Cabrera, A. L. 28493

Isoetes gardneriana Kunze ex Mett.

Hierba acuática. Nativa. 0-500 m.

Distribución: IGU.

Ejemplar de referencia: Keller, H. A. 13501 (CTES,SI).

\section{LINDSAEACEAE}

Lindsaea Dryand. ex Sm.

Lindsaea quadrangularis Raddi subsp. terminalis K.U. Kramer $\left({ }^{* *}\right)$

Hierba. Perenne. Nativa. 0-500 m.

Distribución: GMB, IGU.

Ejemplar de referencia: Múlgura, M. E. 575 (SI).

\section{LYCOPODIACEAE}

\section{Lycopodiella Holub}

Lycopodiella longipes (Hook. \& Grev.) Holub

Hierba. Perenne. Nativa. 0-500 m.

Distribución: IGU.

Ejemplar de referencia: Denis, L. s.n. (LIL).

\section{Lycopodium L.}

\section{Lycopodium clavatum $\mathrm{L}$.}

Hierba. Perenne. Nativa. 0-500 m.

Distribución: GMB, GUA.

Ejemplar de referencia: Keller, H. A. 49 (CTES, SI). 
Palhinhaea Vasc. \& Franco

Palhinhaea camporum (B. Øllg. \& Windisch) Holub $\left({ }^{* * *}\right)$

Hierba. Perenne. Nativa. 0-500 m.

Distribución: IGU.

Ejemplar de referencia: Beaufils, 17 (SI).

Palhinhaea cernua (L.) Vasc. \& Franco

Hierba. Perenne. Nativa. 0-500 m.

Distribución: GUA, SIG.

Ejemplar de referencia: Guaglianone, E. R. 3007 (SI).

\section{Phlegmariurus (Herter) Holub}

Phlegmariurus acerosus (Sw.) B. Øllg. (***)

Hierba. Perenne. Nativa. 0-500 m.

Distribución: IGU.

Ejemplar de referencia: Rodríguez, F. M. s.n. (SI).

Phlegmariurus heterocarpon (Fée) B. Øllg. ${ }^{* * *}$ ) Hierba epífita. Perenne. Nativa. 0-500 m.

Distribución: SPE.

Ejemplar de referencia: Spegazzini, C. L. s.n. (BA, BAB, BAF).

Phlegmariurus mandiocanus (Raddi) B. Øllg. Hierba epífita. Perenne. Nativa. 0-500 m.

Distribución: GUA, SPE.

Ejemplar de referencia: Deginani, N. B. 1807 (SI).

\section{LYGODIACEAE}

Lygodium Sw., nom. cons.

\section{Lygodium venustum $S$.}

Enredadera. Perenne. Nativa. 0-500 m.

Distribución: IGU, SPE.

Ejemplar de referencia: Meza Torres, E. I. 1645 (CTES).

\section{Lygodium volubile $S w .\left({ }^{* * *}\right)$}

Enredadera. Perenne. Nativa. 0-500 m.

Distribución: CAN, ELD, IGU, GSM, SIG.

Ejemplar de referencia: Biganzoli, F. 416 (SI).

\section{MARATTIACEAE}

Eupodium J. Sm.

Eupodium kaulfussii (J. Sm.) J. Sm. $\left.{ }^{* * *}\right)$

Hierba. Perenne. Nativa. 0-500 m.

Distribución: GMB.

Ejemplar de referencia: Guaglianone, E. R. 2975 (SI).

\section{MARSILEACEAE}

\section{Marsilea L.}

Marsilea ancylopoda A. Braun

Hierba acuática. Perenne. Nativa. 0-500 m.

Distribución: SIG.

Ejemplar de referencia: Múlgura, M. E. 2143 (SI).

\section{NEPHROLEPIDACEAE}

\section{Nephrolepis Schott}

Nephrolepis cordifolia (L.) C. Presl

Hierba terrestre o epífita. Perenne. Nativa.

0-500 m.

Distribución: CAI.

Ejemplar de referencia: Keller, H. A. 4075 (CTES).

\section{OPHIOGLOSSACEAE}

Ophioderma (Blume) Endl.

Ophioderma palmata (L.) Nakai $\left(^{* * *}\right)$

Hierba epífita. Perenne. Nativa. 0-800 m.

Distribución: GMB.

Ejemplar de referencia: Chaves, H. s.n. (SI).

\section{Ophioglossum L.}

Ophioglossum crotalophoroides Walter Hierba. Perenne. Nativa. 0-500 m. 
Distribución: CON, ELD, GUA, IGU, SIG.

Ejemplar de referencia: Keller, H. A. 3606 (CTES).

Ophioglossum nudicaule L. $\mathrm{f}$.

Hierba. Perenne. Nativa. 0-500 m.

Distribución: OBE, SIG.

Ejemplar de referencia: Meza Torres, E. I. 1408 (CTES).

\section{Ophioglossum reticulatum $\mathrm{L}$.}

Hierba. Perenne. Nativa. 0-500 m.

Distribución: GMB, GUA, SIG.

Ejemplar de referencia: Keller, H. A. 42 (CTES).

\section{OSMUNDACEAE}

\section{Osmunda L.}

Osmunda spectabilis Willd.

Hierba. Perenne. Nativa. 0-500 m.

Distribución: APO, GMB, GUA, IGU, SIG, SPE.

Ejemplar de referencia: Johnson, A. E. 23 (CTES, SI).

\section{POLYPODIACEAE}

\section{Campyloneurum C. Presl}

Campyloneurum atlanticum R.C. Moran \& Labiak $\left.{ }^{* * *}\right)$

Hemiepífita. Perenne. Nativa. 100-500 m.

Distribución: CAI, ELD, OBE, SPE.

Ejemplar de referencia: Morrone, O. 1329 (SI,MO).

\section{Campyloneurum austrobrasilianum (Alston)} de la Sota $\left({ }^{* *}\right)$

Hierba epífita. Perenne. Nativa. 0-500 m.

Distribución: CAI, GMB, GUA, SPE.

Ejemplar de referencia: Guaglianone, E. R. 2969 (SI).

\section{Campyloneurum lapathifolium (Poir.) Ching} $\left({ }^{* *}\right)$

Hierba epipétrica. Perenne. Nativa. 0-500 m.

Distribución: SIG.

Ejemplar de referencia: Keller, H. A. 7456 (CTES).

\section{Campyloneurum minus Fée $\left({ }^{* * *}\right)$}

Hierba epífita. Perenne. Nativa. 100-500 m.

Distribución: CAI, GSM, GUA.

Ejemplar de referencia: Biganzoli, F. 194 (SI).

Campyloneurum nitidum (Kaulf.) C. Presl

Hierba epífita. Perenne. Nativa. 300-700 m.

Distribución: CAI, GSM, GUA, OBE, SIG, SPE.

Ejemplar de referencia: Morrone, O. 765 (NY, SI).

Campyloneurum repens (Aubl.) C. Presl ${ }^{* * *}$ )

Hierba. Nativa. 100-500 m.

Distribución: IGU, MON, OBE, SPE.

Ejemplar de referencia: Cabral, E. L. 706 (CTES,SI).

\section{Campyloneurum tucumanense (Hieron.)}

Ching

Hierba terrestre. Perenne. Nativa. 0-800 m.

Distribución: GSM, SPE.

Ejemplar de referencia: Krapovickas, A. 26638 (MO).

\section{Microgramma C. Presl}

Microgramma lindbergii (Mett.) de la Sota $\left({ }^{* * *}\right)$

Hierba epífita. Perenne. Nativa. 0-800 m.

Distribución: CAN, ELD, IGU, SIG.

Ejemplar de referencia: Biganzoli, F. 440 (SI).

Microgramma mortoniana de la Sota

Hierba epífita. Perenne. Nativa. 0-500 m.

Distribución: GMB, IGU.

Ejemplar de referencia: Zuloaga, F. O. 5271 (SI).

Microgramma squamulosa (Kaulf.) de la Sota

Hierba epífita. Perenne. Nativa. 0-800 m.

Distribución: CAI, CAP, GMB, GSM, GUA, SIG, SPE.

Ejemplar de referencia: Deginani, N. B. 1209 (SI).

Microgramma vacciniifolia (Langsd. \& Fisch.) Copel.

Hierba epífita. Perenne. Nativa. 0-500 m.

Distribución: IGU, SIG, SPE.

Ejemplar de referencia: Niederlein, G. s.n. (SI). 
Niphidium J. Sm.

Niphidium crassifolium (L.) Lellinger

Hierba epífita. Perenne. Nativa. 0-400 m.

Distribución: CAI, GUA, SPE.

Ejemplar de referencia: Capurro, R. H. 1054 (BA, LIL).

Niphidium rufosquamatum Lellinger $\left({ }^{* * *}\right)$

Hierba epífita. Perenne. Nativa. 300-500 m.

Distribución: GMB, SPE.

Ejemplar de referencia: Morrone, O. 2137 (SI).

\section{Pecluma M.G. Price}

Pecluma filicula (Kaulf.) M.G. Price

Hierba epífita. Perenne. Nativa. 0-700 m.

Distribución: CAI, CON, ELD, GUA, IGU, SIG, SPE.

Ejemplar de referencia: Morrone, O. 1263 (SI).

\section{Pecluma pectinatiformis (Lindm.) M.G. Price} Hierba epífita. Perenne. Nativa. 0-500 m. Distribución: GUA, IGU, SPE.

Ejemplar de referencia: Tressens, S. G. 5794 (CTES, SI).

Pecluma robusta (Fée) M. Kessler \& A.R. Sm. Hierba terrestre. Perenne. Nativa. 0-500 m. Distribución: GMB, GUA, IGU, SPE.

Ejemplar de referencia: Morrone, O. 2135 (SI).

\section{Pecluma sicca (Lindm.) M.G. Price}

Hierba epífita. Perenne. Nativa. 0-600 m.

Distribución: CAI, GMB, GUA, IGU, OBE, SPE.

Ejemplar de referencia: Vanni, R. O. 3009 (CTES, SI).

\section{Pecluma singeri (de la Sota) M.G. Price ${ }^{* * *}$ )}

Hierba epífita. Perenne. Nativa. 0-800 m.

Distribución: CAI, CON, ELD, GSM, GUA, SIG.

Ejemplar de referencia: Biganzoli, F. 143 (SI).

\section{Pecluma truncorum (Lindm.) M.G. Price $\left(^{* * *}\right.$}

Hierba epífita. Perenne. Nativa. 200-600 m.

Distribución: IGU, SPE.

Ejemplar de referencia: Zuloaga, F. O. 5268 (SI).
Pecluma venturii (de la Sota) M.G. Price

Hierba epífita. Perenne. Nativa. 0-500 m.

Distribución: GUA.

Ejemplar de referencia: Pérez, A. 53 (G, MNES).

Phlebodium (R. Br.) J. Sm.

Phlebodium areolatum (Humb. \& Bonpl. ex Willd.) J. Sm.

Hierba epífita. Perenne. Nativa. 0-500 m.

Distribución: CAN, SIG.

Ejemplar de referencia: Biganzoli, F. 392 (SI).

Pleopeltis Humb. \& Bonpl. ex Willd.

Pleopeltis hirsutissima (Raddi) de la Sota

Hierba terrestre o epífita. Perenne. Nativa. 0-500 m.

Distribución: CAI, GUA, SPE.

Ejemplar de referencia: Zuloaga, F. O. 6488 (SI).

Pleopeltis macrocarpa (Bory ex Willd.) Kaulf.

Hierba epífita o terrestre. Perenne. Nativa. 0-500 m.

Distribución: IGU.

Ejemplar de referencia: Diem, J. 1452 (SI).

Pleopeltis minima (Bory) J. Prado \& R.Y. Hirai Hierba epífita o terrestre. Perenne. Nativa. 0-800 m.

Distribución: CAI, ELD, GMB, GSM, GUA, IGU, LNA, OBE, SPE.

Ejemplar de referencia: Morrone, O. 2156 (SI).

Pleopeltis pleopeltifolia (Raddi) Alston

Hierba epífita. Perenne. Nativa. 0-500 m.

Distribución: CAI, CAN, CON, GMB, GSM, GUA, IGU, OBE, SIG, SPE.

Ejemplar de referencia: Deginani, N. B. 1195 (SI).

Serpocaulon A.R. Sm.

Serpocaulon catharinae (Langsd. \& Fisch.) A.R. Sm. $\left({ }^{* *}\right)$

Hierba epífita. Perenne. Nativa. 0-200 m.

Distribución: SPE. 
Ejemplar de referencia: Seijo, G. J. 197 (CTES, MNES).

Serpocaulon latipes (Langsd. \& Fisch.) A.R. Sm. Hierba terrestre o epipétrica. Perenne. Nativa. 0-500 m.

Distribución: SIG.

Ejemplar de referencia: Keller, H. A. 5827 (CTES).

Serpocaulon vacillans (Link) A.R. Sm.

Hierba terrestre. Perenne. Nativa. 0-500 m.

Distribución: CAI, SIG.

Ejemplar de referencia: Schwindt, E. 4691 (LIL, RB).

\section{PSILOTACEAE}

\section{Psilotum Sw.}

Psilotum nudum (L.) P. Beauv.

Hierba epífita. Perenne. Nativa. 0-500 m.

Distribución: GUA, MON, SPE.

Ejemplar de referencia: Múlgura, M. E. 2649 (SI).

\section{PTERIDACEAE}

\section{Acrostichum L.}

Acrostichum danaeifolium Langsd. \& Fisch.

Hierba palustre. Perenne. Nativa. 0-500 m.

Distribución: CAP.

Ejemplar de referencia: Kermes, E. s.n. (SI).

\section{Adiantopsis Fée}

Adiantopsis $\times$ australopedata Hickey, M.S. Barker \& Ponce $\left({ }^{* * *}\right)$

HIerba. Perenne. Nativa. 0-500 m.

Distribución: CAI.

Ejemplar de referencia: Biganzoli, F. 168 (SI).

\section{Adiantopsis chlorophylla (Sw.) Fée}

Hierba. Perenne. Nativa. 0-600 m.

Distribución: CAI, ELD, GSM, GUA, SIG, SJA, SPE. Ejemplar de referencia: Arbo, M. M. 5881 (CTES, SI).
Adiantopsis dichotoma (Sw.) T. Moore

Hierba. Perenne. Nativa. 0-700 m.

Distribución: APO, CAI, CAN, CON, LNA, SIG.

Ejemplar de referencia: Deginani, N. B. 1359 (SI).

Adiantopsis radiata (L.) Fée

Hierba. Perenne. Nativa. 0-500 m.

Distribución: APO, CAI, GMB, GSM, GUA, IGU, MON, SIG. (SI).

Ejemplar de referencia: Deginani, N. B. 1448

Adiantopsis recurvata (Baker) Ponce \& Scataglini $\left.{ }^{* * *}\right)$

Hierba. Perenne. Nativa. 0-500 m.

Distribución: CAN.

Ejemplar de referencia: Albov, N. M. s.n. (SI).

Adiantopsis tweediana (Hook.) Link-Pérez \& Hickey

Hierba. Perenne. Nativa. 0-500 m.

Distribución: IGU, SIG.

Ejemplar de referencia: Biganzoli, F. 1232 (SI).

\section{Adiantum L.}

\section{Adiantum deflectens Mart.}

Hierba. Perenne. Nativa. 0-600 m.

Distribución: CAP.

Ejemplar de referencia: Parodi, L. R. 135 (SI).

\section{Adiantum latifolium Lam.}

Hierba. Perenne. Nativa. 0-700 m.

Distribución: IGU, SIG, SPE.

Ejemplar de referencia: Biganzoli, F. 438 (SI).

\section{Adiantum lorentzii Hieron.}

Hierba. Perenne. Nativa. 0-700 m.

Distribución: APO, CAI, CAN, GMB, GUA, IGU, LNA, SIG, SPE.

Ejemplar de referencia: Tressens, S. G. 4415 (CTES).

\section{Adiantum macrophyllum $\left.\mathrm{Sw} .{ }^{* * *}\right)$}

Hierba. Perenne. Nativa. 0-500 m.

Distribución: IGU.

Ejemplar de referencia: Rodríguez, F. M. 417 (LP). 
Adiantum poiretii Wikstr. f. poiretii

Hierba. Perenne. Nativa. 0-500 m.

Distribución: SPE.

Ejemplar de referencia: Guillén, R. 214 (CTES, G, MNES).

\section{Adiantum pseudotinctum Hieron.}

Hierba. Perenne. Nativa. 0-800 m.

Distribución: APO, CAI, CAN, ELD, GMB, GSM, GUA, IGU, SIG, SPE.

Ejemplar de referencia: Zuloaga, F. O. 5410 (SI).

\section{Adiantum raddianum $C$. Presl}

Hierba. Perenne. Nativa. 0-700 m.

Distribución: APO, CAI, CAP, CON, ELD, GMB, GUA, IGU, OBE, SIG, SPE.

Ejemplar de referencia: Tressens, S. G. 5792 (CTES, SI).

\section{Adiantum tetraphyllum Willd. ${ }^{* * *}$ )}

Hierba. Perenne. Nativa. 0-500 m.

Distribución: ELD, GMB, IGU.

Ejemplar de referencia: Cabrera, A. L. 204 (LP).

Cheilanthes Sw., nom. cons.

\section{Cheilanthes micropteris $\mathrm{Sw}$.}

Hierba. Perenne. Nativa. 0-500 m.

Distribución: APO, CAN, CON, LNA.

Ejemplar de referencia: Zuloaga, F. O. 5777 (SI).

Doryopteris J. Sm., nom. cons.

\section{Doryopteris arifolia Christ}

Hierba. Perenne. Nativa. 0-500 m.

Distribución: CAN, CAP, ELD, SPE.

Ejemplar de referencia: Zuloaga, F. O. 5472 (SI).

Doryopteris collina (Raddi) J. Sm. ${ }^{* * *}$ )

Hierba. Perenne. Nativa. 0-500 m.

Distribución: SIG.

Ejemplar de referencia: Keller, H. A. 6530 (CTES).

Doryopteris concolor (Langsd. \& Fisch.) Kuhn Hierba. Perenne. Nativa. 0-700 m.

Distribución: CAI, CAN, CAP, CON, GMB, GUA, SIG, SPE.

Ejemplar de referencia: Deginani, N. B. 1155 (SI).
Doryopteris lorentzii (Hieron.) Diels

Hierba. Perenne. Nativa. 0-500 m.

Distribución: CAN, SIG.

Ejemplar de referencia: Burkart, A. 1365 (G).

Doryopteris nobilis (T. Moore) C. Chr.

Hierba. Perenne. Nativa. 0-800 m.

Distribución: CAI, CAN, GMB, GSM, GUA, IGU, SIG, SPE.

Ejemplar de referencia: Zuloaga, F. O. 5472 (SI).

Doryopteris pentagona Pic.Serm.

Hierba. Perenne. Nativa. 0-800 m.

Distribución: APO, CAI, CON, GMB, GSM, GUA, IGU, SPE.

Ejemplar de referencia: Zuloaga, F. O. 5467 (SI).

Doryopteris triphylla (Lam.) Christ

Hierba. Perenne. Nativa. 0-500 m.

Distribución: CAI, GMB, GUA, IGU, SPE.

Ejemplar de referencia: Zuloaga, F. O. 6510 (SI).

Gastoniella Li Bing Zhang \& Liang Zhang

Gastoniella chaerophylla (Desv.) Li Bing Zhang \& Liang Zhang

Hierba. Anual. Nativa. 0-600 m.

Distribución: CAI, CON, GMB, SIG, SPE.

Ejemplar de referencia: Múlgura, M. E. 2126 (SI).

\section{Hemionitis L.}

Hemionitis tomentosa (Lam.) Raddi

Hierba. Perenne. Nativa. 0-700 m.

Distribución: APO, CAI, CAN, CON, ELD, GMB, GSM, GUA, IGU, SIG, SPE.

Ejemplar de referencia: Biganzoli, F. 291 (SI).

Lytoneuron (Klotzsch) Yesilyurt

Lytoneuron lomariaceum (Klotzsch) Yesilyurt $(* * *)$

Hierba. Perenne. Nativa. 200-500 m.

Distribución: MON.

Ejemplar de referencia: Keller, H. A. 2291 (CTES). 


\section{Pityrogramma Link}

Pityrogramma calomelanos (L.) Link

Hierba. Perenne. Nativa. 0-500 m.

Distribución: GSM, IGU, MON, SIG.

Ejemplar de referencia: Morrone, O. 1848 (SI).

Pityrogramma trifoliata (L.) R.M. Tryon

Hierba. Perenne. Nativa. 0-500 m.

Distribución: CAI, IGU, LNA.

Ejemplar de referencia: Múlgura, M. E. 1952 (SI).

\section{Pteris L.}

\section{Pteris brasiliensis Raddi}

Hierba. Perenne. Nativa. 0-700 m.

Distribución: CAN, CAP, ELD, IGU, SIG, SPE.

Ejemplar de referencia: Schwarz, G. J. 10370 (BM, LIL).

\section{Pteris deflexa Link}

Hierba. Perenne. Nativa. 0-700 m. SPE.

Distribución: CAI, CAN, GMB, GUA, IGU,

Ejemplar de referencia: Zuloaga, F. O. 5260 (SI).

\section{Pteris denticulata Sw. var. denticulata}

Hierba. Perenne. Nativa. 0-700 m.

Distribución: APO, CAI, ELD, GMB, GSM, GUA, IGU, OBE, SPE.

Ejemplar de referencia: Zuloaga, F. O. 5633 (SI).

\section{Pteris denticulata Sw. var. tristicula (Raddi)}

J. Prado

Hierba. Perenne. Nativa. 0-600 m.

Distribución: CAN, CON, IGU, SPE.

Ejemplar de referencia: Zanotti, C. A. 464 (SI).

\section{Pteris lechleri Mett. $\left.{ }^{* * *}\right)$}

Hierba. Perenne. Nativa. 100-600 m.

Distribución: GMB, GUA, SPE.

Ejemplar de referencia: Zuloaga, F. O. 5535 (SI).

\section{Pteris vittata $\mathrm{L}$.}

Hierba. Perenne. Introducida. 0-500 m.

Distribución: CAP, GSM.

Ejemplar de referencia: Pérez 173 (CTES).

\section{Vittaria Sm.}

Vittaria graminifolia Kaulf.

Hierba epífita. Perenne. Nativa. 0-500 m.

Distribución: GMB, IGU.

Ejemplar de referencia: Kermes, E. 36961 (SI).

Vittaria lineata (L.) Sm.

Hierba epífita. Perenne. Nativa. 0-500 m.

Distribución: CAN, GMB, GUA, SIG, SPE.

Ejemplar de referencia: Biganzoli, F. 641 (SI).
Actinostachys Wall. ex Hook.

Actinostachys pennula (Sw.) Hook. ${ }^{* * *}$ )

Hierba. Perenne. Nativa. 0-500 m.

Distribución: IGU.

Ejemplar de referencia: Keller, H. A. 13637 (CTES).

\section{SELAGINELLACEAE}

Selaginella P. Beauv., nom. cons.

Selaginella marginata (Humb. \& Bonpl. ex Willd.) Spring

Hierba. Perenne. Nativa. 0-800 m.

Distribución: CON, SPE.

Ejemplar de referencia: Belgrano, M. J. 705 (SI).

Selaginella microphylla (Kunth) Spring

Hierba. Perenne. Nativa. 0-700 m.

Distribución: CAI, GUA.

Ejemplar de referencia: Biganzoli, F. 1321 (SI).

Selaginella muscosa Spring

Hierba. Perenne. Nativa. 0-500 m.

Distribución: CAN, CON, GMB, GUA, IGU, SPE. Ejemplar de referencia: Guaglianone, E. R. 2827 (SI).

\section{Selaginella sellowii Hieron.}

Hierba. Perenne. Nativa. 0-500 m.

Distribución: APO, CAN, CAP, SIG.

Ejemplar de referencia: Múlgura, M. E. 2139 (SI). 
Selaginella sulcata (Desv. ex Poir.) Spring ex Mart. Hierba. Perenne. Nativa. 0-700 m. Distribución: ELD, GMB, GUA, IGU, SIG, SPE. Ejemplar de referencia: Guaglianone, E. R. 2981 (SI).

\section{TECTARIACEAE}

\section{Tectaria Cav.}

\section{Tectaria incisa Cav.}

Hierba. Perenne. Nativa. 0-500 m.

Distribución: CAI, CAN, GMB, GSM, GUA, IGU, OBE, SIG.

Ejemplar de referencia: Denham, S. S. 35 (CTES, SI).

\section{THELYPTERIDACEAE}

\section{Amauropelta Kunze}

Amauropelta amambayensis (Christ) Salino \& T.E. Almeida $\left.{ }^{* * *}\right)$

Hierba. Perenne. Nativa. 100-400 m.

Distribución: GMB, GSM, IGU, SPE.

Ejemplar de referencia: Morrone, O. 1402 (SI).

Amauropelta decurtata (Link) Salino \& T.E. Almeida

Hierba. Perenne. Nativa. 0-500 m.

Distribución: GMB, SJA.

Ejemplar de referencia: Krapovickas, A. 15251 (LP).

Amauropelta metteniana (Ching) Salino \& T.E. Almeida $\left.{ }^{* * *}\right)$

Hierba. Perenne. Nativa. 100-600 m.

Distribución: GMB, SPE.

Ejemplar de referencia: Diem, J. 1537 (BAB, SI).

Amauropelta opposita (Vahl) Pic.Serm. $\left({ }^{* * *}\right)$

Hierba. Perenne. Nativa. 0-500 m.

Distribución: IGU.

Ejemplar de referencia: Zanotti, C. A. 497 (SI).

Amauropelta recumbens (Rosenst.) Salino \& T.E. Almeida (***)

Hierba. Perenne. Nativa. 100-700 m.
Distribución: CAI, GMB, GUA, IGU, SPE.

Ejemplar de referencia: Tressens, S. G. 5862 (CTES, SI).

Amauropelta regnelliana (C. Chr.) Salino \& T.E. Almeida $\left({ }^{* * *}\right)$

Hierba. Perenne. Nativa. 0-600 m.

Distribución: GMB.

Ejemplar de referencia: Morrone, O. 1456 (SI).

Amauropelta rivularioides (Fée) Salino \& T.E. Almeida

Hierba. Perenne. Nativa. 0-700 m.

Distribución: CAP, GMB, GSM, GUA, IGU, LNA, SIG.

Ejemplar de referencia: Morrone, O. 1458 (SI).

Amauropelta sanctae-catharinae (Rosenst.) Salino \& T.E. Almeida (***)

Hierba. Perenne. Nativa. 0-500 m.

Distribución: GUA, SPE.

Ejemplar de referencia: Guaglianone, E. R. 2821 (SI).

\section{Christella H. Lév.}

Christella conspersa (Schrad.) A. Löve \& D. Löve

Hierba. Perenne. Nativa. 0-600 m.

Distribución: GMB, GSM, IGU, MON.

Ejemplar de referencia: Morrone, O. 1461 (SI).

Christella dentata (Forssk.) Brownsey \& Jermy Hierba. Perenne. Introducida. 0-800 m.

Distribución: APO, CAI, CAP, CON, ELD, GMB, GSM, GUA, IGU, LNA, SIG, SPE.

Ejemplar de referencia: Zuloaga, F. O. 5102 (SI).

Christella hispidula (Decne.) Holttum

Hierba. Perenne. Nativa. 0-600 m.

Distribución: CAN, GMB, GSM, GUA, IGU, OBE, SIG, SPE.

Ejemplar de referencia: Morrone, O. 1659 (SI).

\section{Cyclosorus Link}

Cyclosorus interruptus (Willd.) H. Itô

Hierba. Perenne. Nativa. 0-500 m 
Distribución: GUA, LNA, MON.

Ejemplar de referencia: Guillén, R. 318 (MNES, SI).

\section{Goniopteris C. Presl}

Goniopteris burkartii C. Chr. ex Abbiatti

Hierba. Perenne. Nativa. 0-600 m.

Distribución: IGU.

Ejemplar de referencia: Capurro, R. H. 861 $(B A)$.

Goniopteris riograndensis (Lindm.) Ching

Hierba. Perenne. Nativa. 0-800 m.

Distribución: CAI, CAN, CAP, ELD, GMB, GUA, OBE, SIG, SPE.

Ejemplar de referencia: Tressens, S. G. 5720 (CTES, SI).

Goniopteris scabra (C. Presl) Brade

Hierba. Perenne. Nativa. 0-800 m.

Distribución: CAI, ELD, GMB, GUA, IGU, SPE.

Ejemplar de referencia: Morrone, O. 1317 (SI).

\section{Macrothelypteris (H. Itô) Ching}

Macrothelypteris torresiana (Gaudich.)

Ching

Hierba. Perenne. Introducida. 0-800 m.

Distribución: APO, CAI, CON, GMB, GUA, IGU, SJA, SPE.

Ejemplar de referencia: Zuloaga, F. O. 4937 (SI).
Meniscium Schreb.

Meniscium serratum Cav.

Hierba. Perenne. Nativa. 0-700 m.

Distribución: CAP, IGU.

Ejemplar de referencia: Capurro, R. H. 754 (BA).

Thelypteris Schmidel, nom. cons.

Thelypteris berroi (C. Chr.) C.F. Reed

Hierba. Perenne. Nativa. 0-500 m.

Distribución: CAN.

Ejemplar de referencia: Ekman, E. L. 10 (S).

Thelypteris patens (Sw.) Small var. dissimilis (Schrad.) A.R. Sm. ${ }^{* * *}$ )

Hierba. Perenne. Nativa. 0-500 m.

Distribución: IGU.

Ejemplar de referencia: Múlgura, M. E. 576 (SI).

Thelypteris patens (Sw.) Small var. patens

Hierba. Perenne. Nativa. 0-500 m.

Distribución: GUA.

Ejemplar de referencia: Tressens, S. G. 6765 (CTES,SI).

Thelypteris schizotis (Hook.) M. Kessler \& A. R. Sm.

Hierba. Perenne. Nativa. 0-500 m.

Distribución: ELD, IGU.

Ejemplar de referencia: Tell Bertoni, G. 996 (SI).

\section{GYMNOSPERMAE}

\section{ARAUCARIACEAE}

\section{Araucaria Juss.}

Araucaria angustifolia (Bertol.) Kuntze (***)

Árbol. Nativa. 0-700 m.

Distribución: CAN, GMB, SPE.

Ejemplar de referencia: Hauman, L. L. s.n. (BA).

\section{PODOCARPACEAE}

Podocarpus L'Hér. ex Pers., nom. cons.

Podocarpus lambertii Klotzsch ex Endl. $\left({ }^{*}\right)$

Árbol. Introducida. 0-500 m.

Distribución: OBE.

Ejemplar de referencia: Keller, H. A. 10336 (CTES). 


\section{ANGIOSPERMAE (MONOCOTYLEDONEAE Y DICOTYLEDONEAE)}

\section{ACANTHACEAE}

\section{Aphelandra $\mathrm{R}$. Br.}

Aphelandra longiflora (Lindl.) Profice $\left(^{* * *}\right)$

Arbusto. Nativa. 0-500 m.

Distribución: GMB, IGU.

Ejemplar de referencia: Morrone, O. 1952 (SI).

\section{Dicliptera Juss.}

\section{Dicliptera squarrosa Nees}

Hierba. Nativa. 0-700 m.

Distribución: APO, CAI, CAN, GMB, GUA, IGU, SIG, SPE.

Ejemplar de referencia: Deginani, N. B. 1415 (SI).

\section{Dyschoriste Ness}

Dyschoriste hygrophiloides (Nees) Kuntze

Hierba. Perenne. Nativa. 0-500 m.

Distribución: SIG.

Ejemplar de referencia: Keller, H. A. 7804 (CTES).

\section{Dyschoriste lavandulacea (Nees) Kuntze}

Hierba. Perenne. Nativa. 0-500 m.

Distribución: CAN, SPE.

Ejemplar de referencia: Niederlein, G. 42 (B, BA, CORD).

\section{Hygrophila R. Br.}

Hygrophila costata Nees \& T. Nees

Hierba acuática. Nativa. 0-700 m.

Distribución: APO, CAP, ELD, GMB, GSM, GUA, SIG, SJA, SPE.

Ejemplar de referencia: Morrone, O. 965 (SI).

Justicia L., nom. cons.

Justicia axillaris (Nees) Lindau Hierba. Perenne. Nativa. 0-700 m.
Distribución: APO, CAN, CAP, SJA.

Ejemplar de referencia: Krapovickas, A. 28844 (CTES).

\section{Justicia brasiliana Roth}

Hierba o arbusto. Perenne. Nativa. 0-700 m.

Distribución: APO, CAI, CAP, ELD, GMB, GSM, GUA, IGU, LNA, OBE, SIG, SJA, SPE.

Ejemplar de referencia: Morrone, O. 931 (SI).

Justicia carnea Lindl. $\left.{ }^{* * *}\right)$

Hierba o arbusto. Perenne. Nativa. 0-700 m.

Distribución: GMB, GUA, IGU, SJA, SPE.

Ejemplar de referencia: Morrone, O. 691 (SI).

Justicia comata (L.) Lam.

Hierba. Perenne. Nativa. 0-700 m.

Distribución: CAN, IGU, SIG, SJA.

Ejemplar de referencia: Herrera, J. 163 (CTES).

Justicia floribunda (C. Koch) Wassh. $\left.{ }^{* * *}\right)$

Arbusto. Nativa. 0-500 m.

Distribución: GMB, GUA, SPE.

Ejemplar de referencia: Tressens, S. G. 5647 (CTES).

Justicia laevilinguis (Nees) Lindau

Hierba palustre. Perenne. Nativa. 0-500 m.

Distribución: CAN, CAP, GUA.

Ejemplar de referencia: Seijo, G. J. 822 (CTES, G, MNES).

Justicia lythroides (Nees) V.A.W. Graham $\left(^{* * *}\right)$ Hierba. Perenne. Nativa. 0-500 m.

Distribución: CAI, ELD, GMB, GUA, IGU, MON, SIG, SPE.

Ejemplar de referencia: Burkart, A. 14603 (SI).

\section{Justicia oblonga (Nees) Lindau}

Hierba. Perenne. Nativa. 0-500 m.

Distribución: APO, CAI, CAN, LNA, SIG, SJA.

Ejemplar de referencia: Krapovickas, A. 15021 (CTES, SI).

Justicia ramulosa (Morong) C. Ezcurra Hierba o subarbusto. Perenne. Nativa. 0-500 m. Distribución: IGU.

Ejemplar de referencia: Lillo, M. 10520 (LIL). 
Justicia yhuensis Lindau

Hierba. Perenne. Nativa. 0-800 m.

Distribución: GUA, MAY, SJA.

Ejemplar de referencia: Deginani, N. B. 1546 (SI).

\section{Pachystachys Nees}

Pachystachys dubiosa (Lindau) A.L.A. Côrtes

Subarbusto. Nativa. 0-500 m.

Distribución: IGU.

Ejemplar de referencia: Keller, H. A. 63 (CTES, SI).

\section{Poikilacanthus Lindau}

Poikilacanthus glandulosus (Nees) Ariza

Subarbusto. Nativa. 0-500 m.

Distribución: APO, CAI, CAN, CAP, CON, ELD, GMB, GUA, IGU, LNA, MON, SIG.

Ejemplar de referencia: Biganzoli, F. 942 (SI).

\section{Ruellia L.}

Ruellia angustiflora (Nees) Lindau ex Rambo Arbusto. Nativa. 0-500 m.

Distribución: APO, CAI, CAN, CAP, CON, ELD, GMB, GSM, GUA, IGU, LNA, OBE, SIG, SJA, SPE. (SI).

Ejemplar de referencia: Deginani, N. B. 1123

\section{Ruellia brachysiphon (Nees) Hiern}

Hierba. Perenne. Nativa. 0-500 m.

Distribución: CAN, ELD, IGU, SIG. (SI).

Ejemplar de referencia: Zuloaga, F. O. 6540

\section{Ruellia brevicaulis (Nees) Lindau}

Hierba. Perenne. Nativa. 0-800 m.

Distribución: APO, CAN, CON, LNA, SIG, SPE.

Ejemplar de referencia: Cabrera, A. L. 28593 (SI).

\section{Ruellia brevifolia (Pohl) C. Ezcurra}

Hierba o subarbusto. Perenne. Nativa. 0-700 m. Distribución: APO, IGU.

Ejemplar de referencia: Cabrera, A. L. 28593 (SI).

\section{Ruellia bulbifera Lindau}

Hierba. Perenne. Nativa. 0-500 m.

Distribución: APO, CAN, CAP, CON, SIG.

Ejemplar de referencia: Arbo, M. M. 5970 (CTES, SI).

Ruellia epallocaulos Leonard ex C. Ezcurra \& Wassh. $\left({ }^{* * *}\right)$

Subarbusto. Nativa. 0-500 m.

Distribución: CAP, GSM, MON, SIG.

Ejemplar de referencia: Biganzoli, F. 512 (SI).

Ruellia erythropus (Nees) Lindau

Hierba o subarbusto. Perenne. Nativa. 0-800 m.

Distribución: APO, CAN, CON, GMB, IGU, SIG, SJA.

Ejemplar de referencia: Morrone, O. 1107 (SI).

\section{Ruellia geminiflora Kunth}

Hierba o subarbusto. Perenne. Nativa. 0-500 m. Distribución: CAN, GMB, IGU, SIG, SPE.

Ejemplar de referencia: Múlgura, M. E. 2182 (SI).

Ruellia hygrophila Mart.

Hierba. Perenne. Nativa. 0-500 m.

Distribución: SIG.

Ejemplar de referencia: Alboff, N. 22 (LP).

Ruellia hypericoides (Nees) Lindau

Hierba. Perenne. Nativa. 0-500 m.

Distribución: APO, CAN, CAP, CON, GMB, SIG.

Ejemplar de referencia: Biganzoli, F. 1739 (SI).

\section{Ruellia morongii Britton}

Hierba. Perenne. Nativa. 0-500 m.

Distribución: APO, CAN, CAP, CON, SIG, SJA.

Ejemplar de referencia: Cabrera, A. L. 28479 (SI).

\section{Ruellia multifolia (Nees) Lindau var. multifolia} $(* * *)$

Hierba o subarbusto. Perenne. Nativa. 0-500 m.

Distribución: CAN, SIG.

Ejemplar de referencia: Zuloaga, F. O. 3299 (SI).

Ruellia multifolia (Nees) Lindau var. viscosissima (Nees) C. Ezcurra

Hierba. Perenne. Nativa. 0-500 m. 
Distribución: CAN.

Ejemplar de referencia: Krapovickas, A. 28729 (SI).

\section{Ruellia sanguinea Griseb.}

Arbusto o subarbusto. Nativa. 0-500 m.

Distribución: IGU.

Ejemplar de referencia: Cristóbal, C. L. 2275 (CTES).

\section{Ruellia simplex $C$. Wright}

Hierba. Perenne. Nativa. 0-500 m.

Distribución: CAN, SIG.

Ejemplar de referencia: Zuloaga, F. O. 3179 (SI)

\section{Stenandrium Nees}

\section{Stenandrium diphyllum Nees}

Hierba. Nativa. 0-500 m.

Distribución: CAN, SPE.

Ejemplar de referencia: Múlgura, M. E. 2264 (SI).

\section{Stenandrium dulce (Cav.) Nees}

Hierba. Perenne. Nativa. 0-700 m.

Distribución: APO, CAN, CON.

Ejemplar de referencia: Biganzoli, F. 1659 (SI).

\section{Stenandrium mandioccanum Nees}

Hierba. Perenne. Nativa. 0-500 m.

Distribución: ELD, GMB, GSM, IGU, SPE.

Ejemplar de referencia: Cristóbal, C. L. 2273 (CTES, SI).

\section{Thunbergia Retz.}

Thunbergia alata Bojer ex Sims

Enredadera. Introducida. 0-500 m.

Distribución: CAI, ELD, IGU.

Ejemplar de referencia: Morrone, O. 639 (SI).

\section{Thyrsacanthus Moric.}

Thyrsacanthus sulcatus (Nees) C. Ezcurra \& A.L.A. Côrtes

Hierba. Nativa. 0-500 m.

Distribución: GUA.

Ejemplar de referencia: Keller, H. A. 346 (CTES).

\section{ACHATOCARPACEAE}

\section{Achatocarpus Triana}

Achatocarpus balansae Schinz \& Autran $\left({ }^{* * *}\right)$

Árbol. Nativa. 0-500 m.

Distribución: CAN, GMB.

Ejemplar de referencia: Rodríguez, F. M. 674 (SI).

Achatocarpus praecox Griseb. f. obovatus (Schinz \& Autran) Hauman

Arbusto o árbol. Nativa. 0-500 m.

Distribución: SIG.

Ejemplar de referencia: Hauman, L. L. s.n (SI).

Achatocarpus praecox Griseb. var. bicornutus (Schinz \& Autran) Botta

Arbusto o árbol. Nativa. 0-500 m.

Distribución: GUA.

Ejemplar de referencia: Tressens, S. G. 6113 (CTES, NY).

Achatocarpus praecox Griseb. var. praecox Arbusto o árbol. Nativa. 0-500 m.

Distribución: CON, ELD, GMB.

Ejemplar de referencia: Biganzoli, F. 1748 (SI).

\section{ALISMATACEAE}

Echinodorus Rich. ex Engelm.

Echinodorus floribundus (Seub.) Seub.

Hierba palustre. Nativa. 0-500 m.

Distribución: CAN, CAP.

Ejemplar de referencia: Bertoni, M. S. 3490 (G).

Echinodorus grandiflorus (Cham. \& Schltdl.) Micheli

Hierba palustre. Perenne. Nativa. 0-700 m.

Distribución: APO, CAP, GUA, OBE, SJA.

Ejemplar de referencia: Múlgura, M. E. 2578 (SI).

\section{Echinodorus longipetalus Micheli $\left(^{* * *}\right)$}

Hierba palustre. Perenne. Nativa. 0-500 m.

Distribución: GUA, SIG.

Ejemplar de referencia: Hauman, L. L. 1918 (BA). 
Echinodorus paniculatus Micheli emend. Rataj Hierba palustre. Anual. Nativa. 0-500 m. Distribución: GSM, MAY.

Ejemplar de referencia: Krapovickas, A. 15634 (SI).

\section{Echinodorus reptilis Lehtonen}

Hierba palustre. Perenne. Nativa. 0-100 m.

Distribución: CAP.

Ejemplar de referencia: Gerling, G. 10963 (BA).

\section{Echinodorus uruguayensis Arechav. $\left({ }^{* * *}\right)$}

Hierba palustre. Perenne. Nativa. 0-700 m.

Distribución: APO, CAI, CAP, GMB, GSM, GUA, IGU, MON.

Ejemplar de referencia: Zuloaga, F. O. 8852 (SI).

Helanthium (Benth. \& Hook. f.) Engelm. ex J.G. Sm.

Helanthium bolivianum (Rusby) Lehtonen \& Myllys

Hierba acuática. Anual. Nativa. 0-500 m.

Distribución: GUA.

Ejemplar de referencia: Deginani, N. B. 1152 (SI).

\section{Sagittaria L.}

Sagittaria montevidensis Cham. \& Schltdl. Hierba palustre. Perenne. Nativa. 0-500 m. Distribución: CAP.

Ejemplar de referencia: Sesmero, E. s.n. (A).

\section{ALSTROEMERIACEAE}

\section{Alstroemeria L.}

\section{Alstroemeria apertiflora Baker $\left.{ }^{* * *}\right)$}

Hierba. Perenne. Nativa. 0-500 m.

Distribución: CAN.

Ejemplar de referencia: Keller, H.A. 11730 (SI, CTES).

\section{Alstroemeria isabellana Herb.}

Hierba. Perenne. Nativa. 0-500 m.

Distribución: CAN, SJA.

Ejemplar de referencia: Schwartz, 3870 (LIL).
Alstroemeria psittacina Lehm.

Hierba. Perenne. Nativa. 0-800 m.

Distribución: CAN, CAP, ELD, IGU, LNA, GSM, MON, SIG, SPE.

Ejemplar de referencia: Jörgensen, P. 30240 (BAB, SI).

Bomarea Mirb.

Bomarea edulis (Tussac) Herb.

Hierba. Perenne. Nativa. 0-700 m.

Distribución: GMB, GUA, IGU, MON, SPE.

Ejemplar de referencia: Guaglianone, E. R. 2915 (SI).

\section{AMARANTHACEAE}

\section{Alternanthera Forssk.}

Alternanthera brasiliana (L.) Kuntze var. villosa (Moq.) Kuntze

Hierba. Perenne. Nativa. 0-500 m.

Distribución: IGU, MON.

Ejemplar de referencia: Krapovickas, A. 2442 (SI).

Alternanthera ficoidea (L.) $\left.\mathrm{Sm} .{ }^{* * *}\right)$

Hierba o subarbusto. Perenne. Nativa. 0-500 m.

Distribución: IGU.

Ejemplar de referencia: Vanni, R. O. 3603 (CTES).

Alternanthera kurtzii Schinz ex Pedersen

Hierba. Perenne. Nativa. 0-500 m.

Distribución: IGU, SPE. (SI).

Ejemplar de referencia: dos Santos Silva, F. 111

Alternanthera malmeana R.E. Fr. var. malmeana $(* * *)$

Hierba. Nativa. 0-500 m.

Distribución: CAN, SIG.

Ejemplar de referencia: Cabrera, A. L. 29355 (SI).

Alternanthera malmeana R.E. Fr. var. straminea (Chodat) Pedersen ${ }^{* * *}$ )

Hierba. Nativa. 0-500 m.

Distribución: SIG.

Ejemplar de referencia: Chodat, R. H. s.n. (G). 
Alternanthera micrantha R.E. Fr. $\left({ }^{* *}\right)$

Hierba. Nativa. 0-800 m.

Distribución: APO, CAI, CAN, GMB, GUA, SPE.

Ejemplar de referencia: Deginani, N. B. 1263 (SI).

Alternanthera paronychioides A. St.-Hil. subsp. pilosa (Moq.) Pedersen

Hierba. Nativa. 0-500 m.

Distribución: CAP.

Ejemplar de referencia: Holmberg, E. L. 10619 (SI).

Alternanthera philoxeroides (Mart.) Griseb. Hierba acuática. Perenne. Nativa. 0-500 m. Distribución: SIG.

Ejemplar de referencia: Keller, H. A. 10493 (CTES).

Alternanthera puberula (Mart.) D. Dietr.

Hierba. Nativa. 0-500 m.

Distribución: CAP, IGU, MON.

Ejemplar de referencia: Vanni, R. O. 2792 (SI).

\section{Alternanthera pungens Kunth}

Hierba. Perenne. Nativa. 0-500 m.

Distribución: CAN, IGU.

Ejemplar de referencia: Montes, J. E. 55b (SI).

\section{Alternanthera reineckii Briq.}

Hierba. Anual. Nativa. 0-500 m.

Distribución: CAP, IGU.

Ejemplar de referencia: Burkart, A. 14094 (SI).

\section{Alternanthera rufa (Mart.) D. Dietr. $\left({ }^{* * *}\right)$}

Hierba o subarbusto. Perenne. Nativa. 0-500 m. Distribución: SIG.

Ejemplar de referencia: Nicora, E. G. 8022 (CTES).

\section{Amaranthus L.}

Amaranthus hybridus L. subsp. cruentus (L.) Thell. (***)

Hierba. Anual. Introducida. 0-500 m.

Distribución: GUA.

Ejemplar de referencia: Tressens, S. G. 6527 (CTES).

\section{Amaranthus hybridus L. subsp. hybridus}

Hierba. Anual. Introducida. 0-500 m.
Distribución: CAI, GMB, GUA, SIG, SPE.

Ejemplar de referencia: Biganzoli, F. 807 (SI).

Amaranthus spinosus L.

Hierba. Nativa. 0-700 m.

Distribución: CON, ELD, GUA, IGU, SPE.

Ejemplar de referencia: Dematteis, M. 94 (CTES, G, MNES, SI).

\section{Amaranthus viridis $\mathrm{L}$.}

Hierba. Perenne. Nativa. 0-500 m.

Distribución: GMB, GUA.

Ejemplar de referencia: Zuloaga, F. O. 5131 (SI).

Chamissoa Kunth, nom. cons.

\section{Chamissoa acuminata Mart.}

Hierba o subarbusto. Perenne. Nativa. 0-700 m.

Distribución: CAI, GMB, GUA, SPE.

Ejemplar de referencia: Deginani, N. B. 1014 (SI).

Chamissoa altissima (Jacq.) Kunth

Arbusto o liana. Nativa. 0-700 m.

Distribución: CAN, GMB, GSM, GUA, IGU, MON, SIG, SPE.

Ejemplar de referencia: Cabrera, A. L. 29232 (SI).

\section{Chamissoa maximiliani Moq.}

Hierba o subarbusto. Perenne. Nativa. 0-500 m. Distribución: GUA.

Ejemplar de referencia: Tressens, S. E. 5083 (CTES).

\section{Froelichia Moench}

Froelichia procera (Seub.) Pedersen

Hierba. Perenne. Nativa. 0-500 m.

Distribución: CAN, SIG.

Ejemplar de referencia: Múlgura, M. E. 1624 (SI).

\section{Gomphrena L.}

Gomphrena celosioides Mart. f. aureiflora (Chodat \& Hassl.) Pedersen

Hierba. Perenne. Nativa. 0-500 m.

Distribución: CAN, GMB.

Ejemplar de referencia: Morrone, O. 821 (SI). 
Gomphrena celosioides Mart. f. roseiflora (Chodat \& Hassl.) Pedersen

Hierba. Perenne. Nativa. 0-500 m.

Distribución: IGU.

Ejemplar de referencia: Kujawska, M. 195 (CTES).

Gomphrena celosioides Mart. var. celosioides Hierba. Perenne. Nativa. 0-500 m.

Distribución: APO, CAI, CAN, CAP, ELD, GMB, GUA, MON, SIG, SPE.

Ejemplar de referencia: Burkart, A. 14095 (SI).

\section{Gomphrena elegans Mart. var. elegans}

Hierba o subarbusto. Perenne. Nativa. 0-800 m. Distribución: CAI, CAN, CAP, CON, GUA, SIG, SPE. Ejemplar de referencia:Dematteis, M.114(MNES, SI).

\section{Gomphrena graminea Moq.}

Hierba. Perenne. Nativa. 0-500 m.

Distribución: APO, CAN, CON, SIG.

Ejemplar de referencia: Krapovickas, A. 28704 (SI).

\section{Gomphrena paraguayensis $C$ hodat}

Hierba. Perenne. Nativa. 0-500 m.

Distribución: APO.

Ejemplar de referencia: Cabrera, A. L. 28517 (SI).

\section{Hebanthe Mart.}

Hebanthe eriantha (Poir.) Pedersen ${ }^{* * *}$ )

Liana. Perenne. Nativa. 0-700 m.

Distribución: CAI, ELD, GMB, GUA, IGU.

Ejemplar de referencia: Johnson, A. E. 913 (SI).

\section{Herbstia Sohmer}

Herbstia brasiliana (Moq.) Sohmer

Hierba o subarbusto. Perenne. Nativa. 0-500 m. Distribución: CAN, CAP.

Ejemplar de referencia: Ekman, E. L. 127 (MO).

\section{Iresine P. Browne}

Iresine diffusa Humb. \& Bonpl. ex Willd.

Subarbusto. Nativa. 0-800 m.

Distribución: CAI, CAN, CAP, GMB, GSM,
GUA, IGU, MON, SIG, SPE.

Ejemplar de referencia: Cabrera, A. L. 29209 (SI).

Pfaffia Mart.

Pfaffia glabrata Mart.

Hierba o subarbusto. Perenne. Nativa. 0-500 m. Distribución: IGU.

Ejemplar de referencia: Rodríguez, F. M. 781 (SI).

Pfaffia glomerata (Spreng.) Pedersen

Hierba o subarbusto. Perenne. Nativa. 0-800 m. Distribución: CAN, CAP, ELD, IGU, SIG.

Ejemplar de referencia: Morrone, O. 1094 (SI).

Pfaffia gnaphaloides (L. f.) Mart.

Hierba. Perenne. Nativa. 0-500 m.

Distribución: CAN, CON, SIG.

Ejemplar de referencia: Zuloaga, F. O. 6548 (SI).

Pfaffia helichrysoides (Mart.) Kuntze (***)

Hierba. Perenne. Nativa. 0-500 m.

Distribución: CAN, SIG.

Ejemplar de referencia: Zardini, E. M. 699 (SI).

Pfaffia tuberosa (Spreng.) Hicken $\mathrm{f}$. tuberosa

Hierba. Perenne. Nativa. 0-500 m.

Distribución: CAN, SIG.

Ejemplar de referencia: Zuloaga, F. O. 6460 (SI).

\section{Pseudoplantago Suess.}

\section{Pseudoplantago friesii Suess. $\left.{ }^{(* *}\right)$}

Hierba o subarbusto. Nativa. 0-800 m.

Distribución: CAI, CAN, CAP, GMB, GUA, SPE.

Ejemplar de referencia: Deginani, N. B. 1337 (SI).

\section{AMARYLLIDACEAE}

\section{Beauverdia Herter}

Beauverdia dialystemon (Guagl.) Sassone \& Guagl. Hierba. Perenne. Nativa. 0-500 m.

Distribución: CAP.

Ejemplar de referencia: Scala, A. C. 155 (LP). 


\section{Crinum L.}

\section{Crinum americanum $\mathrm{L}$.}

Hierba. Perenne. Nativa. 0-500 m.

Distribución: ELD, GMB.

Ejemplar de referencia: Hunziker, J. H. 11042 (SI).

\section{Habranthus Herb.}

Habranthus robustus Herb. ex Sweet Hierba. Perenne. Nativa. 0-500 m. Distribución: CAP, OBE.

Ejemplar de referencia: Spegazzini, C. L. 22032 (SI).

Hippeastrum Herb., nom. cons.

\section{Hippeastrum angustifolium $\mathrm{Pax}$}

Hierba. Perenne. Nativa. 0-500 m.

Distribución: APO, CAN, CAP, LNA.

Ejemplar de referencia: de Llamas, A. 26616 (SI).

Hippeastrum glaucescens (Mart.) Herb. $\left(^{* * *}\right)$

Hierba. Perenne. Nativa. 0-800 m.

Distribución: GUA, IGU, OBE, SIG, SPE.

Ejemplar de referencia: Zuloaga, F. O. 6456 (SI).

Hippeastrum reticulatum (L'Hér.) Herb. $\left({ }^{* * *}\right)$

Hierba. Perenne. Nativa. 0-700 m.

Distribución: GMB.

Ejemplar de referencia: Spegazzini, C. L. s.n. (SI).

Hippeastrum striatum (Lam.) H. E. Moore

Hierba. Perenne. Nativa. 0-800 m.

Distribución: CAI, ELD, GMB.

Ejemplar de referencia: Ravenna, P. F. 1951

(Herb. Ravenna).

Hippeastrum vittatum (L'Hér.) Herb. ${ }^{* * *}$ )

Hierba. Perenne. Nativa. 0-500 m.

Distribución: CAN, CAP, CON.

Ejemplar de referencia: Seijo, G. J. 1972 (CTES).

\section{Nothoscordum Kunth}

Nothoscordum bonariense (Pers.) Beauverd Hierba. Perenne. Nativa. 0-800 m.
Distribución: CAI.

Ejemplar de referencia: Múlgura, M. E. 2464 (SI).

Nothoscordum gracile (Dryand. ex Aiton) Stearn

Hierba. Perenne. Nativa. 0-600 m.

Distribución: CAN.

Ejemplar de referencia: Morrone, O. 1790 (SI).

Nothoscordum moconense Ravenna ${ }^{* *}$ )

Hierba. Perenne. Endémica. 0-300 m.

Distribución: SPE.

Ejemplar de referencia: Zuloaga, F. O. 718 (SI).

Nothoscordum montevidense Beauverd

Hierba. Perenne. Nativa. 0-500 m.

Distribución: APO, CAN.

Ejemplar de referencia: Zuloaga, F. O. 5773 (SI).

Zephyranthes Herb., nom. cons.

Zephyranthes bifida (Herb.) Nic.García \& S.C. Arroyo

Hierba. Perenne. Nativa. 0-500 m.

Distribución: APO, CAN.

Ejemplar de referencia: Zuloaga, F. O. 1011 (SI).

Zephyranthes candida (Herb. ex Lindl.) Herb. Hierba. Perenne. Nativa. 0-500 m.

Distribución: GUA.

Ejemplar de referencia: Keller, H. A. 470 (CTES).

Zephyranthes flavissima Ravenna Hierba. Perenne. Nativa. 0-800 m. Distribución: MON, SPE.

Ejemplar de referencia: Porta, R. E. 168 (SI).

Zephyranthes mesochloa Herb. ex Lindl.

Hierba. Perenne. Nativa. 0-500 m.

Distribución: CAI, GMB.

Ejemplar de referencia: Montes, J. E. 7262 (SI).

Zephyranthes pedunculosa (Herb.) Nic.García \& S.C. Arroyo

Hierba. Perenne. Nativa. 0-500 m.

Distribución: CAN, CAP, CON, SIG.

Ejemplar de referencia: Biganzoli, F. 1743 (SI). 
Zephyranthes schulziana (Ravenna) S. C. Arroyo

Hierba. Perenne. Nativa. 0-500 m.

Distribución: CAN.

Ejemplar de referencia: Keller, H. A. 11898 (CTES).

\section{Zephyranthes seubertii E.P. Hume}

Hierba. Perenne. Nativa. 0-300 m.

Distribución: CAN, CAP.

Ejemplar de referencia: Daviña, J. 322 (B).

\section{ANACARDIACEAE}

\section{Astronium Jacq.}

\author{
Astronium fraxinifolium Schott var. glabrum \\ Engl. (***) \\ Árbol. Nativa. 0-500 m. \\ Distribución: IGU. \\ Ejemplar de referencia: Rodríguez $\left\{2^{\circ}\right.$ serie $\}$, \\ F. M.s.n. (BAF).
}

\section{Lithraea Miers}

Lithraea brasiliensis Marchand

Árbol. Nativa. 0-500 m.

Distribución: APO, GUA, SPE.

Ejemplar de referencia: Niederlein, G. 713 (BA, SI).

Lithraea molleoides (Vell.) Engl.

Árbol. Nativa. 0-500 m.

Distribución: APO, CAI, CAN, CAP, CON, ELD, IGU, GSM, OBE, SIG.

Ejemplar de referencia: Burkart, A. 14255 (SI).

\section{Myracrodruon Allemão}

Myracrodruon balansae (Engl.) Santin

Árbol. Nativa. 0-700 m.

Distribución: CAN, CAP, CON, LNA, GSM, SIG, SJA.

Ejemplar de referencia: Burkart, A. 14465 (SI).
Schinus L.

Schinus areira $\mathrm{L}$.

Árbol. Nativa. 0-500 m.

Distribución: CAP, IGU.

Ejemplar de referencia: Denis, L. 3 (BA).

Schinus ferox Hassl. $\left(^{(* *}\right)$

Arbusto o arbolito. Nativa. 0-500 m.

Distribución: SPE.

Ejemplar de referencia: Niederlein, G. 1578b (SI).

Schinus lentiscifolius Marchand $\left({ }^{* *}\right)$

Árbol. Nativa. 0-500 m.

Distribución: CAN, GMB, SIG.

Ejemplar de referencia: Eskuche, U. G. 267 (SI).

Schinus longifolius (Lindl.) Speg.

Arbusto o árbol. Nativa. 0-700 m.

Distribución: CAI, CAN, GMB, GSM, SIG.

Ejemplar de referencia: Rodríguez $\left\{2^{\circ}\right.$ serie $\}, F$. M. 51 (BA, BAB, SI).

\section{Schinus molle L.}

Árbol. Nativa. 0-500 m.

Distribución: APO, CAI, CAN, CAP, CON, ELD, GSM, GUA, SIG, SPE.

Ejemplar de referencia: Burkart, A. 14251 (SI).

Schinus terebinthifolius Raddi var. acutifolius Engl. $(* * *)$

Arbusto o árbol. Nativa. 0-500 m.

Distribución: APO, CAN, OBE.

Ejemplar de referencia: Denham, S. S. 44 (SI).

Schinus terebinthifolius Raddi var. pohlianus Engl.

Árbol. Nativa. 0-800 m.

Distribución: APO, CAI, CAN, CAP, CON, ELD, GMB, GSM, GUA, IGU, LNA, MON, OBE, SIG, SJA, SPE.

Ejemplar de referencia: Cabrera, A. L. 28317 (SI).

Schinus weinmannifolius Engl. var. dubius F.A. Barkley

Arbusto. Nativa. 0-500 m.

Distribución: APO, CAN, CAP, CON, SIG, SJA.

Ejemplar de referencia: Vattuone, I. C. 126 (BAF). 


\author{
Schinus weinmannifolius Engl. var. \\ riedelianus Engl. \\ Arbusto o árbol. Nativa. 0-700 m. \\ Distribución: APO, CAN, CAP, CON, ELD, \\ GUA, IGU, SIG, SPE. \\ Ejemplar de referencia: Meyer, T. 11603 (LIL).
}

Schinus weinmannifolius Engl. var. weinmannifolius

Arbusto. Nativa. 0-700 m.

Distribución: APO, CAI, CAN, CAP, SIG.

Ejemplar de referencia: Quiroga, H. 7680 (LIL, SI).

\section{ANNONACEAE}

\section{Annona L.}

Annona neosalicifolia $H$. Rainer

Arbusto o árbol. Nativa. 0-500 m.

Distribución: CAI, CAN, GUA, IGU, SIG, SPE. (SI).

Ejemplar de referencia: Guaglianone, E. R. 2902

Annona rugulosa (Schltdl.) H. Rainer

Arbusto o árbol. Nativa. 0-500 m.

Distribución: GUA, IGU, SPE.

Ejemplar de referencia: Niederlein, G. 1189 $(B A)$.

\section{Xylopia L.}

Xylopia brasiliensis Spreng. $\left.{ }^{* * *}\right)$

Árbol. Nativa. 0-500 m.

Distribución: SIG.

Ejemplar de referencia: Biganzoli, F. 609 (SI).

\section{APIACEAE}

\section{Ammi L.}

\section{Ammi majus L.}

Hierba. Anual. Introducida. 0-500 m.

Distribución: SPE.

Ejemplar de referencia: Zuloaga, F. O. 9928 (SI).
Anethum L.

\section{Anethum graveolens $\mathrm{L}$.}

Hierba. Anual. Introducida. 0-500 m.

Distribución: GUA, IGU.

Ejemplar de referencia: Martínez Crovetto, $R$. 5796 (BAB).

Bowlesia Ruiz \& Pav.

Bowlesia incana Ruiz \& Pav.

Hierba. Anual. Nativa. 0-500 m.

Distribución: CAN, ELD, GUA.

Ejemplar de referencia: Llamas, A. 119 (SI).

\section{Centella L.}

Centella asiatica (L.) Urb.

Hierba. Perenne. Nativa. 0-500 m.

Distribución: CAN, ELD, SIG.

Ejemplar de referencia: Pérez-Moreau, R. A. $31(B A)$.

\section{Coriandrum L.}

\section{Coriandrum sativum $\mathrm{L}$.}

Hierba. Anual. Introducida. 0-500 m.

Distribución: CAP, GMB, GUA, SIG.

Ejemplar de referencia: Rodríguez, F. M. 62 (SI).

\section{Cyclospermum Lag.}

Cyclospermum leptophyllum (Pers.) Britton \& P. Wilson var. leptophyllum

Hierba. Anual. Nativa. 0-800 m.

Distribución: APO, CAI, CAN, GMB, GUA, IGU, SIG, SPE.

Ejemplar de referencia: Morrone, O. 687 (SI).

Cyclospermum leptophyllum (Pers.) Britton \& P. Wilson var. multisectum (Mathias \& Constance) Constance (***)

Hierba. Anual. Nativa. 0-500 m.

Distribución: GUA.

Ejemplar de referencia: Tressens, S. G. 5916 (CTES). 


\section{Daucus L.}

\section{Daucus pusillus Michx.}

Hierba. Anual. Nativa. 0-800 m.

Distribución: GMB, OBE, SIG.

Ejemplar de referencia: Morrone, O. 1498 (SI).

\section{Eryngium L.}

Eryngium chamissonis Urb.

Hierba. Perenne. Nativa. 0-800 m.

Distribución: APO, CON, OBE.

Ejemplar de referencia: Biganzoli, F. 1640 (SI).

Eryngium divaricatum Hook. \& Arn.

Hierba. Perenne. Nativa. 0-500 m.

Distribución: CAN, CAP.

Ejemplar de referencia: Jörgensen, P. 415 $(B A B)$.

\section{Eryngium ebracteatum Lam.}

Hierba. Perenne. Nativa. 0-800 m. SIG.

Distribución: CAI, CAN, CAP, CON, ELD, IGU,

Ejemplar de referencia: Biganzoli, F. 1648 (SI).

\section{Eryngium eburneum Decne.}

Hierba. Perenne. Nativa. 0-500 m.

Distribución: CAN.

Ejemplar de referencia: Rodríguez, 752 (SI).

\section{Eryngium echinatum Urb.}

Hierba. Perenne. Nativa. 0-500 m.

Distribución: ELD.

Ejemplar de referencia: Schinini, A. 5935 (CTES).

\section{Eryngium ekmanii $\mathrm{H}$. Wolff}

Hierba. Anual o Bianual. Nativa. 0-700 m.

Distribución: CAI, CAN, CAP, ELD, GMB, GSM, GUA, IGU, LNA, OBE, SIG, SPE.

Ejemplar de referencia: Deginani, N. B. 1283 (SI).

Eryngium elegans Cham. \& Schltdl.

Hierba. Perenne. Nativa. 0-500 m.

Distribución: CAN, SIG, SJA, SPE.

Ejemplar de referencia: Montes, J. E. 7003 (SI).
Eryngium eriophorum Cham. \& Schltdl.

Hierba. Perenne. Nativa. 0-500 m.

Distribución: APO.

Ejemplar de referencia: Cabrera, A. L. 29184 (SI).

Eryngium floribundum Cham. \& Schltdl.

Hierba. Perenne. Nativa. 0-500 m.

Distribución: APO, CAN, CAP.

Ejemplar de referencia: Cabrera, A. L. 28768 (SI).

Eryngium horridum Malme

Hierba. Perenne. Nativa. 0-500 m.

Distribución: APO, CAN, LNA.

Ejemplar de referencia: Calviño, C. 644 (SI).

Eryngium juncifolium (Urb.) Mathias \& Constance $\left.{ }^{* * *}\right)$

Hierba. Perenne. Nativa. 0-500 m.

Distribución: CAN, SIG.

Ejemplar de referencia: Zuloaga, F. O. 5331 (SI).

Eryngium luzulaefolium Cham. \& Schltdl.

Hierba. Perenne. Nativa. 0-500 m.

Distribución: CAN.

Ejemplar de referencia: Jörgensen, P. 507 (SI).

Eryngium nudicaule Lam.

Hierba. Perenne. Nativa. 0-800 m.

Distribución: APO, CAN, CAP, CON, IGU, SIG.

Ejemplar de referencia: Múlgura, M. E. 2358 (SI).

Eryngium pandanifolium Cham. \& Schltdl.

Hierba. Perenne. Nativa. 0-500 m.

Distribución: CAN, GMB, GSM, GUA.

Ejemplar de referencia: Keller, H. A. 205 (CTES, SI).

Eryngium pristis Cham. \& Schltdl.

Hierba. Perenne. Nativa. 0-700 m.

Distribución: APO, CAI, CAN, CON, SIG.

Ejemplar de referencia: Morrone, O. 1039 (SI).

\section{Eryngium regnelli Malme}

Hierba. Perenne. Nativa. 0-500 m.

Distribución: CAI, IGU, LNA.

Ejemplar de referencia: Calviño, C. I. 643 (SI). 
Eryngium sanguisorba $\mathrm{Cham}$. \& Schltdl.

Hierba. Perenne. Nativa. 0-800 m.

Distribución: APO, CAI, CAN, SPE.

Ejemplar de referencia: Montes, J. E. 7004 (SI).

\section{Eryngium stenophyllum Urb.}

Hierba. Perenne. Nativa. 0-500 m.

Distribución: IGU, LNA.

Ejemplar de referencia: Calviño, C. 643 (SI).

\section{Foeniculum Mill.}

Foeniculum vulgare Mill.

Hierba. Perenne. Introducida. 0-500 m.

Distribución: CAP, GUA, OBE, SPE.

Ejemplar de referencia: Keller, H. A. 3493 (CTES).

\section{Lilaeopsis Greene}

Lilaeopsis brasiliensis (Glaz.) Affolter

Hierba. Perenne. Nativa. 0-500 m.

Distribución: APO, CAP. (SI).

Ejemplar de referencia: Spegazzini, C. L. 0187

Lilaeopsis carolinensis J.M. Coult. \& Rose

Hierba. Perenne. Nativa. 0-500 m.

Distribución: CAP.

Ejemplar de referencia: Ekman, E. L. $1553($ MO, NY).

\section{APOCYNACEAE}

\section{Araujia Brot.}

\section{Araujia plumosum Schltdl.}

Enredadera. Perenne. Nativa. 0-500 m.

Distribución: CAP. ELD.

Ejemplar de referencia: Keller, H. A. 13255 (CTES).

\section{Araujia sericifera Brot.}

Enredadera. Perenne. Nativa. 0-700 m.

Distribución: CAI, GMB, GUA, LNA, MAY, SIG, SPE.

Ejemplar de referencia: Denham, S. S. 52 (SI).

\section{Asclepias L.}

Asclepias aequicornu E. Fourn. $\left({ }^{* * *}\right)$

Hierba. Perenne. Nativa. 0-500 m.

Distribución: CAP.

Ejemplar de referencia: Ekman, E. L. 404 (S).

Asclepias candida Vell. (***)

Hierba. Perenne. Nativa. 0-500 m.

Distribución: CAN, SJA.

Ejemplar de referencia: Keller, H. A. 13099 (CTES).

\section{Asclepias curassavica L.}

Hierba. Perenne. Introducida. 0-700 m.

Distribución: CAI, CAN, CAP, ELD, GMB, GSM, GUA, IGU, OBE.

Ejemplar de referencia: Deginani, N. B. 1033 (SI).

Asclepias mellodora A. St.-Hil.

Hierba. Perenne. Nativa. 0-700 m.

Distribución: APO, CAI, CAN, CAP, CON, GUA.

Ejemplar de referencia: Rodríguez, M. 213 (MNES, SI).

Aspidosperma Mart. \& Zucc.

Aspidosperma australe Müll. Arg.

Árbol. Nativa. 0-500 m.

Distribución: CAN, ELD, SPE.

Ejemplar de referencia: Zuloaga, F. O. 5584 (SI).

Aspidosperma polyneuron Müll. Arg. $\left.{ }^{* * *}\right)$

Árbol. Nativa. 0-500 m.

Distribución: IGU, SPE.

Ejemplar de referencia: Keller, H. A. 3043 (CTES).

Austrochthamalia Morillo \& Fontella

Austrochthamalia teyucuarensis H.A. Keller $(* *)$

Subarbusto. Endémica. 0-500 m.

Distribución: SIG.

Ejemplar de referencia: Keller, H. A. 12323 (CTES,SI). 


\section{Blepharodon Decne.}

\section{Blepharodon lineare (Decne.) Decne.}

Hierba o subarbusto. Perenne. Nativa. 0-700 m. Distribución: APO, CAN, GMB, SIG, SJA.

Ejemplar de referencia: Zuloaga, F. O. 3183 (SI).

\section{Brargentina Morillo \& H.A. Keller}

Brargentina bornmulleri (Schltr. Ex Malme) Morillo \& H.A. Keller

Hierba. Perenne. Nativa. 0-500 m.

Distribución: CAN, CON, SJA.

Ejemplar de referencia: Keller, H. A. 13092 (CTES).

\section{Condylocarpon Desf.}

Condylocarpon isthmicum (Vell.) A.DC.

Liana. Perenne. Nativa. 0-700 m.

Distribución: ELD, GMB, GUA, IGU, SIG, SPE.

Ejemplar de referencia: Zuloaga, F. O. 6465 (SI).

Cristobalia Morillo, S.A. Cáceres \& H.A. Keller

Cristobalia bella Morillo, H.A. Keller \& S.A. Cáceres

Subarbusto. Nativa. 200-700 m.

Distribución: CAI, OBE, SIG, SPE.

Ejemplar de referencia: Keller, H. A. 5276 (CTES).

\section{Cynanchum L.}

Cynanchum caudiculatum Rapini ${ }^{* * *}$ )

Enredadera. Nativa. 0-500 m.

Distribución: CAN, ELD, GUA, SJA.

Ejemplar de referencia: Bertoni, M. S. 3368 (LIL).

\section{Cynanchum montevidense Spreng.}

Enredadera. Nativa. 0-500 m.

Distribución: CAN, CAP, SIG.

Ejemplar de referencia: Zuloaga, F. O. 3200 (SI).
Ditassa R. Br.

Ditassa burchellii Hook. \& Arn.

Enredadera. Perenne. Nativa. 0-800 m.

Distribución: CAN, SIG, SPE.

Ejemplar de referencia: Rodríguez, F. M. 726 (LIL, SI).

Ditassa hispida (Vell.) Fontella $\left(^{* * *}\right)$

Enredadera. Nativa. 0-500 m.

Distribución: SJA.

Ejemplar de referencia: Molfino, J. F. s.n. (LIL).

\section{Fischeria DC.}

Fischeria stellata (Vell.) E. Fourn. $\left.{ }^{* * *}\right)$

Enredadera o liana. Perenne. Nativa. 0-700 m.

Distribución: ELD, GMB, GUA, IGU, SPE.

Ejemplar de referencia: Zuloaga, F. O. 5594 (SI).

Forsteronia G. Mey.

Forsteronia glabrescens Müll. Arg.

Liana. Perenne. Nativa. 0-800 m.

Distribución: APO, CAI, CAN, CON, GUA, IGU, LNA, SIG.

Ejemplar de referencia: Morrone, O. 1637 (SI).

Forsteronia pubescens A.DC.

Liana. Perenne. Nativa. 0-500.

Distribución: APO.

Ejemplar de referencia: Zanotti, C. A. 351 (SI).

Forsteronia refracta Müll. Arg. $\left({ }^{* * *}\right)$

Liana. Perenne. Nativa. 0-500 m.

Distribución: GMB, GUA, IGU, SPE.

Ejemplar de referencia: Tressens, S. G. 6504 (CTES).

Forsteronia thyrsoidea (Vell.) Müll. Arg.

Liana. Perenne. Nativa. 0-700 m.

Distribución: CAI, CAN, GSM, SPE.

Ejemplar de referencia: Zuloaga, F. O. 6440 (SI).

Funastrum E. Fourn.

Funastrum clausum (Jacq.) Schltr. Enredadera. Perenne. Nativa. 0-500 m. 
Distribución: IGU, SIG.

Ejemplar de referencia: Keller, H. A. 12740 (CTES).

Funastrum flavum (Decne.) Malme

Enredadera. Perenne. Nativa. 0-500 m.

Distribución: CAN, CAP

Ejemplar de referencia: Keller, H.A. 13882 (CTES).

Gomphocarpus $\mathrm{R}$. Br.

Gomphocarpus physocarpus E. Mey. $\left(^{*}\right)$

Subarbusto. Introducida. 0-500 m.

Distribución: SPE.

Ejemplar de referencia: Keller, H. A. 12280 (SI, CTES).

\section{Gonolobus Michx.}

Gonolobus parviflorus Decne.

Enredadera. Perenne. Nativa. 0-500 m.

Distribución: GUA, IGU, SIG.

Ejemplar de referencia: Keller, H. A. 1648 (CTES, SI).

Gonolobus rostratus (Vahl) Roem. \& Schult.

Enredadera. Perenne. Nativa. 0-600 m.

Distribución: CAN, GMB, IGU, SPE.

Ejemplar de referencia: Deginani, N. B. 1217 (SI).

Jobinia E. Fourn.

Jobinia latipes (Decne.) Liede \& Meve $\left(^{* * *}\right)$

Enredadera. Perenne. Nativa. 0-500 m.

Distribución: GUA, SPE.

Ejemplar de referencia: Tressens, S. G. 5472 (CTES).

Jobinia lindbergii E. Fourn. $\left.{ }^{* * *}\right)$

Liana. Nativa. 0-500 m.

Distribución: GMB.

Ejemplar de referencia: Guaglianone, E. R. 2963 (SI).

\section{Macropharynx Rusby}

Macropharynx peltata (Vell.) J.F. Morales, M.E. Endress \& Liede

Liana. Perenne. Nativa. 0-700 m.
Distribución: GMB, GUA, IGU, SIG, SPE.

Ejemplar de referencia: Zuloaga, F. O. 6493 (SI).

\section{Macroscepis Kunth}

Macroscepis elliptica N.E. Br.

Liana. Nativa. 0-500 m.

Distribución: ELD, GMB, GUA, MON, SPE

Ejemplar de referencia: Deginani, N. B. 1745 (SI).

Mandevilla Lindl.

Mandevilla coccinea (Hook. \& Arn.) Woodson $(* * *)$

Hierba o subarbusto. Perenne. Nativa.

Distribución: en el sur de la provincia.

Ejemplar de referencia: Bertoni, M. S. 691 (LIL).

Mandevilla emarginata (Vell.) C. Ezcurra

Hierba o subarbusto. Perenne. Nativa. 0-500 m. Distribución: CAI, OBE.

Ejemplar de referencia: Niederlein, G. 174 (BA, SI).

Mandevilla longiflora (Desf.) Pichon

Hierba. Perenne. Nativa. 0-500 m.

Distribución: APO, CON, SIG, SJA.

Ejemplar de referencia: Morrone, O. 1014 (SI).

Mandevilla petraea (A. St.-Hil.) Pichon

Hierba. Perenne. Nativa. 0-500 m.

Distribución: CAN, CAP, CON.

Ejemplar de referencia: Fontana, J. L. 49 (CTESN, SI).

Mandevilla undulata (C. Ezcurra) A.O. Simões, Kin.-Gouv. \& M.E. Endress $\left.{ }^{* * *}\right)$

Hierba. Perenne. Nativa. 0-500 m.

Distribución: CAN, SIG.

Ejemplar de referencia: Zuloaga, F. O. 3185 (SI).

Mandevilla virescens (A. St.-Hil.) Pichon $\left(^{* * *}\right.$ ) Hierba. Perenne. Nativa

Distribución: SIG.

Ejemplar de referencia: Zanotti, C. A. 406 (SI). 


\section{Matelea Aubl.}

Matelea balansae Morillo \& Fontella (***)

Hierba o enredadera. Perenne. Nativa. 0-300 m.

Distribución: GMB, GUA.

Ejemplar de referencia: Keller, H. A. 13687 (CTES).

\section{Minaria T.U.P. Konno \& Rapini}

Minaria acerosa (Mart.) T.U.P. Konno \& Rapini $(* * *)$

Subarbusto. Nativa. 0-500 m.

Distribución: SIG.

Ejemplar de referencia: Morrone, O. 1674 (MO, SI).

\section{Nautonia Decne.}

Nautonia nummularia Decne.

Hierba. Nativa. 0-500 m.

Distribución: APO, CAI, CAN, CAP, GMB, SIG, SJA.

Ejemplar de referencia: Biganzoli, F. 432 (SI).

\section{Orthosia Decne.}

\section{Orthosia congesta Decne. $\left({ }^{*}\right)$}

Enredadera. Perenne. Nativa. 0-500 m.

Distribución: ELD, MON.

Ejemplar de referencia: Keller, H. A. 13903 (CTES).

\section{Orthosia scoparia (Nutt.) Liede \& Meve}

Enredadera. Perenne. Nativa. 0-500 m.

Distribución: CAN, ELD, GMB, GUA, SIG, SPE.

Ejemplar de referencia: Zuloaga, F. O. 6711 (SI).

Orthosia urceolata E. Fourn. $\left.{ }^{* * *}\right)$

Enredadera. Perenne. Nativa. 0-500 m.

Distribución: CON, GSM, GUA.

Ejemplar de referencia: Tressens, S. G. 6383 (CTES).

Orthosia virgata (Poir.) E. Fourn.

Enredadera. Perenne. Nativa. 0-500 m.

Distribución: GSM, GUA.

Ejemplar de referencia: Tressens, S. G. 6280 (CTES).
Oxypetalum R. Br.

Oxypetalum appendiculatum Mart.

Enredadera. Perenne. Nativa. 0-800 m.

Distribución: APO, CAI, GMB, GUA, IGU, LNA,

SIG, SJA, SPE.

Ejemplar de referencia: Morrone, O. 1345 (SI).

Oxypetalum arnottianum $\mathrm{H}$. Buek

Hierba o subarbusto. Perenne. Nativa. 0-700 m. Distribución: APO, CAN, CAP, CON, SIG, SJA. Ejemplar de referencia: Burkart, A. 14167 (SI).

Oxypetalum balansae Malme

Enredadera. Perenne. Nativa. 0-500 m.

Distribución: IGU, SIG, SPE.

Ejemplar de referencia: Barboza, G. E. 468 (CORD, SI).

Oxypetalum capitatum Mart.

Hierba. Perenne. Nativa. 0-500 m.

Distribución: CAN, CAP, SIG, SJA.

Ejemplar de referencia: Fontana, J. L. 621 (CTESN, SI).

Oxypetalum charrua T. Mey. $\left({ }^{* * *}\right)$

Hierba. Perenne. Nativa. 0-500 m.

Distribución: CAN.

Ejemplar de referencia: Spegazzini, C. L. s.n. (LPS).

Oxypetalum coccineum Griseb.

Hierba. Perenne. Nativa. 0-500 m.

Distribución: CAN, SJA.

Ejemplar de referencia: Keller, H. A. 13095 (CTES).

Oxypetalum chodatianum Malme

Hierba. Perenne. Nativa. 0-500 m.

Distribución: CAN.

Ejemplar de referencia: Keller, H. A. 9549 (CTES).

Oxypetalum confusum Malme

Hierba. Perenne. Nativa. 0-500 m.

Distribución: CAP, SJA.

Ejemplar de referencia: Keller, H.A. 12491 (SI, CTES).

Oxypetalum crispum Wight ex Hook. \& Arn.

Hierba. Perenne. Nativa. 0-500 m.

Distribución: CAN, IGU, SIG.

Ejemplar de referencia: Montes, J. E. 3482 (SI). 
Oxypetalum erianthum Decne.

Liana. Nativa. 0-500 m.

Distribución: CAN, CAP, IGU.

Ejemplar de referencia: Morrone, O. 1933 (SI).

Oxypetalum guaraniticum (Malme) Malme $(* *)$

Hierba. Perenne. Endémica. 0-500 m.

Distribución: CAN.

Ejemplar de referencia: Ekman, E. L. 1564 (S).

Oxypetalum incanum E. Fourn.

Hierba. Nativa. 0-500 m.

Distribución: APO.

Ejemplar de referencia: Huidobro, 4810 (LIL).

\section{Oxypetalum joergensenii T. Mey.}

Hierba. Nativa. 0-500 m.

Distribución: APO, CAN, CON.

Ejemplar de referencia: Burkart, A. 14394 (SI).

\section{Oxypetalum lynchianum $\mathrm{T}$. Mey.}

Hierba. Perenne. Nativa. 0-500 m.

Distribución: SJA.

Ejemplar de referencia: Keller, H. A. 12089 (CTES).

Oxypetalum macrolepis (Hook. \& Arn.) Decne. Enredadera. Perenne. Nativa. 0-500 m.

Distribución: CAN, SIG.

Ejemplar de referencia: Keller, H. A. 12374 (SI).

\section{Oxypetalum megapotamicum Spreng. $\left.{ }^{* * *}\right)$}

Enredadera. Perenne. Nativa. 0-500 m.

Distribución: CAI, LNA, OBE, SIG.

Ejemplar de referencia: Biganzoli, F. 343 (SI).

Oxypetalum microphyllum Hook. \& Arn.

Hierba. Perenne. Nativa. 0-500 m.

Distribución: CAP.

Ejemplar de referencia: Spegazzini, C. L. s.n. (BAB).

Oxypetalum molle Hook. \& Arn. $\left({ }^{* *}\right)$

Enredadera. Perenne. Nativa. 0-700 m.

Distribución: CAI, CAN, GMB, GUA, MON, SIG, SPE.

Ejemplar de referencia: Vanni, R. O. 684 (CTES, $G H, K, L I L, V E N)$.

\section{Oxypetalum mosenii Malme ${ }^{* * *}$ )}

Enredadera. Perenne. Nativa. 0-600 m.

Distribución: CAI, LNA, OBE, SIG, SJA.

Ejemplar de referencia: Burkart, A. 15407 (SI).

\section{Oxypetalum ostenii Malme $\left({ }^{* * *}\right)$}

Enredadera. Nativa. 0-500 m.

Distribución: GMB, IGU.

Ejemplar de referencia: Osten, C. 8083 (MVM).

\section{Oxypetalum pannosum Decne.}

Enredadera. Perenne. Nativa. 0-600 m.

Distribución: CAI, CAN, CAP, GMB, GUA, LNA, SIG.

Ejemplar de referencia: Montes, J. E. 7088 (SI).

Oxypetalum radinsii H.A. Keller $\left(^{* *}\right)$

Subarbusto. Endémica. 200-300 m.

Distribución: CAI, CAN, SIG.

Ejemplar de referencia: Keller, H. A. 12770 (CTES).

Oxypetalum solanoides Hook. \& Arn.

Hierba. Perenne. Nativa. 0-500 m.

Distribución: APO, CON, GUA.

Ejemplar de referencia: Zuloaga, F. O. 8120 (SI).

\section{Oxypetalum stipatum Malme}

Enredadera. Perenne. Nativa. 0-500 m.

Distribución: APO, ELD, GMB, GUA, MON.

Ejemplar de referencia: Zuloaga, F. O. 6714 (SI).

\section{Oxypetalum sublanatum Malme $\left.{ }^{* * *}\right)$}

Enredadera. Perenne. Nativa. 0-500 m.

Distribución: APO.

Ejemplar de referencia: Molfino, J. F. s.n. (LIL).

Oxypetalum sylvestre (Hook. \& Arn.) Goyder \& Rapini

Enredadera. Perenne. Nativa. 0-500 m.

Distribución: CAN, CAP.

Ejemplar de referencia: Zuloaga, F. O. 5781 (SI).

Oxypetalum teyucuarense Farinaccio \& H.A. Keller (**)

Hierba. Perenne. Endémica. 100-200 m.

Distribución: SIG.

Ejemplar de referencia: Keller, H. A. 7740 (CTES). 
Oxypetalum wightianum Hook. \& Arn. $\left({ }^{* * *}\right)$

Enredadera. Perenne. Nativa. 0-500 m.

Distribución: IGU.

Ejemplar de referencia: Keller, H. A. 1528 (CTES).

Phaeostemma E. Fourn.

Phaeostemma kelleri Morillo $\left({ }^{* *}\right)$

Liana. Nativa. 0-500 m.

Distribución: GMB, GUA, SPE.

Ejemplar de referencia: Morrone, O. 1528 (SI).

\section{Plumeria L.}

Plumeria rubra L. $\left({ }^{* * *}\right)$

Arbolito. Introducida. 0-500 m.

Distribución: SIG.

Ejemplar de referencia: Deginani, N. B. 1434 (SI).

Prestonia R. Br., nom. cons.

Prestonia calycina Müll. Arg.

Liana. Nativa. 0-500 m.

Distribución: CAN, ELD, SIG.

Ejemplar de referencia: Múlgura, M. E. 2114 (SI).

Prestonia coalita (Vell.) Woodson $\left({ }^{* *}\right)$

Liana. Nativa. 0-500 m.

Distribución: ELD, IGU.

Ejemplar de referencia: Keller, H. A. 57 (CTES, SI).

\section{Prestonia cyaniphylla (Rusby) Woodson}

Enredadera o liana. Perenne. Nativa. 0-700 m. Distribución: APO, CAI, CAN, GMB, LNA, OBE, SIG.

Ejemplar de referencia: Zuloaga, F. O. 5310 (SI).

Prestonia lagoensis (Müll. Arg.) Woodson (***) Enredadera. Perenne. Nativa. 0-500 m.

Distribución: SPE.

Ejemplar de referencia: Montes, J. E. 1700 (CR, K,LIL, RB).

\section{Prestonia quinquangularis (Jacq.) Spreng.}

Liana. Nativa. 0-500 m.

Distribución: IGU, SPE.

Ejemplar de referencia: Meyer, T. 6506 (SI).

\section{Rauvolfia L.}

Rauvolfia sellowii Müll. Arg. $\left.{ }^{(* *}\right)$

Árbol. Nativa. 0-500 m.

Distribución: ELD, GMB, IGU, MON, SPE.

Ejemplar de referencia: Morrone, O. 1504 (SI).

Rhabdadenia Müll. Arg.

Rhabdadenia madida (Vell.) Miers

Hierba o enredadera. Perenne. Nativa. 0-500 m. Distribución: IGU, SIG.

Ejemplar de referencia: Keller, H. A. 13177 (CTES).

Rhodocalyx Müll. Arg.

Rhodocalyx riedelii (Müll. Arg.) J.F.Morales, M.E. Endress \& Liede

Liana. Nativa. 0-500 m.

Distribución: CAI, ELD, IGU, SIG.

Ejemplar de referencia: Morrone, O. 1641 (SI).

Ruehssia H. Karst.

Ruehssia macrophylla (Humb. \& Bonpl. ex Schult.) H. Karst. (***)

Enredadera. Perenne. Nativa. 0-500 m.

Distribución: ELD, GMB, IGU.

Ejemplar de referencia: Guaglianone, E. R. 2998 (SI).

Ruehssia montana (Malme) F. Esp.Santo \& Rapini (***)

Enredadera o liana. Perenne. Nativa. 0-500 m. Distribución: GUA.

Ejemplar de referencia: Keller, H. A. 494 (CTES).

Schubertia Mart., nom. cons.

Schubertia grandiflora Mart.

Enredadera. Perenne. Nativa. 0-500 m.

Distribución: APO, CAP, SIG.

Ejemplar de referencia: Seijo, G. J. 300 (MNES, SI). 


\section{Tabernaemontana L.}

Tabernaemontana catharinensis A. DC.

Arbusto o árbol. Nativa. 0-500 m.

Distribución: APO, CAI, CAN, CAP, CON, ELD, GMB, GUA, IGU, SIG, SPE.

Ejemplar de referencia: Morrone, O. 1617 (SI).

Topea H.A. Keller

Topea micrantha (H.A. Keller) H.A. Keller $\left(^{* *}\right.$ ) Enredadera. Perenne. Endémica. 200-400 m. Distribución: IGU.

Ejemplar de referencia: Keller, H. A. 13591 (CTES).

Topea patens (H.A. Keller) H.A. Keller $\left(^{* * *}\right)$

Enredadera. Perenne. Nativa. 100-500 m.

Distribución: IGU.

Ejemplar de referencia: Keller, H. A. 13910 (CTES).

\section{AQUIFOLIACEAE}

\section{Ilex L.}

Ilex affinis Gardner $\left(^{* * *}\right)$

Arbusto o árbol. Nativa. 0-500 m.

Distribución: SIG.

Ejemplar de referencia: Keller, H. A. 9583 (BAF, CTES).

Ilex brasiliensis (Spreng.) Loes. ${ }^{* * *}$ )

Arbusto o árbol. Nativa. 0-500 m.

Distribución: IGU.

Ejemplar de referencia: Kricun, 402 (BACP).

\section{Ilex brevicuspis Reissek}

Árbol. Nativa. 0-700 m.

Distribución: CAN, GMB, GUA, IGU, SIG.

Ejemplar de referencia: Múlgura, M. E. 2513 (SI).

Ilex dumosa Reissek var. guaranina Loes.

Arbusto o arbolito. Nativa. 0-500 m.

Distribución: ELD, IGU.

Ejemplar de referencia: Hunziker, J. H. 11909 (SI).
Ilex paraguariensis $\mathrm{A}$. St.-Hil.

Arbusto o árbol. Nativa. 0-700 m.

Distribución: APO, CAN, CAP, ELD, GMB, GUA, IGU, LNA, MON, SIG, SPE.

Ejemplar de referencia: Correa, M. N. 5476 (BAA, BAB, SI).

Ilex theezans Mart. ex Reissek $\left.{ }^{(* *}\right)$

Arbusto o árbol. Nativa. 0-500 m.

Distribución: GMB.

Ejemplar de referencia: Giberti, G. 261 (BACP, SI).

\section{ARACEAE}

Asterostigma Fisch. \& C.A. Mey.

Asterostigma lividum (Lodd.) Endl. ${ }^{* * *}$ )

Palmera. Nativa. 0-500 m.

Distribución: GMB.

Ejemplar de referencia: Keller, H. A. 11593 (CTES).

\section{Colocasia Schott}

Colocasia esculenta (L.) Schott

Hierba. Perenne. Introducida. 0-500 m.

Distribución: IGU.

Ejemplar de referencia: Vanni, R. O. 3152 (CTES).

\section{Dieffenbachia Schott}

Dieffenbachia aglaonematifolia Engl.

Hierba o subarbusto. Perenne. Nativa. 0-500 m. Distribución: SIG.

Ejemplar de referencia: Guaglianone, E. R. 3022 (SI).

\section{Gorgonidium Schott}

Gorgonidium vermicidum (Speg.) Bogner \& Nicolson

Hierba. Perenne. Nativa. 0-500 m.

Distribución: ELD.

Ejemplar de referencia: Crisci, J. V. 286 (LP). 
Lemna L., nom. cons.

\section{Lemna valdiviana Phil.}

Hierba acuática. Perenne. Nativa. 0-500 m.

Distribución: IGU.

Ejemplar de referencia: Cabrera, A. 28921 (SI).

Philodendron Schott, nom. cons.

Philodendron imbe (Schott) Schott $\left.{ }^{(* *}\right)$

Hierba. Perenne. Nativa. 0-500 m.

Distribución: IGU.

Ejemplar de referencia: Hauman, L. L. s.n. $(B A)$.

Philodendron missionum (Hauman) Hauman $\left({ }^{* * *}\right)$

Hierba. Perenne. Nativa. 0-500 m.

Distribución: IGU. (BA).

Ejemplar de referencia: Van de Venne, H. s.n.

\section{Pistia L.}

\section{Pistia stratiotes L.}

Hierba acuática. Perenne. Nativa. 0-500 m.

Distribución: CAN, SIG, SPE.

Ejemplar de referencia: Deginani, N. B. 1244 (SI).

\section{Spathicarpa Hook.}

Spathicarpa hastifolia Hook.

Hierba. Perenne. Nativa. 0-800 m.

Distribución: APO, CAI, CAN, CAP, ELD, GMB, GSM, IGU, MON, SIG, SJA, SPE.

Ejemplar de referencia: Morrone, O. 1655 (MO, SI).

\section{Spirodela Schleid.}

Spirodela intermedia $W$. Koch

Hierba acuática. Perenne. Nativa. 0-500 m.

Distribución: CAN.

Ejemplar de referencia: Giardelli, M. L. 539 (SI).
Taccarum Brongn. ex Schott

Taccarum peregrinum (Schott) Endl. $\left({ }^{* *}\right)$

Hierba. Perenne. Nativa. 0-700 m.

Distribución: GMB, GUA, IGU, MON, SPE.

Ejemplar de referencia: Múlgura, M. E. 2676 (SI).

\section{Thaumatophyllum Schott}

Thaumatophyllum bipinnatifidum (Schott ex Endl.) Sakur., Calazans \& Mayo

Hierba epífita. Perenne. Nativa. 0-700 m.

Distribución: CAN, ELD, GMB, GSM, IGU, OBE, SJA.

Ejemplar de referencia: Múlgura, M. E. 1892 (SI).

Thaumatophyllum undulatum (Engl.) Sakur., Calazans \& Mayo

Hierba. Perenne. Nativa. 0-500 m.

Distribución: SIG.

Ejemplar de referencia: Hauman, L. L. s.n. (BA).

Wolffia Horkel ex Schleid., nom. cons.

Wolffia brasiliensis Wedd.

Hierba acuática. Perenne. Nativa. 0-500 m.

Distribución: GUA.

Ejemplar de referencia: Schinini, A. 28741 (CTES).

\section{ARALIACEAE}

\section{Aralia L.}

Aralia warmingiana (Marchal) J. Wen

Árbol. Nativa. 0-500 m.

Distribución: CAN, GUA, LNA.

Ejemplar de referencia: Buchinger, M. s.n. (BA, BAI).

Dendropanax Decne. \& Planch.

Dendropanax affinis (Marchal) Gamerro \& Zuloaga Árbol. Nativa. 0-500 m.

Distribución: CAI, IGU, LNA, SPE.

Ejemplar de referencia: Zuloaga, F. O. 7010 (SI). 
Dendropanax cuneatus (DC.) Decne. \& Planch. Árbol. Nativa. 0-500 m.

Distribución: CAP, IGU, LNA, SIG.

Ejemplar de referencia: Morrone, O. 1618 (SI).

Didymopanax Decne. \& Planch.

Didymopanax calvus (Cham.) Decne. \& Planch. $(* * *)$

Árbol. Nativa. 0-500 m.

Distribución: IGU, SPE.

Ejemplar de referencia: Montes, J. E. 27482 (NY).

\section{Hydrocotyle L.}

\section{Hydrocotyle bonariensis Lam.}

Hierba. Perenne. Nativa. 0-500 m.

Distribución: APO, IGU.

Ejemplar de referencia: Múlgura, M. E. 2367 (SI).

Hydrocotyle bowlesioides Mathias \& Constance Hierba. Perenne. Nativa. 0-500 m.

Distribución: GUA, SIG.

Ejemplar de referencia: Tressens, S. G. 6387 (CTES).

\section{Hydrocotyle callicephala Cham.}

Hierba. Perenne. Nativa. 0-700 m.

Distribución: CAI, CAN, GMB, GUA, IGU, SIG, SJA, SPE.

Ejemplar de referencia: Zuloaga, F. O. 6623 (SI).

Hydrocotyle exigua (Urb.) Malme

Hierba. Perenne. Nativa. 0-500 m.

Distribución: CAN, CAP, ELD, GUA, SIG, SPE.

Ejemplar de referencia: Zuloaga, F. O. 8062 (SI).

Hydrocotyle leucocephala Cham. \& Schltdl.

Hierba. Perenne. Nativa. 0-700 m.

Distribución: APO, CAI, CAN, CAP, GMB, GUA, IGU, SIG, SJA, SPE.

Ejemplar de referencia: Spegazzini, C. L. 0185 (SI).

\section{Hydrocotyle pusilla A. Rich.}

Hierba. Perenne. Nativa. 0-500 m.

Distribución: APO, CON, GUA.

Ejemplar de referencia: Deginani, N. B. 1539 (SI).
Schefflera J.E. Forst. \& G. Forst.

Schefflera morototoni (Aubl.) Maguire, Steyerm. \& Frodin

Árbol. Nativa. 0-500 m.

Distribución: ELD, GMB, IGU, LNA, OBE, SPE.

Ejemplar de referencia: Zuloaga, F. O. 7023 (SI).

\section{ARECACEAE}

\section{Acrocomia Mart.}

Acrocomia aculeata (Jacq.) Lodd. ex Mart.

Palmera. Nativa. 100-200 m.

Distribución: CAP, ELD, SIG.

Ejemplar de referencia: Xifreda, C. C. 1286 (SI)

\section{Allagoptera Nees}

Allagoptera campestris (Mart.) Kuntze

Palmera acaule. Nativa. 0-200 m.

Distribución: CAN, SIG.

Ejemplar de referencia: Morrone, O. 2216 (SI).

Allagoptera leucocalyx (Drude) Kuntze $\left(^{* * *}\right)$

Palmera acaule. Nativa. 100-500 m.

Distribución: CAN, SIG.

Ejemplar de referencia: Krapovickas, A. 18265 (SI).

Butia (Becc.) Becc.

Butia eriospatha (Mart. ex Drude) Becc. $\left(^{* * *}\right)$

Palmera. Nativa. 0-500 m.

Distribución: GMB.

Ejemplar de referencia: Krapovickas, A. 47926 (CTES).

Butia exilata Deble \& Marchiori $\left(^{*}\right)$

Palmera. Nativa. 0-500 m.

Distribución: CAI.

Ejemplar de referencia: Keller, H. A. 13727 (CTES). 
Butia paraguayensis (Barb. Rodr.) L.H. Bailey

Palmera. Nativa. 100-300 m.

Distribución: APO, CAN, SIG.

Ejemplar de referencia: Marcato, A. C. 320 (SI).

Butia poni Burret $\left({ }^{* *}\right)$

Palmera. Endémica. 0-500 m.

Distribución: CAN, SIG.

Ejemplar de referencia: Keller, H. A. 13022 (CTES,SI).

Butia yatay (Mart.) Becc.

Palmera. Nativa. 0-500 m.

Distribución: CAN, SIG.

Ejemplar de referencia: Zuloaga, F. O. 5674 (SI).

Euterpe Mart., nom. cons.

\section{Euterpe edulis Mart. $\left({ }^{* * *}\right)$}

Palmera. Nativa. 0-500 m.

Distribución: GUA, IGU.

Ejemplar de referencia: Mroginsky, L. 359 (F, GH, MO).

\section{Syagrus Mart.}

Syagrus romanzoffiana (Cham.) Glassman

Palmera. Nativa. 0-500 m.

Distribución: ELD, GSM, GUA, IGU, SPE.

Ejemplar de referencia: Múlgura, M. E. 2520 (SI).

\section{ARISTOLOCHIACEAE}

\section{Aristolochia Tourn. ex L.}

\section{Aristolochia angustifolia Cham.}

Hierba. Perenne. Nativa. 0-500 m.

Distribución: CAN.

Ejemplar de referencia: Ekman, E. L. 1603 (F).

Aristolochia castellanosii Ahumada

Hierba. Perenne. Nativa. 0-500 m.

Distribución: CAN, CON, SIG.

Ejemplar de referencia: Múlgura, M. E. 1719 (SI).

\section{Aristolochia ceresensis Kuntze}

Hierba. Perenne. Nativa. 0-500 m.

Distribución: SJA.

Ejemplar de referencia: Eskuche, U. G. 4083

(BM, CTES, F, G, US).

\section{Aristolochia elegans Mast.}

Enredadera. Perenne. Nativa. 0-800 m.

Distribución: CAI, CAN, LNA, SIG.

Ejemplar de referencia: Biganzoli, F. 815 (SI).

Aristolochia fimbriata Cham.

Hierba. Perenne. Nativa. 0-500 m.

Distribución: CON, LNA, OBE.

Ejemplar de referencia: Morrone, O. 575 (SI).

Aristolochia melastoma Silva Manso ex Duch. (***)

Enredadera. Perenne. Nativa. 0-500 m.

Distribución: IGU.

Ejemplar de referencia: Vanni, R. O. 3559 (CTES, MBM, SI).

\section{Aristolochia odoratissima L. $\left({ }^{* * *}\right)$}

Enredadera. Perenne. Nativa. 0-500 m.

Distribución: IGU.

Ejemplar de referencia: Tressens, S. G. 5580 (CTES).

Aristolochia sessilifolia (Klotzsch) Duch.

Hierba. Perenne. Nativa. 0-500 m.

Distribución: CAN, CAP, GSM.

Ejemplar de referencia: Ahumada, L. Z. 99 (LIL).

Aristolochia stomachoidis Hoehne $\left.{ }^{* * *}\right)$

Enredadera. Perenne. Nativa. 0-500 m.

Distribución: SIG.

Ejemplar de referencia: Keller, H. A. 5675 (CTES).

\section{Aristolochia stuckertii Speg.}

Hierba. Perenne. Nativa. 0-500 m.

Distribución: CAN, CON.

Ejemplar de referencia: Biganzoli, F. 1728 (SI).

Aristolochia triangularis $\mathrm{Cham}$.

Enredadera. Perenne. Nativa. 0-800 m.

Distribución: CAI, CAN, CON, ELD, GMB, GSM, GUA, MON, SIG, SPE.

Ejemplar de referencia: Múlgura, M. E. 1809 (SI). 


\section{Aristolochia trilobata L.}

Enredadera. Perenne. Nativa. 0-500 m.

Distribución: CAN, CAP, IGU.

Ejemplar de referencia: Cristóbal, C. L. 256 (LIL).

Prosopanche de Bary

\section{Prosopanche bonacinai Speg.}

Hierba parásita. Perenne. Nativa. 0-500 m.

Distribución: ELD, GMB, SIG.

Ejemplar de referencia: Keller, H. A. 11982 (CTES).

\section{ASPARAGACEAE}

\section{Clara Kunth}

Clara ophiopogonoides Kunth (***)

Hierba. Perenne. Nativa. 0-500 m.

Distribución: CAN, SJA.

Ejemplar de referencia: Burkart, A. 14775 (SI).

Clara stricta (L.B. Sm.) R.C. Lopes \& Andreata $(* * *)$

Hierba. Perenne. Nativa. 0-500 m.

Distribución: SIG.

Ejemplar de referencia: Burkart, A. 15456 (SI).

Cordyline Comm. ex R. Br., nom. cons.

\section{Cordyline sellowiana Kunth}

Hierba. Perenne. Introducida. 200-300 m.

Distribución: APO, CAN, ELD, GMB, GUA, IGU, SPE. (SI).

Ejemplar de referencia: Múlgura, M. E. 1800

\section{Herreria Ruiz \& Pav.}

\section{Herreria bonplandii Lecomte}

Hierba o enredadera. Perenne. Nativa. 0-800 m.

Distribución: APO, CAI, CAN, CAP, GUA, SIG, SJA, SPE.

Ejemplar de referencia: Biganzoli, F. 159 (SI).
Herreria montevidensis Klotzsch ex Griseb.

Hierba o enredadera. Perenne. Nativa. 0-400 m.

Distribución: APO, CAN, CAP, GUA, SJA, SPE.

Ejemplar de referencia: Burkart, A. 15472 (SI).

\section{ASTERACEAE}

Acanthospermum Schrank, nom. cons.

Acanthospermum australe (Loefl.) Kuntze

Hierba. Anual. Nativa. 0-500 m.

Distribución: GUA, SIG.

Ejemplar de referencia: Deginani, N. B. 1622 (SI).

\section{Acanthospermum hispidum $D C$.}

Hierba. Anual. Nativa. 0-500 m.

Distribución: CAI.

Ejemplar de referencia: Deginani, N. B. 977 (SI).

Acanthostyles R.M. King \& H. Rob.

Acanthostyles buniifolius (Hook. \& Arn.) R.M. King \& H. Rob.

Arbusto. Nativa. 0-500 m.

Distribución: APO.

Ejemplar de referencia: Martínez Crovetto, $R$. 9476 (SI,CTES).

\section{Achillea L.}

\section{Achillea millefolium L.}

Hierba. Perenne. Introducida. 0-500 m.

Distribución: GUA.

Ejemplar de referencia: Keller, H. A. 3274 (CTES).

\section{Achyrocline (Less.) DC.}

Achyrocline alata (Kunth) DC.

Subarbusto. Nativa. 0-500 m.

Distribución: CAN, CAP.

Ejemplar de referencia: Schinini, A. 4887 (LP). 
Achyrocline flaccida (Weinm.) DC.

Hierba o subarbusto. Perenne. Nativa. 0-500 m.

Distribución: APO, CAN, CAP, GMB, GUA, IGU, LNA, MON, OBE, SIG, SJA.

Ejemplar de referencia: Burkart, A. 1411 (SI).

Achyrocline satureioides (Lam.) DC.

Subarbusto. Nativa. 0-600 m.

Distribución: APO, CAN, CAP, GMB, IGU, SPE.

Ejemplar de referencia: Morrone, O. 2113 (SI).

\section{Achyrocline tomentosa Rusby}

Subarbusto. Nativa. 0-700 m.

Distribución: CAI, CAN, GMB, IGU, LNA, SIG.

Ejemplar de referencia: Biganzoli, F. 870 (SI).

\section{Acilepidopsis H. Rob.}

Acilepidopsis echitifolia (Mart. ex DC.) H. Rob. Subarbusto. Nativa. 0-500 m.

Distribución: CAN.

Ejemplar de referencia: Spegazzini, C. L. s.n. (BAB).

Acmella Rich. ex Pers.

Acmella bellidioides (Sm.) R.K. Jansen

Hierba. Perenne. Nativa. 0-500 m.

Distribución: CON, GSM, GUA.

Ejemplar de referencia: Rodríguez, M. 750 (MNES, SI).

Acmella decumbens (Sm.) R.K. Jansen

Hierba. Perenne. Nativa. 0-500 m.

Distribución: CAN, GUA.

Ejemplar de referencia: Fontana, J. L. 37-16 (CTESN).

Acmella grisea (Chodat) R.K. Jansen

Hierba. Perenne. Nativa. 0-500 m.

Distribución: APO, CAN, SIG.

Ejemplar de referencia: Renvoize, S. A. 3164 (SI).

\section{Acmella leptophylla (DC.) R.K. Jansen (***)}

Hierba. Perenne. Nativa. 0-500 m.

Distribución: CAI, CAN, SIG.

Ejemplar de referencia: Biganzoli, F. 529 (SI).
Acmella oppositifolia (Lam.) R.K. Jansen

Hierba. Perenne. Nativa. 0-600 m.

Distribución: CON.

Ejemplar de referencia: Keller, H. A. 5648 (CTES).

Acmella pusilla (Hook. \& Arn.) R.K. Jansen Hierba. Perenne. Nativa. 0-500 m.

Distribución: CAP, SIG.

Ejemplar de referencia: Morrone, O. 1893 (SI).

Acmella serratifolia R.K. Jansen

Hierba. Perenne. Nativa. 0-700 m.

Distribución: CAI, GUA, IGU, GSM, MON, SIG.

Ejemplar de referencia: Zuloaga, F. O. 692 (SI).

Adenostemma J.R. Forst. \& G. Forst.

Adenostemma brasilianum (Pers.) Cass.

Hierba. Anual. Nativa. 0-700 m.

Distribución: CAI, CAN, ELD, GMB, GUA, SPE.

Ejemplar de referencia: Biganzoli, F. 939 (SI).

Adenostemma verbesina (L.) Kuntze $\left({ }^{* * *}\right)$

Hierba. Anual. Nativa. 0-700 m.

Distribución: ELD, GMB, GUA, IGU, MON, SPE.

Ejemplar de referencia: Morrone, O. 763 (SI).

\section{Ageratum L.}

Ageratum conyzoides L.

Hierba. Anual. Nativa. 0-800 m.

Distribución: CAN, CAP, ELD, GMB, GUA, IGU, MON, SIG, SPE.

Ejemplar de referencia: Daviña, J. 188 (CTES, MNES).

\section{Aldama La Llave}

Aldama anchusifolia (DC.) E.E.Schill. \& Panero var. anchusifolia

Subarbusto. Nativa. 0-500 m.

Distribución: CAN.

Ejemplar de referencia: Cabrera, A. L. 12456 (LP). 
Aldama anchusifolia (DC.) E.E.Schill. \& Panero var. imarginata (S.F. Blake) Magenta \& Pirani

Subarbusto. Nativa. 0-500 m.

Distribución: CAN.

Ejemplar de referencia: Aguilera, P. M. 18 (SI).

Aldama misionensis (A.A. Sáenz) Freire $\left(^{* *}\right.$ )

Subarbusto. Endémica. 0-500 m.

Distribución: APO, CON, SIG.

Ejemplar de referencia: Cabrera, A. L. 28299 (CTES, LP, SI).

Aldama pilosa (Baker) E.E.Schill. \& Panero

Hierba. Nativa. 300-500 m.

Distribución: APO, CAN, SIG.

Ejemplar de referencia: Cuadrado, G. A. 348 (CTES, SI).

Aldama tuberosa (Griseb.) E.E.Schill. \& Panero var. guaranitica (Chodat) Magenta

Hierba. Perenne. Nativa. 0-700 m.

Distribución: APO, CAN, SIG.

Ejemplar de referencia: Schulz, A. G. 108 $(L P)$.

\section{Ambrosia L.}

\section{Ambrosia artemisiifolia L.}

Hierba. Anual. Nativa. 0-600 m.

Distribución: CAI, CAP, CON, GUA, IGU, SPE.

Ejemplar de referencia: Ariza Espinar, L. 1193 (CORD).

Ambrosia tenuifolia Spreng.

Hierba. Perenne. Nativa. 0-500 m.

Distribución: CAP, MON.

Ejemplar de referencia: Rodríguez, F. M. 33 (SI).

\section{Anthemis L.}

\section{Anthemis cotula L.}

Hierba. Anual. Introducida. 0-500 m.

Distribución: CAN.

Ejemplar de referencia: de Llamas, A. 287 $(B A B)$.

\section{Apopyros G.L. Nesom}

Apopyros corymbosus (Hook. \& Arn.) G.L. Nesom

Hierba. Perenne. Nativa. 0-500 m.

Distribución: APO, CAN.

Ejemplar de referencia: Ekman, E. L. 1057 (LP).

\section{Artemisia L.}

\section{Artemisia absinthium L.}

Subarbusto. Introducida. 0-500 m.

Distribución: GUA.

Ejemplar de referencia: Keller, H. A. 3500 (CTES).

\section{Artemisia verlotorum Lamotte}

Hierba. Perenne. Introducida. 0-500 m.

Distribución: GMB, IGU.

Ejemplar de referencia: Volkart de Hualde, I. s.n. (LPAG).

\section{Aspilia Thouars}

Aspilia linearis S.F. Blake

Hierba. Perenne. Nativa. 100-300 m.

Distribución: APO, CAI, CAN, CON, SIG.

Ejemplar de referencia: Biganzoli, F. 834 (SI).

Aspilia montevidensis (Spreng.) Kuntze

Hierba. Perenne. Nativa. 0-500 m.

Distribución: APO, CAN, CAP, CON, IGU, OBE, SIG.

Ejemplar de referencia: Morrone, O. 1138 (SI).

Aspilia pascalioides Griseb.

Hierba. Perenne. Nativa. 0-500 m.

Distribución: APO, GUA.

Ejemplar de referencia: Cabrera, A. L. 29167 (SI).

Aspilia silphioides (Hook. \& Arn.) Benth. \& Hook. f.

Hierba. Perenne. Nativa. 0-500 m.

Distribución: CAI, CAN.

Ejemplar de referencia: Fontana, 110 (SI). 
Austroeupatorium R.M. King \& H. Rob.

Austroeupatorium inulifolium (Kunth) R.M. King \& H. Rob.

Arbusto. Nativa. 0-800 m.

Distribución: CAI, CAN, CAP, ELD, GMB, GSM, GUA, IGU, SIG, SJA.

Ejemplar de referencia: Zuloaga, F. O. 6638 (SI).

Austroeupatorium laetevirens (Hook. \& Arn.) R.M. King \& H. Rob.

Subarbusto. Nativa. 0-500 m.

Distribución: CAN.

Ejemplar de referencia: Grüner, G. 1345 (LP).

Austroeupatorium picturatum (Malme) R.M. King \& H. Rob. $\left({ }^{* * *}\right)$

Hierba. Perenne. Nativa. 0-600 m.

Distribución: ELD, GSM, GUA, IGU.

Ejemplar de referencia: Burkart, A. 14670 (LP, SI).

Ayapanopsis R.M. King \& H. Rob.

Ayapanopsis esperanzae (Hassl.) R.M. King \& H. Rob. $\left.{ }^{* * *}\right)$

Subarbusto. Nativa. 0-500 m.

Distribución: SIG.

Ejemplar de referencia: Múlgura, M. E. 2101 (SI).

Baccharis L., nom. cons.

Baccharis anomala $D C$.

Arbusto. Nativa. 0-600 m.

Distribución: CAI, CAN, ELD, GUA, SIG, SPE.

Ejemplar de referencia: Zuloaga, F. O. 675 (SI).

\section{Baccharis argentina Heering}

Arbusto. Nativa. 0-500 m.

Distribución: CAI, CAN, GSM, SIG.

Ejemplar de referencia: Biganzoli, F. 160 (SI).

Baccharis articulata (Lam.) Pers.

Arbusto. Nativa. 0-800 m.

Distribución: APO, CAI, CAN, CAP, CON, GSM, GUA, IGU, LNA, MON, OBE, SIG.

Ejemplar de referencia: Biganzoli, F. 261 (SI)
Baccharis brachylaenoides DC. var. brachylaenoides $\left.{ }^{* * *}\right)$

Arbusto. Nativa. 0-500 m.

Distribución: GUA, SPE.

Ejemplar de referencia: Cabrera, A. L. 28900 (LP, SI).

Baccharis brachylaenoides DC. var. polycephala (I.L.Teodoro) G.M. Barroso ${ }^{* * *}$ )

Arbusto. Nativa. 0-300 m.

Distribución: GUA.

Ejemplar de referencia: Tressens, S. G. 6078 (CTES).

Baccharis breviseta $D C$.

Subarbusto. Nativa. 0-500 m.

Distribución: CAN.

Ejemplar de referencia: Ekman, E. L. 1082 (LP).

Baccharis camporum $D C$.

Subarbusto. Nativa. 0-500 m.

Distribución: CAN, SIG.

Ejemplar de referencia: Grüner, G. 1294 (LP).

Baccharis caprariifolia $D C$.

Arbusto. Nativa. 0-600 m.

Distribución: APO, CAN, CON, LNA, OBE, SIG, SPE.

Ejemplar de referencia: Montes, J. E. 2546 (LP).

Baccharis cognata $D C$.

Arbusto. Nativa. 0-800 m.

Distribución: CAI, CAN, CAP, SIG.

Ejemplar de referencia: Montes, J. E. 697 (LP).

Baccharis coridifolia DC.

Arbusto. Nativa. 0-500 m.

Distribución: CAN, SIG.

Ejemplar de referencia: Montes, J. E. 881 (LP).

Baccharis crispa Spreng.

Arbusto o subarbusto. Nativa. 0-500 m.

Distribución: SIG.

Ejemplar de referencia: Montes, J. E. 15160 (SI).

Baccharis dentata (Vell.) G.M. Barroso (***)

Arbusto. Nativa. 0-500 m.

Distribución: GUA, IGU, OBE.

Ejemplar de referencia: Rodríguez, 468c (SI). 
Baccharis dracunculifolia DC.

Arbusto o arbolito. Nativa. 0-800 m.

Distribución: APO, CAI, CAN, CAP, CON, GMB, GSM, GUA, IGU, LNA, OBE, SIG, SJA, SPE.

Ejemplar de referencia: Biganzoli, F. 872 (SI).

\section{Baccharis glutinosa Pers.}

Hierba. Perenne. Nativa. 0-700 m.

Distribución: CAN, CAP, GSM, GUA, MON, SIG.

Ejemplar de referencia: Burkart, A. 14471 (SI).

\section{Baccharis helichrysoides DC. $\left.{ }^{* * *}\right)$ \\ Subarbusto. Nativa. 0-500 m. \\ Distribución: CAN, GMB, GSM, SPE. \\ Ejemplar de referencia: Zuloaga, F. O. 822 (SI).}

Baccharis linearifolia (Lam.) Pers. subsp. linearifolia

Arbusto. Nativa. 0-700 m. SPE.

Distribución: APO, CAI, CAN, CON, SIG,

Ejemplar de referencia: Montes, J. E. 27644 (SI).

Baccharis linearifolia (Lam.) Pers. subsp. polycephala (Wedd.) Joch.Müll.

Arbusto. Nativa. 0-500 m.

Distribución: CAN.

Ejemplar de referencia: Pérez-Moreau, R. L. s.n. (BA, LP).

\section{Baccharis megapotamica Spreng. $\left.{ }^{(* *}\right)$}

Arbusto. Nativa. 0-500 m.

Distribución: CAI, CAN, SIG.

Ejemplar de referencia: Krapovickas, A. 15725 (CTES, LP).

Baccharis microcephala (Less.) DC.

Arbusto o subarbusto. Nativa. 0-500 m.

Distribución: APO, CAN, SIG.

Ejemplar de referencia: Grüner, G. 1311 (LP).

\section{Baccharis microdonta DC.}

Arbusto. Nativa. 0-800 m.

Distribución: CAI, CAN, ELD, GMB, GSM, GUA, IGU, OBE, SIG, SJA, SPE.

Ejemplar de referencia: Morrone, O. 2129 (SI).
Baccharis montana DC. $\left({ }^{* * *}\right)$

Arbusto. Nativa. 0-700 m.

Distribución: ELD, GMB, GUA, IGU, MON, OBE, SPE.

Ejemplar de referencia: Morrone, O. 1555 (SI).

\section{Baccharis oxyodonta DC. $\left({ }^{* *}\right)$}

Arbusto. Nativa. 0-800 m.

Distribución: CAI, CAN, CAP, CON, ELD, GMB, GSM, GUA, IGU, MON, OBE, SIG, SPE.

Ejemplar de referencia: Hunziker, J. H. 803b (SI).

\section{Baccharis petraea Heering $\left({ }^{* * *}\right)$}

Arbusto. Nativa. 0-500 m.

Distribución: CAI, CAN, ELD, GSM, SIG.

Ejemplar de referencia: Fontana, J. L. 119 (SI).

Baccharis phyteumoides (Less.) DC.

Subarbusto. Nativa. 0-500 m.

Distribución: CAN, SIG.

Ejemplar de referencia: Alboff, N. s.n. (LP).

Baccharis punctulata $D C$.

Arbusto. Nativa. 0-700 m.

Distribución: APO, CAI, CAN, CAP, GSM, GUA, IGU, OBE, SIG.

Ejemplar de referencia: Biganzoli, F. 110 (SI).

Baccharis salicifolia (Ruiz \& Pav.) Pers.

Arbusto. Nativa. 0-500 m.

Distribución: GMB, IGU.

Ejemplar de referencia: Vanni, R. O. 3584 (CTES).

\section{Baccharis semiserrata DC. $\left({ }^{* * *}\right)$}

Arbusto. Nativa. 0-500 m.

Distribución: CAN, GMB, IGU, SPE.

Ejemplar de referencia: Morrone, O. 1776 (SI).

\section{Baccharis tridentata Vahl}

Arbusto o subarbusto. Nativa. 0-500 m.

Distribución: APO, CAN, CON, SIG.

Ejemplar de referencia: Cabrera, A. L. 29302 (SI).

Baccharis trimera (Less.) DC.

Subarbusto. Nativa. 0-700 m.

Distribución: CAI, CAN, CAP, ELD, GUA, OBE, SIG, SJA.

Ejemplar de referencia: Zuloaga, F. O. 6712 (SI). 
Baccharis trinervis Pers. var. debilis (Rusby) Joch.Müll.

Arbusto. Nativa. 0-500 m.

Distribución: LNA.

Ejemplar de referencia: Krapovickas, A. 15927 (LP).

\section{Baccharis trinervis Pers. var. trinervis}

Arbusto. Nativa. 0-700 m.

Distribución: CAI, CAN, ELD, GMB, GUA, LNA, SIG, SPE.

Ejemplar de referencia: Montes, J. E. 832 (SI).

\section{Baccharis vulneraria Baker}

Subarbusto. Nativa. 0-600 m.

Distribución: CAI, CAN, GMB, SPE.

Ejemplar de referencia: Guaglianone, E. R. 1158 (SI).

Barrosoa R.M. King \& H. Rob.

Barrosoa candolleana (Hook. \& Arn.) R.M. King \& H. Rob.

Hierba. Perenne. Nativa. 0-800 m.

Distribución: APO, CAI, CAN, CAP, GUA, IGU, LNA, SIG.

Ejemplar de referencia: Zuloaga, F. O. 6692 (SI).

\section{Bidens L.}

Bidens hassleriana (Chodat) A.A. Sáenz $\left(^{* * *}\right)$

Hierba. Anual. Nativa. 0-500 m.

Distribución: APO, CAN, LNA, SIG, SJA. (SI).

Ejemplar de referencia: Zuloaga, F. O. 4924

Bidens laevis (L.) Britton, Stern \& Poggenb.

Hierba. Perenne. Nativa. 0-700 m.

Distribución: GMB.

Ejemplar de referencia: Zuloaga, F. O. 8286 (SI).

\section{Bidens pilosa $\mathrm{L}$.}

Hierba. Anual. Nativa. 0-800 m.

Distribución: CAI, CAN, ELD, GSM, GUA, IGU, SJA.

Ejemplar de referencia: Honfi, A. I. 491 (CTES, MNES).
Bidens reptans (L.) G. Don

Subarbusto. Nativa. 0-500 m.

Distribución: CAN, SIG.

Ejemplar de referencia: Morrone, O. 1850 (SI).

Bidens riedelii Baker $\left(^{* * *}\right)$

Hierba. Perenne. Nativa. 0-500 m.

Distribución: APO, CAN.

Ejemplar de referencia: Eskuche, U. G. 2862 (CTESN).

Bidens subalternans DC. var. simulans Sherff Hierba. Anual. Nativa. 0-500 m.

Distribución: CAP.

Ejemplar de referencia: Romanczuk, M. C. 432 (SI).

Bidens subalternans $D C$. var. subalternans

Hierba. Anual. Nativa. 0-500 m.

Distribución: APO, GUA, SIG.

Ejemplar de referencia: Cabrera, A. L. 29155 (SI).

\section{Calea L.}

Calea asclepiaefolia Hassl. $\left.{ }^{* * *}\right)$

Subarbusto. Nativa. 0-500 m.

Distribución: GMB.

Ejemplar de referencia: Ahumada, L. Z. 4148 (CTES, SI).

\section{Calea clematidea Baker}

Arbusto. Nativa. 0-500 m.

Distribución: CAN, CAP, IGU, SIG.

Ejemplar de referencia: Morrone, O. 2220 (SI).

Calea cymosa Less.

Hierba. Perenne. Nativa. 0-500 m.

Distribución: CAN, CAP, CON, SIG.

Ejemplar de referencia: Grüner, G. 1335 (LP).

\section{Calea hassleriana Chodat}

Hierba. Perenne. Nativa. 0-500 m.

Distribución: CAN, SIG.

Ejemplar de referencia: Morrone, O. 1697 (SI).

\section{Calea kristineae Pruski $\left(^{*}\right)$}

Arbusto. Nativa. 0-500 m.

Distribución: CAN.

Ejemplar de referencia: Keller, H. A. 13894 (CTES). 
Calea mediterranea (Vell.) Pruski

Subarbusto. Nativa. 0-500 m.

Distribución: CAN, SIG.

Ejemplar de referencia: Grüner, G. 1239 (LP).

Calea pinnatifida (R. Br.) Less.

Liana. Nativa. 0-500 m.

Distribución: APO, CAN, CAP, GUA, IGU, SIG.

Ejemplar de referencia: Morrone, O. 1631 (SI).

\section{Calea uniflora Less.}

Hierba. Perenne. Nativa. 0-500 m.

Distribución: CAP, SIG. (SI).

Ejemplar de referencia: Zuloaga, F. O. 6629

Calea verticillata (Klatt) Pruski

Subarbusto. Nativa. 0-500 m.

Distribución: SIG.

Ejemplar de referencia: Morrone, O. 1591 (SI).

\section{Calyptocarpus Less.}

Calyptocarpus biaristatus (DC.) H. Rob.

Hierba. Perenne. Nativa. 0-500 m.

Distribución: APO, CAI, CAN, CAP, CON, ELD, GMB, GSM, GUA, IGU, SIG.

Ejemplar de referencia: Biganzoli, F. 260 (SI).

Campovassouria R.M. King \& H. Rob.

Campovassouria cruciata (Vell.) R.M. King \& H. Rob.

Arbusto. Nativa. 0-500 m.

Distribución: APO, CAI, CAN, GMB, SIG.

Ejemplar de referencia: Rodríguez, F. M. 107 (SI).

\section{Campuloclinium DC.}

\section{Campuloclinium macrocephalum (Less.)} DC.

Hierba. Perenne. Nativa. 0-700 m.

Distribución: APO, CAI, CAN, CAP, CON, ELD, SIG, SJA, SPE.

Ejemplar de referencia: Morrone, O. 1503 (SI).
Campuloclinium purpurascens (Sch. Bip. ex Baker) R.M. King \& H. Rob.

Hierba. Perenne. Nativa. 0-500 m.

Distribución: CAI, IGU, MON.

Ejemplar de referencia: Swartz, 10392 (LP).

\section{Carthamus L.}

\section{Carthamus tinctorius $\mathrm{L}$.}

Hierba. Anual. Introducida. 0-300 m.

Distribución: ELD.

Ejemplar de referencia: Keller, H. A. 5460 (CTES).

Centaurea L., nom. cons.

\section{Centaurea calcitrapa L.}

Hierba. Anual o bianual. Introducida. 0-500 m. Distribución: CAP.

Ejemplar de referencia: Rodríguez, F. M. 445 (BA).

\section{Centaurea melitensis L.}

Hierba. Anual o bianual. Introducida. 0-500 m. Distribución: CAP, MON.

Ejemplar de referencia: Rodríguez, 390 (BA).

\section{Centratherum Cass.}

Centratherum camporum (Hassl.) Malme Subarbusto. Nativa. 0-500 m.

Distribución: CAN.

Ejemplar de referencia: Morrone, O. 1147 (SI).

Centratherum punctatum Cass.

Subarbusto. Nativa. 0-500 m.

Distribución: CAI, CAN, CAP, ELD, GMB, GSM, GUA, IGU, MON, SIG, SJA, SPE.

Ejemplar de referencia: Biganzoli, F. 108 (SI).

Chacoa R.M. King \& H. Rob.

Chacoa pseudoprasiifolia (Hassl.) R.M. King \& H. Rob.

Arbusto. Nativa. 0-500 m. 
Distribución: CAI, CAN, CON, IGU, LNA, SJA, SPE.

Ejemplar de referencia: Arbo, M. M. 5935 (CTES, SI).

Chaptalia Vent., nom. cons.

Chaptalia arechavaletae Hieron. ex Arechav. Hierba. Perenne. Nativa. 0-500 m.

Distribución: CAP, IGU. (LP).

Ejemplar de referencia: Ekman, E. L. 1012

\section{Chaptalia integerrima (Vell.) Burkart}

Hierba. Perenne. Nativa. 0-600 m.

Distribución: APO, CAN, CON, IGU, OBE, SPE.

Ejemplar de referencia: Cabrera, A. L. 28688 (SI).

Chaptalia nutans (L.) Pol.

Hierba. Perenne. Nativa. 0-800 m.

Distribución: APO, CAI, CAN, CAP, ELD, GMB, GSM, GUA, IGU, LNA, MON, OBE, SIG, SPE.

Ejemplar de referencia: Morrone, O. 790 (SI).

Chaptalia piloselloides (Vahl) Baker

Hierba. Perenne. Nativa. 0-500 m.

Distribución: CAI, CAN, SIG.

Ejemplar de referencia: Eskuche, U. G. 3194 (SI).

Chaptalia sinuata (Less.) Baker

Hierba. Perenne. Nativa. 0-500 m.

Distribución: CAN, CAP, OBE.

Ejemplar de referencia: Burkart, A. 1550 (SI).

\section{Chevreulia Cass.}

Chevreulia acuminata Less.

Hierba. Perenne. Nativa. 0-500 m.

Distribución: APO, CAN, CAP, GUA.

Ejemplar de referencia: Cabrera, A. L. 28455 (SI).

Chevreulia sarmentosa (Pers.) S.F. Blake

Hierba. Perenne. Nativa. 0-500 m.

Distribución: APO, GUA.

Ejemplar de referencia: Múlgura, M. E. 2328 (SI).

\section{Chromolaena DC.}

Chromolaena arnottiana (Griseb.) R.M. King \& H. Rob.

Subarbusto. Nativa. 0-500 m.

Distribución: APO, SIG.

Ejemplar de referencia: Keller, H. A. 8697 (CTES).

Chromolaena caaguazuensis (Hieron.) R.M. King \& H. Rob. $\left.{ }^{* * *}\right)$

Subarbusto. Nativa. 0-500 m.

Distribución: CAN, SIG.

Ejemplar de referencia: Fontana, J. L. 114 (CTESN, SI).

Chromolaena christieana (Baker) R.M. King \& H. Rob.

Subarbusto. Nativa. 0-100 m.

Distribución: CAN, GUA.

Ejemplar de referencia: Montes, J. E. 27389 (SI).

Chromolaena elliptica (Hook. \& Arn.) R.M. King \& H. Rob.

Subarbusto. Nativa. 0-500 m.

Distribución: APO, CAN.

Ejemplar de referencia: Fontana, J. L. 128 (SI).

Chromolaena hirsuta (Hook. \& Arn.) R.M. King \& H. Rob.

Subarbusto. Nativa. 0-700 m.

Distribución: APO, CAI, CAN, CAP, CON, IGU, SJA.

Ejemplar de referencia: Montes, J. E. 602 (SI).

Chromolaena ivifolia (L.) R.M. King \& H. Rob. Subarbusto. Nativa. 0-500 m.

Distribución: CAN, CAP, CON, SIG.

Ejemplar de referencia: Grüner, G. 1374 (LP).

Chromolaena laevigata (Lam.) R.M. King \& H. Rob. var. arenaria (Baker) S.E. Freire \& Ariza

Arbusto. Nativa. 0-500 m.

Distribución: CAN.

Ejemplar de referencia: Sotelo, J. B. 10008 (LP).

Chromolaena laevigata (Lam.) R.M. King \& H. Rob. var. laevigata

Arbusto. Nativa. 0-600 m.

Distribución: CAI, CAN, CAP, IGU, SIG.

Ejemplar de referencia: Morrone, O. 1978 (SI). 
Chromolaena odorata (L.) R.M. King \& H. Rob. Arbusto. Nativa. 0-700 m.

Distribución: CAI, CAN, CAP, GSM, GUA, IGU, SPE.

Ejemplar de referencia: Rodríguez, F. M. 277 (SI).

Chromolaena pedunculosa (Hook. \& Arn.) R.M. King \& H. Rob.

Subarbusto. Nativa. 0-700 m.

Distribución: CAI, CAN, CAP, GMB, GSM, GUA, LNA.

Ejemplar de referencia: Biganzoli, F. 863 (SI).

Chromolaena squarrulosa (Hook. \& Arn.) R.M. King \& H. Rob.

Subarbusto. Nativa. 0-600 m.

Distribución: CAN, CAP, GUA, SIG.

Ejemplar de referencia: Fontana, J. L. 128 (SI).

Chromolaena squarrosoramosa (Hieron.)

R.M. King \& H. Rob.

Hierba. Perenne. Nativa. 0-500 m.

Distribución: CAN, CON.

Ejemplar de referencia: Keller, H. A. 6027 (CTES).

Chromolaena verbenacea (DC.) R.M. King \& H. Rob.

Subarbusto. Nativa. 0-600 m.

Distribución: APO, CAI, CAN, CAP, LNA, SIG, SJA.

Ejemplar de referencia: Fontana, J. L. 67 (SI).

Chrysolaena H. Rob.

Chrysolaena cognata (Less.) Dematteis

Subarbusto. Nativa. 0-700 m.

Distribución: APO, CAI, CAN, GMB, SIG, SJA.

Ejemplar de referencia: Krapovickas, A. 28816 (SI).

\section{Chrysolaena cordifolia Dematteis}

Subarbusto. Nativa. 0-500 m.

Distribución: GMB.

Ejemplar de referencia: Keller, H. A. 3975 (CTES,CORD,SI).

Chrysolaena flexuosa (Sims) H. Rob.

Hierba. Perenne. Nativa. 0-500 m.

Distribución: APO, CAN, CAP, CON, SJA.

Ejemplar de referencia: Zuloaga, F. O. 1006 (SI).
Chrysolaena lithospermifolia (Hieron.) $\mathrm{H}$. Rob.

Hierba. Perenne. Nativa. 0-500 m.

Distribución: CAN, CAP, SIG.

Ejemplar de referencia: Krapovickas, A. 44612 (CTES, SI).

Chrysolaena platensis (Spreng.) $\mathrm{H}$. Rob.

Subarbusto. Nativa. 0-600 m.

Distribución: CAI, CAP, GMB, GUA, SIG, SJA, SPE.

Ejemplar de referencia: Tressens, S. G. 5801 (CTES).

Chrysolaena propinqua (Hieron.) $\mathrm{H}$. Rob. var. canescens (Chodat) Dematteis ${ }^{* * *}$ )

Hierba. Perenne. Nativa. 0-500 m.

Distribución: CAP.

Ejemplar de referencia: Ekman, E. L. 1150 (S).

Chrysolaena propinqua (Hieron.) $\mathrm{H}$. Rob. var. propinqua

Hierba. Perenne. Nativa. 0-500 m.

Distribución: CAN, CAP, CON, GMB, SJA.

Ejemplar de referencia: Dematteis, M. 371 (CTES).

Chrysolaena verbascifolia (Less.) H. Rob.

Subarbusto. Nativa. 0-700 m.

Distribución: APO, CAI, CAN, CAP, GMB, SIG, SJA.

Ejemplar de referencia: Krapovickas, A. 28828 (SI)

\section{Cirsium Mill.}

Cirsium vulgare (Savi) Ten.

Hierba. Anual o Bianual. Introducida. 0-500 m. Distribución: GUA.

Ejemplar de referencia: Keller, H. A. 129 (CTES).

Conyza Less., nom. cons.

Conyza blakei (Cabrera) Cabrera

Hierba. Anual. Nativa. 0-500 m.

Distribución: CAN, CAP.

Ejemplar de referencia: Montes, J. E. 27514 (SI). 

Conyza bonariensis (L.) Cronquist var.
angustifolia (Cabrera) Cabrera

Hierba. Anual. Nativa. 0-500 m.

Distribución: CAP, IGU.

Ejemplar de referencia: Nuñez, 205 (LP).

\section{Conyza bonariensis (L.) Cronquist var.} bonariensis

Hierba. Anual. Nativa. 0-600 m.

Distribución: CAI, CAN, CAP, GUA, IGU.

Ejemplar de referencia: Montes, J. E. 6945 (SI).

\section{Conyza catharinensis Cabrera $\left({ }^{* * *}\right)$}

Hierba. Anual. Nativa. 0-500 m.

Distribución: CAN, OBE.

Ejemplar de referencia: Fontana, J. L. 151-51 (LP, SI).

\section{Conyza glandulitecta $\mathrm{Cabrera}$}

Hierba. Anual. Nativa. 0-500 m.

Distribución: CAP, LNA, SIG.

Ejemplar de referencia: Schinini, A. 21909 (LP).

Conyza pampeana (Parodi) Cabrera

Hierba. Anual. Nativa. 0-500 m.

Distribución: APO.

Ejemplar de referencia: Cabrera, A. L. 28575 (SI).

Conyza primulifolia (Lam.) Cuatrec. \& Lourteig

Hierba. Bianual. Nativa. 0-500 m.

Distribución: CAN, GUA, OBE, SPE.

Ejemplar de referencia: Ekman, E. L. 1086 (NY).

Conyza sumatrensis (Retz.) E. Walker var. leiotheca (S.F. Blake) Pruski \& G. Sancho

Hierba. Anual. Nativa. 0-700 m.

Distribución: CAI, CAN, GUA, IGU, OBE, SPE.

Ejemplar de referencia: Schinini, A. 5984 (LP, SI).

Conyza sumatrensis (Retz.) E. Walker var. sumatrensis

Hierba. Anual. Nativa. 0-700 m.

Distribución: CAI, CAN, GMB, GSM, IGU, OBE, SIG.

Ejemplar de referencia: Montes, J. E. 6945 (SI).

\section{Coreopsis L.}

\section{Coreopsis lanceolata L.}

Hierba. Perenne. Introducida. 0-700 m.

Distribución: CAI, ELD, GMB, GSM, GUA, IGU, LNA, MON, OBE, SPE.

Ejemplar de referencia: Cabrera, A. L. 28633 (SI).

\section{Coreopsis tinctoria Nutt.}

Hierba. Anual. Introducida. 0-500 m.

Distribución: GUA.

Ejemplar de referencia: Rodríguez, M. 425 (CTES, MNES).

\section{Cosmos Cav.}

\section{Cosmos sulphureus Cav.}

Hierba. Anual. Nativa. 0-500 m.

Distribución: APO, CAN, CAP, IGU, LNA.

Ejemplar de referencia: Cabrera, A. L. 29125 (SI).

\section{Criscia Katinas}

Criscia stricta (Spreng.) Katinas

Hierba. Perenne. Nativa. 0-500 m.

Distribución: APO, CAN, CAP, SIG.

Ejemplar de referencia: Burkart, A. 14258 (SI).

\section{Critonia P. Browne}

Critonia megaphylla (Baker) R.M. King \& H. Rob.

Hierba o subarbusto. Perenne. Nativa. 0-600 m. Distribución: CAI, CAP, ELD, GSM, IGU.

Ejemplar de referencia: Biganzoli, F. 357 (SI).

\section{Cyrtocymura H. Rob.}

\section{Cyrtocymura scorpioides (Lam.) H. Rob.}

Arbusto. Nativa. 0-700 m.

Distribución: APO, CAI, CAN, CAP, ELD, GUA, IGU, SIG.

Ejemplar de referencia: Morrone, O. 1629 (SI). 
Dasyphyllum Kunth

Dasyphyllum brasiliense (Spreng.) Cabrera var. brasiliense

Arbusto. Nativa. 0-600 m.

Distribución: CAN, CAP, GSM, GUA, OBE, SIG.

Ejemplar de referencia: Grüner, G. 393 (LP).

\section{Dasyphyllum tomentosum (Spreng.) Cabrera} var. multiflorum (Baker) Cabrera ${ }^{* * *}$ )

Árbol. Nativa. 0-500 m.

Distribución: GUA, IGU.

Ejemplar de referencia: Deginani, N. B. 1076 (SI).

Dasyphyllum tomentosum (Spreng.) Cabrera var. tomentosum $\left.{ }^{* * *}\right)$

Árbol. Nativa. 0-500 m.

Distribución: GMB, GUA.

Ejemplar de referencia: Deginani, N. B. 1076 (SI).

\section{Dendrophorbium (Cuatrec.) C. Jeffrey}

Dendrophorbium catharinense (Dusén ex Cabrera) C. Jeffrey (***)

Hierba. Perenne. Nativa. 0-500 m.

Distribución: SPE.

Ejemplar de referencia: Pérez-Moreau, R. A. s.n. (LP).

Dendrophorbium pluricephalum (Cabrera) $C$. Jeffrey $\left({ }^{* * *}\right)$

Arbusto. Nativa. 0-500 m.

Distribución: CAN, ELD, GUA.

Ejemplar de referencia: Eskuche, U. G. 815 (SI).

\section{Dimerostemma Cass.}

Dimerostemma apense (Chodat) M.D. Moraes

Hierba. Perenne. Nativa. 0-500 m.

Distribución: CAN.

Ejemplar de referencia: Schinini, A. 27596 (CTES).

Dimerostemma arnottii (Baker) M.D. Moraes Subarbusto. Nativa. 0-700 m.

Distribución: APO, CAN, CAP, GMB, SIG, SPE. Ejemplar de referencia: Morrone, O. 1593 (SI).
Dimerostemma aspilioides (Griseb.) M.D. Moraes Subarbusto. Nativa. 0-500 m.

Distribución: CAI.

Ejemplar de referencia: Fontana, J. L. 211 (CTESN).

Dimerostemma reitzii (H. Rob.) M.D. Moraes ${ }^{* * *}$ ) Subarbusto. Nativa. 0-500 m.

Distribución: SIG.

Ejemplar de referencia: Zuloaga, F. O. 9958 (SI).

Disynaphia Hook. \& Arn. ex DC.

Disynaphia filifolia (Hassl.) R.M. King \& H. Rob. $\left.{ }^{* * *}\right)$

Subarbusto. Nativa. 0-500 m.

Distribución: CAN, CAP, SIG.

Ejemplar de referencia: Morrone, O. 1031 (SI).

Disynaphia multicrenulata (Sch. Bip. ex Baker) R.M. King \& H. Rob.

Subarbusto. Nativa. 0-500 m.

Distribución: APO, CAN, CAP, LNA, SIG.

Ejemplar de referencia: Martínez Crovetto, $R$. 9581 (SI).

Disynaphia radula (Chodat) R.M. King \& H. Rob. Hierba. Perenne. Nativa. 0-500 m.

Distribución: CAN, SIG.

Ejemplar de referencia: Fontana, J. L. 187 (SI).

Eclipta L., nom. cons.

Eclipta prostrata (L.) L.

Hierba. Anual. Nativa. 0-800 m.

Distribución: CAI, CAN, CON, ELD, GMB, GSM, GUA, IGU, LNA, MON, OBE, SIG, SPE.

Ejemplar de referencia: Cabrera, A. L. 28823 (SI).

\section{Elephantopus L.}

\section{Elephantopus mollis Kunth}

Hierba. Perenne. Nativa. 0-800 m.

Distribución: APO, CAI, CAN, CAP, CON, GMB, GSM, GUA, IGU, SIG, SPE.

Ejemplar de referencia: Morrone, O. 795 (SI). 


\section{Emilia Cass.}

\section{Emilia fosbergii Nicolson $\left({ }^{* * *}\right)$}

Hierba. Anual. Introducida. 0-500 m.

Distribución: CAN, ELD, GMB, IGU, SIG.

Ejemplar de referencia: Tressens, S. G. 5575 (CTES).

\section{Erechtites Raf.}

Erechtites hieraciifolius (L.) Raf. ex DC. var. cacalioides (Fisch. ex Spreng.) Griseb.

Hierba. Anual. Introducida. 0-600 m.

Distribución: CAN, CAP, GSM, GUA, SIG, SPE. Ejemplar de referencia: Xifreda, C. C. 556 (SI).

\section{Erechtites missionus Malme $\left.{ }^{(* * *}\right)$}

Hierba. Anual. Nativa. 0-500 m.

Distribución: CAN.

Ejemplar de referencia: Ekman, E. L. 1104 (LP, S).

Erechtites valerianifolius (Link ex Spreng.) Less. ex DC.

Hierba. Anual. Nativa. 0-800 m.

Distribución: APO, CAI, CAN, CAP, ELD, GMB, GSM, GUA, IGU, SIG.

Ejemplar de referencia: Biganzoli, F. 134 (SI).

\section{Exostigma G. Sancho}

Exostigma notobellidiastrum (Griseb.) G. Sancho

Hierba. Perenne. Nativa. 0-700 m.

Distribución: CAI, CAN, CAP, ELD, GMB, GSM, GUA, IGU, SIG.

Ejemplar de referencia: Tressens, S. G. 6035 (CTES).

Exostigma rivulare (Gardner) G. Sancho

Hierba. Perenne. Nativa. 0-500 m.

Distribución: APO, CAI, CAN, ELD, GMB, GUA, IGU.

Ejemplar de referencia: Xifreda, C. C. 480 (SI).

\section{Facelis Cass.}

Facelis retusa (Lam.) Sch. Bip. Hierba. Anual. Nativa. 0-500 m.
Distribución: APO, CAN, CAP, ELD, OBE.

Ejemplar de referencia: Cabrera, A. L. 28720 (SI).

Flaveria Juss.

Flaveria bidentis (L.) Kuntze

Hierba. Anual. Nativa. 0-500 m.

Distribución: APO, GMB.

Ejemplar de referencia: Keller, H. A. 5079 (CTES,SI).

Fleischmannia Sch. Bip.

Fleischmannia prasiifolia (Griseb.) R.M. King \& H. Rob.

Subarbusto. Nativa. 0-500 m.

Distribución: CAN, GUA, SIG.

Ejemplar de referencia: Grüner, G. 1355 (LP).

Galinsoga Ruiz \& Pav.

Galinsoga parviflora Cav.

Hierba. Anual. Nativa. 0-500 m.

Distribución: ELD, GUA, IGU, LNA, SPE.

Ejemplar de referencia: Cabrera, A. L. 29229 (SI).

\section{Gamochaeta Wedd.}

Gamochaeta americana (Mill.) Wedd.

Hierba. Bianual o perenne. Nativa. 0-700 m.

Distribución: CAN, CAP, GUA, IGU, SIG.

Ejemplar de referencia: Montes, J. E. 2238 (SI).

Gamochaeta calviceps (Fernald) Cabrera

Hierba. Anual o perenne. Nativa. 0-500 m.

Distribución: CAI, CAN, IGU.

Ejemplar de referencia: Montes, J. E. 2522 $(L P)$.

Gamochaeta filaginea (DC.) Cabrera

Hierba. Perenne. Nativa. 0-500 m.

Distribución: CAN, CON, GMB, SIG.

Ejemplar de referencia: Johnson, A. E. 905 (SI). 
Gamochaeta pensylvanica (Willd.) Cabrera

Hierba. Bianual o perenne. Nativa. 0-700 m.

Distribución: CAI, CAN, CON, GUA, IGU, SIG, SPE.

Ejemplar de referencia: Montes, J. E. 4297 (LP).

Gamochaeta simplicicaulis (Willd. ex Spreng.)

Cabrera

Hierba. Anual. Nativa. 0-500 m.

Distribución: GUA. (SI).

Ejemplar de referencia: Múlgura, M. E. 2496

Grazielia R.M. King \& H. Rob.

Grazielia serrata (Spreng.) R.M. King \& H. Rob. $(* * *)$

Arbusto. Nativa. 0-700 m.

Distribución: CAI, CAN, GMB, GSM, SIG.

Ejemplar de referencia: Xifreda, C. C. 551 (SI).

\section{Grindelia Willd.}

Grindelia scorzonerifolia Hook. \& Arn.

Subarbusto. Nativa.

Distribución: probablemente en los campos al Sur de la provincia.

Ejemplar de referencia: Goby, s.n. (BA).

\section{Gymnocoronis DC.}

Gymnocoronis spilanthoides (D. Don ex Hook. \& Arn.) DC.

Hierba. Perenne. Nativa. 0-500 m.

Distribución: CAN, ELD, GMB.

Ejemplar de referencia: Hicken, C. M. 9080 (SI).

\section{Gyptis (Cass.) Cass.}

Gyptis crassipes (Hieron.) R.M. King \& H. Rob. Hierba. Perenne. Nativa. 0-500 m.

Distribución: APO, CAN, SIG.

Ejemplar de referencia: Grüner, G. 1337 (LP).
Gyptis lanigera (Hook. \& Arn.) R.M. King \& H. Rob.

Hierba. Perenne. Nativa. 0-500 m.

Distribución: APO, CAN, SIG, SJA, SPE.

Ejemplar de referencia: Fontana, J. L. 61 (SI).

Gyptis tanacetifolia (Gillies ex Hook. \& Arn.) D.J.N. Hind \& Flann

Subarbusto. Nativa. 0-500 m.

Distribución: APO, CAN, GMB.

Ejemplar de referencia: Eskuche, U. G. 2776 (SI).

Hatschbachiella R.M. King \& H. Rob.

Hatschbachiella tweedieana (Hook. \& Arn.) R.M. King \& H. Rob.

Subarbusto. Nativa. 0-600 m.

Distribución: APO, CAI, CAN, CAP, LNA, SIG.

Ejemplar de referencia: Fontana, J. L. 117 (SI).

Heterocondylus R.M. King \& H. Rob.

Heterocondylus grandis (Sch. Bip. ex Baker) R.M. King \& H. Rob.

Hierba. Perenne. Nativa. 0-500 m.

Distribución: CAN, SIG, SJA.

Ejemplar de referencia: Grüner, G. 1305 (LP).

Heterocondylus vitalbae (DC.) R.M. King \& $\mathrm{H}$. Rob. $\left({ }^{* * *}\right)$

Arbusto. Nativa. 0-500 m.

Distribución: ELD, IGU, SIG.

Ejemplar de referencia: Keller, H. A. 1155 (CTES, SI).

\section{Hieracium L.}

Hieracium commersonii Monnier

Hierba. Perenne. Nativa. 0-500 m.

Distribución: APO, CAN, CAP, SIG.

Ejemplar de referencia: Cabrera, A. L. 29252 (SI).

\section{Hieracium mosenii Malme $\left({ }^{* * *}\right)$}

Hierba. Perenne. Nativa. 100-300 m.

Distribución: CAI, CAP, GSM.

Ejemplar de referencia: Ekman, E. L. 1226 (S). 


\section{Holocheilus Cass.}

\section{Holocheilus brasiliensis (L.) Cabrera}

Hierba. Perenne. Nativa.

Distribución: probablemente en los campos al Sur de la provincia. (LP).

Ejemplar de referencia: Spegazzini, C. L. s.n.

\section{Holocheilus illustris (Vell.) Cabrera ${ }^{(* * *}$ )}

Hierba. Perenne. Nativa. 0-500 m.

Distribución: CAP, GMB.

Ejemplar de referencia: Schinini, A. 10696 (SI).

Holocheilus schulzii (Cabrera) Cabrera

Hierba. Perenne. Nativa. 0-500 m.

Distribución: APO, CAI, CAN.

Ejemplar de referencia: Cabrera, A. L. 28531 (SI).

\section{Hypochaeris L.}

Hypochaeris albiflora (Kuntze) C.F. AzevêdoGonçalves \& Matzenb.

Hierba. Perenne. Nativa. 0-500 m.

Distribución: APO, CAN, CAP, SJA.

Ejemplar de referencia: Burkart, A. 14092 (SI).

Hypochaeris chillensis (Kunth) Hieron.

Hierba. Perenne. Nativa. 0-700 m.

Distribución: APO, CAN, GMB, GSM, GUA, IGU, SPE.

Ejemplar de referencia: Montes, J. E. 1314a (SI).

Hypochaeris microcephala (Sch. Bip.) Cabrera Hierba. Perenne. Nativa. 0-500 m.

Distribución: CAI, CAP, IGU.

Ejemplar de referencia: Barboza, G. E. 409 (CORD).

\section{Hypochaeris tropicalis Cabrera}

Hierba. Perenne. Nativa. 0-500 m.

Distribución: CAN, CAP, IGU.

Ejemplar de referencia: Montes, J. E. 10273 (LP).

Hypochaeris variegata (Lam.) Baker

Hierba. Perenne. Nativa. 200-800 m.

Distribución: CAN.

Ejemplar de referencia: Grüner, G. 1440 (LP).

\section{Hysterionica Willd.}

Hysterionica nidorelloides (DC.) Baker

Hierba. Anual. Nativa. 0-700 m.

Distribución: APO, CAI, CAN, CAP, CON, IGU, OBE, SIG, SJA.

Ejemplar de referencia: Biganzoli, F. 1737 (SI).

\section{Isostigma Less.}

Isostigma dissitifolium Baker $\left.{ }^{(* *}\right)$

Hierba o subarbusto. Perenne. Nativa. 0-500 m.

Distribución: CAN, SIG.

Ejemplar de referencia: Molfino, J. F. s.n. (F).

Isostigma peucedanifolium (Spreng.) Less. var. crithmifolium (Less.) G. Peter

Hierba. Perenne. Nativa. 0-500 m.

Distribución: CAP, SIG.

Ejemplar de referencia: Morrone, O. 1869 (SI).

Isostigma peucedanifolium (Spreng.) Less. var. peucedanifolium

Hierba. Perenne. Nativa. 0-500 m.

Distribución: CAI, CAN, SIG.

Ejemplar de referencia: Zuloaga, F. O. 5748 (SI).

Isostigma peucedanifolium (Spreng.) Less. var. speciosum (Less.) G. Peter $\left(^{* * *}\right)$

Hierba. Perenne. Nativa. 0-700 m.

Distribución: CAN, SIG.

Ejemplar de referencia: Montes, J. E. 14903 (SI).

\section{Jaegeria Kunth}

Jaegeria hirta (Lag.) Less.

Hierba. Anual. Nativa. 0-700 m.

Distribución: APO, CAN, CAP, GUA, SIG, SPE.

Ejemplar de referencia: Burkart, A. 14030 (SI).

Jungia L. f.

Jungia floribunda Less.

Hierba o subarbusto. Perenne. Nativa. 0-500 m. Distribución: APO, CAN, CAP, CON, GUA, SIG. Ejemplar de referencia: Quiroga, H. 445 (BA). 


\author{
Jungia sellowii Less. $\left({ }^{* * *}\right)$ \\ Hierba o subarbusto. Perenne. Nativa. 0-500 m. \\ Distribución: GUA, IGU. \\ Ejemplar de referencia: Keller, H. A. 1742 \\ (CTES).
}

Kaunia R.M. King \& H. Rob.

Kaunia rufescens (P.W. Lund ex DC.) R.M. King \& H. Rob. $\left.{ }^{(* *}\right)$

Arbusto o árbol. Nativa. 0-500 m.

Distribución: IGU, OBE.

Ejemplar de referencia: Vanni, R. O. 3441 (CTES).

\section{Koanophyllon Arruda}

Koanophyllon simillimum (B.L. Rob.) R.M. King \& H. Rob.

Arbusto o árbol. Nativa. 0-500 m.

Distribución: CAN, IGU, SIG.

Ejemplar de referencia: Pedersen, T. M. 9306 (CTES).

\section{Lactuca L.}

\section{Lactuca serriola $\mathrm{L}$.}

Hierba. Anual o bianual. Introducida. 0-500 m.

Distribución: GUA, SPE.

Ejemplar de referencia: Keller, H. A. 3603 (CTES).

\section{Lactuca virosa $\left.\mathrm{L} . \mathrm{(}^{*}\right)$}

Hierba. Anual o bianual. Introducida. 0-500 m.

Distribución: GSM, GUA, IGU, MON.

Ejemplar de referencia: Keller, H. A. 1673 (CTES).

\section{Lepidaploa (Cass.) Cass.}

Lepidaploa balansae (Hieron.) $\mathrm{H}$. Rob.

Arbusto. Nativa. 0-700 m.

Distribución: CAI, CAN, ELD, GSM, GUA, IGU, MON, OBE, SIG, SJA, SPE.

Ejemplar de referencia: Diem, J. 1397 (ICN, SI).
Lepidaploa pseudomuricata $\mathrm{H}$. Rob. $\left({ }^{* * *}\right)$

Subarbusto. Nativa. 0-700 m.

Distribución: SPE.

Ejemplar de referencia: Keller, H. A. 3921 (CTES).

Lepidaploa psilostachya (DC.) H. Rob. $\left(^{* * *}\right)$

Subarbusto. Nativa. 0-500 m.

Distribución: SIG.

Ejemplar de referencia: Krapovickas, A. 28694 (SI).

Lepidaploa remotiflora (Rich.) H. Rob.

Hierba. Anual. Nativa. 0-700 m.

Distribución: APO, CAI, CAN, CAP, CON, ELD, LNA, SIG, SJA.

Ejemplar de referencia: Zuloaga, F. O. 4939 (SI).

\section{Leptostelma D. Don}

\section{Leptostelma maxima D. Don $\left({ }^{* * *}\right)$}

Hierba o subarbusto. Perenne. Nativa. 0-500 m. Distribución: GMB.

Ejemplar de referencia: Guaglianone, E. R. 1155 (SI).

Leptostelma tweediei (Hook. \& Arn.) D. J. N. Hind \& G.L. Nesom

Hierba. Perenne. Nativa. 0-500 m.

Distribución: APO, CAN, CON, GMB, SJA.

Ejemplar de referencia: Cabrera, A. L. 29092 (SI).

\section{Lessingianthus $\mathrm{H}$. Rob.}

Lessingianthus brevifolius (Less.) H. Rob. Subarbusto. Nativa. 0-600 m.

Distribución: CAN, CAP, GMB, SIG.

Ejemplar de referencia: Morrone, O. 1606 (SI).

Lessingianthus cataractum (Hieron.) H. Rob. $\left({ }^{* * *}\right)$

Arbusto. Nativa. 0-500 m.

Distribución: GUA, IGU, SPE.

Ejemplar de referencia: Rodríguez, M. 1005 (G, MNES). 
Lessingianthus glabratus (Less.) H. Rob. var. angustifolius (Cabrera) Dematteis

Arbusto. Nativa. 0-600 m.

Distribución: CAI, CAN, CAP, GSM.

Ejemplar de referencia: Biganzoli, F. 136 (SI).

Lessingianthus glabratus (Less.) H. Rob. var. glabratus

Subarbusto. Nativa. 0-700 m.

Distribución: CAI, CAN, CAP, CON, GSM, MON, SIG, SPE.

Ejemplar de referencia: Biganzoli, F. 204 (SI).

Lessingianthus laniferus (Cristóbal \&

Dematteis) M.B. Angulo

Subarbusto. Nativa. 0-500 m.

Distribución: CAN, GMB, SIG, SJA.

Ejemplar de referencia: Maruñak, V. 119 (C, CTES, LP, MBM, TEX, UC).

Lessingianthus lorentzii (Hieron.) H. Rob.

Subarbusto. Nativa. 0-500 m.

Distribución: CAI, CAN, CAP, SIG.

Ejemplar de referencia: Biganzoli, F. 832 (SI).

Lessingianthus mollissimus (D. Don ex Hook. \& Arn.) H. Rob. var. microcephalus (Hieron.)

Dematteis

Subarbusto. Nativa. 0-500 m.

Distribución: APO, CAI, CAN, CAP, CON, SIG, SJA.

Ejemplar de referencia: Krapovickas, A. 15468 (BAA, BAB, CTES, LP).

Lessingianthus mollissimus (D. Don ex Hook. \& Arn.) H. Rob. var. mollisimus

Subarbusto. Nativa. 0-500 m.

Distribución: APO, CAN, CAP, CON, GSM, SJA.

Ejemplar de referencia: Montes, J. E. 3342 (SI).

Lessingianthus niederleinii (Hieron.) H. Rob. Arbusto. Nativa. 0-500 m.

Distribución: APO, CAI, CAN, CAP, GMB, GUA, SJA.

Ejemplar de referencia: Cabrera, A. L. 29054 (SI).

Lessingianthus plantaginodes (Less.) $\mathrm{H}$. Rob. Subarbusto. Nativa. 0-500 m.
Distribución: CAP, SJA.

Ejemplar de referencia: Krapovickas, A. 15246 (CTES).

Lessingianthus polyphyllus (Sch. Bip. ex Baker) H. Rob.

Subarbusto. Nativa. 0-500 m.

Distribución: CAN, CAP, SIG.

Ejemplar de referencia: Morrone, O. 999 (SI).

Lessingianthus rubricaulis (Bonpl.) $\mathrm{H}$. Rob.

Subarbusto. Nativa. 0-700 m.

Distribución: APO, CAN, CAP, GMB, GUA, LNA, SIG, SJA.

Ejemplar de referencia: Dematteis, M. 595 (CTES, FCQ).

Lessingianthus sellowii (Less.) H. Rob.

Hierba o subarbusto. Perenne. Nativa. 0-600 m.

Distribución: APO, CAN, CAP, CON, SJA, SPE.

Ejemplar de referencia: Cabrera, A. L. 29078 (SI).

Lessingianthus spicatus (Cabrera) Dematteis $(* *)$

Hierba o subarbusto. Perenne. Endémica. 0-500 m.

Distribución: SIG.

Ejemplar de referencia: Schwarz, G. J. 5394 (LIL).

\section{Lessingianthus teyucuarensis (Cabrera)}

Dematteis $(* *)$

Subarbusto. Endémica. 0-500 m.

Distribución: CAN, SIG.

Ejemplar de referencia: Morrone, O. 1019 (SI).

\section{Lucilia Cass.}

Lucilia acutifolia (Poir.) Cass.

Hierba. Perenne. Nativa. 0-600 m.

Distribución: APO, CAN, CAP, CON, SJA.

Ejemplar de referencia: Krapovickas, A. 28786 (SI).

Lucilia nitens Less.

Hierba. Perenne. Nativa. 0-500 m.

Distribución: APO, CAN.

Ejemplar de referencia: Burkart, A. 14439 (SI). 
Macropodina R.M. King \& H. Rob.

Macropodina blumenavii (Hieron.) R.M. King \& H. Rob. $\left.{ }^{* * *}\right)$

Subarbusto. Nativa. 0-500 m.

Distribución: CAP, GUA, IGU.

Ejemplar de referencia: Ferraro, L. I. 2474 (SI).

\section{Malmeanthus R.M. King \& H. Rob.}

Malmeanthus subintegerrimus (Malme) R.M. King \& H. Rob.

Arbusto. Nativa. 0-700 m.

Distribución: CAN, GMB, IGU, SIG.

Ejemplar de referencia: Martínez, G. 40 (SI).

Matricaria L., nom. cons.

\section{Matricaria chamomilla L.}

Hierba. Anual. Nativa. 0-500 m.

Distribución: ELD, GSM, GUA.

Ejemplar de referencia: Tressens, S. G. 6356 (CTES).

\section{Melanthera Rohr}

Melanthera latifolia (Gardner) Cabrera

Hierba. Nativa. 0-500 m.

Distribución: CAN, CAP, ELD, GSM, GUA, IGU, MON, SIG, SPE.

Ejemplar de referencia: Zuloaga, F. O. 5015 (SI).

\section{Micropsis DC.}

Micropsis dasycarpa (Griseb.) Beauverd

Hierba. Anual. Nativa. 0-500 m.

Distribución: CAN, CAP.

Ejemplar de referencia: Burkart, A. 14031 (SI).

\section{Mikania Willd.}

Mikania burchellii Baker ${ }^{* * *}$ )

Hierba o subarbusto. Perenne. Nativa. 0-700 m.

Distribución: CAI, GUA, IGU, SIG, SPE.

Ejemplar de referencia: Montes, J. E. 27459 (SI).
Mikania campanulata Gardner $\left({ }^{* *}\right)$

Enredadera. Perenne. Nativa. 0-800 m.

Distribución: GUA, SIG.

Ejemplar de referencia: Tressens, S. G. 5828 (CORD, CTES).

Mikania capricorni B.L. Rob. $\left.{ }^{* * *}\right)$

Enredadera. Perenne. Nativa. 0-500 m.

Distribución: SIG.

Ejemplar de referencia: Grüner, G. 1303 (LP).

\section{Mikania chlorolepis Baker $\left({ }^{* * *}\right)$}

Enredadera. Perenne. Nativa. 0-800 m.

Distribución: GUA, SPE.

Ejemplar de referencia: Zuloaga, F. O. 5548 (SI).

Mikania cordifolia (L. f.) Willd.

Enredadera. Perenne. Nativa. 0-800 m.

Distribución: APO, IGU, CAI, CAN, GUA, GMB, SIG, SPE.

Ejemplar de referencia: Cabrera, A. L. 29304 (SI).

Mikania daspitti W.C. Holmes \& McDaniel $\left({ }^{* *}\right)$ Enredadera. Perenne. Nativa. 0-500 m.

Distribución: CAN, GMB, IGU.

Ejemplar de referencia: Montes, J. E. 2285 (SI).

Mikania euryanthela (Malme) W.C. Holmes

Enredadera. Perenne. Nativa. 0-500 m.

Distribución: GSM, IGU.

Ejemplar de referencia: Dimitri, s.n. (LPAG).

Mikania fulva (Hook. \& Arn.) Baker

Subarbusto. Nativa. 0-500 m.

Distribución: CON.

Ejemplar de referencia: Fontana, J. L. 266-44 (SI).

Mikania glomerata Spreng. $\left.{ }^{* * *}\right)$

Liana. Nativa. 0-800 m.

Distribución: CON, GUA, IGU, SIG, SPE.

Ejemplar de referencia: Rodríguez, F. M. 370 (SI).

Mikania hemisphaerica Sch. Bip. ex Baker Enredadera. Perenne. Nativa. 0-800 m. Distribución: GMB.

Ejemplar de referencia: Cabrera, A. L. 135 (LP). 
Mikania hirsutissima DC.

Liana. Nativa. 0-700 m.

Distribución: CAI, ELD, GUA, IGU, MON, SIG, SPE.

Ejemplar de referencia: Rodríguez, F. M. 416 (SI).

\section{Mikania lindleyana DC. (***)}

Enredadera. Perenne. Nativa. 0-500 m.

Distribución: GMB, GUA, IGU.

Ejemplar de referencia: Keller, H. A. 68 (CTES, SI).

\section{Mikania lundiana DC. $\left({ }^{* * *}\right)$}

Liana. Nativa. 0-500 m.

Distribución: IGU.

Ejemplar de referencia: Cámara Hernández, J.

A. s.n. (BAA).

\section{Mikania micrantha Kunth}

Enredadera. Perenne. Nativa. 0-700 m.

Distribución: APO, CAI, CAN, CON, GSM, GUA, IGU, LNA, OBE, SIG, SJA, SPE.

Ejemplar de referencia: Zuloaga, F. O. 6613 (SI).

\section{Mikania oblongifolia DC. $\left({ }^{* * *}\right)$}

Subarbusto. Nativa. 0-500 m.

Distribución: GMB.

Ejemplar de referencia: Krapovickas, A. 23363 (SI).

Mikania periplocifolia Hook. \& Arn.

Enredadera. Perenne. Nativa.

Distribución: en el Sur de la provincia.

Ejemplar de referencia: Fontana, J. L. 226-15 (SI).

\section{Mikania pinnatiloba $\mathrm{DC}$.}

Hierba. Perenne. Nativa. 0-500 m.

Distribución: CON.

Ejemplar de referencia: Fontana, J. L. 258-25 (CTESN).

\section{Mikania summinima W.C. Holmes $\left({ }^{* *}\right)$ \\ Subarbusto. Endémica. 0-500 m. \\ Distribución: APO, SIG. \\ Ejemplar de referencia: Martínez Crovetto, $R$. 9626 (SI).}

\footnotetext{
Mikania ternata (Vell.) B.L. Rob. $\left.{ }^{(* *}\right)$

Enredadera. Perenne. Nativa. 0-500 m.

Distribución: GMB, GUA.

Ejemplar de referencia: Tressens, S. G. 6120 (CTES, NY).
}

Mikania thapsoides $D C$.

Subarbusto. Nativa. 0-500 m.

Distribución: APO, CAN, CAP, IGU, SIG, SJA, SPE.

Ejemplar de referencia: Cabrera, A. L. 29063 (SI).

Mikania trachypleura B.L. Rob.

Enredadera. Perenne. Nativa. 0-500 m.

Distribución: CAI, MON.

Ejemplar de referencia: Porta, R. E. 187 (SI).

Mikania urticifolia Hook. \& Arn.

Enredadera. Perenne. Nativa. 0-500 m.

Distribución: ELD, GUA, SIG.

Ejemplar de referencia: Montes, J. E. 14926 (NY, SI).

\section{Mikania variifolia Hieron.}

Enredadera o liana. Perenne. Nativa. 0-500 m.

Distribución: CAN, GUA, IGU.

Ejemplar de referencia: Vanni, R. O. 2842 (CTES).

Mikania vitifolia $\left.\mathrm{DC} .{ }^{* * *}\right)$

Enredadera. Perenne. Nativa. 0-500 m.

Distribución: GUA, IGU.

Ejemplar de referencia: Rodríguez, F. M. 479a (SI).

Montanoa La Llave \& Lex.

Montanoa bipinnatifida (Kunth) K. Koch (***) Arbusto o subarbusto. Introducida. 0-500 m. Distribución: IGU, MON.

Ejemplar de referencia: Keller, H. A. 2133 (CTES,SI).

Moquiniastrum (Cabrera) G. Sancho

Moquiniastrum polymorphum (Less.) G. Sancho subsp. ceanothifolium (Less.) G. Sancho

Árbol. Nativa. 0-500 m.

Distribución: CAN, CON, GUA, IGU, OBE, SIG.

Ejemplar de referencia: Tressens, S. G. 5785 (CTES). 


\section{Mutisia L. f.}

\section{Mutisia campanulata Less. $\left.{ }^{(* *}\right)$}

Arbusto. Nativa. 0-700 m.

Distribución: CAI, CAP, GMB, GUA, SIG, SPE.

Ejemplar de referencia: Zuloaga, F. O. 6888 (SI).

Mutisia coccinea A. St.-Hil. var. dealbata (Less.) Cabrera

Enredadera. Perenne. Nativa. 0-700 m.

Distribución: APO, CAI, CAP, CON, GMB, GUA, IGU, LNA, MON, OBE, SIG, SPE.

Ejemplar de referencia: Zuloaga, F. O. 6450 (SI).

Mutisia speciosa Aiton ex Hook. (***)

Subarbusto. Nativa. 0-500 m.

Distribución: GUA, MON, SIG, SPE.

Ejemplar de referencia: Seijo, G. J. 218 (BAB, CTES, G, MNES, SI).

Neocabreria R.M. King \& H. Rob.

Neocabreria malacophylla (Klatt) R.M. King \& H. Rob. $\left.{ }^{* * *}\right)$

Arbusto. Nativa. 0-700 m.

Distribución: CAN, GMB, GSM, SIG, SPE.

Ejemplar de referencia: Grüner, G. 468 (LP).

\section{Noticastrum DC.}

Noticastrum acuminatum (DC.) Cuatrec.

Hierba. Perenne. Nativa. 0-500 m.

Distribución: SIG.

Ejemplar de referencia: Zuloaga, F. O. 5316 (SI).

Noticastrum gnaphalioides (Baker) Cuatrec.

Hierba. Perenne. Nativa. 0-500 m.

Distribución: CAN, GMB, SIG, SJA.

Ejemplar de referencia: Montes, J. E. 14854 (SI).

Noticastrum macrocephalum (Baker) Cuatrec.

Hierba. Perenne. Nativa. 0-500 m.

Distribución: APO, CAI, CAN, CAP, SIG, SPE.

Ejemplar de referencia: Morrone, O. 1016 (SI).

\section{Orthopappus Gleason}

Orthopappus angustifolius (Sw.) Gleason

Hierba. Perenne. Nativa. 0-700 m.

Distribución: APO, CAI, CAN, CAP, CON, GMB, GUA, LNA, SIG, SJA, SPE.

Ejemplar de referencia: Biganzoli, F. 901 (SI).

\section{Panphalea Lag.}

Panphalea heterophylla Less.

Hierba. Anual. Nativa. 0-500 m.

Distribución: CAI, CAN, SIG.

Ejemplar de referencia: Schwartz, E. A. 6324 (LIL).

Panphalea missionum Cabrera

Hierba. Anual. Nativa. 0-500 m.

Distribución: CAN, CAP, IGU, SPE.

Ejemplar de referencia: Rodríguez, F. M. 97 (SI).

\section{Pectis L.}

Pectis odorata Griseb.

Hierba. Anual. Nativa. 0-500 m.

Distribución: SIG.

Ejemplar de referencia: Keller, H. A. 436 (CTES).

\section{Perezia Lag.}

Perezia squarrosa (Vahl) Less.

Hierba. Perenne. Nativa. 0-400 m.

Distribución: APO, CON.

Ejemplar de referencia: Cabrera, A. L. 28725 (LP).

Picrosia D. Don

Picrosia cabreriana A.G. Schulz

Hierba. Perenne. Nativa. 0-500 m.

Distribución: APO, ELD.

Ejemplar de referencia: Renvoize, S. A. 3085 (SI).

\section{Picrosia longifolia D. Don}

Hierba. Perenne. Nativa. 0-600 m.

Distribución: APO, CAI, CAN, CAP, IGU, MON, SIG. Ejemplar de referencia: Renvoize, S. A. 3311 (SI). 
Piptocarpha R. Br.

Piptocarpha angustifolia Dusén ex Malme (***) Árbol. Nativa. 300-800 m.

Distribución: GMB.

Ejemplar de referencia: Keller, H. A. 9462 (CTES).

Piptocarpha sellowii (Sch. Bip.) Baker

Liana. Perenne. Nativa. 100-600 m.

Distribución: CAI, CAN, CON, GUA, IGU, OBE, SIG, SJA, SPE.

Ejemplar de referencia: Zuloaga, F. O. 5520 (SI).

\section{Plagiocheilus Arn. ex DC.}

Plagiocheilus tanacetoides Haenke ex DC.

Hierba. Anual. Nativa. 0-500 m.

Distribución: CAP.

Ejemplar de referencia: Rodríguez, F. M. 99a (SI).

\section{Plectocephalus D. Don}

Plectocephalus tweediei (Hook. \& Arn.) N. Garcia \& Susanna

Hierba. Anual. Nativa. 0-500 m.

Distribución: CAI, CAN, CAP, CON, ELD, LNA, OBE, SIG.

Ejemplar de referencia: Burkart, A. 14781 (SI).

\section{Pluchea Cass.}

Pluchea sagittalis (Lam.) Cabrera

Hierba. Perenne. Nativa. 0-600 m.

Distribución: APO, CAN, CAP, GSM, GUA, IGU, SIG.

Ejemplar de referencia: Rodríguez, M. 419 (CTES, G, MNES).

\section{Podocoma Cass.}

\section{Podocoma blanchetiana Baker}

Hierba. Perenne. Nativa. 0-500 m.

Distribución: CAI, SJA.

Ejemplar de referencia: Krapovickas, A. 15191 (LP).
Podocoma hieracifolia (Poir.) Cass.

Hierba. Perenne. Nativa. 0-500 m.

Distribución: CAN.

Ejemplar de referencia: Martínez Crovetto, $R$. 9491 (CTES).

Podocoma hirsuta (Hook. \& Arn.) Baker

Hierba. Perenne. Nativa. 0-500 m.

Distribución: CAI, CAN.

Ejemplar de referencia: Montes, J. E. 4114 (LP).

\section{Porophyllum Guett.}

Porophyllum angustissimum Gardner

Hierba o subarbusto. Perenne. Nativa. 100$500 \mathrm{~m}$.

Distribución: OBE, SIG.

Ejemplar de referencia: Krapovickas, A. 28745 (CTES, SI).

\section{Porophyllum lanceolatum DC.}

Hierba. Anual. Nativa. 0-500 m.

Distribución: CAP, GMB.

Ejemplar de referencia: Múlgura, M. E. 3810 (MNES, SI).

Porophyllum linifolium (Ard.) DC.

Hierba o subarbusto. Perenne. Nativa. 0-500 m. Distribución: SIG.

Ejemplar de referencia: Keller, H. A. 13056 (CTES).

Porophyllum obscurum (Spreng.) DC.

Hierba o subarbusto. Perenne. Nativa. 0-700 m. Distribución: APO, CAN, CON, SIG.

Ejemplar de referencia: Morrone, O. 1695 (SI).

Porophyllum ruderale (Jacq.) Cass.

Hierba. Perenne. Nativa. 0-700 m.

Distribución: CAI, CAN, ELD, GSM, GUA, IGU, LNA, SIG, SJA.

Ejemplar de referencia: Montes, J. E. 14937 (NY, SI).

\section{Praxelis Cass.}

Praxelis clematidea R.M. King \& H. Rob. Hierba. Perenne. Nativa. 0-500 m. 

SJA.

Distribución: CAN, ELD, GSM, IGU, OBE,

Ejemplar de referencia: Romanczuk, M. C. 722 (SI).

Praxelis kleinioides (Kunth) Sch. Bip. $\left({ }^{* *}\right)$

Hierba. Anual. Nativa. 0-500 m.

Distribución: GUA, SPE.

Ejemplar de referencia: Zuloaga, F. O. 722 (SI).

Praxelis missiona (Malme) R.M. King \& $\mathrm{H}$. Rob.

Hierba. Perenne. Nativa. 0-700 m.

Distribución: APO, CAI, CAN, CAP, GUA, LNA, OBE, SIG, SJA.

Ejemplar de referencia: Morrone, O. 1669 (SI).

\section{Pseudognaphalium Kirp.}

\section{Pseudognaphalium gaudichaudianum (DC.)} Anderb.

Hierba. Anual. Nativa. 0-700 m.

Distribución: CAN, CAP, CON, GMB, GUA.

Ejemplar de referencia: Morrone, O. 1703 (SI).

\section{Pseudogynoxys (Greenm.) Cabrera}

\section{Pseudogynoxys benthamii Cabrera}

Arbusto. Nativa. 0-600 m.

Distribución: CAN, ELD, GMB, IGU, SIG.

Ejemplar de referencia: Múlgura, M. E. 3962 (MNES, SI).

\section{Pterocaulon Elliott}

Pterocaulon alopecuroides (Lam.) DC.

Hierba. Perenne. Nativa. 0-700 m.

Distribución: APO, CAI, CAN, CAP, CON, GSM, GUA, IGU, LNA, MON, SIG.

Ejemplar de referencia: Zuloaga, F. O. 875 (SI).

\section{Pterocaulon angustifolium $\mathrm{DC}$.}

Hierba. Perenne. Nativa. 0-500 m.

Distribución: CAN, CAP. (SI).

\section{Pterocaulon balansae Chodat}

Hierba. Perenne. Nativa. 0-700 m.

Distribución: APO, CAN, CON, GUA, SIG.

Ejemplar de referencia: Zuloaga, F. O. 863 (SI).

\section{Pterocaulon lorentzii Malme}

Hierba. Anual. Nativa. 0-500 m.

Distribución: CAN, GMB, SIG.

Ejemplar de referencia: Zuloaga, F. O. 6731 (SI).

Pterocaulon polypterum (DC.) Cabrera

Hierba. Perenne. Nativa. 0-500 m.

Distribución: APO.

Ejemplar de referencia: Schulz, A. G. 6904 (LP).

\section{Pterocaulon polystachyum DC.}

Hierba. Perenne. Nativa. 0-500 m.

Distribución: APO, CAI, CAN, CAP, LNA, MON, SIG.

Ejemplar de referencia: Biganzoli, F. 868 (SI).

Pterocaulon rugosum (Vahl) Malme

Hierba. Perenne. Nativa. 0-500 m.

Distribución: CAN, SIG.

Ejemplar de referencia: Morrone, O. 2197 (SI).

Raulinoreitzia R.M. King \& H. Rob.

Raulinoreitzia crenulata (Spreng.) R.M. King \& H. Rob.

Arbusto. Nativa. 0-500 m.

Distribución: APO, CAN, CAP, IGU, SIG.

Ejemplar de referencia: Montes, J. E. 14874 (SI).

\section{Senecio L.}

Senecio bonariensis Hook. \& Arn.

Hierba. Perenne. Nativa. 0-500 m.

Distribución: CON.

Ejemplar de referencia: Keller, H. A. 6112 (CTES).

Senecio brasiliensis (Spreng.) Less. var. brasiliensis

Hierba. Perenne. Nativa. 0-800 m. 
Distribución: APO, CAI, GSM, GUA, IGU, LNA, MON, SIG.

Ejemplar de referencia: Biganzoli, F. 564 (SI).

Senecio brasiliensis (Spreng.) Less. var. tripartitus (DC.) Baker

Hierba. Perenne. Nativa. 0-700 m.

Distribución: APO, CAI, CAN, ELD, GSM, GUA, LNA, SIG.

Ejemplar de referencia: Biganzoli, F. 275 (SI).

\section{Senecio cisplatinus Cabrera}

Subarbusto. Nativa. 0-500 m.

Distribución: CAN.

Ejemplar de referencia: Renvoize, S. A. 2926 (SI, K).

\section{Senecio heterotrichus $D C$.}

Hierba. Perenne. Nativa. 0-500 m.

Distribución: APO, CAN.

Ejemplar de referencia: Cabrera, A. L. 29290 $(S I)$.

Senecio icoglossus DC. var. splendens Cabrera Hierba. Perenne. Nativa. 0-500 m.

Distribución: APO, CAI, GMB, OBE.

Ejemplar de referencia: Cabrera, A. L. 28732 (SI).

\section{Senecio paraguariensis Mattf.}

Hierba. Perenne. Nativa. 0-500 m.

Distribución: APO, CAN.

Ejemplar de referencia: Grüner, G. 537 (LP).

\section{Senecio pinnatus Poir.}

Subarbusto. Nativa. 0-500 m.

Distribución: SIG.

Ejemplar de referencia: Keller, H. A. 9581 (CTES).

\section{Senecio pterophorus DC.}

Hierba. Perenne. Nativa. 0-600 m.

Distribución: APO, CAN, CAP, GUA, SIG.

Ejemplar de referencia: Múlgura, M. E. 2088 (SI).

\footnotetext{
Senecio trichocaulon Baker $\left({ }^{* * *}\right)$

Hierba. Anual. Nativa. 0-500 m.

Distribución: CAN.

Ejemplar de referencia: Spegazzini, C. 1001 (BAB, LP).
}

\section{Senecio trichocodon Baker $\left({ }^{* * *}\right)$}

Hierba. Anual. Nativa. 0-500 m.

Distribución: CAN.

Ejemplar de referencia: Grüner, G. 1169 (LP).

\section{Smallanthus Mack.}

Smallanthus connatus (Spreng.) H. Rob.

Hierba. Nativa. 200-500 m.

Distribución: APO, CAN, CON, GMB, GUA, IGU, LNA, SIG, SJA, SPE.

Ejemplar de referencia: Zuloaga, F. O. 708 (SI).

Smallanthus macroscyphus (Baker) A. Grau ex A.A. Sáenz

Hierba. Perenne. Nativa. 0-500 m.

Distribución: SPE.

Ejemplar de referencia: Seijo, G. J. 223 (CTES, G, MNES).

\section{Solidago L.}

\section{Solidago chilensis Meyen}

Hierba. Perenne. Nativa. 0-600 m.

Distribución: CAI, CAN, CON, MON.

Ejemplar de referencia: Biganzoli, F. 874 (SI),

\section{Solidago microglossa DC.}

Hierba. Perenne. Nativa. 0-500 m.

Distribución: APO, CAI, CAN, CAP, CON, GSM, GUA, LNA, SIG, SPE.

Ejemplar de referencia: Cuadrado, G. A. 290 (CTES, SI).

\section{Soliva Ruiz \& Pav.}

Soliva anthemifolia (Juss.) Sweet

Hierba. Anual. Nativa. 0-500 m.

Distribución: CAN, ELD, IGU.

Ejemplar de referencia: Montes, J. E. 3931 (LP).

Soliva sessilis Ruiz \& Pav.

Hierba. Anual. Nativa. 0-500 m.

Distribución: CAN, CAP, ELD, GUA, IGU, SIG.

Ejemplar de referencia: Tressens, S. G. 5917 (CTES). 


\section{Sonchus L.}

\section{Sonchus asper (L.) Hill}

Hierba. Anual o bianual. Introducida. 0-700 m.

Distribución: CAI, CAN, ELD, GSM, GUA, IGU, SPE.

Ejemplar de referencia: Grüner, G. 1192 (LP).

\section{Sonchus oleraceus L.}

Hierba. Anual. Introducida. 0-500 m.

Distribución: CAN, CAP, IGU, SIG.

Ejemplar de referencia: Vanni, R. O. 3857 (CTES).

\section{Sphagneticola O. Hoffm.}

\section{Sphagneticola brachycarpa (Baker) Pruski}

Hierba. Perenne. Nativa. 0-300 m.

Distribución: APO, SIG.

Ejemplar de referencia: Crisci, J. V. 285 (LP).

\section{Stenachaenium Benth.}

\section{Stenachaenium campestre Baker}

Hierba. Perenne. Nativa. 0-500 m.

Distribución: APO, CAN, CON, LNA, SIG.

Ejemplar de referencia: Guaglianone, E. R. 3009 (SI).

\section{Stenachaenium riedelii Baker}

Hierba. Perenne. Nativa. 0-700 m.

Distribución: APO, CAN, CAP, CON, ELD, SIG.

Ejemplar de referencia: Cabrera, A. L. 28694 (SI).

\section{Stenocephalum Sch. Bip.}

Stenocephalum hexanthum Sch. Bip.

Subarbusto. Nativa. 0-700 m.

Distribución: APO, CAN, CAP, CON, GMB, SIG.

Ejemplar de referencia: Rodríguez, F. M. s.n. (SI)

Stenocephalum megapotamicum (Spreng.) Sch. Bip.

Subarbusto. Nativa. 0-500 m.

Distribución: CAN, CAP, SIG.

Ejemplar de referencia: Burkart, A. 14505 (SI).
Stevia Cav.

Stevia aristata D. Don ex Hook. \& Arn.

Hierba. Perenne. Nativa. 0-500 m.

Distribución: CAN.

Ejemplar de referencia: Montes, J. E. 768 (LP).

Stevia cinerascens Sch. Bip. ex Baker $\left.{ }^{* * *}\right)$

Hierba. Perenne. Nativa. 0-500 m.

Distribución: CAN.

Ejemplar de referencia: Montes, J. E. 797 (LP).

Stevia entreriensis Hieron.

Hierba. Perenne. Nativa. 0-500 m.

Distribución: GUA, OBE.

Ejemplar de referencia: Keller, H. A. 4734 (CTES).

\section{Stevia lundiana DC.}

Hierba. Perenne. Nativa. 0-500 m.

Distribución: APO, CAN.

Ejemplar de referencia: Llamas, A. s.n. (BAB).

Stevia multiaristata Spreng.

Subarbusto. Nativa. 0-500 m.

Distribución: SIG.

Ejemplar de referencia: Biganzoli, F. 58 (SI).

Stevia selloi (Spreng.) Sch. Bip. ex Baker

Subarbusto. Nativa. 0-500 m.

Distribución: CAN, SIG, SPE.

Ejemplar de referencia: Montes, J. E. 860 $(L P)$.

Stevia villaricensis (B.L. Rob.) Cabrera \& Vittet $(* * *)$

Subarbusto. Nativa. 0-500 m.

Distribución: CAN.

Ejemplar de referencia: Jörgensen, P. s.n. (LP).

Stomatanthes R.M. King \& H. Rob.

Stomatanthes oblongifolius (Spreng.) R.M. King \& H. Rob.

Subarbusto. Nativa. 100-300 m.

Distribución: APO, CAN.

Ejemplar de referencia: Cabrera, A. L. 28714 (SI). 


\section{Symphyotrichum Nees}

Symphyotrichum graminifolium (Spreng.)

G.L. Nesom

Hierba. Perenne. Nativa. 0-500 m.

Distribución: SIG, SPE.

Ejemplar de referencia: Keller, H. A. 13021 (CTES).

\section{Symphyotrichum regnellii (Baker) G.L. Nesom \\ Hierba. Perenne. Nativa. 0-500 m. \\ Distribución: CAN, SIG. \\ Ejemplar de referencia: Fontana, J. L. 196-16 (CTESN).}

Symphyotrichum squamatum (Spreng.) G.L. Nesom

Hierba. Perenne. Nativa. 0-500 m.

Distribución: CAN, CAP, SIG.

Ejemplar de referencia: Montes, J. E. 2273 (LP).

\section{Synedrellopsis Hieron. \& Kuntze}

Synedrellopsis grisebachii Hieron. \& Kuntze ex Kuntze

Hierba. Perenne. Nativa. 0-500 m.

Distribución: ELD.

Ejemplar de referencia: Keller, H. A. 14021 (CTES).

\section{Tagetes L.}

\section{Tagetes minuta $\mathrm{L}$.}

Hierba. Anual. Nativa. 0-600 m.

Distribución: CAN, GUA, OBE, SIG, SJA.

Ejemplar de referencia: Daviña, J. 183 (CTES, $G, M N E S$ ).

Taraxacum F.H. Wigg., nom. cons.

Taraxacum officinale F.H. Wigg.

Hierba. Perenne. Introducida. 0-500 m.

Distribución: ELD, GSM, GUA, IGU.

Ejemplar de referencia: Tressens, S. G. 5875 (CTES).
Tessaria Ruiz \& Pav.

Tessaria integrifolia Ruiz \& Pav.

Arbusto o árbol. Nativa. 0-500 m.

Distribución: CAN.

Ejemplar de referencia: Sotelo, J. B. 10011 (LP).

Tilesia G. Mey.

Tilesia baccata (L.) Pruski $\left({ }^{* * *}\right)$

Arbusto. Nativa. 0-600 m.

Distribución: GMB, IGU, SJA, SPE.

Ejemplar de referencia: Morrone, O. 2075 (SI).

Tithonia Desf. ex Juss.

Tithonia rotundifolia (Mill.) S.F. Blake

Hierba. Anual. Introducida. 0-500 m.

Distribución: ELD.

Ejemplar de referencia: Cabrera, A. L. 23594 (SI).

\section{Trichocline Cass.}

Trichocline macrocephala Less. $\left.{ }^{* * *}\right)$

Hierba. Perenne. Nativa. 0-600 m.

Distribución: CAI, CAN, GMB, SJA.

Ejemplar de referencia: Maruñak, V. 116 (LP).

Trichocline speciosa Less.

Hierba. Perenne. Nativa. 0-500 m.

Distribución: CAI, CAN.

Ejemplar de referencia: Krapovickas, A. 28773 (SI).

\section{Tridax L.}

Tridax procumbens $\mathrm{L}$.

Hierba. Anual. Nativa. 0-500 m.

Distribución: ELD.

Ejemplar de referencia: Keller, H. A. 7455 (CTES).

Trixis P. Browne

Trixis divaricata (Kunth) Spreng.

Arbusto. Nativa. 0-700 m. 
Distribución: CAI, CAN, CAP, GSM, MON, SPE. SI).

Ejemplar de referencia: Montes, J. E. 15431 (LP,

\section{Trixis nobilis (Vell.) Katinas}

Subarbusto. Nativa. 0-700 m.

Distribución: APO, CAI, CAN, CAP, CON, SIG, SJA.

Ejemplar de referencia: Bridarolli, A. J. 2673 (LP).

\section{Trixis pallida Less.}

Subarbusto. Nativa. 0-500 m.

Distribución: APO, CAN.

Ejemplar de referencia: Fontana, J. L. 98-47 (CTESN, SI).

\section{Trixis praestans (Vell.) Cabrera}

Arbusto. Nativa. 0-500 m.

Distribución: ELD, IGU, SPE.

Ejemplar de referencia: Correa, M. N. 7944 (LP).

Urolepis (DC.) R.M. King \& H. Rob.

Urolepis hecatantha (DC.) R.M. King \& H. Rob. Hierba. Anual. Nativa. 0-700 m.

Distribución: APO, CAI, CAN, CAP, ELD, GMB, GUA, IGU, LNA, OBE, SIG, SPE.

Ejemplar de referencia: Morrone, O. 751 (SI).

Verbesina L., nom. cons.

Verbesina subcordata $D C$.

Subarbusto. Nativa. 0-500 m.

Distribución: APO, CAN, CAP, IGU, SIG, SPE.

Ejemplar de referencia: Cabrera, A. L. 29052 (SI).

\section{Vernonanthura H. Rob.}

Vernonanthura chamaedrys (Less.) H. Rob.

Subarbusto. Nativa. 0-500 m.

Distribución: APO, CAN, CAP, CON, SIG.

Ejemplar de referencia: Cabrera, A. L. 28302 (SI).
Vernonanthura cupularis (Chodat) $H$. Rob.

Subarbusto. Nativa. 0-500 m.

Distribución: CAN, SIG.

Ejemplar de referencia: Montes, J. E. 636 (SI).

Vernonanthura loretensis (Hieron.) H. Rob.

Subarbusto. Nativa. 100-300 m.

Distribución: CAN, SIG.

Ejemplar de referencia: Zuloaga, F. O. 5342 (SI).

Vernonanthura lucida (Less.) H. Rob.

Subarbusto. Nativa. 100-500 m.

Distribución: CAN, CAP, SPE.

Ejemplar de referencia: Montes, J. E. 15109 (ICN).

Vernonanthura montevidensis (Spreng.) $\mathrm{H}$. Rob.

Arbusto. Nativa. 0-500 m.

Distribución: CAN, CAP, IGU, SIG.

Ejemplar de referencia: Zardini, E. M. 655 (SI).

Vernonanthura oligactoides (Less.) H. Rob. $\left({ }^{* * *}\right)$

Arbusto. Nativa. 0-500 m.

Distribución: GMB.

Ejemplar de referencia: Dematteis, M. 2611 (CTES).

Vernonanthura petiolaris (DC.) H. Rob. $\left(^{* * *}\right)$

Árbol. Nativa. 0-500 m.

Distribución: CAI, GMB, GUA, IGU, SPE.

Ejemplar de referencia: Correa, M. N. 5474

(BAA, BAB, SI).

Vernonanthura tweedieana (Baker) H. Rob.

Arbusto. Nativa. 0-700 m.

Distribución: APO, CAI, CAN, CAP, GMB, GUA, IGU, LNA, SIG, SPE.

Ejemplar de referencia: Zuloaga, F. O. 4905 (SI).

Vernonia Schreb., nom. cons.

Vernonia echioides Less.

Hierba. Perenne. Nativa. 0-600 m.

Distribución: APO, CAN, CAP, CON, LNA, SIG.

Ejemplar de referencia: Cabrera, A. L. 29095 (SI). 
Vernonia incana Less.

Hierba. Perenne. Nativa. 0-500 m.

Distribución: CAN.

Ejemplar de referencia: Sotelo, J. B. 10009 (LP).

Wedelia Jacq., nom. cons.

\section{Wedelia brachylepis Griseb.}

Hierba. Perenne. Nativa. 0-500 m.

Distribución: LNA.

Ejemplar de referencia: Fontana, J. L. 211 (SI).

\section{Wedelia kerrii N.E. Br.}

Hierba. Anual. Nativa. 0-500 m.

Distribución: CAI, CAN, CAP, CON, ELD, GMB, GSM, GUA, IGU, MON, SIG, SPE.

Ejemplar de referencia: Zuloaga, F. O. 6841 (SI).

\section{Wedelia subvelutina $\mathrm{DC} .\left({ }^{* * *}\right)$}

Hierba. Perenne. Nativa. 0-500 m.

Distribución: APO, CAN, CAP, GMB, IGU, SJA. Ejemplar de referencia: Cabrera, A. L. 29076 (SI).

\section{Xanthium L.}

\section{Xanthium strumarium L.}

Hierba. Anual. Nativa. 0-500 m.

Distribución: ELD, GUA, IGU, SIG, SPE.

Ejemplar de referencia: Rodríguez, M. 427 (CTES, G, MNES).

\section{Youngia Cass.}

Youngia japonica (L.) DC.

Hierba. Anual. Introducida. 0-500 m.

Distribución: CAN, ELD, MAY, SIG.

Ejemplar de referencia: Keller, H. A. 2364 (CTES)

Zinnia L., nom. cons.

\section{Zinnia peruviana (L.) L.}

Hierba. Anual. Introducida. 0-500 m.

Distribución: SPE.

Ejemplar de referencia: Keller, H. A. 2846 (CTES).

\section{BALANOPHORACEAE}

\section{Lophophytum Schott \& Endl.}

Lophophytum leandri Eichler

Hierba parásita. Perenne. Nativa. 0-500 m.

Distribución: CAN, CAP, CON, OBE, SIG.

Ejemplar de referencia: Kelsey, G. s.n. (SI).

\section{BALSAMINACEAE}

\section{Impatiens L.}

\section{Impatiens walleriana Hook. f. $\left({ }^{* * *}\right)$}

Hierba. Perenne. Introducida. 0-500 m.

Distribución: CAI, GMB, OBE, SPE.

Ejemplar de referencia: Deginani, N. B. 1101 (MU, SI).

\section{BASELLACEAE}

Anredera Juss.

Anredera cordifolia (Ten.) Steenis

Enredadera. Perenne. Nativa. 0-700 m.

Distribución: CAI, CAP, CON, GMB, GUA, IGU, SJA, SPE.

Ejemplar de referencia: Deginani, N. B. 1066 (SI).

\section{BEGONIACEAE}

\section{Begonia L.}

Begonia cucullata Willd.

Hierba. Perenne. Nativa. 0-700 m.

Distribución: APO, CAI, CAN, CON, GMB, GUA, IGU, SIG, SPE.

Ejemplar de referencia: Deginani, N. B. 975 (SI).

Begonia descoleana L.B. Sm. \& B.G. Schub. Hierba. Perenne. Nativa. 0-500 m.

Distribución: IGU.

Ejemplar de referencia: Meyer, T. 11857 (LIL). 
Begonia fischeri Schrank $\left({ }^{* *}\right)$

Hierba. Perenne. Nativa. 0-800 m.

Distribución: GUA, SIG, SPE.

Ejemplar de referencia: Deginani, N. B. 1322 (SI).

\section{Begonia fruticosa A. DC. $\left({ }^{* * *}\right)$}

Hierba. Perenne. Nativa. 0-500 m.

Distribución: GUA, MON, SPE.

Ejemplar de referencia: Morrone, O. 2131 (SI).

\section{Begonia hassleri C. DC. $\left.{ }^{* *}\right)$}

Hierba. Perenne. Endémica. 200-500 m.

Distribución: SIG.

Ejemplar de referencia: Hassler, E. 460 (G).

\section{Begonia per-dusenii Brade $\left(^{* *}\right)$}

Hierba. Perenne. Nativa. 200-500 m.

Distribución: GUA.

Ejemplar de referencia: Keller, H. A. 328 (CTES).

\section{Begonia subvillosa Klotzsch $\left({ }^{* *}\right)$}

Hierba. Perenne. Nativa. 0-800 m.

Distribución: CAI, CAN, ELD, GMB, GUA, IGU, MON, SIG, SPE.

Ejemplar de referencia: Hunziker, J. H. 10822 (SI).

\section{BERBERIDACEAE}

\section{Berberis L.}

Berberis laurina Thunb. $\left({ }^{* *}\right)$

Arbusto. Nativa. 0-500 m.

Distribución: LNA, OBE, SPE.

Ejemplar de referencia: Keller, H. A. 4256 (CTES).

\section{BIGNONIACEAE}

\section{Adenocalymma Mart. ex Meisn.}

Adenocalymma marginatum (Cham.) DC.

Liana. Nativa. 0-700 m.

Distribución: APO, CAN, CAP, ELD, GMB, GSM, GUA, IGU, LNA, SIG, SJA, SPE.

Ejemplar de referencia: Zuloaga, F. O. 6829 (SI).
Adenocalymma paulistarum Bureau ex $\mathrm{K}$. Schum. $\left.{ }^{* * *}\right)$

Liana. Nativa. 0-700 m.

Distribución: CAI, GMB, GSM, IGU, MON, SIG.

Ejemplar de referencia: Biganzoli, F. 200 (SI).

\section{Amphilophium Kunth}

Amphilophium carolinae (Lindl.) L. G. Lohmann

Liana. Nativa. 0-500 m.

Distribución: CAN, ELD, MON.

Ejemplar de referencia: Pierotti, A. 4169 (K, LIL).

Amphilophium crucigerum (L.) L.G. Lohmann Liana. Nativa. 0-700 m.

Distribución: APO, CAI, CAN, CON, GMB, GUA, IGU, MON, SIG, SPE. (SI).

Ejemplar de referencia: Guaglianone, E. R. 2830

Amphilophium paniculatum (L.) Kunth

Liana. Nativa. 100-500 m.

Distribución: CAN, CAP, GUA, IGU, OBE.

Ejemplar de referencia: Zuloaga, F. O. 8370 (SI).

\section{Amphilophium vauthieri $D C$.}

Liana. Nativa. 0-500 m.

Distribución: IGU.

Ejemplar de referencia: Vanni, R. O. 2960 (CTES).

\section{Bignonia L.}

Bignonia binata Thunb.

Liana. Nativa. 0-400 m.

Distribución: CAN, CAP, GMB, GSM, GUA, IGU, SIG.

Ejemplar de referencia: Morrone, O. 1057 (SI).

Bignonia callistegioides $\mathrm{Cham}$.

Liana. Nativa. 0-500 m.

Distribución: APO, GMB, GSM, OBE.

Ejemplar de referencia: Múlgura, M. E. 3916 (MNES, SI). 
Bignonia sciuripabulum (Hovel.) L.G. Lohmann $\left({ }^{* * *}\right)$

Liana. Nativa. 0-800 m.

Distribución: CAI, CAN, CAP, GMB, GSM, GUA, IGU, LNA, OBE, SIG, SJA, SPE.

Ejemplar de referencia: Morrone, O. 899 (SI).

Cuspidaria DC., nom. cons.

Cuspidaria convoluta (Vell.) A.H. Gentry

Liana. Nativa. 0-800 m.

Distribución: APO, CAI, CAN, CAP, CON, GSM, GUA, IGU, LNA, OBE, SIG, SPE.

Ejemplar de referencia: Zuloaga, F. O. 8749 (SI).

\section{Dolichandra Cham.}

\section{Dolichandra cynanchoides Cham.}

Liana. Nativa. 500-800 m.

Distribución: CAI, CAN.

Ejemplar de referencia: Biganzoli, F. 337 (SI).

Dolichandra dentata (K. Schum.) L.G.

Lohmann

Enredadera o liana. Nativa. 0-400 m.

Distribución: APO, CAN, CAP, CON, ELD, GMB, GUA, SJA, SPE.

Ejemplar de referencia: Morrone, O. 2828 (SI).

Dolichandra hispida (DC.) L.H. Fonseca \& L.G. Lohmann

Liana. Nativa. 0-700 m.

Distribución: CAN, CAP, ELD, GUA, IGU, SIG.

Ejemplar de referencia: Deginani, N. B. 1630 (SI,MO).

Dolichandra quadrivalvis (Jacq.) L.G. Lohmann Liana. Nativa. 0-800 m.

Distribución: CAI, ELD, GUA, IGU, SIG, SPE. (SI).

Ejemplar de referencia: Zuloaga, F. O. 6490

Dolichandra uncata (Andrews) L.G. Lohmann Enredadera o liana. Nativa. 0-700 m.
Distribución: CAN, ELD, GUA, IGU, SIG, SJA. Ejemplar de referencia: Guaglianone, E. R. 2870 (SI).

Dolichandra unguis-cati (L.) L.G. Lohmann

Enredadera o liana. Nativa. 0-800 m.

Distribución: APO, CAN, CAP, CON, GMB, GSM, GUA, IGU, MAY, SIG, SJA, SPE.

Ejemplar de referencia: Morrone, O. 1664 (SI).

\section{Fridericia Mart.}

Fridericia caudigera (S. Moore) L.G. Lohmann Liana. Nativa. 0-800 m.

Distribución: CAI, CAN, CAP, GMB, GUA, IGU, SIG.

Ejemplar de referencia: Zuloaga, F. O. 6887 (SI).

Fridericia chica (Bonpl.) L.G. Lohmann

Liana. Nativa. 0-800 m.

Distribución: APO, CAI, CAN, CON, GMB, GUA, IGU, MON, OBE, SIG, SJA, SPE. (SI).

Ejemplar de referencia: Zuloaga, F. O. 5062

Fridericia florida (DC.) L.G. Lohmann $\left(^{* * *}\right)$

Liana. Nativa. 100-500 m.

Distribución: IGU.

Ejemplar de referencia: Zuloaga, F. O. 5222 (SI).

Fridericia mutabile (Bureau \& K. Schum.) Frazão \& L.G. Lohmann

Liana. Nativa. 0-500 m.

Distribución: GSM, GUA, IGU, SIG.

Ejemplar de referencia: Biganzoli, F. 445 (SI).

Fridericia samydoides (Cham.) L.G. Lohmann

Liana. Nativa. 0-500 m.

Distribución: IGU.

Ejemplar de referencia: Vanni, R. O. 3538 (CTES, MO).

Fridericia triplinervia (Mart. ex DC.) L.G. Lohmann $\left.{ }^{* * *}\right)$

Liana. Nativa. 100-800 m.

Distribución: GMB, IGU.

Ejemplar de referencia: Vanni, R. O. 3544 (CTES, GH, LIL, MO). 


\section{Handroanthus Mattos}

Handroanthus albus (Cham.) Mattos ( $\left.{ }^{* * *}\right)$

Árbol. Nativa. 200-600 m.

Distribución: CAI, GMB, GUA, IGU, OBE.

Ejemplar de referencia: Krapovickas, A. 18612 (CTES).

Handroanthus heptaphyllus (Vell.) Mattos

Árbol. Nativa. 100-800 m.

Distribución: CAI, CAN, CAP, CON, GSM, GUA, IGU, SIG, SJA.

Ejemplar de referencia: Biganzoli, F. 608 (SI).

Handroanthus pulcherrimus (Sandwith) S. Grose

Árbol. Nativa. 0-300 m.

Distribución: APO, CAI, CAN, CAP, GSM, GUA, IGU, SIG, SJA.

Ejemplar de referencia: Cabrera, A. L. 28924 (SI).

\section{Jacaranda Juss.}

Jacaranda micrantha Cham.

Árbol. Nativa. 100-800 m.

Distribución: APO, CAI, ELD, GMB, GSM, GUA, IGU, SPE.

Ejemplar de referencia: Deginani, N. B. 1220 (SI),

\section{Jacaranda puberula Cham.}

Arbolito o árbol. Nativa. 0-500 m.

Distribución: GMB, IGU, SPE.

Ejemplar de referencia: Deginani, N. B. 1740 (SI).

\section{Mansoa DC.}

Mansoa difficilis (Cham.) Bureau \& K. Schum.

Liana. Nativa. 0-500 m.

Distribución: CAI, CAN, CAP, ELD, GMB, GUA, IGU, LNA, MON, SIG, SPE.

Ejemplar de referencia: Zuloaga, F. O. 6832 (SI).

\section{Pyrostegia C. Presl}

Pyrostegia venusta (Ker Gawl.) Miers

Liana. Nativa. 0-800 m.
Distribución: CAN, CAP, ELD, GMB, GSM, GUA, IGU, LNA, SIG, SPE.

Ejemplar de referencia: Morrone, O. 1886 (SI).

\section{Tanaecium Sw.}

Tanaecium dichotomum (Jacq.) Kaehler \& L.G. Lohmann

Liana. Nativa. 0-500 m.

Distribución: CAN, IGU.

Ejemplar de referencia: Zuloaga, F. O. 5284 (SI).

Tanaecium selloi (Spreng.) L.G. Lohmann

Liana. Nativa. 0-800 m.

Distribución: CAN, GMB, GUA, IGU, OBE, SIG, SJA, SPE.

Ejemplar de referencia: Morrone, O. 2157 (SI).

Tecoma Juss.

Tecoma stans (L.) Juss. ex Kunth

Arbusto o arbolito. Nativa. 0-700 m.

Distribución: APO, ELD, LNA.

Ejemplar de referencia: Múlgura, M. E. 2920 (SI).

\section{Tynanthus Miers}

Tynanthus micranthus Corr. Méllo ex $\mathrm{K}$. Schum. $\left.{ }^{(* *}\right)$

Liana. Nativa. $100-800 \mathrm{~m}$.

Distribución: GMB, IGU. (SI).

Ejemplar de referencia: Guaglianone, E. R. 2137

\section{BORAGINACEAE}

\section{Borago L.}

\section{Borago officinalis $\mathrm{L}$.}

Hierba. Anual. Introducida. 0-500 m.

Distribución: CAN, SIG.

Ejemplar de referencia: Martínez Crovetto, $R$. 10043 (BAB). 


\section{Cordia L.}

Cordia americana (L.) Gottschling \& J.S. Mill. Árbol. Nativa. 0-800 m.

Distribución: CAN, CAP, CON, ELD, GMB, GUA, IGU, SIG, SJA.

Ejemplar de referencia: Zuloaga, F. O. 7193 (SI).

Cordia ecalyculata Vell.

Árbol. Nativa. 0-500 m.

Distribución: CAN, CAP, ELD, GMB, GUA, IGU, SIG.

Ejemplar de referencia: Morrone, O. 739 (SI).

Cordia trichotoma (Vell.) Arráb. ex Steud.

Árbol. Nativa. 0-800 m.

Distribución: CAN, CAP, ELD, GMB, GUA, IGU, LNA, SIG, SPE.

Ejemplar de referencia: Zuloaga, F. O. 5441 (SI).

\section{Echium L.}

\section{Echium plantagineum L.}

Hierba. Introducida. 0-500 m.

Distribución: CON, GUA, LNA.

Ejemplar de referencia: Maruñak, V. 155 (BAB).

\section{Euploca Nutt.}

Euploca filiformis (Lehm.) J.I. M. Melo \& Semir Hierba. Anual. Nativa. 0-500 m.

Distribución: CAN, LNA. (SI).

Ejemplar de referencia: Zuloaga, F. O. 2208

Euploca ocellata (Cham.) J.I. M. Melo \& Semir

Hierba. Perenne. Nativa. 0-500 m.

Distribución: APO, CAI, CAN, CON.

Ejemplar de referencia: Burkart, A. 15430 (SI).

\section{Euploca procumbens (Mill.) Diane \& Hilger}

Hierba. Perenne. Nativa. 0-700 m.

Distribución: CAN, CAP, GUA, IGU, LNA, SIG, SPE.

Ejemplar de referencia: Deginani, N. B. 1426 (SI).
Euploca salicoides (Cham.) J.I. M. Melo \& Semir Hierba. Perenne. Nativa. 0-500 m.

Distribución: CAN, SIG.

Ejemplar de referencia: Múlgura, M. E. 2266 (SI).

\section{Heliotropium L.}

\section{Heliotropium amplexicaule Vahl}

Hierba o subarbusto. Perenne. Nativa. 100$800 \mathrm{~m}$.

Distribución: APO, CAP, SJA.

Ejemplar de referencia: Correa, M. N. 6998 (BAB).

Heliotropium curassavicum L. var. argentinum I.M. Johnst.

Hierba. Perenne. Nativa. 0-500 m.

Distribución: IGU.

Ejemplar de referencia: Spegazzini, C. L. s.n. (BAB).

Heliotropium elongatum Hoffm. ex Roem. \& Schult.

Hierba o subarbusto. Perenne. Nativa. 0-500 m.

Distribución: CAP, IGU, OBE, SIG.

Ejemplar de referencia: Martínez Crovetto, $R$. 5096 (BAB).

\section{Heliotropium indicum $\mathrm{L}$.}

Hierba. Anual. Nativa. 0-500 m.

Distribución: IGU, SIG, SPE.

Ejemplar de referencia: Morrone, O. 1895 (SI).

\section{Heliotropium leiocarpum Morong}

Subarbusto. Nativa. 0-500 m.

Distribución: CAI, CAN, CAP, GMB, IGU, SIG.

Ejemplar de referencia: Morrone, O. 1983 (SI).

Heliotropium nicotianaefolium Poir.

Subarbusto. Nativa. 0-500 m.

Distribución: CAN, IGU.

Ejemplar de referencia: Cocucci, A. A. 3220 (CORD, CTES, SI).

\section{Heliotropium phylicoides Cham.}

Subarbusto. Nativa. 0-200 m.

Distribución: CAN, CAP.

Ejemplar de referencia: Martínez Crovetto, $R$. 9502 (BAB). 
Heliotropium tiaridioides Cham.

Hierba o arbusto. Perenne. Nativa. 0-800 m. Distribución: APO, CAI, CAN, CON, ELD, GMB, GSM, GUA, IGU, LNA, MAY, OBE, SIG, SJA, SPE.

Ejemplar de referencia: Zuloaga, F. O. 5518 (SI).

\section{Heliotropium transalpinum Vell.}

Arbusto. Nativa. 0-800 m.

Distribución: CAI, CAN, CAP, CON, ELD, GMB, GSM, GUA, IGU, MON, OBE, SIG, SJA, SPE.

Ejemplar de referencia: Morrone, O. 1483 (SI).

\section{Myriopus Small}

Myriopus breviflorus (DC.) Luebert

Arbusto. Nativa. 0-500 m.

Distribución: CAI, GUA.

Ejemplar de referencia: Montes, J. E. 4181 (LP).

\section{Myriopus paniculatus (Cham.) Feuillet}

Arbusto. Nativa. 0-500 m.

Distribución: CAI, ELD, GMB, GUA, IGU, SIG, SPE.

Ejemplar de referencia: Guaglianone, E. R. 2898 (SI).

Myriopus rubicundus (Salzm. ex DC.) Luebert Arbusto. Nativa. 0-800 m.

Distribución: APO, CAI, SIG.

Ejemplar de referencia: Biganzoli, F. 1525 (SI).

Myriopus villosus (Salzm. ex DC.) J.I. M. Melo

Arbusto. Nativa. 0-500 m.

Distribución: IGU, SPE.

Ejemplar de referencia: Cabrera, A. L. 28898 (SI).

\section{Thaumatocaryon Baill.}

Thaumatocaryon dasyanthum (Cham.) I.M. Johnst. $\left.{ }^{(* *}\right)$

Hierba. Perenne. Nativa. 0-500 m.

Distribución: GMB.

Ejemplar de referencia: Keller, H. A. 4213 (CTES).

Thaumatocaryon tetraquetrum (Cham.) I.M. Johnst. $\left.{ }^{(* *}\right)$

Hierba. Perenne. Nativa. 0-800 m.

Distribución: GMB, IGU, MON, OBE.

Ejemplar de referencia: Guaglianone, E. R. 2945 (SI).
Varronia P. Browne

Varronia axillaris (I.M. Johnst.) Borhidi (***)

Arbusto. Nativa. 200-300 m.

Distribución: IGU.

Ejemplar de referencia: Morrone, O. 1214 (SI).

Varronia curassavica Jacq.

Arbusto. Nativa. 0-500 m.

Distribución: CAN, CON, LNA.

Ejemplar de referencia: Zuloaga, F. O. 6558 (SI).

Varronia dichotoma Ruiz \& Pav.

Arbusto o árbol. Nativa. 0-700 m.

Distribución: CAN, CON, ELD, GMB, GUA, IGU, MON, SJA.

Ejemplar de referencia: Barboza, G. E. 418 (CORD, CTES, SI).

Varronia paucidentata (Fresen.) Friesen

Arbusto. Nativa. 0-500 m.

Distribución: APO, CAN, CAP, CON.

Ejemplar de referencia: Biganzoli, F. 1608 (SI).

\section{BRASSICACEAE}

\section{Brassica L.}

\section{Brassica bonariensis Pers.}

Hierba. Perenne. Nativa. 0-500 m.

Distribución: GUA.

Ejemplar de referencia: Keller, H. A. 1299 (CTES).

\section{Brassica juncea $L$.}

Hierba. Anual o Bianual. Introducida. 0-500 m.

Distribución: CAP, ELD.

Ejemplar de referencia: Keller, H. A. 11579 (CTES).

\section{Brassica rapa $\mathrm{L}$.}

Hierba. Anual o Bianual. Introducida. 0-500 m.

Distribución: IGU.

Ejemplar de referencia: Hunziker, A. T. 15509 (CORD). 


\section{Capsella Medik}

\section{Capsella bursa-pastoris (L.) Medik}

Hierba anual o bianual. Introducida. 0-500 m. Distribución: GMB, GUA.

Ejemplar de referencia: Keller, H. A. 3778 (CTES).

\section{Cardamine L.}

\section{Cardamine africana $\mathrm{L}$.}

Hierba. Perenne. Introducida. 0-500 m.

Distribución: GMB.

Ejemplar de referencia: Morrone, O. 1378 (MO, SI).

\section{Cardamine bonariensis Pers.}

Hierba. Perenne. Nativa. 0-500 m.

Ejemplar de referencia: Keller, H. A. 1299 (CTES).

\section{Cardamine chenopodiifolia Pers.}

Hierba. Anual. Nativa. 0-800 m.

Distribución: IGU, SPE.

Ejemplar de referencia: Hunziker, A. T. 15481 (CORD).

Descurainia Webb \& Berthel., nom. cons.

Descurainia erodiifolia (Phil.) Prantl ex Reiche

Hierba. Bianual. Nativa. 0-500 m.

Distribución: SPE.

Ejemplar de referencia: Zardini, E. M. 921 (SI).

\section{Lepidium L.}

Lepidium auriculatum Regel \& Körn.

Hierba. Anual. Nativa. 0-700 m.

Distribución: APO, CAI, CAN, ELD, GMB, IGU, SIG, SPE.

Ejemplar de referencia: Múlgura, M. E. 492 (SI).

\section{Lepidium bonariense $\mathrm{L}$.}

Hierba. Anual. Nativa. 0-700 m.

Distribución: APO, ELD, IGU, MON.

Ejemplar de referencia: Correa, M. N. 5441 (SI).

\section{Lepidium didymum $\mathrm{L}$.}

Hierba. Anual o bianual. Nativa. 0-500 m.

Distribución: ELD, GUA, IGU.

Ejemplar de referencia: Krapovickas, A. 2503 (SI).

Lepidium pedersenii Al-Shehbaz

Hierba. Anual. Nativa. 0-300 m.

Distribución: CAI, CAN, CAP, GMB, GSM, IGU, SJA.

Ejemplar de referencia: Cabrera, A. L. 29475 (SI).

\section{Raphanus L.}

\section{Raphanus raphanistrum $\mathrm{L}$.}

Hierba. Anual o bianual. Introducida. 0-600 m. Distribución: LNA.

Ejemplar de referencia: Ohashi 38 (CTES).

\section{Raphanus sativus L.}

Hierba. Anual o bianual. Introducida. 0-600 m. Distribución: LNA.

Ejemplar de referencia: Krapovickas, A. 29742 (SI).

Rapistrum Crantz, nom. cons.

Rapistrum rugosum (L.) All.

Hierba. Anual. Introducida. 0-500 m.

Distribución: GMB.

Ejemplar de referencia: Keller, H. A. 5081 (CTES).

\section{Rorippa Scop.}

Rorippa bonariensis (Poir.) Macloskie

Hierba. Anual o Bianual. Nativa. 0-500 m.

Distribución: GMB.

Ejemplar de referencia: Zardini, E. M. 921 (SI).

Rorippa burkartii (Mart.-Laborde) Al-Shehbaz Hierba. Anual o Bianual. Nativa. 0-500 m.

Distribución: APO.

Ejemplar de referencia: Cabrera, A. L. 29287 (SI).

\section{Rorippa dubia (Pers.) H. Hara}

Hierba. Anual. Introducida. 0-500 m.

Distribución: CAP.

Ejemplar de referencia: Burkart, A. 14464 (SI). 
Rorippa palustris (L.) Besser

Hierba. Anual. Introducida. 100-600 m.

Distribución: GSM.

Ejemplar de referencia: Cabrera, A. L. 28807 (SI).

\section{Sinapis L.}

\section{Sinapis arvensis $\mathrm{L}$.}

Hierba. Anual. Introducida. 0-500 m.

Distribución: SPE.

Ejemplar de referencia: Cabrera, A. L. 28824 (SI).

\section{BROMELIACEAE}

\section{Acanthostachys Klotzsch}

Acanthostachys strobilacea (Schult. f.) Klotzsch $(* * *)$

Hierba epífita. Perenne. Nativa. 700-800 m.

Distribución: CAI, IGU.

Ejemplar de referencia: Belgrano, M. J. 326 (SI).

Aechmea Ruiz \& Pav., nom. cons.

Aechmea bromeliifolia (Rudge) Baker

Hierba epífita o terrestre. Perenne. Nativa. 0-600 m.

Distribución: CAN, IGU, SPE.

Ejemplar de referencia: Hauman, L. L. s.n. (BA).

Aechmea calyculata (E. Morren) Baker ${ }^{* * *}$ )

Hierba epífita o terrestre. Perenne. Nativa. 100-700 m.

Distribución: GUA, SPE.

Ejemplar de referencia: Deginani, N. B. 1018 (SI).

\section{Aechmea distichantha Lem. f. distichantha}

Hierba epífita o terrestre. Perenne. Nativa. 0-700 m.

Distribución: CAN, GMB, GSM, IGU, SIG, SPE.

Ejemplar de referencia: Gamerro, J. C. 80 (SI).

\section{Aechmeadistichantha Lem.var. schlumbergeri}

E. Morren ex Mez

Hierba epífita o terrestre. Perenne. Nativa. 0-800 m.
Distribución: CAP, GMB, GUA, IGU, SIG, SPE.

Ejemplar de referencia: Morrone, O. 1216 (SI).

Aechmea recurvata (Klotzsch) L.B. Sm.

Hierba epífita. Perenne. Nativa. 0-800 m.

Distribución: CAI, CAN, GMB, GSM, GUA, IGU, SIG, SPE.

Ejemplar de referencia: Honfi, A. I. 464 (MNES, SI).

\section{Ananas Mill.}

Ananas bracteatus (Lindl.) Schult. $\mathrm{f}$.

Hierba. Perenne. Nativa. 0-700 m.

Distribución: SIG.

Ejemplar de referencia: Múlgura, M. E. 1635 (SI).

Billbergia Thunb.

Billbergia nutans $H$. Wendl. ex Regel

Hierba epífita. Perenne. Nativa. 0-800 m.

Distribución: CAI, CAN, ELD, GMB, GUA, IGU, OBE, SIG, SJA, SPE.

Ejemplar de referencia: Biganzoli, F. 179 (SI).

Billbergia zebrina (Herb.) Lindl.

Hierba epífita. Perenne. Nativa. 0-300 m.

Distribución: CAP, GMB, GSM, GUA, IGU.

Ejemplar de referencia: Biganzoli, F. 281 (SI).

Bromelia Plum. ex L.

\section{Bromelia balansae $\mathrm{Mez}$}

Hierba. Perenne. Nativa. 0-800 m.

Distribución: CAI, CAN, CAP, GUA, IGU, SIG.

Ejemplar de referencia: Deginani, N. B. 991 (SI).

Dyckia Schult. f.

\section{Dyckia choristaminea $\mathrm{Mez}\left({ }^{*}\right)$}

Hierba. Perenne. Nativa. 0-300 m.

Distribución: CAI.

Ejemplar de referencia: Keller, H. A. 13416 (CTES). 
Dyckia distachya Hassl. ( $\left.{ }^{* *}\right)$

Hierba. Perenne. Nativa. 0-500 m.

Distribución: IGU, SIG.

Ejemplar de referencia: Tressens, S. G. 4573 (CTES, SI).

\section{Dyckia ferox Mez}

Hierba. Perenne. Nativa. 0-500 m.

Distribución: CAN.

Ejemplar de referencia: Rodríguez, F. M. 701 (SI).

Dyckia microcalyx Baker var. ostenii L.B. Sm. $\left({ }^{* * *}\right)$

Hierba. Perenne. Nativa. 0-300 m.

Distribución: IGU, SIG.

Ejemplar de referencia: Zanotti, C. A. 482 (SI).

\section{Dyckia mitis A. Cast. (**)}

Hierba terrestre o rupícola. Perenne. Endémica. 0-300 m.

Distribución: CAI.

Ejemplar de referencia: Spegazzini, C. L. s.n. (BA).

\section{Dyckia niederleinii Mez $\left({ }^{* *}\right)$}

Hierba. Perenne. Endémica. 0-300 m.

Distribución: CAN, IGU, SIG, SPE.

Ejemplar de referencia: Burkart, A. 1529 (BAA).

Dyckia remotiflora Otto \& A. Dietr. var. montevidensis (K. Koch) L.B. Sm.

Hierba. Perenne. Nativa. 0-100 m.

Distribución: APO, CAN.

Ejemplar de referencia: Zuloaga, F. O. 6568 (SI).

\section{Dyckia subinermis $\mathrm{Mez}\left({ }^{* *}\right)$}

Hierba. Perenne. Endémica. 0-300 m.

Distribución: SPE.

Ejemplar de referencia: Niederlein, G. 1205 (B).

\section{Pseudananas Hassl. ex Harms}

Pseudananas sagenarius (Arruda) Camargo

Hierba. Perenne. Nativa. 0-500 m.

Distribución: CAN, IGU, SIG.

Ejemplar de referencia: Burkart, A. 15290 (SI).

\section{Tillandsia L.}

Tillandsia geminiflora Brongn. $\left.{ }^{(* *}\right)$

Hierba epífita. Perenne. Nativa. 0-500 m.

Distribución: GMB, IGU, OBE, SPE.

Ejemplar de referencia: Morrone, O. 850 (SI).

Tillandsia lorentziana Griseb.

Hierba epífita. Perenne. Nativa. 0-500 m.

Distribución: SIG.

Ejemplar de referencia: Zuloaga, F. O. 6472 (SI).

Tillandsia meridionalis Baker

Hierba epífita. Perenne. Nativa. 0-500 m.

Distribución: CAN.

Ejemplar de referencia: Descole, H. R. 3022 (LIL).

\section{Tillandsia pohliana $\mathrm{Mez}$}

Hierba epífita. Perenne. Nativa. 0-500 m.

Distribución: CAN, SIG.

Ejemplar dereferencia: Schinini, A. 5501 (CTES).

Tillandsia recurvata (L.) L.

Hierba epífita. Perenne. Nativa. 0-500 m.

Distribución: GMB, IGU, SIG.

Ejemplar de referencia: Denham, S. S. 240 (SI).

\section{Tillandsia streptocarpa Baker}

Hierba epífita. Perenne. Nativa. 0-500 m.

Distribución: CAN, ELD, SIG.

Ejemplar de referencia: Keller, H. A. 2550 (CTES).

Tillandsia stricta Sol. ex Sims

Hierba epífita. Perenne. Nativa. 0-700 m.

Distribución: APO, GMB, GUA, IGU, OBE, SPE. Ejemplar de referencia: Morrone, O. 1364 (SI).

\section{Tillandsia tenuifolia $\mathrm{L}$.}

Hierba epífita. Perenne. Nativa. 0-700 m.

Distribución: APO, GMB, GSM, GUA, IGU, SPE.

Ejemplar de referencia: Morrone, O. 1390 (SI).

\section{Tillandsia tricholepis Baker}

Hierba epífita. Perenne. Nativa. 0-500 m.

Distribución: GMB.

Ejemplar de referencia: Múlgura, M. E. 1917 (SI). 
Vriesea Lindl., nom. cons.

Vriesea friburgensis Mez var. tucumanensis (Mez) L.B. Sm.

Hierba epífita o terrestre. Perenne. Nativa. 0-800 m.

Distribución: GMB, GUA, IGU, SIG, SJA, SPE.

Ejemplar de referencia: Deginani, N. B. 1200 (SI).

\section{Vriesea platynema Gaudich.}

Hierba epífita. Perenne. Nativa. 300-500 m.

Distribución: GMB, GUA, SPE.

Ejemplar de referencia: Morrone, O. 1550 (SI).

\section{BURMANNIACEAE}

\section{Apteria Nutt.}

Apteria aphylla (Nutt.) Barnhart ex Small

Saprófita. Perenne. Nativa. 0-500 m.

Distribución: IGU.

Ejemplar de referencia: Keller, H. A. 13636 (CTES).

\section{Burmannia L.}

\section{Burmannia flava Mart.}

Hierba. Anual. Nativa.

Distribución: en el centro-este de la provincia.

Ejemplar de referencia: Sagastizabal, F. s.n. (BAF).

\section{Gymnosiphon Blume}

Gymnosiphon divaricatus (Benth.) Benth. \& Hook. (**)

Saprófita. Perenne. Nativa. 0-500 m.

Distribución: GMB.

Ejemplar de referencia: Keller, H. A. 9751 (CTES).

\section{CACTACEAE}

\section{Brasiliopuntia A. Berger}

Brasiliopuntia schulzii (A. Cast. \& Lelong) Backeb. Arbolito. Nativa. 0-500 m.
Distribución: APO, SIG.

Ejemplar de referencia: Morrone, O. 1884 (SI).

Cereus Mill.

Cereus stenogosus $\mathrm{K}$. Schum

Árbol suculento. Nativa. 0-500 m.

Distribucón: GUA.

Ejemplar de referencia: Tressens, S. G. 5852 (CTES).

Epiphyllum Haw.

Epiphyllum phyllanthus (L.) Haw.

Hierba epífita. Perenne. Nativa. 0-500 m.

Distribución: SIG.

Ejemplar de referencia: Múlgura, M. E. 2203 (SI).

\section{Frailea Britton \& Rose}

Frailea castanea Backeb.

Hierba suculenta. Perenne. Nativa. 0-500 m.

Distribución: CAN.

Frailea schilinzkyana (F. Haage ex K. Schum.) Britton \& Rose

Hierba suculenta. Perenne. Nativa. 0-500 m.

Distribución: CAI, CAN.

Ejemplar de referencia: Biganzoli, F. 185 (SI).

\section{Lepismium Pfeiff.}

Lepismium lineare (K. Schum.) Barthlott

Hierba epífita. Nativa. 0-500 m.

Distribución: GUA, IGU.

Ejemplar de referencia: Vanni, R. O. 3417 (CTES).

\section{Monvillea Britton \& Rose}

Monvillea cavendischii (Monv.) Britton \& Rose Subarbusto suculento. Nativa. 0-500 m.

Distribución: SIG.

Ejemplar de referencia: Zuloaga, F. O. 6603 (SI). 
Monvillea euchlora (F.A.C. Weber ex K. Schum.) Backeb.

Arbusto suculento. Nativa. 0-500 m.

Distribución: SIG.

Ejemplar de referencia: Morrone, O. 1613 (SI).

\section{Opuntia Mill.}

Opuntia elata Salm-Dyck var. cardiosperma (K. Schum.) R. Kiesling

Hierba suculenta. Perenne. Nativa. 0-500 m.

Distribución: SIG.

Ejemplar de referencia: Keller, H. A. 11926 (CTES).

\section{Parodia Speg.}

Parodia linkii (Lehm.) R. Kiesling

Hierba suculenta. Perenne. Nativa. 0-500 m.

Distribución: APO, CAN, CAP, CON, ELD, LNA.

Ejemplar de referencia: Keller, H. A. 6409 (CTES).

Parodia ottonis (Lehm.) N.P. Taylor

Hierba suculenta. Perenne. Nativa. 0-500 m.

Distribución: CAI, SIG.

Ejemplar de referencia: Biganzoli, F. 374 (SI).

Parodia schumanniana (Nicolai) F.H. Brandt $\left({ }^{* * *}\right)$

Hierba suculenta. Perenne. Nativa. 0-500 m.

Distribución: SIG.

Ejemplar de referencia: Biganzoli, F. 606 (SI).

\section{Pereskia Mill.}

Pereskia aculeata Mill.

Arbusto suculento. Nativa. 0-700 m.

Distribución: CAI, ELD, GMB, GSM, GUA, IGU, MON, SIG, SPE.

Ejemplar de referencia: Deginani, N. B. 1070 (SI).

\section{Pereskia nemorosa Rojas Acosta}

Arbusto suculento. Nativa. 0-500 m.

Distribución: CAP.

Ejemplar de referencia: Bertoni 14 (LIL).

\section{Rhipsalis Gaertn.}

Rhipsalis baccifera (J.S. Muell.) Stearn

Hierba epífita. Perenne. Nativa. 0-500 m.

Distribución: GMB, IGU, SIG.

Ejemplar de referencia: Morrone, O. 1883 (SI).

Rhipsalis cereuscula Haw.

Hierba epífita. Perenne. Nativa. 0-700 m.

Distribución: APO, CAI, ELD, GMB, GUA, IGU, SPE.

Ejemplar de referencia: Zuloaga, F. O. 6827 (SI).

Rhipsalis cruciforme (Vell.) A. Cast.

Hierba epífita. Perenne. Nativa. 0-800 m.

Distribución: GMB, GUA, IGU, OBE, SIG, SPE.

Ejemplar de referencia: Deginani, N. B. 1710 (SI).

Rhipsalis floccosa Pfeiff. subsp. hohenauensis

(F. Ritter) Barthlott \& N.P. Taylor $\left({ }^{* * *}\right)$

Hierba epífita. Perenne. Nativa. 0-700 m.

Distribución: APO, CAI, GMB, GUA, SPE.

Ejemplar de referencia: Zuloaga, F. O. 6993 (SI).

Rhipsalis houlletiana Lem. ${ }^{* * *}$ )

Hierba epífita. Perenne. Nativa. 0-500 m.

Distribución: GUA, SPE.

Ejemplar de referencia: Morrone, O. 1541 (SI).

Rhipsalis lumbricoides (Lem.) Lem. ex Salm-Dyck Hierba epífita. Perenne. Nativa. 0-500 m.

Distribución: CAI, GUA, IGU, OBE, SPE.

Ejemplar de referencia: Rodríguez, M. 782 (MNES, SI).

\section{CALCEOLARIACEAE}

\section{Calceolaria L.}

Calceolaria chelidonioides Kunth

Hierba. Perenne. Nativa. 0-500 m.

Distribución: GUA, IGU.

Ejemplar de referencia: Múlgura, M. E. 555 (SI).

Calceolaria tripartita Ruiz \& Pav. ${ }^{* * *}$

Anual. Nativa. 0-500 m.

Distribución: GUA, SPE.

Ejemplar de referencia: Keller, H. A. 3078 (CTES, SI). 


\section{CALOPHYLLACEAE}

\section{Calophyllum L.}

Calophyllum brasiliense Cambess.

Árbol. Nativa. 0-500 m.

Distribución: SIG.

Ejemplar de referencia: Krauczuk, E. R. 32 (CTES).

\section{CALYCERACEAE}

\section{Acicarpha Juss.}

\section{Acicarpha tribuloides Juss.}

Hierba. Anual. Nativa. 0-500 m.

Distribución: CAN, CAP, CON. (SI).

Ejemplar de referencia: Múlgura, M. E. 2053

\section{CAMPANULACEAE}

\section{Lobelia L.}

\section{Lobelia camporum Pohl (***)}

Subarbusto. Nativa. 0-500 m.

Distribución: GMB. SI).

Ejemplar de referencia: Maruñak, V. 95 (CTES,

\section{Lobelia hassleri Zahlbr.}

Subarbusto. Nativa. 200-700 m.

Distribución: APO, CAI, CAN, GMB, GUA, SPE.

Ejemplar de referencia: Gamerro, J. C. 176 (SI).

\section{Lobelia hederacea Cham.}

Hierba. Perenne. Nativa. 0-500 m.

Distribución: GUA, SIG, SPE. (SI).

Ejemplar de referencia: Múlgura, M. E. 1652

Lobelia nummularioides $\mathrm{Cham}$.

Hierba. Anual. Nativa. 0-700 m.
Distribución: GMB, GUA.

Ejemplar de referencia: Guaglianone, E. R. 2964 (SI).

Lobelia reniformis Cham. $\left.{ }^{* * *}\right)$

Hierba. Perenne. Nativa. 0-500 m.

Distribución GMB.

Ejemplar de referencia: Keller, H. A. 4212 (CTES).

\section{Lobelia xalapensis Kunth}

Hierba. Anual. Nativa. 0-700 m.

Distribución: CAI, CAN, ELD, IGU, SIG.

Ejemplar de referencia: Múlgura, M. E. 2193 (SI).

\section{Siphocampylus Pohl}

Siphocampylus verticillatus (Cham.) G. Don $\left({ }^{* * *}\right)$

Subarbusto. Nativa. 0-500 m.

Distribución: GMB.

Ejemplar de referencia: Guaglianone, E. R. 2956 (SI).

Siphocampylus yerbalensis E. Wimm. $\left.{ }^{* *}\right)$

Subarbusto. Endémica. 0-500 m.

Distribución: GUA, SPE.

Ejemplar de referencia: Morrone, O. 2167 (SI).

\section{Triodanis Raf.}

Triodanis perfoliata (L.) Nieuwl. var. biflora (Ruiz \& Pav.) T.R. Bradley

Hierba. Anual. Nativa. 0-800 m.

Distribución: CAI, CAN, CAP, CON, GMB, GSM, GUA, LNA, SIG, SPE.

Ejemplar de referencia: Biganzoli, F. 554 (SI).

Wahlenbergia Schrad. ex Roth, nom. cons.

Wahlenbergia linarioides (Lam.) A. DC.

Hierba. Perenne. Nativa. 0-800 m.

Distribución: APO, CAI, CAN, GSM, GUA, LNA, MAY, MON, SIG, SPE. 

(SI).

Ejemplar de referencia: Deginani, N. B. 1180

\section{CANNABACEAE}

\section{Celtis L.}

Celtis iguanaea (Jacq.) Sarg.

Arbusto o árbol. Nativa. 0-800 m.

Distribución: APO, CAI, CAN, CON, GMB, GUA, IGU, SIG, SJA, SPE.

Ejemplar de referencia: Deginani, N. B. 1038 (SI).

\section{Trema L.}

Trema micrantha (L.) Blume

Arbusto o árbol. Nativa. 0-800 m.

Distribución: CAI, CAN, CAP, ELD, GMB, GSM, GUA, IGU, LNA, MON, OBE, SIG, SPE. (SI).

Ejemplar de referencia: Cabrera, A. L. 28819

\section{CANNACEAE}

\section{Canna L.}

\section{Canna glauca L.}

Hierba. Perenne. Nativa. 0-500 m.

Distribución: IGU, OBE.

Ejemplar de referencia: Guaglianone, R. E. 1587 (SI, NY).

\section{Canna indica $\mathrm{L}$.}

Hierba. Perenne. Nativa. 0-800 m.

Distribución: CAI, GMB, GSM, GUA, IGU, OBE, SPE.

Ejemplar de referencia: Morrone, O. 2158 (SI).

\section{Canna paniculata Ruiz \& Pav.}

Hierba. Perenne. Nativa. 0-800 m.

Distribución: GMB, GSM, IGU, OBE, SJA, SPE.

Ejemplar de referencia: Bertoni, M. S. 2774 (LIL).

\section{CAPPARACEAE}

Capparidastrum (DC.) Hutch.

Capparidastrum humilis (Hassl.) X. Cornejo \& H.H. Iltis

Subarbusto. Nativa. 0-500 m.

Distribución: APO, CAN, GMB, LNA, SIG.

Ejemplar de referencia: Biganzoli, F. 405 (SI).

\section{Cynophalla (DC.) J. Presl}

Cynophalla flexuosa (L.) J. Presl

Árbol. Nativa. 0-500 m.

Distribución: CAN, GUA, SIG.

Ejemplar de referencia: Keller, H. A. 13696 (CTES).

\section{CAPRIFOLIACEAE}

\section{Valeriana L.}

Valeriana kurtziana Borsini $\left({ }^{* *}\right)$

Hierba. Nativa. 0-600 m.

Distribución: APO, CAN, SPE.

Ejemplar de referencia: Zuloaga, F. O. 8229 (SI).

Valeriana scandens Loefl. ex L.

Hierba o enredadera. Perenne. Nativa. 0-600 m.

Distribución: CAN, GMB, GUA, IGU, SPE. SI).

Ejemplar de referencia: Tur, N. M. 2007 (LP,

\section{CARDIOPTERIDACEAE}

\section{Citronella D. Don}

Citronella gongonha (Mart.) R.A. Howard

Arbusto o árbol. Nativa. 0-500 m.

Distribución: CAN, CON. (SI). 
Citronella paniculata (Mart.) R.A. Howard $(* * *)$

Árbol. Nativa. 200-500 m.

Distribución: CAI, CAN, GUA, IGU.

Ejemplar de referencia: Burkart, A. 25552 (SI).

\section{CARICACEAE}

\section{Carica L.}

\section{Carica papaya $\mathrm{L}$.}

Arbolito. Nativa. 0-500 m.

Distribución: SIG.

Ejemplar de referencia: Morrone, O. 1881 (SI).

\section{Jacaratia A. DC.}

Jacaratia spinosa (Aubl.) A. DC.

Árbol. Nativa. 0-600 m.

Distribución: ELD, GMB, GUA, SIG, SPE.

Ejemplar de referencia: Zuloaga, F. O. 6805 (SI).

Vasconcellea A. St.-Hil.

Vasconcellea quercifolia A. St.-Hil.

Árbol. Nativa. 0-700 m.

Distribución: CAI, CAN, CAP, CON, GMB, GUA, SPE.

Ejemplar de referencia: Zuloaga, F. O. 6904 (SI).

\section{CARYOPHYLLACEAE}

\section{Cerastium L.}

Cerastium commersonianum Ser. ex DC.

Hierba. Anual. Nativa. 0-500 m.

Distribución: CAN, SIG.

Ejemplar de referencia: Montes, J. E. s.n. (BA).

Cerastium glomeratum Thuill.

Hierba. Anual. Introducida. 0-500 m.

Distribución: CON.

Ejemplar de referencia: Keller, H. A. 6034 (CTES).
Cerastium rivulare Cambess.

Hierba. Anual. Nativa. 0-600 m.

Distribución: APO, CAN, GUA, IGU.

Ejemplar de referencia: Múlgura, M. E. $2010 b$ (SI).

Cerastium rivulariastrum Möschl \& Pedersen Hierba. Anual. Nativa. 0-700 m.

Distribución: APO, CAN, CON, GSM, GUA, IGU, SIG.

Ejemplar de referencia: Biganzoli, F. 258 (SI).

\section{Paronychia Mill.}

Paronychia brasiliana $D C$.

Hierba. Perenne. Nativa. 0-500 m.

Distribución: GMB, GUA.

Ejemplar de referencia: Zuloaga, F. O. 689 (LP, SI).

\section{Paronychia communis Cambess.}

Hierba. Perenne. Nativa. 0-600 m.

Distribución: APO, GUA, SIG, SPE.

Ejemplar de referencia: Zuloaga, F. O. 5576 (SI).

Paronychia setigera (Gillies ex Hook. \& Arn.) F. Herm. subsp. cordobensis Chaudhri

Hierba. Perenne. Nativa. 0-500 m.

Distribución: SPE.

Ejemplar de referencia: Montes, J.E. 27469 (MO, SI).

Paronychia setigera (Gillies ex Hook. \& Arn.) F. Herm. subsp. setigera

Hierba. Perenne. Nativa. 0-500 m.

Distribución: APO.

Ejemplar de referencia: Múlgura, M. E. 2012 (LP, SI).

Polycarpaea Lam., nom. cons.

Polycarpaea corymbosa (L.) Lam.

Hierba. Nativa. 0-500 m.

Distribución: SIG.

Ejemplar de referencia: Múlgura, M. E. 2139 (SI).

Polycarpon Loefl. ex L.

Polycarpon apurense Kunth

Hierba. Nativa. 0-500 m. 
Distribución: CAP.

Ejemplar de referencia: Burkart, A. 14046 (SI).

Silene L., nom. cons.

\section{Silene antirrhina $\mathrm{L}$.}

Hierba. Anual. Introducida. 0-500 m.

Distribución: CAN, GMB, GUA, MON, SIG.

Ejemplar de referencia: Morrone, O. 1771 (SI).

Stellaria L., nom. cons.

\section{Stellaria media (L.) Cirillo}

Hierba. Anual. Introducida. 0-500 m.

Distribución: SIG.

Ejemplar de referencia: Keller, H. A. 5664 (CTES).

\section{CELASTRACEAE}

\section{Elachyptera A.C. Sm.}

Elachyptera micrantha (Cambess.) A.C. Sm. $(* * *)$

Liana. Perenne. Nativa. 0-800 m.

Distribución: IGU.

Ejemplar de referencia: Hunziker, J. H. 11864 (SI).

\section{Hippocratea L.}

\section{Hippocratea volubilis $L$.}

Liana. Perenne. Nativa. 0-800 m.

Distribución: CAN, CAP, GSM, IGU, MON, SIG.

Ejemplar de referencia: Zuloaga, F. O. 3180 (SI).

\section{Maytenus Molina}

\section{Maytenus boaria Molina}

Árbol. Nativa. 0-500 m.

Distribución: APO.

Ejemplar de referencia: Spegazzini, C. L. s.n.
Monteverdia A. Rich.

Monteverdia aquifolia (Mart.) Biral (***)

Arbusto o árbol. Nativa. 100-500 m.

Distribución: IGU.

Ejemplar de referencia: Romanczuk, M. C. 661 (SI).

Monteverdia evonymoides (Reissek) Biral $(* * *)$

Árbol. Nativa. 0-500 m.

Distribución: CAP.

Ejemplar de referencia: Ekman, E. L. 1421 $(N Y)$.

Monteverdia ilicifolia (Mart. ex Reissek) Biral Arbusto o árbol. Nativa. 0-700 m.

Distribución: APO, CAI, CAN, CAP, CON, ELD, GMB, GUA, IGU, OBE, SIG, SJA.

Ejemplar de referencia: Keller, H. A. 325 (CTES, SI).

\section{Pristimera Miers}

Pristimera celastroides (Kunth) A.C. Sm.

Liana. Perenne. Nativa. 0-600 m.

Distribución: APO, CAI, CAN, GUA, IGU, SJA, SPE.

Ejemplar de referencia: Zuloaga, F. O. 6445 (SI).

\section{Schaefferia Jacq.}

Schaefferia argentinensis Speg.

Arbusto o árbol. Nativa. 300-700 m.

Distribución: CAI, CAN, GMB, GUA, SJA.

Ejemplar de referencia: Jörgensen, P. 170 (BAB).

\section{CHENOPODIACEAE}

\section{Chenopodium L.}

\section{Chenopodium album $\mathrm{L}$.}

Hierba. Anual. Introducida. 0-500 m.

Distribución: CAP, SPE.

Ejemplar de referencia: Keller, H. A. 710 (CTES). 


\section{Dysphania R. Br.}

Dysphania ambrosioides (L.) Mosyakin \& Clemants

Hierba. Perenne. Nativa. 0-500 m.

Distribución: CAI, ELD, GUA, SIG.

Ejemplar de referencia: Zuloaga, F. O. 2045 (SI).

Dysphania carinata (R. Br.) Mosyakin \& Clemants Hierba. Anual. Introducida. 0-500 m.

Distribución: GUA, SIG.

Ejemplar de referencia: Ariza Espinar, L. 1108 (CORD).

Dysphania pumilio (R. Br.) Mosyakin \& Clemants Hierba. Anual. Introducida. 0-500 m.

Distribución: ELD, SPE.

Ejemplar de referencia: Burkart, A. 28299 (SI).

\section{CISTACEAE}

\section{Crocanthemum Spach}

Crocanthemum brasiliense (Lam.) Spach

Hierba o subarbusto. Perenne. Nativa. 0-500 m. Distribución: SIG.

Ejemplar de referencia: Múlgura, M. E. 2181 (SI).

\section{CLEOMACEAE}

\section{Melidiscus Raf.}

Melidiscus giganteus (L.) Raf.

Arbusto. Nativa. 0-700 m.

Distribución: CAI, ELD, GMB, GUA, SPE.

Ejemplar de referencia: Morrone, O. 2150 (SI).

\section{Tarenaya Raf.}

Tarenaya hassleriana (Chodat) H.H. Iltis

Hierba. Nativa. 0-800 m.

Distribución: CAI, CAN, CAP, ELD, GSM, IGU, SIG, SPE.

Ejemplar de referencia: Morrone, O. 1161 (SI).
Tarenaya parviflora (Kunth) H.H. Iltis

Hierba. Nativa. 0-700 m.

Distribución: CAI, GUA, IGU, SIG, SJA, SPE.

Ejemplar de referencia: Zuloaga, F. O. 2146 (SI).

Tarenaya rosea (Vahl ex DC.) Soares Neto \& Roalson

Hierba. Nativa. 0-700 m.

Distribución: CAI, CAN, CON, GMB, SIG, SJA.

Ejemplar de referencia: Zuloaga, F. O. 6436 (SI).

Tarenaya trachycarpa (Klotzsch ex Eichler) Soares Neto \& Roalson

Hierba. Nativa. 0-500 m.

Distribución: GMB, SIG.

Ejemplar de referencia: Schwarz, G. J. 6222 (LIL).

\section{CLUSIACEAE}

\section{Garcinia L.}

Garcinia guacopary (S. Moore) M. Nee

Árbol. Nativa. 0-500 m.

Distribución: IGU, SIG.

Ejemplar de referencia: Guaglianone, E. R. 2155 (SI).

\section{COMBRETACEAE}

\section{Combretum Loefl., nom. cons.}

Combretum fruticosum (Loefl.) Stuntz

Arbusto o liana. Nativa. 0-800 m.

Distribución: APO, CAI, CAP, CON, ELD, GMB, GUA, IGU, LNA, MON, OBE, SIG, SJA, SPE.

Ejemplar de referencia: Morrone, O. 2169 (SI).

\section{Combretum laxum Jacq.}

Arbusto o liana. Nativa. 0-700 m.

Distribución: CAI, CAN, CAP, SIG. (SI).

Ejemplar de referencia: Guaglianone, E. R. 3026

Combretum mellifluum Eichler $\left.{ }^{* * *}\right)$

Arbusto. Nativa. 0-500 m. 
Distribución: IGU.

Ejemplar de referencia: Muniez, A. A. s.n. (BAF).

Terminalia L., nom. cons.

Terminalia australis Cambess.

Árbol. Nativa. 0-500 m.

Distribución: CAI, CAN, CON, GMB, GUA, IGU, MON, OBE, SIG, SPE.

Ejemplar de referencia: Correa, M. N. 5382 (BAA, BAB, SI).

Terminalia triflora (Griseb.) Lillo

Arbusto o árbol. Nativa. 300-700 m.

Distribución: APO, CAN, CAP, IGU, SIG.

Ejemplar de referencia: Xifreda, C. C. 349 (SI).

\section{COMMELINACEAE}

\section{Callisia Loefl.}

Callisia monandra (Sw.) Schult. \& Schult. f.

Hierba. Perenne. Nativa. 0-800 m.

Distribución: IGU, SPE.

Ejemplar de referencia: Múlgura, M. E. 507 (SI).

\section{Callisia repens (Jacq.) L.}

Hierba. Perenne. Nativa. 0-500 m.

Distribución: CAN, IGU, SIG.

Ejemplar de referencia: Biganzoli, F. 388 (SI).

\section{Commelina L.}

\section{Commelina benghalensis $\mathrm{L}$. $\left.{ }^{* * *}\right)$}

Hierba perenne. Introducida. 0-500 m.

Distribución: GUA.

Ejemplar de referencia: Grabiele, M. 94 (MNES,SI).

\section{Commelina diffusa Burm. $\mathrm{f}$.}

Hierba. Perenne. Nativa. 0-800 m.

Distribución: CAI, GUA, SPE. (SI).

\section{Commelina erecta L.}

Hierba. Perenne. Nativa. 0-700 m.

Distribución: ELD, CON, GMB, SIG.

Ejemplar de referencia: Morrone, O. 1693 (SI).

Commelina longicaulis Jacq.

Hierba. Perenne. Nativa. 0-800 m.

Distribución: CAP, ELD, SIG, SPE.

Ejemplar de referencia: Keller, H. A. 3487 (CTES).

\section{Commelina obliqua Vahl}

Hierba. Perenne. Nativa. 0-500 m.

Distribución: GUA, IGU, LNA, SIG, SPE.

Ejemplar de referencia: Zuloaga, F. O. 5569 (SI).

Commelina platyphylla Klotzsch ex Seub.

Hierba. Perenne. Nativa. 0-500 m.

Distribución: APO, CAN, CAP, GMB, GSM.

Ejemplar de referencia: Montes, J. E. 7252 (SI).

Dichorisandra J.C. Mikan, nom. cons.

Dichorisandra hexandra (Aubl.) Standl.

Hierba. Perenne. Nativa. 0-800 m.

Distribución: CAI, GMB, GSM, GUA, IGU, SIG, SPE.

Ejemplar de referencia: Zuloaga, F. O. 5040 (SI).

Floscopa Lour.

Floscopa glabrata (Kunth) Hassk.

Hierba. Perenne. Nativa. 0-600 m.

Distribución: GUA, IGU, SIG.

Ejemplar de referencia: Krapovickas, A. 15728 (CTES, SI).

\section{Tradescantia L.}

Tradescantia cerinthoides Kunth

Hierba. Perenne. Nativa. 0-700 m.

Distribución: ELD, GUA.

Ejemplar de referencia: Gabriele, M. 1 (SI). 
Tradescantia crassula Link \& Otto $\left.{ }^{* * *}\right)$

Hierba. Perenne. Nativa. 0-800 m.

Distribución: CAI, GMB, SPE.

Ejemplar de referencia: Morrone, O. 1356 (SI).

Tradescantia cymbispatha C.B. Clarke

Hierba perenne. Nativa. 0-800 m.

Distribución: APO, CAP, ELD, GMB, GSM, GUA, IGU, SIG, SJA, SPE.

Ejemplar de referencia: Múlgura, M.E. 391 (BAB, SI).

\section{Tradescantia fluminensis Vell.}

Hierba. Perenne. Nativa. 0-800 m.

Distribución: APO, CAI, CAP, GMB, GSM, GUA, IGU, LNA, SIG, SPE.

Ejemplar de referencia: Morrone, O. 747 (SI).

\section{Tradescantia mundula Kunth}

Hierba perenne. Nativa. 0-500 m.

Distribución: APO, CON, GUA, IGU.

Ejemplar de referencia: Tressens, S. G. 6037 (CTES).

\section{Tradescantia pallida (Rose) D.R. Hunt ${ }^{* * *}$ )}

Hierba. Perenne. Nativa. 0-500 m.

Distribución: CAP, IGU, SPE.

Ejemplar de referencia: Zuloaga, F. O. 5573 (SI).

\section{Tradescantia tenella Kunth}

Hierba. Perenne. Nativa. 0-500 m.

Distribución: APO, CAI, CAN, CAP, GUA, IGU, OBE, SIG, SPE.

Ejemplar de referencia: Hojsgaard, D. 307 (SI, CTES).

\section{Tradescantia tucumanensis Pellegr.}

Hierba. Perenne. Nativa. 0-500 m.

Distribución: GMB.

Ejemplar de referencia: Múlgura, M. E. 417 (SI).

Tradescantia umbraculifera Hand.-Mazz.

Hierba perenne. Nativa. 0-700 m.

Distribución: CAI, CAN, ELD, GUA, LNA, MON, SIG.

Ejemplar de referencia: Schulz, A. G. 6979 (SI, CTES).

\section{Tradescantia zebrina Bosse}

Hierba. Perenne. Introducida. 400-700 m.

Distribución: CAP, ELD, IGU.

Ejemplar de referencia: Cabral, E. L. 56 (CTES).

\section{Tripogandra Raf.}

Tripogandra diuretica (Mart.) Handlos

Hierba. Perenne. Nativa. 0-800 m.

Distribución: CAI, CAN, CAP, ELD, GMB, GSM, GUA, IGU, SIG, SPE.

Ejemplar de referencia: Zuloaga, F. O. 6634 (SI).

Tripogandra glandulosa (Seub.) Rohweder

Hierba. Perenne. Nativa. 0-700 m.

Distribución: CAI, CAN, ELD.

Ejemplar de referencia: Zuloaga, F. O. 6576 (SI).

Tripogandra purpurascens (Schauer) Handlos subsp. australis Handlos

Hierba. Anual. Nativa. 200-500 m.

Distribución: APO.

Ejemplar de referencia: Múlgura, M. E. 2881 (SI).

\section{CONVOLVULACEAE}

\section{Aniseia Choisy}

Aniseia argentina (N.E. Br.) O’Donell

Enredadera. Perenne. Nativa. 0-500 m.

Distribución: CAN, CAP.

Ejemplar de referencia: Burkart, A. 14481 (SI).

Aniseia luxurians (Moric.) Athiê-Souza \& Buril Enredadera. Perenne. Nativa. 0-500 m.

Distribución: CAN, CAP, GMB, SIG.

Ejemplar de referencia: Barboza, G. E. 1045 (CORD).

Aniseia martinicensis (Jacq.) Choisy $\left({ }^{* * *}\right)$

Enredadera. Perenne. Nativa. 0-500 m

Distribución: CAI, SIG.

Ejemplar de referencia: Galeano, C. s.n. (SI).

\section{Camonea Raf.}

Camonea umbellata (L.) Simões \& Staples

Enredadera. Perenne. Nativa. 0-500 m.

Distribución: IGU, SIG.

Ejemplar de referencia: Morrone, O. 2045 (SI). 


\section{Convolvulus L.}

\section{Convolvulus bonariensis $\mathrm{Cav}$.}

Enredadera. Perenne. Nativa. 0-700 m.

Distribución: CAI, CAN, GMB, GUA, OBE.

Ejemplar de referencia: Biganzoli, F. 1462 (SI).

Convolvulus crenatifolius Ruiz \& Pav. subsp. crenatifolius

Enredadera. Nativa. 0-700 m.

Distribución: APO, CAI, CAN, GUA, SIG, SPE.

Ejemplar de referencia: Tell Bertoni, G. 362 (SI).

Convolvulus crenatifolius Ruiz \& Pav. subsp. montevidensis (Spreng.) J.R.I. Wood \& R.W. Scotland

Enredadera. Nativa. 0-500 m.

Distribución: CAN.

Ejemplar de referencia: Gallardo, C. s.n. (SI).

Convolvulus hasslerianus (Chodat) O'Donell

Enredadera. Nativa. 0-800 m.

Distribución: APO.

Ejemplar de referencia: Pierotti, S. A. 5511 (LIL).

\section{Convolvulus hermanniae L'Hér.}

Enredadera. Perenne. Nativa. 0-500 m.

Distribución: CAN, CAP.

Ejemplar de referencia: Jörgensen, P. 635 (BAB).

\section{Convolvulus lilloi O’Donell}

Enredadera. Nativa. 0-500 m.

Distribución: CAP.

Ejemplar de referencia: Holmberg, E. L. s.n. (SI).

\section{Convolvulus schulzei O’Donell}

Enredadera. Perenne. Nativa. 0-500 m.

Distribución: CON.

Ejemplar de referencia: Keller, H. A. 5727 (CTES).

\section{Cuscuta L.}

Cuscuta boliviana Yunck. var. paranensis Hunz. $\left.{ }^{* * *}\right)$

Enredadera parásita. Anual. Nativa. 0-500 m.

Distribución: CAP.

Ejemplar de referencia: Burkart, A. 1626 (BAA).
Cuscuta indecora Choisy var. longisepala Yunck.

Enredadera parásita. Anual. Nativa. 0-500 m.

Distribución: APO, CAP.

Ejemplar de referencia: Ibarrola, T. 1020 (LIL).

\section{Cuscuta obtusiflora Kunth}

Enredadera parásita. Anual. Nativa. 0-500 m.

Distribución: CAN, CAP.

Ejemplar de referencia: Niederlein, G. 1304 (BA).

\section{Cuscuta platyloba Progel}

Enredadera parásita. Anual. Nativa. 0-500 m.

Distribución: APO, CAI, CAN, GUA, OBE, SIG, SPE.

Ejemplar de referencia: Guaglianone, E. R. 3025 (SI).

Cuscuta xanthochortos Mart. ex Engelm. var. lanceolata Yunck.

Enredadera parásita. Anual. Nativa. 0-500 m.

Distribución: SIG.

Ejemplar de referencia: Burkart, A. 15311 (SI).

Cuscuta xanthochortos Mart. ex Engelm. var. xanthochortos

Enredadera parásita. Anual. Nativa. 0-500 m.

Distribución: CAN, CAP, SIG.

Ejemplar de referencia: Naboulet, L. R. 7 (BA).

Dichondra J.R. Forst. \& G. Forst.

Dichondra macrocalyx Meisn.

Hierba. Perenne. Nativa. 0-500 m.

Distribución: CAN, GUA, SPE.

Ejemplar de referencia: Tressens, S. G. 6442 (CTES, SI).

Dichondra microcalyx (Hallier f.) Fabris

Hierba. Perenne. Nativa. 0-500 m.

Distribución: CAN, GUA.

Ejemplar de referencia: Tressens, S. G. 6358 (CTES).

\section{Dichondra sericea Sw.}

Hierba. Perenne. Nativa. 0-500 m.

Distribución: CAI, CAN, CAP, GMB, GSM, SIG. Ejemplar de referencia: Biganzoli, F. 569 (SI). 


\section{Distimake Raf.}

Distimake aegyptius (L.) Simões \& Staples

Enredadera. Anual. Nativa. 0-500 m.

Distribución: ELD, SIG, SPE.

Ejemplar de referencia: Morrone, O. 988 (SI).

Distimake cissoides (Lam.) Simões \& Staples

Enredadera. Anual. Nativa. 0-600 m

Distribución: GSM, IGU, SIG.

Ejemplar de referencia: Morrone, O. 1681 (SI).

Distimake dissectus (Jacq.) Simões \& Staples

Enredadera. Perenne. Nativa. 0-500 m.

Distribución: CAI, CAN, CAP, GSM, GUA, IGU, SIG, SPE.

Ejemplar de referencia: Múlgura, M. E. 1751 (SI).

Distimake hasslerianus (Chodat) Simões \& Staples $\left({ }^{* * *}\right)$

Enredadera. Perenne. Nativa. 0-500 m.

Distribución: IGU, SIG.

Ejemplar de referencia: Morrone, O. 1691 (SI).

Distimake macrocalyx (Ruiz \& Pav.) Simões \& Staples $\left({ }^{* * *}\right)$

Enredadera. Perenne. Nativa. 0-600 m.

Distribución: CAN, GMB, IGU, SIG.

Ejemplar de referencia: Morrone, O. 2068 (SI).

\section{Evolvulus L.}

\section{Evolvulus sericeus $\mathrm{S} w$.}

Hierba. Perenne. Nativa. 0-700 m.

Distribución: APO, CAI, CAP, GMB, SIG.

Ejemplar de referencia: Biganzoli, F. 530 (SI).

Evolvulus tenuis Mart. ex Choisy var. cinereus Chodat \& Hassl.

Subarbusto. Nativa. 0-500 m.

Distribución: APO.

Ejemplar de referencia: Molfino, J. F. s.n. (BA, BAB).

Ipomoea L. nom. cons.

Ipomoea acutisepala O'Donell (***)

Enredadera. Perenne. Nativa. 0-500 m.
Distribución: CAN, GSM, SIG.

Ejemplar de referencia: Schwarz, G. J. 1812 (LIL).

Ipomoea aequiloba J.R.I. Wood \& R.W. Scotland $\left({ }^{* * *}\right)$

Enredadera. Perenne. Nativa. 100-200 m.

Distribución: CAN.

Ejemplar de referencia: Keller, H. A. 13355 (CTES).

Ipomoea alba L.

Enredadera. Perenne. Nativa. 0-500 m.

Distribución: CAP, ELD, GMB, GSM, GUA, IGU, MON.

Ejemplar de referencia: Biganzoli, F. 97 (SI).

Ipomoea aristolochiifolia G. Don

Enredadera. Perenne. Nativa. 0-500 m.

Distribución: MON.

Ejemplar de referencia: Keller, H. A. 922 (CTES).

Ipomoea bonariensis Hook.

Enredadera. Perenne. Nativa. 0-500 m.

Distribución: APO, CAI, CAN, CAP, ELD, GMB, GSM, IGU, LNA, MON, SIG.

Ejemplar de referencia: Morrone, O. 1053 (SI).

Ipomoea cairica (L.) Sweet

Enredadera. Perenne. Nativa. 0-500 m.

Distribución: APO, CAI, CAN, CAP, ELD, GSM, SIG.

Ejemplar de referencia: Schwarz, G. J. 4436 (LIL).

Ipomoea carnea Jacq. subsp. fistulosa (Mart. ex Choisy) D.F. Austin

Arbusto. Nativa. 0-500 m.

Distribución: SPE.

Ejemplar de referencia: Zuloaga, F. O. 9927 (SI).

\section{Ipomoea descolei O’Donell}

Hierba. Perenne. Nativa. 0-500 m.

Distribución: APO, CAI, CAN, CAP, CON, SIG, SPE.

Ejemplar de referencia: Zuloaga, F. O. 6780 (SI).

\section{Ipomoea fimbriosepala Choisy}

Enredadera. Anual. Nativa. 0-500 m.

Distribución: CAP, MON, SIG.

Ejemplar de referencia: O’Donell, C. A. 5602 (LIL). 
Ipomoea grandifolia (Dammer) O’Donell

Enredadera. Anual. Nativa. 0-500 m.

Distribución: APO, CAI, CAN, GMB, GSM, GUA, IGU, SIG, SPE.

Ejemplar de referencia: Biganzoli, F. 84 (SI).

Ipomoea indica (Burm.) Merr.

Enredadera. Perenne. Nativa. 0-800 m.

Distribución: APO, CAI, CAN, CAP, ELD, GMB, GSM, GUA, IGU, MON, OBE, SIG, SJA, SPE.

Ejemplar de referencia: Biganzoli, F. 100 (SI).

Ipomoea indivisa (Vell.) Hallier $\mathrm{f}$.

Enredadera. Anual. Nativa. 0-500 m.

Distribución: CAN, GUA, IGU, SPE.

Ejemplar de referencia: Cabrera, A. L. 29370 (SI).

\section{Ipomoea lanuginosa O'Donell $\left({ }^{* * *}\right)$}

Hierba. Perenne. Nativa. 0-500 m.

Distribución: SIG.

Ejemplar de referencia: O’Donell, C. A. 5611 (LIL).

\section{Ipomoea malpighipila O'Donell}

Subarbusto. Nativa. 0-500 m.

Distribución: CAI, CAN, CAP, ELD, IGU, MON, SIG, SJA.

Ejemplar de referencia: Morrone, O. 1666 (SI).

\section{Ipomoea malvaeoides Meisn.}

Subarbusto. Nativa. 0-500 m.

Distribución: CAN, CAP, SPE.

Ejemplar de referencia: Tell Bertoni, G. 730 (SI).

\section{Ipomoea maurandioides Meisn.}

Hierba. Perenne. Nativa. 0-500 m.

Distribución: APO.

Ejemplar de referencia: Bertoni, 2568 (LIL).

Ipomoea mirabilis P.P.A. Ferreira \& Sim.Bianch. $\left.{ }^{* * *}\right)$

Enredadera o liana. Perenne. Nativa. 0-500 m.

Distribución: GUA, IGU, MON.

Ejemplar de referencia: Keller, H. A. 13252 (CTES).

Ipomoea nil (L.) Roth

Enredadera. Anual. Nativa. 0-700 m.
Distribución: APO, CAP, CON, GUA, SJA, SPE.

Ejemplar de referencia: Ruíz Huidobro, A. M. 5062 (LIL).

Ipomoea nitida Griseb. subsp. krapovickasii J.R.I. Wood \& R. W. Scotland

Hierba. Perenne. Nativa. 0-500 m.

Distribución: APO, CAP.

Ejemplar de referencia: Cristóbal, C. L. 1910 (CTES).

Ipomoea nitida Griseb. subsp. nitida

Hierba. Perenne. Nativa. 0-500 m.

Distribución: APO, CAP, CON.

Ejemplar de referencia: Schulz, A. G. 6881 (LIL).

Ipomoea padillae O'Donell

Hierba. Perenne. Nativa. 0-500 m.

Distribución: APO, CAN, CAP, CON, LNA.

Ejemplar de referencia: Biganzoli, F. 1671 (SI).

Ipomoea paludosa O'Donell

Subarbusto. Nativa. 0-500 m.

Distribución: APO, CAI, CAN, CAP, GMB, LNA, MON, SIG, SJA. (SI).

Ejemplar de referencia: Múlgura, M. E. 1955

Ipomoea paranaensis Hoehne

Hierba. Nativa. 0-500 m.

Distribución: GMB.

Ejemplar de referencia: Keller, H. A. 3733 (CTES).

Ipomoea procumbens Mart. ex Choisy

Enredadera. Nativa. 0-500 m.

Distribución: CAI, CAN, CAP, ELD, SIG, SJA.

Ejemplar de referencia: Rodríguez, F. M. 798 (SI).

Ipomoea purpurea (L.) Roth

Enredadera. Anual. Nativa. 0-700 m.

Distribución: CAN, ELD, GMB, MON.

Ejemplar de referencia: Schwindt, E. 1790 (LIL).

\section{Ipomoea quamoclit $\mathrm{L}$.}

Enredadera. Anual. Introducida. 0-500 m.

Distribución: APO, SIG.

Ejemplar de referencia: Zuloaga, F. O. 5722 (SI). 
Ipomoea rubens Choisy

Enredadera. Nativa. 0-500 m.

Distribución: CAP, SIG.

Ejemplar de referencia: O’Donell, C. A. 5601 (LIL).

\section{Ipomoea saopaulista O'Donell $\left({ }^{* * *}\right)$}

Enredadera. Nativa. 100-500 m.

Distribución: GMB, GUA, IGU, LNA.

Ejemplar de referencia: Múlgura, M. E. 3075 (SI).

\section{Ipomoea setifera Poir.}

Enredadera. Nativa. 0-500 m.

Distribución: CAI, CAN, CAP, ELD, GSM, IGU, MON, SIG, SPE.

Ejemplar de referencia: Zuloaga, F. O. 5655 (SI).

\section{Ipomoea subrevoluta Choisy}

Enredadera. Nativa. 0-500 m.

Distribución: CAP.

Ejemplar de referencia: Bertoni, 5831 (LIL).

\section{Ipomoea syringifolia Meisn. $\left(^{* * *}\right)$}

Enredadera. Nativa. 0-500 m.

Distribución: ELD, GMB, GUA, IGU, SPE.

Ejemplar de referencia: Zuloaga, F. O. 5125 (SI).

\section{Jacquemontia Choisy}

Jacquemontia blanchetii Moric.

Enredadera. Nativa. 0-800 m.

Distribución: APO, CAI, CAN, CAP, IGU, LNA, SIG, SPE.

Ejemplar de referencia: Zuloaga, F. O. 6575 (SI).

Jacquemontia cataractae Krapov. $\left.{ }^{(* *}\right)$

Hierba. Perenne. Nativa. 0-500 m.

Distribución: IGU.

Ejemplar de referencia: Ferrucci, M. S. 3360 (CTES).

Jacquemontia evolvuloides (Moric.) Meisn.

Enredadera. Perenne. Nativa. 0-500 m.

Distribución: CAN, CAP, LNA, SIG.

Ejemplar de referencia: Zuloaga, F. O. 6581 (SI).
Jacquemontia heterotricha O'Donell

Subarbusto. Nativa. 0-500 m.

Distribución: CAI, CAP.

Ejemplar de referencia: Biganzoli, F. 839 (SI).

Jacquemontia selloi (Meisn.) Hallier $\mathrm{f}$.

Subarbusto. Nativa. 0-500 m.

Distribución: APO, CAI, CAN, CON, IGU, LNA, OBE, SIG, SPE.

Ejemplar de referencia: Montes, J. E. 2339 (SI).

Jacquemontia sphaerostigma (Cav.) Rusby

Enredadera. Nativa. 0-500 m.

Distribución: CAN, IGU, SIG.

Ejemplar de referencia: Schwarz, G. J. 2211 (LIL).

\section{COSTACEAE}

\section{Costus L.}

Costus arabicus $\mathrm{L}$.

Hierba. Perenne. Nativa. 0-500 m.

Distribución: CAN, IGU.

Ejemplar de referencia: Delucchi, G. 546 (LP, SI).

\section{CRASSULACEAE}

\section{Bryophyllum Salisb.}

Bryophyllum daigremontianum (Raym.Hamet \& H. Perrier) A. Berger

Hierba perenne. Introducida. 0-500 m.

Distribución: CAN, MON.

Ejemplar de referencia: Zuloaga, F. O. 8067 (SI).

Bryophyllum fedtschenkoi (Raym.-Hamet \& H. Perrier) Lauz.-March.

Hierba perenne. Introducida. 0-500 m.

Distribución: CON.

Ejemplar de referencia: Stampella, P. 154 (LP).

Bryophyllum pinnatum (Lam.) Oken

Hierba. Perenne. Introducida. 0-500 m.

Distribución: CON.

Ejemplar de referencia: Stampella, P. 153 (LP). 


\section{Crassula L.}

Crassula drummondii (Torr. \& A. Gray) Fedde Hierba. Anual. Nativa. 0-500 m.

Distribución: APO.

Ejemplar de referencia: Cabrera, A. L. 28564 (SI).

\section{Crassula peduncularis $(\mathrm{Sm}$.) F. Meigen}

Hierba. Anual. Nativa. 0-500 m.

Distribución: APO, CON. (SI).

Ejemplar de referencia: Renvoize, S. A. 3050

\section{CUCURBITACEAE}

\section{Apodanthera Arn.}

Apodanthera laciniosa (Schltdl.) Cogn. $\left.{ }^{* * *}\right)$

Enredadera. Perenne. Nativa. 200-500 m.

Distribución: CAN.

Ejemplar de referencia: Niederlein, G. 1309 (BR).

\section{Apodanthera linearis (Cogn.) Mart.Crov.}

Enredadera. Perenne. Nativa. 0-500 m.

Distribución: CAN.

Ejemplar de referencia: Jörgensen, P. 459 (BAB).

Cayaponia Silva Manso, nom. cons.

\section{Cayaponia bonariensis (Mill.) Mart.Crov.}

Enredadera. Perenne. Nativa. 0-500 m.

Distribución: GMB, GUA, IGU. (SI).

Ejemplar de referencia: Deginani, N. B. 1643

Cayaponia citrullifolia (Griseb.) Cogn. ex Griseb.

Enredadera. Perenne. Nativa. 0-500 m.

Distribución: CAI, ELD.

Ejemplar de referencia: Martínez Crovetto, $R$. 8704 (SI).

Cayaponia martiana (Cogn.) Cogn.

Enredadera. Perenne. Nativa. 0-600 m.
Distribución: GMB, GUA, SIG, SPE.

Ejemplar de referencia: Deginani, N. B. 1037 (SI).

\section{Cayaponia palmata Cogn. $\left.{ }^{(* *}\right)$}

Enredadera. Perenne. Nativa. 200-500 m.

Distribución: CAP.

Ejemplar de referencia: Kermes, E. s.n. (BAB).

\section{Cayaponia pilosa (Vell.) Cogn. $\left({ }^{* * *}\right)$}

Enredadera. Perenne. Nativa. 200-800 m.

Distribución: GUA, SPE.

Ejemplar de referencia: Tressens, S. G. 5754 (CTES, SI).

Ceratosanthes Burm. ex Adans.

\section{Ceratosanthes hilariana Cogn.}

Enredadera. Perenne. Nativa. 0-500 m.

Distribución: CAI.

Ejemplar de referencia: Biganzoli, F. 528 (SI).

\section{Ceratosanthes multiloba Cogn.}

Enredadera. Perenne. Nativa. 0-500 m.

Distribución: CAN, SIG.

Ejemplar de referencia: Meyer, T. 17180 (CTES).

Citrullus Schrad. ex Eckl. \& Zeyh., nom. cons.

Citrullus lanatus (Thunb.) Matsum. \& Nakai subsp. vulgaris (Schrad. ex Eckl. \& Zeyh.) Fursa

Enredadera. Anual. Introducida. 0-500 m.

Distribución: IGU.

Ejemplar de referencia: Morrone, O. 2008 (SI).

\section{Cucurbitella Walp.}

Cucurbitella asperata (Gillies ex Hook. \& Arn.) Walp.

Enredadera. Perenne. Nativa. 0-500 m.

Distribución: GUA.

Ejemplar de referencia: Tressens, S. G. 4981 (CTES). 


\section{Cyclanthera Schrad.}

\author{
Cyclanthera hystrix (Gillies) Arn. \\ Enredadera. Anual. Nativa. 0-700 m. \\ Distribución: GMB, GUA, IGU. \\ Ejemplar de referencia: Vanni, R. O. 3934 \\ (CTES, SI).
}

\section{Cyclanthera oligoechinata L.F.P. Lima \&} Pozner $\left({ }^{* *}\right)$

Enredadera. Anual. Nativa. 100-500 m.

Distribución: CAI, SJA.

Ejemplar de referencia: Krapovickas, A. 28878 (CTES,SI).

\section{Cyclanthera quinquelobata (Vell.) Cogn.}

Enredadera. Anual. Nativa. 300-700 m.

Distribución: CAI, GMB, GUA.

Ejemplar de referencia: Morrone, O. 637 (SI).

\section{Cyclanthera tenuisepala Cogn. $\left({ }^{* * *}\right)$}

Enredadera. Anual. Nativa. 0-800 m.

Distribución: CAI, GMB, GUA, IGU, SPE.

Ejemplar de referencia: Biganzoli, F. 790 (SI).

\section{Fevillea L.}

\section{Fevillea trilobata L. $\left({ }^{* * *}\right)$}

Liana. Perenne. Nativa. 0-500 m.

Distribución: SPE.

Ejemplar de referencia: Daviña, J. 196 (CTES, SI).

\section{Lagenaria Ser.}

Lagenaria siceraria (Molina) Standl.

Hierba o enredadera. Anual. Introducida. 0-500 m.

Distribución: IGU.

Ejemplar de referencia: Sanzin, R. 1275 (MOY).

\section{Luffa Mill.}

\section{Luffa aegyptiaca Mill.}

Enredadera. Anual. Introducida. 0-500 m.

Distribución: GUA.

Ejemplar de referencia: Zuloaga, F. O. 5482 (SI).

\section{Melothria L.}

Melothria campestris (Naudin) H. Schaef. \& S.S. Renner

Hierba. Perenne. Nativa. 0-500 m.

Distribución: CAP, CON.

Ejemplar de referencia: Keller, H. A. 12488 (CTES).

\section{Melothria candolleana Cogn.}

Enredadera. Anual. Nativa. 0-500 m.

Distribución: CAN, SIG.

Ejemplar de referencia: Keller, H. A. 13831 (CTES).

\section{Melothria cucumis Vell.}

Enredadera. Anual. Nativa. 0-800 m.

Distribución: APO, CAI, CAN, CAP, ELD, GMB, GSM, GUA, IGU, LNA, SIG, SJA, SPE.

Ejemplar de referencia: Morrone, O. 2151 (SI).

\section{Melothria pendula $\mathrm{L}$.}

Enredadera. Anual. Nativa. 0-800 m.

Distribución: APO, CAI, CAN, CAP, CON, GMB, GSM, GUA, IGU, MAY, OBE, SIG, SJA, SPE.

Ejemplar de referencia: Biganzoli, F. 212 (SI).

\section{Melothria warmingii Cogn.}

Enredadera. Anual. Nativa. 0-500 m.

istribución: IGU, SIG.

Ejemplar de referencia: Schwarz, G. J. 5616 (LIL).

\section{Sicydium Schltdl.}

\section{Sicydium gracile Cogn. $\left({ }^{* * *}\right)$}

Enredadera. Perenne. Nativa. 0-500 m.

Distribución: ELD, GMB, SPE.

Ejemplar de referencia: Keller, H. A. 13410 (CTES).

\section{Sicyos L.}

\section{Sicyos polyacanthus Cogn.}

Enredadera. Anual. Nativa. 0-700 m.

Distribución: CAI, ELD, GMB, GSM, GUA, IGU, MAY, MON, SIG, SPE.

Ejemplar de referencia: Deginani, N. B. 1138 (SI). 


\section{Wilbrandia Silva Manso}

Wilbrandia ebracteata Cogn. var. bracteata Mart. Crov. (**)

Enredadera. Perenne. Endémica. 300-500 m.

Distribución: GMB, SIG, SPE.

Ejemplar de referencia: Martínez Crovetto, $R$. 6062 (BAB).

\section{Wilbrandia ebracteata Cogn. var. ebracteata \\ Enredadera. Perenne. Nativa. 0-500 m. \\ Distribución: APO, CAI, CAN, ELD, GMB, GUA, IGU, LNA, MON, OBE, SIG, SJA, SPE. \\ Ejemplar de referencia: Johnson, A. E. 841 (SI).}

\section{Wilbrandia ebracteata Cogn. var. pedunculata} Mart. Crov. $\left.{ }^{* * *}\right)$

Enredadera. Perenne. Nativa. 0-500 m.

Distribución: CAI, CAN, LNA.

Ejemplar de referencia: Martínez Crovetto, $R$. $6218(B A B)$.

\section{CYPERACEAE}

\section{Abildgaardia Vahl}

\section{Abildgaardia ovata (Burm. f.) Kral}

Hierba. Perenne. Nativa. 0-500 m.

Distribución: CAN, CAP.

Ejemplar de referencia: Burkart, A. 14176 (SI).

\section{Bulbostylis Kunth}

\section{Bulbostylis aspera M.G. López}

Hierba. Anual. Nativa. 0-500 m.

Distribución: SIG.

Ejemplar de referencia: Schwarz, G. J. 5867 (CTES, SI).

\section{Bulbostylis brevifolia Palla}

Hierba. Perenne. Nativa. 0-800 m.

Distribución: IGU, SIG.

Ejemplar de referencia: Biganzoli, F. 10 (SI).

Bulbostylis capillaris (L.) C.B. Clarke Hierba. Anual. Nativa. 0-700 m.
Distribución: CAN, ELD, GUA, SIG.

Ejemplar de referencia: Zuloaga, F. O. 6647 (SI).

Bulbostylis communis M.G. López \& D.A. Simpson var. scabrida M.G. López \& D.A. Simpson

Hierba. Anual. Nativa. 0-500 m.

Distribución: CAI, IGU.

Ejemplar de referencia: Vanni, R. O. 2894 (CTES).

Bulbostylis communis M.G. López \& D.A. Simpson var. communis

Hierba. Anual. Nativa. 0-500 m.

Distribución: CAN, SIG.

Ejemplar de referencia: Morrone, O. 1011 (SI).

\section{Bulbostylis guaglianoneae M.G. López $\left(^{* *}\right)$}

Hierba. Perenne. Endémica. 0-600 m.

Distribución: APO.

Ejemplar de referencia: Guaglianone, E. R. 863 (SI).

Bulbostylis hirtella (Schrad. ex Schult.) Urb. Hierba. Perenne. Nativa. 0-500 m.

Distribución: SIG.

Ejemplar de referencia: Morrone, O. 1889 (SI).

Bulbostylis juncoides (Vahl) Kük. ex Herter $\mathrm{f}$. subfimbriata Kük. ex Osten

Hierba. Perenne. Nativa. 0-500 m.

Distribución: CAN, SJA.

Ejemplar de referencia: Montes, J. E. 2309 (SI).

Bulbostylis juncoides (Vahl) Kük. ex Herter var. juncoides

Hierba. Perenne. Nativa. 0-600 m.

Distribución: APO, CAI, GMB.

Ejemplar de referencia: Biganzoli, F. 838 (SI).

Bulbostylis juncoides (Vahl) Kük. ex Herter var. lorentzii (Boeck.) Kük. ex Osten

Hierba. Perenne. Nativa. 0-500 m.

Distribución: APO, CAN, CON, OBE, SJA.

Ejemplar de referencia: Biganzoli, F. 1651 (SI).

Bulbostylis loefgrenii (Boeck.) Prata \& M.G. López $\left.{ }^{* * *}\right)$

Hierba. Perenne. Nativa. 0-500 m.

Distribución: GMB.

Ejemplar de referencia: López, M. G. 400 (CTES). 
Bulbostylis rugosa M.G. López

Hierba. Perenne. Nativa. 0-500 m.

Distribución: CAN.

Ejemplar de referencia: Keller, H. A. 8120 (CTES, SI).

Bulbostylis scabra (J. Presl \& C. Presl) C.B. Clarke

Hierba. Perenne. Nativa. 100-700 m.

Distribución: SIG.

Ejemplar de referencia: Keller, H. A. 5687 (CTES).

\section{Bulbostylis subtilis M.G. López}

Hierba. Anual. Nativa. 0-600 m.

Distribución: APO, CAN, ELD, IGU.

Ejemplar de referencia: Peichoto, M. C. 258 (CTES).

Bulbostylis sphaerocephala (Boeck.) C.B. Clarke Hierba. Perenne. Nativa. 0-600 m.

Distribución: CAN, SIG.

Ejemplar de referencia: Zuloaga, F. O. 5743 (SI).

\section{Bulbostylis sphaerolepis (Boeck.) Beetle}

Hierba. Perenne. Nativa. 0-700 m.

Distribución: CAN, CAP, CON, SIG, SJA, SPE.

Ejemplar de referencia: Zuloaga, F. O. 6786 (SI).

Bulbostylis wanderleyana Prata \& M.G. López $(* * *)$

Hierba. Perenne. Nativa. 0-600 m.

Distribución: GMB.

Ejemplar de referencia: Keller, H. A. 3705 (CTES).

\section{Carex L.}

Carex aureolensis Steud. (***)

Hierba. Perenne. Nativa.

Distribución: Desconocida.

Ejemplar de referencia: Schwarz, G. J. 3615 (LIL).

\section{Carex brasiliensis A. St.-Hil.}

Hierba. Perenne. Nativa. 0-800 m.

Distribución: CAP, GMB, IGU, SPE.

Ejemplar de referencia: Morrone, O. 1369 (SI).

\section{Carex brongniartii Kunth}

Hierba. Perenne. Nativa. 0-700 m.

Distribución: SPE.

Ejemplar de referencia: Morrone, O. 1289 (SI).
Carex feddeana $\mathrm{H}$. Pfeiff.

Hierba. Perenne. Nativa. 0-600 m.

Distribución: APO, SPE.

Ejemplar de referencia: Guaglianone, E. R. 2912 (SI).

\section{Carex hilairei Boott ${ }^{* * *}$ )}

Hierba. Perenne. Nativa. 0-500 m.

Distribución: IGU.

Ejemplar de referencia: Hatschbach, G. 4316 (SI).

Carex longii Mack. subsp. meridionalis (Kük.) Luceño \& M.V. Alves

Hierba. Perenne. Nativa. 0-500 m.

Distribución: CAI, GUA.

Ejemplar de referencia: Guaglianone, E. R. 2893 (SI).

Carex phalaroides Kunth subsp. paraguayensis (Maury) Luceño \& M.V. Alves (***)

Hierba. Perenne. Nativa. 0-500 m.

Distribución: GUA.

Ejemplar de referencia: Múlgura, M. E. 2516 (SI).

Carex phalaroides Kunth subsp. phalaroides

Hierba. Perenne. Nativa. 0-300 m.

Distribución: CAI, GMB, GUA.

Ejemplar de referencia: Morrone, O. 1474 (SI).

Carex polysticha Boeck.

Hierba. Perenne. Nativa. 0-800 m.

Distribución: APO, GUA, SPE.

Ejemplar de referencia: Morrone, O. 2890 (SI).

Carex sellowiana Schltdl.

Hierba. Perenne. Nativa. 0-800 m.

Distribución: GMB, GSM, GUA, SPE.

Ejemplar de referencia: Deginani, N. B. 1092 (SI).

\section{Carex sororia Kunth}

Hierba. Perenne. Nativa. 0-700 m.

Distribución: APO, GMB, GSM, MON, SIG.

Ejemplar de referencia: Deginani, N. B. 1774 (SI).

\section{Carex uruguensis Boeck.}

Hierba. Perenne. Nativa. 0-500 m.

Distribución: CAI, GMB, SPE.

Ejemplar de referencia: Zuloaga, F. O. 6498 (SI). 


\section{Cyperus L.}

Cyperus aggregatus (Willd.) Endl.

Hierba. Perenne. Nativa. 0-500 m.

Distribución: APO, CAN, ELD, SIG.

Ejemplar de referencia: Morrone, O. 1090 (SI).

\section{Cyperus andreanus Mauryvar. capitinduensis}

(Maury) Kük.

Hierba. Perenne. Nativa. 100-800 m.

Distribución: CAI, CAN, ELD, GMB, GUA, IGU, SIG, SPE. (SI).

Ejemplar de referencia: Guaglianone, E. R. 2862

Cyperus andreanus Maury var. yguazuensis Kük. $\left.{ }^{* *}\right)$

Hierba. Perenne. Endémica. 0-500 m.

Distribución: IGU.

Ejemplar de referencia: Osten, C. 8035 (SI).

Cyperus brasiliensis (Kunth) Bauters

Hierba. Perenne. Nativa. 0-500 m.

Distribución: APO, CON, SPE. (SI).

Ejemplar de referencia: Guaglianone, E. R. 2848

Cyperus brevifolius (Rottb.) Hassk.

Hierba palustre. Perenne. Nativa. 0-800 m.

Distribución: CAI, GUA, IGU, SIG.

Ejemplar de referencia: Deginani, N. B. 1433 (SI).

\section{Cyperus burkartii Guagl. (***)}

Hierba. Perenne. Nativa. 200-400 m.

Distribución: SPE.

Ejemplar de referencia: Zuloaga, F. O. 735 (NY, SI).

\section{Cyperus chalaranhus J. Presl \& C. Presl}

Hierba. Perenne. Nativa. 0-500 m.

Distribución: GUA, IGU, SIG.

Ejemplar de referencia: Keller, H. A. 5819 (CTES).

\section{Cyperus cornelii-ostenii Kük.}

Hierba. Perenne. Nativa. 100-600 m.

Distribución: IGU, SIG.

Ejemplar de referencia: Zardini, E. M. 763 (SI).

Cyperus densicaespitosus Mattf. \& Kük.

Hierba. Anual. Nativa. 0-600 m.
Distribución: GMB, GSM, IGU, SIG, SPE.

Ejemplar de referencia: Guaglianone, E. R. 2846 (SI).

Cyperus digitatus Roxb.

Hierba palustre. Perenne. Nativa. 0-500 m.

Distribución: CAI.

Ejemplar de referencia: Schwarz, G. J. 6712 (SI).

\section{Cyperus entrerianus Boeck. var. entrerianus}

Hierba palustre. Perenne. Nativa. 0-800 m.

Distribución: CAN, CAP, GMB, SIG. (SI).

Ejemplar de referencia: Deginani, N. B. 1394

\section{Cyperus entrerianus Boeck. var. parvicapitulatus Kük. \\ Hierba. Perenne. Nativa. 0-800 m. \\ Distribución: IGU. \\ Ejemplar de referencia: Guaglianone, E. R. 985 (SI).}

\section{Cyperus eragrostis Lam.}

Hierba. Perenne. Nativa. 0-700 m.

Distribución: CAP, ELD, GSM, GUA, IGU.

Ejemplar de referencia: Cabrera, A. L. 28815 (SI).

\section{Cyperus esculentus L. var. leptostachyus} Boeck.

Hierba. Perenne. Introducida. 0-700 m.

Distribución: CAN, GSM, IGU, SPE.

Ejemplar de referencia: Zuloaga, F. O. 5238 (SI).

\section{Cyperus fraternus Kunth}

Hierba. Perenne. Nativa. 0-500.

Distribución: CAN.

Ejemplar de referencia: Keller, H. A. 13664 (CTES).

\section{Cyperus friburgensis Boeck.}

Hierba palustre. Perenne. Nativa. 0-800 m.

Distribución: CAI, CAN, GMB, GSM, GUA, IGU, SIG, SPE.

Ejemplar de referencia: Burkart, A. 28262 (SI).

\section{Cyperus giganteus Vahl}

Hierba palustre. Perenne. Nativa. 0-500 m.

Distribución: CAN.

Ejemplar de referencia: Montes, J. E. 103b (SI). 
Cyperus haspan L. var. amplissimus Kük.

Hierba. Perenne. Nativa. 0-700 m.

Distribución: APO, IGU, SIG.

Ejemplar de referencia: Parodi, L. R. 6861 (SI).

\section{Cyperus haspan L. var. haspan}

Hierba. Anual o perenne. Nativa. 0-800 m.

Distribución: GMB, IGU, SIG, SPE.

Ejemplar de referencia: Morrone, O. 2106 (SI).

Cyperus hermaphroditus (Jacq.) Standl.

Hierba. Perenne. Nativa. 0-800 m.

Distribución: CAI, CAN, GMB, GSM, IGU, SJA.

Ejemplar de referencia: Biganzoli, F. 954 (SI).

\section{Cyperus hieronymi Boeck.}

Hierba. Perenne. Nativa. 0-500 m.

Distribución: IGU, MON, SIG.

Ejemplar de referencia: Keller, H. A. 13643 (CTES).

\section{Cyperus imbricatus Retz.}

Hierba. Perenne. Nativa. 0-500 m.

Distribución: GSM, SIG.

Ejemplar de referencia: Cabrera, A. L. 28814 (SI).

\section{Cyperus incomtus Kunth}

Hierba. Perenne. Nativa. 0-800 m.

Distribución: APO, CAI, CAN, CON, ELD, GMB, IGU, SIG, SPE.

Ejemplar de referencia: Cabrera, A. L. 28778 (SI).

Cyperus intricatus Schrad. ex Schult.

Hierba. Perenne. Nativa. 0-500 m.

Distribución: GMB, IGU. (SI).

Ejemplar de referencia: Guaglianone, E. R. 1139

\section{Cyperus iria L.}

Hierba. Anual. Introducida. 0-500 m.

Distribución: CAN, GUA.

Ejemplar de referencia: Seijo, G. J. 835 (MNES, SI).

\section{Cyperus lanceolatus Poir.}

Hierba acuática. Perenne. Nativa. 0-700 m.

Distribución: APO, CAP, CON, GMB, IGU, SIG.

Ejemplar de referencia: Zuloaga, F. O. 5657 (SI).

\section{Cyperus laxus Lam.}

Hierba palustre. Perenne. Nativa. 0-500 m.
Distribución: IGU, MON, SIG.

Ejemplar de referencia: Guaglianone, E. R. 2173 (SI).

Cyperus megapotamicus Kunth var. jaeggii (Boeck.) Kük. ex Barros

Hierba acuática. Perenne. Nativa. 0-300 m.

Distribución: CAP, GMB.

Ejemplar de referencia: Guaglianone, E. R. 1093 (SI,CTES).

Cyperus megapotamicus Kunth var. megapotamicus

Hierba palustre. Perenne. Nativa. 0-500 m.

Distribución: APO, CAN.

Ejemplar de referencia: Guaglianone, E. R. 845 (SI).

\section{Cyperus meyenianus Kunth}

Hierba palustre. Perenne. Nativa. 0-700 m.

Distribución: APO, CAN, CAP, GMB, GUA, SIG.

Ejemplar de referencia: Morrone, O. 1155 (SI).

Cyperus muniziae G.C. Tucker $\left.{ }^{(* *}\right)$

Hierba. Perenne. Nativa. 0-500 m.

Distribución: SIG.

Ejemplar de referencia: Morrone, O. 1081 (SI,MO).

\section{Cyperus niederleinianus Boeck.}

Hierba. Perenne. Nativa. 0-600 m.

Distribución: APO, CAN.

Ejemplar de referencia: Biganzoli, F. 1697 (SI).

Cyperus obtusatus (J. Presl \& C. Presl) Mattf. \& Kük.

Hierba palustre. Perenne. Nativa. 0-700 m.

Distribución: GUA, SPE.

Ejemplar de referencia: Zuloaga, F. O. 6893 (SI).

\section{Cyperus odoratus L.}

Hierba palustre. Perenne. Nativa. 0-500 m.

Distribución: APO, IGU.

Ejemplar de referencia: Guaglianone, E. R. 1021 (SI).

\section{Cyperus pohlii (Nees) Steud.}

Hierba. Perenne. Nativa. 0-700 m.

Distribución: APO, CAN, CON, IGU, OBE.

Ejemplar de referencia: Keller, H. A. 13678 (CTES). 
Cyperus prolixus Kunth

Hierba. Perenne. Nativa. 0-800 m.

Distribución: APO, CAI, CAN, GUA, IGU, MAY, SPE.

Ejemplar de referencia: Seijo, G. J. 220 (CTES, MNES).

\section{Cyperus reflexus Vahl}

Hierba. Perenne. Nativa. 0-500 m.

Distribución: CAN, CAP, IGU.

Ejemplar de referencia: Zuloaga, F. O. 5787 (SI).

Cyperus rigens J. Presl var. impolitus (Kunth) Hefler \& Longhi-Wagner

Hierba acuática. Perenne. Nativa. 0-800 m.

Distribución: APO, CAN, GUA, IGU, SJA.

Ejemplar de referencia: Guaglianone, E. R. 869 (SI).

Cyperus rigens $J$. Presl \& $C$. Presl var. rigens

Hierba. Perenne. Nativa. 0-500 m.

Distribución: APO, CAN, SIG. (SI).

Ejemplar de referencia: Guaglianone, E. R. 3028

\section{Cyperus rotundus $\mathrm{L}$.}

Hierba palustre. Perenne. Nativa. 0-500 m.

Distribución: GUA, SIG.

Ejemplar de referencia: Morrone, O. 1901 (SI).

\section{Cyperus sellowianus (Kunth) T. Koyama}

Hierba palustre. Perenne. Nativa. 0-800 m.

Distribución: CAN, CAP, IGU.

Ejemplar de referencia: Biganzoli, F. 1716 (SI).

Cyperus sesquiflorus (Torr.) Mattf. \& Kük. ex Kük.

Hierba palustre. Perenne. Nativa. 0-800 m.

Distribución: APO, CAN, GMB, GSM, GUA, IGU, LNA, SIG.

Ejemplar de referencia: Morrone, O. 1097 (SI).

\section{Cyperus surinamensis Rottb.}

Hierba. Anual o perenne. Nativa. 0-500 m.

Distribución: ELD, GUA, IGU, SIG.

Ejemplar de referencia: Morrone, O. 1906 (SI).

Cyperus uncinulatus Schrad. ex Nees

Hierba palustre. Anual o perenne. Nativa. 0-200 m.
Distribución: CAN.

Ejemplar de referencia: Rodríguez, F. M. 340 (SI).

Cyperus unicolor Boeck.

Hierba. Anual. Nativa. 0-500 m.

Distribución: ELD.

Ejemplar de referencia: Burkart, A. 14536 (SI).

\section{Cyperus unioloides $\mathrm{R}$. $\mathrm{Br}$.}

Hierba acuática. Perenne. Nativa. 0-500 m.

Distribución: CAP, CON, ELD.

Ejemplar de referencia: Biganzoli, F. 1639 (SI).

Cyperus virens Michx. var. montanus (Boeck.) Denton

Hierba. Perenne. Nativa. 0-500 m.

Distribución: CAI, GMB, GUA, SJA.

Ejemplar de referencia: Guaglianone, E. R. 3001 (SI).

\section{Cyperus virens Michx. var. virens}

Hierba palustre. Perenne. Nativa. 0-500 m.

Distribución: CAI, GMB, SIG.

Ejemplar de referencia: Biganzoli, F. 955 (SI).

\section{Eleocharis R. Br.}

Eleocharis acutangula (Roxb.) Schult.

Hierba acuática. Perenne. Nativa. 0-500 m.

Distribución: APO, CAN, CAP, MON.

Ejemplar de referencia: Guaglianone, E. R. 867 (SI).

Eleocharis bicolor Chapm.

Hierba palustre. Perenne. Nativa.

Distribución: Desconocida.

Ejemplar de referencia: Hauman, L. L. s.n. (BA).

\section{Eleocharis bonariensis Nees}

Hierba palustre. Perenne. Nativa. 0-500 m.

Distribución: APO.

Ejemplar de referencia: Cabrera, A. L. 28763 (SI).

\section{Eleocharis capillacea Kunth $\left(^{* * *}\right)$}

Hierba acuática. Perenne. Nativa. 200-500 m.

Distribución: APO, CAN.

Ejemplar de referencia: Fontana, J. L. 171-2 (CTESN, SI). 
Eleocharis confervoides (Poir.) Steud. $\left({ }^{* *}\right)$ Hierba acuática. Perenne. Nativa. 0-500 m. Distribución: CAP.

Ejemplar de referencia: Molfino, J. F. 3692 (SI).

\section{Eleocharis contracta Maury}

Hierba acuática. Perenne. Nativa. 0-500 m.

Distribución: APO, CAN. (SI).

Ejemplar de referencia: Renvoize, S. A. $3105 a$

Eleocharis elegans (Kunth) Roem. \& Schult. Hierba acuática. Perenne. Nativa. 0-500 m. Distribución: APO, SIG.

Ejemplar de referencia: Keller, H. A. 8039 (CTES).

Eleocharis flavescens (Poir.) Urb.

Hierba acuática. Perenne. Nativa. 0-400 m.

Distribución: IGU. (SI).

Ejemplar de referencia: Zuloaga, F. O. 5250

\section{Eleocharis minima Kunth}

Hierba palustre. Anual. Nativa. 0-500 m.

Distribución: GUA, SIG, SPE. (SI).

Ejemplar de referencia: Deginani, N. B. 1100

Eleocharis montana (Kunth) Roem. \& Schult.

Hierba acuática. Perenne. Nativa. 0-700 m.

Distribución: CAI, CON, GMB, GSM, GUA, IGU, SIG, SPE. (SI).

Ejemplar de referencia: Guaglianone, E. R. 2849

Eleocharia nudipes (Kunth) Palla

Hierba palustre. Perenne. Nativa. 0-500 m.

Distribución: SIG.

Ejemplar de referencia: Keller, H. A. 5704 (CTES).

\section{Eleocharis quinquangularis Boeck.}

Hierba palustre. Perenne. Nativa. 0-800 m.

Distribución: CAN.

Ejemplar de referencia: Niederlein, G. 595 (SI).

Eleocharis radicans (Poir.) Kunth

Hierba acuática. Perenne. Nativa. 0-700 m.
Distribución: ELD, GUA.

Ejemplar de referencia: Deginani, N. B. 1586 (SI).

Eleocharis sellowiana Kunth

Hierba. Perenne. Nativa. 0-800 m.

Distribución: CAN, GMB.

Ejemplar de referencia: Múlgura, M. E. 2234 (SI).

\section{Fimbristylis Vahl}

Fimbristylis autumnalis (L.) Roem. \& Schult. Hierba palustre. Anual o perenne. Nativa. 0-500 m.

Distribución: CAN, CON, IGU.

Ejemplar de referencia: Guaglianone, E. R. 1004 (CTES, MO, MU, SI).

Fimbristylis complanata (Retz.) Link

Hierba. Perenne. Nativa. 0-500 m.

Distribución: APO, CAN, IGU, SIG.

Ejemplar de referencia: Keller, H. A. 11784 (CTES).

\section{Fimbristylis dichotoma (L.) Vahl}

Hierba acuática. Perenne. Introducida. 0-700 m.

Distribución: CAI, CAN, CON, ELD, GMB, IGU, LNA, SIG, SPE.

Ejemplar de referencia: Dematteis, M. 663 (CTES, SI).

\section{Fimbristylis squarrosa Vahl}

Hierba. Perenne. Nativa. 0-500 m.

Distribución: ELD.

Ejemplar de referencia: Cabrera, A. L. 28850 (SI).

Fuirena Rottb.

\section{Fuirena incompleta Nees}

Hierba palustre. Perenne. Nativa. 0-800 m.

Distribución: APO, CAP, SIG.

Ejemplar de referencia: Guaglianone, E. R. 850 (SI).

Fuirena umbellata Rottb.

Hierba. Perenne. Nativa. 0-500 m.

Distribución: IGU.

Ejemplar de referencia: Zuloaga, F. O. 5656 (SI). 


\section{Rhynchospora Vahl}

\section{Rhynchospora albiceps Kunth $\left(^{* * *}\right)$}

Hierba. Perenne. Nativa. 0-600 m.

Distribución: Desconocida.

Ejemplar de referencia: Irwin, J. B. 27290 (LIL).

Rhynchospora asperula (Nees) Steud.

Hierba. Perenne. Nativa. 0-800 m.

Distribución: APO, CAN, CAP, CON, ELD, GMB, GUA, IGU, SIG, SPE. (SI).

Ejemplar de referencia: Guaglianone, E. R. 2825

\section{Rhynchospora barrosiana Guagl.}

Hierba. Perenne. Nativa. 0-600 m.

Distribución: CAP, GUA, IGU, SPE.

Ejemplar de referencia: Guaglianone, E. R. 2932 (SI).

Rhynchospora brownii Roem. \& Schult. subsp. americana Guagl.

Hierba. Perenne. Nativa. 0-700 m.

Distribución: APO, CAN, CAP, CON, GUA, IGU. (SI).

Ejemplar de referencia: Guaglianone, E. R. 3053

Rhynchospora caduciglumis $\mathrm{P}$. Weber \& $\mathrm{R}$. Trevis. $\left.{ }^{* * *}\right)$

Hierba. Perenne. Nativa. 0-500 m.

Distribución: IGU.

Ejemplar de referencia: Keller, H. A. 13507 (CTES, SI).

Rhynchospora conferta (Nees) Boeck.

Hierba. Perenne. Nativa. 0-800 m.

Distribución: APO.

Ejemplar de referencia: Bruhl, J. J. 2317 (SI, NSW, NY).

Rhynchospora confinis (Nees) C.B. Clarke

Hierba. Perenne. Nativa. 0-500 m.

Distribución: CON, SIG.

Ejemplar de referencia: Biganzoli, F. 1699 (SI).

\section{Rhynchospora corymbosa (L.) Britton}

Hierba. Perenne. Nativa. 0-800 m.

Distribución: APO, CAN, CAP, CON, GMB, GUA, IGU, MON, SIG, SPE.

Ejemplar de referencia: Morrone, O. 2170 (SI).
Rhynchospora emaciata (Nees) Boeck.

Hierba. Perenne. Nativa. 0-700 m.

Distribución: APO, CAN.

Ejemplar de referencia: Guaglianone, E. R. 865 (SI).

Rhynchospora glaziovii Boeck. $\left.{ }^{* * *}\right)$

Hierba. Perenne. Nativa. 0-500 m.

Distribución: GMB.

Ejemplar de referencia: Morrone, O. 1435 (SI).

Rhynchospora globosa (Kunth) Roem. \& Schult. Hierba. Perenne. Nativa. 0-500 m.

Distribución: SJA.

Ejemplar de referencia: Montes, J. E. s.n. (BAF)

Rhynchospora hieronymi Boeck. subsp. montevidensis (Barros) Guagl.

Hierba. Perenne. Nativa. 0-500 m.

Distribución: GMB, GUA.

Ejemplar de referencia: Guaglianone, E. R. 2946 (SI).

Rhynchospora loefgrenii Boeck.

Hierba. Perenne. Nativa. 200-800 m.

Distribución: APO.

Ejemplar de referencia: Guaglianone, E. R. 842 (NY).

\section{Rhynchospora marisculus Nees}

Hierba. Perenne. Nativa. 0-700 m.

Distribución: GMB, GUA, IGU.

Ejemplar de referencia: Guaglianone, E. R. 2948 (SI).

Rhynchospora megapotamica (A. Spreng.) H. Pfeiff.

Hierba. Perenne. Nativa. 0-800 m.

Distribución: CAP, IGU, OBE, SPE.

Ejemplar de referencia: Guaglianone, E. R. 2851 (SI).

Rhynchospora nervosa (Vahl) Boeck.

Hierba. Perenne. Nativa. O-500 m.

Distribución: SIG.

Ejemplar de referencia: Hauman, L. L. s.n. (SI).

Rhynchospora organensis $C$. B. Clarke

Hierba. Perenne. Nativa. 0-500 m.

Distribución: SIG, SJA.

Ejemplar de referencia: Cabrera, A. L. 29463 (SI). 


\section{Rhynchospora praecincta Maury}

Hierba. Perenne. Nativa. 0-500 m.

Distribución: APO, CAN, ELD, SIG.

Ejemplar de referencia: Cabrera, A. L. 28562 (SI).

\section{Rhynchospora pungens Liebm}

Hierba. Perenne. Nativa. 0-500 m.

Distribución: CAP, CAN, IGU.

Ejemplar de referencia: Keller, H. A. 10389 (CTES).

\section{Rhynchospora robusta (Kunth) Boeck.}

Hierba. Perenne. Nativa. 0-500 m.

Distribución: CAP, SIG.

Ejemplar de referencia: Rodríguez $\left\{2^{\circ}\right.$ serie $\}, F$. M. 483 (BA, SI).

\section{Rhynchospora rugosa (Vahl) Gale}

Hierba. Perenne. Nativa. 0-500 m.

Distribución: CAP, SJA.

Ejemplar de referencia: Molfino, J. F. s.n. (BAF).

\section{Rhynchospora scutellata Griseb.}

Hierba. Perenne. Nativa. 0-500 m.

Distribución: SIG, SPE. (SI).

Ejemplar de referencia: Guaglianone, E. R. 2845

\section{Rhynchospora setigera (Kunth) Boeck.}

Hierba. Perenne. Nativa. 0-700 m.

Distribución: APO, CAN, CON, LNA, SIG.

Ejemplar de referencia: Biganzoli, F. 447 (SI).

\section{Rhynchospora splendens Lindm. $\left.{ }^{* * *}\right)$}

Hierba. Perenne. Nativa. 0-500 m.

Distribución: GMB. (SI).

Ejemplar de referencia: Múlgura, M. E. 1888

Rhynchospora tenuis Link subsp. austrobrasiliensis $\mathrm{T}$. Koyama $\left.{ }^{* * *}\right)$

Hierba. Perenne. Nativa. 0-500 m.

Distribución: CAN.

Ejemplar de referencia: Ekman, E. L. 1300 (NY, S).

\section{Rhynchospora tenuis Link subsp. tenuis}

Hierba. Perenne. Nativa. 0-500 m.

Distribución: APO, CAI, CAP, GUA, SIG.

Ejemplar de referencia: Guaglianone, E. R. 862 (SI).
Rhynchospora velutina (Kunth) Boeck.

Hierba. Perenne. Nativa. 0-400 m.

Distribución: CAN, CAP, SIG.

Ejemplar de referencia: Guaglianone, E. R. 3051 (SI).

\section{Scleria Bergius}

Scleria ciliata Michx.

Hierba. Perenne. Nativa. 0-500 m.

Distribución: APO, CAN, CAP, SIG. (SI).

Ejemplar de referencia: Guaglianone, E. R. 3049

Scleria composita (Nees) Boeck.

Hierba. Perenne. Nativa. 0-500 m.

Distribución: CAP.

Ejemplar de referencia: Ekman, E. L. 1297.

Scleria distans Poir.

Hierba. Perenne. Nativa. 0-500 m.

Distribución: CAI, CAN, CAP, SIG.

Ejemplar de referencia: Keller, H. A. 10812 (CTES).

\section{Scleria gaertneri Raddi}

Hierba. Perenne. Nativa. 0-500 m.

Distribución: CAP, ELD, GUA, IGU, SIG, SJA.

Ejemplar de referencia: Morrone, O. 1087 (SI).

\section{Scleria latifolia $\mathrm{S} w$.}

Hierba. Perenne. Nativa. 0-500 m.

Distribución: CAN, GMB, IGU, SIG, SPE. (SI).

Ejemplar de referencia: Guaglianone, E. R. 2911

\section{Scleria leptostachya Kunth}

Hierba. Perenne. Nativa. 0-500 m.

Distribución: CAN, ELD.

Ejemplar de referencia: Ekman, E. L. 1309 (*, NY).

\section{Scleria longigluma Kük. $\left.{ }^{* * *}\right)$}

Hierba. Perenne. Nativa. 0-500 m.

Distribución: APO, CAP.

Ejemplar de referencia: Guaglianone, E. R. 843 (SI).

\section{Scleria panicoides Kunth $\left({ }^{* * *}\right)$}

Hierba. Perenne. Nativa. 0-500 m.

Distribución: CAI, GMB, GUA, IGU, SIG, SPE. Ejemplar de referencia: Deginani, N. B. 1268 (SI). 
Scleria plusiophylla Steud. $\left.{ }^{* * *}\right)$

Hierba. Perenne. Nativa. 0-500 m.

Distribución: GMB, IGU, SIG, SPE.

Ejemplar de referencia: Krapovickas, A. 28753 (CTES, NY, SI).

\section{Scleria reticularis Michx.}

Hierba. Perenne. Nativa. 0-500 m.

Distribución: APO.

Ejemplar de referencia: Ahumada, O. 3457 (JUA).

\section{Scleria secans (L.) Urb. $\left.{ }^{* * *}\right)$}

Hierba. Perenne. Nativa. 0-500 m.

Distribución: IGU, SIG.

Ejemplar de referencia: Zuloaga, F. O. 5717 (SI).

\section{Scleria sellowiana Kunth}

Hierba. Perenne. Nativa. 0-500 m.

Distribución: CAN.

Ejemplar de referencia: Montes, J. E. 15255 (SI).

\section{Scleria variegata (Nees) Steud. $\left(^{* * *}\right)$}

Hierba. Perenne. Nativa. 0-800 m.

Distribución: IGU.

Ejemplar de referencia: Guaglianone, E. R. 2504 (SI).

\section{DILLENIACEAE}

\section{Tetracera L.}

\section{Tetracera oblongata $\mathrm{DC} .\left({ }^{* * *}\right)$}

Liana. Perenne. Nativa. 0-500 m.

Distribución: IGU.

Ejemplar de referencia: Keller, H. A. 13571 (CTES).

\section{DIOSCOREACEAE}

\section{Dioscorea L.}

Dioscorea amaranthoides $C$. Presl $\left({ }^{* * *}\right)$

Enredadera. Perenne. Nativa.

Distribución: Desconocida.

Ejemplar de referencia: Meyer, T. 5903 (A, LIL).
Dioscorea campestris Griseb.

Enredadera. Perenne. Nativa. 0-800 m.

Distribución: APO, CAI, CAN, CAP, CON, GUA, IGU, SIG, SJA.

Ejemplar de referencia: Herrera, J. 43 (CTES, SI).

Dioscorea ceratandra $R$. Knuth

Enredadera. Perenne. Nativa. 0-800 m.

Distribución: CAP, GMB, IGU, SIG.

Ejemplar de referencia: Parodi, L. R. 4465 $(B A A)$.

Dioscorea cinnamomifolia Hook. $\left.{ }^{(* *}\right)$

Hierba. Perenne. Nativa. 0-300 m.

Distribución: CAP, IGU.

Ejemplar de referencia: Zanotti, C. A. 481 (SI).

\section{Dioscorea coronata Hauman}

Enredadera. Perenne. Nativa. 0-500 m.

Distribución: APO, CAN, GMB, IGU.

Ejemplar de referencia: Tressens, S. G. 5538 (CTES, SI).

\section{Dioscorea demourae R. Knuth}

Enredadera. Perenne. Nativa. 0-700 m.

Distribución: CAI, CAN, CAP, GMB, GUA.

Ejemplar de referencia: Tressens, S. G. 6443 (SI).

\section{Dioscorea dodecaneura Vell.}

Enredadera. Perenne. Nativa. 0-500 m.

Distribución: GUA, IGU, SIG.

Ejemplar de referencia: Zuloaga, F. O. 5037 (SI).

Dioscorea furcata Griseb.

Enredadera. Perenne. Nativa. 0-500 m.

Distribución: APO, IGU.

Ejemplar de referencia: Krapovickas, A. 31389 (BAB).

Dioscorea glandulosa Klotzsch ex Kunth

Enredadera. Perenne. Nativa. 300-700 m.

Distribución: CAI, CAN, GUA, IGU, MON, SIG.

Ejemplar de referencia: Deginani, N. B. 1588 (SI).

\section{Dioscorea microbotrya Griseb.}

Enredadera. Perenne. Nativa. 0-500 m. 
Distribución: CAN.

Ejemplar de referencia: Montes, J. E. 619 (BA).

Dioscorea multiflora Mart. ex Griseb.

Enredadera. Perenne. Nativa. 0-700 m.

Distribución: APO, CAI, CAN, GMB, GUA, IGU, MON, SIG, SJA.

Ejemplar de referencia: Tressens, S. G. 5594 (CTES, SI).

\section{Dioscorea sinuata Vell.}

Enredadera. Perenne. Nativa. 0-500 m.

Distribución: APO, ELD, GSM, IGU, SIG.

Ejemplar de referencia: Xifreda, C. C. 339 (SI).

Dioscorea subhastata Vell.

Enredadera. Perenne. Nativa. 0-500 m.

Distribución: GMB, IGU.

Ejemplar de referencia: Zanotti, C. A. 475 (SI).

\section{DROSERACEAE}

\section{Drosera L.}

Drosera brevifolia Pursh

Hierba. Anual. Nativa. 0-500 m.

Distribución: CAN. (LP).

Ejemplar de referencia: Spegazzini, C. L. s.n.

Drosera communis A. St.-Hil.

Hierba. Perenne. Nativa. 0-500 m.

Distribución: SIG. (BA).

Ejemplar de referencia: Hauman, L. L. s.n.

\section{EBENACEAE}

\section{Diospyros L.}

Diospyros inconstans Jacq. subsp. obovata (Mart. ex Miq.) B. Walln.

Árbol. Nativa. 0-500 m.

Distribución: CAN, GUA, IGU, SIG, SJA.

Ejemplar de referencia: Rojas, T. 4586 (BAF).

\section{ELAEOCARPACEAE}

Sloanea L.

Sloanea lasiocoma K. Schum. $\left.{ }^{* * *}\right)$

Árbol. Nativa. 0-500 m.

Distribución: GMB.

Ejemplar de referencia: Keller, H. A. 9461 (CTES).

\section{ELATINACEAE}

\section{Elatine L.}

Elatine lorentziana Hunz.

Hierba. Perenne. Nativa. 0-500 m.

Distribución: GMB, SIG.

Ejemplar de referencia: Deginani, N. B. 1404 (SI).

\section{ERICACEAE}

Agarista D. Don ex G. Don

Agarista paraguayensis (Sleumer) Judd $\left({ }^{* * *}\right)$

Arbusto. Nativa. 0-500 m.

Distribución: CAN, SIG.

Ejemplar de referencia: Zuloaga, F. O. 6468 (SI).

\section{ERIOCAULACEAE}

\section{Eriocaulon L.}

\section{Eriocaulon arechavaletae Herter}

Hierba. Perenne. Nativa. 0-800 m.

Distribución: GMB.

Ejemplar de referencia: Guaglianone, E. R. 2955 (K, SI).

\section{Eriocaulon crassiscapum Bong.}

Hierba. Perenne. Nativa. 0-500 m.

Distribución: CAN.

Ejemplar de referencia: Muniez, A. A. s.n. (BA). 


\section{Eriocaulon leptophyllum Kunth}

Hierba. Perenne. Nativa. 0-500 m.

Distribución: APO.

Ejemplar de referencia: Cardozo, A. 282 (MNES, SI).

\section{Eriocaulon missionum A. Cast. $\left.{ }^{* *}\right)$}

Hierba. Perenne. Endémica. 200-500 m.

Distribución: APO, CAP.

Ejemplar de referencia: Guaglianone, E. R. 905 (SI).

\section{Syngonanthus Ruhland}

Syngonanthus anthemidiflorus (Bong.) Ruhland $\left({ }^{* * *}\right)$

Hierba. Perenne. Nativa. 0-500 m.

Distribución: CAN.

Ejemplar de referencia: de Llamas, A. s.n. (BAF).

\section{Syngonanthus caulescens (Poir.) Ruhland}

Hierba. Perenne. Nativa. 0-600 m.

Distribución: APO, CAN, CAP, IGU, MON, SIG.

Ejemplar de referencia: Biganzoli, F. 1709 (SI).

\section{ERYTHROXYLACEAE}

\section{Erythroxylum P. Browne}

Erythroxylum argentinum O.E. Schulz

Arbusto o árbol. Nativa. 0-800 m.

Distribución: APO, CAI, IGU, SIG, SJA, SPE. (SI).

Ejemplar de referencia: Zuloaga, F. O. 6506

Erythroxylum cuneifolium (Mart.) O.E. Schulz Arbusto. Nativa. 0-800 m.

Distribución: CAI, GMB, GUA, IGU, MAY, MON.

Ejemplar de referencia: Belgrano, M. J. 367 (SI, WIS).

\section{Erythroxylum deciduum A. St.-Hil.}

Árbol. Nativa. 0-500 m.

Distribución: CAN, GUA, IGU.

Ejemplar de referencia: Johnson, A. E. 1077 (SI).

\section{Erythroxylum myrsinites Mart.}

Arbusto. Nativa. 0-500 m.

Distribución: ELD, IGU, MAY.

Ejemplar de referencia: Zuloaga, F. O. 4977 (SI).

\section{ESCALLONIACEAE}

\section{Escallonia Mutis ex L.f.}

Escallonia bifida Link \& Otto $\left(^{* * *}\right)$

Árbol. Nativa. 0-700 m.

Distribución: APO, CAN, CAP, GMB, SPE.

Ejemplar de referencia: Bridarolli, A. J. 2521 $(L P)$.

Escallonia megapotamica Spreng.

Arbusto. Nativa. 0-800 m.

Distribución: APO, SPE.

Ejemplar de referencia: Zuloaga, F. O. 7011 (SI).

\section{EUPHORBIACEAE}

\section{Acalypha L.}

Acalypha brasiliensis Müll. Arg.

Arbusto. Nativa. 0-700 m.

Distribución: GMB, GUA, IGU, SPE.

Ejemplar de referencia: Guaglianone, E. R. 2875 (SI).

Acalypha communis Müll. Arg. subsp. apicalis (N.E. Br.) Cardiel \& P. Muñoz

Hierba o subarbusto. Nativa. 0-800 m.

Distribución: APO, CAI, CAN, ELD, GSM, GUA, IGU, LNA, MON, SIG, SPE.

Ejemplar de referencia: Bernardi, L. 18873 $(B M, F, M O, N Y)$.

Acalypha communis Müll. Arg. subsp. communis

Hierba o subarbusto. Nativa. $0-800 \mathrm{~m}$.

Distribución: CAI, CAN, CON, ELD, GMB, IGU, SIG, SPE.

Ejemplar de referencia: Zuloaga, F. O. 8047 (SI). 
Acalypha communis Müll. Arg. subsp. paraguariensis (Chodat \& Hassl.) Cardiel \& $\mathrm{P}$. Muñoz

Hierba o subarbusto. Nativa. 100-200 m.

Distribución: APO, CAP, SIG.

Ejemplar de referencia: Keller, H. A. 10461 (CTES).

\section{Acalypha gracilis Spreng.}

Hierba o subarbusto. Nativa. 100-500 m.

Distribución: APO, CAI, CON, ELD, GMB, GSM, GUA, IGU, MON, SIG, SPE.

Ejemplar de referencia: Deginani, N. B. 1763 (SI).

Acalypha herzogiana Pax \& K. Hoffm. $\left({ }^{* *}\right)$

Hierba. Nativa. 0-400 m.

Distribución: APO, CAN, ELD, SIG, SJA.

Ejemplar de referencia: Keller, H. A. 10603 (CTES).

Acalypha multicaulis Müll. Arg.

Hierba. Nativa. 0-500 m.

Distribución: CAN, GUA, IGU, SIG.

Ejemplar de referencia: Deginani, N. B. 1409 (SI).

\section{Acalypha senilis Baill.}

Hierba o subarbusto. Perenne. Nativa. 0-200 m. Distribución: SIG.

Ejemplar de referencia: Medina, B. R. $142(W)$.

\section{Acalypha striolata Lingelsh.}

Hierba. Nativa. 100-300 m.

Distribución: SIG.

Ejemplar de referencia: Schwarz, G. J. 3167 (LIL, W).

\section{Actinostemon Mart. ex Klotzsch}

Actinostemon concolor (Spreng.) Müll. Arg.

Arbusto o árbol. Nativa. 0-800 m.

Distribución: GMB, GSM, GUA, IGU, SIG, SPE.

Ejemplar de referencia: Biganzoli, F. 245 (SI).

Adelia L. nom. cons.

Adelia membranifolia (Müll. Arg.) Chodat \& Hassl.

Arbusto o árbol. Nativa. 0-500 m.

Distribución: CAI.

Ejemplar de referencia: Morrone, O. 1582 (SI).
Alchornea Sw.

Alchornea glandulosa Poepp. subsp. iricurana (Casar.) Secco $\left({ }^{* *}\right)$

Árbol. Nativa. 0-800 m.

Distribución: GUA, IGU, SIG, SPE.

Ejemplar de referencia: Burkart, A. 14635 (SI).

Alchornea sidifolia Müll. Arg. $\left.{ }^{* * *}\right)$

Árbol. Nativa. 0-500 m.

Distribución: GMB, IGU, SJA.

Ejemplar de referencia: Bertoni, M. S. 3133 (W).

Alchornea triplinervia (Spreng.) Müll. Arg.

Arbusto o árbol. Nativa. 0-500 m.

Distribución: GUA, IGU, SIG, SPE.

Ejemplar de referencia: Múlgura, M. E. 1787 (SI).

Aleurites J.R. Forst. \& G. Forst.

Aleurites moluccanus (L.) Willd.

Árbol. Introducida. 0-200 m.

Distribución: ELD, SIG.

Ejemplar de referencia: Keller, H. A. 10666 (CTES).

\section{Astraea Klotzsch}

Astraea lobata (L.) Klotzsch

Hierba o subarbusto. Perenne. Nativa. 0-700 m. Distribución: CAI, GMB, GUA. (SI).

Ejemplar de referencia: Zuloaga, F. O. 6842

Bernardia Mill., nom. cons.

Bernardia multicaulis Müll. Arg.

Subarbusto. Nativa. 0-500 m.

Distribución: APO, CAI, CAN.

Ejemplar de referencia: Biganzoli, F. 1422 (SI).

\section{Bernardia odonellii Villa $\left(^{* *}\right)$}

Arbusto o subarbusto. Endémica. 0-500 m.

Distribución: SIG.

Ejemplar de referencia: Schwarz, G. J. 5015 (LIL). 
Bernardia pulchella (Baill.) Müll. Arg.

Arbusto o árbol. Nativa. 0-800 m.

Distribución: CAI, CAN, ELD, GMB, GUA, IGU, LNA, SIG, SPE, MAY, MON.

Ejemplar de referencia: Morrone, O. 820 (SI).

\section{Bia Klotzsch}

Bia alienata Didr.

Hierba voluble. Perenne. Nativa. 0-500 m.

Distribución: IGU. (SI).

Ejemplar de referencia: Romanczuk, M. C. 591

Caperonia A. St.-Hil.

Caperonia castaneifolia (L.) A. St.-Hil.

Hierba. Perenne. Nativa. 0-700 m.

Distribución: CAN, CAP.

Ejemplar de referencia: Burkart, A. 14104 (SI).

Caperonia cordata A. St.-Hil.

Hierba o subarbusto. Perenne. Nativa. 0-500 m.

Distribución: CAN, CAP, IGU, OBE, SIG.

Ejemplar de referencia: Zuloaga, F. O. 6530 (SI).

Caperonia palustris (L.) A. St.-Hil.

Hierba acuática. Perenne. Nativa. 0-500 m.

Distribución: APO.

Ejemplar de referencia: Pedersen, T. M. 15777 (CTES).

\section{Chiropetalum A. Juss.}

\section{Chiropetalum griseum Griseb.}

Subarbusto. Nativa. 0-500 m.

Distribución: SPE.

Ejemplar de referencia: Keller, H. A. 3752 (CTES).

Chiropetalum tricoccum (Vell.) Chodat \& Hassl.

Subarbusto. Nativa. 0-500 m.

Distribución: GMB, IGU.

Ejemplar de referencia: Morrone, O. 1479 (SI).

\section{Cnidoscolus Pohl}

Cnidoscolus albomaculatus ( $\mathrm{Pax}$ ) I.M. Johnst. Hierba o arbusto. Perenne. Nativa. 0-700 m. Distribución: APO, CAN, CON, SIG.

Ejemplar de referencia: Múlgura, M. E. 2028 $(S I)$.

Cnidoscolus appendiculatus ( $\mathrm{Pax} \& \mathrm{~K}$. Hoffm.) Pax \& K. Hoffm

Hierba. Perenne. Nativa. 0-600 m.

Distribución: APO.

Ejempar de referencia: Cabrera, A. L. 29315 (MO, SI).

Cnidoscolus loasoides ( $\mathrm{Pax}$ ) I.M. Johnst.

Subarbusto. Nativa. 0-500 m.

Distribución: CAN, SIG.

Ejemplar de referencia: Morrone, O. 2201 (SI).

Cnidoscolus urens (L.) Arthur

Arbusto. Nativa. 0-500 m.

Distribución: CAP.

Ejemplar de referencia: Rodríguez $\left\{2^{\circ}\right.$ serie $\}, F$. M. 36 (BA, SI).

\section{Croton L.}

Croton aberrans Müll. Arg.

Subarbusto. Nativa. 0-500 m.

Distribución: CAP, CON, GMB, SIG.

Ejemplar de referencia: Múlgura, M. E. 1654 (SI).

Croton apostolon Radcl.-Sm. \& Govaerts $\left({ }^{* * *}\right)$ Subarbusto. Nativa. 0-500 m.

Distribución: CAN, SIG.

Ejemplar de referencia: Rodríguez, F. M. 236 (GH, SI).

\section{Croton bonplandianus Baill.}

Hierba. Perenne. Nativa. 0-500 m.

Distribución: APO, CAP.

Ejemplar de referencia: Cabrera, A. L. 28457 (SI).

\section{Croton calonervosus G.L. Webster $\left.{ }^{(* *}\right)$}

Arbusto. Nativa. 0-500 m.

Distribución: GMB, GUA.

Ejemplar de referencia: Deginani, N. B. 1140 (SI). 
Croton didrichsenii G.L. Webster

Hierba o subarbusto. Perenne. Nativa. 0-600 m. Distribución: APO, CAN, SIG.

Ejemplar de referencia: Montes, J. E. 449 b (SI).

Croton doratophylloides (Croizat) G.L. Webster Arbusto. Nativa. 0-500 m.

Distribución: CAN, IGU.

Ejemplar de referencia: Rodríguez $\left\{2^{\circ}\right.$ serie $\}, F$. M. 438 (A).

Croton echinulatus (Griseb.) Croizat

Arbusto o subarbusto. Nativa. 0-500 m.

Distribución: CAN.

Ejemplar de referencia: Keller, H. A. 13860 (CTES).

\section{Croton flavispicatus Rusby}

Arbusto. Nativa. 0-500 m.

Distribución: CAN, CAI, SPE, ELD, SIG.

Ejemplar de referencia: Montes, J. E. 250 (CTES).

\section{Croton glandulosus $\mathrm{L}$.}

Hierba. Perenne. Nativa. 0-500 m.

Distribución: APO, GSM, IGU, SIG, SPE.

Ejemplar de referencia: Morrone, O. 2207 (SI).

\section{Croton glechomifolius Müll. Arg. ${ }^{* * *}$}

Hierba. Perenne. Nativa. 0-500 m.

Distribución: GMB, SIG.

Ejemplar de referencia: Ahumada, L. Z. 6782 (CTESN).

\section{Croton grandivelus Baill.}

Subarbusto. Nativa. 0-500 m.

Distribución: APO, CAP, SIG.

Ejemplar de referencia: Zuloaga, F. O. 8912 (SI).

\section{Croton hirtus L'Hér.}

Hierba. Anual. Nativa. 0-700 m.

Distribución: APO, CAN, CON, SIG. (SI).

Ejemplar de referencia: Múlgura, M. E. 1654

Croton lachnostachyus Baill.

Arbusto. Nativa. 0-500 m.

Distribución: SPE.

Ejemplar de referencia: Keller, H. A. 738 (CTES).
Croton lanatus Lam. var. astrogynus (Baill.)

P.E. Berry

Arbusto. Nativa. 0-500 m.

Distribución: CAN, SIG, SPE.

Ejemplar de referencia: Montes, J. E. 7006 (SI).

\section{Croton landoltii Ahumada}

Arbusto. Nativa. 0-500 m.

Distribución: APO, CAN, CAP.

Ejemplar de referencia: Ahumada, L. Z. 6578 (CTESN, SI).

Croton missionum Croizat

Arbusto. Nativa. 0-500 m.

Distribución: CAN, GMB.

Ejemplar de referencia: Montes, J. E. 7049 (SI).

Croton reitzii L.B. Sm. \& Downs (***)

Arbolito. Nativa. 0-500 m.

Distribución: GUA, IGU, SPE.

Ejemplar de referencia: Zuloaga, F. O. 8011 (SI).

Croton serratifolius Baill.

Arbusto. Nativa. 0-500 m.

Distribución: CAN, GUA, SJA.

Ejemplar de referencia: Montes, J. E. 2243 (SI).

Croton spissirameus Radcl.-Sm. \& Govaerts $(* *)$

Subarbusto. Nativa. 0-700 m.

Distribución: CAN, CON, IGU, SIG.

Ejemplar de referencia: Rodríguez, F. M. 559 (GH).

Croton subpannosus Müll. Arg. ex Griseb.

Subarbusto. Nativa. 0-500 m.

Distribución: APO, CAP. IGU, SIG.

Ejemplar de referencia: Burkart, A. 14395 (SI).

Croton triqueter Lam.

Arbusto. Nativa. 0-600 m.

Distribución: GMB, GSM, IGU, MAY, SPE.

Ejemplar de referencia: Guaglianone, E. R. 2835 (SI).

\section{Croton urucurana Baill.}

Arbolito o árbol. Nativa. 0-600 m.

Distribución: CAN, CAP, ELD, IGU, LNA, SIG, SJA, SPE.

Ejemplar de referencia: Morrone, O. 1098 (SI). 
Croton uruguayensis Baill.

Arbusto. Nativa. 0-500 m.

Distribución: CAN, CAP, GMB.

Ejemplar de referencia: Schinini, A. 5410 (SI).

\section{Dalechampia L.}

Dalechampia glechomifolia Baill. $\left(^{* *}\right)$

Subarbusto. Nativa. 0-700 m.

Distribución: APO, SJA.

Ejemplar de referencia: Cabrera, A. 28453 (SI).

\section{Dalechampia micromeria Baill.}

Hierba voluble. Perenne. Nativa. 0-800 m.

Distribución: CAP, GMB.

Ejemplar de referencia: Ekman, E. L. 510 (S).

\section{Dalechampia scandens L.}

Hierba voluble. Perenne. Nativa. 0-500 m.

Distribución: CAI, GMB, GUA, SPE.

Ejemplar de referencia: Zuloaga, F. O. 6698 (SI).

\section{Dalechampia stenosepala Müll. Arg.}

Hierba voluble. Perenne. Nativa. 0-500 m.

Distribución: CAI, CAN, GMB, GSM, GUA, SIG, SPE.

Ejemplar de referencia: Zuloaga, F. O. 5765 (SI).

Dalechampia stipulacea Müll. Arg.

Hierba voluble. Perenne. Nativa. 0-500 m.

Distribución: CAN, GMB, GUA, IGU, SPE.

Ejemplar de referencia: Zuloaga, F. O. 5043 (SI).

Dalechampia ulmifolia Chodat \& Hassl.

Subarbusto. Nativa. 0-500 m.

Distribución: APO, SIG.

Ejemplar de referencia: Renvoize, S. A. 3073 (SI).

Ditaxis Vahl ex A. Juss.

Ditaxis acaulis Herter ex Arechav.

Hierba. Perenne. Nativa. 0-500 m.

Distribución: APO, CAN, SIG.

Ejemplar de referencia: Jörgensen, P. 667 (BAB).

Ditaxis rosularis Pax $\& \mathrm{~K}$. Hoffm. $\left.{ }^{(* *}\right)$

Hierba. Perenne. Nativa. 0-500 m.
Distribución: CAN.

Ejemplar de referencia: Niederlein, G. 87.

\section{Euphorbia L.}

Euphorbia adenoptera Bertol. Hierba. Anual. Nativa. 0-500 m.

Distribución: APO.

Ejemplar de referencia: Molfino, J. F. s.n. (BAA).

Euphorbia cyathophora Murray

Hierba. Anual. Introducida. 0-500 m.

Distribución: CAP, ELD, IGU.

Ejemplar de referencia: Eskuche, U. G. 371 (CORD).

\section{Euphorbia helioscopia L.}

Hierba. Anual o Bianual. Introducida. 0-500 m. Distribución: GMB.

Ejemplar de referencia: Múlgura, M. E. 2722 (SI).

\section{Euphorbia heterophylla L.}

Hierba. Anual. Nativa. 0-800 m.

Distribución: APO, CAI, CAP, ELD, GMB, GUA, IGU, LNA, SIG, SJA, SPE.

Ejemplar de referencia: Deginani, N. B. 1156 (SI).

\section{Euphorbia hirta L. var. hirta}

Hierba. Anual. Introducida. 0-800 m.

Distribución: ELD, GMB, GSM, SJA.

Ejemplar de referencia: Zuloaga, F. O. 5082 (SI).

Euphorbia hirta L. var. ophthalmica (Pers.) Allem \& Irgang

Hierba. Anual. Introducida. 0-500 m.

Distribución: GUA.

Ejemplar de referencia: Keller, H. A. 147 (CTES).

\section{Euphorbia hypericifolia L.}

Hierba. Perenne. Nativa. 0-700 m.

Distribución: CAI, CAN, LNA, SPE.

Ejemplar de referencia: Morrone, O. 2171 (SI).

\section{Euphorbia insulana Vell.}

Hierba. Anual. Nativa. 0-500 m.

Distribución: SPE.

Ejemplar de referencia: Schwindt, E. 1538 (LIL). 
Euphorbia klotzschii Oudejans var. argentina (Müll. Arg. ex Griseb.) Oudejans

Hierba. Anual. Nativa. 0-500 m.

Distribución: CAI, IGU, GSM, SIG.

Ejemplar de referencia: Bertoni, M. S. 1076 (LIL).

\section{Euphorbia klotzschii Oudejans var. klotzschii}

Hierba. Anual. Nativa. 0-500 m.

Distribución: CAP, LNA, SIG.

Ejemplar de referencia: Múlgura, M. E. 2298 (SI).

\section{Euphorbia kurtzii Subils}

Hierba. Anual. Nativa. 0-500 m.

Distribución: CAI.

Ejemplar de referencia: Zuloaga, F. O. 5395 (SI).

\section{Euphorbia maculata L.}

Hierba. Perenne. Introducida. 0-500 m.

Distribución: CAI, CAP, GUA, IGU. (LIL).

Ejemplar de referencia: Bertoni, M. S. 3854

Euphorbia papillosa A. St.-Hil. var. cabrerae Subils

Hierba. Perenne. Nativa. 0-500 m.

Distribución: CAN, SIG, SJA.

Ejemplar de referencia: Rodríguez, F. M. 566 (SI).

Euphorbia papillosa A. St.-Hil. var. papillosa

Hierba. Perenne. Nativa. 0-800 m.

Distribución: APO, CAI, CAN, CAP, CON, IGU, SIG, SJA, SPE.

Ejemplar de referencia: Zuloaga, F. O. 7217 (SI).

\section{Euphorbia pentadactyla Griseb.}

Hierba. Anual. Nativa. 0-500 m.

Distribución: IGU.

Ejemplar de referencia: Hunziker, J. H. 10983 (SI).

\section{Euphorbia peplus L.}

Hierba. Anual. Introducida. 0-500 m.

Distribución: GMB.

Ejemplar de referencia: Keller, H. A. 11714 (CTES).

\section{Euphorbia potentilloides Boiss.}

Hierba. Perenne. Nativa. 300-700 m.

Distribución: APO, CAI, CAN, CAP, SIG.

Ejemplar de referencia: Biganzoli, F. 616 (SI).
Euphorbia sciadophila Boiss.

Hierba. Anual. Nativa. 0-800 m.

Distribución: CAI, CAN, ELD, GMB, GUA, IGU, MON, SPE.

Ejemplar de referencia: Biganzoli, F. 1268 (SI).

Euphorbia selloi (Klotzsch \& Garcke) Boiss.

Hierba. Perenne. Nativa. 0-500 m.

Distribución: APO, CAI, CAN, CAP, CON, IGU, SPE.

Ejemplar de referencia: Morrone, O. 1733 (SI).

Euphorbia serpens Kunth var. microphylla Müll. Arg.

Hierba. Perenne. Nativa. 0-500 m.

Distribución: SIG.

Ejemplar de referencia: Schwarz, G. J. 2176 (LIL).

Euphorbia serpens Kunth var. serpens

Hierba. Perenne. Nativa. 0-800 m.

Distribución: CAP, GMB, GSM, SIG.

Ejemplar de referencia: Múlgura, M. E. 2768 (SI).

Euphorbia spathulata Lam.

Hierba. Anual. Introducida. 0-700 m.

Distribución: APO, CAN, LNA, SIG.

Ejemplar de referencia: Schwarz, G. J. 5088 (LIL).

Euphorbia stenophylla (Klotzsch \& Garcke) Boiss.

Hierba. Perenne. Nativa. 0-500 m.

Distribución: APO, CAN, CON, GMB.

Ejemplar de referencia: Montes, J. E. 1613 (LIL).

\section{Gymnanthes Sw.}

Gymnanthes discolor (Spreng.) Müll. Arg.

Arbusto. Nativa. 0-500 m.

Distribución: GMB.

Ejemplar de referencia: Johnson, A. E. 896 (SI).

\section{Jatropha L.}

\section{Jatropha curcas L.}

Arbusto o arbolito. Introducida. 0-500 m.

Distribución: CAP, SPE.

Ejemplar de referencia: Keller, H. A. 4170 (CTES). 


\section{Manihot Mill.}

Manihot grahamii Hook.

Arbusto o arbolito. Nativa. 0-700 m.

Distribución: CAI, CAN, ELD, GMB, IGU, SIG, SPE.

Ejemplar de referencia: Daviña, J. 106 (CTES, G, MNES).

\section{Manihot hunzikeriana Mart. Crov. $\left.{ }^{* * *}\right)$}

Subarbusto. Nativa. 0-500 m.

Distribución: APO, CAI, CAN, CAP, SIG, SJA.

Ejemplar de referencia: Múlgura, M. E. 2321 (SI).

\section{Microstachys A. Juss.}

Microstachys hispida (Mart.) Govaerts

Arbusto o subarbusto. Nativa. 0-500 m.

Distribución: GMB, SIG, SPE.

Ejemplar de referencia: Múlgura, M. E. 2066 (SI).

\section{Philyra Klotzsch}

\section{Philyra brasiliensis Klotzsch}

Arbusto o árbol. Nativa. 0-500 m.

Distribución: GSM, GUA, SPE.

Ejemplar de referencia: Pérez, A. 29 (G,LP, MNES).

\section{Ricinus L.}

\section{Ricinus communis $\mathrm{L}$.}

Hierba o subarbusto. Perenne. Introducida. 0-500 m.

Distribución: ELD, GUA, IGU, MON, SPE.

Ejemplar de referencia: Vanni, R. O. 3798 (CTES).

\section{Sapium P. Browne}

\section{Sapium glandulosum (L.) Morong}

Arbusto o árbol. Nativa. 0-700 m.

Distribución: GMB, GSM, GUA, IGU.

Ejemplar de referencia: Múlgura, M. E. 2664 (SI).

Sapium haematospermum Müll. Arg. Arbusto o árbol. Nativa. 0-700 m.
Distribución: CAN, IGU, SIG, SJA.

Ejemplar de referencia: Morrone, O. 1743 (SI).

\section{Sebastiania Spreng.}

Sebastiania brasiliensis Spreng.

Arbusto o árbol. Nativa. 0-800 m.

Distribución: APO, CAI, CAN, CAP, ELD, GMB, GSM, GUA, IGU, LNA, MON, SIG, SPE.

Ejemplar de referencia: Biganzoli, F. 450 (SI).

Sebastiania commersoniana (Baill.) L.B. Sm. \& Downs

Arbusto o árbol. Nativa. 0-700 m.

Distribución: CAI, CAP, CON, GMB, GSM, GUA, MON, SIG, SPE.

Ejemplar de referencia: Guaglianone, E. R. 2901 (SI).

Sebastiania schottiana (Müll. Arg.) Müll. Arg. Arbusto. Nativa. 0-800 m.

Distribución: GMB, GUA, SIG, SPE.

Ejemplar de referencia: Deginani, N. B. 1185 (SI).

Sebastiania serrata (Baill. ex Müll. Arg.) Müll. Arg. Arbusto o árbol. Nativa. 0-500 m.

Distribución: IGU, SIG.

Ejemplar de referencia: Morrone, O. 1076 (SI).

Sebastiania subsessilis (Müll. Arg.) $\mathrm{Pax}\left({ }^{* * *}\right)$

Arbusto. Nativa. 0-500 m.

Distribución: CAN, CON, SIG.

Ejemplar de referencia: Múlgura, M. E. 2041 (SI).

\section{Stillingia L.}

Stillingia salpingadenia (Müll. Arg.) Huber Arbusto o subarbusto. Nativa. 0-500 m. Distribución: CAI, CAN, CAP, SIG. Ejemplar de referencia: Schwarz, E. A. 1354 (LIL).

\section{Tetrorchidium Poepp.}

\section{Tetrorchidium rubrivenium Poepp. $\left.{ }^{* * *}\right)$}

Árbol. Nativa. 0-500 m.

Distribución: ELD, GMB, IGU, SPE.

Ejemplar de referencia: Herrera, J. 243 (SI). 


\section{Tragia L.}

Tragia giardelliae M.M. Gut. \& Múlgura $\left(^{* * *}\right)$ Hierba voluble. Perenne. Nativa. 0-500 m. Distribución: ELD, GMB, GUA, IGU, SIG.

Ejemplar de referencia: Zuloaga, F. O. 6685 (SI).

\section{Tragia melochioides Griseb.}

Subarbusto. Nativa. 0-500 m.

Distribución: APO, CAN, SIG, SJA.

Ejemplar de referencia: Zuloaga, F. O. 5340 (SI).

Tragia paxii Lourteig \& O’Donell (**)

Hierba voluble. Perenne. Nativa. 0-500 m.

Distribución: CAI, CAN, ELD, GMB, IGU, LNA, SIG, SPE.

Ejemplar de referencia: Hunziker, J. H. 825 (CORD, LIL).

\section{Tragia polyandra Vell. (***)}

Hierba voluble. Perenne. Nativa. 0-800 m.

Distribución: CAI, CAN, CON, GSM, LNA, SIG, SPE.

Ejemplar de referencia: Montes, J. E. 4023 (LP).

Tragia uberabana Müll. Arg.

Hierba. Perenne. Nativa. 0-700 m.

Distribución: APO, CAI, CAN, CAP, CON, SIG, SJA, SPE. (SI).

Ejemplar de referencia: Zuloaga, F. O. 5785

\section{Tragia volubilis $L$.}

Hierba voluble. Perenne. Nativa. 0-600 m.

Distribución: APO, CAI, CAN, GMB, GSM, GUA, IGU, LNA, MON, SIG, SPE.

Ejemplar de referencia: Biganzoli, F. 1396 (SI).

\section{Vernicia Lour.}

Vernicia fordii (Hemsl.) Airy Shaw ${ }^{* * *}$ )

Árbol. Introducida. 0-200 m.

Distribución: CAI, CAN, GSM, GUA, LNA, MON, SIG, SJA.

Ejemplar de referencia: Keller, H. A. 4293 (CTES).

\section{FABACEAE}

\section{Acosmium Schott}

Acosmium subelegans (Mohlenbr.) Yakovlev $\left({ }^{* *}\right)$

Árbol. Nativa. 0-500 m.

Distribución: CAN, SIG.

Ejemplar de referencia: Múlgura, M. E. 2847 (SI).

Adesmia DC., nom. cons.

Adesmia bicolor (Poir.) DC.

Hierba. Perenne. Nativa. 0-500 m.

Distribución: SIG.

Ejemplar de referencia: Keller, H. A. 1290 (CTES).

Adesmia globosa Davyt \& Izag.

Hierba. Perenne. Nativa. 0-500 m.

Distribución: CAP, CON, SJA. (SI).

Ejemplar de referencia: Cabrera, A. L. 28650

\section{Adesmia incana Vogel}

Hierba. Perenne. Nativa. 0-500 m.

Distribución: APO, CAN.

Ejemplar de referencia: Múlgura, M. E. 2903 (SI).

Adesmia punctata (Poir.) DC.

Hierba. Perenne. Nativa. 0-300 m.

Distribución: CAP.

Ejemplar de referencia: Seijo, G. J. 650 (MNES, SI).

\section{Aeschynomene L.}

\section{Aeschynomene americana L.}

Subarbusto. Nativa. 0-500 m.

Distribución: SPE.

Ejemplar de referencia: Keller, H. A. 726 (CTES).

\section{Aeschynomene denticulata Rudd}

Hierba. Anual o Bianual. Nativa. 0-500 m.

Distribución: CAP, SIG, SPE.

Ejemplar de referencia: Burkart, A. 14040 (SI). 
Aeschynomene evenia $C$. Wright

Hierba. Nativa. 0-500 m.

Distribución: SIG.

Ejemplar de referencia: Keller, H. A. 7819 (CTES).

Aeschynomene falcata (Poir.) DC.

Hierba. Perenne. Nativa. 0-800 m.

Distribución: APO, CAN, CAP, MON, SIG.

Ejemplar de referencia: Seijo, G. J. 299 (MNES, SI).

Aeschynomene histrix Poir. var. incana (Vogel) Benth.

Hierba. Perenne. Nativa. 0-500 m.

Distribución: CAN, SIG.

Ejemplar de referencia: Grüner, G. 1049 (SI).

Aeschynomene histrix Poir. var. multijuga (Chodat \& Hassl.) Rudd (***)

Hierba. Perenne. Nativa. 0-500 m.

Distribución: CAN, SIG.

Ejemplar de referencia: Burkart, A. 15344 (US).

Aeschynomene lorentziana Bacigalupo \& Vanni Hierba. Perenne. Nativa. 0-500 m.

Distribución: SIG.

Ejemplar de referencia: Eskuche, U. G. 5228 (SI).

\section{Aeschynomene montevidensis Vogel}

Hierba o arbusto. Perenne. Nativa. 0-500 m.

Distribución: CAN, CAP, IGU, SIG, SJA.

Ejemplar de referencia: Grüner, G. 536 (SI).

\section{Aeschynomene sensitiva $S w$.}

Arbusto o arbolito. Nativa. 0-500 m.

Distribución: CAP.

Ejemplar de referencia: Honfi, A. I. 956 (SI, MNES).

\section{Albizia Durazz.}

Albizia edwallii (Hoehne) Barneby \& J.W. Grimes (***)

Árbol. Nativa. 200-600 m.

Distribución: GUA, IGU.

Ejemplar de referencia: Vanni, R. O. 3309 (BAB, CTES).

Albizia inundata (Mart.) Barneby \& J.W. Grimes Árbol. Nativa. 0-500 m.
Distribución: CAN.

Ejemplar de referencia: Rodríguez, F. M. 111 (SI).

Albizia niopoides (Spruce ex Benth.) Burkart

Árbol. Nativa. 0-700 m.

Distribución: CAN, CAP, GMB, LNA, OBE, SIG.

Ejemplar de referencia: Burkart, A. 14236 (SI.

\section{Anadenanthera Speg.}

Anadenanthera colubrina (Vell.) Brenan var. cebil (Griseb.) Altschul

Arbusto o árbol. Nativa. 0-500 m.

Distribución: CAN, SIG.

Ejemplar de referencia: Grüner, G. s.n. (SI).

Ancistrotropis A. Delgado

Ancistrotropis clitorioides (Mart. ex Benth.)

A. Delgado

Enredadera. Perenne. Nativa. 0-500 m.

Distribución: APO, CAN, SIG, SJA, SPE.

Ejemplar de referencia: Zuloaga, F. O. 6651 (SI).

Andira Lam., nom. cons.

Andira inermis (W. Wright) DC. $\left({ }^{* * *}\right)$

Árbol. Introducida. 0-500 m.

Distribución: SIG.

Ejemplar de referencia: Keller, H. A. 10696 (CTES).

Apuleia Mart., nom. cons.

Apuleia leiocarpa (Vogel) J.F. Macbr.

Árbol. Nativa. 100-700 m.

Distribución: ELD, GUA, IGU, OBE, SJA, SIG, SPE. Ejemplar de referencia: Biganzoli, F. 1285 (SI).

\section{Arachis L.}

Arachis burkartii Handro

Hierba. Perenne. Nativa. 0-500 m.

Distribución: APO, CAN.

Ejemplar de referencia: Krapovickas, A. 29116 (SI). 
Arachis glabrata Benth.

Hierba. Perenne. Nativa. 0-500 m.

Distribución: APO, CAN, SIG.

Ejemplar de referencia: Krapovickas, A. 7934 (CTES, SI).

Ateleia (DC.) Benth.

Ateleia glazioveana Baill. $\left.{ }^{* * *}\right)$

Árbol. Nativa. 0-700 m. SPE.

Distribución: CAI, GMB, GUA, MON, SJA,

Ejemplar de referencia: Niederlein, G. 2189 (BA, SI).

\section{Bauhinia L.}

Bauhinia affinis Vogel $(* * *)$

Arbusto. Nativa. 0-800 m.

Distribución: GMB, IGU.

Ejemplar de referencia: Morrone, O. 2003 (SI).

Bauhinia forficata Link subsp. pruinosa (Vogel) Fortunato \& Wunderlin

Arbusto o árbol. Nativa. 0-800 m.

Distribución: APO, CAI, CAN, CON, ELD, GMB, GSM, GUA, IGU, OBE, SIG.

Ejemplar de referencia: Zuloaga, F. O. 5495 (SI).

Bauhinia uruguayensis Benth.

Arbusto o árbol. Nativa. 0-500 m.

Distribución: MAY, SJA.

Ejemplar de referencia: Schwarz, G. J. 4117 (LIL, SI).

\section{Calliandra Benth.}

Calliandra brevicaulis Micheli var. brevicaulis

Subarbusto. Nativa. 0-500 m.

Distribución: CAN, CAP, SIG.

Ejemplar de referencia: Zuloaga, F. O. 7212 (SI).

Calliandra brevicaulis Micheli var. glabra Chodat \& Hassl.

Subarbusto. Nativa. 100-300 m.

Distribución: CAN, CAP, SIG.

Ejemplar de referencia: Burkart, A. 15242 (SI).
Calliandra brevipes Benth. $\left.{ }^{* * *}\right)$

Arbusto. Nativa. 0-500 m.

Distribución: CAI, ELD, GUA, MON, SPE.

Ejemplar de referencia: Daviña, J. 272 (MNES, SI).

Calliandra foliolosa Benth. $\left.{ }^{* * *}\right)$

Árbol. Nativa. 0-800 m.

Distribución: APO, CAI, CAN, ELD, GMB, GUA, IGU, LNA, MAY, MON, SIG, SJA, SPE.

Ejemplar de referencia: Niederlein, G. 315 (BA, SI).

Calliandra longipes Benth. $\left({ }^{* * *}\right)$

Arbusto. Nativa. 200-800 m.

Distribución: SIG.

Ejemplar de referencia: Biganzoli, F. 612 (SI).

Calliandra parvifolia (Hook. f. \& Arn.) Speg.

Arbusto. Nativa. 0-700 m.

Distribución: APO, GSM, GUA, IGU, LNA, SIG.

Ejemplar de referencia: Schinini, A. 19886 (CTES).

Calliandra tweedii Benth.

Arbusto. Nativa. 0-600 m.

Distribución: APO, CAI, CAN, GUA, IGU, MON, OBE, SJA, SPE.

Ejemplar de referencia: Biganzoli, F. 653 (SI).

\section{Calopogonium Desv.}

Calopogonium caeruleum (Benth.) C. Wright ex Sauv.

Liana. Perenne. Nativa. 0-700 m.

Distribución: CAP, SIG.

Ejemplar de referencia: Rodríguez, 205 (SI).

Calopogonium sericeum (Benth.) Chodat \& Hassl.

Hierba o subarbusto. Nativa. 0-500 m.

Distribución: CAI, CAN, CAP, IGU, SIG.

Ejemplar de referencia: Fortunato, R. H. 8048 (BAB, SI).

Camptosema Hook. \& Arn.

Camptosema rubicundum Hook. \& Arn.

Liana. Perenne. Nativa. 0-500 m.

Distribución: APO, CAN.

Ejemplar de referencia: Zuloaga, F. O. 8106 (SI). 
Camptosema scarlatinum (Mart. ex Benth.) Burkart $\left.{ }^{(* *}\right)$

Enredadera. Perenne. Nativa. 0-500 m.

Distribución: CAN, GSM, SIG.

Ejemplar de referencia: Zuloaga, F. O. 5302 (SI).

\section{Canavalia DC.}

Canavalia brasiliensis Mart. ex Benth.

Liana. Perenne. Nativa. 0-500 m.

Distribución: SIG, SPE.

Ejemplar de referencia: Morrone, O. 1856 (SI).

\section{Canavalia ensiformis (L.) DC.}

Liana. Perenne. Introducida. 0-500 m.

Distribución: GUA, SPE.

Ejemplar de referencia: Deginani, N. B. 1169 (SI).

\section{Centrosema (DC.) Benth.}

Centrosema brasilianum (L.) Benth. $\left.{ }^{(* *}\right)$

Hierba o subarbusto. Perenne. Nativa. 0-500 m. Distribución: CAN, IGU, LNA.

Ejemplar de referencia: Fortunato, R. H. 8006 $(B A B)$.

Centrosema sagittatum (Humb. \& Bonpl. ex Willd.) Brandegee ex L. Riley

Hierba o subarbusto. Perenne. Nativa. 0-500 m.

Distribución: CAI, IGU, SIG.

Ejemplar de referencia: Biganzoli, F. 902 (SI).

Centrosema virginianum (L.) Benth.

Hierba o subarbusto. Perenne. Nativa. 0-700 m. Distribución: CAI, CAN, CAP, ELD, IGU.

Ejemplar de referencia: Keller, H. A. 80 (CTES, SI).

\section{Chamaecrista Moench}

Chamaecrista desvauxii (Collad.) Killip var. latistipula (Benth.) G.P. Lewis

Hierba o subarbusto. Perenne. Nativa. 100$700 \mathrm{~m}$

Distribución: SIG.

Ejemplar de referencia: Zuloaga, F. O. 5315 (SI).
Chamaecrista desvauxii (Collad.) Killip var. mollissima (Benth.) H.S. Irwin \& Barneby $\left({ }^{* * *}\right)$

Arbusto o subarbusto. Nativa. 0-500 m.

Distribución: SIG.

Ejemplar de referencia: Zuloaga, F. O. 5323 (SI).

\section{Chamaecrista flexuosa (L.) Greene}

Hierba o subarbusto. Perenne. Nativa. 0-500 m. Distribución: SJA.

Ejemplar de referencia: Morrone, O. 603 (SI).

Chamaecrista nictitans (L.) Moench var. patellaria (DC. ex Collad.) Kartesz \& Gandhi

Hierba. Anual. Nativa. 0-500 m.

Distribución: APO, CAI, CAP, CON, IGU, LNA, SIG, SJA.

Ejemplar de referencia: Dematteis, M. 221 (MNES, SI).

Chamaecrista repens (Vogel) H.S. Irwin \& Barneby

Hierba o subarbusto. Perenne. Nativa. 0-500 m.

Distribución: CAN, CAP, CON, SIG.

Ejemplar de referencia: Morrone, O. 1744 (SI).

Chamaecrista rotundifolia (Pers.) Greene

Hierba o subarbusto. Perenne. Nativa. 0-500 m. Distribución: APO, CAN.

Ejemplar de referencia: Keller, H. A. 8113 (CTES).

\section{Chamaecrista serpens (L.) Greene}

Hierba o subarbusto. Perenne. Nativa. 0-500 m. Distribución: CAP.

Ejemplar de referencia: Dematteis, M. 194 (CTES).

\section{Chloroleucon Britton \& Rose}

Chloroleucon tenuiflorum (Benth.) Barneby \& J.W. Grimes

Árbol. Nativa. 0-500 m.

Distribución: CAP.

Ejemplar de referencia: Rodríguez, F. M. 24 (BA).

\section{Clitoria L.}

Clitoria epetiolata Burkart

Hierba o subarbusto. Perenne. Nativa. 0-500 m. 
Distribución: SJA.

Ejemplar de referencia: Keller, H. A. 4831 (CTES).

\section{Clitoria nana Benth.}

Hierba o subarbusto. Perenne. Nativa. 0-500 m.

Distribución: APO, CON.

Ejemplar de referencia: Biganzoli, F. 1674 (SI).

\section{Cochliasanthus Trew}

\section{Cochliasanthus caracalla (L.) Trew}

Hierba o enredadera. Perenne. Nativa. 0-500 m. Distribución: CAN, GSM, LNA, SIG, SPE.

Ejemplar de referencia: Guillén, R. 197 (CTES, $G, M N E S, S I$ ).

\section{Collaea DC}

Collaea argentina Griseb.

Subarbusto. Nativa. 0-500 m.

Distribución: APO, SJA.

Ejemplar de referencia: Múlgura, M. E. 4375 (SI).

Collaea paraguariensis (Hassl.) R. García $\left({ }^{* * *}\right)$

Hierba o subarbusto. Nativa. 0-500 m.

Distribución: APO, CAI, SIG.

Ejemplar de referencia: Fortunato, R. H. 8043 (BAB, SI).

Collaea stenophylla (Hook. \& Arn.) Benth.

Subarbusto. Nativa. 0-500 m. SIG.

Distribución: APO, CAN, CAP, CON, SJA,

Ejemplar de referencia: Morrone, O. 1121 (SI).

\section{Condylostylis Piper}

Condylostylis candida (Vell.) A. Delgado

Enredadera. Perenne. Nativa. 0-500 m.

Distribución: CAI, GMB, GUA, LNA, MON, SIG, SJA, SPE.

Ejemplar de referencia: Morrone, O. 731 (SI).

\section{Copaifera L.}

Copaifera langsdorffii Desf. (**)

Árbol. Nativa. 0-500 m.

Distribución: IGU.

Ejemplar de referencia: Rodríguez, F. M. 795 (SI).

Cratylia Mart. ex Benth.

Cratylia intermedia (Hassl.) L.P. Queiroz \& R. Monteiro ${ }^{* * *}$ )

Liana. Nativa. 0-500 m.

Distribución: GMB, IGU.

Ejemplar de referencia: Vanni, R. O. 2781 (CTES).

\section{Crotalaria L.}

\section{Crotalaria balansae Micheli}

Hierba o subarbusto. Perenne. Nativa. 0-500 m. Distribución: CAN, SJA.

Ejemplar de referencia: Burkart, A. 15403 (SI).

\section{Crotalaria incana $\mathrm{L}$.}

Hierba o arbusto. Perenne. Nativa. 0-800 m.

Distribución: APO, CAI, CAN, CAP, CON, GMB, GSM, GUA, LNA, MAY, SIG, SPE.

Ejemplar de referencia: Cabrera, A. L. 29060 (SI).

Crotalaria martiana Benth. subsp. mohlenbrockii (Windler \& S.G. Skinner) Planchuelo ${ }^{* * *}$ )

Subarbusto. Nativa. 0-500 m.

Distribución: CAP.

Ejemplar de referencia: Molfino J. F. s.n. (BAF).

Crotalaria micans Link

Hierba o subarbusto. Perenne. Nativa. 0-700 m.

Distribución: CAN, CAP, ELD, GMB, IGU, SIG, SPE.

Ejemplar de referencia: Rodríguez, M. 886 (MNES, SI).

\section{Crotalaria ochroleuca G. Don $\left(^{*}\right)$}

Subarbusto. Introducida. 0-500 m.

Distribución: ELD.

Ejemplar de referencia: Keller, H. A. 14022 (CTES). 


\section{Crotalaria spectabilis Roth}

Hierba o arbusto. Perenne. Introducida. 0-500 m.

Distribución: CAN, ELD.

Ejemplar de referencia: Schinini, A. 19848 (CTES).

\section{Crotalaria stipularia Desv.}

Hierba. Anual. Nativa. 0-500 m.

Distribución: CAN, CAP, CON, GSM, IGU, SIG.

Ejemplar de referencia: Krapovickas, A. 12110 (CTES, SI).

\section{Crotalaria tweediana Benth.}

Hierba o arbusto. Perenne. Nativa. 0-500 m.

Distribución: CAN, SIG, SJA. (SI).

Ejemplar de referencia: Montes, J. E. 1374

Crotalaria vespertilio Benth.

Arbusto o subarbusto. Nativa. 200-800 m.

Distribución: CAN, CAP, IGU, SIG.

Ejemplar de referencia: Zuloaga, F. O. 5258 (SI,CTES).

\section{Cyclolobium Benth.}

Cyclolobium brasiliense Benth. ${ }^{* * *}$ )

Árbol. Nativa. 100-800 m.

Distribución: GMB.

Ejemplar de referencia: Herrera, J. 328 (SI).

\section{Dahlstedtia Malme}

Dahlstedtia burkartii M.J. Silva \& A.M.G. Azevedo (**)

Subarbusto. Endémica. 0-200 m.

Distribución: SPE.

Ejemplar de referencia: Keller, H. A. 2826 (CTES,K).

Dahlstedtia muehlbergiana (Hassl.) M.J. Silva \& A.M.G. Azevedo

Árbol. Nativa. 0-500m.

Distribución: CAI, CAN, CAP, ELD.

Ejemplar de referencia: Lillo, M. 10574 (LIL, SI).

\section{Dalbergia L. f.}

Dalbergia frutescens (Vell.) Britton

Liana. Perenne. Nativa. 0-700 m.

Distribución: CAI, CON, GMB, GUA, IGU, LNA, SIG, SJA, SPE.

Ejemplar de referencia: Deginani, N. B. 1056 (SI).

\section{Desmanthus Willd.}

Desmanthus hexapetalus (Micheli) J.F. Macbr. $(* * *)$

Hierba o subarbusto. Perenne. Nativa. 200$500 \mathrm{~m}$.

Distribución: CAI, SIG.

Ejemplar de referencia: Pérez-Moreau, R. A. 14456 (SI).

Desmanthus paspalaceus (Lindm.) Burkart

Hierba o subarbusto. Perenne. Nativa. 0-500 m.

Distribución: CAI, CON.

Ejemplar de referencia: Biganzoli, F. 1653 (SI).

Desmanthus tatuhyensis Hoehne

Hierba o subarbusto. Perenne. Nativa. 0-500 m.

Distribución: APO, CAN, CON, SPE.

Ejemplar de referencia: Burkart, A. 14379 (SI).

Desmanthus virgatus (L.) Willd.

Hierba o subarbusto. Perenne. Nativa. 0-700 m.

Distribución: APO, CAI, CAN, CON, SIG.

Ejemplar de referencia: Morrone, O. 571 (SI).

Desmodium Desv., nom. cons.

Desmodium adscendens (Sw.) DC. ${ }^{* * *}$ )

Hierba. Perenne. Nativa. 0-500 m.

Distribución: ELD.

Ejemplar de referencia: Schwindt, E. 2294 (CTES).

Desmodium affine Schltdl.

Hierba. Perenne. Nativa. 0-700 m.

Distribución: CAI, CON, ELD, GMB, GSM, GUA, IGU, MAY, SIG, SPE.

Ejemplar de referencia: Zuloaga, F. O. 6625 (SI). 


\section{Desmodium arechavaletae Burkart}

Subarbusto. Nativa. 0-500 m.

Distribución: APO, CON, SJA.

Ejemplar de referencia: Krapovickas, A. 28855

(CTES, G, MBM, MO, SI).

\section{Desmodium barbatum (L.) Benth.}

Hierba. Perenne. Nativa. 0-500 m.

Distribución: CAN, CAP, GUA, LNA, SIG, SPE.

Ejemplar de referencia: Mutinelli, A. 15 (SI).

\section{Desmodium cuneatum Hook. \& Arn.}

Subarbusto. Nativa. 0-500 m.

Distribución: APO, CAN, CON, GSM, IGU, LNA, SIG, SJA.

Ejemplar de referencia: Zuloaga, F. O. 4900 (SI).

\section{Desmodium hassleri (Schindl.) Burkart}

Subarbusto. Nativa. 0-500 m.

Distribución: CAI, CAN, ELD, MON, OBE, SIG.

Ejemplar de referencia: Pedersen, T. M. 3219 (SI).

\section{Desmodium incanum $D C$.}

Hierba. Perenne. Nativa. 0-700 m.

Distribución: APO, CAI, CAN, CAP, CON, ELD, GMB, GSM, IGU, LNA, SIG, SJA.

Ejemplar de referencia: Morrone, O. 1058 (SI).

Desmodium leiocarpum (Spreng.) G. Don (***)

Subarbusto. Nativa. 0-500 m.

Distribución: CAN, GSM, IGU, MON, SIG, SPE.

Ejemplar de referencia: Biganzoli, F. 38 (SI).

Desmodium microcarpum (Rusby) L.C.P.

Lima, Tozzi \& L.P. Queiroz

Subarbusto. Nativa. 0-600 m.

Distribución: CAN, CON, ELD, GSM, IGU, SIG.

Ejemplar de referencia: Mutinelli, A. 18 (SI).

\section{Desmodium pachyrrhizum Vogel}

Hierba. Perenne. Nativa. 0-500 m.

Distribución: CAI, CAN, CON, IGU, SJA.

Ejemplar de referencia: Montes, J. E. 9646 (CTES).
Desmodium polygaloides Chodat \& Hassl.

Hierba. Perenne. Nativa. 0-500 m.

Distribución: CAN, CAP, LNA.

Ejemplar de referencia: Burkart, A. 14771 (SI).

Desmodium tortuosum (Sw.) DC.

Subarbusto. Nativa. 0-500 m.

Distribución: CAN, ELD, LNA.

Ejemplar de referencia: Schinini, A. 18945 (CTES, K).

Desmodium uncinatum (Jacq.) DC.

Hierba o subarbusto. Perenne. Nativa. 0-600 m. Distribución: CAI, CAN, CAP, IGU, GMB, GSM, GUA, MON, OBE, SIG, SPE.

Ejemplar de referencia: Deginani, N. B. 1650 (SI).

\section{Desmodium venosum Vogel}

Hierba o subarbusto. Perenne. Nativa. 0-500 m. Distribución: CON, ELD.

Ejemplar de referencia: Schwindt, E. 2815 (LIL).

\section{Dioclea Kunth}

Dioclea burkartii R.H. Maxwell

Liana. Perenne. Nativa. 0-500 m.

Distribución: CAP.

Ejemplar de referencia: Rodríguez, F. M. 203 (SI).

Dioclea violacea Mart. ex Benth.

Liana. Nativa. 0-500 m.

Distribución: ELD, IGU, SIG.

Ejemplar de referencia: Spegazzini, C. L. 372 (LPS).

Dioclea virgata (Rich.) Amshoff (***)

Liana. Perenne. Nativa. 0-500 m.

Distribución: CAN.

Ejemplar de referencia: Spegazzini, C. L. s.n. (BAB, LPS).

Discolobium Benth.

Discolobium psoraleaefolium Benth.

Subarbusto. Nativa. 0-500 m.

Distribución: CAP.

Ejemplar de referencia: Seijo, G. J. 638 (MNES, SI). 


\section{Enterolobium Mart.}

Enterolobium contortisiliquum (Vell.) Morong Árbol. Nativa. 0-700 m.

Distribución: CAI, CAN, ELD, GUA, SIG.

Ejemplar de referencia: Biganzoli, F. 583 (SI).

\section{Eriosema (DC.) Rchb.}

\section{Eriosema campestre Benth. ${ }^{* * *}$ )}

Hierba o subarbusto. Perenne. Nativa. 0-500 m. Distribución: CAI.

Ejemplar de referencia: Bertoni, M. S. 648 (LIL).

\section{Eriosema glabrum Mart. ex Benth. $\left.{ }^{* * *}\right)$}

Hierba o subarbusto. Perenne. Nativa. 0-500 m.

Distribución: CAN.

Ejemplar de referencia: de Llamas, A. 413 (G).

\section{Eriosema platycarpon Micheli}

Hierba o subarbusto. Perenne. Nativa. 0-500 m.

Distribución: CAN, SIG.

Ejemplar de referencia: Montes, J. E. 825 (SI).

Eriosema rufum (Kunth) G. Don var. macrostachyum (DC.) G. Don $\left(^{* * *}\right)$

Subarbusto. Nativa. 0-500 m.

Distribución: CAI.

Ejemplar de referencia: Martínez Crovetto, $R$. 8717 (SI).

\section{Eriosema tacuaremboense Arechav.}

Hierba. Perenne. Nativa. 0-500 m.

Distribución: APO, CAN, CON, SIG.

Ejemplar de referencia: Múlgura, M. E. 2035 (SI).

\section{Erythrina L.}

\section{Erythrina crista-galli L.}

Arbusto o árbol. Nativa. 0-500 m.

Distribución: CAP, SIG, SPE.

Ejemplar de referencia: Cabrera, A. L. 28999 (SI).

\section{Erythrina falcata Benth.}

Árbol. Nativa. 0-500 m.

Distribución: CAP, ELD, IGU.

Ejemplar de referencia: Burkart, A. 2566 (SI).

\section{Galactia P. Browne}

Galactia australis (Malme) Ceolin \& Miotto

Hierba o subarbusto. Perenne. Nativa. 0-500 m.

Distribución: CAP.

Ejemplar de referencia: sin colector s.n. (LPS).

\section{Galactia benthamiana Micheli}

Hierba o enredadera. Perenne. Nativa. 0-500 m.

Distribución: CAN, CAP, SIG.

Ejemplar de referencia: Biganzoli, F. 622 (SI).

Galactia boavista (Vell.) Burkart

Hierba. Perenne. Nativa. 0-500 m.

Distribución: CAN, CON, GMB, SIG, SJA.

Ejemplar de referencia: Morrone, O. 1029 (SI).

Galactia dimorphophylla Fortunato, S. Sede \& Luckow

Subarbusto. Nativa. 0-300 m.

Distribución: APO, CAP, SJA.

Ejemplar de referencia: Fortunato, R. H. 9066 (BAB).

Galactia gracillima Benth.

Hierba, subarbusto o enredadera. Perenne. Nativa. 0-800 m.

Distribución: CAN, CAP, SJA.

Ejemplar de referencia: Martínez Crovetto, $R$. 8113 (BAB).

\section{Galactia marginalis Benth.}

Hierba o subarbusto. Perenne. Nativa. 0-500 m. Distribución: CAN, GMB, SIG.

Ejemplar de referencia: Biganzoli, F. 60 (SI).

Galactia martioides Burkart $\left({ }^{* *}\right)$

Hierba o enredadera. Perenne. Nativa. 0-500 m. Distribución: CAN, SIG.

Ejemplar de referencia: Van de Venne, H. s.n. (SI).

\section{Galactia neesii DC. $\left.{ }^{* * *}\right)$}

Hierba o subarbusto. Perenne. Nativa. 0-800 m. Distribución: CAI.

Ejemplar de referencia: Pierotti, A. 6591 (SI).

\section{Galactia pretiosa Burkart $\left(^{* * *}\right)$}

Hierba o subarbusto. Perenne. Nativa. 0-500 m.

Distribución: CAN, SIG.

Ejemplar de referencia: Morrone, O. 1668 (SI). 
Galactia striata (Jacq.) Urb.

Hierba o enredadera. Perenne. Nativa. 0-500 m. Distribución: CAI, CAN, CAP, ELD, GSM, IGU, SIG.

Ejemplar de referencia: Bossi, F. 14 (MNES, SI).

\section{Gleditsia L.}

Gleditsia amorphoides (Griseb.) Taub.

Árbol. Nativa. 0-500 m.

Distribución: CAN, ELD.

Ejemplar de referencia: Rodríguez, F. M. 608 (SI).

\section{Holocalyx Micheli}

\section{Holocalyx balansae Micheli}

Árbol. Nativa. 0-500 m.

Distribución: CAN, ELD, GMB, GSM, IGU, OBE, SIG.

Ejemplar de referencia: Biganzoli, F. 313 (SI).

\section{Hymenaea L.}

Hymenaea martiana Hayne $\left(^{* * *}\right)$

Árbol. Nativa. 0-500 m.

Distribución: LNA.

Ejemplar de referencia: Barreiro, J. s.n. (SI).

\section{Indigofera L.}

Indigofera asperifolia Bong. ex Benth.

Hierba. Perenne. Nativa. 0-500 m.

Distribución: APO, CAN, CAP, CON.

Ejemplar de referencia: Morrone, O. 1141 (SI).

\section{Indigofera bongardiana (Kuntze) Burkart}

Hierba. Perenne. Nativa. 0-500 m.

Distribución: CON.

Ejemplar de referencia: Keller, H. A. 5750 (CTES).

\section{Indigofera latifolia Micheli}

Hierba. Perenne. Nativa. 0-500 m.

Distribución: CAN, SIG.

Ejemplar de referencia: de Llamas, A. 617 (BAB).
Indigofera suffruticosa Mill.

Subarbusto. Nativa. 0-800 m.

Distribución: APO, CAI, CAN, CON, GSM, IGU, MON, SIG, SJA.

Ejemplar de referencia: Biganzoli, F. 223 (SI).

Inga Mill.

Inga affinis $D C$.

Árbol. Nativa. 0-500 m.

Distribución: CAN, IGU, SIG, SJA, SPE.

Ejemplar de referencia: Hoc, P. S. S. 38 (BAFC).

Inga marginata Willd.

Árbol. Nativa. 0-700 m.

Distribución: APO, CAI, CAN, CAP, ELD, GMB, GSM, GUA, IGU, LNA, MAY, MON, OBE, SIG, SJA, SPE.

Ejemplar de referencia: Zuloaga, F. O. 6641 (SI).

Inga uraguensis Hook. \& Arn.

Árbol. Nativa. 0-500 m.

Distribución: CAN, CAP, CON, ELD, GSM, IGU, MAY, SIG, SJA, SPE.

Ejemplar de referencia: Zuloaga, F. O. 6471 (SI).

Inga virescens Benth. $\left({ }^{* * *}\right)$

Arbolito. Nativa. 0-800 m.

Distribución: CAI, CAN, GUA, IGU, SIG.

Ejemplar de referencia: Grüner, G. 22 (SI).

\section{Kummerowia Schindl.}

Kummerowia striata (Thunb.) Schindl. $\left(^{(* *}\right)$

Hierba. Anual. Introducida. 0-500 m.

Distribución: ELD, MAY.

Ejemplar de referencia: Keller, H. A. 9814 (CTES).

\section{Lathyrus L.}

Lathyrus crassipes Gillies ex Hook. \& Arn.

Hierba. Anual. Nativa. 0-100 m.

Distribución: CAN, CON, OBE.

Ejemplar de referencia: Seijo, J. G. 4237 (CTES, SI). 
Lathyrus hasslerianus Burkart $\left({ }^{* * *}\right)$

Hierba. Perenne. Nativa. 0-500 m.

Distribución: GUA, IGU, SPE.

Ejemplar de referencia: Seijo, G. J. 2000 (SI).

Lathyrus macrostachys Vogel

Hierba o enredadera. Perenne. Nativa.

0-500 m.

Distribución: CAN, CAP, LNA, SIG.

Ejemplar de referencia: Zuloaga, F. O. 7137 (SI).

\section{Lathyrus nervosus Lam.}

Hierba. Perenne. Nativa. 0-500 m.

Distribución: SPE.

Ejemplar de referencia: Morrone, O. 1559 (SI).

\section{Lathyrus subulatus Lam.}

Hierba. Perenne. Nativa. 0-500 m.

Distribución: SIG.

Ejemplar de referencia: Hassler, E. 420 (SI).

\section{Leptospron (Benth.) A. Delgado}

Leptospron adenanthum (G. Mey.) A. Delgado

Enredadera. Perenne. Nativa. 0-500 m.

Distribución: CON, GMB, SPE.

Ejemplar de referencia: Zuloaga, F. O. 9828 (SI).

\section{Leucaena Benth.}

Leucaena leucocephala (Lam.) de Wit subsp. glabrata (Rose) Zárate

Arbusto o árbol. Introducida. 0-500 m.

Distribución: ELD.

Ejemplar de referencia: Keller, H. A. 5902 (CTES).

\section{Lonchocarpus Kunth}

Lonchocarpus nitidus (Vogel) Benth.

Árbol. Nativa. 0-500 m.

Distribución: APO, CAI, CAN, CAP, IGU, LNA, SJA, SPE.

Ejemplar de referencia: Dematteis, M. 276 (MNES, SI).

\section{Lupinus L.}

Lupinus gibertianus C.P. Sm. var. berroanus (C.P. Sm.) Planchuelo \& E. Fuentes

Hierba. Anual. Nativa. 0-500 m.

Distribución: APO.

Ejemplar de referencia: Arbo, M. M. 2300 (CTES).

\section{Lupinus gibertianus C.P. $\mathrm{Sm}$. var. gibertianus} Hierba. Anual. Nativa. 0-500 m.

Distribución: APO, CAN, OBE. (SI).

Ejemplar de referencia: Zuloaga, F. O. 6564

\section{Lupinus gibertianus $C$.P. Smvar. reineckianus}

(C.P. Sm.) Planchuelo \& E. Fuentes

Hierba. Anual. Nativa. 0-500 m.

Distribución: GSM, SIG.

Ejemplar de referencia: Schwarz, G. J. 10685 (LIL).

Lupinus guaraniticus (Hassl.) C.P. Sm.

Hierba o subarbusto. Perenne. Nativa. 0-500 m. Distribución: GUA, SPE.

Ejemplar de referencia: Niederlein, G. 1129 (SI).

Lupinus lanatus Benth. $\left.{ }^{* * *}\right)$

Hierba. Perenne. Nativa. 0-500 m.

Distribución: CAI.

Ejemplar de referencia: Bertoni, M. S. 638 (LIL).

\section{Lupinus linearis Desr.}

Hierba. Anual. Nativa. 0-500 m.

Distribución: APO, CAI, CAP, GSM.

Ejemplar de referencia: Seijo, G. J. 634 (MNES, SI).

Lupinus paraguariensis Chodat \& Hassl.

Hierba o subarbusto. Perenne. Nativa. 0-500 m. Distribución: APO, CAN, GMB, SIG, SJA.

Ejemplar de referencia: Morrone, O. 1684 (SI).

\section{Lupinus sellowianus Harms $\left({ }^{* * *}\right)$}

Hierba. Perenne. Nativa. 0-500 m.

Distribución: SPE.

Ejemplar de referencia: Krapovickas, A. 23381 (CTES). 


\section{Machaerium Pers.}

\section{Machaerium acutifolium Vogel $(* * *)$}

Árbol. Nativa. 0-500 m.

Distribución: CAP.

Ejemplar de referencia: Parodi, L. R. 4432 (SI).

\section{Machaerium brasiliense Vogel $\left({ }^{* *}\right)$}

Árbol. Nativa.

Distribución: Desconocida.

Ejemplar de referencia: Kermes, E. s.n. (BAB).

\section{Machaerium hirtum (Vell.) Stellfeld $\left({ }^{* *}\right)$}

Árbol. Nativa. 0-500 m.

Distribución: CAP, GUA, IGU, SIG.

Ejemplar de referencia: Grüner, G. 807 (SI).

Machaerium nyctitans (Vell.) Benth.

Árbol. Nativa. 0-500 m.

Distribución: LNA, MON, SPE. (SI).

Ejemplar de referencia: Múlgura, M. E. 3215

\section{Machaerium paraguariense Hassl.}

Árbol. Nativa. 0-500 m.

Distribución: CAP, CON, ELD, GMB, GUA, IGU, SIG, SPE.

Ejemplar de referencia: Zuloaga, F. O. 5438 (SI).

\section{Machaerium stipitatum (DC.) Vogel (***)}

Árbol. Nativa. 0-500 m.

Distribución: CAN, CAP, ELD, GUA, SIG, SPE.

Ejemplar de referencia: Burkart, A. 14724 (SI).

\section{Macroptilium (Benth.) Urb.}

Macroptilium erythroloma (Mart. ex Benth.) Urb.

Hierba o enredadera. Anual. Nativa. 0-800 m.

Distribución: APO, CAI, CAN, CON, ELD, GSM, OBE, SIG, SJA, SPE.

Ejemplar de referencia: Biganzoli, F. 225 (SI).

\section{Macroptilium lathyroides (L.) Urb.}

Hierba. Anual. Nativa. 0-700 m.

Distribución: ELD, CAI, CAP, GMB.

Ejemplar de referencia: Morrone, O. 648 (SI).
Macroptilium longepedunculatum (C. Mart. ex Benth.) Urb.

Enredadera. Nativa. 0-500 m.

Distribución: SJA.

Ejemplar de referencia: Keller, H. A. 4808 (CTES).

Macroptilium panduratum (C. Mart. ex Benth.) Maréchal \& Baudet

Hierba. Perenne. Nativa. 200-700 m.

Distribución: APO, CAN, CON. (SI).

Ejemplar de referencia: Krapovickas, A. 15136

Macroptilium prostratum (Benth.) Urb.

Hierba. Perenne. Nativa. 0-500 m.

Distribución: CAN, SJA.

Ejemplar de referencia: Xifreda, C. C. 550 (SI).

Macroptilium psammodes (Lindm.) S.I. Drewes \& R.A. Palacios

Hierba. Perenne. Nativa. 0-500 m.

Distribución: CON, SIG.

Ejemplar de referencia: Seijo, G. J. 719 (MNES, SI).

\section{Medicago L.}

\section{Medicago lupulina $\mathrm{L}$.}

Hierba. Anual o Bianual. Introducida. 0-500 m. Distribución: GUA.

Ejemplar de referencia: Seijo, G. J. 823 (CTES, $G, M N E S$ ).

\section{Medicago polymorpha $\mathrm{L}$.}

Hierba. Anual. Introducida. 0-500 m.

Distribución: ELD.

Ejemplar de referencia: Keller, H. A. 5903 (CTES).

\section{Melilotus Mill.}

\section{Melilotus albus Desr.}

Hierba o subarbusto. Bianual. Introducida. 0-500 m.

Distribución: CAP, SPE.

Ejemplar de referencia: Bossi, F. 24 (MNES, SI). 
Melilotus officinalis (L.) Lam.

Hierba o subarbusto. Anual o Bianual. Introducida. 0-500 m.

Distribución: GMB.

Ejemplar de referencia: Keller, H. A. 5075 (CTES).

\section{Mimosa L.}

Mimosa adpressa Hook. \& Arn.

Arbusto o subarbusto. Nativa. 0-500 m.

Distribución: CAN.

Ejemplar de referencia: Cabrera, A. L. 29470 (SI).

\section{Mimosa amphigena Burkart}

Arbusto. Nativa. 0-500 m.

Distribución: APO.

Ejemplar de referencia: Spegazzini, C. L. s.n. (LP).

\section{Mimosa balansae Micheli}

Hierba o subarbusto. Perenne. Nativa. 0-400 m. Distribución: CON.

Ejemplar de referencia: Cabrera, A. 28318 (SI).

\section{Mimosa bifurca Benth. var. bifurca}

Arbusto. Nativa. 0-500 m.

Distribución: SIG.

Ejemplar de referencia: Keller, H. A. 7602 (CTES).

Mimosa bifurca Benth. var. desmanthoides (Hoehne) Barneby ${ }^{* * *}$ )

Arbusto o subarbusto. Nativa. 200-300 m.

Distribución: CAN.

Ejemplar de referencia: Montes, J. E. 341 (NY).

Mimosa bimucronata (DC.) Kuntze

Arbusto o árbol. Nativa. 0-800 m.

Distribución: APO, CAN, CAP, GUA, IGU, SIG, SJA, SPE.

Ejemplar de referencia: Deginani, N. B. 1109 (SI).

Mimosa brevipetiolata Burkart var. brevipetiolata $\left.{ }^{* *}\right)$

Hierba o subarbusto. Perenne. Endémica. 200$300 \mathrm{~m}$.

Distribución: CAN, SIG.

Ejemplar de referencia: Llamas, A. 506 (SI).
Mimosa brevipetiolata Burkart var. hirtula (Burkart) Barneby

Hierba o subarbusto. Perenne. Nativa. 100-400 m.

Distribución: CAN, CON.

Ejemplar de referencia: Burkart, A. 15270 (GH, SI).

Mimosa caaguazuensis Barneby $\left(^{* * *}\right)$

Hierba o subarbusto. Perenne. Nativa. 200$500 \mathrm{~m}$.

Distribución: SIG, SPE.

Ejemplar de referencia: Biganzoli, F. 614 (SI).

Mimosa cainguensis Burkart $\left({ }^{* * *}\right)$

Hierba o subarbusto. Perenne. Nativa. 300$400 \mathrm{~m}$.

Distribución: CAN, OBE.

Ejemplar de referencia: Pierotti, A. 5512 (LIL).

\section{Mimosa candollei R. Grether}

Hierba o subarbusto. Perenne. Nativa. 0-500 m.

Distribución: CAP, ELD, IGU, SIG.

Ejemplar de referencia: Rodríguez, F. M. 179 (SI).

Mimosa cruenta Benth.

Arbusto o subarbusto. Nativa. 0-300 m.

Distribución: CAP, SJA.

Ejemplar de referencia: Rodríguez $\left\{2^{\circ}\right.$ serie $\}, F$. M. 426 (BAF, SI).

Mimosa daleoides Benth.

Arbusto. Nativa. 0-500 m.

Distribución: CAN.

Ejemplar de referencia: Seijo, J. G. 536 (CTES).

Mimosa debilis Humb. \& Bonpl. ex Willd.

Subarbusto. Nativa. 0-500 m.

Distribución: CAN, SIG.

Ejemplar de referencia: Zuloaga, F. O. 5332 (SI).

Mimosa diplotricha $C$. Wright ex Sauvalle

Hierba o subarbusto. Perenne. Nativa. 0-500 m.

Distribución: CAN, ELD, IGU.

Ejemplar de referencia: Burkart, A. 14774 (SI).

\section{Mimosa diversipila Micheli var. diversipila}

Hierba o subarbusto. Perenne. Nativa. 100$400 \mathrm{~m}$.

Distribución: CAN.

Ejemplar de referencia: Burkart, A. 14773 (SI). 
Mimosa diversipila Micheli var. subglabriseta Barneby \& Fortunato

Hierba o subarbusto. Perenne. Nativa. 100$300 \mathrm{~m}$.

Distribución: CAN, CAP.

Ejemplar de referencia: Krapovickas, A. 12094 (CTES, US).

Mimosa dolens Vell. var. foliolosa (Benth.) Barneby Hierba o subarbusto. Perenne. Nativa. 200$800 \mathrm{~m}$.

Distribución: CAN.

Ejemplar de referencia: Zuloaga, F. O. 9861 (SI).

Mimosa dolens Vell. var. latifolia (Benth.) Barneby

Hierba o subarbusto. Perenne. Nativa. 200$800 \mathrm{~m}$.

Distribución: CAN, SIG.

Ejemplar de referencia: Xifreda, C. C. 525 (SI).

Mimosa dolens Vell. subsp. acerba (Benth.) Barneby $\left({ }^{*}\right)$

Subarbusto. Nativa. 0-500 m.

Distribución: GMB.

Ejemplar de referencia: Keller, H. A. 3639 (CTES, SI).

Mimosa dolens Vell. subsp. callosa (Benth.)

Barneby

Hierba o subarbusto. Perenne. Nativa. 100$500 \mathrm{~m}$.

Distribución: SIG.

Ejemplar de referencia: Múlgura, M. E. 1666 (SI).

Mimosa dolens Vell. subsp. rigida (Benth.) Barneby

Subarbusto. Nativa. 0-500 m.

Distribución: CAN.

Ejemplar de referencia: Meyer, T. 11362 (MVFA).

Mimosa glanduliseta Burkart $\left.{ }^{(* *}\right)$

Arbusto. Nativa. 0-300 m.

Distribución: CAN.

Ejemplar de referencia: Ekman, E. L. 1630 (MO, $N Y, S)$.

Mimosa gracilis Benth. var. leiocarpa (Burkart) Barneby

Hierba o subarbusto. Perenne. Nativa. 0-800 m.
Distribución: CAN.

Ejemplar de referencia: Burkart, A. 14733 (SI).

Mimosa gracilis Benth. subsp. capillipes (Benth.) Barneby

Hierba o subarbusto. Perenne. Nativa. 0-500 m.

Distribución: CAI, GMB, SPE.

Ejemplar de referencia: Keller, H. A. 3717 (SI, CTES).

Mimosa guaranitica Chodat \& Hassl.

Subarbusto. Nativa.

Distribución: en el centro-este y sur de la provincia.

Ejemplar de referencia: Molfino, J. F. s.n. (BAF).

Mimosa hexandra Micheli

Arbusto. Nativa. 0-500 m.

Distribución: CAN.

Ejemplar de referencia: Hauman, L. L. s.n. (BA).

Mimosa hirsutissima Mart.

Hierba o subarbusto. Perenne. Nativa. 200$800 \mathrm{~m}$.

Distribución: CAN, SIG.

Ejemplar de referencia: Martínez Crovetto, $R$. 8257 (SI).

Mimosa longiracemosa (Burkart) Barneby $\left({ }^{* *}\right)$

Hierba o subarbusto. Perenne. Endémica. 200$300 \mathrm{~m}$.

Distribución: CAN.

Ejemplar de referencia: Rodríguez, F. M. 626 $(F, S I)$.

Mimosa macrocalyx Micheli

Arbusto o subarbusto. Nativa. 200-400 m.

Distribución: APO, CAN.

Ejemplar de referencia: Cabrera, A. 29166 (SI).

Mimosa myriophylla Bong. ex Benth.

Arbusto o subarbusto. Nativa. 0-500 m.

Distribución: GMB.

Ejemplar de referencia: Zuloaga, F. O. 5107 (SI).

Mimosa niederleinii Burkart ${ }^{* * *}$ )

Hierba o subarbusto. Perenne. Nativa. 0-500 m.

Distribución: GUA.

Ejemplar de referencia: Niederlein, G. s.n. (SI). 
Mimosa nuda Benth. var. glaberrima (Chodat \& Hassl.) Barneby $\left({ }^{* * *}\right)$

Hierba o subarbusto. Perenne. Nativa. 0-500 m. Distribución: SIG.

Ejemplar de referencia: Keller, H. A. 8030 (CTES).

\section{Mimosa obstrigosa Burkart}

Arbusto. Nativa. 0-200 m.

Distribución: APO, CAN, OBE.

Ejemplar de referencia: Fortunato, R. H. 8077 $(B A B)$.

\section{Mimosa oligophylla Micheli}

Hierba o subarbusto. Perenne. Nativa. 200$300 \mathrm{~m}$

Distribución: APO, CAN.

Ejemplar de referencia: Burkart, A. 15512 (SI).

\section{Mimosa paraguariae Micheli}

Arbusto. Nativa. 100-800 m.

Distribución: CAN, SIG.

Ejemplar de referencia: Grüner, G. 707 (SI).

\section{Mimosa parvipinna Benth. $\left.{ }^{* * *}\right)$}

Subarbusto. Nativa. 0-400 m.

Distribución: CAN, CAP, CON, SIG.

Ejemplar de referencia: Burkart, A. 15444 (GH, $K, S I)$.

\section{Mimosa paupera Benth.}

Hierba o subarbusto. Perenne. Nativa. 0-400 m. Distribución: APO, CAI, CAN, LNA, SIG, SJA. Ejemplar de referencia: Biganzoli, F. 934 (SI).

Mimosa pauperoides (Burkart) Fortunato

Hierba o subarbusto. Perenne. Nativa. 0-500 m. Distribución: SPE.

Ejemplar de referencia: Bertoni, 2000 (LIL).

\section{Mimosa pedersenii Barneby}

Hierba o subarbusto. Perenne. Nativa. 0-500 m.

Distribución: LNA, SJA.

Ejemplar de referencia: Burkart, A. 15452 (SI).

\section{Mimosa pigra $\mathrm{L}$.}

Arbusto o subarbusto. Nativa. 0-700 m.

Distribución: CAN, CAP, ELD, IGU, SIG, SPE.

Ejemplar de referencia: Vanni, R. O. 2806 (CTES).
Mimosa polycarpa Kunth var. spegazzini (Pirotta ex Hook. f.) Burkart

Arbusto. Nativa. 0-400 m.

Distribución: APO, CAI, CAN, ELD, GMB, GUA, IGU, LNA, MON, SJA, SPE. (SI).

Ejemplar de referencia: Múlgura, M. E. 2870

Mimosa regnellii Benth. var. exuta Barneby $\left({ }^{* *}\right)$

Arbusto. Nativa. 0-800 m.

Distribución: GMB.

Ejemplar de referencia: Morrone, O. 913 (SI).

Mimosa somnians Humb. \& Bonpl. ex Willd

Arbusto o subarbusto. Nativa. 0-500 m.

Distribución: CAN, CAP, ELD, IGU, SIG.

Ejemplar de referencia: Honfi, A. I. 798 (MNES, SI).

Mimosa subsericea Benth.

Hierba o subarbusto. Perenne. Nativa. 0-500 m.

Distribución: CAN, SIG.

Ejemplar de referencia: Burkart, A. 15389 (SI).

Mimosa uliginosa Chodat \& Hassl.

Arbusto o arbolito. Nativa. $100-300 \mathrm{~m}$.

Distribución: APO, CAN, CON, GUA, LNA, MON, SIG.

Ejemplar de referencia: Burkart, A. 14742 (SI).

Mimosa uraguensis Hook. \& Arn.

Arbusto. Nativa. 0-500 m.

Distribución: SPE.

Ejemplar de referencia: Zuloaga, F. O. 7013 (SI).

Mimosa velloziana Mart.

Arbusto o subarbusto. Nativa. 0-500 m.

Distribución: CAN, ELD, IGU, SIG.

Ejemplar de referencia: Morrone, O. 1158 (SI).

\section{Mucuna Adans.}

Mucuna sloanei Fawc. \& Rendle $(* * *)$

Liana. Perenne. Nativa. 0-500 m.

Distribución: IGU, SIG.

Ejemplar de referencia: Krapovickas, A. 15777 (SI). 


\section{Muellera L. f.}

Muellera campestris (Mart. ex Benth.) M.J.

Silva \& A.M.G. Azevedo

Árbol. Nativa. 0-500 m.

Distribución: CAN, CAP, CON, ELD, IGU, MON, OBE, SIG, SPE.

Ejemplar de referencia: Rodríguez, F. M. 506 (BAF, SI).

\section{Myrocarpus Allemão}

Myrocarpus frondosus Allemão

Árbol. Nativa. 0-500 m.

Distribución: APO, ELD, GMB, OBE.

Ejemplar de referencia: Zuloaga, F. O. 6431 (SI).

\section{Neptunia Lour.}

Neptunia pubescens Benth.

Hierba subleñosa. Perenne. Nativa. 0-500 m.

Distribución: CAN, OBE.

Ejemplar de referencia: Keller, H. A. 10846 (CTES).

Nissolia Jacq., nom. cons.

Nissolia brasiliensis (Vogel) T.M. Moura \& Fort.-Perez

Enredadera. Perenne. Nativa. 0-800 m.

Distribución: CAN, CAP, ELD, GSM, SIG.

Ejemplar de referencia: Zuloaga, F. O. 5686 (SI).

Nissolia fruticosa Jacq. var. guatemalensis (Rose) Rudd

Enredadera. Perenne. Nativa. 0-500 m.

Distribución: ELD, SJA.

Ejemplar de referencia: Keller, H. A. 3023 (CTES).

Nissolia longiflora (Benth. ex A. Gray) T.M.

Moura \& Fort.-Perez

Enredadera. Perenne. Nativa. 0-700 m.

Distribución: CAI, CAN, GMB, GUA, IGU, OBE, SIG, SPE.

Ejemplar de referencia: Vanni, R. O. 4003 (CTES).
Nissolia nigricans (Burkart) T.M. Moura \& Fort.-Perez

Enredadera. Perenne. Nativa. 0-700 m.

Distribución: CAI, CAN, ELD, GMB, GSM, GUA, IGU, MAY.

Ejemplar de referencia: Morrone, O. 1162 (SI).

\section{Parapiptadenia Brenan}

Parapiptadenia rigida (Benth.) Brenan

Árbol. Nativa. 0-700 m.

Distribución: APO, CAI, CAN, CAP, CON, ELD, GUA, IGU, SIG, SPE.

Ejemplar de referencia: Schwarz, G. J. 5182 (SI).

Peltophorum (Vogel) Benth.

Peltophorum dubium (Spreng.) Taub.

Árbol. Nativa. 0-500 m.

Distribución: CAI, CAN, CAP, IGU, SIG, SPE. (SI).

Ejemplar de referencia: Wolffhügel, K. 124

\section{Phaseolus L.}

Phaseolus lunatus L. var. sylvester Baudet Hierba o enredadera. Anual. Nativa. 0-500 m. Distribución: CAN, SIG.

Ejemplar de referencia: Morrone, O. 1929 (SI).

\section{Poecilanthe Benth.}

Poecilanthe parvifolia Benth.

Arbusto o árbol. Nativa. 0-500 m.

Distribución: APO, SIG.

Ejemplar de referencia: Zanotti, C. A. 360 (SI).

\section{Poiretia Vent.}

\section{Poiretia latifolia Vogel}

Subarbusto. Nativa. 0-500 m.

Distribución: APO, CAN, CON, SJA.

Ejemplar de referencia: Zuloaga, F. O. 6545 (SI). 


\section{Pomaria Cav.}

Pomaria rubicunda (Vogel) B.B. Simpson \& G.P. Lewis var. hauthalii (Harms) B.B. Simpson \& G.P. Lewis $\left({ }^{* * *}\right)$

Arbusto. Nativa. 0-500 m.

Distribución: CAI, CAN, SIG.

Ejemplar de referencia: Schwarz, E. A. 10842 (CTES, LIL).

Pomaria rubicunda (Vogel) B.B. Simpson \& G.P. Lewis var. rubicunda

Arbusto. Nativa. 0-700 m.

Distribución: APO, CAN, CAP, CON, LNA, SIG. Ejemplar de referencia: Biganzoli, F. 1689 (SI).

\section{Pterogyne Tul.}

\section{Pterogyne nitens Tul.}

Árbol. Nativa. 0-500 m.

Distribución: SIG.

Ejemplar de referencia: Keller, H. A. 8321 (CTES).

\section{Pueraria DC.}

Pueraria montana (Lour.) Merr. var. lobata (Willd.) Maesen \& S.M. Almeida ex Sanjappa \& Predeep ${ }^{* * *}$ )

Enredadera o liana. Perenne. Introducida. 0-500 m.

Distribución: CAN, ELD, SPE.

Ejemplar de referencia: Keller, H. A. 7843 (CTES).

\section{Rhynchosia Lour.}

\section{Rhynchosia arenicola Hassl.}

Hierba o enredadera. Perenne. Nativa. 0-500 m.

Distribución: APO, SIG.

Ejemplar de referencia: Zuloaga, F. O. 6474 (SI).

\section{Rhynchosia balansae Micheli}

Hierba. Perenne. Nativa. 0-500 m.

Distribución: SIG.

Ejemplar de referencia: Fortunato, R. H. 8024 (BAB, SI).
Rhynchosia corylifolia Mart. ex Benth.

Hierba. Perenne. Nativa. 0-600 m.

Distribución: APO, CAN, CON.

Ejemplar de referencia: Zuloaga, F. O. 4898 (SI).

Rhynchosia diversifolia Micheli

Hierba. Perenne. Nativa. 0-500 m.

Distribución: LNA, SJA.

Ejemplar de referencia: Burkart, A. 15467 (SI).

Rhynchosia edulis Griseb.

Enredadera. Perenne. Nativa. 0-700 m.

Distribución: MON, SIG, SJA.

Ejemplar de referencia: Cuadrado, G. 338 (CTES).

Rhynchosia hauthalii Harms ex Kuntze

Hierba. Perenne. Nativa. 400-500 m.

Distribución: CAN.

Ejemplar de referencia: Burkart, A. 15436 (SI).

Rhynchosia lateritia Burkart

Hierba. Perenne. Nativa. 0-500 m.

Distribución: APO, CAN.

Ejemplar de referencia: Múlgura, M. E. 2037 (SI).

Rhynchosia lineata Benth.

Hierba o subarbusto. Perenne. Nativa. 0-500 m. Distribución: CAN.

Ejemplar de referencia: Burkart, A. 15448bis (SI).

\section{Rhynchosia melanocarpa Grear ${ }^{* * *}$ )}

Hierba o enredadera. Perenne. Nativa. 200$700 \mathrm{~m}$.

Distribución: CAN, IGU, SPE.

Ejemplar de referencia: Keller, H. A. 56 (CTES, SI).

Rhynchosia minima (L.) DC.

Enredadera. Perenne. Nativa. 0-500 m.

Distribución: SIG.

Ejemplar de referencia: Guaglianone, E. R. 1198 (SI).

\section{Rhynchosia rojasii Hassl.}

Enredadera. Perenne. Nativa. 0-500 m.

Distribución: CAN, GUA.

Ejemplar de referencia: Montes, J. E. 367 (SI). 
Schnella Raddi

\section{Schnella microstachya Raddi}

Arbusto. Nativa. 0-500 m.

Distribución: CAI, CAN, GUA, SIG.

Ejemplar de referencia: Zuloaga, F. O. 6831 (SI).

\section{Senegalia Raf.}

Senegalia bonariensis (Gillies ex Hook. \& Arn.) Seigler \& Ebinger

Arbusto o árbol. Nativa. 0-500 m.

Distribución: CAI, CAP, ELD.

Ejemplar de referencia: Morrone, O. 659 (SI).

Senegalia gilliesii (Steud.) Seigler \& Ebinger Arbusto o árbol. Nativa. 0-500 m.

Distribución: CAN.

Ejemplar de referencia: Jörgensen, P. 291 (SI).

Senegalia nitidifolia (Speg.) Seigler \& Ebinger $(* * *)$

Arbusto. Nativa. 0-800 m.

Distribución: CAI, ELD, GMB, GSM, GUA, IGU, MON, SPE.

Ejemplar de referencia: Deginani, N. B. 1022 (SI).

Senegalia polyphylla (DC.) Britton \& Rose ex Britton \& Killip (***)

Árbol. Nativa. 0-500 m.

Distribución: CAP, GMB, IGU.

Ejemplar de referencia: Zuloaga, F. O. 5160 (SI).

Senegalia recurva (Benth.) Seigler \& Ebinger

Arbusto. Nativa. 0-800 m.

Distribución: CAI, ELD; GMB, GSM, GUA, IGU, SJA, SPE.

Ejemplar de referencia: Morrone, O. 806 (SI).

Senegalia tucumanensis (Griseb.) Seigler \& Ebinger

Arbusto o árbol. Nativa. 0-800 m.

Distribución: CAI, CAN, CAP, GSM, GUA, IGU, LNA, SIG.

Ejemplar de referencia: Morrone, O. 656 (SI).

Senegalia velutina (DC.) Seigler \& Ebinger Arbusto. Nativa. 100-800 m.
Distribución: CAN, GMB, GUA, IGU, OBE, SIG, SPE.

Ejemplar de referencia: Múlgura, M. E. 1753 (SI).

\section{Senna Mill.}

Senna alata (L.) Roxb.

Arbusto o subarbusto. Introducida. 0-500 m.

Distribución: GMB, LNA.

Ejemplar de referencia: Maruñak, V. 964 (CTES).

Senna corymbosa (Lam.) H.S. Irwin \& Barneby Arbusto o árbol. Nativa. 0-500 m.

Distribución: APO.

Ejemplar de referencia: Burkart, A. 14298 (SI).

Senna hirsuta (L.) H.S. Irwin \& Barneby var. streptocarpa H.S. Irwin \& Barneby $\left.{ }^{* * *}\right)$

Hierba o subarbusto. Perenne. Nativa. 0-400 m. Distribución: APO, CAI, CON, GSM, LNA, SIG. Ejemplar de referencia: Biganzoli, F. 911 (SI).

Senna nana (Benth.) H.S. Irwin \& Barneby $\left({ }^{* * *}\right)$ Hierba o subarbusto. Perenne. Nativa. 0-300 m. Distribución: CAN, SIG.

Ejemplar de referencia: Keller, H. A. 13071 (CTES).

Senna oblongifolia (Vogel) H.S. Irwin \& Barneby $\left.{ }^{* * *}\right)$

Arbusto. Nativa. 0-500 m.

Distribución: GMB, SPE.

Ejemplar de referencia: Morrone, O. 860 (SI).

Senna obtusifolia (L.) H.S. Irwin \& Barneby Hierba o subarbusto. Perenne. Nativa. 0-500 m. Distribución: CAI, GUA, IGU, SPE.

Ejemplar de referencia: Deginani, N. B. 1030 (SI).

Senna occidentalis (L.) Link

Hierba o subarbusto. Anual. Nativa. 0-500 m.

Distribución: GSM, GUA, IGU, SIG.

Ejemplar de referencia: Deginani, N. B. 1031 (SI).

Senna pendula (Willd.) H.S. Irwin \& Barneby var. missionum H.S. Irwin \& Barneby

Arbusto. Nativa. 200-400 m.

Distribución: CAI, CAN, CAP, SIG.

Ejemplar de referencia: Montes, J. E. 138 (NY). 
Senna pendula (Willd.) H.S. Irwin \& Barneby var. paludicola H.S. Irwin \& Barneby

Arbusto. Nativa. 0-500 m.

Distribución: GMB.

Ejemplar de referencia: Zuloaga, F. O. 9932 (SI).

Senna pilifera (Vogel) H.S. Irwin \& Barneby Hierba o subarbusto. Perenne. Nativa. 0-500 m. Distribución: CAN, SIG.

Ejemplar de referencia:Seijo, G. J. 100 (MNES, SI).

\section{Sesbania Scop.}

Sesbania punicea (Cav.) Benth.

Árbol. Nativa. 0-500 m.

Distribución: APO, CAP, CON, GMB, IGU, MAY, SPE.

Ejemplar de referencia: Morrone, O. 1521 (SI).

\author{
Sesbania virgata (Cav.) Pers. \\ Arbusto. Nativa. 0-500 m. \\ Distribución: ELD, SIG, SPE. \\ Ejemplar de referencia: Guillén, R. 202 (CTES, \\ $G, M N E S)$.
}

\section{Stylosanthes Sw.}

Stylosanthes guianensis (Aubl.) Sw. var. gracilis (Kunth) Vogel ${ }^{* * *}$ )

Hierba. Perenne. Nativa. 100-500 m.

Distribución: CAN, GUA, SIG, SPE.

Ejemplar de referencia: Montes, J. E. 14901 (NY).

Stylosanthes guianensis (Aubl.) Sw. var. guianensis

Hierba. Perenne. Nativa. 0-500 m.

Distribución: CAN, CON, SIG.

Ejemplar de referencia: Cabral, E. L. 370 (CTES, SI).

Stylosanthes hippocampoides Mohlenbr.

Hierba. Perenne. Nativa. 0-500 m.

Distribución: SIG.

Ejemplar de referencia: Zuloaga, F. O. 6644 (SI).

\section{Stylosanthes montevidensis Vogel}

Hierba o subarbusto. Perenne. Nativa. 0-500 m.

Distribución: APO, CAN, CON, SIG, SJA.

Ejemplar de referencia: Zuloaga, F. O. 6917 (SI).
Stylosanthes viscosa (L.) Sw.

Hierba o subarbusto. Perenne. Nativa. 0-500 m. Distribución: SIG.

Ejemplar de referencia: Schinini, A. 21901 (CTES).

Sweetia Spreng., nom. cons.

Sweetia fruticosa Spreng. $\left.{ }^{* * *}\right)$

Árbol. Nativa. 0-500 m.

Distribución: CAI.

Ejemplar de referencia: Spegazzini, C. L. s.n. (BAB).

\section{Tephrosia Pers.}

Tephrosia adunca Benth.

Hierba. Perenne. Nativa. 0-500 m.

Distribución: CAI, CAP, CON, SIG, SJA.

Ejemplar de referencia: Biganzoli, F. 1581 (SI).

Tephrosia cinerea (L.) Pers.

Hierba. Perenne. Nativa. 0-500 m.

Distribución: LNA, SIG.

Ejemplar de referencia: Fortunato, R. H. 7970 (BAB).

Tephrosia fertilis R.T. Queiroz \& A.M.G. Azevedo (**)

Subarbusto. Endémica. 0-500 m.

Distribución: CAN, MON, SIG.

Ejemplar de referencia: Rodríguez, F. M. 654 (SI).

Tephrosia hassleri Chodat

Hierba. Perenne. Nativa. 0-500 m.

Distribución: CAN.

Ejemplar de referencia: Keller, H. A. 10838 (CTES).

Tephrosia marginata Hassl.

Hierba. Perenne. Nativa. 0-500 m.

Distribución: CAN, CON.

Ejemplar de referencia: Zuloaga, F. O. 8116 (SI).

\section{Trifolium L.}

\section{Trifolium polymorphum Poir.}

Hierba. Perenne. Nativa. 0-500 m.

Distribución: CON, SIG.

Ejemplar de referencia: Diem, J. 1570 (SI). 
Trifolium riograndense Burkart $\left.{ }^{* * *}\right)$

Hierba. Perenne. Nativa. 0-500 m.

Distribución: GMB.

Ejemplar de referencia: Gamerro, J. C. 101 (SI).

\section{Vachellia Wight \& Arn.}

Vachellia caven (Molina) Seigler \& Ebinger

Arbusto o arbolito. Nativa. 0-500 m.

Distribución: CAN, CAP, SJA.

Ejemplar de referencia: Burkart, A. 14734 (SI).

Vachellia farnesiana (L.) Wight \& Arn.

Arbusto o arbolito. Nativa. 0-500 m.

Distribución: IGU.

Ejemplar de referencia: Vanni, R. O. 2862 (CTES, F).

\section{Vicia L.}

Vicia benghalensis $\left.\mathrm{L} .{ }^{(* *}\right)$

Hierba. Anual. Introducida. 0-500 m.

Distribución: SIG.

Ejemplar de referencia: Keller, H. A. 7450 (CTES).

\section{Vicia epetiolaris Burkart var. epetiolaris}

Enredadera. Anual. Nativa. 0-500 m.

Distribución: CAN, CAP, CON.

Ejemplar de referencia: Spegazzini, R. A. 1682 (SI).

Vicia epetiolaris Burkart var. microcarpa Vanni \& D. Kurtz

Enredadera. Anual. Nativa. 0-500 m.

Distribución: APO, CAN, CAP, ELD, LNA, OBE, SIG, SJA.

Ejemplar de referencia: Múlgura, M. E. 3801 (MNES, SI).

\section{Vicia graminea $\mathrm{Sm}$.}

Hierba. Anual o Bianual. Nativa. 0-500 m.

Distribución: OBE, SJA.

Ejemplar de referencia: Múlgura, M. E. 2295 (SI).

\section{Vicia nana Vogel}

Enredadera. Anual. Nativa.

Distribución: Desconocida.

Ejemplar de referencia: Spegazzini, R. A. 1680 (SI).

\section{Vicia stenophylla Vogel}

Enredadera. Perenne. Nativa. 0-500 m.

Distribución: APO, CAN, CAP, OBE, SIG, SJA.

Ejemplar de referencia: Schinini, A. 5416 (CTES, $G, K, M O, S I$ ).

\section{Vicia tephrosoides Vogel}

Enredadera. Nativa. 0-500 m.

Distribución: APO, CAP, CON, IGU.

Ejemplar de referencia: Guaglianone, E. R. 892 (SI).

\section{Vigna Savi}

Vigna lasiocarpa (Mart. ex Benth.) Verdc.

Enredadera. Nativa. 0-500 m.

Distribución: CAN, CAP, LNA, SIG.

Ejemplar de referencia: Burkart, A. 15424 (SI).

Vigna longifolia (Benth.) Verdc.

Enredadera. Perenne. Nativa. 0-500 m.

Distribución: APO, CAN, SIG.

Ejemplar de referencia: Zuloaga, F. O. 4913 (SI).

Vigna luteola (Jacq.) Benth.

Hierba o enredadera. Anual. Nativa. 0-500 m.

Distribución: GMB.

Ejemplar de referencia: Pedersen, T. M. 13689 (SI).

\section{Zapoteca H.M. Hern.}

Zapoteca portoricensis (Jacq.) H.M. Hern. subsp. flavida (Urb.) H.M. Hern. ${ }^{* * *}$ )

Arbusto. Nativa. 0-500 m.

Distribución: IGU.

Ejemplar de referencia: Spegazzini, C. L. 435 (LP).

Zornia J.F. Gmel.

\section{Zornia burkartii Vanni}

Hierba. Nativa. 0-500 m.

Distribución: APO.

Ejemplar de referencia: Ibarrola, T. 1107 (CTES, LIL).

Zornia crinita (Mohlenbr.) Vanni

Hierba. Nativa. 0-500 m. 
Distribución: GUA.

Ejemplar de referencia: Keller, H. A. 3262 (CTES).

\section{Zornia cryptantha Arechav.}

Hierba. Perenne. Nativa. 0-500 m.

Distribución: APO, CAI, CON, OBE.

Ejemplar de referencia: Schwarz, G. J. 2538 (LIL).

\section{Zornia latifolia $\mathrm{Sm}$.}

Hierba. Perenne. Nativa. 0-500 m.

Distribución: ELD.

Ejemplar de referencia: Keller, H. A. 2819 (CTES).

\section{Zornia orbiculata Mohlenbr.}

Hierba. Perenne. Nativa. 0-500 m.

Distribución: CAN, CAP, GUA, MON, OBE, SIG, SPE.

Ejemplar de referencia: Múlgura, M. E. 1752 (SI).

\section{Zornia pardina Mohlenbr.}

Hierba. Perenne. Nativa. 0-600 m.

Distribución: CAI, CAN, CON, SIG.

Ejemplar de referencia: Rodríguez, F. M. 280 (BAF, SI).

\section{Zornia reticulata $\mathrm{Sm}$.}

Hierba. Perenne. Nativa. 0-500 m.

Distribución: APO, CAI, CAN, SIG.

Ejemplar de referencia: Múlgura, M. E. 2850 (SI).

\section{Zornia sericea Moric.}

Hierba. Perenne. Nativa. 0-500 m.

Distribución: CAN, CON, SIG, SJA.

Ejemplar de referencia: Krapovickas, A. 15141 (CTES).

\section{Zornia trachycarpa Vogel}

Hierba. Perenne. Nativa. 0-500 m.

Distribución: CAN, CON, SIG.

Ejemplar de referencia: Morrone, O. 1034 (SI).

\section{Zygia P. Browne}

\author{
Zygia cataractae (Kunth) L. Rico \\ Arbusto o árbol. Nativa. 0-500 m. \\ Distribución: GSM, SIG. \\ Ejemplar de referencia: Keller, H. A. 6547 \\ (CTES, SI).
}

\section{FRANCOACEAE}

\section{Viniania Cav.}

Viviania albiflora (Cambess.) Reiche $\left({ }^{* * *}\right)$

Arbusto. Nativa. 0-600 m.

Distribución: CAI, GUA, MAY, SPE.

Ejemplar de referencia: Deginani, N. B. 1626 (SI).

\section{GENTIANACEAE}

\section{Chelonanthus Gilg.}

Chelonanthus viridiflorus (Mart.) Gilg.

Hierba. Anual. Nativa. 0-500 m.

Distribución: CAP, SIG.

Ejemplar de referencia: Keller, H. A. 9943 (CTES).

\section{Helia Mart.}

Helia oblongifolia Mart. $\left({ }^{* *}\right)$

Hierba. Anual. Nativa. 0-500 m.

Distribución: CAN.

Ejemplar de referencia: Fontana, J. L. 433 (CTESN).

Schultesia Mart., nom. cons.

Schultesia australis Griseb.

Hierba. Anual. Nativa. 0-500 m.

Distribución: CAP, CON.

Ejemplar de referencia: Biganzoli, F. 1643 (SI).

Schultesia guianensis (Aubl.) Malme ${ }^{(* * *}$ )

Hierba. Anual. Nativa. 0-500 m

Distribución: CAP.

Ejemplar de referencia: Molfino, J. F. s.n. (BA, BAF).

\section{Voyria Aubl.}

\section{Voyria aphylla (Jacq.) Pers. ${ }^{* * *}$ )}

Saprófita. Perenne. Nativa. 0-500 m.

Distribución: IGU.

Ejemplar de referencia: Keller, H. A. 13635 (CTES). 
Zygostigma Griseb.

Zygostigma australe (Cham. \& Schltdl.) Griseb.

Hierba. Perenne. Nativa. 0-500 m.

Distribución: CAN, CON.

Ejemplar de referencia: Keller, H. A. 6033 (CTES).

\section{GERANIACEAE}

\section{Erodium L’Hér. ex Aiton}

\section{Erodium cicutarium (L.) L'Hér. ex Aiton}

Hierba. Anual o bianual. Introducida. 0-500 m.

Distribución: ELD.

Ejemplar de referencia: Keller, H. A. 13414 (CTES).

\section{Geranium L.}

\section{Geranium dissectum L.}

Hierba. Anual. Introducida. 0-500 m.

Distribución: MON.

Ejemplar de referencia: Keller, H. A. 1978 (CTES).

\section{GESNERIACEAE}

\section{Sinningia Nees}

Sinningia allagophylla (Mart.) Wiehler

Hierba. Perenne. Nativa. 0-700 m.

Distribución: CAI, CAN, GUA, SIG.

Ejemplar de referencia: Zuloaga, F. O. 5336 (SI).

Sinningia douglasii (Lindl.) Chautems $\left(^{* * *}\right.$ )

Hierba epífita. Perenne. Nativa. 0-700 m.

Distribución: CAI, GUA, SPE.

Ejemplar de referencia: Morrone, O. 1530 (SI).

Sinningia elatior (Kunth) Chautems

Hierba. Perenne. Nativa. 0-700 m.
Distribución: CAI, CAP, CON, GMB, IGU, SIG.

Ejemplar de referencia: Zuloaga, F. O. 5234 (SI).

Sinningia sellowii (Mart.) Wiehler

Hierba o subarbusto. Perenne. Nativa. 100$600 \mathrm{~m}$

Distribución: CAI, GSM, IGU, SIG.

Ejemplar de referencia: Morrone, O. 630 (SI).

Sinningia warmingii (Hiern) Chautems

Hierba. Perenne. Nativa. 0-700 m.

Distribución: CAI, GMB, IGU.

Ejemplar de referencia: Biganzoli, F. 1521 (SI).

\section{HALORAGACEAE}

\section{Myriophyllum L.}

Myriophyllum aquaticum (Vell.) Verdc.

Hierba acuática. Perenne. Nativa. 0-500 m.

Distribución: SPE.

Ejemplar de referencia: Keller, H. A. 4434 (CTES).

\section{HELICONIACEAE}

Heliconia L., nom. cons.

Heliconia brasiliensis Hook. $\mathrm{f}$.

Hierba. Perenne. Nativa. 0-300 m.

Distribución: IGU.

Ejemplar de referencia: Vanni, R. O. 3592 (CTES).

Heliconia hirsuta L. f. (***)

Hierba. Perenne. Nativa.

Distribución: Desconocida.

Ejemplar de referencia: Schulz, A. G. 5141 $(N Y)$.

Heliconia subulata Ruiz \& Pav. subsp. gracilis (Petersen) L.Andersson

Hierba. Perenne. Nativa. 0-500 m.

Distribución: IGU.

Ejemplar de referencia: Pingitore, E. s.n. (SI). 


\section{HYDROCHARITACEAE}

Apalanthe Planch.

Apalanthe granatensis (Humb. \& Bonpl.)

Planch.

Hierba acuática. Perenne. Nativa. 0-700 m.

Distribución: GMB.

Ejemplar de referencia: Morrone, O. 878 (SI).

\section{Egeria Planch.}

Egeria densa Planch.

Hierba acuática. Perenne. Nativa. 0-800 m.

Distribución: SJA.

Ejemplar de referencia: Cabrera, A. L. 28670 (SI).

Egeria najas Planch.

Hierba acuática. Perenne. Nativa. 0-200 m.

Distribución: CON.

Ejemplar de referencia: Keller, H. A. 5618 (CTES).

\section{Ottelia Pers.}

Ottelia brasiliensis (Planch.) Walp.

Hierba. Anual. Nativa.

Distribución: Desconocida.

Ejemplar de referencia: Parodi, D. 65 (BAF).

\section{HYDROLEACEAE}

Hydrolea L., nom. cons.

\section{Hydrolea elatior Schott}

Hierba. Perenne. Nativa. 0-500 m.

Distribución: IGU, SPE.

Ejemplar de referencia: Zuloaga, F. O. 5247 (SI).

Hydrolea spinosa L. var. paraguayensis (Chodat) Davenp.

Hierba o subarbusto. Perenne. Nativa. 0-500 m.

Distribución: CAP, CON, SIG.

Ejemplar de referencia: Montes, J. E. 2432 (SI).

\section{Hydrolea spinosa L. var spinosa}

Hierba o subarbusto. Perenne. Nativa. 0-500 m. Distribución: GMB.

Ejemplar de referencia: Guaglianone, R. E. 1107 (SI).

\section{HYPERICACEAE}

\section{Hypericum L.}

\section{Hypericum brasiliense Choisy}

Arbusto o subarbusto. Nativa. 0-700 m.

Distribución: APO, CAI, CAN, CAP, GMB, GSM, GUA, IGU, LNA, SIG, SJA, SPE.

Ejemplar de referencia: Guaglianone, E. R. 2867 (SI).

Hypericum campestre Cham. \& Schltdl. subsp. campestre

Hierba o subarbusto. Perenne. Nativa. 0-300 m. Distribución: GSM, SJA.

Ejemplar de referencia: Mroginski, L. 375 (ZT).

Hypericum campestre Cham. \& Schltdl. subsp. tenue N. Robson

Subarbusto. Nativa. 0-500 m.

Distribución: CAN, ELD.

Ejemplar de referencia: Morrone, O. 1635 (SI).

Hypericum caprifoliatum Cham. \& Schltdl.

Hierba o subarbusto. Perenne. Nativa. 0-700 m. Distribución: SJA, SPE.

Ejemplar de referencia: Zuloaga, F. O. 5451 (SI).

Hypericum carinatum Griseb.

Hierba o subarbusto. Perenne. Nativa.

0-500 m.

Distribución: APO, CAP, GMB, GUA.

Ejemplar de referencia: Keller, H. A. 200 (CTES, SI).

Hypericum connatum Lam.

Hierba o arbusto. Perenne. Nativa. 0-700 m.

Distribución: APO, CAI, CAN, CON, ELD, GUA, MON, SIG, SJA.

Ejemplar de referencia: Burkart, A. 15409 (SI). 
Hypericum denudatum A. St.-Hil. $\left(^{(* *}\right)$

Arbusto o subarbusto. Nativa. 0-700 m.

Distribución: CAP.

Ejemplar de referencia: Rodríguez, F. M. 84 (P, SI).

Hypericum lorentzianum Gilg ex R. Keller

Hierba o subarbusto. Perenne. Nativa. 0-500 m.

Distribución: APO, SIG, SPE. $(N Y)$.

Ejemplar de referencia: Bertoni, M. S. 1008

\section{Hypericum mutilum L.}

Hierba. Perenne. Nativa. 0-500 m.

Distribución: CAP.

Ejemplar de referencia: Burkart, A. 14021 (SI).

Hypericum myrianthum Cham. \& Schltdl.

Subarbusto. Nativa. 0-500 m.

Distribución: APO, CAN, CAP.

Ejemplar de referencia: Ibarrola, T. 1149 (BM, S).

Hypericum robsonii H.A. Keller \& S. Crockett $(* *)$

Hierba. Perenne. Endémica. 0-500 m.

Distribución: CAN.

Ejemplar de referencia: Keller, H. A. 11752 (CTES).

\section{HYPOXIDACEAE}

\section{Hypoxis L.}

Hypoxis decumbens $\mathrm{L}$.

Hierba. Perenne. Nativa. 0-800 m.

Distribución: CAI, CAN, CAP, GMB, GSM, GUA, IGU, MON, OBE, SIG, SPE.

Ejemplar de referencia: Biganzoli, F. 326 (SI).

\section{IRIDACEAE}

\section{Babiania Ker Gawl.}

\section{Babiania angustifolia Sweet $(*)$}

Hierba. Perenne. Introducida. 0-500 m.

Distribución: GMB.

Ejemplar de referencia: Keller, H. A. 11713 (CTES).
Calydorea Herb.

Calydorea approximata R.C. Foster

Hierba. Perenne. Nativa. 0-500 m.

Distribución: CAI, CAN, CON.

Ejemplar de referencia: Biganzoli, F. 370 (SI).

Calydorea minima Roitman \& A. Castillo

Hierba. Perenne. Nativa. 0-500 m.

Distribución: APO, CAN.

Ejemplar de referencia: Cabrera, A. L. 29307 (SI).

Crocosmia Planch.

Crocosmia crocosmiiflora (Lemoine ex Burbidge \& Dean) N.E. Br.

Hierba. Perenne. Introducida. 0-500 m.

Distribución: CAI, GMB, SIG.

Ejemplar de referencia: Rodríguez, M. 451 (MNES, SI).

\section{Cypella Herb.}

Cypella armosa Ravenna

Hierba. Perenne. Nativa. 0-500 m.

Distribución: APO, CAI.

Ejemplar de referencia: Cabrera, A. L. 28556 (SI).

\section{Cypella coelestis (Lehm.) Diels}

Hierba. Perenne. Nativa. 0-500 m.

Distribución: APO, CAN, GUA.

Ejemplar de referencia: Zuloaga, F. O. 980 (SI).

Cypella crenata (Vell.) Ravenna

Hierba. Perenne. Nativa. 100-500 m.

Distribución: CAN.

Ejemplar de referencia: Roitman, G. s.n. (BAA).

Cypella hauthalii (Kuntze) R.C. Foster subsp. hauthalii

Hierba. Perenne. Nativa. 0-500 m.

Distribución: APO.

Ejemplar de referencia: Morrone, O. 1101 (SI).

Cypella hauthalii (Kuntze) R.C. Foster subsp. opalina Ravenna

Hierba. Perenne. Nativa. 0-400 m. 
Distribución: APO.

Ejemplar de referencia: Múlgura, M. E. 2016 (SI).

Cypella herbertii (Lindl.) Herb. subsp. brevicristata Ravenna $(*)$

Hierba. Perenne. Nativa. 0-200 m.

Distribución: CAP.

Ejemplar de referencia: Keller, H. A. 13108 (CTES).

Cypella herbertii (Lindl.) Herb. subsp. herbertii

Hierba. Perenne. Nativa. 0-500 m.

Distribución: APO, CAN.

Ejemplar de referencia: Múlgura, M. E. 2971 (SI).

\section{Cypella lapidosa Ravenna}

Hierba. Perenne. Nativa. 0-500 m.

Distribución: APO, LNA.

Ejemplar de referencia: Deble, L. P. 15571 (SI).

Cypella ravenniana Deble \& F.S. Alves

Hierba. Perenne. Nativa. 100-300 m.

Distribución: CAN, SIG.

Ejemplar de referencia: Hauman, L. L. 73 (BA, SI).

\section{Cypella suffusa Ravenna (**)}

Hierba. Perenne. Endémica. 0-300 m.

Distribución: CAI, LNA.

Ejemplar de referencia: Keller, H. A. 12745 (CTES).

Cypella uliginosa Deble \& F.S. Alves

Hierba. Perenne. Nativa. 0-500 m.

Distribución: CAN.

Ejemplar de referencia: Keller, H. A. 13683 (CTES).

\section{Gelasine Herb.}

\section{Gelasine caerulea (Vell.) Ravenna ${ }^{* * *}$ )}

Hierba. Perenne. Nativa. 0-500 m.

Distribución: CAN, GMB.

Ejemplar de referencia: Jörgensen, P. 285 (SI).

\section{Gladiolus L.}

Gladiolus dalenii Van Geel (***)

Hierba. Perenne. Introducida. 0-500 m.

Distribución: ELD, MON.

Ejemplar de referencia: Keller, H. A. 13415 (CTES).

\section{Herbertia Sweet}

Herbertia darwinii Roitman \& A. Castillo

Hierba. Perenne. Nativa. 0-500 m.

Distribución: IGU.

Ejemplar de referencia: Keller, H. A. 12275 (CTES).

Herbertia lahue (Molina) Goldblatt subsp. amoena (Griseb.) Goldblatt

Hierba. Perenne. Nativa. 0-500 m.

Distribución: CAN, SIG.

Ejemplar de referencia: Keller, H. A. 13665 (CTES).

\section{Neomarica Sprague}

Neomarica caerulea (Ker Gawl.) Sprague (***)

Hierba. Perenne. Nativa. 0-500 m.

Distribución: IGU.

Ejemplar de referencia: Xifreda, C. C. 1259 (SI).

\section{Neomarica candida (Hassl.) Sprague}

Hierba. Perenne. Nativa. 0-500 m.

Distribución: IGU, SIG, SPE.

Ejemplar de referencia: Zuloaga, F. O. 6463 (SI).

\section{Sisyrinchium L.}

Sisyrinchium alatum Hook.

Hierba. Perenne. Nativa. 0-800 m.

Distribución: CAN, SIG.

Ejemplar de referencia: Zuloaga, F. O. 7190 (SI).

Sisyrinchium bromelioides R.C. Foster subsp. angustius Ravenna

Hierba. Perenne. Nativa. 0-800 m.

Distribución: CAI, GUA.

Ejemplar de referencia: Zuloaga, F. O. 8174 (SI).

\section{Sisyrinchium chilense Hook.}

Hierba. Perenne. Nativa. 0-500 m.

Distribución: APO, GUA.

Ejemplar de referencia: Zardini, E. M. 1043 (SI).

\section{Sisyrinchium commutatum Klatt}

Hierba. Perenne. Nativa. 0-500 m.

Distribución: CAN, SIG.

Ejemplar de referencia: Jörgensen, P. 292a (SI). 
Sisyrinchium dasypathum (Ravenna) Ravenna $\left({ }^{* * *}\right)$

Hierba. Perenne. Nativa. 0-500 m.

Distribución: CAN, OBE, SIG.

Ejemplar de referencia: Mroginsky, L. 778 (CTES, Herb. Ravenna).

\section{Sisyrinchium fasciculatum Klatt}

Hierba. Perenne. Nativa. 0-500 m.

Distribución: CAN, CAP, SIG.

Ejemplar de referencia: Krapovickas, A. 15499 (CTES).

\section{Sisyrinchium hasslerianum Baker}

Hierba. Perenne. Nativa. 0-700 m.

Distribución: APO, CAI, CAN, SIG.

Ejemplar de referencia: Morrone, O. 1769 (SI).

\section{Sisyrinchium incurvatum Gardner $\left.{ }^{* * *}\right)$}

Hierba. Perenne. Nativa.

Distribución: Desconocida.

Ejemplar de referencia: Niederlein, G. s.n.

\section{Sisyrinchium latiusculum Ravenna $\left.{ }^{(* *}\right)$}

Hierba. Perenne. Endémica. 0-500.

Distribución: CAN.

Ejemplar de referencia: Krapovickas, A. 15498 (CTES).

\section{Sisyrinchium luzula Klotzsch}

Hierba. Perenne. Nativa. 0-700 m.

Distribución: APO, CAN, SIG.

Ejemplar de referencia: Jörgensen, P. 34486 (SI).

\section{Sisyrinchium marchioides Ravenna}

Hierba. Perenne. Nativa. 0-500 m.

Distribución: CAN, SIG.

Ejemplar de referencia: Keller, H. A. 9226 (CTES).

\section{Sisyrinchium micranthum $\mathrm{Cav}$.}

Hierba. Perenne. Nativa. 0-700 m.

Distribución: GSM, GUA, MON, SIG.

Ejemplar de referencia: Biganzoli, F. 263a (SI).

Sisyrinchium minus Engelm. \& A. Gray subsp. everrucosum Ravenna

Hierba. Anual. Nativa. 0-500 m.

Distribución: GUA, SIG.

Ejemplar de referencias: Keller, H. A. 9223 (CTES).

\section{Sisyrinchium minutiflorum Klatt}

Hierba. Anual. Nativa.

Distribución: Desconocida.

Ejemplar de referencia: Grondona, E. M. 1264 (BAB).

\section{Sisyrinchium pachyrhizum Baker}

Hierba. Perenne. Nativa. 0-500 m.

Distribución: CAN, CAP.

Ejemplar de referencia: Cabrera, A. L. 29371 (SI).

\section{Sisyrinchium palmifolium $\mathrm{L}$.}

Hierba. Perenne. Nativa. 0-800 m.

Distribución: CAI, CAN, CAP, GMB, GUA, SIG, SPE.

Ejemplar de referencia: Lirussi, I. 493 (MNES, SI).

\section{Sisyrinchium platycaule Baker}

Hierba. Perenne. Nativa. 0-800 m.

Distribución: CAI, CAN, GMB, SIG.

Ejemplar de referencia: Zuloaga, F. O. 8329 (SI).

Sisyrinchium scariosum I.M. Johnst. $\left.{ }^{* * *}\right)$

Hierba. Perenne. Nativa. 0-500 m.

Distribución: APO, CAN, CON.

Ejemplar de referencia: Keller, H. A. 13663 (CTES).

\section{Sisyrinchium sellowianum Klatt}

Hierba. Perenne. Nativa. 0-800 m.

Distribución: CAI, CAN, GSM, GUA, IGU, MON, OBE, SIG.

Ejemplar de referencia: Zuloaga, F. O 13329 (SI, MU).

\section{Sisyrinchium setaceum Klatt}

Hierba. Perenne. Nativa. 0-800 m.

Distribución: APO, CAI, CAN, GMB, GUA, OBE, SIG, SPE.

Ejemplar de referencia: Gamerro, J. C. 56 (SI).

\section{Sisyrinchium soboliferum Ravenna}

Hierba. Perenne. Nativa. 0-500 m.

Distribución: GUA, IGU.

Ejemplar de referencia: Ravenna, P. F. 1045

(BA, C, G, Herb. Ravenna, K, NY, P, RB).

Sisyrinchium subnudum I.M. Johnst. Hierba. Perenne. Nativa. 0-500 m. 
Distribución: CAI, SJA.

Ejemplar de referencia: Krapovickas, A. 15232 (CTES).

\section{Sisyrinchium vaginatum Spreng.}

Hierba. Perenne. Nativa. 0-700 m.

Distribución: APO, CAN, CAP, SIG.

Ejemplar de referencia: Morrone, O. 1912 (SI).

Trimezia Salisb. ex Herb.

Trimezia spathata (Klatt) Baker subsp. martii (Baker) Ravenna

Hierba. Perenne. Nativa. 0-500 m.

Distribución: CAN, GSM, GUA.

Ejemplar de referencia: Biganzoli, F. 280 (SI).

\section{JUGLANDACEAE}

Carya Nutt., nom. cons.

Carya illinoinensis (Wangenh.) C. Koch

Árbol. Introducida. 0-200 m.

Distribución: ELD, MON.

Ejemplar de referencia: Keller, H. A. 9149 (CTES, LP).

\section{JUNCACEAE}

\section{Juncus L.}

\section{Juncus densiflorus Kunth}

Hierba palustre. Perenne. Nativa. 0-500 m.

Distribución: APO, CAN.

Ejemplar de referencia: Clos, E. C. 2066 (BAB).

\section{Juncus imbricatus Laharpe}

Hierba. Perenne. Nativa. 0-500 m.

Distribución: GMB, SIG.

Ejemplar de referencia: Guaglianone, E. R. 2960 (SI).

\section{Juncus marginatus Rostk.}

Hierba. Perenne. Nativa. 0-500 m.

Distribución: CAN, SIG.

Ejemplar de referencia: Quiroga, H. s.n. (BA).
Juncus microcephalus Kunth

Hierba. Perenne. Nativa. 0-500 m.

Distribución: CAN, GUA, SIG, SPE.

Ejemplar de referencia: Jörgensen, P. 245 (BAB).

\section{Juncus pallescens Lam.}

Hierba. Perenne. Nativa. 0-500 m.

Distribución: SIG.

Ejemplar de referencia: Keller, H. A. 7607 (CTES).

\section{Juncus tenuis Willd.}

Hierba. Perenne. Nativa. 0-500 m.

Distribución: CAP, GMB.

Ejemplar de referencia: Zuloaga, F. O. 9940 (SI).

\section{LACISTEMATACEAE}

\section{Lacistema Sw.}

Lacistema hasslerianum $C$ hodat

Árbol. Nativa. 0-500 m.

Distribución: IGU.

Ejemplar de referencia: Keller, H. A. 6125 (CTES).

\section{LAMIACEAE}

\section{Aegiphila Jacq.}

Aegiphila brachiata Vell.

Arbusto o árbol. Nativa. 100-800 m.

Distribución: APO, CAI, CAN, GMB, GUA, IGU, MON, SIG, SJA, SPE.

Ejemplar de referencia: Morrone, O. 1117 (SI).

Aegiphila mediterranea Vell. ${ }^{* * *}$ )

Árbol. Nativa. 0-500 m.

Distribución: IGU.

Ejemplar de referencia: Amarilla, L. 100 (CTES).

Aegiphila vitelliniflora Klotzsch ex Walp.

Arbusto o liana. Nativa. 0-500 m.

Distribución: SIG.

Ejemplar de referencia: Keller, H. A. 6545 (CTES). 
Cantinoa Harley \& J.F.B. Pastore

Cantinoa althaeifolia (Pohl ex Benth.) Harley \& J.F.B. Pastore

Hierba o subarbusto. Perenne. 0-700 m.

Distribución: APO, CAN, CAP, MON, SIG.

Ejemplar de referencia: Ekman, E. L. 1828 (BR, $F, K, M O, N Y, S, U S$ ).

Cantinoa mutabilis (Rich.) Harley \& J.F.B. Pastore

Hierba. Perenne. Nativa. 0-800 m.

Distribución: APO, CAI, CAN, CAP, CON, ELD, GSM, GUA, MON, SIG, SJA, SPE.

Ejemplar de referencia: Múlgura, M. E. 1838 (SI).

Cantinoa stricta (Benth.) Harley \& J.F.B. Pastore Hierba. Perenne. Nativa. 0-500 m.

Distribución: CAN, GMB, SIG.

Ejemplar de referencia: Montes, J. E. 15121 (SI).

\section{Clerodendrum L.}

Clerodendrum ekmani Moldenke $\left(^{* * *}\right)$

Árbol. Nativa. 0-500 m.

Distribución: CAN, SIG.

Ejemplar de referencia: Keller, H. A. 10957 (SI).

\section{Clinopodium L.}

Clinopodium brownei (Sw.) Kuntze

Hierba o arbusto. Perenne. Nativa. 0-500 m.

Distribución: CAP, CAN.

Ejemplar de referencia: Keller, H. A. 13668 (CTES).

\section{Condea Adans.}

Condea elegans (Briq.) Harley \& J.F.B. Pastore Hierba. Perenne. Nativa. 0-500 m.

Distribución: ELD, GMB, IGU, SJA.

Ejemplar de referencia: Zuloaga, F. O. 5076 (SI).

Condea fastigiata (Benth.) Harley \& J.F.B. Pastore Hierba. Perenne. Nativa. 0-500 m.

Distribución: IGU, SIG.

Ejemplar de referencia: Keller, H. A. 3520 (CTES).
Condea floribunda (Briq.) Harley \& J.F.B. Pastore

Hierba o subarbusto. Perenne. Nativa. 0-700 m. Distribución: CAI, CAN, CAP, IGU, MON, SIG.

Ejemplar de referencia: Montes, J. E. 15200 (SI).

Condea undulata (Schrank) Harley \& J.F.B. Pastore

Hierba. Perenne. Nativa. 0-500 m.

Distribución: IGU, SIG, SPE.

Ejemplar de referencia: Montes, J. E. 2113 (SI).

Cunila D. Royen ex L.

Cunila angustifolia Benth. $\left({ }^{* * *}\right)$

Arbusto. Nativa. 200-500 m.

Distribución: CAN.

Ejemplar de referencia: Medán, D. 92 (BAA).

Cunila incana Benth.

Arbusto. Nativa. 0-500 m.

Distribución: CAI, CAN, CAP, CON, IGU, LNA, SIG, SJA.

Ejemplar de referencia: Cabrera, A. L. 29327 (SI).

Cunila spicata Benth.

Hierba. Perenne. Nativa. 0-600 m.

Distribución: APO, CAI, CAN, CAP.

Ejemplar de referencia: Rodríguez, F. M. 527 (SI).

\section{Glechon Spreng.}

Glechon ciliata Benth.

Hierba o arbusto. Perenne. Nativa. 0-700 m.

Distribución: APO, CAI, CAN, CON, SIG, SJA, SPE.

Ejemplar de referencia: Múlgura, M. E. 2239 (SI).

Glechon marifolia Benth. $\left.{ }^{* * *}\right)$

Hierba o arbusto. Perenne. Nativa. 0-500 m.

Distribución: CAI, GUA, SPE.

Ejemplar de referencia: Zuloaga, F. O. 8172 (SI).

\section{Glechon thymoides Spreng. $\left({ }^{* *}\right)$}

Hierba. Perenne. Nativa. 0-500 m.

Distribución: CAI, CON.

Ejemplar de referencia: Keller, H. A. 13540 (CTES). 


\section{Hedeoma Pers.}

Hedeoma teyucuarensis H.A. Keller \& Tressens $(* *)$

Subarbusto. Endémica. 100-200 m.

Distribución: SIG.

Ejemplar de referencia: Keller, H. A. 7559 (CTES).

\section{Hyptis Jacq.}

Hyptis australis Epling $\left(^{* *}\right)$

Hierba. Perenne. Endémica. 0-500 m.

Distribución: CAN, SIG.

Ejemplar de referencia: Keller, H. A. 13275 (CTES).

\section{Hyptis balansae Briq.}

Hierba. Perenne. Nativa. 0-600 m.

Distribución: APO, CAI, CAN, CAP, SIG, SPE.

Ejemplar de referencia: Montes, J. E. 15155 (SI).

\section{Hyptis brevipes Poit.}

Hierba. Perenne. Nativa. 0-600 m.

Distribución: APO, CAN, CAP, CON, GUA, SIG.

Ejemplar de referencia: Zuloaga, F. O. 936 (SI).

Hyptis comaroides (Briq.) Harley \& J.F.B. Pastore

Hierba. Perenne. Nativa. 0-700 m.

Distribución: APO, CAN, CAP, CON, SIG, SJA.

Ejemplar de referencia: Zuloaga, F. O. 5670 (SI).

Hyptis lagenaria A. St.-Hil. ex Benth. $\left.{ }^{(* *}\right)$

Hierba. Perenne. Nativa. 0-500 m.

Distribución: ELD, GUA.

Ejemplar de referencia: Keller, H. A. 1686 (CTES).

\section{Hyptis lappacea Benth.}

Hierba. Perenne. Nativa. 0-700 m.

Distribución: APO, CAP, ELD, GSM, SIG.

Ejemplar de referencia: Rodríguez $\left\{2^{\circ}\right.$ serie $\}, F$. M. 109 (SI).

Hyptis lappulacea Mart. ex Benth.

Hierba. Perenne. Nativa. 0-800 m.

Distribución: CAI, SIG, SPE.

Ejemplar de referencia: Biganzoli, F. 788 (SI).
Hyptis lorentziana O. Hoffm.

Hierba. Anual. Nativa. 0-600 m.

Distribución: CAP, GUA, IGU, SPE.

Ejemplar de referencia: Spegazzini, C. L.s.n. (BAB).

Hyptis muelleri Briq.

Hierba. Perenne. Nativa. 0-500 m.

Distribución: CAN, CON.

Ejemplar de referencia: Niederlein, G. 523 (SI).

Hyptis uliginosa A. St.-Hil. ex Benth. $\left({ }^{* *}\right)$

Hierba. Perenne. Nativa. 0-600 m.

Distribución: GMB, GUA, IGU, OBE, SPE.

Ejemplar de referencia: Keller, H. A. 141 (CTES).

\section{Lamium L.}

Lamium amplexicaule $\mathrm{L}$.

Hierba. Anual. Introducida. 100-500 m.

Distribución: CAP, MON.

Ejemplar de referencia: Keller, H. A. 241 (CTES, SI).

Leonotis (Pers.) R. Br.

Leonotis nepetifolia (L.) R. Br.

Hierba. Anual. Introducida. 0-600 m.

Distribución: CAN, CAP, GSM, GUA, SIG, SJA, SPE.

Ejemplar de referencia: Deginani, N. B. 1582 (SI).

\section{Leonurus L.}

Leonurus japonicus Houtt.

Hierba. Bianual. Introducida. 0-700 m.

Distribución: CAI, CAN, CAP, ELD, GMB, GUA, IGU, LNA, MON, OBE, SIG, SJA, SPE.

Ejemplar de referencia: Morrone, O. 2145 (SI).

Marsypianthes Mart. ex Benth.

Marsypianthes chamaedrys (Vahl) Kuntze

Hierba. Anual. Nativa. 0-500 m.

Distribución: ELD, IGU, GSM, SPE.

Ejemplar de referencia: Vanni, R. O. 4049 (CTES, K, LIL). 


\section{Marsypianthes hassleri Briq. $\left.{ }^{* * *}\right)$}

Hierba. Perenne. Nativa. 0-500 m.

Distribución: SIG.

Ejemplar de referencia: Zanotti, C. A. 390 (SI).

\section{Mentha L.}

\section{Mentha pulegium L.}

Hierba. Perenne. Introducida. 0-500 m.

Distribución: GUA, LNA.

Ejemplar de referencia: Keller, H. A. 3272 (CTES).

\section{Nepeta L.}

\section{Nepeta cataria L.}

Hierba. Perenne. Introducida. 0-500 m.

Distribución: CAN.

Ejemplar de referencia: Llamas, A. 730 (BAB).

\section{Ocimum L.}

Ocimum carnosum (Spreng.) Link \& Otto ex Benth

Hierba. Perenne. Nativa. 0-700 m.

Distribución: CAI, CAN, CAP, CON, GMB, GSM, GUA, IGU, LNA, MON, OBE, SIG, SJA, SPE.

Ejemplar de referencia: Morrone, O. 712 (SI).

\section{Ocimum nudicaule Benth.}

Hierba. Perenne. Nativa. 0-600 m.

Distribución: APO, CAN, CAP, CON, SIG, SJA.

Ejemplar de referencia: Morrone, O. 2228 (SI).

\section{Ocimum ovatum Benth.}

Hierba. Perenne. Nativa. 0-500 m.

Distribución: APO, CAN, CON, IGU, SIG.

Ejemplar de referencia: Montes, J. E. 650 (LA).

\section{Prunella L.}

\section{Prunella vulgaris $\mathrm{L}$.}

Hierba. Perenne. Introducida. 0-500 m.

Distribución: CAN.

Ejemplar de referencia: Schwarz, G. J. 682 (SI).
Rhabdocaulon (Benth.) Epling

Rhabdocaulon strictus (Benth.) Epling

Hierba. Perenne. Nativa. 0-500 m.

Distribución: CAP.

Ejemplar de referencia: Romanczuk, M. C. 330 (SI).

\section{Salvia L.}

Salvia coccinea Etl.

Hierba. Anual. Nativa. 0-500 m.

Distribución: CAP, SIG.

Ejemplar de referencia: Krapovickas, A. 2408 (SI).

Salvia guaranitica A. St.-Hil. ex Benth.

Hierba o subarbusto. Perenne. Nativa. 0-700 m.

Distribución: CAI, CAN, CAP, ELD, GMB, GSM, GUA, IGU, SIG, SPE.

Ejemplar de referencia: Zuloaga, F. O. 5028 (SI).

Salvia microphylla Kunth

Subarbusto. Introducida. 0-500 m.

Distribución: SIG.

Ejemplar de referencia: Gallardo, C. 883 (BA).

Salvia nervosa Benth.

Subarbusto. Nativa. 0-700 m.

Distribución: CAI, CAP.

Ejemplar de referencia: Spegazzini, C. L.s.n. (BAB).

Salvia ovalifolia A. St.-Hil. ex Benth. var. nitidula (Briq.) E.P. Santos

Subarbusto. Nativa. 200-400 m.

Distribución: CAN, CAP, LNA.

Ejemplar de referencia: Krapovickas, A. 15015 (CTES, MBM, P).

Salvia ovalifolia A. St.-Hil. ex Benth. var. ovalifolia

Subarbusto. Nativa. 200-300 m.

Distribución: APO, CAI, CAN, CAP, GSM, IGU, LNA, SPE.

Ejemplar de referencia: Schwindt, E. 3949 (SI, US).

Salvia pallida Benth.

Hierba. Perenne. Nativa. 0-500 m.

Distribución: CAN, CAP.

Ejemplar de referencia: Rodríguez, F. M. 13 (BAB). 
Salvia rosmarinoides A. St.-Hil. ex Benth.

Hierba o subarbusto. Perenne. Nativa. 0-500 m. Distribución: CAP, LNA, SJA.

Ejemplar de referencia: Krapovickas, A. 28842 (CTES).

Salvia subrotunda A. St.-Hil. ex Benth.

Hierba. Anual. Nativa. 0-500 m.

Distribución: CAN, CAP, GUA, SPE.

Ejemplar de referencia: Guaglianone, E. R. 2937 (SI).

\section{Salvia uliginosa Benth.}

Hierba o subarbusto. Perenne. Nativa. 0-500 m. Distribución: APO, CAN.

Ejemplar de referencia: Zuloaga, F. O. 6920 (SI).

\section{Scutellaria L.}

\section{Scutellaria platensis Speg.}

Hierba. Perenne. Nativa. 0-500 m.

Distribución: GUA.

Ejemplar de referencia: Tressens, S. G. 6522 (CTES).

\section{Scutellaria racemosa Pers.}

Hierba. Perenne. Nativa. 0-700 m.

Distribución: APO, CAI, CAN, CAP, GMB, GSM, GUA, IGU, SIG, SPE.

Ejemplar de referencia: Morrone, O. 1233 (SI).

Scutellaria uliginosa A. St.-Hil. ex Benth. $\left.{ }^{* * *}\right)$ Hierba. Perenne. Nativa. 0-700 m.

Distribución: GUA, IGU, SPE.

Ejemplar de referencia: Rodríguez, M. 560 (CTES, G, MNES, SI).

\section{Stachys L.}

\section{Stachys arvensis (L.) L.}

Hierba. Anual. Introducida. 0-500 m.

Distribución: ELD, GMB, OBE.

Ejemplar de referencia: Schinini, A. 5453 (BAB).

\section{Stachys gilliesii Benth.}

Hierba. Perenne. Nativa. 0-500 m.

Distribución: CAP.

Ejemplar de referencia: Muniez, A. A. 18 (BAB).

\section{Teucrium L.}

Teucrium cubense Jacq.

Hierba. Perenne. Nativa. 0-500 m.

Distribución: CON, CAN, SIG.

Ejemplar de referencia: Jörgensen, P. 267 $(B A B)$.

\section{Teucrium vesicarium Mill.}

Hierba. Perenne. Nativa. 0-700 m.

Distribución: CAI, CAN, CAP, GUA, OBE, SIG.

Ejemplar de referencia: Daviña, J. 287 (MNES, SI).

\section{Vitex L.}

Vitex megapotamica (Spreng.) Moldenke

Árbol. Nativa. 0-500 m.

Distribución: CAI, ELD, GUA, OBE, SIG, SPE.

Ejemplar de referencia: Morrone, O. 744 (SI).

\section{LAURACEAE}

\section{Aiouea Aubl.}

Aiouea amoena (Nees \& Mart.) R. Rohde ${ }^{* * *}$ )

Arbusto o árbol. Nativa. 0-500 m.

Distribución: GUA.

Ejemplar de referencia: Múlgura, M. E. 2563 (SI).

\section{Cinnamomum Schaeff.}

Cinnamomum verum $J$. Presl $\left({ }^{* * *}\right)$

Árbol. Introducida. 0-500 m.

Distribución: CAN, ELD.

Ejemplar de referencia: Keller, H. A. 12138 (CTES).

Cryptocarya R. Br.

\section{Cryptocarya aschersoniana $\mathrm{Mez}\left({ }^{* * *}\right)$}

Arbusto o árbol. Nativa. 0-500 m.

Distribución: GMB, SPE.

Ejemplar de referencia: Gamerro, J. C. 154 (SI). 
Endlicheria Nees, nom. cons.

Endlicheria paniculata (Spreng.) J.F. Macbr. $\left(^{*}\right)$ Árbol. Nativa. 0-500 m.

Distribución: IGU.

Ejemplar de referencia: Keller, H. A. 10420 (CTES).

Nectandra Rol. ex Rottb., nom. cons.

Nectandra angustifolia (Schrad.) Nees \& Mart. Arbusto o árbol. Nativa. 0-500 m.

Distribución: APO, CAI, CAN, CAP, CON, ELD, GMB, GSM, GUA, IGU, LNA, MON, OBE, SIG, SJA, SPE. Ejemplar de referencia: Montes, J. E. 803 (BAB, SI).

Nectandra lanceolata Nees \& Mart.

Árbol. Nativa. 0-500 m.

Distribución: GMB, GUA, IGU, MON, SIG, SPE. Ejemplar de referencia: Guaglianone, E. R. 2820 (SI).

Ocotea Aubl.

Ocotea acutifolia (Nees) Mez

Árbol. Nativa. 0-800 m.

Distribución: GMB, SIG.

Ejemplar de referencia: Múlgura, M. E. 1739 (SI).

Ocotea diospyrifolia (Meisn.) Mez

Árbol. Nativa. 0-800 m.

Distribución: CAI, ELD, GMB, GUA, IGU, MAY, OBE, SIG, SPE.

Ejemplar de referencia: Guaglianone, E. R. 2906 (SI).

Ocotea lancifolia (Schott) Mez

Arbusto o árbol. Nativa. 0-800 m.

Distribución: CAI, CAN, CAP, CON, GSM, MON, SIG.

Ejemplar de referencia: Zuloaga, F. O. 5736 (SI).

Ocotea puberula (Rich.) Nees

Árbol. Nativa. 0-800 m.

Distribución: CAN, CAP, CON, ELD, GMB, GUA, IGU, LNA, MAY, MON, SIG, SPE.

Ejemplar de referencia: Guaglianone, E. R. 2894 (SI).

Ocotea pulchella (Nees \& Mart.) Mez $\left(^{(* *}\right)$

Árbol. Nativa. 0-700 m.
Distribución: CAN, GMB, IGU.

Ejemplar de referencia: Ragonese, A. E. 107 (BAB).

Persea Mill., nom. cons.

Persea americana Mill.

Árbol. Introducida. 0-500 m.

Distribución: ELD.

Ejemplar de referencia: Keller, H. A. 2398 (CTES).

\section{LENTIBULARIACEAE}

\section{Utricularia L.}

\section{Utricularia foliosa L.}

Hierba acuática. Perenne. Nativa. 0-500 m.

Distribución: CAP, SIG.

Ejemplar de referencia: Rodríguez $\left\{2^{\circ}\right.$ serie $\}, F$. M. 447 (BA, BAF).

\section{Utricularia gibba $L$.}

Hierba acuática. Perenne. Nativa. 0-500 m.

Distribución: GMB, IGU.

Ejemplar de referencia: Múlgura, M. E. 1858 (SI).

Utricularia laxa A. St.-Hil. \& Girard

Hierba. Perenne. Nativa. 0-500 m.

Distribución: APO, CON, IGU, SIG.

Ejemplar de referencia: Biganzoli, F. 1714 (SI).

Utricularia nervosa G. Weber ex Benj.

Hierba. Perenne. Nativa. 0-500 m.

Distribución: CAP.

Ejemplar de referencia: Muniez, A. A. 33 (BA, $B A F)$.

Utricularia praelonga A. St.-Hil. \& Girard

Hierba. Perenne. Nativa. 0-500 m.

Distribución: GUA, SIG.

Ejemplar de referencia: Hauman, L. L. s.n. (BA).

Utricularia tricolor A. St.-Hil.

Hierba. Perenne. Nativa. 0-500 m.

Distribución: CON, IGU, SIG.

Ejemplar de referencia: Biganzoli, F. 1706 (SI). 


\section{Utricularia tridentata Sylvén}

Hierba. Perenne. Nativa. 0-500 m.

Distribución: GMB, SPE.

Ejemplar de referencia: Spegazzini, C. L. s.n. (LPS).

\section{LILIACEAE}

\section{Lilium L.}

Lilium regale E.H. Wilson $\left({ }^{* * *}\right)$

Hierba. Perenne. Introducida. 700-800 m.

Distribución: GMB.

Ejemplar de referencia: Keller, H. A. 4115 (CTES).

\section{LINACEAE}

\section{Clioccoca Bab.}

Cliococca selaginoides (Lam.) C.M. Rogers \& Mildner

Hierba. Perenne. Nativa. 0-500 m.

Distribución: CAP. CAN., SIG.

Ejemplar de referencia: Keller, H. A. 13709 (CTES).

\section{Linum L.}

Linum carneum A. St.-Hil.

Hierba. Perenne. Nativa. 0-500 m.

Distribución: ELD.

Ejemplar de referencia: Keller, H. A. 2810 (CTES).

\section{LINDERNIACEAE}

\section{Micranthemum Michx., nom. cons.}

Micranthemum umbrosum (J.F. Gmel.) S.F. Blake

Hierba. Anual. Nativa. 0-700 m.

Distribución: CAI, CAN, CAP, GMB, GUA, IGU, MON, SIG.

Ejemplar de referencia: Morrone, O. 1994 (SI).

\section{LOASACEAE}

Blumenbachia Schrad., nom. cons.

Blumenbachia latifolia Cambess.

Hierba. Anual o Bianual. Nativa. 0-700 m.

Distribución: CAI, CON, ELD, GUA, MON, SPE.

Ejemplar de referencia: Biganzoli, F. 555 (SI).

\section{LOGANIACEAE}

\section{Spigelia L.}

Spigelia caaguazuensis Kraenzl. ${ }^{* * *}$

Subarbusto. Nativa. 0-500 m.

Distribución: SIG.

Ejemplar de referencia: Schwarz, G. J. 1173 (LIL).

\section{Spigelia insignis Progel $\left({ }^{* *}\right)$}

Hierba. Perenne. Nativa. 0-500 m.

Distribución: SIG.

Ejemplar de referencia: Muniez, A. A. 116 (BA).

Spigelia martiana Cham. \& Schltdl.

Hierba. Perenne. Nativa. 0-600 m.

Distribución: CAN, MON, SIG, SPE.

Ejemplar de referencia: Zuloaga, F. O. 3181 (SI).

\section{Spigelia paraguariensis Chodat $\left({ }^{* * *}\right)$}

Hierba. Perenne. Nativa. 0-500 m.

Distribución: SIG.

Ejemplar de referencia: Quiroga, H. s.n. (BA).

Spigelia scabra Cham. \& Schltdl.

Hierba. Perenne. Nativa. 0-700 m.

Distribución: CAI, CAN, CAP, CON, ELD, GMB, GSM, GUA, IGU, SIG, SJA, SPE.

Ejemplar de referencia: Burkart, A. 14214 (SI).

\section{Spigelia stenophylla Progel}

Hierba. Perenne. Nativa. 0-500 m.

Distribución: CAN, LNA, SJA.

Ejemplar de referencia: Zuloaga, F. O. 6553 (SI). 


\section{Strychnos L.}

Strychnos brasiliensis (Spreng.) Mart.

Arbusto. Nativa. 0-700 m.

Distribución: APO, CAI, CAN, CAP, CON, ELD, GMB, GUA, IGU, MON, SIG, SJA, SPE.

Ejemplar de referencia: Deginani, N. B. 1064 (SI).

\section{LORANTHACEAE}

\section{Struthanthus Mart., nom. cons.}

Struthanthus uraguensis (Hook. \& Arn.) G. Don Arbusto parásito. Nativa. 0-500 m.

Distribución: APO.

Ejemplar de referencia: Burkart, A. 14297 (SI).

\section{Tripodanthus (Eichler) Tiegh.}

Tripodanthus acutifolius (Ruiz \& Pav.) Tiegh.

Arbusto parásito. Nativa. 0-500 m.

Distribución: CON, SPE.

Ejemplar de referencia: Morrone, O. 594 (SI).

\section{LYTHRACEAE}

\section{Cuphea P. Browne}

\section{Cuphea bonplandii Lourteig}

Hierba. Perenne. Nativa. 0-500 m.

Distribución: IGU, SIG.

Ejemplar de referencia: Ferrucci, M. S. 3466 (CTES, SI).

Cuphea calophylla Cham. \& Schltdl. subsp. mesostemon (Koehne) Lourteig

Hierba. Perenne. Nativa. 0-600 m.

Distribución: CAI, CAN, GMB, SIG, SPE.

Ejemplar de referencia: Biganzoli, F. 56 (SI).

\section{Cuphea carthagenensis (Jacq.) J.F. Macbr.}

Hierba o subarbusto. Perenne. Nativa. 0-500 m. Distribución: CAI, GMB, GUA, SIG, SPE.

Ejemplar de referencia: Biganzoli, F. 1488 (SI).
Cuphea confertiflora A. St.-Hil. $\left.{ }^{* * *}\right)$

Hierba. Perenne. Nativa. 0-500 m.

Distribución: CAN.

Ejemplar de referencia: Jörgensen, P. 141 (BAB).

\section{Cuphea fruticosa Spreng.}

Hierba o subarbusto. Perenne. Nativa. 0-500 m. Distribución: GMB, GUA, IGU, SPE.

Ejemplar de referencia: Zuloaga, F. O. 5253 (SI).

\section{Cuphea glaziovii Koehne $\left({ }^{* *}\right)$}

Arbusto. Nativa. 0-500 m.

Distribución: SPE.

Ejemplar de referencia: Bertoni, M. S. 998 (LIL).

\section{Cuphea glutinosa Cham. \& Schltdl.}

Hierba o subarbusto. Perenne. Nativa. 0-700 m.

Distribución: APO, CAI, CAN, CON, GSM, OBE, SIG.

Ejemplar de referencia: Biganzoli, F. 406 (SI).

\section{Cuphea hirsutissima Lourteig $\left({ }^{* * *}\right)$}

Subarbusto. Nativa. 200-500 m.

Distribución: APO, CON, SIG.

Ejemplar de referencia: Ibarrola, T. 962 (LIL).

Cuphea ingrata Cham. \& Schltdl.

Hierba o subarbusto. Perenne. Nativa. 0-700 m.

Distribución: APO, CAN, CAP, ELD, GUA, IGU,

OBE, SIG.

Ejemplar de referencia: Zuloaga, F. O. 5486 (SI).

\section{Cuphea laeviuscula Bacig.}

Hierba. Perenne. Nativa. 0-500 m.

Distribución: CAN, CAP.

Ejemplar de referencia: Guillén, R. 362 (MNES, SI).

Cuphea linarioides Cham. \& Schltdl.

Hierba. Perenne. Nativa. 0-500 m.

Distribución: CAI, CAN, LNA, OBE, SIG.

Ejemplar de referencia: Zuloaga, F. O. 6650 (SI).

Cuphea lysimachioides Cham. \& Schltdl.

Hierba. Perenne. Nativa. 0-700 m.

Distribución: APO, CAI, CAN, CAP, SIG, SPE.

Ejemplar de referencia: Zuloaga, F. O. 7170 (SI).

Cuphea melvilla Lindl.

Arbusto. Nativa. 0-500 m. 
Distribución: CAN, CAP, SIG.

Ejemplar de referencia: Burkart, A. 14475 (SI).

\section{Cuphea niederleinii Koehne $\left(^{* * *}\right)$}

Subarbusto. Nativa. 0-500 m.

Distribución: GMB, SPE.

Ejemplar de referencia: Múlgura, M. E. 2659 (SI).

Cuphea racemosa (L. f.) Spreng. var. ramosior (Koehne) Duré \& Molero

Hierba. Perenne. Nativa. 0-500 m.

Distribución: APO, CAI, CAN, CAP, CON, ELD, GSM, GMB, SIG.

Ejemplar de referencia: Biganzoli, F. 1350 (SI).

Cuphea racemosa (L. f.) Spreng. subsp. longiflora (Koehne) Duré \& Molero

Hierba. Nativa. 0-500 m.

Distribución: APO, CAI, CAN, GMB, GUA, IGU, LNA.

Ejemplar de referencia: Biganzoli, F. 551 (SI).

Cuphea racemosa (L. f.) Spreng. subsp. racemosa Hierba o subarbusto. Perenne. Nativa. 0-700 m. Distribución: CAN, ELD, GMB, GSM, GUA, SIG, SPE. Ejemplar de referencia: Deginani, N. B. 1159 (SI).

Cuphea thymoides Cham. \& Schltdl.

Hierba o subarbusto. Perenne. Nativa. 0-500 m. Distribución: CAN, GUA.

Ejemplar de referencia: Keller, H. A. 204 (CTES, SI).

Cuphea tuberosa Cham. \& Schltdl.

Hierba. Perenne. Nativa. 0-500 m.

Distribución: SIG.

Ejemplar de referencia: Schwarz, G. J. 1324 (LIL).

\section{Heimia Link \& Otto}

Heimia apetala (Spreng.) S.A. Graham \& Gandhi Arbusto. Nativa. 0-600 m.

Distribución: GSM, GUA, IGU, SIG, SJA.

Ejemplar de referencia: Meyer, T. 5345 (LIL).

\section{Heimia salicifolia (Kunth) Link}

Arbusto o subarbusto. Nativa. 0-600 m.

Distribución: APO, CAN, GMB.

Ejemplar de referencia: Morrone, O. 1760 (SI).

\section{Lafoensia Vand.}

Lafoensia nummularifolia A. St.-Hil. $\left.{ }^{* * *}\right)$

Arbusto o arbolito. Nativa. 0-500 m.

Distribución: SPE.

Ejemplar de referencia: Rodríguez, M. 1003

(CTES, G, MNES, SI).

\section{Rotala L.}

Rotala mexicana Cham. \& Schltdl.

Hierba. Anual. Nativa. 0-500 m.

Distribución: APO, CAN.

Ejemplar de referencia: Cabrera, A. L. 28502 (SI).

\section{MALPIGHIACEAE}

\section{Alicia W.R. Anderson}

Alicia anisopetala (A. Juss.) W.R. Anderson

Liana. Nativa. 0-500 m.

Distribución: CAI, IGU, SIG.

Ejemplar de referencia: Morrone 1875 (SI).

\section{Amorimia W.R. Anderson}

Amorimia exotropica (Griseb.) W.R. Anderson $(* * *)$

Liana. Nativa. 0-500 m.

Distribución: IGU.

Ejemplar de referencia: Keller 13575 (CTES).

\section{Aspicarpa Rich.}

Aspicarpa pulchella (Griseb.) O’Donell \& Lourteig

Hierba. Nativa. 0-500 m.

Distribución: APO, CAP, CON, SIG.

Ejemplar de referencia: Múlgura 2346 (SI).

\section{Aspicarpa salicifolia (Chodat) Nied.}

Hierba. Nativa. 0-500 m.

Distribución: CAN, SIG.

Ejemplar de referencia: Múlgura 2261 (SI). 
Banisteriopsis C.B. Rob.

Banisteriopsis muricata (Cav.) Cuatrec.

Arbusto o liana. Nativa. 0-700 m.

Distribución: GUA, ELD, IGU, SIG.

Ejemplar de referencia: Vanni 3572 (CTES, SI).

Bunchosia Rich. ex Kunth

Bunchosia pallescens Skottsb.

Árbol. Nativa. 0-500 m.

Distribución: GMB, GUA, IGU, SPE.

Ejemplar de referencia: Zuloaga 5613 (SI).

\section{Callaeum Small}

Callaeum psilophyllum (A. Juss.) D.M. Johnson

Enredadera o liana. Nativa. 0-500 m.

Distribución: CON, GUA, SJA.

Ejemplar de referencia: Deginani 1028 (SI).

\section{Dicella Griseb.}

\section{Dicella nucifera Chodat}

Liana. Perenne. Nativa. 0-700 m.

Distribución: CAI, CAN, ELD, GMB, GUA, IGU, SIG, SJA, SPE.

Ejemplar de referencia: Morrone. 745 (SI).

\section{Galphimia Cav.}

Galphimia australis Chodat

Subarbusto. Nativa. 0-700 m.

Distribución: CAP, GUA, MAY.

Ejemplar de referencia: Zuloaga, F. O. 8751 (SI).

Heladena A. Juss.

Heladena multiflora (Hook. \& Arn.) Nied.

Liana. Nativa. 0-500 m.

Distribución: CAN, CAP, SIG.

Ejemplar de referencia: Múlgura, M. E. 2093 (SI).
Heteropterys Kunth, nom. cons.

Heteropterys amplexicaulis Morong $\left(^{* * *}\right)$

Arbusto o liana. Nativa. 0-500 m.

Distribución: SIG.

Ejemplar de referencia: Keller, H. A. 13603 (CTES).

Heteropterys argyrophaea A. Juss.

Liana. Nativa. 0-500 m.

Distribución: CAI, GUA, SIG.

Ejemplar de referencia: Honfi, A. I. 528 (G, MNES).

Heteropterys bicolor A. Juss. (***)

Arbusto o liana. Nativa. 0-500 m.

Distribución: GMB, GUA.

Ejemplar de referencia: Múlgura, M. E. 2734 (SI).

Heteropterys glabra Hook. \& Arn.

Arbusto. Nativa. 0-500 m.

Distribución: APO, CAN, CAP, IGU, SPE.

Ejemplar de referencia: Burkart, A. 15482 (SI).

Heteropterys hypericifolia A. Juss.

Arbusto. Nativa. 0-500 m.

Distribución: CAI.

Ejemplar de referencia: Biganzoli, F. 1426 (SI).

Heteropterys intermedia (A. Juss.) Griseb.

Liana. Nativa. 0-500 m.

Distribución: APO, CAI, GMB, GSM, GUA, IGU, LNA, MAY, OBE, SIG, SPE.

Ejemplar de referencia: Deginani, N. B. 1473 (SI).

Heteropterys leschenaultiana A. Juss.

Arbusto o liana. Nativa. 0-500 m.

Distribución: GUA.

Ejemplar de referencia: Keller, H. A. 1613 (CTES).

Heteropterys microcarpa Skottsb. $\left({ }^{* * *}\right)$

Liana. Nativa. 400-800 m.

Distribución: CAN.

Ejemplar de referencia: Xifreda, C. C. 402 (SI).

Heteropterys mulgurae W.R. Anderson $\left(^{(* *}\right)$

Liana. Nativa. 0-500 m. 
Distribución: CON, IGU.

Ejemplar de referencia: Vanni, R. O. 3374 (CTES, MICH).

\section{Heteropterys syringifolia Griseb.}

Arbusto. Nativa. 0-500 m.

Distribución: CAI, CAN, CON, GMB, GUA, IGU, SIG.

Ejemplar de referencia: González, A. M. 36 (CTES, SI).

\section{Hiraea Jacq.}

Hiraea fagifolia (DC.) A. Juss.

Arbusto o liana. Nativa. 0-500 m.

Distribución: CAI, CON, IGU, SIG.

Ejemplar de referencia: Zuloaga, F. O. 5199 (SI).

\section{Janusia A. Juss.}

Janusia guaranitica (A. St.-Hil.) A. Juss.

Enredadera. Nativa. 0-800 m.

Distribución: APO, CAI, CAN, CON, GSM, LNA, SIG, SPE.

Ejemplar de referencia: Morrone, O. 586 (SI).

Janusia linearifolia (A. St.-Hil.) A. Juss. ${ }^{(* * *}$ )

Hierba. Nativa. 0-500 m.

Distribución: SIG.

Ejemplar de referencia: Zanotti, C. A. 401 (SI).

\section{Mascagnia (Bertero ex DC.) Colla}

Mascagnia divaricata (Kunth) Nied.

Liana. Nativa. 0-500 m.

Distribución: CAI, ELD, GMB, GUA, IGU, MAY, OBE, SIG, SPE.

Ejemplar de referencia: Zuloaga, F. O. 6610 (SI).

\section{Niedenzuella W.R. Anderson}

Niedenzuella sericea (A. Juss.) W.R. Anderson Arbusto o liana. Nativa. 0-500 m.

Distribución: IGU, SIG.

Ejemplar de referencia: Zuloaga, F. O. 5737 (SI).
Stigmaphyllon A. Juss.

Stigmaphyllon bonariense (Hook. \& Arn.) C.

E. Anderson

Liana. Nativa. 0-500 m.

Distribución: IGU, MON, SIG.

Ejemplar de referencia: Zuloaga, F. O. 5249 (SI).

Stigmaphyllon jatrophifolium A. Juss.

Liana. Nativa. 0-500 m.

Distribución: CAI, CAN, ELD, GSM, GUA, IGU, LNA, MON, SIG.

Ejemplar de referencia: Zuloaga, F. O. 5292 (SI).

Tetrapterys Cav., nom. cons.

Tetrapterys ambigua (A. Juss.) Nied.

Arbusto o subarbusto. Nativa. 0-500 m.

Distribución: CAN.

Ejemplar de referencia: Keller, H. A. 13030 (CTES).

Tetrapterys mollis Griseb. $\left.{ }^{* * *}\right)$

Arbusto o liana. Nativa. 0-500 m.

Distribución: CAI, GUA.

Ejemplar de referencia: Zuloaga, F. O. 6889 (SI).

\section{MALVACEAE}

\section{Abutilon Mill.}

Abutilon affine (Spreng.) G. Don

Arbusto. Nativa. 0-500 m.

Distribución: APO, CAN, SJA.

Ejemplar de referencia: Rodríguez, F. M. 749 (SI).

Abutilon grandifolium (Willd.) Sweet

Arbusto o subarbusto. Nativa. 0-600 m.

Distribución: CAN, CAP, IGU.

Ejemplar de referencia: Montes, J. E. 358 (BAB, CTES).

Abutilon malachroides A. St.-Hil. \& Naudin $(* * *)$

Subarbusto. Nativa. 0-500 m.

Distribución: CAI, SIG.

Ejemplar de referencia: Vanni, R. O. 681 (CTES, NY). 
Abutilon ramiflorum A. St.-Hil.

Arbusto. Nativa. 0-500 m.

Distribución: CAP.

Ejemplar de referencia: sin colector s.n. (BAB).

Abutilon terminale (Cav.) A. St.-Hil.

Subarbusto. Nativa. 0-500 m.

Distribución: APO, OBE.

Ejemplar de referencia: Cabrera, A. L. 28722 (SI).

Abutilon umbelliflorum A. St.-Hil.

Arbusto. Nativa. 0-700 m.

Distribución: APO, CAI, CAN, CAP, ELD, GMB, GSM, GUA, MAY, OBE, SPE.

Ejemplar de referencia: Morrone, O. 1827 (SI).

\section{Ayenia L.}

\section{Ayenia aprica Cristóbal}

Subarbusto. Nativa. 0-500 m.

Distribución: CAN, SIG.

Ejemplar de referencia: Montes, J. E. 10037 (LIL).

Ayenia mansfeldiana (Herter) Herter ex Cristóbal

Subarbusto. Nativa. 0-500 m.

Distribución: CAN, CAP, LNA.

Ejemplar de referencia: Ekman, E. L. 195 (NY, $S, U S)$.

\section{Ayenia nummularia Cristóbal}

Subarbusto. Nativa. 0-500 m.

Distribución: CAP, SIG.

Ejemplar de referencia: Schwarz, G. J. 6429 (LIL).

\section{Ayenia praecipua Cristóbal}

Subarbusto. Nativa. 0-500 m.

Distribución: CAN, SIG.

Ejemplar de referencia: Montes, J. E. 10031 (LIL).

\section{Bastardiopsis (K. Schum.) Hassl.}

Bastardiopsis densiflora (Hook. \& Arn.) Hassl. Árbol. Nativa. 0-800 m.

Distribución: CAI, CAN, ELD, GSM, GUA, IGU, SPE.

Ejemplar de referencia: Hunziker, J. H. 2620 (SI).
Byttneria Loefl., nom. cons.

Byttneria australis A. St.-Hil.

Arbusto. Nativa. 0-700 m.

Distribución: GUA, IGU, SJA, SPE.

Ejemplar de referencia: Rodríguez, M. 545 (CTES, G, MNES).

Byttneria catalpifolia Jacq. subsp. sidifolia (A. St.-Hil.) Cristóbal (**)

Liana. Nativa. 0-500 m.

Distribución: ELD, IGU.

Ejemplar de referencia: Schinini, A. 5944 (CTES).

Byttneria gracilipes Decne. ex Baill.

Arbusto. Nativa. 0-500 m.

Distribución: CAI, CAN, CAP, ELD, GUA, LNA, MON, SIG, SPE.

Ejemplar de referencia: Zuloaga, F. O. 5044 (SI).

\section{Byttneria pedersenii Cristóbal}

Subarbusto. Nativa. 0-500 m.

Distribución: APO, CAI, CAN, CAP, ELD, GSM, IGU, SIG, SJA.

Ejemplar de referencia: Montes, J. E. 9472 (LIL).

\section{Byttneria ramosissima Pohl $\left({ }^{* *}\right)$}

Subarbusto. Nativa. 0-500 m.

Distribución: CAP, IGU.

Ejemplar de referencia: Van de Venne, H. s.n. (LIL).

\section{Byttneria rojassi Cristóbal (***)}

Subarbusto. Nativa. 0-500 m.

Distribución: IGU.

Ejemplar de referencia: Keller, H. A. 1772 (CTES).

\section{Byttneria scabra $\mathrm{L}$.}

Arbusto. Nativa. 0-500 m.

Distribución: CAN, CAP, ELD, IGU, SIG.

Ejemplar de referencia: Keller, H. A. 76 (CTES, SI).

Byttneria urticifolia K. Schum.

Arbusto. Nativa. 0-500 m.

Distribución: CAN, CON, LNA, MON.

Ejemplar de referencia: Krapovickas, A. 15069 (CTES). 


\section{Callianthe Donnell}

Callianthe amoena (K. Schum.) Donnell (***)

Arbusto. Nativa. 0-500 m.

Distribución: ELD, MON, SPE.

Ejemplar de referencia: Deginani, N. B. 1662 (SI).

Callianthe pauciflora (A. St.-Hil.) Dorr

Arbusto. Nativa. 0-500 m.

Distribución: APO, CAN, SIG.

Ejemplar de referencia: Montes, J. E. 55 (BAB, CTES, MARY).

Callianthe picta (Gillies ex Hook. \& Arn.) Donnell

Arbusto. Nativa. 0-700 m.

Distribución: CAI, CAN, GMB, GSM, GUA, IGU, LNA, SIG, SJA, SPE.

Ejemplar de referencia: Deginani, N. B. 1021 (CTES, SI).

\section{Ceiba Mill.}

Ceiba pubiflora (A. St.-Hil.) K. Schum. $\left({ }^{* * *}\right)$

Árbol. Nativa. 0-500 m.

Distribución: CAN.

Ejemplar de referencia: Jörgensen, P. 729 (BAB, $M O, N Y$ ).

Ceiba speciosa (A. St.-Hil.) Ravenna

Árbol. Nativa. 0-700 m.

Distribución: CAN, CAP, GUA, SJA.

Ejemplar de referencia: Holmberg, E. L. 24 (SI).

\section{Corchorus L.}

Corchorus argutus Kunth

Hierba o subarbusto. Nativa. 0-500 m.

Distribución: APO, CAN, CAP, CON, ELD, GUA, IGU, LNA, OBE, SIG, SJA, SPE.

Ejemplar de referencia: Cabrera, A. L. 29134 (SI).

\section{Corchorus hirtus L.}

Hierba o subarbusto. Nativa. 0-700 m.

Distribución: CAN, SPE.

Ejemplar de referencia: Múlgura 3217 (SI).
Gaya Humb., Bonpl. \& Kunth

Gaya gracilipes K. Schum.

Subarbusto. Nativa. 0-700 m.

Distribución: APO, CAI, CAN, CAP, ELD, IGU, MON, SIG.

Ejemplar de referencia: Montes, J. E. 15124 (CTES, LIL).

\section{Gaya kelleri Krapov. ${ }^{* *}$ )}

Arbusto. Endémica. 0-200 m.

Distribución: SIG.

Ejemplar de referencia: Keller, H. A. 10806 (CTES, G, K, LIL, SI).

Gaya pilosa K. Schum.

Subarbusto. Nativa. 0-600 m.

Distribución: CAI, CAN, CAP, ELD, LNA, SIG, SJA.

Ejemplar de referencia: Schwindt, E. 4662 (CTES, LIL).

\section{Guazuma Mill.}

Guazuma ulmifolia Lam. var. tomentella K. Schum.

Árbol. Nativa. 0-500 m.

Distribución: CAN, ELD, SIG.

Ejemplar de referencia: Rodríguez, F. M. 11 (SI).

\section{Heliocarpus L.}

Heliocarpus popayanensis Kunth

Árbol. Nativa. 0-700 m.

Distribución: ELD, GUA, IGU, SIG, SPE.

Ejemplar de referencia: Dematteis, M. 123

(CTES, G, MNES).

\section{Herissantia Medik.}

Herissantia intermedia (Hassl.) Krapov.

Subarbusto. Nativa. 0-500 m.

Distribución: IGU.

Ejemplar de referencia: Keller, H. A. 9667 (CTES). 
Herissantia nemoralis (A. St.-Hil.) Brizicky

Arbusto. Nativa. 0-500 m.

Distribución: CAN, ELD, SIG.

Ejemplar de referencia: Krapovickas, A. 28764 (CTES, SI).

\section{Hibiscus L.}

Hibiscus bifurcatus $C a v$.

Arbusto. Nativa. 0-500 m.

Distribución: SIG.

Ejemplar de referencia: Keller, H. A. 12212 (CTES).

\section{Hibiscus furcellatus Desr.}

Arbusto. Nativa. 0-500 m.

Distribución: CAP.

Ejemplar de referencia: Molfino, J. F. s.n. (BAF).

\section{Hibiscus sororius $\mathrm{L}$.}

Arbusto. Nativa. 0-500 m.

Distribución: CAN.

Ejemplar de referencia: Molfino, J. F. s.n. (BAF).

\section{Hibiscus striatus Cav.}

Arbusto. Nativa. 0-700 m.

Distribución: CAP, SPE.

Ejemplar de referencia: Deginani, N. B. 1686 (SI).

Hibiscus urticifolium A. St.-Hil. \& Naudin

Arbusto. Nativa. 0-500 m.

Distribución: CAN.

Ejemplar de referencia: Rodríguez, F. M. 644 (SI).

\section{Hochreutinera Krapov.}

Hochreutinera hasslerana (Hochr.) Krapov.

Subarbusto. Nativa. 0-500 m.

Distribución: CAI, CAN, CAP, CON, IGU, SPE.

Ejemplar de referencia: Seijo, G. J. 1981 (CTES, SI).

\section{Krapovickasia Fryxell}

Krapovickasia flavescens (Cav.) Fryxell

Hierba. Nativa. 0-500 m.

Distribución: CAN.

Ejemplar de referencia: Montes, J. E. 522 (SI).
Krapovickasia macrodon (DC.) Fryxell

Hierba. Nativa. 0-800 m.

Distribución: CAN, CON, GMB.

Ejemplar de referencia: Keller, H. A. 3704 (CTES).

Krapovickasia urticifolia (A. St.-Hil.) Fryxell

Hierba. Nativa. 0-800 m.

Distribución: APO, CAI, GSM, MON, SIG, SJA.

Ejemplar de referencia: Krapovickas, A. 15189 (CTES).

\section{Luehea Willd.}

Luehea candicans Mart.

Árbol. Nativa. 0-500 m.

Distribución: IGU, MON, SIG.

Ejemplar de referencia: Keller, H. A. 206 (CTES).

Luehea divaricata Mart.

Árbol. Nativa. 0-500 m.

Distribución: APO, CAI, CAN, CAP, CON, ELD, GMB, GSM, GUA, IGU, LNA, MON, OBE, SIG, SJA, SPE.

Ejemplar de referencia: Zuloaga, F. O. 5051 (SI).

Luehea microcarpa R.E. Fr. $\left({ }^{* * *}\right)$

Árbol. Nativa. 0-500 m.

Distribución: GMB.

Ejemplar de referencia: Gallardo, C. s.n. (BAF).

Luehea paniculata Mart. $\left(^{* *}\right)$

Árbol. Nativa. 0-500 m.

Distribución: SIG.

Ejemplar de referencia: Vattuone, I. C. s.n. (BAF).

Malvastrum A. Gray, nom. cons.

Malvastrum americanum (L.) Torr.

Subarbusto. Nativa. 0-500 m.

Distribución: CAN, CAP.

Ejemplar de referencia: Ekman, E. L. 132 (MO, NY).

Malvastrum coromandelianum (L.) Garcke

Subarbusto. Nativa. 0-700 m.

Distribución: CAN, CON, GSM, GUA, OBE, SJA.

Ejemplar de referencia: Krapovickas, A. 47657

(CTES, SI). 
Malvastrum uniapiculatum Krapov. $\left.{ }^{(* *}\right)$

Arbusto. Endémica. 100-200 m.

Distribución: CON, SJA.

Ejemplar de referencia: Keller, H. A. 8000 (CTES, CORD, LP, MBM).

\section{Melochia L.}

Melochia argentina R.E. Fr.

Subarbusto. Nativa. 600-800 m.

Distribución: SPE.

Ejemplar de referencia: Deginani, N. B. 1178 (SI).

Melochia chamaedrys A. St.-Hil. var. chamaedrys

Subarbusto. Nativa. 0-800 m.

Distribución: APO, CAI, CAN, CAP, GSM, LNA, SIG, SJA.

Ejemplar de referencia: Zuloaga, F. O. 4892 (SI).

Melochia chamaedrys A. St.-Hil. var. decumbens (R.E. Fr.) Goldberg

Subarbusto. Nativa. 0-500 m.

Distribución: APO, CAI, CAN.

Ejemplar de referencia: Biganzoli, F. 822 (SI).

\section{Melochia goldbergii Cristóbal}

Subarbusto. Nativa. 0-500 m.

Distribución: CON.

Ejemplar de referencia: Biganzoli, F. 1736 (SI).

\section{Melochia hassleriana Chodat}

Subarbusto. Nativa. 0-700 m.

Distribución: APO, CAN, CAP, GUA, SIG. (SI).

Ejemplar de referencia: Zuloaga, F. O. 8163

Melochia hermannioides A. St.-Hil.

Hierba o subarbusto. Nativa. 0-500 m.

Distribución: CAN.

Ejemplar de referencia: Cocucci, A. A. 3243 (CORD, SI).

Melochia morongii Britton (***)

Subarbusto. Nativa. 0-500 m.

Distribución: CAN, SIG.

Ejemplar de referencia: Biganzoli, F. 1435 (SI).
Melochia pilosa (Mill.) Fawc. \& Rendle

Subarbusto. Nativa. 0-800 m.

Distribución: APO, CAN, CAP, CON, GUA, IGU, SIG.

Ejemplar de referencia: Cocucci, A. A. 3165 (CORD, CTES, SI).

\section{Melochia pyramidata $\mathrm{L}$.}

Subarbusto. Nativa. 0-800 m.

Distribución: CAI, CAN, CAP, GUA, IGU, OBE, SIG, SPE.

Ejemplar de referencia: Burkart, A. 15192 (SI).

Melochia simplex A. St.-Hil.

Subarbusto. Nativa. 0-500 m.

Distribución: CAN, CAP, IGU, SIG.

Ejemplar de referencia: Krapovickas, A. 12031 (CTES).

Melochia villosa (Mill.) Fawc. \& Rendle var. tomentosa (K. Schum.) Goldberg

Subarbusto. Nativa. 0-500 m.

Distribución: CAI, CAN.

Ejemplar de referencia: Schwindt, E. 3323 (CTES).

Modiola Moench.

Modiola carolinianum (L.) G. Don

Hierba. Perenne. Nativa. 0-500 m.

Distribución: CAI, CAN, CON, IGU.

Ejemplar de referencia: Keller, H. A. 4888 (CTES).

\section{Modiolastrum K. Schum.}

Modiolastrum lateritium (Hook.) Krapov.

Hierba. Nativa. 0-500 m.

Distribución: CAN, CAP, ELD, GUA, SPE.

Ejemplar de referencia: Guaglianone, E. R. 2888 (SI).

Modiolastrum malvifolium (Griseb.) K. Schum.

Hierba. Nativa. 0-700 m.

Distribución: APO, CAN, GMB, SPE.

Ejemplar de referencia: Morrone, O. 1154 (SI). 


\section{Pavonia Cav.}

Pavonia angustipetala Krapov. \& Cristóbal (***) Arbusto. Nativa. 0-500 m.

Distribución: CAI, CAN.

Ejemplar de referencia: Schwarz, G. J. 10601 (LIL).

Pavonia aurigloba Krapov. \& Cristóbal

Arbusto. Nativa. 0-500 m.

Distribución: GMB.

Ejemplar de referencia: Keller, H. A. 3713 (CTES).

Pavonia betonicaefolia $C$. Presl

Arbusto. Nativa. 0-500 m.

Distribución: CAN, CAP, LNA.

Ejemplar de referencia: Schinini, A. 5540 (CTES).

Pavonia communis A. St.-Hil.

Arbusto. Nativa. 0-800 m.

Distribución: CAN, GMB, IGU, MON, SIG.

Ejemplar de referencia: Vanni, R. O. 1421 (CLEMS, CTES, F, LIL, NY).

Pavonia distinguenda A. St.-Hil. \& Naudin

Subarbusto. Nativa. 0-700 m.

Distribución: CON.

Ejemplar de referencia: Schwarz, E. A. 3631 (LIL).

\section{Pavonia flavispina Miq. $\left.{ }^{(* *}\right)$}

Subarbusto. Nativa. 0-500 m.

Distribución: ELD, GSM, OBE, SIG, SJA.

Ejemplar de referencia: Krapovickas, A. 2302 (CTES, MO, SI).

\section{Pavonia glechomoides A. St.-Hil.}

Hierba. Perenne. Nativa. 0-500 m.

Distribución: CON.

Ejemplar de referencia: Keller, H. A. 5721 (CTES).

Pavonia glutinosa Krapov. \& Cristóbal

Subarbusto. Nativa. 0-500 m.

Distribución: CAN.

Ejemplar de referencia: Bertoni, M. S. 98416 (U).

\section{Pavonia guerkeana R.E. Fr.}

Arbusto. Nativa. 0-700 m.

Distribución: GMB, GUA.

Ejemplar de referencia: Krapovickas, A. 23384 (BAA, BAB, CTES).

\section{Pavonia hastata Cav.}

Subarbusto. Nativa. 0-800 m.

Distribución: CAI, CAN, CAP, CON, LNA, MON, OBE, SIG, SJA.

Ejemplar de referencia: Morrone, O. 598 (SI).

Pavonia laxifolia A. St.-Hil. $\left.{ }^{* * *}\right)$

Arbusto. Nativa. 200-600 m.

Distribución: CAN.

Ejemplar de referencia: Ekman, E. L. 174 (MO).

Pavonia missionum Ekman

Arbusto. Nativa. 0-800 m.

Distribución: APO, CAI, CAN, CAP, GMB, GUA, IGU, MON, OBE, SIG, SPE.

Ejemplar de referencia: Seijo, G. J. 1975 (CTES, SI).

\section{Pavonia narcissi Krapov.}

Arbusto. Nativa. 0-500 m.

Distribución: CAI.

Ejemplar de referencia: Schwindt, E. 4451 $(C A S)$

Pavonia psilophylla Ekman $\left({ }^{* * *}\right)$

Arbusto. Nativa. 0-700 m.

Distribución: CAN, OBE, SIG, SJA, SPE.

Ejemplar de referencia: Morrone, O. 2173 (SI).

\section{Pavonia restiaria Bertoni}

Arbusto. Nativa. 0-500 m.

Distribución: CAN, CAP, ELD, GSM, IGU, MON, SIG.

Ejemplar de referencia: Krapovickas, A. 2354 (CTES, NY).

Pavonia rubra Krapov. \& Cristóbal

Arbusto. Nativa. 100-300 m.

Distribución: SIG.

Ejemplar de referencia: Schwarz, E. A. 5637 (LIL).

Pavonia sepium A. St.-Hil.

Arbusto. Nativa. 0-800 m.

Distribución: CAI, CAP, GMB, GSM, GUA, IGU, MON, OBE, SIG, SJA, SPE.

Ejemplar de referencia: Daviña, J. 207 (CTES, $M N E S)$. 
Pavonia sidifolia Kunth

Arbusto o subarbusto. Nativa. 0-800 m.

Distribución: ELD, MON.

Ejemplar de referencia: Krapovickas, A. 43577 (CTES, SI).

Pavonia subrotunda A. St.-Hil. \& Naudin

Subarbusto. Nativa. 0-500 m.

Distribución: SIG.

Ejemplar de referencia: Zanotti, C. A. 394 (SI).

Pavonia vitifolia Hochr. ex Chodat \& Hassl.

Arbusto. Nativa. 100-600 m.

Distribución: SJA.

Ejemplar de referencia: Bertoni, M. S. 5870 (CTES).

\section{Pavonia xanthogloea Ekman}

Arbusto. Nativa. 0-500 m.

Distribución: APO, CAN, CON, GSM, LNA, OBE, SIG, SJA.

Ejemplar de referencia: Krapovickas, A. 28833 (CTES, G, NY, SI).

Peltaea (C. Presl) Standl.

Peltaea edouardii (Hochr.) Krapov. \& Cristóbal

$\left(^{*}\right)$

Subarbusto. Nativa. 0-500 m.

Distribución: GMB.

Ejemplar de referencia: Keller, H. A. 3548 (CTES).

\section{Pseudabutilon R.E. Fr.}

Pseudabutilon aristulosum (K. Schum.)

Krapov.

Arbusto. Nativa. 0-500 m.

Distribución: CON, IGU.

Ejemplar de referencia: Keller, H. A. 5780 (CTES).

Pseudabutilon balansae (Hassl.) H.A. Keller $\left({ }^{* *}\right)$

Subarbusto. Nativa. 0-500 m.

Distribución: CAI.

Ejemplar de referencia: Keller, H. A. 12744 (CTES).
Sida L.

Sida anomala A. St.-Hil.

Hierba. Nativa. 0-500 m.

Distribución: CAP.

Ejemplar de referencia: Molfino, J. F. s.n. (BAF).

Sida argentina K. Schum.

Hierba. Nativa. 0-500 m.

Distribución: CAN.

Ejemplar de referencia: Jörgensen, P. 281 (BAB).

Sida caudata A. St.-Hil. \& Naudin

Hierba. Nativa. 500-700 m.

Distribución: CAN, IGU.

Ejemplar de referencia: Vanni, R. O. 2793 (CTES).

Sida confusa Hassl.

Hierba. Perenne. Nativa. 0-500 m.

Distribución: APO, CAN.

Ejemplar de referencia: Burkart, A. 14382 (SI).

\section{Sida cordifolia L.}

Subarbusto. Nativa. 0-700 m.

Distribución: ELD, IGU, SIG.

Ejemplar de referencia: Vanni, R. O. 4020 (CTES).

Sida honoriana Krapov. $\left({ }^{* * *}\right)$

Subarbusto. Nativa. 0-500 m.

Distribucion: GMB, GUA, SPE.

Ejemplar de referencia: Keller, H. A. 2153 (CTES, SI, TEX).

Sida ignaciana Krapov. $\left.{ }^{(* *}\right)$

Arbusto. Endémica. 0-500 m.

Distribución: SIG.

Ejemplar de referencia: Keller, H. A. 8623 (CTES).

Sida kelleri Krapov. $\left({ }^{* * *}\right)$

Arbusto. Nativa. 0-500 m.

Distribución: CAN, GUA, SIG.

Ejemplar de referencia: Keller, H. A. 9700 (CTES).

Sida lilianae Krapov. $\left({ }^{* * *}\right)$

Subarbusto. Nativa. 0-500 M.

Distribución: CAP.

Ejemplar de referencia: Keller, H. A. 8815 (CTES, NY, SI). 
Sida loretana Krapov. $\left({ }^{* *}\right)$

Subarbusto. Endémica. 0-500 m.

Distribución: CAN.

Ejemplar de referencia: Keller, H. A. 8365 (CTES).

Sida nemorensis Mart. ex Colla $\left(^{*}\right)$

Subarbusto. Nativa. 0-500 m.

Distribución: ELD, GMB, SPE.

Ejemplar de referencia: Keller, H. A. 4974 (CTES).

\section{Sida palustris Krapov. $\left({ }^{* *}\right)$}

Arbusto. Endémica. 0-500 m.

Distribución: CAN.

Ejemplar de referencia: Keller, H. A. 13284 (CTES).

\section{Sida pindapoyensis Krapov. $\left(^{* *}\right)$}

Subarbusto. Endémica. 0-200 m.

Distribución: CAP.

Ejemplar de referencia: Krapovickas, A. 7935 (CTES, LIL, SI).

\section{Sida planicaulis Cav. $\left.{ }^{* * *}\right)$}

Subarbusto. Nativa. 0-300 m.

Distribución: GUA.

Ejemplar de referencia: Keller, H. A. 3684 (CTES).

Sida poeppigiana (K. Schum.) Fryxell

Subarbusto. Nativa. 0-700 m.

Distribución: GUA.

Ejemplar de referencia: Keller, H. A. 644 (SI).

Sida potentilloides A. St.-Hil.

Subarbusto. Nativa. 0-800 m.

Distribución: APO, CAN, CON, OBE, SIG.

Ejemplar de referencia: Keller, H. A. 13699 (CTES).

Sida ramoniana Krapov. $\left({ }^{* *}\right)$

Arbusto. Endémica. 0-500 m.

Distribución: OBE.

Ejemplar de referencia: Keller, H. A. 10825

(CTES, FCQ, K, SI, LIL).

Sida riedelii $\mathrm{K}$. Schum.

Subarbusto. Nativa. 0-500 m.

Distribución: CAN, GMB, OBE, SIG.

Ejemplar de referencia: Keller, H. A. 4743 (CTES).

Sida regnellii R.E. Fr.

Subarbusto. Nativa. 0-500 m.
Distribución: CAN, SIG, SJA.

Ejemplar de referencia: Keller, H. A. 119 (CTES).

Sida rhizomatosa Krapov. $\left(^{* *}\right)$

Hierba. Endémica. 100-200 m.

Distribución: CAN.

Ejemplar de referencia: Keller, H. A. 10962 (CTES,NY,SI).

\section{Sida rhombifolia L.}

Hierba o subarbusto. Nativa. 0-700 m.

Distribución: APO, CAI, CAN, GMB, GSM, IGU, MON, OBE, SPE.

Ejemplar de referencia: Biganzoli, F. 230 (SI).

Sida rufescens A. St.-Hil. $\left.{ }^{* * *}\right)$

Hierba. Nativa. 100-500 m.

Distribución: CAN.

Ejemplar de referencia: Schwindt, E. 92 (CTES, $N Y$ ).

Sida santaremensis Monteiro

Subarbusto. Nativa. 0-600 m.

Distribución: ELD, SIG, SPE.

Ejemplar de referencia: Tressens, S. G. 5550 (CTES).

\section{Sida spinosa $L$.}

Subarbusto. Nativa. 0-500 m.

Distribución: CAP, GUA, MON.

Ejemplar de referencia: Keller, H. A. 837 (CTES).

Sida tuberculata R.E. Fr. var. pseudorhombifolia Monteiro

Subarbusto. Nativa. 0-500 m.

Distribución: CAI, CAN, CAP, CON, GMB, SIG.

Ejemplar de referencia: Montes, J. E. 27624 (CTES, NY).

\section{Sida urens $\mathrm{L}$.}

Hierba. Nativa. 0-600 m.

Distribución: CAI, CAP, ELD, GSM, GUA, IGU, MON, SIG.

Ejemplar de referencia: Deginani, N. B. 1502 (SI).

\section{Sida vespertina Ekman}

Subarbusto. Nativa. 0-500 m.

Distribución: CAN, SIG.

Ejemplar de referencia: Zuloaga, F. O. 6627 (SI). 
Sida viarum A. St.-Hil.

Subarbusto. Nativa. 0-700 m.

Distribución: CAN, GSM, GUA, SIG.

Ejemplar de referencia: Deginani, N. B. $1072 a$ (SI).

\section{Triumfetta L.}

\section{Triumfetta bogotensis DC.}

Arbusto. Nativa. 0-500 m.

Distribución: GUA, SIG, SPE.

Ejemplar de referencia: Morrone, O. 794 (SI).

\section{Triumfetta guaranitica Villa $\left.{ }^{(* *}\right)$}

Arbusto. Nativa. 600-800 m.

Distribución: GMB, IGU, SPE.

Ejemplar de referencia: Zuloaga, F. O. 4994 (SI).

Triumfetta obscura A.St.-Hil.,A. Juss. \& Cambess. $\left.{ }^{* * *}\right)$

Arbusto. Nativa. 500-800 m.

Distribución: CAN.

Ejemplar de referencia: Ekman, E. L. 200 (S).

\section{Triumfetta semitriloba Jacq.}

Arbusto. Nativa. 100-700 m.

Distribución: APO, CAN, CAP, GMB, GSM, GUA, IGU, LNA, SIG, SJA.

Ejemplar de referencia: Zuloaga, F. O. 5290 (SI).

\section{Waltheria L.}

Waltheria communis A. St.-Hil.

Hierba. Nativa. 0-700 m.

Distribución: CON, GUA, SIG, SPE.

Ejemplar de referencia: Deginani, N. B. 1647 (SI).

\section{Waltheria indica $\mathrm{L}$.}

Subarbusto. Nativa. 0-800 m.

Distribución: ELD, IGU, SIG, SPE.

Ejemplar de referencia: Vanni, R. O. 2791 (CTES).

\section{Wissadula Medik.}

Wissadula densiflora R.E. Fr.

Subarbusto. Nativa. 0-600 m.

Distribución: SIG.

Ejemplar de referencia: Zuloaga, F. O. 9967 (SI).
Wissadula excelsior (Cav.) C. Presl (***)

Arbusto. Nativa. 100-500 m.

Distribución: GUA, IGU, SPE.

Ejemplar de referencia: Deginani, N. B. 1607 (SI).

Wissadula glechomaefolia (A. St.-Hil.) R.E. Fr. Hierba. Nativa. 0-500 m.

Distribución: CAN.

Ejemplar de referencia: Keller, H. A. 10959 (CTES).

Wissadula hernandioides (L'Hér.) Garcke

Arbusto. Nativa. 0-700 m.

Distribución: CAI, CAN, CON, ELD, GSM, IGU, MON, SIG, SJA, SPE.

Ejemplar de referencia: Morrone, O. 1073 (SI).

Wissadula parviflora (A. St.-Hil.) R.E. Fr. $\left(^{* * *}\right)$

Subarbusto. Nativa. 0-800 m.

Distribución: CAN, IGU.

Ejemplar de referencia: Morrone, O. 2015 (SI).

\section{MARANTACEAE}

\section{Ctenanthe Eichler}

\section{Ctenanthe muelleri Petersen $\left({ }^{* * *}\right)$}

Hierba. Perenne. Nativa. 0-800 m.

Distribución: CAI, ELD, GMB, GUA, IGU, SIG, SPE. Ejemplar de referencia: Morrone, O. 1577 (SI).

\section{Goeppertia Ness}

Goeppertia eichleri (Petersen) Borchs. \& S. Suárez

Hierba. Perenne. Nativa. 0-700 m.

Distribución: CAI, GMB, GSM, IGU, SIG, SPE.

Ejemplar de referencia: Guaglianone, E. R. 2855 (SI).

\section{Maranta L.}

Maranta sobolifera L.Andersson

Hierba. Perenne. Nativa. 0-700 m.

Distribución: APO, CAI, GMB, GUA, IGU, SIG, SJA, SPE.

Ejemplar de referencia: Morrone, O. 823 (SI). 
Saranthe (Regel \& Körn.) Eichler

\section{Saranthe eichleri Petersen $\left({ }^{* * *}\right)$}

Hierba. Perenne. Nativa. 0-500 m.

Distribución: GUA, IGU, SPE.

Ejemplar de referencia: Bertoni, M. S. s.n. (BA).

\section{Thalia L.}

\section{Thalia geniculata $\mathrm{L}$.}

Hierba palustre. Perenne. Nativa. 0-100 m.

Distribución: CON, SIG, SJA.

Ejemplar de referencia: Keller, H. A. 8133 (CTES).

\section{MARTYNIACEAE}

\section{Craniolaria L.}

Craniolaria integrifolia Cham.

Hierba. Nativa. 0-600 m.

Distribución: CAN, SIG.

Ejemplar de referencia: Zuloaga, F. O. 5669 (SI).

\section{MAYACACEAE}

\section{Mayaca Aubl.}

\section{Mayaca sellowiana Kunth}

Hierba. Nativa. 0-800 m.

Distribución: CAN, CON, IGU, MON, SIG.

Ejemplar de referencia: Biganzoli, F. 1701 (SI).

\section{MAZACEAE}

\section{Mazus Lour.}

Mazus japonicus (Thunb.) Kuntze

Hierba. Perenne. Introducida. 0-500 m.

Distribución: ELD.

Ejemplar de referencia: Keller, H. A. 13907 (CTES).

\section{MELASTOMATACEAE}

Acisanthera P. Browne

Acisanthera alsinaefolia (DC.) Triana

Hierba. Nativa. 0-700 m.

Distribución: CAN, GMB, SIG. (SI).

Ejemplar de referencia: Guaglianone, E. R. 1154

Acisanthera paraguayensis (Hook. f.) Cogn.

Arbusto. Nativa. 0-500 m.

Distribución: GUA, SPE.

Ejemplar de referencia: Zuloaga, F. O. 6881 (SI, MO).

\section{Chaetogastra DC.}

\section{Chaetogastra debilis Cham.}

Hierba o subarbusto. Nativa. 0-600 m.

Distribución: CAI, CON, SJA.

Ejemplar de referencia: Krapovickas, A. 28819 (CTES).

\section{Chaetogastra gracilis (Bonpl.) DC.}

Hierba o subarbusto. Nativa. 0-700 m.

Distribución: APO, CAN, CAP, CON, GMB, IGU, SIG.

Ejemplar de referencia: Guaglianone, E. R. 912 (SI).

Chaetogastra herbacea (DC.) P.J.F. Guim. \& Michelangeli

Hierba o subarbusto. Perenne. Nativa. 0-500 m.

Distribución: CAN, GUA, IGU, SIG, SPE.

Ejemplar de referencia: Zuloaga, F. O. 6694 (SI).

Chaetogastra longipilosa (Cogn.) P.J.F. Guim. \& Michelangeli $(* * *)$

Subarbusto. Nativa. 0-500 m.

Distribución: GMB, GUA, IGU, SPE.

Ejemplar de referencia: Múlgura, M.E. 3353 (SI).

Chaetogastra nitida (Graham) P.J.F. Guim. \& Michelangeli

Hierba o arbusto. Perenne. Nativa 0-500 m.

Distribución: MON.

Ejemplar de referencia: Montes, J. E. 15210 (SI). 
Chaetogastra paratropica (Griseb.) P.J.F. Guim. \& Michelangeli

Subarbusto. Nativa. 0-500 m.

Distribución: IGU.

Ejemplar de referencia: Bottino, O. 389 (LP).

\section{Clidemia D. Don}

Clidemia hirta (L.) D. Don $\left(^{* * *}\right)$

Arbusto. Nativa. 0-500 m.

Distribución: SIG.

Ejemplar de referencia: Biganzoli, F. 439 (NY, SI).

Miconia Ruiz \& Pav., nom. cons.

Miconia alterninervia (Cogn.) R. Goldenb.

Arbusto. Nativa. 0-500 m.

Distribución: ELD, GMB, GUA, IGU, OBE, SPE. $(S I)$.

Ejemplar de referencia: Deginani, N. B. 1262

Miconia australis (Cham.) R. Goldenb.

Arbolito. Nativa. 0-500 m.

Distribución: GMB, GUA, IGU, SIG, SJA, SPE.

Ejemplar de referencia: Morrone, O. 772 (SI).

Miconia balansae (Cogn.) R. Goldenb.

Arbusto. Nativa. 0-800 m.

Distribución: GMB, GUA, IGU, SPE.

Ejemplar de referencia: Morrone, O. 1452 (SI).

Miconia biserrata (DC.) Michelangeli ${ }^{* * *}$ )

Arbusto. Nativa. 0-500 m.

Distribución: SIG.

Ejemplar de referencia: Biganzoli, F. 454 (SI).

Miconia cinerascens Miq. $\left.{ }^{* * *}\right)$

Arbusto o subarbusto. Nativa. 0-700 m.

Distribución: GMB, GUA, IGU, SPE.

Ejemplar de referencia: Zuloaga, F. O. 6733 (SI).

\section{Miconia collatata Wurdack $\left({ }^{* * *}\right)$}

Arbusto o árbol. Nativa. 0-800 m.

Distribución: GUA, IGU, SPE.

Ejemplar de referencia: Zuloaga, F. O. 6494 (SI).
Miconia discolor DC. $\left(^{* * *}\right)$

Arbusto. Nativa. 0-500 m.

Distribución: GMB, IGU, MON.

Ejemplar de referencia: Morrone, O. 1170 (SI).

Miconia erostrata (DC.) R. Goldenb.

Arbusto. Nativa. 0-800 m.

Distribución: SPE.

Ejemplar de referencia: Niederlein, G. s.n. (BA).

Miconia leacrenata R. Goldenb. \& Michelangeli Arbusto. Nativa. 0-500 m.

Distribución: GMB, SPE.

Ejemplar de referencia: Zanotti, C. A. 522 (SI).

Miconia leamarginata R. Goldenb. $\left.{ }^{* * *}\right)$

Arbusto. Nativa. 0-500 m.

Distribución: CAN, IGU, SIG.

Ejemplar de referencia: Morrone, O. 1197 (SI).

Miconia niederleinii (Cogn.) R. Goldenb. $\left.{ }^{(* *}\right)$

Hierba. Endémica. 0-500 m.

Distribución: SPE.

Ejemplar de referencia: Niederlein, G. 1878 (P).

Miconia petropolitana $\left.\operatorname{Cogn} .{ }^{(* * *}\right)$

Arbusto o árbol. Nativa. 200-800 m.

Distribución: SPE.

Ejemplar de referencia: Legname, P. R. 5556 (LIL).

Miconia pusilliflora (DC.) Naudin

Árbol. Nativa. 0-500 m.

Distribución: GMB, GUA, IGU, SIG, SPE.

Ejemplar de referencia: Morrone, O. 2128 (SI).

Miconia xanthocoma (Naudin) R. Goldenb. $\left({ }^{* * *}\right)$

Arbusto. Nativa. 0-600.

Distribución: GUA, IGU, SPE.

Ejemplar de referencia: Zuloaga, F. O. 5517 (SI).

Pterolepis (DC.) Miq., nom. cons.

Pterolepis repanda (DC.) Triana

Subarbusto. Nativa. 0-500 m.

Distribución: APO, SIG.

Ejemplar de referencia: Zuloaga, F. O. 3193 (SI). 
Rhynchanthera $\mathrm{DC}$., nom. cons.

\section{Rhynchanthera verbenoides Cham.}

Hierba. Nativa. 0-500 m.

Distribución: CAN, SIG.

Ejemplar de referencia: Keller, H. A. 12806 (CTES).

\section{MELIACEAE}

Cabralea A. Juss.

Cabralea canjerana (Vell.) Mart.

Árbol. Nativa. 0-800 m.

Distribución: CAI, ELD, GMB, GUA, IGU, LNA, SIG, SPE.

Ejemplar de referencia: Morrone, O. 1516 (SI).

Cedrela P. Browne

Cedrela fissilis Vell.

Árbol. Nativa. 0-8000 m.

Distribución: CAI, CAN, CAP, ELD, GMB, GUA, IGU, GSM, LNA, MAY, MON, SJA, SPE.

Ejemplar de referencia: Zuloaga, F. O. 5593 (SI).

\section{Cedrela odorata L.}

Árbol. Nativa. 0-800 m.

Distribución: GMB, SPE.

Ejemplar de referencia: Keller, H. A. 10482 (CTES).

Guarea F. Allam. ex L.

Guarea guidonia (L.) Sleumer $\left(^{* * *}\right)$

Árbol. Nativa. 0-500 m.

Distribución: CAP.

Ejemplar de referencia: Ekman, E. L. 2005 (F, $G H, N Y, S)$.

Guarea kunthiana A. Juss. ${ }^{* * *}$ )

Árbol. Nativa. 0-800 m.

Distribución: ELD, GMB, IGU.

Ejemplar de referencia: Burkart, A. 14558 (SI).
Guarea macrophylla Vahl subsp. spiciflora (A. Juss.) T.D. Penn.

Árbol. Nativa. 0-800 m.

Distribución: CAI, CAN, CAP, ELD, GUA, IGU, LNA, MON, SIG, SPE.

Ejemplar de referencia: Zuloaga, F. O. 2273 (SI).

Guarea macrophylla Vahl subsp. tuberculata (Vell.) T.D. Penn. $\left.{ }^{* * *}\right)$

Árbol. Nativa. 0-800 m.

Distribución: CAP, ELD, GMB, GSM, SIG.

Ejemplar de referencia: Hunziker, J. H. 11841 (SI).

Melia L.

Melia azedarach L.

Árbol. Introducida. 0-500 m.

Distribución: GUA, IGU.

Ejemplar de referencia: Tressens, S. G. 6057 (CTES).

Trichilia P. Browne

Trichilia catigua A. Juss.

Árbol. Nativa. 100-600 m.

Distribución: CAI, CAN, CAP, ELD, GMB, GSM, GUA, IGU, SIG, SPE.

Ejemplar de referencia: Zuloaga, F. O. 739 (SI).

Trichilia claussenii $C$. DC.

Árbol. Nativa. 200-800 m.

Distribución: CAI, GMB, GSM, IGU, SPE.

Ejemplar de referencia: Morrone, O. 1568 (SI).

Trichilia elegans A. Juss.

Árbol. Nativa. 100-700 m.

Distribución: APO, CAI, CAN, ELD, GMB, GSM, GUA, IGU, MON, SIG, SPE.

Ejemplar de referencia: Deginani, N. B. 1277 (SI).

Trichilia pallida $S w$.

Árbol. Nativa. 0-800 m. 
Distribución: IGU, SIG.

Ejemplar de referencia: Hunziker, J. H. 11957 (SI).

\section{MENISPERMACEAE}

\section{Cissampelos L.}

Cissampelos andromorpha DC.

Enredadera. Nativa. 0-300 m.

Distribución: GUA, IGU.

Ejemplar de referencia: Keller, H. A. 3239 (CTES).

\section{Cissampelos ovalifolia DC. $\left(^{* * *}\right)$}

Enredadera. Nativa. 0-800 m.

Distribución: GMB, SPE.

Ejemplar de referencia: Keller, H. A. 3796 (CTES).

\section{Cissampelos pareira $\mathrm{L}$.}

Enredadera. Nativa. 0-700 m.

Distribución: CAN, CAP, GSM, LNA, SPE.

Ejemplar de referencia: Romanczuk, M. C. 560 (BAB, SI).

Hyperbaena Miers ex Benth.

Hyperbaena oblongifolia (Mart.) Chodat \& Hassl. $\left.{ }^{* * *}\right)$

Enredadera. Nativa. 0-500 m.

Distribución: GUA, SIG, SPE.

Ejemplar de referencia: Schwindt, E. 4105 (US).

\section{Odontocarya Miers}

\section{Odontocarya acuparata Miers}

Liana o epífita. Nativa. 0-800 m.

Distribución: APO, GUA.

Ejemplar de referencia: Zuloaga, F. O. 7179 (SI).

\section{Odontocarya asarifolia Barneby}

Liana o epífita. Nativa. 0-500 m.

Distribución: IGU, MON.

Ejemplar de referencia: Keller, H. A. 25 (CTES).

\section{MENYANTHACEAE}

\section{Nymphoides Ség.}

\section{Nymphoides indica (L.) Kuntze}

Hierba acuática. Nativa. 0-500 m.

Distribución: CAN, CAP.

Ejemplar de referencia: Zanotti, C. A. 418 (SI).

\section{MICROTEACEAE}

\section{Microtea Sw.}

Microtea scabrida Urb.

Hierba. Nativa. 0-500 m.

Distribución: CAN, CAP, ELD, GSM, IGU, LNA, MON, SIG, SJA, SPE.

Ejemplar de referencia: Biganzoli, F. 410 (SI).

\section{MOLLUGINACEAE}

\section{Glinus L.}

Glinus radiatus (Ruiz \& Pav.) Rohrb.

Hierba. Nativa. 0-500 m.

Distribución: CAP.

Ejemplar de referencia: Burkart, A. 1605 (SI).

\section{Mollugo L.}

\section{Mollugo verticillata $L$.}

Hierba. Nativa. 0-800 m.

Distribución: CAP, ELD, GUA, IGU, SIG.

Ejemplar de referencia: Cabrera, A. L. 28839 (SI).

\section{MONIMIACEAE}

Hennecartia J. Poiss.

Hennecartia omphalandra J. Poiss. Arbusto o arbolito. Nativa. 0-800 m. 
Distribución: CAI, CAN, CON, ELD, GMB, GSM, GUA, IGU, OBE, SIG.

Ejemplar de referencia: Morrone, O. 1484 (SI).

\section{MORACEAE}

Broussonetia L'Hér. ex Vent., nom. cons.

Broussonetia papyrifera (L.) Vent.

Árbol. Introducida. 0-500 m.

Distribución: MON.

Ejemplar de referencia: Keller, H. A. 2596 (CTES).

Dorstenia Plum. ex L.

\section{Dorstenia brasiliensis Lam.}

Hierba. Nativa. 0-500 m.

Distribución: CAI, CON, MON, SIG.

Ejemplar de referencia: Biganzoli, F. 155 (SI).

\section{Dorstenia tenuis Bonpl. ex Bureau}

Hierba. Perenne. Nativa. 0-500 m.

Distribución: CAI, CAN, CON, GSM, GUA, IGU, SPE.

Ejemplar de referencia: Zuloaga, F. O. 6677 (SI).

\section{Ficus L.}

Ficus adhatodifolia Schott

Árbol. Nativa. 0-800 m.

Distribución: SIG.

Ejemplar de referencia: Biganzoli, F. 389 (SI).

Ficus calyptroceras (Miq.) Miq. $\left.{ }^{(* *}\right)$

Árbol. Nativa. 0-500 m.

Distribución: CAI.

Ejemplar de referencia: Spegazzini, C. L. s.n. $(B A F)$.

\section{Ficus citrifolia Mill.}

Árbol. Nativa. 0-700 m.
Distribución: CAN, IGU.

Ejemplar de referencia: Tressens, S. G. 4548 (CTES).

\section{Ficus eximia Schott $\left({ }^{*}\right)$}

Árbol. Nativa. 0-800 m.

Distribución: CAI, ELD, IGU, SIG, SPE.

Ejemplar de referencia: Keller, H. A. 570 (CTES).

Ficus luschnathiana (Miq.) Miq.

Árbol. Nativa. 0-800 m.

Distribución: APO, CAI, CON, ELD, GMB, GUA, IGU, SIG, SPE.

Ejemplar de referencia: Morrone, O. 1588 (SI).

Ficus obtusiuscula (Miq.) Miq.

Árbol. Nativa. 0-700 m.

Distribución: ELD, IGU.

Ejemplar de referencia: Keller, H. A. 1152 (CTES).

Maclura Nutt., nom. cons.

Maclura tinctoria (L.) Steud.

Árbol. Nativa. 0-700 m.

Distribución: ELD, GUA, IGU, SPE.

Ejemplar de referencia: Deginani, N. B. 1161 (SI).

\section{Morus L.}

Morus alba L.

Árbol. Introducida. 0-700 m.

Distribución: GUA, IGU.

Ejemplar de referencia: Keller, H. A. 209 (CTES).

Sorocea A. St.-Hil.

Sorocea bonplandii (Baill.) W.C.Burger, Lanj. \& Wess.Boer

Arbusto o árbol. Nativa. 0-500 m.

Distribución: APO, CAI, CAN, CON, ELD, GMB, GUA, IGU, MON, SIG, SPE.

Ejemplar de referencia: Zuloaga, F. O. 5539 (SI). 


\section{MYRTACEAE}

Acca O. Berg

Acca sellowiana (O. Berg) Burret ${ }^{* * *}$ )

Arbusto o árbol. Nativa.

Distribución: CAI, CAN, LNA, OBE, SIG.

Ejemplar de referencia: Keller, H. A. 395 (ASU, CTES).

\section{Blepharocalyx O. Berg}

Blepharocalyx salicifolius (Kunth) O. Berg

Arbusto o árbol. Nativa. 0-500 m.

Distribución: CAN, GSM, IGU.

Ejemplar de referencia: Rodríguez, F. M. 337 (SI).

\section{Campomanesia Ruiz \& Pav.}

Campomanesia aurea $\mathrm{O}$. Berg

Arbusto o subarbusto. Nativa. 0-500 m.

Distribución: SIG.

Ejemplar de referencia: Krapovickas, A. 44649 (CTES).

Campomanesia guaviroba (DC.) Kiaersk. ${ }^{* * *}$ ) Árbol. Nativa. 0-700 m.

Distribución: GSM, GUA.

Ejemplar de referencia: Tressens, S. G. 6450 (CTES, SI).

Campomanesia guazumifolia (Cambess.) O. Berg Arbusto o árbol. Nativa. 100-800 m.

Distribución: APO, CAN, CAP, ELD, GMB, GUA, SIG, SJA, SPE.

Ejemplar de referencia: Cabrera, A. L. 28791 (SI).

\section{Campomanesia xanthocarpa $\mathrm{O}$. Berg}

Arbusto o árbol. Nativa. 0-800 m.

Distribución: CAI, ELD, GMB, IGU, SIG, SJA.

Ejemplar de referencia: Ahumada, O. 1484 (SI).

\section{Eugenia L.}

Eugenia anomala D. Legrand

Subarbusto. Nativa. 0-600 m.
Distribución: CAN, SIG.

Ejemplar de referencia: Keller, H. A. 7599 (CTES).

Eugenia burkartiana (D. Legrand) D. Legrand Árbol. Nativa. 0-800 m.

Distribución: CAI, ELD, GSM, GUA, SJA, SPE.

Ejemplar de referencia: Rodríguez, M. 565 (BAB, CTES, G, MNES).

Eugenia hyemalis Cambess. var. marginata (O. Berg) D. Legrand

Arbusto o árbol. Nativa. 0-800 m.

Distribución: APO, CAN, GMB, IGU, MON, SIG, SPE.

Ejemplar de referencia: Morrone, O. 1696 (SI).

Eugenia involucrata $D C$.

Árbol. Nativa. 0-800 m.

Distribución: CAI, CAN, CAP, ELD, GUA, IGU, LNA. (SI).

Ejemplar de referencia: Cabrera, A. L. 28939

Eugenia lilloana D. Legrand $(* *)$

Subarbusto. Endémica. 0-500 m.

Distribución: CAI, IGU, SIG.

Ejemplar de referencia: Múlgura, M. E. 1619 (SI).

Eugenia mansoni $O$. Berg

Árbol. Nativa. 0-500 m.

Distribución: SIG.

Ejemplar de referencia: Denis, L. s.n. (LIL).

Eugenia moraviana $\mathrm{O}$. Berg

Arbusto o árbol. Nativa. 0-700 m.

Distribución: ELD, IGU, SIG.

Ejemplar de referencia: Tressens, S. G. 4543 (CTES).

Eugenia myrcianthes Nied.

Arbusto o árbol. Nativa. 0-500 m.

Distribución: APO, CAN, CAP, ELD, SIG.

Ejemplar de referencia: Biganzoli, F. 413 (SI).

Eugenia pitanga (O. Berg) Kiaersk.

Arbusto. Nativa. 0-500 m.

Distribución: APO, CAN, SIG.

Ejemplar de referencia: Schinini, A. 5594 (CTES). 
Eugenia pyriformis Cambess.

Árbol. Nativa. 0-800 m.

Distribución: CAI, CAN, CAP, GMB, GUA, IGU, SIG, SPE.

Ejemplar de referencia: Guaglianone, E. R. 3031 (SI).

Eugenia ramboi D. Legrand $(* * *)$

Árbol. Nativa. 0-800 m.

Distribución: GUA.

Ejemplar de referencia: Keller, H. A. 10 (CTES, ICN).

Eugenia repanda $O$. Berg

Arbusto o árbol. Nativa. 0-600 m.

Distribución: CAN, GMB, ELD, SIG.

Ejemplar de referencia: Guaglianone, E. R. 3027 (SI).

\section{Eugenia subterminalis $D C$.}

Árbol. Nativa. 0-800 m.

Distribución: CAN, GMB, IGU.

Ejemplar de referencia: Ferrucci, M. S. 3445 (CTES, SI).

\section{Eugenia uniflora L.}

Arbusto o árbol. Nativa. 0-800 m.

Distribución: APO, CAI, CAN, GMB, GSM, IGU, SIG, SJA, SPE.

Ejemplar de referencia: Cabrera, A. L. 28782 (SI).

Eugenia uruguayensis Cambess.

Árbol. Nativa. 0-800 m.

Distribución: SIG, SJA, SPE.

Ejemplar de referencia: Daviña, J. 186 (CTES, $G, M N E S$ ).

\section{Myrceugenia O. Berg}

Myrceugenia euosma (O. Berg) D. Legrand (***) Arbusto o árbol. Nativa. 0-500 m.

Distribución: GUA, SJA.

Ejemplar de referencia: Múlgura, M. E. 2622 (SI).

\section{Myrceugenia glaucescens (Cambess.) D.}

Legrand \& Kausel

Arbusto o árbol. Nativa. 0-800 m.

Distribución: APO, ELD, GMB, IGU, MON.

Ejemplar de referencia: Burkart, A. 14301 (SI).
Myrcia DC. ex Guill.

Myrcia anomala Cambess.

Subarbusto. Nativa. 0-500 m.

Distribución: SIG.

Ejemplar de referencia: Burkart, A. 15306 (SI).

Myrcia cruciflora A.R. Lourenço \& E. Lucas

Árbol. Nativa. 0-800 m.

Distribución: CAI, GMB, GUA, IGU, SIG, SPE.

Ejemplar de referencia: Rodríguez, M. E. 575 (MNES, SI).

\section{Myrcia oblongata DC.}

Arbusto o árbol. Nativa. 0-800 m.

Distribución: APO, CAI, CAN, CAP, CON, GMB, GSM, GUA, LNA, MON, SIG, SJA, SPE.

Ejemplar de referencia: Morrone, O. 2237 (SI).

Myrcia palustris $\mathrm{DC}$.

Arbusto o árbol. Nativa

Distribución: CAI, GMB, GUA, MON, SIG. (SI).

Ejemplar de referencia: Múlgura, M. E. 1716

Myrcia selloi (Spreng.) N. Silveira

Arbusto o árbol. Nativa. 0-500 m.

Distribución: CAI, CAN, CAP, GMB, GSM, IGU, MON, SJA, SPE.

Ejemplar de referencia: Morrone, O. 1384 (SI).

Myrcia tricona (D. Legrand) A.R. Lourenço \& E. Lucas $(* * *)$

Árbol. Nativa. 0-500 m.

Distribución: GUA, SPE.

Ejemplar de referencia: Honfi, A. I. 539 (CTES, MNES, SI).

\section{Myrcianthes O. Berg}

Myrcianthes gigantea (D. Legrand) D. Legrand $(* * *)$

Árbol. Nativa. 0-700 m.

Distribución: GSM, IGU, LNA, SPE.

Ejemplar de referencia: Cabrera, A. L. 28920 (SI). 
Myrcianthes pungens $(\mathrm{O}$. Berg) $\mathrm{D}$. Legrand

Árbol. Nativa. 0-800 m.

Distribución: CAN, ELD, GSM.

Ejemplar de referencia: Martínez Crovetto, $R$. 35 (CTES).

\section{Myrciaria O. Berg}

Myrciaria delicatula (DC.) O. Berg

Arbusto o árbol. Nativa. 0-500 m.

Distribución: CAN, LNA, SIG, SJA.

Ejemplar de referencia: Krapovickas, A. 15022 (CTES, SI).

Myrciaria tenella (DC.) O. Berg

Arbusto o árbol. Nativa. 0-800 m.

Distribución: GUA, SPE.

Ejemplar de referencia: Zuloaga, F. O. 728 (SI).

\section{Myrrhinium Schott}

\section{Myrrhinium atropurpureum Schott}

Arbusto o árbol. Nativa. 0-500 m.

Distribución: CON, SIG.

Ejemplar de referencia: Keller, H. A. 6023 (CTES).

\section{Plinia L.}

Plinia peruviana (Poir.) Govaerts

Árbol. Nativa.

Distribución: GUA, IGU, SPE.

Ejemplar de referencia: Keller, H. A. 3378 (CTES).

Plinia rivularis (Cambess.) Rotman

Árbol. Nativa. 0-800 m.

Distribución: CAI, ELD, GMB, GUA, IGU, SIG.

Ejemplar de referencia: Cabrera, A. L. 28776 (SI).

\section{Psidium L.}

Psidium australe Cambess.

Arbusto o subarbusto. Nativa. $100-800$ m.
Distribución: CAI, CAN, GMB, MON.

Ejemplar de referencia: Krapovickas, A. 23378 (CTES, MO).

Psidium grandifolium $D C$.

Arbusto. Nativa. 100-700 m.

Distribución: CAI, ELD, MON, SIG.

Ejemplar de referencia: Landrum, L. R. 5731 (ASU, CTES).

\section{Psidium guajava $\mathrm{L}$.}

Arbusto o árbol. Nativa. 0-800 m.

Distribución: CAI, CAP, ELD, GUA, MON, SIG, SPE.

Ejemplar de referencia: Morrone, O. 1095 (SI).

Psidium guineense $\mathrm{S} w$.

Arbusto o árbol. Nativa. 0-500 m.

Distribución: CAN, CAP, IGU, SIG.

Ejemplar de referencia: Zuloaga, F. O. 6556 (SI).

Psidium missionum D. Legrand $\left({ }^{* *}\right)$

Arbusto o subarbusto. Nativa. 100-500 m.

Distribución: CAI, CAN, SIG.

Ejemplar de referencia: Biganzoli, F. 1235 (SI).

Psidium salutare (Humb., Bonpl. \& Kunth) O. Berg var. mucronatum (Cambess.) Landrum

Subarbusto. Nativa. 0-600 m.

Distribución: APO, CAI, CAN, CAP.

Ejemplar de referencia: Krapovickas, A. 28785 (CTES).

Psidium salutare (Humb., Bonpl. \& Kunth) O. Berg var. sericeum (Cambess.) Landrum

Subarbusto. Nativa. 0-500 m.

Distribución: APO, CAN, CAP.

Ejemplar de referencia: Ekman, E. L. 2056 (MICH, NY).

Syzygium Gaertn., nom. cons.

Syzygium jambos (L.) Alston ${ }^{* * *}$ )

Árbol. Introducida. 0-500 m.

Distribución: SIG.

Ejemplar de referencia: Keller, H. A. 12198 (CTES). 


\section{NYCTAGINACEAE}

\section{Boerhavia L.}

\section{Boerhavia diffusa $L$.}

Hierba. Perenne. Introducida. 0-700 m.

Distribución: CAN, CAP, GSM, IGU, SIG.

Ejemplar de referencia: Krapovickas, A. 2461 (BA, SI).

\section{Mirabilis L.}

\section{Mirabilis jalapa L.}

Hierba. Introducida. 0-600 m.

Distribución: ELD, GMB, GSM.

Ejemplar de referencia: Zuloaga, F. O. 7993 (SI).

\section{Pisonia L.}

\section{Pisonia aculeata $L$.}

Arbusto o árbol. Nativa. 0-700 m.

Distribución: CAN, IGU, LNA, SIG, SPE.

Ejemplar de referencia: Dematteis, $M .112$ (G, MNES).

\section{Pisonia zapallo Griseb.}

Árbol. Nativa. 0-700 m.

Distribución: CAN, ELD, GSM, GUA, IGU, SIG.

Ejemplar de referencia: Zuloaga, F. O. 6640 (SI).

\section{Reichenbachia Spreng.}

Reichenbachia paraguayensis (D. Parodi)

Dugand \& Daniel (***)

Arbusto o árbol. Nativa. 0-500 m.

Distribución: CAN.

Ejemplar de referencia: De Sagastizábal s.n. (BA, BAF).

\section{NYMPHAEACEAE}

Nymphaea L. nom. cons.

Nymphaea caerulea Savigny $\left({ }^{* * *}\right)$

Hierba acuática. Perenne. Introducida. 200-700 m.
Distribución: GMB, IGU.

Ejemplar de referencia: Guaglianone, E. R. 1036 (SI).

Nymphaea gardneriana Planch.

Hierba acuática. Nativa. 0-500 m.

Distribución: CAP.

Ejemplar de referencia: Rodríguez, F. M. 98 (SI).

\section{OCHNACEAE}

\section{Sauvagesia L.}

\section{Sauvagesia erecta $L$.}

Hierba. Nativa. 0-500 m.

Distribución: SIG.

Ejemplar de referencia: Keller, H. A. 10826 (CTES).

\section{Sauvagesia racemosa A. St.-Hil. $\left.{ }^{* * *}\right)$}

Hierba. Nativa. 0-500 m.

Distribución: CAP.

Ejemplar de referencia: Gülich s.n. (LP).

\section{OLEACEAE}

\section{Chionanthus L.}

Chionanthus trichotomus (Vell.) P.S. Green

Árbol. Nativa. 0-500 m.

Distribución: IGU.

Ejemplar de referencia: Placci, G. 181 (SI).

\section{Ligustrum L.}

Ligustrum sinense Lour.

Arbusto o árbol. Introducida. 0-500 m.

Distribución: MON, SIG.

Ejemplar de referencia: Keller, H. A. 7665 (CTES).

Menodora Bonpl.

Menodora integrifolia (Cham. \& Schltdl.) Steud. Hierba o arbusto. Nativa. 0-500 m. 
Distribución: APO, CAI, CAN, CON, ELD, GUA, IGU, LNA, MON, SIG, SPE.

Ejemplar de referencia: Montes, J.E. 15569 (LIL).

\section{ONAGRACEAE}

\section{Ludwigia L.}

Ludwigia bonariensis (Micheli) $\mathrm{H}$. Hara

Hierba. Perenne. Nativa. 0-500 m.

Distribución: SIG.

Ejemplar de referencia: Keller, H. A. 10815 (CTES).

Ludwigia caparosa (Cambess.) H. Hara

Arbusto o subarbusto. Nativa. 0-600 m.

Distribución: APO, CON.

Ejemplar de referencia: Zuloaga, F. O. 4903 (SI).

\section{Ludwigia decurrens Walter}

Hierba. Nativa. 0-500 m.

Distribución: CAI, CAN, CAP, GSM, IGU, SIG, SJA, SPE.

Ejemplar de referencia: Zuloaga, F. O. 5586 (SI).

Ludwigia elegans (Cambess.) H. Hara

Hierba o subarbusto. Nativa. 0-500 m.

Distribución: CAN, CAP, ELD, IGU, MON, SIG, SPE.

Ejemplar de referencia: Morrone, O. 1762 (SI).

\section{Ludwigia grandiflora (Michx.) Greuter \&} Burdet

Hierba o subarbusto. Perenne. Nativa. 0-500 m. Distribución: SIG.

Ejemplar de referencia: Keller, H. A. 10808 (CTES).

Ludwigia hassleriana (Chodat) Ramamoorthy Arbusto o subarbusto. Nativa. 0-500 m.

Distribución: SIG.

Ejemplar de referencia: Schwarz, G. J. 5159 (TEX, W).

\section{Ludwigia hookeri (Micheli) H. Hara}

Hierba. Nativa. 0-500 m.

Distribución: GMB.

Ejemplar de referencia: Múlgura, M. E. 1859 (SI).
Ludwigia irwinii Ramamoorthy

Subarbusto. Nativa. 0-500 m.

Distribución: CAN, CAP, IGU, SIG.

Ejemplar de referencia: Montes, J. E. 27551 (CTES).

Ludwigia leptocarpa (Nutt.) H. Hara

Hierba o subarbusto. Nativa. 0-500 m.

Distribución: CON, ELD, SIG.

Ejemplar de referencia: Deginani, N. B. 1789 (SI).

Ludwigia longifolia (DC.) H. Hara

Hierba. Nativa. 0-700 m.

Distribución: CAN, CAP, CON, SIG.

Ejemplar de referencia: Martínez Crovetto, $R$. 8057 (CTES).

Ludwigia major (Micheli) Ramamoorthy

Hierba. Nativa. 0-500 m.

Distribución: APO, CAI, CAP, SIG.

Ejemplar de referencia: Guaglianone, E. R. 861 (SI).

Ludwigia martii (Micheli) Ramamoorthy

Hierba o subarbusto. Nativa. 0-500 m.

Distribución: CAN, GSM, IGU, SIG, SJA, SPE.

Ejemplar de referencia: Crisci, J. V. 309 (CTES).

Ludwigia neograndiflora (Munz) $\mathrm{H}$. Hara

Hierba. Nativa. 0-500 m.

Distribución: APO, CAN, CAP, CON, SIG.

Ejemplar de referencia: Guaglianone, E. R. 898 (SI).

Ludwigia octovalvis (Jacq.) P.H. Raven

Hierba o arbusto. Nativa. 0-700 m.

Distribución: CAI, CAP, GMB, GSM, GUA, IGU, SIG, SPE. (SI).

Ejemplar de referencia: Guaglianone, E. R. 1144

Ludwigia peruviana (L.) H. Hara

Hierba o arbusto. Nativa. 0-800 m.

Distribución: CAI, CAN, CON, GSM, GUA, SPE.

Ejemplar de referencia: Zuloaga, F. O. 6690 (SI).

Ludwigia pseudonarcissus (Chodat \& Hassl.) Ramamoorthy

Hierba o subarbusto. Nativa. 0-500 m.

Distribución: CAP.

Ejemplar de referencia: Krapovickas, A. 12107 (CTES, DS). 
Ludwigia sericea (Cambess.) H. Hara

Arbusto o subarbusto. Nativa. 0-500 m.

Distribución: APO, CAN, CAP, CON, GMB, GSM, IGU, SIG, SJA.

Ejemplar de referencia: Zuloaga, F. O. 6919 (SI).

\section{Oenothera L.}

Oenothera affinis Cambess.

Hierba. Nativa. 0-800 m.

Distribución: CAN, CAP, GMB, GUA, IGU, SIG, SPE.

Ejemplar de referencia: Schwindt, E. 2743 (LIL).

Oenothera indecora Cambess.

Hierba. Nativa. 0-700 m.

Distribución: APO, CAI, CAN, CAP, ELD, LNA, MON, SIG.

Ejemplar de referencia: Krapovickas, A. 13714 (BAB, C, CTES, UC).

\section{Oenothera mollissima $\mathrm{L}$.}

Hierba. Anual. Nativa. 0-500 m.

Distribución: CAI, CAN, ELD, GMB, SIG.

Ejemplar de referencia: Zuloaga, F. O. 5085 (SI).

\section{Oenothera parodiana Munz}

Hierba. Nativa. 0-700 m.

Distribución: CAN, ELD, IGU.

Ejemplar de referencia: Tressens, S. G. 5565 (CTES).

Oenothera ravenii $W$. Dietr.

Hierba. Nativa. 0-800 m. SJA.

Distribución: APO, CAN, CON, ELD, IGU, SIG,

Ejemplar de referencia: Montes, J. E. 9591 (LIL).

\section{OPILIACEAE}

\section{Agonandra Miers ex Hook. f.}

Agonandra excelsa Griseb.

Arbusto o arbolito. Nativa. 0-700 m.

Distribución: CAN, GMB, MON.

Ejemplar de referencia: Keller, H. A. 5692 (CTES).

\section{ORCHIDACEAE}

\section{Acianthera Scheidw.}

Acianthera aphthosa (Lindl.) Pridgeon \& M.W. Chase ${ }^{* * *}$ )

Hierba epífita. Nativa. 0-700 m.

Distribución: GMB, IGU.

Ejemplar de referencia: Johnson, A. E. 472 (CTES, SI).

Acianthera aurantio-lateritia (Speg.)

Pridgeon \& M.W. Chase

Hierba epífita. Nativa. 0-800 m.

Distribución: GMB.

Ejemplar de referencia: Spegazzini, C. L.s.n. (BA).

Acianthera hatschbachii (Schltr.) Chiron \& C. Berg $\left({ }^{* * *}\right)$

Hierba epífita. Nativa. 0-600 m.

Distribución: CAI, GMB, GUA, SPE.

Ejemplar de referencia: Johnson, A. E. 109 (SI).

Acianthera hygrophila (Barb. Rodr.) Pridgeon \& M.W. Chase $\left.{ }^{* * *}\right)$

Hierba epífita. Nativa. 0-600 m.

Distribución: ELD, GSM, SPE.

Ejemplar de referencia: Krapovickas, A. 15808 (CTES).

Acianthera hystrix (Kraenzl.) F. Barros $\left(^{* * *}\right)$

Hierba epífita. Nativa. 0-500 m.

Distribución: GMB.

Ejemplar de referencia: Insaurralde, I. S. 17 $(B A B)$.

Acianthera klingelfusii Luer, Toscano \& D.H. Baptista $\left.{ }^{* * *}\right)$

Hierba epífita. Nativa. 300-800 m.

Distribución: GMB, SPE.

Ejemplar de referencia: Johnson, A. E. 144 (CTES, MO, SI).

Acianthera klotzscheana (Rchb. f.) Pridgeon \& M.W. Chase $\left(^{* * *}\right)$

Hierba epífita. Nativa. 0-500 m.

Distribución: GMB, IGU.

Ejemplar de referencia: Johnson, A. E. 95 (SI). 
Acianthera luteola (Lindl.) Pridgeon \& M.W. Chase $\left.{ }^{* * *}\right)$

Hierba epífita. Nativa. 0-800 m.

Distribución: SPE.

Ejemplar de referencia: Johnson, A. E. 148 (CTES, SI).

Acianthera macuconensis (Barb. Rodr.) F. Barros (***)

Hierba epífita. Nativa. 200-800 m.

Distribución: GMB, IGU, SPE.

Ejemplar de referencia: Johnson, A. E. 431 (BAB, CTES).

Acianthera muscosa (Barb. Rodr.) Pridgeon \& M.W. Chase $\left.{ }^{(* *}\right)$

Hierba epífita. Nativa. 0-500 m.

Distribución: IGU.

Ejemplar de referencia: Johnson, A. E. 152 (SI).

Acianthera pubescens (Lindl.) Pridgeon \& M.W. Chase $\left(^{* * *}\right)$

Hierba epífita. Nativa. 0-700 m.

Distribución: CAN, ELD, GMB, IGU.

Ejemplar de referencia: Zuloaga, F. O. 5645 (SI).

Acianthera recurva (Lindl.) Pridgeon \& M.W. Chase

Hierba epífita. Nativa. 0-500 m.

Distribución: CAI, GUA, IGU, SPE.

Ejemplar de referencia: Johnson, A. E. 442 (BAB, CTES, SI).

Acianthera rhabdosepala (Schltr.) Chiron \& C. Berg

Hierba epífita. Nativa. 0-700 m.

Distribución: APO, GMB.

Ejemplar de referencia: Morrone, O. 1459 (SI).

Acianthera saundersiana (Rchb. f.) Pridgeon \& M.W. Chase $\left.{ }^{* * *}\right)$

Hierba epífita. Nativa. 0-800 m.

Distribución: GMB, IGU.

Ejemplar de referencia: Johnson, A. E. 372 (CTES, SI).

Acianthera sonderana (Rchb. f.) Pridgeon \& M.W. Chase $\left.{ }^{(* *}\right)$

Hierba epífita. Nativa. 0-800 m.

Distribución: GMB, SPE.

Ejemplar de referencia: Correa, M. N. 10530 (BAB).
Anathallis Barb. Rodr.

Anathallis johnsonii Luer \& Toscano $\left.{ }^{* * *}\right)$

Hierba epífita. Nativa. 0-500 m.

Distribución: SPE.

Ejemplar de referencia: Johnson, A. E. 171 (MO).

Anathallis linearifolia (Cogn.) Pridgeon \& M.W. Chase $\left({ }^{* * *}\right)$

Hierba epífita. Nativa. 0-800 m.

Distribución: CAI, ELD, GMB, GUA, IGU, SPE.

Ejemplar de referencia: Johnson, A. E. 295 (BAB, SI).

Anathallis marginata (Barb. Rodr.) F. Barros \& Barberena ${ }^{* * *}$ )

Hierba epífita. Nativa. 0-500 m.

Distribución: CAI, IGU.

Ejemplar de referencia: Johnson, A. E. 254 (CTES).

Anathallis microphyta (Barb. Rodr.) C.O. Azevedo \& C. Berg $\left.{ }^{* * *}\right)$

Hierba epífita. Nativa. 400-800 m.

Distribución: IGU, SIG, SPE.

Ejemplar de referencia: Johnson, A. E. 438 (BAB).

Anathallis obovata (Lindl.) Pridgeon \& M.W. Chase

Hierba epífita. Nativa. 0-700 m.

Distribución: GMB, IGU, SPE.

Ejemplar de referencia: Johnson, A. E. 461 (BAB).

\section{Aspidogyne Garay}

Aspidogyne bidentifera (Schltr.) Garay

Hierba. Nativa. 0-500 m.

Distribución: GUA, IGU.

Ejemplar de referencia: Keller, H. A. 97 (CTES, SI).

Aspidogyne bruxellii (Pabst) Garay

Hierba. Perenne. Nativa. 0-800 m.

Distribución: GMB, OBE.

Ejemplar de referencia: Correa, M. N. 5962 (BAB).

Aspidogyne kuczynskii (Porsch) Garay

Hierba. Nativa. 0-800 m.

Distribución: CAI, GMB, IGU.

Ejemplar de referencia: Hunziker, J. H. 11040 (SI). 
Aspidogyne lindleyana (Cogn.) Garay $\left({ }^{* * *}\right)$

Hierba. Nativa. 0-800 m.

Distribución: SPE.

Ejemplar de referencia: Morrone, O. 2118 (SI).

\section{Aspidogyne malmei (Kraenzl.) Garay $\left({ }^{* * *}\right)$}

Hierba. Perenne. Nativa. 0-600 m.

Distribución: GUA, SPE.

Ejemplar de referencia: Johnson, A. E. 358 (BAB).

\section{Barbosella Schltr.}

Barbosella cogniauxiana (Speg. \& Kraenzl.) Schltr. $\left({ }^{* * *}\right)$

Hierba epífita. Nativa. 0-800 m.

Distribución: GMB, GUA, SPE.

Ejemplar de referencia: Correa, M. N. 7023 (BAB).

\section{Brachystele Schltr.}

Brachystele burkartii M.N. Correa (**)

Hierba. Endémica. 0-500 m.

Distribución: CAP.

Ejemplar de referencia: Burkart, A. 14501 (SI).

Brachystele camporum (Lindl.) Schltr.

Hierba. Nativa. 0-500 m.

Distribución: APO, CAN, CAP.

Ejemplar de referencia: Jörgensen, P. 171 (BA, BAB, SI).

Brachystele cyclochila (Kraenzl.) Schltr.

Hierba. Nativa. 0-500 m.

Distribución: CAP.

Ejemplar de referencia: Ekman, E. L. 429 (S).

Brachystele dilatata (Lindl.) Schltr.

Hierba. Nativa. 0-500 m.

Distribución: CAN.

Ejemplar de referencia: Jörgensen, P. s.n. (BAB).

Brasiliorchis R.B. Singer, S. Koehler \& Carnevali

Brasiliorchis chrysantha (Barb. Rodr.) R.B. Singer, S. Koehler \& Carnevali $\left(^{* * *}\right)$

Hierba epífita. Nativa. 0-600 m.
Distribución: GMB, IGU, SPE.

Ejemplar de referencia: Johnson, A. E. 391 (SI).

Brasiliorchis picta (Hook.) R.B. Singer, S. Koehler \& Carnevali $\left({ }^{* * *}\right)$

Hierba epífita. Nativa. 0-500 m.

Distribución: GMB, GUA, IGU, SPE.

Ejemplar de referencia: Johnson, A. E. 547 (SI).

Brassavola R. Br., nom. cons.

Brassavola tuberculata Hook.

Hierba epífita. Nativa. 0-700 m.

Distribución: APO, CAN, CON, IGU, SIG.

Ejemplar de referencia: Morrone, O. 1262 (SI).

\section{Bulbophyllum Thouars}

Bulbophyllum napelli Lindl. $\left.{ }^{(* *}\right)$

Hierba epífita. Nativa. 0-800 m.

Distribución: GMB, SPE. (SI).

Ejemplar de referencia: Zuloaga, F. O. 7017

Bulbophyllum regnellii Rchb. f. $\left.{ }^{* * *}\right)$

Hierba epífita. Nativa. 700-800 m.

Distribución: GMB, SPE.

Ejemplar de referencia: Johnson, A. E. 453 (SI).

Bulbophyllum tripetalum Lindl. ${ }^{(* *}$ )

Hierba epífita. Nativa. 0-500 m.

Distribución: GMB, IGU.

Ejemplar de referencia: Johnson, A. E. 515 (MU, SI).

Campylocentrum Benth.

Campylocentrum densiflorum Cogn.

Hierba epífita. Nativa. 0-800 m.

Distribución: CAN, IGU, SPE.

Ejemplar de referencia: Johnson, A. E. 217 (BAB, CTES).

\section{Campylocentrum grisebachii Cogn.}

Hierba epífita. Nativa. 0-800 m.

Distribución: CAI, CAN, GMB, GUA, IGU.

Ejemplar de referencia: Johnson, A. E. 1058 (SI). 
Campylocentrum neglectum (Rchb. f. \& Warm.) Cogn.

Hierba epífita. Nativa. 0-500 m.

Distribución: GUA, SIG, SPE.

Ejemplar de referencia: Tressens, S. G. 5804 (CTES, SI).

Campylocentrum pauloense Hoehne \& Schltr. $(* * *)$

Hierba epífita. Nativa. 0-500 m.

Distribución: IGU.

Ejemplar de referencia: Correa, M. N. 8416 $(B A B)$.

\section{Campylocentrum ulaei $\left.\operatorname{Cogn} .{ }^{* * *}\right)$}

Hierba epífita. Nativa. 0-500 m.

Distribución: GMB, IGU, SPE.

Ejemplar de referencia: Deginani, N. B. 1083 (SI).

Capanemia Barb. Rodr.

Capanemia micromera Barb. Rodr.

Hierba epífita. Nativa. 0-800 m.

Distribución: CAN, CAP, CON, ELD, GMB, GUA, IGU, MON, SIG.

Ejemplar de referencia: Johnson, A. E. 194 (SI).

\section{Capanemia superflua (Rchb. f.) Garay $\left({ }^{* *}\right)$}

Hierba epífita. Nativa. 0-700 m.

Distribución: CAI, CAN, GMB, GUA, SPE.

Ejemplar de referencia: Morrone, O. 1565 (SI).

Catasetum Rich. ex Kunth

Catasetum fimbriatum (C. Morren) Lindl. \& Paxton

Hierba epífita. Nativa. 0-800 m.

Distribución: IGU, SIG, SPE.

Ejemplar de referencia: Johnson, A. E. 426 $(B A B)$.

Cattleya Lindl.

Cattleya cernua (Lindl.) C. Berg

Hierba epífita. Nativa. 0-600 m.
Distribución: GMB, IGU, SPE.

Ejemplar de referencia: Seijo, G. J. 205 (G, MNES).

Cattleya lundii (Rchb. f. \& Warm.) Van den Berg Hierba epífita. Nativa. 0-500 m.

Distribución: IGU.

Ejemplar de referencia: Correa, M. N. 10529 (BAB).

Cleistes Rich. ex Lindl.

Cleistes ramboi Pabst $\left.{ }^{* * *}\right)$

Hierba. Nativa. 0-500 m.

Distribución: CAP.

Ejemplar de referencia: Insaurralde, I. S. 596 (CTES, MNES).

\section{Cohniella Pfitzer}

Cohniella cepula (Hoffmanns.) Carnevali \& G.

A. Romero

Hierba. Nativa. 0-700 m.

Distribución: IGU.

Ejemplar de referencia: Johnson, A. E. 430 (CTES).

Cohniella jonesiana (Rchb. f.) Christenson

Hierba epífita. Nativa. 0-600 m.

Distribución: SIG.

Ejemplar de referencia: sin colector 4464 (SI)

\section{Corymborkis Thouars}

\section{Corymborkis flava (Sw.) Kuntze}

Hierba. Nativa. 0-500 m.

Distribución: CAI, ELD, GMB, IGU, OBE, SPE.

Ejemplar de referencia: Daviña, J. 208 (MNES).

\section{Cranichis Sw.}

Cranichis candida (Barb. Rodr.) Cogn.

Hierba. Nativa. 0-500 m.

Distribución: IGU.

Ejemplar de referencia: Johnson, A. E. 459 (BAB, CTES). 


\section{Cyclopogon C. Presl}

Cyclopogon bicolor (Ker Gawl.) Schltr.

Hierba. Nativa. 0-600 m.

Distribución: CAI.

Ejemplar de referencia: Spegazzini, C. L. s.n. (SI).

Cyclopogon calophyllus (Barb. Rodr.) Barb. Rodr. Hierba. Nativa. 0-500 m.

Distribución: IGU, SPE.

Ejemplar de referencia: Johnson, A. E. 474 (BAB, SI).

\section{Cyclopogon congestus (Vell.) Hoehne}

Hierba terrestre o epífita. Nativa. 0-800 m.

Distribución: CAI, CAN, ELD, GMB, GUA, IGU, MON, SIG, SPE.

Ejemplar de referencia: Diem, J. 1482 (SI).

Cyclopogon elatus (Sw.) Schltr.

Hierba. Nativa. 0-600 m.

Distribución: CAI, CAN, CAP, ELD, GMB, GSM, GUA, IGU, SIG.

Ejemplar de referencia: Biganzoli, F. 516 (SI).

\section{Cyclopogon elegans Hoehne}

Hierba. Nativa. 0-800 m.

Distribución: GMB, GUA.

Ejemplar de referencia: Tressens, S. G. 6335 (CTES, SI).

Cyclopogon longibracteatus (Barb. Rodr.) Schltr.

Hierba. Nativa. 100-500 m.

Distribución: GSM.

Ejemplar de referencia: Biganzoli, F. 243 (SI).

Cyclopogon oliganthus (Hoehne) Hoehne \& Schltr.

Hierba. Nativa. 0-600 m.

Distribución: CAI, SJA.

Ejemplar de referencia: Biganzoli, F. 305 (SI).

Cyclopogon polyaden (Vell.) F.S. Rocha \& J.L. Waechter

Hierba. Nativa. 0-500 m.

Distribución: IGU.

Ejemplar de referencia: Correa, M. N. 5960 (BAB, SI).
Cyclopogon subalpestris Schltr.

Hierba. Nativa. 0-500 m.

Distribución: GUA.

Ejemplar de referencia: Tressens, S. G. 6024 (CTES).

Cyclopogon variegatus Barb. Rodr. $\left.{ }^{* * *}\right)$

Hierba. Nativa. 0-500 m.

Distribución: CAN, IGU.

Ejemplar de referencia: Johnson, A. E. 488 (SI).

Cyrtopodium R. Br.

Cyrtopodium hatschbachii Pabst

Hierba. Nativa. 0-500 m.

Distribución: APO.

Ejemplar de referencia: Zuloaga, F. O. 7156 (SI).

Cyrtopodium palmifrons Rchb. f. \& Warm.

Hierba epífita. Nativa. 0-500 m.

Distribución: IGU, MON.

Ejemplar de referencia: Cabrera, A. L. 28887 (SI).

Eltroplectris Raf.

Eltroplectris schlechterana (Porto \& Brade) Pabst $\left.{ }^{* * *}\right)$

Hierba. Nativa. 0-500 m.

Distribución: IGU, SIG.

Ejemplar de referencia: Correa, M. N. 5965 (BAB).

Eltroplectris triloba (Lindl.) Pabst

Hierba. Nativa. 0-800 m.

Distribución: CAN, IGU.

Ejemplar de referencia: Johnson, A. E. 289 (SI).

Epidendrum L., nom. cons.

Epidendrum andres-johnsonii Hágsater \& E. Santiago $\left({ }^{* * *}\right)$

Hierba epífita. Nativa. 0-500 m.

Distribución: IGU.

Ejemplar de referencia: Johnson, A. E. 288 (SI). 
Epidendrum densiflorum Hook.

Hierba epífita. Nativa. 0-800 m.

Distribución: ELD, GMB, GUA, IGU, SPE.

Ejemplar de referencia: Johnson, A. E. 192 (SI).

\section{Epidendrum hassleri Cogn. $\left.{ }^{(* *}\right)$}

Hierba epífita o epipétrica. Nativa. 0-500 m.

Distribución: IGU, SPE.

Ejemplar de referencia: Johnson, A. E. 322 (SI).

\section{Epidendrum rigidum Jacq.}

Hierba epífita. Nativa. 0-800 m.

Distribución: GMB, IGU.

Ejemplar de referencia: Johnson, A. E. 263 (BAB, CTES, MU, SI).

\section{Epistephium Kunth}

Epistephium sclerophyllum Lindl.

Hierba perenne. Nativa. 0-500 m.

Distribución: CAN.

Ejemplar de referencia: Vanni, R. O. 1083 (CTES).

Eulophia R. Br., nom. cons.

Eulophia alta (L.) Fawc. \& Rendle

Hierba terrestre. Nativa. 0-500 m.

Distribución: GSM, IGU.

Ejemplar de referencia: Johnson, A. E. 335 (SI).

\section{Eurystyles Wawra}

Eurystyles actinosophila (Barb. Rodr.) Schltr. $\left({ }^{* * *}\right)$

Hierba epífita. Nativa. 0-500 m.

Distribución: ELD, GMB, IGU, SPE.

Ejemplar de referencia: Johnson, A. E. 379 (SI)

\section{Galeandra Lindl.}

\section{Galeandra beyrichii Rchb. $\mathrm{f}$.}

Hierba terrestre. Nativa. 0-500 m.

Distribución: GMB, IGU, OBE, SPE.

Ejemplar de referencia: Múlgura, M. E. 3258 (SI).
Gomesa R. Br.

Gomesa bifolia (Sims) M.W. Chase \& N.H. Williams

Hierba epífita. Nativa. 0-700 m.

Distribución: IGU.

Ejemplar de referencia: Johnson, A. E. 269 (SI).

Gomesa cornigera (Lindl.) M.W. Chase \& N.H. Williams

Hierba epífita. Nativa. 0-300 m.

Distribución: CAN, GMB, IGU, SIG, SPE.

Ejemplar de referencia: Correa, M. N. 5514 (BAB).

Gomesa longicornu (Mutel) M.W. Chase \& N.H. Williams

Hierba epífita. Nativa. 0-800 m.

Distribución: ELD, GMB, GSM, GUA, IGU, MON, SPE.

Ejemplar de referencia: Johnson, A. E. 121 (SI).

Gomesa longipes (Lindl. \& Paxton) M.W. Chase \& N.H. Williams

Hierba epífita. Nativa. 0-800 m.

Distribución: GMB, IGU, SIG.

Ejemplar de referencia: Johnson, A. E. 134 (MU, SI).

Gomesa paranaensoides M.W. Chase N.H. Williams $\left({ }^{* * *}\right)$

Hierba epífita. Nativa. 0-700 m.

Distribución: GMB, GSM, IGU, SPE.

Ejemplar de referencia: Correa, M. N. 5054 (BAB).

Gomesa pubes (Lindl.) M.W. Chase \& N.H. Williams

Hierba epífita. Nativa. 0-500 m.

Distribución: CAN, GUA.

Ejemplar de referencia: Burkart, A. 4931 (SI).

Gomesa recurva $\mathrm{R}$. Br. $\left.{ }^{(* *}\right)$

Hierba epífita. Nativa. 300-800 m.

Distribución: IGU, SPE.

Ejemplar de referencia: Correa, M. N. 10535 (BAB).

Gomesa riograndensis (Cogn.) M.W. Chase \& N.H. Williams

Hierba epífita. Nativa. 0-600 m.

Distribución: ELD, GUA, IGU, SPE.

Ejemplar de referencia: Johnson, A. E. 355 (SI). 


\section{Grandiphyllum Docha Neto}

Grandiphyllum edwallii (Cogn.) Docha Neto Hierba epífita. Nativa. 0-500 m.

Distribución: CAI, GMB, SPE.

Ejemplar de referencia: Johnson, A. E. 140 (SI).

Grandiphyllum hians (Lindl.) Docha Neto $(* * *)$

Hierba epífita. Nativa. 0-600 m.

Distribución: GMB.

Ejemplar de referencia: Valebella, M. 80 (CTES).

Grandiphyllum pulvinatum (Lindl.) Docha Neto

Hierba epífita. Nativa. 0-700 m.

Distribución: GMB, IGU, OBE, SPE.

Ejemplar de referencia: Johnson, A. E. 20 (SI).

\section{Habenaria Willd.}

Habenaria achalensis Kraenzl.

Hierba. Nativa. 0-500 m.

Distribución: CAP.

Ejemplar de referencia: Ekman, E. L. 433

(AMES, HB, HBG, NY, S).

\section{Habenaria bractescens Lindl.}

Hierba. Nativa. 0-700 m.

Distribución: CAN, CAP, IGU.

Ejemplar de referencia: Barboza, G. E. 407 (CORD).

Habenaria ekmaniana Kraenzl.

Hierba terrestre. Nativa. 0-300 m.

Distribución: CAP, CON.

Ejemplar de referencia: Dematteis, M. 254 (CTES).

Habenaria gourlieana Gillies ex Lindl.

Hierba palustre. Nativa. 0-800 m.

Distribución: CAN, CAP, IGU.

Ejemplar de referencia: Zuloaga, F. O. 3154 (SI).

\section{Habenaria hassleriana Cogn. $\left({ }^{* * *}\right)$}

Hierba terrestre. Nativa.

Distribución: Desconocida.

Ejemplar de referencia: Parodi, D. 62 (SI).
Habenaria johannensis Barb. Rodr.

Hierba terrestre. Nativa. 0-800 m.

Distribución: CAN, IGU, SIG.

Ejemplar de referencia: Guaglianone, E. R. 1017 (SI).

Habenaria josephensis Barb. Rodr. $\left.{ }^{* * *}\right)$

Hierba terrestre. Nativa. 300-500 m.

Distribución: IGU.

Ejemplar de referencia: Keller, H. A. 12823 (CTES).

Habenaria parviflora Lindl.

Hierba. Nativa. 0-500 m.

Distribución: CAN.

Ejemplar de referencia: Jörgensen, P. 30659 (BAB).

Habenaria trifida Kunth

Hierba. Nativa. 0-500 m.

Distribución: CAN.

Ejemplar de referencia: Alboff, N. s.n. (BA).

Isabelia Barb. Rodr.

Isabelia virginalis Barb. Rodr. $\left.{ }^{* * *}\right)$

Hierba epífita. Nativa. 0-500 m.

Distribución: GMB.

Ejemplar de referencia: Johnson, A. E. 382 (SI).

Isochilus $\mathrm{R}$. $\mathrm{Br}$.

Isochilus linearis (Jacq.) R. Br. Hierba epífita. Nativa. 0-800 m.

Distribución: CAI, CAP, CON, GMB, IGU, SPE.

Ejemplar de referencia: Seijo, G. J. 206 (CTES, G, MNES).

\section{Lankesterella Ames}

Lankesterella ceracifolia (Barb. Rodr.) Ames

Hierba epífita. Nativa. 0-800 m.

Distribución: GMB, IGU, SIG.

Ejemplar de referencia: Johnson, A. E. 111 (SI). 


\section{Leptotes Lindl.}

\section{Leptotes unicolor Barb. Rodr.}

Hierba epífita. Nativa. 0-500 m.

Distribución: CAN, GMB, IGU, MON, SPE.

Ejemplar de referencia: Honfi, A. I. 536 (MNES).

\section{Ligeophila Garay}

Ligeophila juruensis (Hoehne) Garay $\left(^{* * *}\right.$ )

Hierba terrestre. Nativa. 0-500 m.

Distribución: IGU.

Ejemplar de referencia: Keller, H. A. 13525 (CTES).

\section{Ligeophila rosea (Lindl.) Garay ${ }^{* * *}$ )}

Hierba terrestre. Nativa. 0-500 m.

Distribución: IGU.

Ejemplar de referencia: Johnson, A. E. 516 (SI).

Liparis Rich., nom. cons.

Liparis nervosa (Thunb.) Lindl.

Hierba epífita o epipétrica. Nativa. 0-700 m.

Distribución: CAI, IGU, MON.

Ejemplar de referencia: Correa, M. N. 10523 (BAB).

Malaxis Sol. ex Sw.

Malaxis irmae Radins \& Salazar

Hierba. Perenne. Nativa. 0-200 m.

Distribución: CAP.

Ejemplar de referencia: Radins, J. A. 105 (CTES).

\section{Malaxis parthoni $C$. Morren}

Hierba terrestre. Nativa. 0-700 m.

Distribución: GMB, GUA, IGU, SIG, SPE.

Ejemplar de referencia: Deginani, N. B. 1712 (SI).

\section{Maxillaria Ruiz \& Pav.}

\section{Maxillaria spegazziniana Kraenzl. $\left({ }^{* * *}\right)$}

Hierba epífita. Nativa. 0-500 m.

Distribución: GMB, IGU, MON, SPE.

Ejemplar de referencia: Johnson, A. E. 234 (SI).
Maxillaria vitelliniflora Barb. Rodr. $\left({ }^{* *}\right)$

Hierba epífita. Nativa. 0-800 m.

Distribución: ELD, GMB, GUA, IGU, SPE.

Ejemplar de referencia: Correa, M. N. 5050 (BAB).

\section{Mesadenella Pabst \& Garay}

Mesadenella cuspidata (Lindl.) Garay

Hierba terrestre. Nativa. 0-500 m.

Distribución: ELD, GMB, GSM, GUA, IGU, SPE.

Ejemplar de referencia: Johnson, A. E. 439 (SI).

Microchilus C. Presl

Microchilus arietinus (Rchb. f. \& Warm.) Ormd. $\left({ }^{* * *}\right)$

Hierba terrestre. Nativa. 0-500 m.

Distribución: GMB, IGU.

Ejemplar de referencia: Johnson, A. E. 315 (SI).

Miltonia Lindl., nom. cons.

Miltonia flavescens Lindl. $\left(^{* * *}\right)$

Hierba epífita. Nativa. 0-600 m.

Distribución: ELD, GMB, GSM, GUA, IGU, SIG, SPE.

Ejemplar de referencia: Morrone, O. 1269 (SI).

Octomeria R. Br.

Octomeria chamaeleptotes Rchb. f. $\left.{ }^{* * *}\right)$

Hierba epífita. Nativa. 400-700 m.

Distribución: SPE.

Ejemplar de referencia: Correa, M. N. 5053 (BAB).

Octomeria micrantha Barb. Rodr. $\left.{ }^{* * *}\right)$

Hierba epífita. Nativa. 0-500 m.

Distribución: GMB, IGU.

Ejemplar de referencia: Johnson, A. E. 374 (BAB).

Octomeria warmingii Rchb. f. $\left({ }^{* * *}\right)$

Hierba epífita. Nativa. 0-500 m.

Distribución: GMB, IGU.

Ejemplar de referencia: Johnson, A. E. 300 (BAB, SI). 


\section{Oeceoclades Lindl.}

Oeceoclades maculata (Lindl.) Lindl.

Hierba terrestre. Nativa. 0-500 m.

Distribución: IGU, SIG.

Ejemplar de referencia: Biganzoli, F. 76 (SI).

Oncidium Sw., nom. cons.

Oncidium verrucosissimum $\operatorname{Cogn}$.

Hierba epífita. Nativa. 0-800 m.

Distribución: SPE.

Ejemplar de referencia: Deginani, N. B. 1714 (SI).

Orthochilus Hochst. ex A. Rich.

Orthochilus ruwenzoriensis (Rendle) Bytebier Hierba epífita. Perenne. Introducida. 0-500 m. Distribución: CAN.

Ejemplar de referencia: Keller, H. A. 11954 (CTES).

\section{Pabstiella Brieger \& Senghas}

Pabstiella aveniformis (Hoehne) Luer ${ }^{(* *}$ )

Hierba epífita. Nativa. 0-800 m.

Distribución: GMB, GUA, SPE.

Ejemplar de referencia: Johnson, A. E. 1019 (SI).

Pabstiella matinhensis (Hoehne) Luer $\left({ }^{* * *}\right)$

Hierba epífita. Nativa. 0-500 m.

Distribución: ELD, GMB, GUA, IGU, SPE.

Ejemplar de referencia: Johnson, A. E. 219 (SI).

Pabstiella tripterantha (Rchb. f.) F. Barros $\left({ }^{* * *}\right)$

Hierba epífita. Nativa. 0-800 m.

Distribución: IGU.

Ejemplar de referencia: Johnson, A. E. 487 (SI).

Pelexia Poit. ex Lindl., nom. cons.

Pelexia bonariensis (Lindl.) Schltr.

Hierba terrestre. Nativa

Distribución: SIG.

Ejemplar de referencia: Keller, H. A. 12817 (CTES).
Pelexia ekmanii (Kraenzl.) Schltr.

Hierba terrestre. Nativa. 0-600 m.

Distribución: APO, CAN, CAP.

Ejemplar de referencia: Zuloaga, F. O. 3240 (SI).

Pelexia laxa (Poepp. \& Endl.) Lindl. (***)

Hierba terrestre. Nativa. 0-500 m.

Distribución: GMB, IGU.

Ejemplar de referencia: Johnson, A. E. 499 (SI).

Pelexia lindmanii Kraenzl.

Hierba terrestre. Nativa. 0-500 m.

Distribución: ELD, GMB, IGU.

Ejemplar de referencia: Johnson, A. E. 245 (SI).

Pelexia novofriburgensis (Rchb. f.) Garay

Hierba terrestre. Nativa. 100-700 m.

Distribución: IGU.

Ejemplar de referencia: Johnson, A. E. 390 (SI).

\section{Phymatidium Lindl.}

Phymatidium delicatulum Lindl. $\left.{ }^{(* *}\right)$

Hierba epífita. Nativa. 0-800 m.

Distribución: IGU, SPE.

Ejemplar de referencia: Johnson, A. E. 436 (SI).

Polystachya Hook., nom. cons.

Polystachya concreta (Jacq.) Garay \& Sweet Hierba epífita. Nativa. 0-500 m.

Distribución: ELD, GMB, IGU, SIG.

Ejemplar de referencia: Johnson, A. E. 267 (BAB, SI).

Polystachya foliosa (Hook.) Rchb. f.

Hierba epífita. Nativa. 0-500 m.

Distribución: GUA, SIG, SPE.

Ejemplar de referencia: Correa, M. N. 7019 (BAB).

\section{Prescottia Lindl.}

Prescottia oligantha (Sw.) Lindl.

Hierba terrestre. Nativa. 0-800 m.

Distribución: IGU, SIG, SPE.

Ejemplar de referencia: Johnson, A. E. 292 (SI). 
Prescottia stachyodes (Sw.) Lindl. $\left.{ }^{* * *}\right)$

Hierba terrestre. Nativa. 0-700 m.

Distribución: IGU.

Ejemplar de referencia: Johnson, A. E. 220 (BAB, CTES).

\section{Pteroglossa Schltr.}

Pteroglossa macrantha (Rchb. f.) Schltr. $\left({ }^{* *}\right)$

Hierba terrestre. Nativa. 0-200 m.

Distribución: SIG.

Ejemplar de referencia: Ekman, E. L. 425 (S).

Pteroglossa roseoalba (Rchb. f.) Salazar \& \& M.W. Chase

Hierba. Nativa. 0-500 m.

Distribución: GMB.

Ejemplar de referencia: Johnson, A. E. 170 (BAB).

\section{Rodriguezia Ruiz \& Pav.}

Rodriguezia decora (Lem.) Rchb. f. ${ }^{* * *}$ )

Hierba epífita. Nativa. 0-500 m.

Distribución: IGU.

Ejemplar de referencia: Johnson, A. E. 465 (BAB, SI).

\section{Sacoila Raf.}

Sacoila lanceolata (Aubl.) Garay

Hierba terrestre. Nativa. 0-800 m.

Distribución: APO, CAI, CAN, GMB, GSM, GUA, IGU, SIG, SPE.

Ejemplar de referencia: Morrone, O. 1270 (SI).

\section{Sanderella Kuntze}

Sanderella riograndensis Dutra ex Pabst $\left(^{* *}\right)$

Hierba epífita. Nativa. 0-500 m.

Distribución: IGU.

Ejemplar de referencia: Johnson, A. E. 188 (BAB).

\section{Sarcoglottis C. Presl}

Sarcoglottis fasciculata (Vell.) Schltr. $\left.{ }^{(* *}\right)$

Hierba terrestre. Nativa. 0-500 m.
Distribución: CAI, CAN, IGU, ELD.

Ejemplar de referencia: Johnson, A. E. 521 (SI).

Sarcoglottis grandiflora (Hook.) Klotzsch

Hierba terrestre. Nativa. 0-500 m.

Distribución: SIG.

Ejemplar de referencia: Quiroga, H. s.n. (BA).

Sarcoglottis homalogastra (Rchb. f. \& Warm.) Schltr. $\left.{ }^{* * *}\right)$

Hierba terrestre. Nativa.

Distribución: Desconocida.

Ejemplar de referencia: Spegazzini, C. L. s.n. (BA).

Sarcoglottis ventricosa (Vell.) Hoehne ex Pabst $\left.{ }^{* * *}\right)$

Hierba terrestre. Nativa. 0-500 m.

Distribución: ELD, GMB, IGU, SPE.

Ejemplar de referencia: Johnson, A. E. 509 (CTES, SI).

\section{Skeptrostachys Garay}

Skeptrostachys disoides (Kraenzl.) Garay $\left(^{(* *}\right)$

Hierba terrestre. Nativa. 0-500 m.

Distribución: CAN.

Ejemplar de referencia: Jörgensen, P. 671 (BAB).

Skeptrostachys gigantea ( $\operatorname{Cogn}$.) Garay

Hierba terrestre. Nativa. 0-500 m.

Distribución: CAI, CAN.

Ejemplar de referencia: Biganzoli, F. 842 (SI).

Skeptrostachys paraguayensis (Rchb. f.) Garay

Hierba terrestre. Nativa. 0-500 m.

Distribución: CAI, CAP, SIG.

Ejemplar de referencia: Naboulet, L. R. s.n. (BA).

\section{Specklinia Lindl.}

Specklinia grobyi (Bateman ex Lindl.) F. Barros $(* * *)$

Hierba epífita. Nativa. 0-800 m.

Distribución: GMB, GUA, IGU, SPE.

Ejemplar de referencia: Johnson, A. E. 484 (CTES, SI). 
Trichocentrum Poepp. \& Endl.

Trichocentrum pumilum (Lindl.) M.W. Chase \& N.H. Williams

Hierba epífita. Nativa. 0-800 m.

Distribución: GMB, GSM, GUA, IGU, MON, SIG.

Ejemplar de referencia: Morrone, O. 2022 (SI).

\section{Triphora Nutt.}

Triphora hassleriana (Cogn.) Schltr.

Hierba terrestre. Nativa. 0-800 m.

Distribución: ELD, GMB.

Ejemplar de referencia: Johnson, A. E. 1022 (SI).

\section{Vanilla Mill.}

Vanilla angustipetala Schltr.

Hierba. Nativa. 300-700 m.

Distribución: IGU, SIG.

Ejemplar de referencia: Biganzoli, F. 443 (SI).

\section{Vanilla chamissonis Klotzsch}

Hierba. Nativa. 0-300 m.

Distribución: SIG.

Ejemplar de referencia: Johnson, A. E. 503 (SI).

\section{Vanilla edwallii Hoehne $\left.{ }^{(* *}\right)$}

Hierba. Perenne. Nativa. 0-500 m.

Distribución: GMB, GUA, IGU, SPE.

Ejemplar de referencia: Morrone, O. 2078 (SI).

\section{Vanilla verrucosa Hauman $\left({ }^{* * *}\right)$}

Hierba. Nativa. 0-500 m.

Distribución: MON, SIG.

Ejemplar de referencia: Kermes, E. 127 (BAB).

Warmingia Rchb. f., nom. cons.

Warmingia eugenii Rchb. f. ${ }^{* * *}$ )

Hierba epífita. Nativa. 0-700 m.

Distribución: GMB, IGU.

Ejemplar de referencia: Johnson, A. E. 131 (SI).
Warrea Lindl.

Warrea warreana (Lodd. ex Lindl.) C. Schweinf. $(* * *)$

Hierba epífita. Nativa. 0-500 m.

Distribución: GUA, IGU.

Ejemplar de referencia: Johnson, A. E. 218 (SI).

Wullschlaegelia Rchb. f.

Wullschlaegelia aphylla (Sw.) Rchb. f.

Hierba terrestre. Nativa. 0-500 m.

Distribución: CAI, GMB, IGU, OBE, SIG, SPE.

Ejemplar de referencia: Zuloaga, F. O. 5527 (SI).

\section{Zygopetalum Hook.}

Zygopetalum maxillare Lodd. Hierba epífita. Nativa. 0-500 m.

Distribución: GMB, IGU, SPE.

Ejemplar de referencia: Burkart, A. 28282 (SI).

Zygostates Lindl.

Zygostates alleniana Kraenzl.

Hierba epífita. Nativa. 0-700 m.

Distribución: ELD, IGU, MON, SIG.

Ejemplar de referencia: Johnson, A. E. 559 (SI).

\section{OROBANCHACEAE}

\section{Agalinis Raf.}

Agalinis communis (Cham. \& Schltdl.) D'Arcy

Subarbusto. Nativa. 0-800 m.

Distribución: APO, CAI, CAN, CAP, CON, LNA, SIG, SJA, SPE.

Ejemplar de referencia: Biganzoli, F. 384 (SI).

Agalinis fiebrigii (Diels) D'Arcy

Subarbusto. Nativa. 0-600 m.

Distribución: CAI, SIG.

Ejemplar de referencia: Martínez Crovetto, $R$. 8258 (BAB). 
Agalinis genistifolia (Cham. \& Schltdl.) D'Arcy

Subarbusto parásito. Nativa. 0-600 m.

Distribución: APO, CAI, CAN, LNA, SIG.

Ejemplar de referencia: Zuloaga, F. O. 6646 (SI).

\section{Buchnera L.}

Buchnera longifolia Kunth

Hierba parásita. Nativa. 0-500 m.

Distribución: APO, CAN, CAP, CON, SIG, SJA.

Ejemplar de referencia: Biganzoli, F. 1613 (SI).

\section{Buchnera ternifolia Kunth}

Hierba parásita. Nativa. 0-500 m.

Distribución: CAN, CAP.

Ejemplar de referencia: Gómez, R. s.n. (BAB).

\section{Castilleja Mutis ex L.f.}

Castilleja arvensis Schltdl. \& Cham.

Hierba. Nativa. 0-500 m.

Distribución: APO.

Ejemplar de referencia: Cabrera, A. L. 28715 (SI).

\section{Escobedia Ruiz \& Pav.}

Escobedia grandiflora (L. f.) Kuntze

Subarbusto parásito. Nativa. 0-500 m.

Distribución: APO, CAP.

Ejemplar de referencia: Zuloaga, F. O. 9820 (SI).

\section{Melasma P.J. Bergius}

Melasma melampyroides (Rich.) Pennell

Hierba parásita. Nativa. 0-600 m.

Distribución: CAN, ELD, GMB, IGU, SIG, SPE.

Ejemplar de referencia: Keller, H. A. 4239 (CTES,SI).

Melasma rhinanthoides (Cham.) Benth.

Hierba parásita. Nativa. 0-500 m.

Distribución: CAN, IGU.

Ejemplar de referencia: Rodríguez, F. M. 779 (BA, BAB, LIL, LP, SI).

\section{OXALIDACEAE}

\section{Oxalis L.}

Oxalis articulata Savigny

Hierba. Nativa. 0-500 m.

Distribución: APO, CAI, CAN, CON, IGU.

Ejemplar de referencia: Morrone, O. 578 (SI).

Oxalis bipartita A. St.-Hil. subsp. bipartita Hierba. Nativa. 0-500 m.

Distribución: APO, CAI, CAP, GSM, GUA, LNA, MON, SIG.

Ejemplar de referencia: Cabrera, A. L. 28712 (SI).

Oxalis bipartita A. St.-Hil. subsp. pabstii Lourteig $\left({ }^{* * *}\right)$

Hierba. Nativa. 0-500 m.

Distribución: SIG.

Ejemplar de referencia: Schwindt, E. 5029 (LIL).

Oxalis brasiliensis Lodd., G. Lodd. \& W. Lodd. ex Hildebr.

Hierba. Nativa. 0-500 m.

Distribución: CAI, CAN, SIG.

Ejemplar de referencia: Múlgura, M. E. 2265 (SI).

\section{Oxalis conorrhiza Jacq.}

Hierba. Nativa. 0-600 m.

Distribución: CAI, CAP, CON, SIG.

Ejemplar de referencia: Múlgura, M. E. 2075 (SI).

Oxalis cytisoides $C$. Mart. \& Zucc. $\left.{ }^{* * *}\right)$

Subarbusto. Nativa. 0-800 m.

Distribución: GUA, SPE.

Ejemplar de referencia: Deginani, N. B. 1110 (SI).

Oxalis debilis Kunth

Hierba. Nativa. 0-600 m.

Distribución: APO, ELD, GMB, GUA, IGU, SIG, SPE.

Ejemplar de referencia: Deginani, N. B. 1257 (SI).

\section{Oxalis eriocarpa DC.}

Hierba. Nativa. 0-500 m.

Distribución: APO, CAN, CAP, LNA, SIG.

Ejemplar de referencia: Múlgura, M. E. 2319 (SI). 


\section{Oxalis frutescens $\mathrm{L}$.}

Hierba o arbusto. Nativa. 0-500 m.

Distribución: ELD, SPE.

Ejemplar de referencia: Guillén, R. 174 (G, MNES, SI).

\section{Oxalis geralensis $\mathrm{R}$. Knuth $\left.{ }^{* * *}\right)$}

Hierba. Nativa. 0-800 m.

Distribución: GSM, IGU.

Ejemplar de referencia: Zuloaga, F. O. 5416 (SI).

\section{Oxalis hispidula Zucc.}

Hierba. Perenne. Nativa. 0-700 m.

Distribución: CAI, CAN, CON, GUA, LNA, SIG.

Ejemplar de referencia: Zuloaga, F. O. 5772 (SI).

\section{Oxalis hyalotricha Lourteig}

Hierba o subarbusto. Nativa. 0-500 m.

Distribución: ELD, IGU, SIG.

Ejemplar de referencia: Múlgura, M. E. 2161 (SI).

Oxalis lasiopetala Zucc.

Hierba. Nativa. 0-500 m.

Distribución: APO, CAP.

Ejemplar de referencia: Schinini, A. 20836 (CTES).

\section{Oxalis linarantha Lourteig}

Hierba. Nativa. 0-800 m.

Distribución: CAI, CAN, GMB, GSM, GUA, OBE, SIG, SPE.

Ejemplar de referencia: Keller, H. A. 214 (CTES, SI).

Oxalis niederleiniana Hieron. ex $\mathrm{R}$. Knuth

Hierba. Perenne. Nativa. 0-500 m.

Distribución: GSM, IGU.

Ejemplar de referencia: Zuloaga, F. O. 5640 (SI).

Oxalis niederleinii $\mathrm{R}$. Knuth

Hierba. Nativa. 0-700 m.

Distribución: CAI, CAN, CAP, CON, ELD, GSM, GUA, IGU, LNA, OBE, SIG, SPE.

Ejemplar de referencia: Honfi, A. I. 492 (MNES, SI).

Oxalis paludosa A. St.-Hil.

Hierba. Perenne. Nativa. 0-500 m.

Distribución: GUA.

Ejemplar de referencia: Deginani, N. B. 1154 (SI).
Oxalis perdicaria (Molina) Bertero

Hierba. Nativa. 0-500 m.

Distribución: APO, CAN, LNA, SIG.

Ejemplar de referencia: Cabrera, A. L. 29289 (SI).

Oxalis potamophila Lourteig $\left(^{* * *}\right)$

Hierba. Perenne. Nativa. 400-800 m.

Distribución: IGU, SPE.

Ejemplar de referencia: Morrone, O. 1225 (SI).

Oxalis refracta A. St.-Hil.

Hierba. Perenne. Nativa. 0-500 m.

Distribución: APO, CAN, CAP, SPE.

Ejemplar de referencia: Dematteis, M. 116 (CTES, MNES).

Oxalis rhombeo-ovata A. St.-Hil. $\left.{ }^{(* * *}\right)$

Subarbusto. Nativa. 0-500 m.

Distribución: IGU.

Ejemplar de referencia: Morrone, O. 1202 (SI).

Oxalis sellowiana Zucc. f. alba Herter

Hierba. Perenne. Nativa. 0-500 m.

Distribución: CAN.

Ejemplar de referencia: Keller, H. A. 13899 (CTES).

Oxalis sellowii Spreng. var. rosea (Chodat) Lourteig

Hierba o subarbusto. Nativa. 0-500 m.

Distribución: SIG, SJA, SPE.

Ejemplar de referencia: Bertoni, M. 2060 (LIL).

\section{Oxalis sellowii Spreng. var. sellowii}

Hierba o subarbusto. Nativa. 0-600 m.

Distribución: APO, CAI, CAN, CAP, IGU, SIG.

Ejemplar de referencia: Morrone, O. 1678 (SI).

Oxalis subvillosa Norlind (**)

Hierba. Perenne. Nativa. 0-800 m.

Distribución: GUA, MON.

Ejemplar de referencia: Tressens, S. G. 6510 (CTES, SI).

Oxalis triangularis A. St.-Hil. subsp. papilionacea (Hoffmanns. ex Zucc.) Lourteig

Hierba. Perenne. Nativa. 0-800 m.

Distribución: GMB, IGU, SIG.

Ejemplar de referencia: Zuloaga, F. O. 5194 (SI). 
Oxalis triangularis A. St.-Hil. subsp. triangularis

Hierba. Nativa. 0-700 m.

Distribución: CAI, GMB, GSM, GUA, MON, SPE.

Ejemplar de referencia: Zuloaga, F. O. 5134 (SI).

\section{PAPAVERACEAE}

\section{Argemone L.}

Argemone subfusiformis G.B. Ownbey

Hierba. Nativa. 0-700 m.

Distribución: CAN, IGU.

Ejemplar de referencia: Kujawska, M. 62 (CTES).

\section{Fumaria L.}

Fumaria officinalis L.

Hierba. Anual. Introducida. 0-500 m.

Distribución: MON, OBE.

Ejemplar de referencia: Keller, H. A. 7485 (CTES).

\section{PASSIFLORACEAE}

\section{Passiflora L.}

\section{Passiflora alata Curtis $\left({ }^{* * *}\right)$}

Liana. Nativa. 0-800 m.

Distribución: CAI, CAN, CAP, ELD, GMB, IGU, LNA, MAY, SIG.

Ejemplar de referencia: Zuloaga, F. O. 6729 (SI).

Passiflora amethystina J.C. Mikan

Liana. Nativa. 0-800 m.

Distribución: GSM, IGU, OBE, SPE. (SI).

Ejemplar de referencia: Zuloaga, F. O. 5619

\section{Passiflora caerulea L.}

Liana. Nativa. 0-700 m.
Distribución: APO, CAI, CAN, CAP, CON, ELD, GSM, GUA, IGU, LNA, SIG, SJA, SPE.

Ejemplar de referencia: Zuloaga, F. O. 6910 (SI).

\section{Passiflora capsularis L. $\left.{ }^{* * *}\right)$}

Enredadera. Nativa. 0-800 m.

Distribución: APO, CAI, CAN, CON, ELD, GMB, GSM, GUA, IGU, MON, SIG, SPE.

Ejemplar de referencia: Zuloaga, F. O. 5560 (SI).

\section{Passiflora chrysophylla Chodat}

Liana. Nativa. 0-600 m.

Distribución: APO, CAN, CON, SIG.

Ejemplar de referencia: Martínez Crovetto, $R$. 9586 (CTES).

\section{Passiflora edulis Sims}

Liana. Nativa. 200-700 m.

Distribución: APO, CAI, CAN, CAP, GMB, GSM, MON, SIG.

Ejemplar de referencia: Morrone, O. 1449 (SI).

Passiflora elegans Mast.

Liana. Nativa. 0-500 m.

Distribución: APO, CAP, SIG.

Ejemplar de referencia: Zuloaga, F. O. 7147 (SI).

\section{Passiflora foetida $\mathrm{L}$.}

Liana. Nativa. 0-800 m.

Distribución: APO, CAN, CAP, CON, GSM, IGU, OBE, SIG.

Ejemplar de referencia: Morrone, O. 1882 (SI).

\section{Passiflora giberti N.E. Br.}

Liana. Nativa. 0-800 m.

Distribución: GMB, SJA, SPE.

Ejemplar de referencia: Múlgura, M. E. 3926 (MNES, SI).

Passiflora misera Kunth

Liana. Nativa. 0-700 m.

Distribución: APO, CAN, CAP, GUA, IGU, SIG, SPE.

Ejemplar de referencia: Guaglianone, E. R. 3020 (SI).

\section{Passiflora morifolia Mast.}

Liana. Nativa. 0-800 m.

Distribución: CAN, GMB, LNA, SJA.

Ejemplar de referencia: Morrone, O. 836 (SI). 
Passiflora suberosa L. subsp. litoralis (Kunth) Port.-Utl. ex M.A.M. Azevedo, Baumgratz \& Gonç.-Estev.

Liana. Perenne. Nativa. 0-700 m.

Distribución: APO, CAI, CAN, CAP, CON, LNA, SIG.

Ejemplar de referencia: Martínez Crovetto, $R$. 9635 (CTES).

\section{Passiflora tenuifila Killip}

Liana. Nativa. 200-800 m.

Distribución: APO, CAI, CAN, ELD, GMB, GSM, GUA, IGU, MAY, MON, OBE, SIG, SJA, SPE.

Ejemplar de referencia: Biganzoli, F. 352 (SI).

\section{Passiflora tricuspis Mast. $\left({ }^{* * *}\right)$}

Liana. Nativa. 200-400 m.

Distribución: GUA.

Ejemplar de referencia: Tressens, S. G. 6858 (CTES).

\section{Passiflora tucumanensis Hook.}

Liana. Nativa. 200-500 m.

Distribución: OBE.

Ejemplar de referencia: Pérez-Moreau, R. A. 21155 (US).

\section{Passiflora urnifolia Rusby}

Liana. Nativa. 200-700 m.

Distribución: CAI, ELD, GMB, OBE, SIG, SPE.

Ejemplar de referencia: Zuloaga, F. O. 673 (SI, US).

\section{PHYLLANTHACEAE}

\section{Margaritaria L. f.}

\section{Margaritaria nobilis L. $\mathrm{f}$.}

Arbusto o árbol. Nativa. 0-500 m.

Distribución: CAP.

Ejemplar de referencia: Jeckeln, G. R. s.n. (SI).

\section{Phyllanthus L.}

Phyllanthus caroliniensis Walter subsp. guianensis (Klotzsch) G.L. Webster

Hierba o subarbusto. Nativa. 0-700 m.

Distribución: CAN, ELD, GMB, GUA, IGU, SIG, SPE.

Ejemplar de referencia: Deginani, N. B. 1174 (SI).

\section{Phyllanthus niruri L.}

Hierba. Nativa. 0-800 m.

Distribución: CAI, GMB, GUA, IGU, SIG, SPE.

Ejemplar de referencia: Deginani, N. B. 1132 (SI).

Phyllanthus sellowianus (Klotzsch) Müll. Arg. Arbusto. Nativa. 0-800 m.

Distribución: CAI, GMB, GUA, SIG.

Ejemplar de referencia: Daviña, J. 278 (CTES, G, MNES).

Phyllanthus stipulatus (Raf.) G.L. Webster Hierba. Nativa. 0-500 m.

Distribución: ELD, SIG.

Ejemplar de referencia: Keller, H. A. 170 (CTES).

Phyllanthus tenellus Roxb.

Hierba. Introducida. 0-500 m.

Distribución: APO, ELD, SPE.

Ejemplar de referencia: Keller, H. A. 2224 (CTES).

\section{PHYTOLACCACEAE}

\section{Petiveria L.}

Petiveria alliacea L. var. alliacea

Hierba. Nativa. 0-500 m.

Distribución: APO, CAI, CAN, CAP, CON, GMB, GSM, GUA, IGU, MON, SIG, SJA, SPE.

Ejemplar de referencia: Múlgura, M. E. 3003 (SI).

Petiveria alliacea L. var. tetrandra (B.A. Gomes) Hauman

Hierba. Nativa. 0-500 m.

Distribución: CAN.

Ejemplar de referencia: Rodríguez, F. M. 247 (SI).

\section{Phytolacca L.}

Phytolacca bogotensis Kunth

Subarbusto. Nativa. 0-700 m.

Distribución: CAI, GMB, GUA, IGU, MON, SPE.

Ejemplar de referencia: Cabrera, A. L. 28931 (SI).

Phytolacca dioica L.

Árbol. Nativa. 0-500 m. 
Distribución: APO, CAI, ELD.

Ejemplar de referencia: Morrone, O. 1307 (SI).

Phytolacca thyrsiflora Fenzl ex J.A. Schmidt $\left.{ }^{(* *}\right)$

Hierba o subarbusto. Nativa. 0-700 m.

Distribución: GMB.

Ejemplar de referencia: Keller, H. A. 3939 (CTES).

\section{Rivina L.}

\section{Rivina humilis $\mathrm{L}$.}

Hierba. Nativa. 0-500 m.

Distribución: APO, ELD, GSM, IGU, MON, SIG.

Ejemplar de referencia: Zuloaga, F. O. 5711 (SI).

\section{Seguieria Loefl.}

\section{Seguieria aculeata Jacq.}

Arbusto. Nativa. 0-800 m.

Distribución: APO, GMB, GUA, IGU, MON, OBE, SJA.

Ejemplar de referencia: Deginani, N. B. 1747 (SI).

\section{Trichostigma A. Rich.}

Trichostigma octandrum (L.) H. Walter

Liana. Nativa. 0-500 m.

Distribución: ELD, IGU.

Ejemplar de referencia: Rodríguez, F. M. 447 (LIL, SI).

\section{PICRAMNIACEAE}

\section{Picramnia Sw.}

\section{Picramnia parvifolia Engl.}

Arbusto o arbolito. Nativa. 0-800 m.

Distribución: GMB, MON, SJA, SPE.

Ejemplar de referencia: Múlgura, M. E. 1589 (SI).

\section{Picramnia sellowii Planch.}

Arbusto o arbolito. Nativa. 0-500 m.

Distribución: CAN, CAP, SIG.

Ejemplar de referencia: Burkart, A. 14205 (SI).

\section{PIPERACEAE}

Peperomia Ruiz \& Pav.

Peperomia aceroana C. DC.

Hierba epífita. Nativa. 0-700 m.

Distribución: CAN, GUA, SPE.

Ejemplar de referencia: Zuloaga, F. O. 5530 (SI).

Peperomia arifolia Miq. $\left.{ }^{(* *}\right)$

Hierba. Nativa. 100-800 m.

Distribución: APO, CAI, ELD, GMB, GSM, GUA, IGU, SPE.

Ejemplar de referencia: Morrone, O. 1232 (SI).

Peperomia armondii Yunck. (***)

Hierba. Nativa. 0-100 m.

Distribución: IGU.

Ejemplar de referencia: Vanni, R. O. 3020 (CTES).

Peperomia balansana C. DC.

Hierba suculenta. Nativa. 0-800 m.

Distribución: ELD, GMB, GSM, GUA, IGU, MAY, SJA, SPE.

Ejemplar de referencia: Morrone, O. 2027 (SI).

Peperomia barbarana $C$. DC.

Hierba suculenta. Nativa. 0-500 m.

Distribución: CAI, CAN, CAP, CON, GMB, GSM, GUA, IGU, SIG, SPE.

Ejemplar de referencia: Zuloaga, F. O. 5533 (SI).

\section{Peperomia catharinae Miq.}

Hierba epífita. Nativa. 0-700 m.

Distribución: CAI, ELD, GMB, GUA, IGU, SPE.

Ejemplar de referencia: Morrone, O. 1386 (SI).

\section{Peperomia circinnata Link}

Hierba epífita. Nativa. 0-800 m.

Distribución: APO, CAI, CAN, CAP, GMB, GSM, GUA, IGU, MON, SIG.

Ejemplar de referencia: Cabrera, A. L. 28947 (SI).

\section{Peperomia corcovadensis Gardner (***)}

Hierba epífita. Nativa. 0-400 m.

Distribución: CAI, ELD, GMB, GSM, GUA, SPE. Ejemplar de referencia: Keller, H. A. 2584 (CTES). 
Peperomia delicatula Henschen

Hierba. Nativa. 0-800 m.

Distribución: GUA, SPE.

Ejemplar de referencia: Keller, H. A. 239 (CTES).

\section{Peperomia diaphanoides Dahlst. $\left.{ }^{* * *}\right)$}

Hierba. Nativa. 0-800 m.

Distribución: GUA, SPE.

Ejemplar de referencia: Deginani, N. B. 1743 (SI).

Peperomia hispidula (Sw.) A. Dietr. var. sellowiana (Miq.) Dahlst. (***)

Hierba. Nativa. 400-800 m.

Distribución: GUA, SPE.

Ejemplar de referencia: Tressens, S. G. 4753 (AAU, BH, CTES, GH, MBM, MICH).

\section{Peperomia increscens Miq.}

Hierba epífita. Nativa. 0-700 m.

Distribución: APO, CAI, CAN, CAP, GMB, GSM, GUA, IGU, LNA, MON, SIG, SJA, SPE.

Ejemplar de referencia: Morrone, O. 673 (SI).

\section{Peperomia martiana Miq. $\left({ }^{* * *}\right)$}

Hierba. Nativa. 0-700 m.

Distribución: SPE.

Ejemplar de referencia: Keller, H. A. 2726 (CTES).

Peperomia nitida Dahlst., nom. cons.

Hierba. Nativa. 500-700 m.

Distribución: ELD, IGU, SIG.

Ejemplar de referencia: Múlgura, M. E. 2094 (SI).

\section{Peperomia psilostachya C. DC.}

Hierba epífita. Nativa. 0-800 m.

Distribución: CAI, CAN, CAP, ELD, GSM, GUA, IGU, SJA, SPE.

Ejemplar de referencia: Biganzoli, F. 199 (SI).

\section{Peperomia rotundifolia (L.) Kunth $\left(^{* * *}\right.$ )}

Hierba epífita. Nativa. 0-700 m.

Distribución: GUA, IGU, SPE.

Ejemplar de referencia: Daviña, J. 133 (CTES, MNES).

\section{Peperomia rubricaulis (Nees) A. Dietr. $\left.{ }^{(* *}\right)$}

Hierba. Nativa. 0-700 m.

Distribución: GSM, GUA, IGU, MON, SPE.

Ejemplar de referencia: Deginani, N. B. 1768 (SI).
Peperomia tetraphylla Hook. \& Arn.

Hierba epífita. Nativa. 600-800 m.

Distribución: GMB, GUA, SPE.

Ejemplar de referencia: Morrone, O. 2124 (SI).

Peperomia trineuroides Dahlst. $\left.{ }^{(* *}\right)$

Hierba epífita. Nativa. 0-800 m.

Distribución: GMB, SPE.

Ejemplar de referencia: Biganzoli, F. 634 (SI).

Peperomia urocarpa Fisch. \& C.A. Mey.

Hierba. Nativa. 0-800 m.

Distribución: CAI, CAN, CAP, ELD, GMB, GSM, GUA, IGU, SIG, SPE.

Ejemplar de referencia: Morrone, O. 2136 (SI).

\section{Piper L.}

\section{Piper aduncum L.}

Arbusto o arbolito. Nativa. 0-700 m.

Distribución: GSM, GUA, IGU, SIG.

Ejemplar de referencia: Schwarz, G. J. 6976 (LIL).

\section{Piper amalago L.}

Arbusto o arbolito. Nativa. 0-800 m.

Distribución: CAI, GMB, GSM, GUA, IGU, LNA, OBE, SIG, SJA, SPE.

Ejemplar de referencia: Hunziker, J. H. 10795 (SI).

Piper hieronymi C. DC.

Arbusto o arbolito. Nativa. 400-700 m.

Distribución: APO, CAI, CON, ELD, GMB, GSM, GUA, IGU, MON, SIG, SPE.

Ejemplar de referencia: Zuloaga, F. O. 8358 (MU, SI).

Piper mapirense $C$. DC.

Arbusto. Nativa. 300-800 m.

Distribución: GMB, IGU, MON, SJA.

Ejemplar de referencia: Zuloaga, F. O. 5152 (SI).

Piper mikanianum (Kunth) Steud. ${ }^{* * *}$ )

Arbusto. Nativa. 0-800 m.

Distribución: APO, CAI, CAN, CAP, CON, GMB, GSM, GUA, IGU, LNA, SIG, SPE.

Ejemplar de referencia: Krapovickas, A. 2431 (SI). 
Piper miquelianum $C$. DC. $\left({ }^{* * *}\right)$

Arbusto. Nativa. 0-500 m.

Distribución: IGU.

Ejemplar de referencia: Keller, H. A. 13586 (SI, CTES).

Piper regnellii (Miq.) C. DC.

Hierba o arbusto. Nativa. 100-200 m.

Distribución: CAP, CON, GUA.

Ejemplar de referencia: Keller, H. A. 5709 (CTES).

\section{PLANTAGINACEAE}

\section{Angelonia Bonpl.}

\section{Angelonia integerrima Spreng.}

Hierba. Nativa. 0-700 m.

Distribución: APO, CAI, CAN, CAP, CON, SIG, SJA.

Ejemplar de referencia: Zuloaga, F. O. 6652 (SI).

\section{Angelonia salicariifolia Bonpl.}

Hierba. Nativa. 0-500 m.

Distribución: APO, CAN.

Ejemplar de referencia: Fontana, J. L. 778 (SI).

Bacopa Aubl., nom. cons.

Bacopa congesta Chodat \& Hassl.

Hierba. Nativa. 0-800 m.

Distribución: APO, CAN, CAP.

Ejemplar de referencia: Cabrera, A. L. 29149 (SI).

Bacopa dubia Chodat \& Hassl.

Hierba. Nativa. 0-500 m.

Distribución: GMB.

Ejemplar de referencia: Múlgura, M. E. 3273 (SI).

Bacopa monnierioides (Cham.) B.L. Rob.

Hierba. Nativa. 0-500 m.

Distribución: APO, SPE.

Ejemplar de referencia: Cabrera, A. L. 28754 (SI).

\section{Bacopa paraguariensis (S. Moore) Hassl.}

Subarbusto. Nativa. 0-500 m.

Distribución: CAI.

Ejemplar de referencia: Schwarz 6680 (LIL).
Bacopa salzmannii (Benth.) Wettst. ex Edwall Hierba. Nativa. 0-500 m.

Distribución: CAP, SIG.

Ejemplar de referencia: Keller, H. A. 9330 (CTES).

Bacopa scabra (Benth.) Descole \& Borsini

Hierba. Nativa. 0-500 m.

Distribución: CAP.

Ejemplar de referencia: Denis s.n. (LIL).

Bacopa serpyllifolia (Benth.) Pennell

Hierba. Nativa. 0-500 m.

Distribución: SIG.

Ejemplar de referencia: Quiroga, H. 4269 (BAB).

\section{Callitriche L.}

Callitriche terrestris Raf. subsp. subsessilis (Fassett) Bacigalupo

Hierba. Nativa. 0-500 m.

Distribución: ELD.

Ejemplar de referencia: Cabrera, A. L. 28858 (SI).

Callitriche truncata Guss.

Hierba acuática. Anual. Introducida. 0-500 m. Distribución: CAN.

Ejemplar de referencia: Keller, H. A. 13901 (CTES).

\section{Gratiola L.}

\section{Gratiola peruviana $\mathrm{L}$.}

Hierba. Nativa. 0-500 m.

Distribución: CAN, IGU.

Ejemplar de referencia: Rodríguez, F. M. 603 (SI).

Mecardonia Ruiz \& Pav.

Mecardonia grandiflora (Benth.) Pennell

Hierba. Nativa. 0-700 m.

Distribución: CAN, GUA, IGU, SPE.

Ejemplar de referencia: Deginani, N. B. 1112 (SI).

Mecardonia procumbens (Mill.) Small var.

flagellaris (Cham. \& Schltdl.) V.C. Souza

Hierba. Nativa. 0-700 m. 
Distribución: APO, CAI, CAN, CAP, CON, ELD, GSM, IGU, LNA.

Ejemplar de referencia: Zuloaga, F. O. 5786 (SI).

Mecardonia procumbens (Mill.) Small var. herniarioides (Cham.) V.C. Souza

Hierba. Anual. Nativa. 0-600 m.

Distribución: CAN, CAP, IGU, LNA. (SI).

Ejemplar de referencia: Zuloaga, F. O. 8054

Mecardonia procumbens (Mill.) Small var. tenella (Cham. \& Schltdl.) V.C. Souza

Hierba. Nativa. 0-500 m.

Distribución: APO, CAP.

Ejemplar de referencia: Cabrera, A. L. 28764 (SI).

Mecardonia serpylloides (Cham. \& Schltdl.) Pennell

Hierba. Nativa. 0-500 m.

Distribución: ELD, SIG.

Ejemplar de referencia: Cabrera, A. L. 28843 (SI).

\section{Nuttallanthus D.A. Simpson}

Nuttallanthus canadensis (L.) D.A. Webb

Hierba. Introducida. 0-500 m.

Distribución: CAN, SIG.

Ejemplar de referencia: Biganzoli, F. 395 (SI).

\section{Plantago L.}

Plantago australis Lam. subsp. australis

Hierba. Nativa. 0-600 m.

Distribución: APO, CAI, CAN, ELD, GUA.

Ejemplar de referencia: Cabrera, A. L. 28879 (SI).

Plantago australis Lam. subsp. hirtella (Kunth) Rahn

Hierba. Nativa. 0-500 m

Distribución: IGU, SPE.

Ejemplar de referencia: Perrone, V. s.n. (SI).

\section{Plantago lanceolata $\mathrm{L}$.}

Hierba. Introducida. 0-500 m.

Distribución: CAP.

Ejemplar de referencia: Issouribehere, P. s.n. (SI).

\section{Plantago major L.}

Hierba. Introducida. 0-500 m.

Distribución: SPE.

Ejemplar de referencia: Biganzoli, F. 647 (SI).

Plantago myosuros Lam.

Hierba. Nativa. 0-500 m.

Distribución: OBE.

Ejemplar de referencia: Múlgura, M. E. 2297 (SI).

Plantago napiformis (Rahn) Hassemer

Hierba. Nativa. 0-500 m.

Distribución: SIG.

Ejemplar de referencia: Biganzoli, F. 408 (SI).

Plantago tomentosa Lam.

Hierba. Nativa. 0-700 m.

Distribución: CAN, IGU, GMB, SIG.

Ejemplar de referencia: Múlgura, M. E. 1726 (SI).

\section{Scoparia L.}

Scoparia aemilii Chodat

Hierba. Nativa. 0-500 m.

Distribución: CAN.

Ejemplar de referencia: Martínez Crovetto, $R$. 9504 (CTES).

\section{Scoparia dulcis L.}

Hierba o subarbusto. Nativa. 0-700 m.

Distribución: APO, CAN, CAP, IGU, SIG, SPE.

Ejemplar de referencia: Rodríguez, M. 553 (CTES, G, MNES).

Scoparia ericacea Cham. \& Schltdl.

Hierba o subarbusto. Nativa. 0-500 m.

Distribución: APO, CAN, CON, SIG.

Ejemplar de referencia: Biganzoli, F. 1589 (SI).

\section{Scoparia hassleriana Chodat}

Hierba. Nativa. 0-600 m.

Distribución: APO, CAI, CAN, CAP, CON, LNA, SIG.

Ejemplar de referencia: Cabrera, A. L. 29144 (SI).

Scoparia montevidensis (Spreng.) R.E. Fr.

Hierba. Nativa. 0-600 m.

Distribución: CAI, CAN, CAP, CON, ELD, LNA, SIG. Ejemplar de referencia: Deginani, N. B. 1402 (SI). 
Scoparia plebeja Cham. \& Schltdl.

Hierba. Nativa. 0-500 m.

Distribución: SIG.

Ejemplar de referencia: Pire, S. M. 85 (CTES).

Stemodia L., nom. cons.

Stemodia diplohyptoides M.M. Sosa \& Dematteis $\left({ }^{* *}\right)$

Hierba. Endémica. 100-400 m.

Distribución: ELD, GSM, IGU, MON.

Ejemplar de referencia: Sosa, M. M. 123 (CTES, $\mathrm{MICH).}$

Stemodia hyptoides Cham. \& Schltdl.

Hierba. Nativa. 0-700 m.

Distribución: APO, CAN, CAP, ELD, GSM, GUA, IGU, OBE, MON, SIG.

Ejemplar de referencia: Zuloaga, F. O. 1021 (SI).

\section{Stemodia lanceolata Benth.}

Hierba. Nativa. 0-500 m.

Distribución: APO, CAN, CAP, OBE.

Ejemplar de referencia: Burkart, A. 14033 (SI).

\section{Stemodia palustris A. St.-Hil.}

Hierba. Nativa. 0-700 m.

Distribución: APO, CAP, IGU.

Ejemplar de referencia: Múlgura, M. E. 2914 (SI).

Stemodia stricta Cham. \& Schltdl.

Hierba. Nativa. 0-700 m.

Distribución: APO, CAI, CAN, CAP, ELD, IGU, MON, OBE, SIG, SPE.

Ejemplar de referencia: Zuloaga, F. O. 6526 (SI).

Stemodia verticillata (Mill.) Hassl.

Hierba. Nativa. 0-700 m.

Distribución: APO, CAN, CAP, ELD, GMB, GSM, GUA, LNA, SIG, SJA, SPE.

Ejemplar de referencia: Morrone, O. 727 (SI).

\section{Veronica L.}

\section{Veronica arvensis $\mathrm{L}$.}

Hierba. Introducida. 0-700 m.
Distribución: GUA, IGU.

Ejemplar de referencia: Tressens, S. G. 6350 (CTES).

\section{Veronica chamaedrys L.}

Hierba. Introducida.

Distribución: Desconocida.

Ejemplar de referencia: Schwarz, G. J. 6087 (LIL).

\section{Veronica peregrina $\mathrm{L}$.}

Hierba. Introducida. 0-700 m.

Distribución: GUA, IGU, MON, SIG.

Ejemplar de referencia: Keller, H. A. 13435 (CTES).

\section{Veronica persica Poir.}

Hierba. Introducida. 0-700 m.

Distribución: GUA, MON.

Ejemplar de referencia: Keller, H. A. 4444 (CTES).

\section{Veronica polita Fr.}

Hierba. Introducida. 0-500 m.

Distribución: IGU.

Ejemplar de referencia: Rossow, R. 4817 (BAB).

\section{POACEAE}

\section{Acroceras Stapf}

Acroceras excavatum (Henrard) Zuloaga \& Morrone (***)

Hierba. Nativa. 0-500 m.

Distribución: CAI, CAN, GMB, GSM, GUA, IGU, SIG.

Ejemplar de referencia: Deginani, N. B. 1553 (SI).

Acroceras zizanioides (Kunth) Dandy

Hierba. Nativa. 0-700 m.

Distribución: APO, CAN, CAP, ELD, GSM, IGU, MON, SIG, SJA, SPE.

Ejemplar de referencia: Deginani, N. B. 1103 (SI).

\section{Agenium Nees}

Agenium leptocladum (Hack.) Clayton

Hierba. Nativa. 0-500 m.

Distribución: APO, SIG.

Ejemplar de referencia: Burkart, A. 15356 (SI). 
Agenium villosum (Nees) Pilg.

Hierba. Nativa. 0-500 m.

Distribución: APO, CAI, CAN, CAP, MAY, SIG, SJA.

Ejemplar de referencia: Biganzoli, F. 151 (SI).

Agrostis L., nom. cons.

Agrostis montevidensis Spreng. ex Nees f. montevidensis

Hierba. Nativa. 0-600 m.

Distribución: APO, CAN, CAP, SIG, SPE.

Ejemplar de referencia: Montes, J. E. 12355 (LP).

Agrostis montevidensis Spreng. ex Nees f. submutica (Döll) Kämpf

Hierba. Nativa.

Distribución: APO.

Ejemplar de referencia: Zuloaga, F. O. 4888 (SI).

Aira L., nom. cons.

\section{Aira praecox $\mathrm{L}$.}

Hierba. Introducida. 0-600 m.

Distribución: IGU.

Ejemplar de referencia: Barboza, G. E. 488 (CORD, SI).

\section{Amphibromus Nees}

Amphibromus quadridentulus (Döll) Swallen Hierba. Nativa. 0-700 m.

Distribución: GUA.

Ejemplar de referencia: Keller, H. A. 361 (SI).

Andropogon L., nom. cons.

\section{Andropogon bicornis L.}

Hierba. Nativa. 0-700 m.

Distribución: CAP, CON, ELD, GUA, IGU, OBE, SIG, SPE.

Ejemplar de referencia: Zuloaga, F. O. 5502 (SI).

Andropogon exaratus Hack.

Hierba. Nativa. 0-500 m.
Distribución: CAP, SIG.

Ejemplar de referencia: Norrmann, G. A. 106 (CTES).

Andropogon hypogynus Hack.

Hierba. Nativa. 0-600 m.

Distribución: CAN, SIG.

Ejemplar de referencia: Rodríguez, F. M. 649 (SI).

Andropogon lateralis Nees

Hierba. Nativa. 0-700 m.

Distribución: CAN, CAP, SIG, SPE.

Ejemplar de referencia: Montes, J. E. 15317 (SI).

Andropogon leucostachyus Kunth

Hierba. Nativa. 0-600 m.

Distribución: GMB, GSM, GUA.

Ejemplar de referencia: Keller, H. A. 627 (CTES, SI).

Andropogon macrothrix Trin.

Hierba. Nativa. 0-500 m.

Distribución: CAP.

Ejemplar de referencia: Parodi, L. R. 4196 (BAA).

Andropogon selloanus (Hack.) Hack.

Hierba. Nativa. 0-500 m.

Distribución: APO, CAI, CAN, OBE, SIG.

Ejemplar de referencia: Guaglianone, E. R. 3047 (SI).

Andropogon ternatus (Spreng.) Nees

Hierba. Nativa. 0-500 m.

Distribución: CAP.

Ejemplar de referencia: Honfi, A. I. 389 (MNES, SI).

Andropogon virgatus Desv. ex Ham.

Hierba. Nativa. 0-600 m.

Distribución: APO, CAI, CAN, CAP, ELD, GSM, OBE, SIG, SJA.

Ejemplar de referencia: Krapovickas, A. 12086 (SI).

Anthaenantia P. Beauv.

Anthaenantia lanata (Kunth) Benth.

Hierba. Nativa. 0-600 m.

Distribución: APO, CAN, IGU, SIG.

Ejemplar de referencia: Zuloaga, F. O. 5749 (SI). 
Anthaenantiopsis Mez ex Pilg.

\section{Anthaenantiopsis rojasiana Parodi}

Hierba. Nativa. 0-500 m.

Distribución: CAP.

Ejemplar de referencia: Parodi, L. R. 4552 (BAA).

\section{Aristida L.}

Aristida circinalis Lindm.

Hierba. Nativa. 100-500 m.

Distribución: APO, CAN, CON.

Ejemplar de referencia: Burkart, A. 14386 (SI).

Aristida jubata (Arechav.) Herter

Hierba. Nativa. 0-500 m.

Distribución: APO, CAI, CAN, CAP, CON, SIG, SJA.

Ejemplar de referencia: Múlgura, M. E. 2038 (SI).

Aristida macrophylla Hack.

Hierba. Nativa. 0-500 m.

Distribución: APO, CAN. (SI).

Ejemplar de referencia: Cabrera, A. L. 28467

Aristida megapotamica Spreng. var. longipes Henrard

Hierba. Nativa. 0-800 m.

Distribución: APO, GMB.

Ejemplar de referencia: Keller, H. A. 3851 (CTES).

Aristida megapotamica Spreng. var. megapotamica

Hierba. Nativa. 0-700 m.

Distribución: APO, CAN, CAP, GMB, SIG, SJA.

Ejemplar de referencia: Morrone, O. 997 (SI).

\section{Aristida pallens Cav.}

Hierba. Nativa. 0-700 m.

Distribución: CAN, CAP, GMB, SIG.

Ejemplar de referencia: Zuloaga, F. O. 441 (SI).

\section{Aristida spegazzinii Arechav.}

Hierba. Nativa. 0-500 m.

Distribución: APO, CAI, CAN, SIG.

Ejemplar de referencia: Renvoize, S. A. 5466 (SI).
Aristida valida Henrard (**)

Hierba. Endémica. 0-500 m.

Distribución: APO, CAN, CON, OBE, SIG.

Ejemplar de referencia: Morrone, O. 1768 (SI).

Aristida venustula Arechav.

Hierba. Nativa. 0-500 m.

Distribución: APO, CAP.

Ejemplar de referencia: Parodi, L. R. 6903 (BAA, SI).

\section{Arundinella Raddi}

Arundinella hispida (Humb. \& Bonpl. ex Willd.) Kuntze

Hierba. Nativa. 0-700 m.

Distribución: APO, CAN, CAP, IGU, MON, OBE, SIG, SJA.

Ejemplar de referencia: Zuloaga, F. O. 5338 (SI).

\section{Arundo L.}

\section{Arundo donax L.}

Hierba. Introducida. 0-500 m.

Distribución: ELD, GMB, SIG.

Ejemplar de referencia: Zuloaga, F. O. 5606 (SI).

\section{Avena L.}

Avena strigosa Schreb.

Hierba. Introducida. 0-600 m.

Distribución: CAI.

Ejemplar de referencia: Zuloaga, F. O. 502 (SI).

Axonopus P. Beauv.

Axonopus argentinus Parodi

Hierba. Nativa. 0-800 m.

Distribución: CAP, GUA, SPE.

Ejemplar de referencia: Morrone, O. 720 (SI).

Axonopus compressus (Sw.) P. Beauv.

Hierba. Nativa. 0-700 m.

Distribución: APO, CAI, CAN, CAP, GMB, GUA, SIG.

Ejemplar de referencia: Morrone, O. 684 (SI). 
Axonopus fissifolius (Raddi) Kuhlm.

Hierba. Nativa. 0-700 m.

Distribución: CAN, GMB, GUA, SIG.

Ejemplar de referencia: Deginani, N. B. 1452 (SI).

Axonopus leptostachyus (Flüggé) Hitchc.

Hierba. Nativa. 0-600 m.

Distribución: CAN, SIG.

Ejemplar de referencia: Zuloaga, F. O. 3175 (SI, MO).

\section{Axonopus marginatus (Trin.) Chase $\left({ }^{* *}\right)$}

Hierba. Nativa. 0-500 m.

Distribución: CAN, SIG.

Ejemplar de referencia: Morrone, O. 1604 (SI).

Axonopus obtusifolius (Raddi) Chase $\left(^{* * *}\right)$

Hierba. Nativa. 0-700 m.

Distribución: ELD, GUA.

Ejemplar de referencia: Quarín, C. L. 569 (CTES, SI).

Axonopus siccus (Nees) Kuhlm.

Hierba. Nativa. 100-500 m.

Distribución: APO, CAI, SIG.

Ejemplar de referencia: Morrone, O. 990 (SI).

Axonopus suffultus (J.C. Mikan ex Trin.) Parodi

Hierba. Nativa. 0-700 m.

Distribución: CAI, CAN, CAP, GSM, MON, SIG.

Ejemplar de referencia: Biganzoli, F. 263 (SI).

\section{Bothriochloa Kuntze}

Bothriochloa exaristata (Nash) Henrard

Hierba. Nativa. 0-700 m.

Distribución: GUA.

Ejemplar de referencia: Morrone, O. 721 (SI).

Bothriochloa laguroides (DC.) Herter

Hierba. Nativa. 0-500 m.

Distribución: APO, CAN, ELD, SIG.

Ejemplar de referencia: Zuloaga, F. O. 858 (SI).

Bothriochloa longipaniculata (Gould) Allred \& Gould

Hierba. Nativa. 100-700 m.

Distribución: APO, CAI, CAP, GMB, MAY, SIG.

Ejemplar de referencia: Schwarz, E. A. 7680 (US).
Bothriochloa torreyana (Steud.) Scrivanti \& Anton

Hierba. Nativa. 300-600 m.

Distribución: APO, ELD.

Ejemplar de referencia: Zuloaga, F. O. 4944 (SI).

\section{Bouteloua Lag.}

Bouteloua megapotamica (Spreng.) Kuntze

Hierba. Nativa. 0-500 m.

Distribución: APO.

Ejemplar de referencia: Múlgura, M. E. 2331 (SI).

Briza L.

\section{Briza minor L.}

Hierba. Introducida. 0-500 m.

Distribución: APO, CAN, CAP.

Ejemplar de referencia: Bertoni, M. 4749 (LIL).

\section{Bromidium Nees \& Meyen}

Bromidium tandilense (Kuntze) Rúgolo

Hierba. Nativa. 0-500 m.

Distribución: APO, CAN, SIG.

Ejemplar de referencia: Cabrera, A. L. 28594 (SI).

Bromus L., nom. cons.

Bromus brachyanthera Döll

Hierba. Nativa. 0-500 m.

Distribución: APO, CAN, GMB, GUA, SIG.

Ejemplar de referencia: Tressens, S. G. 6461 (CTES, SI).

\section{Bromus catharticus Vahl}

Hierba. Nativa. 0-500 m.

Distribución: CAI, CAN, SIG.

Ejemplar de referencia: Schwarz, E. A. 6401 (LIL).

\section{Bromus rigidus Roth}

Hierba. Introducida.

Distribución: Desconocida.

Ejemplar de referencia: Kunkel, G. W. H. 2028 (BAA). 


\section{Cenchropsis Nash}

Cenchropsis myosuroides (Kunth) Nash var. longiseta (Caro \& E.A. Sánchez) J.K. Wipff \& R.B. Shaw

Hierba. Nativa. 0-600 m.

Distribución: LNA, SIG.

Ejemplar de referencia: Biganzoli, F. 1 (SI).

\section{Cenchrus L.}

\section{Cenchrus echinatus $\mathrm{L}$.}

Hierba. Nativa. 0-700 m.

Distribución: APO, CAN, CAP, ELD, GMB, GSM, IGU, OBE, SIG, SJA.

Ejemplar de referencia: Cabrera, A. L. 29186 (SI).

Cenchrus latifolius (Spreng.) Morrone

Hierba. Nativa. 0-600 m.

Distribución: APO, CAI, GMB, GSM, GUA, MON, SPE.

Ejemplar de referencia: Zuloaga, F. O. 1910 (SI).

Cenchrus nervosus (Nees) Kuntze

Hierba. Nativa. 0-500 m.

Distribución: ELD, GMB, GSM, SIG, SPE.

Ejemplar de referencia: Deginani, N. B. 1439 (SI).

\section{Cenchrus pilcomayensis (Mez) Morrone}

Hierba. Nativa. 0-600 m.

Distribución: CAI, CAN, ELD.

Ejemplar de referencia: Deginani, N. B. 971 (SI).

Cenchrus purpureus (Schumach.) Morrone

Hierba. Introducida. 0-500 m.

Distribución: APO, LNA, SPE.

Ejemplar de referencia: Cabrera, A. L. 29503 (SI).

\section{Chascolytrum Desv.}

Chascolytrum calotheca (Trin.) Essi, LonghiWagner \& Souza-Chies

Hierba. Nativa. 0-600 m.

Distribución: APO, CAN, CAP, GUA, SIG.

Ejemplar de referencia: Burkart, A. 14331 (SI).
Chascolytrum lamarckianum (Nees) Matthei

Hierba. Nativa. 0-500 m.

Distribución: GMB.

Ejemplar de referencia: Keller, H. A. 11699 (CTES).

Chascolytrum poomorphum (J. Presl) Essi, Longhi-Wagner \& Souza-Chies

Hierba. Nativa. 0-500 m.

Distribución: APO, GMB.

Ejemplar de referencia: Morrone, O. 1347 (SI).

Chascolytrum subaristatum (Lam.) Desv.

Hierba. Nativa. 0-600 m.

Distribución: APO, CAI, CAN, CAP, CON, MON, OBE, SIG, SJA.

Ejemplar de referencia: Morrone, O. 1125 (SI).

Chascolytrum uniolae (Nees) Essi, LonghiWagner \& Souza-Chies

Hierba. Nativa. 0-600 m.

Distribución: APO, CAI, CAN, CAP, IGU, SIG.

Ejemplar de referencia: Morrone, O. 1662 (SI).

\section{Chloris Sw.}

Chloris barbata Sw.

Hierba. Nativa. 100-600 m.

Distribución: CAI, LNA.

Ejemplar de referencia: Morrone, O. 638 (SI).

\section{Chloris ciliata Sw.}

Hierba. Nativa. 0-700 m.

Distribución: GSM.

Ejemplar de referencia: Biganzoli, F. 252 (SI).

\section{Chloris gayana Kunth}

Hierba. Introducida. 0-600 m.

Distribución: CAI, ELD.

Ejemplar de referencia: Burkart, A. 14708 (SI).

\section{Chloris pycnothrix Trin.}

Hierba. Nativa. 0-700 m.

Distribución: CAI, CAN, CAP, GUA, IGU, MON, OBE, SIG, SPE.

Ejemplar de referencia: Seijo, G. J. 847 (CTES, MNES). 
Chrysopogon Trin., nom. cons.

\section{Chrysopogon zizanioides (L.) Roberty}

Hierba. Introducida. 0-600 m.

Distribución: GUA, IGU.

Ejemplar de referencia: Zuloaga, F. O. 680 (SI).

\section{Chusquea Kunth}

\section{Chusquea juergensii Hack. $\left({ }^{* * *}\right)$}

Hierba subleñosa. Nativa. 400-600 m.

Distribución: GUA, OBE.

Ejemplar de referencia: Keller, H. A. 5286 (CTES).

Chusquea ramosissima Lindm.

Hierba subleñosa. Nativa. 0-800 m.

Distribución: APO, CAI, CAN, CAP, CON, GMB, GSM, GUA, IGU, LNA, SIG. (SI).

Ejemplar de referencia: Zuloaga, F. O. 5269

\section{Chusquea tenella Nees $\left.{ }^{* * *}\right)$}

Hierba subleñosa. Nativa. 200-800 m.

Distribución: GUA, IGU, SPE.

Ejemplar de referencia: Deginani, N. B. 1746 (SI).

\section{Cinnagrostis Griseb.}

Cinnagrostis viridiflavescens (Poir.) P.M. Peterson, Soreng, Romasch. \& Barberá var. montevidensis (Nees) P.M. Peterson, Soreng, Romasch. \& Barberá

Hierba. Nativa. 0-600 m.

Distribución: APO, CAI, CAN, ELD, GMB, GUA, OBE, SIG, SJA, SPE.

Ejemplar de referencia: Guaglianone, E. R. 2887 (SI).

Cinnagrostis viridiflavescens (Poir.) P.M. Peterson, Soreng, Romasch. \& Barberá var. viridiflavescens

Hierba. Nativa. 0-700 m.

Distribución: APO, CAI, CAN, CAP, GUA, MON, SIG, SJA, SPE.

Ejemplar de referencia: Daviña, J. 111 (G, MNES).

\section{Coix L.}

\section{Coix lacryma-jobi L.}

Hierba. Introducida. 0-200 m.

Distribución: CAN, GMB, GUA, SPE.

Ejemplar de referencia: Keller, H. A. 21 (CTES).

\section{Colanthelia McClure \& E.W. Smith}

Colanthelia rhizantha (Hack.) McClure ${ }^{* * *}$ )

Hierba subleñosa. Nativa. 100-800 m.

Distribución: GMB, GSM.

Ejemplar de referencia: Morrone, O. 856 (SI).

\section{Coleataenia Griseb.}

\section{Coleataenia prionitis (Nees) Soreng}

Hierba. Perenne. Nativa. 0-100 m.

Distribución: APO, CAN, CAP, GUA, IGU, MON, SIG.

Ejemplar de referencia: Zuloaga, F. O. 6794 (SI).

\section{Cymbopogon Spreng.}

\section{Cymbopogon citratus (DC.) Stapf $\left({ }^{* *}\right)$}

Hierba. Introducida. 0-600 m.

Distribución: GSM, IGU, SIG.

Ejemplar de referencia: Biganzoli, F. 603 (SI).

Cynodon Rich., nom. cons.

Cynodon affinis Caro \& E.A. Sánchez

Hierba. Nativa. 0-500 m.

Distribución: CAP.

Ejemplar de referencia: Naboulet, L. R. 349 (BA).

Cynodon dactylon (L.) Pers. var. dactylon

Hierba. Introducida. 0-800 m.

Distribución: APO, CAI, CAN, IGU, MON, SIG, SPE.

Ejemplar de referencia: Seijo, G. J. 794 (G, MNES).

Cynodon dactylon (L.) Pers. var. longiglumis Caro \& E.A. Sánchez

Hierba. Perenne. Nativa. 0-500 m. 
Distribución: CAI.

Ejemplar de referencia: Montes, J. E. 9679 (LIL).

\section{Cynodon maritimus Kunth}

Hierba. Nativa. 0-500 m.

Distribución: CAP.

Ejemplar de referencia: Holmberg, E. L. s.n. (BA).

Cynodon mucronatus Caro \& E.A. Sánchez $\left({ }^{* *}\right)$

Hierba. Nativa. 100-500 m.

Distribución: MON.

Ejemplar de referencia: Porta, R. E. 85 (BAA).

Cynodon plectostachyus (K. Schum.) Pilg.

Hierba. Introducida. 0-500 m.

Distribución: CAN, SPE.

Ejemplar de referencia: Zuloaga, F. O. 5589 (SI).

Dichanthelium (Hitchc. \& Chase) Gould

Dichanthelium sabulorum (Lam.) Gould \& C.A. Clark var. cordatum (Zuloaga \& Morrone) Zuloaga $\left.{ }^{* * *}\right)$

Hierba. Nativa. 300-700 m.

Distribución: GMB, GUA.

Ejemplar de referencia: Zuloaga, F. O. 5504 (SI).

Dichanthelium sabulorum (Lam.) Gould \& C.A. Clark var. polycladum (Ekman) Zuloaga

Hierba. Nativa. 0-700 m.

Distribución: APO, CAI, CAN, CAP, GMB, GSM, GUA, OBE, SIG, SJA, SPE.

Ejemplar de referencia: Zuloaga, F. O. 5061 (SI).

Dichanthelium sabulorum (Lam.) Gould \& C.A. Clark var. sabulorum

Hierba. Nativa. 0-700 m.

Distribución: APO, CAI, CAN, GMB, GUA, SIG, SPE.

Ejemplar de referencia: Zuloaga, F. O. 6909 (SI).

Digitaria Haller, nom. cons.

Digitaria balansae Henrard

Hierba. Nativa. 0-500 m.

Distribución: APO, SJA.

Ejemplar de referencia: Zuloaga, F. O. 942 (SI).
Digitaria bicornis (Lam.) Roem. \& Schult.

Hierba. Introducida. 100-700 m.

Distribución: GUA.

Ejemplar de referencia: Tressens, S. G. 4878 (CTES).

Digitaria ciliaris (Retz.) Koeler

Hierba. Nativa. 0-700 m.

Distribución: APO, CAI, CAN, ELD, GMB, GSM, IGU, MON, SIG, SPE. (SI).

Ejemplar de referencia: Zuloaga, F. O. 5725

Digitaria eriantha Steud.

Hierba. Introducida. 0-600 m.

Distribución: CAI, SIG.

Ejemplar de referencia: Vanni, R. O. 3854 (CTES).

Digitaria eriostachya Mez.

Hierba. Nativa. 0-500 m.

Distribución: CAN, SIG.

Ejemplar de referencia: Schulz, A. G. 12081 (CTES).

Digitaria horizontalis Willd.

Hierba. Nativa. 0-600 m.

Distribución: CAP, ELD, SIG.

Ejemplar de referencia: Honfi, A. I. 592 (MNES, SI).

Digitaria insularis (L.) Fedde

Hierba. Nativa. 0-700 m.

Distribución: CAI, CAN, CAP, ELD, GMB, IGU, MON, OBE, SIG.

Ejemplar de referencia: Hunziker, J. H. 10856 (SI).

Digitaria laxa (Rchb.) Parodi

Hierba. Nativa. 0-500 m.

Distribución: GSM, SIG.

Ejemplar de referencia: Morrone, O. 1857 (SI).

Digitaria leiantha (Hack.) Parodi

Hierba. Nativa. 0-500 m.

Distribución: CAN.

Ejemplar de referencia: Montes, J. E. 12328 (LP).

Digitaria sacchariflora (Nees) Henrard

Hierba. Nativa. 0-600 m.

Distribución: CAN, GSM, LNA, SIG.

Ejemplar de referencia: Zuloaga, F. O. 6814 (SI). 
Digitaria sanguinalis (L.) Scop.

Hierba. Introducida. 0-700 m.

Distribución: GMB, GUA, OBE, SIG.

Ejemplar de referencia: Montes, J. E. 2428 (SI).

Digitaria similis Beetle ex Gould

Hierba. Nativa. 0-500 m.

Distribución: SIG.

Ejemplar de referencia: Biganzoli, F. 1230 (SI).

\section{Digitaria singularis $\mathrm{Mez}\left({ }^{* * *}\right)$}

Hierba. Nativa. 0-500 m.

Distribución: APO.

Ejemplar de referencia: Martínez Crovetto, $R$. 9411 (BAA).

\section{Digitaria violascens Link}

Hierba. Nativa. 0-500 m.

Distribución: ELD, GMB, GUA, SIG, SPE.

Ejemplar de referencia: Rúgolo de Agrasar, Z. E. 2095 (SI).

\section{Diplachne P. Beauv.}

Diplachne fusca (L.) P. Beauv. ex Roem. \& Schult. subsp. uninervia (J. Presl) P.M. Peterson \& N. Snow

Hierba. Perenne. Nativa. 0-500 m.

Distribución: CAP.

Ejemplar de referencia: Hauman, L. L. s.n. (BAA).

Echinochloa P. Beauv., nom. cons.

Echinochloa colona (L.) Link

Hierba. Nativa. 0-600 m.

Distribución: ELD, LNA, SPE.

Ejemplar de referencia: Zuloaga, F. O. 6768 (SI).

Echinochloa crus-galli (L.) P. Beauv. var. crusgalli

Hierba. Introducida. 0-800 m.

Distribución: GUA.

Ejemplar de referencia: Keller, H. A. 375 (CTES).

Echinochloa crus-galli (L.) P. Beauv. var. mitis (Pursh) Peterm.

Hierba. Introducida. 0-700 m.
Distribución: GUA.

Ejemplar de referencia: Morrone, O. 734 (SI).

Echinochloa crus-pavonis (Kunth) Schult.

Hierba. Nativa. 0-800 m.

Distribución: ELD, GMB, IGU, SIG, SPE.

Ejemplar de referencia: Burkart, A. 14533 (SI).

Echinochloa oryzoides (Ard.) Fritsch

Hierba. Introducida. 0-500 m.

Distribución: CAP.

Ejemplar de referencia: Parodi, L. R. 13917 (BAA).

Echinochloa polystachya (Kunth) Hitchc.

Hierba. Nativa. 0-300 m.

Distribución: CAP, SIG.

Ejemplar de referencia: Schwarz, G. J. 6204 (BAA).

\section{Eleusine Gaertn.}

Eleusine indica (L.) Gaertn.

Hierba. Introducida. 0-500 m.

Distribución: CAP, ELD, GMB, GSM, GUA, IGU.

Ejemplar de referencia: Zuloaga, F. O. 5602 (SI).

Eleusine tristachya (Lam.) Lam.

Hierba. Nativa. 0-600 m.

Distribución: APO, CAN, CAP, SIG, SPE.

Ejemplar de referencia: Honfi, A. I. 474 (CTES, MNES).

Elionurus Humb. \& Bonpl. ex Willd., nom. cons.

Elionurus muticus (Spreng.) Kuntze

Hierba. Nativa. 0-500 m.

Distribución: APO, CAN, SIG.

Ejemplar de referencia: Morrone, O. 1608 (SI).

\section{Eragrostis Wolf}

\section{Eragrostis airoides Nees}

Hierba. Nativa. 0-700 m.

Distribución: APO, CAI, CAN, CAP, GUA, SIG.

Ejemplar de referencia: Seijo, G. J. 815 (CTES,

G, MNES). 
Eragrostis articulata (Schrank) Nees

Hierba. Nativa. 0-800 m.

Distribución: CAI, CAN, ELD, SIG.

Ejemplar de referencia: Quiroga, H. 48 (BA, BAA).

Eragrostis bahiensis Schrad. ex Schult.

Hierba. Nativa. 0-600 m.

Distribución: APO, CAI, CAN, CAP, SJA.

Ejemplar de referencia: Burkart, A. 14336 (SI).

Eragrostis ciliaris (L.) R. Br.

Hierba. Introducida. 0-600 m.

Distribución: GSM.

Ejemplar de referencia: Zuloaga, F. O. 5661 (SI).

Eragrostis hypnoides (Lam.) Britton, Stern \& Poggenb.

Hierba. Nativa. 0-700 m.

Distribución: CAP, ELD, GSM, SIG.

Ejemplar de referencia: Morrone, O. 1891 (SI).

Eragrostis japonica (Thunb.) Trin.

Hierba. Introducida. 0-500 m.

Distribución: ELD, MON, OBE, SJA.

Ejemplar de referencia: Burkart, A. 15460 (SI).

\section{Eragrostis lugens Nees}

Hierba. Nativa. 0-800 m.

Distribución: APO, CAI, CAN, CAP, ELD, GMB, GSM, IGU, MON, SIG, SPE.

Ejemplar de referencia: Biganzoli, F. 250 (SI).

Eragrostis mexicana (Hornem.) Link subsp. virescens (J. Presl) S.D. Koch \& Sanchéz Vega

Hierba. Nativa. 0-700 m.

Distribución: GUA.

Ejemplar de referencia: Keller, H. A. 590 (CTES).

Eragrostis neesii Trin. var. expansiflora Nicora

Hierba. Nativa. 0-300 m.

Distribución: CAI, GMB, SIG.

Ejemplar de referencia: Múlgura, M. E. 1724 (SI).

\section{Eragrostis neesii Trin. var. lindmanii (Hack.)}

Ekman

Hierba. Nativa. 0-500 m.

Distribución: CAN, SIG.

Ejemplar de referencia: Biganzoli, F. 75 (SI).

\section{Eragrostis neesii Trin. var. neesii}

Hierba. Nativa. 0-500 m.

Distribución: APO, CAI, CAN, CAP, SIG.

Ejemplar de referencia: Zuloaga, F. O. 432 (CTES, SI).

\section{Eragrostis perennis Döll}

Hierba. Nativa. 0-600 m.

Distribución: CAN, CAP, GMB, SIG.

Ejemplar de referencia:Zuloaga, F. O. 448 (CTES, SI).

Eragrostis pilosa (L.) P. Beauv.

Hierba. Introducida. 0-600 m.

Distribución: SPE.

Ejemplar de referencia: Honfi, A. I. 558 (MNES).

\section{Eragrostis polytricha Nees}

Hierba. Nativa. 0-700 m.

Distribución: APO, CAI, CAN, CAP, GSM, SIG.

Ejemplar de referencia: Biganzoli, F. 17 (SI).

Eragrostis rojasii Hack.

Hierba. Nativa. 0-500 m.

Distribución: APO, CAN, CAP, GSM.

Ejemplar de referencia: Martínez Crovetto, $R$.

9127 (CTES).

Eragrostis seminuda Trin.

Hierba. Nativa. 0-600 m.

Distribución: CAP, SIG.

Ejemplar de referencia: Parodi, L. R. 5485 (BAA).

Eragrostis tenuifolia (A. Rich.) Hochst. ex Steud.

Hierba. Introducida. 0-700 m.

Distribución: GMB, IGU, MON, SIG.

Ejemplar de referencia: Deginani, N. B. 1776 (SI).

Eragrostis trichocolea Hack. \& Arechav.

Hierba. Nativa. 0-500 m.

Distribución: CAN, SIG.

Ejemplar de referencia: Morrone, O. 1701 (SI).

\section{Eriochloa Kunth}

Eriochloa grandiflora (Trin.) Benth. ${ }^{* * *}$ )

Hierba. Nativa. 0-500 m.

Distribución: APO, CAN.

Ejemplar de referencia: Fontana, J. L. 117-7 (SI). 
Eriochloa montevidensis Griseb.

Hierba. Perenne. Nativa. 0-500 m.

Distribución: ELD.

Ejemplar de referencia: Keller, H. A. 2167 (CTES).

Eriochloa punctata (L.) Desv. ex Ham.

Hierba. Nativa. 0-200 m.

Distribución: CAN, ELD, GMB, GSM, GUA, IGU, SIG, SPE.

Ejemplar de referencia: Zuloaga, F. O. 5494 (SI).

\section{Eriochrysis P. Beauv.}

Eriochrysis cayennensis $P$. Beauv.

Hierba. Nativa. 0-600 m.

Distribución: CAN, CAP, CON, SIG.

Ejemplar de referencia: Múlgura, M. E. 2855 (SI).

\section{Eriochrysis laxa Swallen}

Hierba. Nativa. 0-500 m.

Distribución: CAP.

Ejemplar de referencia: Ekman, E. L. 526 (BAA).

\section{Eustachys Desv.}

Eustachys calvescens (Hack.) Caro \& E.A. Sánchez $\left.{ }^{* * *}\right)$

Hierba. Nativa. 0-500 m.

Distribución: CAN, SIG.

Ejemplar de referencia: Montes, J. E. 2011 (LIL).

\section{Eustachys distichophylla (Lag.) Nees}

Hierba. Nativa. 0-700 m.

Distribución: APO, CAI, CAN, CAP, GSM, LNA, MON, OBE, SIG.

Ejemplar de referencia: Zuloaga, F. O. 4925 (SI).

Eustachys retusa (Lag.) Kunth

Hierba. Nativa. 0-700 m.

Distribución: LNA.

Ejemplar de referencia: Zuloaga, F. O. 13359 (CTES, SI).

Eustachys uliginosa (Hack.) Herter Hierba. Nativa. 100-700 m.
Distribución: APO, CAI, CAN, CAP, SIG.

Ejemplar de referencia: Zuloaga, F. O. 992 (SI).

\section{Festuca L.}

Festuca australis Nees ex Steud.

Hierba. Nativa. 0-500 m.

Distribución: CAN.

Ejemplar de referencia: Montes, J. E. 2198 (SI).

\section{Festuca fimbriata Nees}

Hierba. Nativa. 0-500 m.

Distribución: APO, CON, IGU.

Ejemplar de referencia: Cabrera, A. L. 28766 (SI).

Festuca ulochaeta Nees ex Steud.

Hierba. Nativa. 400-700 m.

Distribución: CAI, GMB, GUA. LIL).

Ejemplar de referencia: Parodi, L. R. 5673 (BAA,

\section{Guadua Kunth}

Guadua chacoensis (Rojas) Londoño \& P.M. Peterson

Hierba subleñosa. Nativa. 0-600 m.

Distribución: ELD, GSM, IGU, MON, SIG.

Ejemplar de referencia: Parodi, L. R. 5543 (BAA).

\section{Guadua paraguayana Döll}

Hierba subleñosa. Nativa. 0-500 m.

Distribución: IGU. (SI).

Ejemplar de referencia: Guaglianone, E. R. 2486

\section{Guadua tagoara (Nees) Kunth $\left({ }^{* * *}\right)$}

Hierba subleñosa. Nativa. 200-400 m.

Distribución: APO, GMB.

Ejemplar de referencia: Lizarazu, M. Á. 63 (SI).

Guadua trinii (Nees) Nees ex Rupr.

Hierba subleñosa. Nativa. 0-800 m.

Distribución: CAN, GMB, IGU, MAY, SJA, SPE.

Ejemplar de referencia: Zuloaga, F. O. 4975 (SI). 
Guadua variegata Lizarazu $\left({ }^{* *}\right)$

Hierba. Endémica. 400-600 m.

Distribución: SPE.

Ejemplar de referencia: Rúgolo, Z. E. 878 (SI).

\section{Gymnopogon P. Beauv.}

Gymnopogon burchellii (Munro ex Döll) Ekman

Hierba. Nativa. 0-500 m.

Distribución: CAN.

Ejemplar de referencia: Montes, J. E. 15256 (SI).

Gymnopogon legrandii Roseng., B.R. Arrill. \& Izag.

Hierba. Nativa. 0-500 m.

Distribución: SIG.

Ejemplar de referencia: Zuloaga, F. O. 5747 (SI).

Gymnopogon spicatus (Spreng.) Kuntze

Hierba. Perenne. Nativa. 0-500 m.

Distribución: SIG.

Ejemplar de referencia: Biganzoli, F. 26 (SI).

\section{Gynerium P. Beauv.}

Gynerium sagittatum (Aubl.) P. Beauv.

Hierba subleñosa. Nativa. 0-500 m.

Distribución: SIG.

Ejemplar de referencia: Martínez Crovetto, $R$. 3408 (SI).

\section{Hemarthria R. Br.}

Hemarthria altissima (Poir.) Stapf \& C.E. Hubb.

Hierba. Introducida. 0-700 m.

Distribución: CAP, IGU.

Ejemplar de referencia: Osten, C. 8178 (MOY).

\section{Heteropogon Pers.}

Heteropogon contortus (L.) P. Beauv. ex Roem. \& Schult.

Hierba. Introducida. 0-500 m.
Distribución: APO, SIG.

Ejemplar de referencia: Burkart, A. 15356 (SI).

Hildaea C. Silva \& R.P. Oliveira

Hildaea pallens (Sw.) C. Silva \& R.P. Oliveira var. major (Nees) C. Silva \& R.P. Oliveira ${ }^{* * *}$ )

Hierba. Nativa. 0-600 m.

Distribución: GMB, IGU, SPE.

Ejemplar de referencia: Zuloaga, F. O. 5475 (SI).

Hildaea pallens (Sw.) C. Silva \& R.P. Oliveira var. pallens

Hierba. Nativa. 0-600 m.

Distribución: CAI, ELD, GMB, GSM, GUA, IGU, LNA, OBE, SIG, SPE.

Ejemplar de referencia: Zuloaga, F. O. 2275 (SI).

Hildaea ruprechtii (Döll) C. Silva \& R.P. Oliveira Hierba. Nativa. 0-500 m.

Distribución: GMB, IGU.

Ejemplar de referencia: Zuloaga, F. O. 2093 (SI).

Hildaea tenuis (J. Presl \& C. Presl) C. Silva \& R.P. Oliveira

Hierba. Nativa. 0-700 m.

Distribución: CAI, CAN, ELD, GMB, GSM, GUA, SPE.

Ejemplar de referencia: Guillén, R. 139 (CTES, G, MNES, SI).

\section{Homolepis Chase}

Homolepis glutinosa (Sw.) Zuloaga \& Soderstr. Hierba. Nativa. 0-700 m.

Distribución: CAN, GMB, GUA, IGU, MON, OBE, SIG, SPE.

Ejemplar de referencia: Zuloaga, F. O. 6799 (SI).

Homolepis villaricensis (Mez) Zuloaga \& Soderstr.

Hierba. Nativa. 0-500 m.

Distribución: APO, CAI, CAN, GMB, GSM, SIG, SPE.

Ejemplar de referencia: Morrone, O. 1885 (SI). 


\section{Hordeum L.}

Hordeum stenostachys Godr.

Hierba. Nativa. 0-500 m.

Distribución: CAN, OBE, SJA.

Ejemplar de referencia: Morrone, O. 1786 (SI).

Hymenachne P. Beauv.

Hymenachne amplexicaulis (Rudge) Nees

Hierba. Nativa. 0-700 m.

Distribución: CAN, CAP, ELD, GMB, GSM, IGU, SPE.

Ejemplar de referencia: Deginani, N. B. 1116 (SI).

Hymenachne donacifolia (Raddi) Chase

Hierba. Nativa. 0-500 m.

Distribución: CAI, CAN, CON, GMB, GSM, GUA, IGU, MON.

Ejemplar de referencia: Deginani, N. B. 1801 (SI).

Hymenachne grumosa (Nees) Zuloaga

Hierba. Nativa. 0-500 m.

Distribución: APO, CAP, IGU, SIG, SJA.

Ejemplar de referencia: Cabrera, A. L. 28940 (SI).

\section{Hymenachne pernambucense (Spreng.)}

Zuloaga

Hierba. Nativa. 0-500 m.

Distribución: APO, CAI, CAN, CAP, CON, ELD, GMB, GSM, IGU, SIG, SJA, SPE.

Ejemplar de referencia: Burkart, A. 15461 (SI).

\section{Hyparrhenia Andersson ex E. Fourn.}

Hyparrhenia rufa (Nees) Stapf

Hierba. Introducida. 0-500 m.

Distribución: APO.

Ejemplar de referencia: Montes, J. E. 15287 (SI).

\section{Ichnanthus P. Beauv.}

Ichnanthus inconstans (Trin. ex Nees) Döll (***)

Hierba. Nativa. 100-700 m.

Distribución: SPE.

Ejemplar de referencia: Zuloaga, F. O. 6485 (SI).
Ichnanthus procurrens (Nees ex Trin.) Stieber var. subaequiglume (Hack.) Killeen \& Kirpes

Hierba. Nativa. 0-800 m.

Distribución: CAI, CAN, CAP, CON, SIG.

Ejemplar de referencia: Parodi, L. R. 6942 (US).

\section{Imperata Cirillo}

Imperata brasiliensis Trin.

Hierba. Nativa. 0-600 m.

Distribución: APO, CAN, IGU, MON, SIG, SPE.

Ejemplar de referencia: Biganzoli, F. 624 (SI).

Lasiacis (Griseb.) Hitchc.

Lasiacis divaricata (L.) Hitchc. var. austroamericana Davidse

Hierba subleñosa. Nativa. 0-500 m.

Distribución: APO, CAI, CAN, ELD, GMB, GSM, GUA, IGU, LNA, MON, SIG, SPE. (SI).

Ejemplar de referencia: Deginani, N. B. 1302

Lasiacis sorghoidea (Desv. ex Ham.) Hitchc. \& Chase

Hierba subleñosa. Nativa. 0-400 m.

Distribución: CAI, CAN, CAP, GMB, GSM, IGU, LNA, MON, SIG, SJA, SPE.

Ejemplar de referencia: Zuloaga, F. O. 5455 (SI).

Leersia Sol. ex Sw.

Leersia hexandra $S w$.

Hierba. Nativa. 0-800 m.

Distribución: APO, CAP, IGU, MON.

Ejemplar de referencia: Deginani, N. B. 1771 (SI).

Leersia ligularis Trin. var. grandiflora (Döll) Pyrah

Hierba. Perenne. Nativa. 100-700 m.

Distribución: CAI, CAN, ELD, GMB, GUA, IGU, OBE, SIG, SPE.

Ejemplar de referencia: Zuloaga, F. O. 6907 (SI). 
Leptochloa P. Beauv.

\section{Leptochloa fusca (L.) Kunth}

Hierba. Nativa. 0-500 m.

Distribución: SIG.

Ejemplar de referencia: Grondona, E. M. 3188 (BAA).

Leptochloa panicea (Retz.) Ohwi subsp. brachiata (Steud.) N.W. Snow

Hierba. Nativa. 0-700 m.

Distribución: CAN, CAP, ELD, LNA, SIG.

Ejemplar de referencia: Schwarz, G. J. 4591 (LIL).

Leptochloa virgata (L.) P. Beauv.

Hierba. Nativa. 0-800 m.

Distribución: APO, CAI, CAN, CAP, ELD, GMB, GSM, GUA, IGU, MON, OBE, SIG, SJA, SPE.

Ejemplar de referencia: Parodi, L. R. 5535 (BAA).

\section{Lithachne P. Beauv.}

Lithachne pauciflora (Sw.) P. Beauv.

Hierba. Nativa. 0-300 m.

Distribución: CAN, CAP, ELD, GMB, GUA, IGU, SIG.

Ejemplar de referencia: Zuloaga, F. O. 650 (SI).

\section{Lolium L.}

\section{Lolium multiflorum Lam.}

Hierba. Introducida. 0-500 m.

Distribución: CAN, CAP, MON.

Ejemplar de referencia: Schwarz, G. J. 6832 (BAA).

\section{Luziola Juss.}

Luziola bahiensis (Steud.) Hitchc.

Hierba. Nativa. 0-700 m.

Distribución: GUA, IGU, MON, SPE.

Ejemplar de referencia: Keller, H. A. 13502 (CTES).

Luziola peruviana Juss. ex J.F. Gmel.

Hierba. Nativa. 0-500 m.

Distribución: APO, CAP, GUA.

Ejemplar de referencia: Múlgura, M. E. 1821 (SI).
Megathyrsus (Pilg.) B.K. Simon \& S.W.L. Jacobs

Megathyrsus maximus (Jacq.) B.K. Simon \& S.W.L. Jacobs var. maximus

Hierba. Introducida. 0-500 m.

Distribución: GMB, GUA, IGU, SIG.

Ejemplar de referencia: Guillén, R. 195 (G, MNES).

Megathyrsus maximus (Jacq.) B.K. Simon \& S.W.L. Jacobs var. pubiglumis (K. Schum.) B.K. Simon \& S.W.L. Jacobs

Hierba. Introducida. 0-500 m.

Distribución: ELD.

Ejemplar de referencia: Quarín, C. L. 334 (CTES).

Melica L., nom. cons.

Melica brasiliana Ard.

Hierba. Nativa. 0-500 m.

Distribución: CAN.

Ejemplar de referencia: Niederlein, G. s.n. (BA).

Melica sarmentosa Nees var. pilosula Döll

Hierba. Nativa. 0-500 m.

Distribución: APO, CAI, CAN, CON, ELD, GMB, GSM, GUA, IGU, MON, OBE, SIG, SJA, SPE.

Ejemplar de referencia: Rúgolo de Agrasar, Z. E. 876 (SI).

Melica sarmentosa Nees var. sarmentosa Hierba. Nativa. 0-700 m.

Distribución: APO, CAI, CAN, GMB, SIG.

Ejemplar de referencia: Morrone, O. 643 (SI).

Melica tenuis Hack. ex Arechav.

Hierba. Nativa. 0-600 m.

Distribución: APO, CAI, CAN, ELD, SIG.

Ejemplar de referencia: Morrone, O. 1792 (SI).

Melinis P. Beauv.

Melinis minutiflora $P$. Beauv. Hierba. Introducida. 0-600 m. Distribución: ELD, SIG.

Ejemplar de referencia: Morrone, O. 1338 (SI). 
Melinis repens (Willd.) Zizka

Hierba. Introducida. 0-600 m.

Distribución: APO, CAN, CAP, CON, ELD, GUA, LNA, SIG.

Ejemplar de referencia: Morrone, O. 1048 (SI).

\section{Merostachys Spreng.}

Merostachys claussenii Munro $\left({ }^{* * *}\right)$

Hierba subleñosa. Nativa. 0-800 m.

Distribución: CAI, CAN, GMB, GSM, GUA, IGU, MON, SIG, SPE.

Ejemplar de referencia: Parodi, L. R. 5586 (BAA).

\section{Merostachys multiramea Hack. $\left({ }^{* * *}\right)$}

Hierba subleñosa. Nativa. 400-700 m.

Distribución: CAI, CAN, GMB, GSM, GUA, IGU, MON, SPE.

Ejemplar de referencia: Romanczuk, M. C. 644 (SI).

\section{Mesosetum Steud.}

\section{Mesosetum comatum Swallen $\left(^{* *}\right)$}

Hierba. Endémica. 0-300 m.

Distribución: SIG.

Ejemplar de referencia: Zuloaga, F. O. 5329 (SI).

\section{Microchloa R. Br.}

Microchloa indica (L. f.) P. Beauv.

Hierba. Perenne. Nativa. 0-500 m.

Distribución: SIG.

Ejemplar de referencia: Montes, J. E. 14846 (NY).

\section{Microchloa kunthii Desv.}

Hierba. Nativa. 200-500 m.

Distribución: APO, CAI.

Ejemplar de referencia: Biganzoli, F. 925 (SI).

\section{Mnesithea Kunth}

Mnesithea aurita (Steud.) de Koning \& Sosef

Hierba. Nativa. 0-300 m.

Distribución: CAP, SIG.

Ejemplar de referencia: Quiroga, H. s.n. (BAA).
Mnesithea balansae (Hack.) de Koning \& Sosef Hierba. Nativa. 0-500 m.

Distribución: CAN, CAP, SIG.

Ejemplar de referencia: Parodi, D. 4074 (BAA).

Mnesithea selloana (Hack.) de Koning \& Sosef Hierba. Nativa. 0-500 m.

Distribución: APO, CAN, CAP, ELD, GSM, OBE. Ejemplar de referencia: Burkart, A. 14089 (SI).

Morronea Zuloaga \& Scataglini

Morronea trichidiachnis (Döll) Zuloaga \& Scataglini $\left({ }^{* * *}\right)$

Hierba. Nativa. 0-500 m.

Distribución: GMB, IGU.

Ejemplar de referencia: Zuloaga, F. O. 5213 (SI).

Muhlenbergia Schreb.

Muhlenbergia schreberi J.F. Gmel.

Hierba. Nativa. 0-600 m.

Distribución: CAI, CAP, MON, SIG.

Ejemplar de referencia: Montes, J. E. 15340 (SI).

Nassella E. Desv. emend. Barkworth

Nassella airoides (Ekman) Barkworth

Hierba. Perenne. Nativa. 0-500 m.

Distribución: CAN, CAP, GUA, OBE.

Ejemplar de referencia: Morrone, O. 1730 (SI).

Nassella filiculmis (Delile) Barkworth

Hierba. Nativa. 0-500 m.

Distribución: APO.

Ejemplar de referencia: Cabrera, A. L. 28589 (SI).

Nassella melanosperma (J. Presl) Barkworth Hierba. Nativa. 0-500 m.

Distribución: APO, CAN.

Ejemplar de referencia: Morrone, O. 1109 (SI).

Nassella neesiana (Trin. \& Rupr.) Barkworth Hierba. Nativa. 0-500 m.

Distribución: APO, CAN, CAP.

Ejemplar de referencia: Burkart, A. 14216 (SI). 
Nassella sellowiana (Nees ex Trin. \& Rupr.) Peñailillo $\left(^{* * *}\right)$

Hierba. Nativa. 200-500 m.

Distribución: APO.

Ejemplar de referencia: Molfino, J. F. s.n. (SI).

\section{Ocellochloa Zuloaga \& Morrone}

Ocellochloa stolonifera (Poir.) Zuloaga \& Morrone

Hierba. Nativa. 0-700 m.

Distribución: CAI, CON, IGU.

Ejemplar de referencia: Vanni, R. O. 3161 (CTES).

\section{Oedochloa C. Silva \& R.P. Oliveira}

Oedochloa minarum (Nees) C. Silva \& R.P. Oliveira

Hierba. Nativa. 500-700 m.

Distribución: GMB, GUA.

Ejemplar de referencia: Morrone, O. 736 (SI).

Oedochloa procurrens (Nees ex Trin.) C. Silva \& R.P. Oliveira

Hierba. Nativa. 0-500 m.

Distribución: APO, SIG.

Ejemplar de referencia: Zuloaga, F. O. 886 (SI).

\section{Olyra L.}

\section{Olyra ciliatifolia Raddi}

Hierba. Nativa. 0-700 m.

Distribución: CAI, CAN, CON, ELD, GMB, GSM, GUA, IGU, MON, OBE, SIG.

Ejemplar de referencia: Burkart, A. 14703 (SI).

\section{Olyra fasciculata Trin.}

Hierba. Perenne. Nativa. 0-800 m.

Distribución: IGU, SPE.

Ejemplar de referencia: Vanni, R. O. 3202 (SI).

\section{Olyra humilis Nees}

Hierba. Nativa. 0-700 m.

Distribución: APO, CAI, CAN, CON, ELD, GMB, GSM, GUA, OBE, SIG, SJA, SPE.

Ejemplar de referencia: Burkart, A. 14619 (SI).

\section{Olyra latifolia $\mathrm{L}$.}

Hierba. Nativa. 0-800 m.

Distribución: CAN, CAP, ELD, GMB, GUA, IGU, SIG, SPE.

Ejemplar de referencia: Burkart, A. 14612 (SI).

Oplismenus P. Beauv., nom. cons.

Oplismenus hirtellus (L.) P. Beauv. subsp. hirtellus

Hierba. Nativa. 0-800 m.

Distribución: CAN, ELD, GMB, GSM, GUA, IGU, SIG, SPE.

Ejemplar de referencia: Parodi, L. R. 5629 (BAA).

Oplismenus hirtellus (L.) P. Beauv. subsp. setarius (Lam.) Mez ex Ekman

Hierba. Nativa. 0-500 m.

Distribución: APO, CAI, CAN, CAP, ELD, GMB, GUA, IGU, LNA, MON, OBE, SIG, SPE.

Ejemplar de referencia: Deginani, N. B. 1108 (SI).

\section{Oryza L.}

\section{Oryza rufipogon Griff.}

Hierba. Introducida. 0-500 m.

Distribución: SJA.

Ejemplar de referencia: Parodi, L. R. 7985 (BAA).

\section{Otachyrium Nees}

Otachyrium versicolor (Döll) Henrard

Hierba. Nativa. 0-500 m.

Distribución: APO, CAN, CAP.

Ejemplar de referencia: Parodi, L. R. 4485 (BAA).

\section{Panicum L.}

\section{Panicum bergii Arechav. var. bergii}

Hierba. Nativa. 0-500 m.

Distribución: APO, CAN, CAP, CON, ELD, IGU, LNA, OBE, SIG.

Ejemplar de referencia: Burkart, A. 14455 (F, SI, US). 
Panicum bergii Arechav. var. pilosissimum Zuloaga

Hierba. Nativa. 0-500 m.

Distribución: APO, CAN, CAP, ELD.

Ejemplar de referencia: Zuloaga, F. O. 3271 (SI).

\section{Panicum dichotomiflorum Michx.}

Hierba. Nativa. 0-600 m.

Distribución: CAN, CAP, ELD, GMB, GSM, GUA, IGU, MON, SIG.

Ejemplar de referencia: Morrone, O. 743 (SI).

\section{Panicum millegrana Poir.}

Hierba. Nativa. 0-800 m.

Distribución: APO, CAI, CAN, GMB, GSM, GUA, IGU, LNA, MON, SIG, SPE.

Ejemplar de referencia: Deginani, N. B. 1780 (SI).

Panicum olyroides Kunth var. hirsutum Henrard Hierba. Nativa. 0-500 m.

Distribución: SIG.

Ejemplar de referencia: Zuloaga, F. O. 7195 (SI).

\section{Panicum olyroides Kunth var. olyroides}

Hierba. Nativa. 0-600 m.

Distribución: APO, CAI, CAN, GSM, SIG.

Ejemplar de referencia: Montes, J. E. 1885 (F, US).

\section{Panicum peladoense Henrard}

Hierba. Nativa. 0-500 m.

Distribución: APO, CAN, CAP, CON, SIG.

Ejemplar de referencia: Zuloaga, F. O. 5794 (SI).

\section{Panicum quadriglume (Döll) Hitchc. $\left({ }^{* *}\right)$}

Hierba. Nativa. 0-500 m.

Distribución: MON.

Ejemplar de referencia: Schwindt, E. 1154 (LIL, MO, US).

\section{Panicum repens $\mathrm{L}$.}

Hierba. Introducida. 0-500 m.

Distribución: CAN.

Ejemplar de referencia: Maidana s.n. (SI).

\section{Panicum sellowii Nees}

Hierba. Nativa. 100-800 m.

Distribución: CAI, CAN, GMB, GSM, GUA, OBE, SIG, SPE.

Ejemplar de referencia: Deginani, N. B. 1514 (SI).

\section{Panicum trichanthum Nees}

Hierba. Nativa. 0-800 m.

Distribución: CAN, CAP, ELD, GSM, GUA, IGU, MON, OBE, SJA, SPE.

Ejemplar de referencia: Zuloaga, F. O. 2074 (SI).

\section{Panicum tricholaenoides Steud.}

Hierba. Nativa. 0-500 m.

Distribución: CAN, MON.

Ejemplar de referencia: Cariola, L. 16 (CTES).

\section{Parodiophyllochloa Zuloaga \& Morrone}

Parodiophyllochloa cordovensis (E. Fourn.) Zuloaga \& Morrone

Hierba. Nativa. 500-700 m.

Distribución: CAP, GMB, GSM, GUA.

Ejemplar de referencia: Zuloaga, F. O. 6718 (SI).

Parodiophyllochloa missiona (Ekman) Zuloaga \& Morrone

Hierba. Nativa. 0-700 m.

Distribución: APO, CAI, CAN, CAP, CON, ELD, GMB, GSM, GUA, IGU, SJA, SPE.

Ejemplar de referencia: Zuloaga, F. O. 5008 (SI).

Parodiophyllochloa ovulifera (Trin.) Zuloaga \& Morrone

Hierba. Nativa. 0-700 m.

Distribución: APO, CAN, ELD, GMB, GSM, GUA, IGU, MON, SIG, SPE.

Ejemplar de referencia: Morrone, O. 754 (SI).

Parodiophyllochloa pantricha (Hack.) Zuloaga \& Morrone ${ }^{* * *}$ )

Hierba. Nativa. 0-800 m.

Distribución: CAN, CAP, ELD, GMB, GSM, GUA, IGU, MON, SIG, SJA, SPE.

Ejemplar de referencia: Morrone, O. 1439 (SI).

Parodiophyllochloa rhizogona (Hack.) Zuloaga \& Morrone ${ }^{* * *}$ )

Hierba. Nativa. 0-800 m.

Distribución: GMB, GUA.

Ejemplar de referencia: Tressens, S. G. 6352 (CTES, SI). 


\section{Paspalum L.}

\section{Paspalum acuminatum Raddi}

Hierba. Perenne. Nativa. 0-500 m.

Distribución: CAN.

Ejemplar de referencia: Mutinelli, A s.n. (SI).

\section{Paspalum almum Chase}

Hierba. Nativa. 0-300 m.

Distribución: APO, CAI, IGU.

Ejemplar de referencia: Zuloaga, F. O. 7149 (SI).

\section{Paspalum arundinellum $\mathrm{Mez}$}

Hierba. Nativa. 0-300 m.

Distribución: CAN, SIG.

Ejemplar de referencia: Zuloaga, F. O. 6810 (SI).

\section{Paspalum bertonii Hack. $\left.{ }^{* * *}\right)$}

Hierba. Nativa. 100-500 m.

Distribución: CAI, CAN, GSM, IGU, MON, SIG.

Ejemplar de referencia: Correa, M. N. 5393 (SI, US).

\section{Paspalum compressifolium Swallen}

Hierba. Nativa. 0-500 m.

Distribución: SIG.

Ejemplar de referencia: Morrone, O. 1682 (SI).

\section{Paspalum conjugatum P.J. Bergius}

Hierba. Nativa. 0-700 m.

Distribución: APO, CAI, CAN, CAP, ELD, GMB, GUA, IGU, LNA, SIG, SJA, SPE.

Ejemplar de referencia: Morrone, O. 686 (SI).

\section{Paspalum conspersum Schrad.}

Hierba. Nativa. 0-700 m.

Distribución: CAP, ELD, GSM, IGU, SIG.

Ejemplar de referencia: Morrone, O. 1070 (SI).

\section{Paspalum cromyorrhizon Trin. ex Döll}

Hierba. Nativa. 0-500 m.

Distribución: CAN.

Ejemplar de referencia: Zuloaga, F. O. 7223 (SI).

\section{Paspalum dedeccae Quarín}

Hierba. Nativa. 0-500 m.

Distribución: APO, CAP.

Ejemplar de referencia: Honfi, A. I. 388 (SI).

\section{Paspalum denticulatum Trin.}

Hierba. Nativa. 0-500 m.

Distribución: CAP.

Ejemplar de referencia: Burkart, A. 14473 (SI).

\section{Paspalum dilatatum Poir.}

Hierba. Nativa. 0-600 m.

Distribución: APO, GMB, GUA, OBE, SPE.

Ejemplar de referencia: Zuloaga, F. O. 5503 (SI).

\section{Paspalum distichum $\mathrm{L}$.}

Hierba. Nativa. 0-500 m.

Distribución: CAP.

Ejemplar de referencia: Hojsgaard, D. 148 (MNES, SI).

\section{Paspalum equitans $\mathrm{Mez}$}

Hierba. Nativa. 0-500 m.

Distribución: APO, CAP.

Ejemplar de referencia: Parodi, L. R. 4181 (BAA).

\section{Paspalum erianthoides Lindm.}

Hierba. Nativa. 0-500 m.

Distribución: APO.

Ejemplar de referencia: Cabrera, A. L. 28759 (SI).

Paspalum falcatum Nees ex Steud.

Hierba. Nativa. 0-500 m.

Distribución: CAN, CAP.

Ejemplar de referencia: Burkart, A. 14770 (SI, US).

Paspalum glabrinode (Hack.) Morrone \& Zuloaga

Hierba. Nativa. 0-300 m.

Distribución: CAN, SIG.

Ejemplar de referencia: Rodríguez, F. M. 721 (BAA, SI, US).

Paspalum glaucescens Hack.

Hierba. Nativa. 0-500 m.

Distribución: CAN, SIG.

Ejemplar de referencia: Montes, J. E. $15363 a$ (SI, US).

Paspalum guenoarum Arechav. var. guenoarum Hierba. Nativa. 0-600 m.

Distribución: APO, CAN, CAP, CON, GSM, GUA, LNA, OBE, SIG.

Ejemplar de referencia: Morrone, O. 1917 (SI). 
Paspalum guenoarum Arechav. var. rojasii (Hack.) Parodi ex Burkart

Hierba. Nativa. 0-500 m.

Distribución: GSM.

Ejemplar de referencia: Montes, J. E. 27648 (MO).

\section{Paspalum haumanii Parodi}

Hierba. Nativa. 0-300 m.

Distribución: CAN, SIG, SPE.

Ejemplar de referencia: Zuloaga, F. O. 7213 (SI).

\section{Paspalum inaequivalve Raddi}

Hierba. Nativa. 0-500 m.

Distribución: APO, CAI, CAN, CAP, CON, ELD, GMB, GSM, GUA, IGU, MON, SIG, SJA.

Ejemplar de referencia: Morrone, O. 1896 (SI).

\section{Paspalum indecorum $\mathrm{Mez}$}

Hierba. Nativa. 0-500 m.

Distribución: APO, CAI, CAN, CAP, CON, ELD, GUA, LNA, OBE, SIG.

Ejemplar de referencia: Montes, J. E. 15327 (MO, SI).

Paspalum intermedium Munro ex Morong \& Britton

Hierba. Nativa. 0-600 m.

Distribución: APO, CAN, CAP, IGU, LNA, SIG, SPE.

Ejemplar de referencia: Cabrera, A. L. 28617 (SI).

\section{Paspalum jesuiticum Parodi $\left.{ }^{(* *}\right)$ \\ Hierba. Nativa. 0-500 m. \\ Distribución: CAN. \\ Ejemplar de referencia: Jörgensen, P. 239 (BAA, $B A B)$.}

Paspalum juergensii Hack.

Hierba. Nativa. 0-500 m.

Distribución: CAP, OBE, SPE.

Ejemplar de referencia: Deginani, N. B. 1696 (SI).

\section{Paspalum lilloi Hack. $\left.{ }^{(* *}\right)$}

Hierba. Nativa. 0-500 m.

Distribución: IGU.

Ejemplar de referencia: Zuloaga, F. O. 5226 (SI).

\section{Paspalum maculosum Trin.}

Hierba. Nativa. 0-500 m.
Distribución: CAN.

Ejemplar de referencia: Burkart, A. 14747 (SI).

Paspalum malacophyllum Trin.

Hierba. Nativa. 0-500 m.

Distribución: CAN, SIG.

Ejemplar de referencia: Hojsgaard, D. 1182 (CTES).

Paspalum mandiocanum Trin. var. mandiocanum

Hierba. Nativa. 0-700 m.

Distribución: CAI, CAN, GUA, IGU, SPE.

Ejemplar de referencia: Zuloaga, F. O. 5018 (SI).

Paspalum mandiocanum Trin. var. subaequiglume Barreto

Hierba. Nativa. 0-500 m.

Distribución: APO, CAI, CAN, ELD, GMB, GSM, GUA, MON, SIG, SPE.

Ejemplar de referencia: Tressens, S. G. 5866 (CTES, SI).

Paspalum notatum Flüggé var. notatum

Hierba. Nativa. 0-600 m.

Distribución: APO, CAN, GSM, GUA, SIG.

Ejemplar de referencia: Guaglianone, E. R. 1205 (SI).

Paspalum notatum Flüggé var. saurae Parodi Hierba. Nativa. 0-600 m.

Distribución: CAI, CAN, GUA, SIG, SJA.

Ejemplar de referencia: Biganzoli, F. 1509 (SI).

Paspalum orbiculatum Poir.

Hierba. Nativa. 0-500 m.

Distribución: CAP, GUA, SIG, SPE.

Ejemplar de referencia: Morrone, O. 1888 (SI).

Paspalum ovale Nees ex Steud.

Hierba. Nativa. 0-500 m.

Distribución: APO, CAN, CAP.

Ejemplar de referencia: Parodi, L. R. 5437 (BAA, CTES, $K, U S, W$ ).

\section{Paspalum paniculatum $\mathrm{L}$.}

Hierba. Nativa. 0-700 m.

Distribución: CAN, ELD, GMB, GUA, IGU, SIG, SPE.

Ejemplar de referencia: Deginani, N. B. 1641 (SI). 
Paspalum pauciciliatum (Parodi) Herter

Hierba. Nativa. 0-500 m.

Distribución: APO, CAI, CAN, CAP, ELD, GUA, IGU, SIG.

Ejemplar de referencia: Múlgura, M. E. 2789 (SI).

\section{Paspalum plenum Chase}

Hierba. Nativa. 0-700 m.

Distribución: CAN, SIG, SPE.

Ejemplar de referencia: Zuloaga, F. O. 5745 (SI).

\section{Paspalum plicatulum Michx.}

Hierba. Nativa. 0-700 m.

Distribución: APO, CAI, CAN, CAP, CON, ELD, GSM, IGU, OBE, SIG, SJA.

Ejemplar de referencia: Montes, J. E. 2181 (MO, SI), Zuloaga, F. O. 5696 (SI).

Paspalum polyphyllum Nees ex Trin.

Hierba. Nativa. 0-500 m.

Distribución: APO, CAN, CAP, SIG. MO).

Ejemplar de referencia: Honfi, A. I. 98 (LIL,

Paspalum quadrifarium Lam.

Hierba. Nativa. 0-500 m.

Distribución: APO, CAN, LNA.

Ejemplar de referencia: Zuloaga, F. O. 4895 (SI).

Paspalum quarinii Morrone \& Zuloaga

Hierba. Nativa. 0-500 m.

Distribución: APO, CAN, CAP, LNA, SIG, SJA.

Ejemplar de referencia: Krapovickas, A. 25693 (BAA, CTES, SI).

\section{Paspalum regnellii $\mathrm{Mez}$}

Hierba. Nativa. 0-700 m.

Distribución: APO, CAI, CAN, CAP, ELD, GMB, GSM, GUA, IGU, MAY, MON, OBE, SIG, SJA, SPE.

Ejemplar de referencia: Morrone, O. 2115 (SI).

Paspalum rufum Nees ex Steud.

Hierba. Nativa. 0-400 m.

Distribución: APO, CAN.

Ejemplar de referencia: Zuloaga, F. O. 3246 (SI).

\section{Paspalum simplex Morong}

Hierba. Perenne. Nativa. 0-500 m.

Distribución: CAI.

Ejemplar de referencia: Keller, H. A. 12762 (CTES).

Paspalum stagnophilum Morrone \& Zuloaga

Hierba. Nativa. 0-600 m.

Distribución: ELD, GMB, GSM.

Ejemplar de referencia: Burkart, A. 14547 $(B A A)$.

Paspalum stellatum Humb. \& Bonpl. ex Flüggé Hierba. Nativa. 0-600 m.

Distribución: APO, CAI, CAN, CAP, GSM, LNA, SIG, SJA.

Ejemplar de referencia: Zuloaga, F. O. 5671 (SI).

\section{Paspalum umbrosum Trin.}

Hierba. Nativa. 0-700 m.

Distribución: CAI, ELD, GUA, IGU, LNA, OBE, SIG, SJA, SPE.

Ejemplar de referencia: Morrone, O. 1079 (SI).

Paspalum urvillei Steud.

Hierba. Nativa. 0-700 m.

Distribución: APO, CAI, CAN, ELD, GMB, GSM, GUA, IGU, SIG, SJA, SPE.

Ejemplar de referencia: Honfi, A. I. 520 (CTES, G, MNES).

Paspalum usteri Hack. $\left({ }^{* * *}\right)$

Hierba. Nativa. 0-700 m.

Distribución: CAI, CAN, GSM, MON, SIG.

Ejemplar de referencia: Zuloaga, F. O. 5695 (SI).

Paspalum vaginatum $\mathrm{Sw}$.

Hierba. Nativa. 0-500 m.

Distribución: CAP.

Ejemplar de referencia: Burkart, A. 14485 (SI).

Phalaris L., nom. cons.

Phalaris angusta Nees ex Trin.

Hierba. Nativa. 0-700 m.

Distribución: APO, CAI, CAN, CAP, GSM, GUA, MON, SIG, SJA, SPE.

Ejemplar de referencia: Biganzoli, F. 268 (SI). 


\section{Pharus P. Browne}

\section{Pharus lappulaceus Aubl.}

Hierba. Nativa. 0-800 m.

Distribución: APO, CAI, CAN, ELD, GMB, GSM, GUA, IGU, LNA, MON, SIG, SPE.

Ejemplar de referencia: Deginani, N. B. 1089 (SI).

Piptochaetium J. Presl, nom. cons.

Piptochaetium lasianthum Griseb.

Hierba. Nativa. 0-500 m.

Distribución: CAN, CAP.

Ejemplar de referencia: Zuloaga, F. O. 7141 (SI).

Piptochaetium medium (Speg.) Torres

Hierba. Nativa. 0-500 m.

Distribución: APO.

Ejemplar de referencia: Burkart, A. 14248 (SI).

Piptochaetium montevidense (Spreng.) Parodi

Hierba. Nativa. 0-600 m.

Distribución: APO, CAN, GMB, OBE, SIG, SJA.

Ejemplar de referencia: Morrone, O. 1789 (SI).

\section{Piptochaetium ruprechtianum E. Desv.}

Hierba. Nativa. 0-500 m.

Distribución: SIG.

Ejemplar de referencia: Grüner, G. 1435 (BAA).

Piptochaetium stipoides (Trin. \& Rupr.) Hack. ex Arechav.

Hierba. Nativa. 0-500 m.

Distribución: APO, CAN, CAP, CON, SIG, SJA.

Ejemplar de referencia: Zuloaga, F. O. 7198 (SI).

Piptochaetium uruguense Griseb.

Hierba. Nativa. 0-500 m.

Distribución: APO, CAN, CAP, OBE, SIG.

Ejemplar de referencia: Zuloaga, F. O. 437 (SI).

\section{Poa L.}

\section{Poa annua $L$.}

Hierba. Introducida. 0-600 m.

Distribución: CAN, GUA, IGU.

Ejemplar de referencia: Llamas, A. s.n. (SI).

\section{Poa lanigera Nees}

Hierba. Nativa. 0-500 m.

Distribución: CON.

Ejemplar de referencia: Múlgura, M. E. 2056 (SI).

\section{Polypogon Desf.}

\section{Polypogon chilensis (Kunth) Pilg.}

Hierba. Nativa. 0-500 m.

Distribución: APO, CAN.

Ejemplar de referencia: Mroginski, L. 444 (BAA, CTES).

\section{Polypogon elongatus Kunth}

Hierba. Nativa. 0-600 m.

Distribución: SPE.

Ejemplar de referencia: Seijo, G. J. 842 (CTES, G, MNES).

\section{Pseudechinolaena Stapf}

Pseudechinolaena polystachya (Kunth) Stapf Hierba. Nativa. 0-700 m.

Distribución: CAI, CAN, ELD, GMB, GSM, GUA, IGU, MON, SIG, SJA, SPE.

Ejemplar de referencia: Deginani, N. B. 1254 (SI).

\section{Rugoloa Zuloaga}

\section{Rugoloa hylaeica (Mez) Zuloaga}

Hierba. Nativa. 0-500 m.

Distribución: CAN, CAP, SIG.

Ejemplar de referencia: Morrone, O. 1860 (SI).

\section{Rugoloa pilosa (Sw.) Zuloaga}

Hierba. Nativa. 0-700 m.

Distribución: CAI, CAN, CAP, ELD, GMB, GSM, GUA, IGU, MAY, MON, OBE, SIG, SJA, SPE.

Ejemplar de referencia: Biganzoli, F. 1393 (SI).

\section{Rugoloa polygonata (Schrad.) Zuloaga $\left(^{* * *}\right.$ )}

Hierba. Nativa. 0-600 m.

Distribución: GUA, IGU, SJA.

Ejemplar de referencia: Zuloaga, F. O. 6901 (SI). 
Saccharum L., nom. cons.

Saccharum angustifolium (Nees) Trin.

Hierba. Nativa. 0-500 m.

Distribución: CAN, CAP.

Ejemplar de referencia: Quarín, C. L. 3447 (CTES, SI).

Saccharum asperum (Nees) Steud.

Hierba. Nativa. 0-500 m.

Distribución: APO, CAN, CAP.

Ejemplar de referencia: Zuloaga, F. O. 4909 (SI).

Saccharum balansae (Hack.) Roberty

Hierba. Nativa. 0-500 m.

Distribución: CAN, CAP.

Ejemplar de referencia: Rodríguez, F. M. 776 (SI).

Saccharum giganteum (Walter) Pers. ${ }^{(* *}$ )

Hierba. Nativa. 0-500 m.

Distribución: CAN, OBE.

Ejemplar de referencia: Zuloaga, F. O. 6532 (SI).

Saccharum intermedium Welker \& Peichoto

Hierba. Nativa. 0-500 m.

Distribución: CAN, LNA.

Ejemplar de referencia: Peichoto, M. C. 262 (CTES).

Saccharum trinii (Hack.) Renvoize

Hierba. Nativa. 0-600 m.

Distribución: CAI, CAN, CAP, GSM, GUA, MAY, SJA, SPE.

Ejemplar de referencia: Parodi, L. R. 5672 (BAA).

\section{Sacciolepis Nash}

Sacciolepis vilvoides (Trin.) Chase

Hierba. Nativa. 0-500 m.

Distribución: APO, CAP, SJA.

Ejemplar de referencia: Biganzoli, F. 1713 (SI).

\section{Schizachyrium Nees}

Schizachyrium bimucronatum Roseng., B.R. Arrill. \& Izag.

Hierba. Nativa. 0-300 m.

Distribución: CAN, CON, SIG, SJA.

Ejemplar de referencia: Peichoto, M. C. 98 (CTES).
Schizachyrium condensatum (Kunth) Nees

Hierba. Perenne. Nativa. 0-600 m.

Distribución: APO, CAI, CAN, CAP, ELD, GMB, GSM, IGU, OBE, SIG.

Ejemplar de referencia: Biganzoli, F. 930 (SI).

\section{Schizachyrium glaziovii Peichoto}

Hierba. Nativa. 0-500 m.

Distribución: CAN, LNA, SIG.

Ejemplar de referencia: Peichoto, M. C. 100 (CTES).

Schizachyrium gracilipes (Hack.) A. Camus

Hierba perenne. Nativa. 0-500 m.

Distribución: CAP.

Ejemplar de referencia: Parodi, L. R. 5451 (BAA).

Schizachyrium hatschbachii Peichoto $\left.{ }^{* * *}\right)$

Hierba. Nativa. 0-700 m.

Distribución: GMB, SPE.

Ejemplar de referencia: Keller, H. A. 5002 (CTES).

Schizachyrium microstachyum (Desv. ex Ham.) Roseng., B.R. Arrill. \& Izag.

Hierba. Nativa. 0-500 m.

Distribución: CAN, GUA, SIG, OBE.

Ejemplar de referencia: Peichoto, M. C. 17 (CTES).

Schizachyrium salzmannii (Trin. ex Steud.) Nash var. aristatum (Hack.) Peichoto

Hierba. Nativa. 0-500 m.

Distribución: APO, CAN, SIG.

Ejemplar de referencia: Morrone, O. 1614 (SI).

Schizachyrium sanguineum (Retz.) Alston

Hierba. Nativa. 0-500 m.

Distribución: CAI, CAP, SIG.

Ejemplar de referencia: Zuloaga, F. O. 5333 (SI).

Schizachyrium scabriflorum (Rupr. ex Hack.) A. Camus

Hierba. Nativa. 0-500 m.

Distribución: APO, CON.

Ejemplar de referencia: Vanni, R. O. 4603 (CTES). 
Schizachyrium spicatum (Spreng.) Herter

Hierba. Nativa. 0-500 m.

Distribución: CAI, CAN.

Ejemplar de referencia: Morrone, O. 1737 (SI).

\section{Schizachyrium tenerum Nees}

Hierba. Nativa. 0-600 m.

Distribución: GMB, SPE.

Ejemplar de referencia: Krapovickas, A. 23343 (CTES).

Setaria P. Beauv., nom. cons.

Setaria fiebrigii R.A.W. Herrm.

Hierba. Nativa. 0-500 m.

Distribución: APO, CAI, CAN, CAP, IGU, GSM, SIG.

Ejemplar de referencia: Burkart, A. 14079 (SI).

Setaria globulifera (Steud.) Griseb.

Hierba. Nativa. 0-500 m.

Distribución: CAN, CAP.

Ejemplar de referencia: Zuloaga, F. O. 3176 (SI).

Setaria hassleri Hack.

Hierba. Nativa. 0-700 m.

Distribución: APO, CAN, CAP, LNA, OBE, SIG, SJA, SPE.

Ejemplar de referencia: Schwindt, E. 4074 (CORD, CTES, LIL).

Setaria italica (L.) P. Beauv.

Hierba. Introducida. 0-500 m.

Distribución: CAN.

Ejemplar de referencia: Hauman, L. L. s.n. (BAA).

Setaria lachnea (Nees) Kunth

Hierba. Nativa. 0-600 m.

Distribución: CAN, CAP, LNA.

Ejemplar de referencia: Zuloaga, F. O. 6758 (SI).

\section{Setaria macrostachya Kunth}

Hierba. Nativa. 0-600 m.

Distribución: CAN, CAP, GSM, SIG.

Ejemplar de referencia: Morrone, O. 1859 (SI).

\section{Setaria parodii Nicora}

Hierba. Nativa. 0-500 m.
Distribución: CAN, CON.

Ejemplar de referencia: Zuloaga, F. O. 995 (SI).

Setaria parviflora (Poir.) Kerguélen

Hierba. Nativa. 0-700 m.

Distribución: CAI, CAN, CAP, ELD, GMB, GSM, GUA, IGU, SIG, SPE.

Ejemplar de referencia: Rodríguez, M. 531 (CTES, G, MNES, SI).

Setaria paucifolia (Morong) Lindm.

Hierba. Nativa. 0-500 m.

Distribución: APO, CAN, CAP, CON, SIG, SJA.

Ejemplar de referencia: Zuloaga, F. O. 4916 (SI).

\section{Setaria rosengurtii Nicora}

Hierba. Perenne. Nativa. 0-500 m.

Distribución: APO.

Ejemplar de referencia: Cabrera, A. L. 28724 (LP, SI).

Setaria scandens Schrad. ex Schult.

Hierba. Nativa. 0-600 m.

Distribución: APO, GMB, SIG, SJA.

Ejemplar de referencia: Zuloaga, F. O. 5113 (SI).

Setaria sphacelata (Schumach.) Stapf \& C.E. Hubb. var. sericea (Stapf) Clayton

Hierba. Introducida. 0-500 m.

Distribución: CAI, CAN, ELD.

Ejemplar de referencia: Morrone, O. 649 (SI).

Setaria sulcata Raddi

Hierba. Nativa. 0-600 m.

Distribución: APO, CAN, ELD, GSM, GUA, IGU, MON, OBE, SIG, SJA, SPE.

Ejemplar de referencia: Schinini, A. 6091 (CTES).

Setaria vaginata Spreng. var. bonariensis Nicora

Hierba. Nativa. 0-300 m.

Distribución: APO.

Ejemplar de referencia: Ibarrola, T. 949 (LIL).

\section{Setaria vaginata Spreng. var. vaginata}

Hierba. Nativa. 0-500 m.

Distribución: APO, CAN, CAP.

Ejemplar de referencia: Burkart, A. 14402 (SI). 
Setaria vulpiseta (Lam.) Roem. \& Schult.

Hierba. Nativa. 0-700 m.

Distribución: APO, CAI, CAN, CAP, ELD, GMB, GSM, GUA, IGU, LNA, SIG, SJA, SPE.

Ejemplar de referencia: Morrone, O. 1086 (SI).

\section{Sorghastrum Nash}

Sorghastrum minarum (Nees) Hitchc.

Hierba. Nativa. 0-500 m.

Distribución: SIG.

Ejemplar de referencia: Zuloaga, F. O. 6812 (SI).

\section{Sorghastrum pellitum (Hack.) Parodi}

Hierba. Nativa. 0-500 m.

Distribución: APO, CAN, CON, OBE.

Ejemplar de referencia: Cabrera, A. L. 28698 (SI).

Sorghastrum setosum (Griseb.) Hitchc.

Hierba. Nativa. 0-500 m.

Distribución: CAN, SIG. (SI).

Ejemplar de referencia: Rodríguez, F. M. 208

\section{Sorghastrum stipoides (Kunth) Nash}

Hierba. Nativa. 0-500 m.

Distribución: APO.

Ejemplar de referencia: Bertoni, M. 2728 (US).

\section{Sorghastrum viride Swallen}

Hierba. Nativa. 0-500 m.

Distribución: CAN, SIG, SJA.

Ejemplar de referencia: Correa, M. N. 7010 (BAB).

\section{Sorghum Moench}

\section{Sorghum halepense (L.) Pers. var. halepense}

Hierba. Introducida. 0-600 m.

Distribución: CAN, ELD, GMB, GUA.

Ejemplar de referencia: Rodríguez, M. 578 (G, MNES, SI).

Sorghum halepense (L.) Pers. var. muticum (Hack.) Hayek

Hierba. Introducida. 0-600 m.
Distribución: CAN, CON, ELD, GMB.

Ejemplar de referencia: Deginani, N. B. 1805 (SI).

Sporobolus R. Br.

Sporobolus aeneus (Trin.) Kunth var. aeneus ${ }^{(* *}$ ) Hierba. Nativa. 0-500 m.

Distribución: SIG.

Ejemplar de referencia: Morrone, O. 1003 (SI).

Sporobolus aeneus (Trin.) Kunth var. angustifolia (Döll) S.S. Denham \& Aliscioni

Hierba. Perenne. Nativa. 0-500 m.

Distribución: SIG.

Ejemplar de referencia: Keller, H. A. 12913 (CTES).

Sporobolus indicus (L.) R. Br.

Hierba. Nativa. 0-700 m.

Distribución: APO, CAI, CAN, CAP, GMB, GSM, GUA, SIG, SJA, SPE.

Ejemplar de referencia: Morrone, O. 713 (SI).

Sporobolus monandrus Roseng., B.R. Arrill. \& Izag.

Hierba. Nativa. 0-500 m.

Distribución: APO.

Ejemplar de referencia: Cabrera, A. L. 28570 (SI).

\section{Sporobolus pseudairoides Parodi $\left({ }^{* * *}\right)$}

Hierba. Nativa. 0-600 m.

Distribución: CAN, GSM, IGU, OBE, SIG, SPE.

Ejemplar de referencia: Cabrera, A. L. 29201 (SI).

Stapfochloa H. Scholz

Stapfochloa canterae (Arechav.) P.M. Peterson Hierba. Nativa. 0-600 m.

Distribución: GSM, IGU, SIG, SPE.

Ejemplar de referencia: Biganzoli, F. 252 (SI).

Stapfochloa elata (Desv.) P.M. Peterson

Hierba. Nativa. 0-700 m.

Distribución: APO, CAI, CAN, CAP, ELD, GSM, GUA, IGU, LNA, MON, OBE, SIG, SPE.

Ejemplar de referencia: Zuloaga, F. O. 1907 (SI). 


\section{Steinchisma Raf.}

Steinchisma decipiens (Nees ex Trin.) W.V. Br. Hierba. Nativa. 0-600 m.

Distribución: APO, CAI, CAN, GUA, OBE, SPE. Ejemplar de referencia: Seijo, G. J. 796 (CTES, MNES).

\section{Steinchisma hians (Elliott) Nash}

Hierba. Nativa. 0-600 m.

Distribución: APO, CAI, CAN, CAP, GMB, GSM, GUA, LNA, MON, OBE, SIG, SPE.

Ejemplar de referencia: Deginani, N. B. 966 (SI).

Steinchisma laxa (Sw.) Zuloaga

Hierba. Nativa. 0-700 m.

Distribución: APO, CAI, CAN, CAP, CON, ELD, GSM, GUA, IGU, LNA, MON, OBE, SIG, SJA, SPE.

Ejemplar de referencia: Deginani, N. B. 1106 (SI).

\section{Steinchisma spathellosa (Döll) Renvoize}

Hierba. Nativa. 0-600 m.

Distribución: APO, CAN, ELD, GMB, GSM, GUA, IGU, LNA, MON, OBE, SIG, SJA, SPE.

Ejemplar de referencia: Zuloaga, F. O. 493 (LP, SI).

\section{Stephostachys Zuloaga \& Morrone}

Stephostachys mertensii (Roth) Zuloaga \& Morrone

Hierba. Nativa. 0-500 m.

Distribución: CAP, SIG.

Ejemplar de referencia: Morrone, O. 1852 (SI).

\section{Streptochaeta Schrad.}

Streptochaeta spicata Schrad. ex Nees $\left(^{* * *}\right)$

Hierba. Nativa.

Distribución: en selvas del norte de la provincia, probablemente extinta en el país.

Ejemplar de referencia: Solomon, A. 6864 (SI).

\section{Taquara I.L..C. Oliveira \& R.P. Oliveira}

Taquara micrantha (Kunth) I.L.C. Oliveira \& R.P. Oliveira $\left.{ }^{* * *}\right)$

Hierba. Perenne. Nativa. 0-500 m.
Distribución: GMB, SPE.

Ejemplar de referencia: Renvoize, S. A. 3232 (K, SI).

\section{Trachypogon Nees}

Trachypogon spicatus (L. f.) Kuntze

Hierba. Nativa. 0-600 m.

Distribución: CAI, CAN, GSM, SIG.

Ejemplar de referencia: Biganzoli, F. 286 (SI).

\section{Trichanthecium Zuloaga \& Morrone}

Trichanthecium caaguazuense (Henrard)

Zuloaga \& Morrone $\left.{ }^{* * *}\right)$

Hierba. Nativa. 0-500 m.

Distribución: CAP, GMB.

Ejemplar de referencia: Parodi, L. R. 5459 (BAA).

Trichanthecium parvifolium (Lam.) Zuloaga \& Morrone

Hierba. Nativa. 0-600 m.

Distribución: GUA, SIG, SJA.

Ejemplar de referencia: Burkart, A. 15465 (SI).

Trichanthecium schwackeanum (Mez)

Zuloaga \& Morrone

Hierba. Nativa. 0-600 m.

Distribución: APO, CAI, CAN, CAP, GMB, IGU, SIG, SJA, SPE.

Ejemplar de referencia: Parodi, L. R. 5613 (BAA, US).

Tridens Roem. \& Schult.

Tridens brasiliensis (Nees ex Steud.) Parodi

Hierba. Nativa. 0-500 m.

Distribución: APO, CAI, CAN, CAP, CON, ELD, MON, OBE, SIG.

Ejemplar de referencia: Biganzoli, F. 329 (SI).

Tripogon Roem. \& Schult.

Tripogon ekmanii Nicora \& Rúgolo ${ }^{* * *}$ )

Hierba. Nativa. 0-500 m.

Distribución: CAI, CAN, CAP.

Ejemplar de referencia: Ekman, E. L. 703 (BAA). 
Tripogonella P.M. Peterson \& Romasch.

Tripogonella spicata (Nees) P.M. Peterson \& Romasch.

Hierba. Nativa. 0-500 m.

Distribución: APO, CAN, CAP, LNA.

Ejemplar de referencia: Morrone, O. 2235 (SI).

\section{Urochloa P. Beauv.}

Urochloa arrecta (Hack. ex T. Durand \& Schinz) Morrone \& Zuloaga

Hierba. Introducida. 0-500 m.

Distribución: GMB.

Ejemplar de referencia: Morrone, O. 2057 (SI).

Urochloa brizantha (Hochst. ex A. Rich.) R.D. Webster

Hierba. Introducida. 0-600 m.

Distribución: CAN, GMB.

Ejemplar de referencia: Morrone, O. 2066 (SI).

Urochloa decumbens (Stapf) R.D. Webster

Hierba. Introducida. 0-500 m.

Distribución: GMB, GUA.

Ejemplar de referencia: Biganzoli, F. 1528 (SI).

Urochloa mutica (Forssk.) T.Q. Nguyen

Hierba. Perenne. Introducida. 0-600 m.

Distribución: CAN, ELD, SPE.

Ejemplar de referencia: Zuloaga, F. O. 7007 (SI).

Urochloa plantaginea (Link) R.D. Webster Hierba. Nativa. 0-500 m.

Distribución: APO, CAN, GUA, LNA, SIG.

Ejemplar de referencia: Deginani, N. B. 1046 (SI).

\section{PODOSTEMACEAE}

\section{Apinagia Tul.}

Apinagia riedelii (Bong.) Tul. ${ }^{* * *}$ )

Hierba acuática. Anual. 0-600 m.

Distribución: IGU, OBE, SIG.

Ejemplar de referencia: Guaglianone, E. R. 1060 (SI).
Marathrum Bonpl.

Marathrum azarensis $\operatorname{Tur}\left({ }^{* * *}\right)$

Hierba acuática. Nativa. 0-300 m.

Distribución: APO.

Ejemplar de referencia: Tur, N. M. 2104 (LP, MEXU, SI).

Mourera Aubl.

Mourera aspera (Bong.) Tul.

Hierba acuática. Nativa. 0-600 m.

Distribución: IGU.

Ejemplar de referencia: Tur, N. M. 2036 (LP, SI).

Podostemum Michx.

Podostemum comatum Hicken

Hierba acuática. Nativa. 0-600 m.

Distribución: APO, GMB, GUA, IGU, SJA.

Ejemplar de referencia: Cabrera, A. L. 58 (CTES, $L P)$.

Podostemum distichum (Cham.) Wedd.

Hierba acuática. Nativa. 0-700 m.

Distribución: APO, GMB, GSM, IGU.

Ejemplar de referencia: Tur, N. M. 1869 (LP, STL).

Podostemum muelleri Warm.

Hierba acuática. Nativa. 0-800 m.

Distribución: CAI, ELD, GSM, GUA, IGU, SIG, SPE.

Ejemplar de referencia: Zuloaga, F. O. 561 (LP, SI).

Podostemum rutifolium Warm.

Hierba acuática. Nativa. 0-700 m.

Distribución: GMB, IGU, SJA.

Ejemplar de referencia: Tur, N. M. 2025 (LP, SI).

Tristicha Thouars, nom. cons.

Tristicha trifaria (Bory ex Willd.) Spreng.

Hierba acuática. Nativa. 0-700 m.

Distribución: APO, CAI, ELD, GMB, GSM, GUA, IGU, SIG, SJA.

Ejemplar de referencia: Tur, N. M. 2045 (SI). 


\section{POLYGALACEAE}

\section{Acanthocladus Klotzsch}

Acanthocladus brasiliensis (A. St.-Hil. \& Moq.) Klotzsch ex Hassk. $\left(^{*}\right)$

Arbolito. Nativa. 0-500 m.

Distribución: IGU.

Ejemplar de referencia: Keller, H. A. 13874 (CTES).

\section{Asemeia Raf.}

Asemeia extraaxillaris (Chodat) J.F.B. Pastore \& J.R. Abbott

Hierba. Nativa. 0-500 m.

Distribución: CAN, CAP, SIG.

Ejemplar de referencia: Parodi, L. R. 4535 (BAA).

\section{Monnina Ruiz \& Pav.}

Monnina oblongifolia Arechav.

Hierba. Nativa. 0-500 m.

Distribución: CAN, SIG, SPE.

Ejemplar de referencia: Montes, J. E. 7000 (SI).

Monnina resedoides A. St.-Hil.

Hierba. Nativa. 0-500 m.

Distribución: APO, CAI, CAN, CAP, SIG, SJA.

Ejemplar de referencia: Biganzoli, F. 181 (SI).

\section{Monnina tristaniana A. St.-Hil.}

Hierba. Nativa. 0-500 m.

Distribución: APO.

Ejemplar de referencia: Zuloaga, F. O. 7152 (SI).

\section{Polygala L.}

Polygala adenophylla A. St.-Hil. \& Moq.

Hierba subleñosa. Nativa. 0-500 m.

Distribución: APO, CAN, CAP, SPE. (SI).

Ejemplar de referencia: Cabrera, A. L. 28713

\section{Polygala aspalatha $\mathrm{L}$.}

Hierba. Nativa. 0-500 m.
Distribución: CAN, SIG.

Ejemplar de referencia: Schwarz, G. J. 1169 (LIL).

Polygala bonariensis Grondona

Hierba. Nativa. 0-500 m.

Distribución: CAN, CAP.

Ejemplar de referencia: Zuloaga, F. O. 6550 (SI).

\section{Polygala brasiliensis L.}

Hierba. Nativa. 0-500 m.

Distribución: CAN.

Ejemplar de referencia: Grüner, G. 1449 (LP).

Polygala duarteana A. St.-Hil. \& Moq.

Hierba. Nativa. 0-500 m.

Distribución: APO, CAN, CAP, CON, SIG, SPE. Ejemplar de referencia: Burkart, A. 15515 (SI).

Polygala lancifolia A. St.-Hil. \& Moq. $\left.{ }^{* * *}\right)$

Hierba subleñosa. Nativa. 0-800 m.

Distribución: GMB, IGU, OBE.

Ejemplar de referencia: Keller, H. A. 66 (CTES, SI).

Polygala leptocaulis Torr. \& A. Gray

Hierba. Nativa. 0-600 m.

Distribución: APO, CAP, SJA.

Ejemplar de referencia: Burkart, A. 14324 (SI).

Polygala linoides Poir. var. laxiflora Grondona $(* *)$

Hierba. Endémica. 0-500 m.

Distribución: CAN, GUA, IGU, SIG.

Ejemplar de referencia: Lourteig, A. 1109 (LIL).

\section{Polygala linoides Poir. var. linoides}

Hierba. Nativa. 0-500 m.

Distribución: APO, CAN, CAP.

Ejemplar de referencia: Burkart, A. 14441 (SI).

Polygala molluginifolia A. St.-Hil. \& Moq.

Hierba. Nativa. 0-600 m.

Distribución: APO, CAI, CAN, CAP, CON, SIG, SJA.

Ejemplar de referencia: Morrone, O. 1115 (SI).

Polygala obovata A. St.-Hil. \& Moq.

Hierba subleñosa. Nativa. 0-500 m.

Distribución: CAN, CAP, MON, SIG.

Ejemplar de referencia: Biganzoli, F. 25 (SI). 
Polygala pulchella A. St.-Hil. \& Moq.

Hierba. Nativa. 0-700 m.

Distribución: APO, CAN, CAP, CON, IGU, OBE, SIG, SJA.

Ejemplar de referencia: Múlgura, M. E. 581 (SI).

\section{Polygala punctata A.W. Benn.}

Hierba. Nativa. 0-500 m.

Distribución: APO, CAI, CAN, CAP, SIG.

Ejemplar de referencia: Biganzoli, F. 183 (SI).

\section{Polygala rojasii Chodat}

Hierba. Nativa. 0-500 m.

Distribución: CAP, SJA.

Ejemplar de referencia: Molfino, J. F. s.n. (BAF).

\section{Polygala timoutoides Chodat}

Hierba. Nativa. 0-500 m.

Distribución: CAN, CAP, SIG.

Ejemplar de referencia: Grondona, E. M. 1633 $(B A B)$.

\section{Securidaca L.}

Securidaca rivinifolia A. St.-Hil. \& Moq. $\left.{ }^{(* * *}\right)$

Arbusto. Nativa. 0-700 m.

Distribución: IGU.

Ejemplar de referencia: Keller, H. A. 13750 (CTES).

\section{POLYGONACEAE}

\section{Coccoloba P. Browne}

\section{Coccoloba argentinensis Speg.}

Arbusto o árbol. Nativa. 0-500 m.

Distribución: ELD, MON.

Ejemplar de referencia: Keller, H. A. 5888 (CTES).

Muehlenbeckia Meisn., nom. cons.

Muehlenbeckia sagittifolia (Ortega) Meisn.

Arbusto. Nativa. 0-500 m.

Distribución: ELD, SIG.

Ejemplar de referencia: Cuadrado, G. 220 (CTES).
Polygonum L., nom. cons.

Polygonum acuminatum Kunth

Hierba. Nativa. 0-700 m.

Distribución: GMB, GUA, IGU, SPE.

Ejemplar de referencia: Deginani, N. B. 1602 (SI).

\section{Polygonum aviculare $\mathrm{L}$.}

Hierba. Introducida. 0-500 m.

Distribución: CAP.

Ejemplar de referencia: Grondona, E. M. 1149 $(B A B)$.

Polygonum convolvulus $\mathrm{L}$.

Hierba. Introducida. 0-500 m.

Distribución: CAN, CAP.

Ejemplar de referencia: Grondona, E. M. 1201 (BAB).

Polygonum ferrugineum Wedd.

Hierba. Nativa.

Distribución: Desconocida.

Ejemplar de referencia: Rodríguez, F. M. 266 (BA, BAB).

Polygonum hydropiperoides Michx. var. hydropiperoides

Hierba. Nativa. 0-800 m.

Distribución: CAI, CAN, CAP, GMB, LNA, SPE.

Ejemplar de referencia: Morrone, O. 1346 (SI).

Polygonum hydropiperoides Michx. var. setaceum (Baldwin ex Elliott) Gleason

Hierba. Nativa. 0-500 m.

Distribución: CAP, CON, GMB, GUA.

Ejemplar de referencia: Guaglianone, E. R. 2959 (SI).

\section{Polygonum lapathifolium $\mathrm{L}$.}

Hierba. Perenne. Introducida. 0-700 m.

Distribución: SPE.

Ejemplar de referencia: Belgrano, M. J. 411 (SI, WIS).

Polygonum meisnerianum Cham. \& Schltdl. Hierba. Nativa. 0-700 m.

Distribución: APO, CAN, GMB, IGU, SIG, SPE. Ejemplar de referencia: Deginani, N. B. 1332 (SI). 
Polygonum minus Huds.

Hierba. Introducida. 0-500 m.

Distribución: CAP.

Ejemplar de referencia: Gerling, G. F. 65 (BAB).

\section{Polygonum persicaria $\mathrm{L}$.}

Hierba. Introducida. 0-800 m.

Distribución: IGU, OBE.

Ejemplar de referencia: Morrone, O. 620 (SI).

\section{Polygonum punctatum Elliott}

Hierba. Nativa. 0-800 m.

Distribución: APO, CAI, CAN, CAP, CON, ELD, GMB, GSM, GUA, IGU, LNA, OBE, SIG, SPE.

Ejemplar de referencia: Biganzoli, F. 409 (SI).

\section{Polygonum stelligerum Cham.}

Hierba. Nativa. 0-500 m.

Distribución: CAN, SIG.

Ejemplar de referencia: Keller, H. A. 10655 (CTES).

\section{Rumex L.}

\section{Rumex crispus L.}

Hierba. Introducida. 0-500 m.

Distribución: SPE.

Ejemplar de referencia: Seijo, G. J. 830 (MNES).

\section{Rumex obovatus Danser}

Hierba. Nativa. 0-500 m.

Distribución: MON.

Ejemplar de referencia: Vanni, R. O. 3833 (CTES).

\section{Rumex obtusifolius L.}

Hierba. Introducida. 0-700 m.

Distribución: GMB, SPE.

Ejemplar de referencia: Múlgura, M. E. 2667 (SI).

\section{Ruprechtia C.A. Mey.}

\section{Ruprechtia brachysepala Meisn.}

Árbol. Nativa.

Distribución: Desconocida.

Ejemplar de referencia: Hauman, L. L. 24-641 (BA).

Ruprechtia laxiflora Meisn.

Árbol. Nativa. 0-800 m.
Distribución: CAN, ELD, GMB, GUA, IGU, MON, OBE, SIG.

Ejemplar de referencia: Schwarz, G. J. 5104 (LIL).

Ruprechtia salicifolia (Cham. \& Schltdl.) C.A.Mey.

Arbusto o árbol. Nativa. 0-100 m.

Distribución: CON, SJA.

Ejemplar de referencia: Schulz, A. G. 7098 (LIL).

\section{PONTEDERIACEAE}

Heteranthera Ruiz \& Pav.

Heteranthera reniformis Ruiz \& Pav.

Hierba palustre. Nativa. 0-500 m.

Distribución: CAN, CAP, GUA, SIG.

Ejemplar de referencia: Múlgura, M. E. 1938 (LP, SI).

Heteranthera zosterifolia Mart.

Hierba acuática. Nativa. 0-700 m.

Distribución: GMB, GUA, SPE.

Ejemplar de referencia: Múlgura, M. E. 1856 (SI).

\section{Pontederia L.}

\section{Pontederia azurea Sw.}

Hierba acuática. Nativa. 0-500 m.

Distribución: CAN, CAP, SIG.

Ejemplar de referencia: Keller, H. A. 10683 (CTES).

\section{Pontederia cordata L.}

Hierba palustre. Nativa. 0-700 m.

Distribución: APO, CON, GMB, IGU, SIG.

Ejemplar de referencia: Cabrera, A. 28675 (SI).

Pontederia crassipes Mart.

Hierba acuática. Nativa. 0-500 m.

Distribución: CAN, CAP.

Ejemplar de referencia: Jörgensen, P. 672 (BAB).

\section{Pontederia rotundifolia L. f.}

Hierba palustre. Nativa. 0-500 m.

Distribución: CAP.

Ejemplar de referencia: Spegazzini, C. L.s.n. (BAB). 
Pontederia subovata (Seub.) Lowden

Hierba acuática. Nativa. 0-500 m.

Distribución: CAP.

Ejemplar de referencia: Molfino, J. F. s.n. (BAF).

\section{PORTULACACEAE}

\section{Portulaca L.}

\section{Portulaca amilis Speg.}

Hierba. Nativa. 0-500 m.

Distribución: CAP, SIG, SJA.

Ejemplar de referencia: Morrone, O. 1908 (SI).

\section{Portulaca cryptopetala Speg.}

Hierba. Nativa. 0-700 m.

Distribución: CAI, CAN, ELD, SIG.

Ejemplar de referencia: Morrone, O. 640 (SI).

\section{Portulaca oleracea L.}

Hierba. Introducida. 0-600 m.

Distribución: CAN, GUA, SIG. (SI).

Ejemplar de referencia: Deginani, N. B. 1441

Portulaca papulifera D. Legrand $\left({ }^{* * *}\right)$

Hierba. Nativa. 0-300 m.

Distribución: APO, CAN.

Ejemplar de referencia: Zuloaga, F. O. 6573 (SI).

\section{Portulaca papulosa Schltdl.}

Hierba. Nativa. 0-500 m.

Distribución: SIG.

Ejemplar de referencia: Múlgura, M. E. 1733 (SI).

\section{POTAMOGETONACEAE}

\section{Potamogeton L.}

Potamogeton polygonus Cham. \& Schltdl.

Hierba acuática. Nativa. 0-500 m.

Distribución: GMB, MON.

Ejemplar de referencia: Renvoize, S. A. 3188 (SI).

\section{PRIMULACEAE}

\section{Anagallis L.}

\section{Anagallis arvensis $\mathrm{L}$.}

Hierba. Introducida. 0-600 m.

Distribución: GUA, MON, SPE.

Ejemplar de referencia: Keller, H. A. 131 (CTES).

\section{Anagallis pumila $S w$.}

Hierba. Nativa. 0-500 m.

Distribución: CAP.

Ejemplar de referencia: Spegazzini, C. L. s.n. (BAB).

\section{Centunculus L.}

\section{Centunculus minimus L.}

Hierba. Introducida. 0-700 m.

Distribución: APO, CON, ELD, IGU, SIG.

Ejemplar de referencia: Vanni, R. O. 3465 (CTES).

\section{Lysimachia L.}

Lysimachia filiformis (Cham. \& Schltdl.) U. Manns \& Anderb. $\left.{ }^{* * *}\right)$

Hierba. Nativa. 0-700 m.

Distribución: GUA, IGU.

Ejemplar de referencia: Keller, H. A. 1464 (CTES).

\section{Myrsine L.}

Myrsine balansae (Mez) Otegui $\left(^{* * *}\right)$

Árbol. Nativa. 0-800 m.

Distribución: CAN, GMB, GSM, GUA, IGU, MON, OBE, SIG, SPE.

Ejemplar de referencia: Burkart, A. 15363 (SI).

Myrsine coriacea (Sw.) R. Br.

Árbol. Nativa. 0-800 m.

Distribución: CAN, GMB, GUA, SIG, SPE.

Ejemplar de referencia: Gamerro, J. C. 170 (SI).

Myrsine laetevirens (Mez) Arechav.

Árbol. Nativa. 0-700 m. 
Distribución: CAN, CAP, CON, ELD, GMB, GSM, IGU, MAY, OBE, SIG, SJA.

Ejemplar de referencia: Morrone, O. 1151 (SI).

Myrsine loefgrenii (Mez) Otegui

Arbusto. Nativa. 0-500 m.

Distribución: CAN, GMB, GUA, IGU, SIG, SPE.

Ejemplar de referencia: Deginani, N. B. 1336 (SI).

\section{Myrsine parvula (Mez) Otegui}

Árbol. Nativa. 0-500 m.

Distribución: APO, CAP, IGU, SPE.

Ejemplar de referencia: Dematteis, M. 91 (CTES, G, MNES).

Myrsine umbellata Mart. (***)

Árbol. Nativa. 0-500 m.

Distribución: CAN, CAP, GMB, GUA, IGU, LNA, OBE, SIG, SJA, SPE.

Ejemplar de referencia: Morrone, O. 1647 (SI).

\section{Pelletiera A. St.-Hil.}

Pelletiera verna A. St.-Hil.

Hierba. Nativa. 0-500 m.

Distribución: CAN.

Ejemplar de referencia: Montes, J. E. 2170 (SI).

\section{PROTEACEAE}

\section{Roupala Aubl.}

Roupala montana Aubl. var. brasiliensis (Klotzsch) K.S. Edwards Árbol. Nativa. 0-600 m. Distribución: GMB, GUA, IGU.

Ejemplar de referencia: Rojas, T. 4420 (BAB, BAF).

\section{QUILLAJACEAE}

Quillaja Molina

Quillaja brasiliensis (A. St.-Hil. \& Tul.) Mart. Árbol. Nativa. 0-700 m.
Distribución: CAN, GUA, LNA.

Ejemplar de referencia: Romanczuk, M. C. 752 (SI).

\section{RANUNCULACEAE}

Anemone L., nom. cons.

\section{Anemone decapetala Ard. var. decapetala}

Hierba. Nativa. 0-500 m.

Distribución: CAN, SJA.

Ejemplar de referencia: Montes, J. E. 2159 (SI).

Anemone decapetala Ard. var. foliolosa Eichler

Hierba. Nativa. 0-500 m.

Distribución: CAI, CAN.

Ejemplar de referencia: Biganzoli, F. 170 (SI).

\section{Clematis L.}

Clematis bonariensis Juss. ex DC.

Liana. Nativa. 0-700 m.

Distribución: APO, CAN, SPE.

Ejemplar de referencia: Morrone, O. 1120 (SI).

Clematis campestris A. St.-Hil. $\left.{ }^{* * *}\right)$

Liana. Nativa. 0-500 m.

Distribución: CAN.

Ejemplar de referencia: Ekman, E. L. 1444 (G, S).

Clematis dioica L. var. australis Eichler

Liana. Nativa. 0-600 m.

Distribución: CAI, CAN, GSM, GUA, IGU, SIG.

Ejemplar de referencia: Deginani, N. B. 1274 (SI).

Clematis dioica L. var. brasiliana (DC.) Eichler $\left({ }^{* * *}\right)$

Liana. Nativa. 0-500 m.

Distribución: SJA.

Ejemplar de referencia: Molfino, J. F. s.n. (BA, G, P).

\section{Clematis montevidensis Spreng.}

Liana. Nativa. 0-600 m.

Distribución: CAN, SPE.

Ejemplar de referencia: Deginani, N. B. 1340 (SI). 


\section{Ranunculus L.}

Ranunculus bonariensis Poir. var. bonariensis Hierba. Nativa. 0-500 m.

Distribución: APO, CAN, CAP.

Ejemplar de referencia: Burkart, A. 14135 (SI).

Ranunculus bonariensis Poir. var. phyteumifolius (A. St.-Hil.) Hicken ex Lourteig

Hierba. Nativa. 0-500 m.

Distribución: CAP, IGU, SPE.

Ejemplar de referencia: Bertoni, M. 1788 (LIL).

Ranunculus bonariensis Poir. var. trisepalus (Gillies ex Hook. \& Arn.) Lourteig

Hierba. Nativa. 0-500 m.

Distribución: CAN.

Ejemplar de referencia: Múlgura, M. E. 2232 (SI).

\section{Ranunculus flagelliformis $\mathrm{Sm}$.}

Hierba. Nativa. 0-700 m.

Distribución: APO, CAN, GUA. (SI).

Ejemplar de referencia: Cabrera, A. L. 28496

\section{Ranunculus muricatus $\mathrm{L}$.}

Hierba. Introducida. 0-600 m.

Distribución: CAP, SJA, SPE.

Ejemplar de referencia: Morrone, O. 1510 (SI).

\section{Ranunculus platensis Spreng.}

Hierba. Anual. Nativa. 0-500 m.

Distribución: ELD, MON.

Ejemplar de referencia: Keller, H. A. 1966 (CTES).

\section{RHAMNACEAE}

\section{Colubrina Brongn.}

Colubrina retusa (Pittier) R.S. Cowan var. latifolia (Reissek) M.C. Johnst.

Arbusto o árbol. Nativa. 0-500 m.

Distribución: CAN, CAP, ELD, GSM, SIG.

Ejemplar de referencia: Burkart, A. 14008 (SI).

\section{Crumenaria Mart.}

Crumenaria lilloi Suess.

Hierba. Nativa. 0-500 m.

Distribución: CAN, SIG.

Ejemplar de referencia: Múlgura, M. E. 2191 (SI).

\section{Gouania Jacq.}

Gouania latifolia Reissek

Liana o epífita. Nativa. 0-500 m.

Distribución: APO, CAI, CAN, CON, ELD, GMB, IGU, LNA, MON.

Ejemplar de referencia: Zuloaga, F. O. 5397 (SI).

Gouania lupuloides (L.) Urb. $\left.{ }^{(* *}\right)$

Liana. Nativa. 0-500 m.

Distribución: GUA, IGU.

Ejemplar de referencia: Vanni, R. O. 2765 (CTES).

Gouania ulmifolia Hook. \& Arn.

Liana o epífita.Nativa. 0-600 m.

Distribución: ELD, GMB, GUA, IGU, OBE, SIG, SPE.

Ejemplar de referencia: Deginani, N. B. 1026 (SI).

\section{Hovenia Thunb.}

Hovenia dulcis Thunb.

Árbol. Introducida. 0-500 m.

Distribución: CAI, CAN, ELD, IGU, SIG, SPE.

Ejemplar de referencia: Morrone, O. 1518 (SI).

\section{Sageretia Brongn.}

Sageretia lehmanii (Hieron.) Radlk.

Arbusto o árbol. Nativa. 0-500 m.

Distribución: CAN, CON, GSM, IGU, OBE, SIG.

Ejemplar de referencia: Montes, J. E. 15079 (SI).

Scutia (Comm. ex DC.) Brongn.

\section{Scutia buxifolia Reissek}

Arbusto o árbol. Nativa. 0-500 m.

Distribución: CAN.

Ejemplar de referencia: Keller, H. A. 13852 (CTES). 


\section{ROSACEAE}

Acaena Mutis ex L.

Acaena eupatoria Cham. \& Schltdl. $\left.{ }^{* * *}\right)$

Hierba. Nativa. 0-500 m.

Distribución: GMB.

Ejemplar de referencia: Bernardi, M. 18819 (NY).

\section{Agrimonia L.}

\section{Agrimonia parviflora Sol.}

Hierba. Introducida. $500 \mathrm{~m}$.

Distribución: GMB.

Ejemplar de referencia: Guaglianone, E. R. 1121 (SI).

Agrimonia villosa Cham. \& Schltdl. $\left.{ }^{* * *}\right)$

Hierba. Nativa. 0-500 m.

Distribución: GMB.

Ejemplar de referencia: Keller, H. A. 3788 (CTES).

\section{Aphanes L.}

\section{Aphanes arvensis L.}

Hierba. Introducida. 0-700 m.

Distribución: GMB.

Ejemplar de referencia: Keller, H. A. 11718 (CTES).

\section{Eriobotrya Lindl.}

Eriobotrya japonica (Thunb.) Lindl. (***)

Arbusto o árbol. Introducida. 0-500 m.

Distribución: CAN, ELD, GSM, IGU, MON, SIG, SPE.

Ejemplar de referencia: Keller, H. A. 8708 (CTES).

\section{Geum L.}

Geum boliviense Focke

Hierba. Perenne. Nativa. 0-500 m.

Distribución: CAP, SIG.

Ejemplar de referencia: Keller, H. A. 4134 (CTES).

\section{Prunus L.}

Prunus brasiliensis (Cham. \& Schltdl.) D. Dietr. $(* * *)$

Arbusto o árbol. Nativa. 0-500 m.

Distribución: APO, CAI, CAN, CAP, ELD, GUA, IGU, OBE, SIG, SPE. (SI).

Ejemplar de referencia: Hunziker, J. H. 11901

Prunus chamissoana Koehne $\left.{ }^{* * *}\right)$

Árbol. Nativa. 0-500 m.

Distribución: IGU.

Ejemplar de referencia: Biloni, A. 59 (SI).

Prunus persica (L.) Batsch

Arbusto o árbol. Introducida. 0-600 m.

Distribución: GMB, GUA.

Ejemplar de referencia: Keller, H. A. 2126 (CTES).

Prunus subcoriacea (Chodat $\&$ Hassl.) Koehne Árbol. Nativa. 0-700 m.

Distribución: CAP, IGU, LNA.

Ejemplar de referencia: Schinini, A. 25444 (CTES, SI).

\section{Rubus L.}

Rubus brasiliensis Mart. (***)

Arbusto. Nativa. 0-700 m.

Distribución: CAI, GMB, SPE.

Ejemplar de referencia: Keller, H. A. 3543 (CTES).

\section{Rubus erythroclados Mart. ( $\left.{ }^{* * *}\right)$}

Arbusto o subarbusto. Nativa. 300-700 m.

Distribución: CAI, GMB.

Ejemplar de referencia: Biganzoli, F. 914 (SI).

Rubus imperialis Cham. \& Schltdl.

Arbusto o subarbusto. Nativa. 0-700 m.

Distribución: GUA, SPE.

Ejemplar de referencia: Guillén, R. 170 (MNES, SI).

\section{Rubus rosifolius Baker}

Arbusto. Introducida. 0-500 m.

Distribución: CAI, ELD, IGU, MON.

Ejemplar de referencia: Biganzoli, F. 576 (SI). 
Rubus schottii Pohl ex Focke $\left(^{* *}\right)$

Arbusto. Nativa. 0-500 m.

Distribución: GUA.

Ejemplar de referencia: Tressens, S. G. 6743 (CTES).

Rubus sellowii Cham. \& Schltdl.

Arbusto subarbusto. Nativa. 0-500 m.

Distribución: ELD, GUA, SPE.

Ejemplar de referencia: Tressens, S. G. 4931 (CTES).

\section{Rubus ulmifolius Schott}

Arbusto. Introducida. 0-500 m.

Distribución: CAN, GUA.

Ejemplar de referencia: Deginani, N. B. 1247 (SI).

\section{Rubus urticifolius Poir.}

Arbusto. Nativa. 0-500 m.

Distribución: ELD, GUA, IGU, SPE.

Ejemplar de referencia: Zuloaga, F. O. 6998 (SI).

\section{RUBIACEAE}

\section{Borreria G. Mey.}

Borreria brachystemonoides Cham. \& Schltdl. Hierba o subarbusto. Nativa. 0-500 m.

Distribución: CON, ELD.

Ejemplar de referencia: Tressens, S. G. 5566 (CTES).

Borreria dasycephala (Cham. \& Schltdl.) Bacigalupo \& E.L. Cabral

Hierba. Anual. Nativa. 0-500 m.

Distribución: GUA.

Ejemplar de referencia: Keller, H. A. 13034 (CTES).

Borreria krapocarmeniana E.L. Cabral \& L. Miguel

Hierba. Nativa. 0-700 m.

Distribución: CAN, CAP, GUA, IGU, SPE.

Ejemplar de referencia: Schwindt, E. 2384 (CTES).

\section{Borreria loretiana E.L. Cabral (**)}

Subarbusto. Endémica. 0-500 m.

Distribución: APO, CAN, CON, GSM, OBE, SIG.

Ejemplar de referencia: Morrone, O. 2213 (SI).
Borreria marticrovettiana E.L. Cabral $\left({ }^{* * *}\right)$

Subarbusto. Nativa. 0-500 m.

Distribución: CAN, SIG.

Ejemplar de referencia: Schinini, A. 36453 (CTES, SI).

Borreria nelidae E.L. Cabral

Hierba. Nativa. 0-500 m.

Distribución: APO, CON, SJA.

Ejemplar de referencia: Keller, H. A. 13098 (CTES).

Borreria orientalis E.L. Cabral, R. Salas \& L. Miguel (***)

Subarbusto. Nativa. 0-500 m.

Distribución: IGU, SIG.

Ejemplar de referencia: Vanni, R. O. 3467

(CTES, F, MBM).

Borreria poaya (A. St.-Hil.) DC. $\left(^{* * *}\right)$

Hierba o arbusto. Nativa. 0-500 m.

Distribución: CAN, SIG.

Ejemplar de referencia: Morrone, O. 1001 (SI).

\section{Borreria quadrifaria E.L. Cabral}

Hierba. Nativa. 0-500 m.

Distribución: IGU.

Ejemplar de referencia: Zuloaga, F. O. 5235 (SI).

Borreria schumannii (Standl. ex Bacigalupo) E.L. Cabral \& Sobrado

Subarbusto. Nativa. 0-600 m.

Distribución: CAN, CAP, GUA, IGU, SIG, SPE.

Ejemplar de referencia: Schwindt, E. 1483 (CTES).

Borreria spinosa Cham. \& Schltdl. ex DC.

Hierba. Nativa. 0-800 m.

Distribución: GUA.

Ejemplar de referencia: Keller, H. A. 353 (CTES).

Borreria tenella (Kunth) Cham. \& Schltdl.

Hierba o subarbusto. Nativa. 0-600 m.

Distribución: CAI, CAN, CAP, CON, SIG.

Ejemplar de referencia: Biganzoli, F. 372 (SI).

Borreria valens Standl. $\left({ }^{* * *}\right)$

Subarbusto. Nativa. 0-500 m.

Distribución: ELD, IGU, SIG.

Ejemplar de referencia: Cabrera, A. L. 47 (CTES, LP, SI). 


\section{Cephalanthus L.}

Cephalanthus glabratus (Spreng.) K. Schum.

Arbusto. Nativa. 0-500 m.

Distribución: IGU, SJA, SIG, SPE.

Ejemplardereferencia:Seijo, G. J. 849 (CTES, G, MNES).

Chiococca P. Browne

Chiococca alba (L.) C.L. Hitchc.

Arbusto o liana. Nativa. 0-500 m.

Distribución: CAI, CAN, GSM, IGU, MON, SIG, SPE.

Ejemplar de referencia: Zuloaga, F. O. 6578 (SI).

\section{Chomelia Jacq.}

Chomelia obtusa Cham. \& Schltdl.

Arbusto o árbol. Nativa. 0-500 m.

Distribución: APO, CAI, CAN, CAP, CON, GMB, GUA, IGU, LNA, MAY, MON, SIG, SJA, SPE.

Ejemplar de referencia: Zuloaga, F. O. 4979 (SI).

\section{Coccocypselum P. Browne}

\section{Coccocypselum hasslerianum Chodat $\left.{ }^{* * *}\right)$}

Hierba. Nativa. 0-500 m.

Distribución: GMB, GUA, IGU, SPE.

Ejemplar de referencia: Zuloaga, F. O. 6487 (SI).

Coccocypselum lanceolatum (Ruiz \& Pav.) Pers.

Hierba. Nativa. 0-700 m.

Distribución: GUA, SPE.

Ejemplar de referencia: Tressens, S. G. 6071 (CTES).

\section{Coccocypselum pulchellum Cham. ${ }^{* * *}$}

Hierba. Nativa. 300-700 m.

Distribución: GMB, GUA, IGU, SIG.

Ejemplar de referencia: Keller, H. A. 3946 (CTES).

Cordiera A. Rich. ex DC.

Cordiera concolor (Cham.) Kuntze (***)

Arbolito o arbusto. Nativa. 0-500 m.

Distribución: SIG.

Ejemplar de referencia: Biganzoli, F. 435 (SI).

\section{Coussarea Aubl.}

Coussarea contracta (Walp.) Benth. \& Hook. ex Müll. Arg. $\left.{ }^{* * *}\right)$

Arbusto o arbolito. Nativa. 0-500 m.

Distribución: GMB, GUA, IGU, MON, SPE.

Ejemplar de referencia: Tressens, S. G. 5074 (CTES, SI).

\section{Coussarea platyphylla Müll. Arg. $\left.{ }^{* * *}\right)$}

Arbusto o arbolito. Nativa. 0-500 m.

Distribución: IGU.

Ejemplar de referencia: Sleumer, H. O. 4750 (CTESN, SI).

\section{Coutarea Aubl.}

Coutarea hexandra (Jacq.) K. Schum.

Arbusto o árbol. Nativa. 0-700 m.

Distribución: CAN, SIG, SPE.

Ejemplar de referencia: Morrone, O. 1041 (SI).

Deppea Cham. \& Schltdl.

Deppea blumenaviensis (K. Schum.) Lorence $(* * *)$

Subarbusto. Nativa. 0-500 m.

Distribución: GUA, SPE.

Ejemplar de referencia: Daviña, J. 126 (CTES, G, MNES, SI).

\section{Diodia L.}

Diodia saponariifolia (Cham. \& Schltdl.) K. Schum. $\left.{ }^{* * *}\right)$

Hierba. Nativa. 0-700 m.

Distribución: GUA, SPE.

Ejemplar de referencia: Guaglianone, E. R. 2868 (SI).

Faramea Aubl.

Faramea hyacinthina Mart.

Arbusto. Nativa. 0-600 m.

Distribución: ELD, IGU, MON, SIG.

Ejemplar de referencia: Morrone, O. 1209 (SI). 


\section{Galianthe Griseb.}

Galianthe brasiliensis (Spreng.) E.L. Cabral \& Bacigalupo

Subarbusto. Nativa. 0-500 m.

Distribución: CAI, CAN, CAP, CON, GMB, GUA, IGU, MAY, SIG, SPE.

Ejemplar de referencia: Morrone, O. 1897 (SI).

Galianthe centranthoides (Cham. \& Schltdl.)

E.L. Cabral

Subarbusto. Nativa. 0-500 m.

Distribución: CAN, CAP, IGU, SIG. (SI).

Ejemplar de referencia: Zuloaga, F. O. 5304

Galianthe dichasia (Sucre \& C.G. Costa) E.L. Cabral (***)

Subarbusto. Nativa. 0-800 m.

Distribución: GMB, GSM, SIG.

Ejemplar de referencia: Guaglianone, E. R. 1147 (CTES, SI).

Galianthe eupatorioides (Cham. \& Schltdl.) E.L. Cabral

Subarbusto. Nativa. 0-800 m.

Distribución: CAI, CAN, CON, GSM, IGU, OBE, SIG, SJA, SPE.

Ejemplar de referencia: Morrone, O. 1017 (SI).

\section{Galianthe fastigiata Griseb.}

Subarbusto. Nativa. 0-800 m.

Distribución: APO, CAN, CAP, CON, GSM, IGU, SIG, SPE. (SI).

Ejemplar de referencia: Múlgura, M. E. 1958

Galianthe hispidula (A. Rich. ex DC.) E.L. Cabral \& Bacigalupo

Subarbusto. Nativa. 0-800 m.

Distribución: CAI, CAN, GMB, GSM, GUA, IGU, SPE.

Ejemplar de referencia: Zuloaga, F. O. 6675 (SI).

Galianthe laxa (Cham. \& Schltdl.) E.L. Cabral Subarbusto. Nativa. 0-600 m.

Distribución: CAN, ELD, GMB, GSM, GUA, IGU, MAY, MON, SIG, SJA, SPE.

Ejemplar de referencia: Zuloaga, F. O. 5186 (SI).
Galianthe linearifolia E.L. Cabral (***)

Subarbusto. Nativa. 0-500 m.

Distribución: APO, CAN.

Ejemplar de referencia: Spegazzini, C. L. s.n. (BAB, LP).

Galianthe montesii E.L. Cabral (***)

Subarbusto. Nativa. 0-300 m.

Distribución: GMB, SIG.

Ejemplar de referencia: Montes, J. E. 7036 (CTES, SI).

Galianthe palustris (Cham. \& Schltdl.) Cabaña Fader \& E.L. Cabral

Subarbusto. Nativa. 0-500 m.

Distribución: SPE.

Ejemplar de referencia: Guaglianone, E. R. 2833 (CTES, SI).

Galianthe thalictroides (K. Schum.) E.L. Cabral

Subarbusto. Nativa. 100-800 m.

Distribución: CAI, CAN, CON, ELD, GSM, OBE.

Ejemplar de referencia: Schwindt, E. 2220 (CTES).

Galianthe valerianoides (Cham. \& Schltdl.)

E.L. Cabral

Subarbusto. Nativa. 0-800 m.

Distribución: APO, CAN, GMB, IGU, SIG.

Ejemplar de referencia: Rodríguez, F. M. 766 (SI).

\section{Galium L.}

Galium atherodes Spreng.

Hierba o subarbusto. Nativa. 0-500 m.

Distribución: CAP.

Ejemplar de referencia: Molfino, J. F. 189 (F).

Galium equisetoides (Cham. \& Schltdl.) Standl. Hierba. Perenne. Nativa. 0-500 m.

Distribución: GMB.

Ejemplar de referencia: Keller, H. A. 4990 (CTES).

\section{Galium hirtum Lam.}

Hierba. Nativa. 0-600 m. 
Distribución: CAP, CON.

Ejemplar de referencia: Múlgura, M. E. 4052 (MNES, SI).

Galium hypocarpium (L.) Endl. ex Griseb.

Hierba. Nativa. 0-600 m.

Distribución: CAI, ELD, GUA, IGU, MON, SIG, SPE.

Ejemplar de referencia: Morrone, O. 1272 (SI).

\section{Galium latoramosum $\mathrm{Clos}$}

Hierba o subarbusto. Nativa. 0-700 m.

Distribución: GMB, GUA, IGU, SPE.

Ejemplar de referencia: Morrone, O. 1282 (SI).

\section{Galium megapotamicum Spreng.}

Hierba o subarbusto. Nativa. 0-500 m.

Distribución: APO, CAN, CON, OBE, SIG.

Ejemplar de referencia: Rodríguez, F. M. 562 $(F, G H)$.

Galium nigroramosum (Ehrend.) Dempster

Hierba. Nativa. 0-700 m.

Distribución: APO, CAI, IGU.

Ejemplar de referencia: Biganzoli, F. 568 (SI).

Galium noxium (A. St.-Hil.) Dempster subsp. valantioides (Cham. \& Schltdl.) Dempster

Hierba o enredadera. Nativa. 0-600 m.

Distribución: CAN, LNA, SIG, SJA.

Ejemplar de referencia: Montes, J. E. 15147 (NY).

Galium richardianum (Gillies ex Hook. \& Arn.) Endl. ex Walp.

Hierba. Nativa. 0-500 m.

Distribución: CAI, CAN, ELD.

Ejemplar de referencia: Biganzoli, F. 182 (SI).

Galium uruguayense Bacigalupo var. echinulatus Bacigalupo (***)

Hierba. Nativa. 0-500 m.

Distribución: GUA.

Ejemplar de referencia: Keller, H. A. 333 (SI).

Galium vile (Cham. \& Schltdl.) Dempster

Hierba. Nativa. 0-500 m.
Distribución: CAP, CON, SIG.

Ejemplar de referencia: Guillén, R. 276 (MNES, SI).

\section{Genipa L.}

Genipa americana $\mathrm{L}$.

Árbol. Nativa. 0-500 m.

Distribución: IGU.

Ejemplar de referencia: Keller, H. A. 13570 (CTES).

\section{Geophila D. Don}

Geophila macropoda (Ruiz \& Pav.) DC. ${ }^{* * *}$ )

Hierba. Nativa. 0-500 m.

Distribución: CAI, GMB, GUA.

Ejemplar de referencia: Biganzoli, F. 572 (SI).

Geophila repens (L.) I.M. Johnst.

Hierba. Nativa. 0-500 m.

Distribución: CAI, CAN, GMB, GSM, GUA, IGU, MON, SPE.

Ejemplar de referencia: Deginani, N. B. 1087 (SI).

\section{Guettarda L.}

Guettarda uruguensis Cham. \& Schltdl.

Arbusto. Nativa. 0-500 m.

Distribución: APO, CAI, CAN, CAP, CON, ELD, GMB, GUA, LNA, MON, SIG, SJA, SPE.

Ejemplar de referencia: Morrone, O. 592 (SI).

\section{Hamelia Jacq.}

Hamelia patens Jacq.

Arbusto. Nativa. 0-700 m.

Distribución: GMB, GUA, IGU, SPE.

Ejemplar de referencia: Múlgura, M. E. 3157 (SI).

\section{Hoffmannia Sw.}

Hoffmannia peckii K. Schum. $\left.{ }^{* * *}\right)$

Arbusto. Nativa. 0-500 m.

Distribución: SPE.

Ejemplar de referencia: Zanotti, C. A. 558 (SI). 


\section{Ixora L.}

Ixora venulosa Benth. $\left.{ }^{* * *}\right)$

Arbusto o árbol. Nativa. 0-500 m.

Distribución: IGU, SPE.

Ejemplar de referencia: Tressens, S. G. 4533 (CTES).

\section{Machaonia Bonpl.}

Machaonia brasiliensis (Hoffmanns. ex Humb.) Cham. \& Schltdl.

Arbusto. Nativa. 0-500 m.

Distribución: APO, OBE.

Ejemplar de referencia: Cabrera, A. L. 29139 (SI).

Machaonia spinosa Cham. \& Schltdl.

Arbusto. Nativa. 0-500 m.

Distribución: APO, CAN, CAP, OBE, SIG, SJA.

Ejemplar de referencia: Zuloaga, F. O. 5776 (SI).

\section{Manettia L.}

Manettia cordifolia Mart.

Liana. Nativa. 0-700 m.

Distribución: CAN, GSM, IGU, SIG, SPE.

Ejemplar de referencia: Zuloaga, F. O. 5013 (SI).

Manettia luteo-rubra (Vell.) Benth.

Liana. Nativa. 0-700 m.

Distribución: CAI, ELD, GMB, GUA, IGU, SIG, SPE.

Ejemplar de referencia: Morrone, O. 1296 (SI).

Manettia tweedieana K. Schum. ${ }^{* * *}$ )

Liana. Nativa. 0-500 m.

Distribución: IGU.

Ejemplar de referencia: Krapovickas, A. 2440 (BA).

Mitracarpus Zucc. ex Schult. \& Schult. f.

Mitracarpus brasiliensis M.L. Porto \& J.L. Waechter $\left({ }^{* *}\right)$

Hierba. Nativa. 0-500 m.

Distribución: GUA, SPE.

Ejemplar de referencia: Tressens, S. G. 6070 (CTES).
Mitracarpus megapotamicus (Spreng.)

Kuntze

Hierba. Nativa. 0-500 m.

Distribución: CAI, CAN.

Ejemplar de referencia: Biganzoli, F. 843 (SI).

Mitracarpus villosus (Sw.) DC.

Hierba. Nativa. 0-700 m.

Distribución: CAN, GMB, GUA, SIG.

Ejemplar de referencia: Zuloaga, F. O. 5512 (SI).

\section{Oldenlandia L.}

Oldenlandia salzmannii (DC.) Benth. \& Hook.

Hierba. Nativa. 0-500 m.

Distribución: CON, IGU.

Ejemplar de referencia: Biganzoli, F. 1710 (SI).

\section{Palicourea Aubl.}

Palicourea brevicollis (Müll. Arg.) C.M. Taylor $\left({ }^{* *}\right)$

Arbusto. Nativa. $100-800 \mathrm{~m}$.

Distribución: GMB, IGU.

Ejemplar de referencia: Morrone, O. 1177 (SI).

Palicourea crocea (Sw.) Roem. \& Schult.

Arbusto o subarbusto. Nativa. 0-8000 m.

Distribución: GMB, IGU.

Ejemplar de referencia: Zanotti, C. A. 456 (SI).

Palicourea macrobotrys (Ruiz \& Pav.) Roem. \& Schult. $\left({ }^{* * *}\right)$

Arbusto. Nativa. 0-500 m.

Distribución: IGU.

Ejemplar de referencia: Correa Luna, H. s.n. $(B A, S I)$.

\section{Psychotria L.}

\section{Psychotria carthagenensis Jacq.}

Arbusto o arbolito. Nativa. 0-700 m.

Distribución: CAN, CON, GMB, GSM, GUA, IGU, SIG, SJA, SPE.

Ejemplar de referencia: Zuloaga, F. O. 5031 (SI). 
Psychotria deflexa DC. $\left.{ }^{* * *}\right)$

Arbusto o subarbusto. Nativa. 0-500 m.

Distribución: CAN.

Ejemplar de referencia: Montes, J. E. 27374 (NY).

Psychotria leiocarpa Cham. \& Schltdl.

Hierba o arbusto. Nativa. 0-800 m.

Distribución: CAI, GMB, GSM, GUA, IGU, SIG, SPE. (SI).

Ejemplar de referencia: Guaglianone, E. R. 2856

Psychotria myriantha Müll. Arg.

Arbusto. Nativa. 0-500 m.

Distribución: CAI, GMB, GSM, GUA, IGU, SIG, SPE.

Ejemplar de referencia: Deginani, N. B. 1084 (SI).

Psychotria nitidula Cham. \& Schltdl. (***)

Subarbusto. Nativa. 0-500 m.

Distribución: IGU, SPE. (SI).

Ejemplar de referencia: Guaglianone, E. R. 2908

Psychotria suterella Müll. Arg. $\left.{ }^{* * *}\right)$

Arbusto o arbolito. Nativa. 0-700 m.

Distribución: GMB, SPE.

Ejemplar de referencia: Deginani, N. B. 1713 (SI).

\section{Randia L.}

Randia brevituba Judkevich \& R.M. Salas $\left(^{* * *}\right)$

Arbolito o árbol. Nativa. 100-500 m.

Distribución: SIG.

Ejemplar de referencia: Keller, H. A. 12253 (CTES, SI).

Randia ferox (Cham. \& Schltdl.) DC.

Arbusto. Nativa. 0-800 m.

Distribución: CAN, CAP, GMB, GUA, IGU, SIG, SJA, SPE.

Ejemplar de referencia: Keller, H. A. 10686 (CTES).

\section{Richardia L.}

Richardia brasiliensis Gomes

Hierba. Nativa. 0-800 m.

Distribución: CAI, CAN, GMB, GSM, GUA, IGU, SPE.

Ejemplar de referencia: Rodríguez, M. 547 (CTES, G, MNES).
Richardia grandiflora (Cham. \& Schltdl.) Steud.

Hierba. Nativa. 0-500 m.

Distribución: CAN, SIG.

Ejemplar de referencia: Múlgura, M. E. 1613 (SI).

Richardia stellaris (Cham. \& Schltdl.) Steud.

Hierba. Nativa. 0-500 m.

Distribución: APO, CAN.

Ejemplar de referencia: Múlgura, M. E. 2976 (SI).

\section{Rudgea Salisb.}

Rudgea jasminoides (Cham.) Müll. Arg. ${ }^{* * *}$ )

Arbusto o arbolito. Nativa. 0-700 m.

Distribución: GUA.

Ejemplar de referencia: Tressens, S. G. 4722 (CTES).

Rudgea parquioides (Cham.) Müll. Arg. (***)

Arbusto. Nativa. 0-500 m.

Distribución: CAI, ELD, GMB, GUA, SPE.

Ejemplar de referencia: Morrone, O. 1533 (SI).

\section{Simira Aubl.}

Simira sampaioana (Standl.) Steyerm. $\left({ }^{* *}\right)$

Árbol. Nativa. 0-500 m.

Distribución: IGU.

Ejemplar de referencia: Vanni, R. O. 3413 (CTES, SI).

\section{Spermacoce L.}

Spermacoce eryngioides (Cham. \& Schltdl.) Kuntze

Hierba o subarbusto. Nativa. 0-500 m.

Distribución: CAP, CAI, CON, SIG.

Ejemplar de referencia: Biganzoli, F. 935 (SI).

\section{Spermacoce glabra Michx.}

Hierba. Nativa. 0-500 m.

Distribución: ELD, IGU, MON.

Ejemplar de referencia: Vanni, R. O. 4010 (CTES).

Spermacoce hassleri E.L. Cabral \& J. Florentin Hierba o subarbusto. Perenne. Nativa. 0-500 m. Distribución: CAP, SIG.

Ejemplar de referencia: Keller, H. A. 13190 (CTES). 
Spermacoce latifolia Aubl. (***)

Subarbusto. Nativa. 0-800 m.

Distribución: GMB.

Ejemplar de referencia: Keller, H. A. 5053 (CTES).

Spermacoce prostrata Aubl.

Hierba. Nativa. 0-500 m.

Distribución: CAN, SJA.

Ejemplar de referencia: Cabral, E. L. 415 (CTES).

\section{Spermacoce verticillata $L$.}

Hierba o subarbusto. Nativa. 0-500 m.

Distribución: APO, CAI, CAN, CON, GMB, SIG, SPE.

Ejemplar de referencia: Biganzoli, F. 306 (SI).

\section{Staelia Cham. \& Schltdl.}

Staelia thymoides Cham. \& Schltdl.

Hierba. Nativa. 0-500 m.

Distribución: CAI, CAN, CAP, ELD, SIG, SPE.

Ejemplar de referencia: Morrone, O. 996 (SI).

\section{RUTACEAE}

Balfourodendron Corr. Méllo ex Oliv.

Balfourodendron riedelianum (Engl.) Engl.

Árbol. Nativa. 0-700 m.

Distribución: CAI, ELD, GMB, GUA, IGU.

Ejemplar de referencia: Morrone, O. 755 (SI).

\section{Citrus L.}

Citrus aurantium $\mathrm{L}$.

Árbol. Introducida. 0-700 m.

Distribución: IGU.

Ejemplar de referencia: Vanni, R. O. 3431 (CTES).

\section{Esenbeckia Kunth}

Esenbeckia densiflora (Chodat \& Hassl.) Hassl. $(* * *)$

$$
\text { Árbol. Nativa. 0-500 m. }
$$

Distribución: IGU.

Ejemplar de referencia: Cocucci, A. A. 3211 (CORD, SI).

Esenbeckia febrifuga (A. St.-Hil.) A. Juss. ex Mart. (***)

Árbol. Nativa. 0-700 m.

Distribución: IGU.

Ejemplar de referencia: Zuloaga, F. O. 5202 (SI).

Esenbeckia grandiflora Mart.

Arbusto o arbolito. Nativa. 0-700 m.

Distribución: IGU, SPE.

Ejemplar de referencia: Zuloaga, F. O. 5639 (SI).

\section{Helietta Tul.}

Helietta apiculata Benth.

Árbol. Nativa. 0-800 m.

Distribución: APO, CAI, CAN, CON, ELD, GMB, GUA, LNA, MON, SIG, SPE.

Ejemplar de referencia: Guaglianone, E. R. 2897 (SI).

\section{Pilocarpus Vahl}

Pilocarpus pennatifolius Lem.

Arbusto o árbol. Nativa. 0-800 m.

Distribución: APO, CAI, CAN, CAP, CON, GMB, GUA, IGU, LNA, SIG, SPE.

Ejemplar de referencia: Deginani, N. B. 1060 (SI).

\section{Zanthoxylum L.}

Zanthoxylum caribaeum Lam. subsp. rugosum (A. St.-Hil. \& Tul.) Reynel

Árbol. Nativa. 0-800 m.

Distribución: CAP, GSM, GUA, IGU.

Ejemplar de referencia: Rojas, T. 4470 (BAF, LIL).

Zanthoxylum fagara (L.) Sarg. subsp. culantrilo (Kunth) Reynel

Árbol. Nativa. 0-500 m.

Distribución: SIG.

Ejemplar de referencia: Mereles, F. 3282 (G). 
Zanthoxylum fagara (L.) Sarg. subsp. fagara

Árbol. Nativa. 0-700 m.

Distribución: CAN, IGU, SIG.

Ejemplar de referencia: Scala, A. C. 194 (LIL, LP).

Zanthoxylum petiolare A. St.-Hil. \& Tul.

Árbol. Nativa. 0-600 m.

Distribución: CAN, OBE, SPE.

Ejemplar de referencia: Zuloaga, F. O. 8181 (SI).

\section{Zanthoxylum rhoifolium Lam.}

Árbol. Nativa. 0-800 m.

Distribución: APO, CAI, CAN, CAP, ELD, GMB, GUA, IGU, SIG, SJA, SPE.

Ejemplar de referencia: Zuloaga, F. O. 6560 (SI).

\section{Zanthoxylum riedelianum Engl.}

Árbol. Nativa. 0-700 m.

Distribución: CAP, IGU.

Ejemplar de referencia: Venturi, S. 158 (BAF, LIL).

\section{SALICACEAE}

\section{Abatia Ruiz \& Pav.}

\section{Abatia angeliana M.H. Alford (***)}

Arbusto. Nativa. 0-800 m.

Distribución: GMB, GUA, SPE.

Ejemplar de referencia: Guaglianone, E. R. 2986 (SI).

\section{Banara Aubl.}

Banara parviflora (A. Gray) Benth. $\left({ }^{* * *}\right.$ )

Arbolito o árbol. Nativa. 0-700 m.

Distribución: IGU.

Ejemplar de referencia: Zuloaga, F. O. 8361 (SI).

\section{Banara tomentosa $\mathrm{Clos}$}

Arbusto o árbol. Nativa. 0-800 m.

Distribución: CAI, CAN, CAP, GMB, GUA, IGU, LNA, SIG, SJA, SPE.

Ejemplar de referencia: Zuloaga, F. O. 5393 (SI).

\section{Casearia Jacq.}

Casearia decandra Jacq. $\left.{ }^{* * *}\right)$

Arbusto o arbolito. Nativa. 0-800 m.

Distribución: CAN, ELD, GMB, GSM, GUA, IGU, SIG, SJA, SPE.

Ejemplar de referencia: Deginani, N. B. 1278 (SI).

\section{Casearia gossypiosperma Briq.}

Árbol. Nativa. 0-400 m.

Distribución: IGU.

Ejemplar de referencia: Keller, H. A. 7048 (CTES).

\section{Casearia lasiophylla Eichler $\left({ }^{* * *}\right)$}

Arbusto o árbol. Nativa. 500-800 m.

Distribución: GMB, SPE.

Ejemplar de referencia: Keller, H. A. 3650 (CTES).

\section{Casearia obliqua Spreng. $\left({ }^{* * *}\right)$}

Arbusto o arbolito. Nativa. 0-700 m.

Distribución: IGU.

Ejemplar de referencia: Keller, H. A. 6442 (CTES,SI).

\section{Casearia sylvestris $\mathrm{Sw}$.}

Arbusto o árbol. Nativa. 0-800 m.

Distribución: APO, CAN, CAP, ELD, GMB, GSM, GUA, IGU, SIG, SPE.

Ejemplar de referencia: Zuloaga, F. O. 6496 (SI).

Prockia P. Browne ex L.

Prockia crucis $\mathrm{P}$. Browne ex $\mathrm{L}$.

Arbusto o arbolito. Nativa. 0-700 m.

Distribución: IGU.

Ejemplar de referencia: Herrera, J. 256 (CTES).

Salix L., nom. cons.

Salix humboldtiana Willd.

Árbol. Nativa. 0-500 m.

Distribución: SIG.

Ejemplar de referencia: Keller, H. A. 10667 (CTES). 
Xylosma G. Forst., nom. cons.

Xylosma pseudosalzmanii Sleumer $\left(^{* * *}\right)$

Arbusto o arbolito. Nativa. 0-500 m.

Distribución: CAN, MON, SIG.

Ejemplar de referencia: Schwindt, E. 1758 (LIL).

\section{Xylosma pubescens Griseb.}

Arbusto o árbol. Nativa. 0-500 m.

Distribución: CAI.

Ejemplar de referencia: Biganzoli, F. 325 (SI).

Xylosma tweediana (Clos) Eichler

Arbusto o arbolito. Nativa. 0-700 m.

Distribución: GMB.

Ejemplar de referencia: Múlgura, M. E. 476 (SI).

\section{Xylosma venosa N.E. Br.}

Arbusto o arbolito. Nativa. 0-500 m.

Distribución: APO, CAN, CAP, GSM, SIG.

Ejemplar de referencia: Montes, J. E. 2106 (SI).

\section{SAPINDACEAE}

\section{Allophylus L.}

Allophylus edulis (A. St.-Hil., A. Juss. \& Cambess.) Hieron. ex Niederl.

Arbusto o árbol. Nativa. 0-800 m.

Distribución: APO, CAI, CAN, ELD, GMB, GUA, IGU, SIG, SPE. (SI).

Ejemplar de referencia: Zuloaga, F. O. 5003

Allophylus guaraniticus (A. St.-Hil.) Radlk.

Arbusto o arbolito. Nativa. 0-800 m.

Distribución: APO, CAI, CAN, CON, GMB, GSM, GUA, IGU, MON, SIG, SJA, SPE.

Ejemplar de referencia: Deginani, N. B. 1246 (CTES, SI).

\section{Cardiospermum L.}

Cardiospermun corindum $L$. var. parviflorum (Cambess.) Ferruci

Liana. Perenne. Nativa. 0-500 m.
Distribución: SIG.

Ejemplares de referencia: Keller, H. A. 7105 (CTES).

\section{Cardiospermum grandiflorum $\mathrm{Sw}$.}

Liana. Nativa. 0-800 m.

Distribución: CON, ELD, GMB, GSM, GUA, SIG, SPE.

Ejemplar de referencia: Morrone, O. 1513 (SI).

\section{Cardiospermum halicacabum L. var. halicacabum \\ Liana. Nativa. 0-600 m. \\ Distribución: CON, GSM, SIG. \\ Ejemplar de referencia: Biganzoli, F. 240 (SI).}

Cardiospermum halicacabum L. var. microcarpum (Kunth) Blume

Liana. Nativa. 0-700 m.

Distribución: APO, ELD, GUA, LNA, SPE.

Ejemplar de referencia: Zuloaga, F. O. 5566 (SI).

\section{Cupania L.}

Cupania vernalis $C$ ambess.

Árbol. Nativa. 0-800 m.

Distribución: APO, CAN, CAP, ELD, GMB, GUA, SIG, SJA, SPE.

Ejemplar de referencia: Hunziker, J. H. 778 (SI).

Diatenopteryx Radlk.

Diatenopteryx sorbifolia Radlk.

Árbol. Nativa. 0-700 m.

Distribución: CAI, CAP, ELD, GMB, GUA, IGU, SIG.

Ejemplar de referencia: Biganzoli, F. 596 (SI).

Matayba Aubl.

Matayba elaeagnoides Radlk.

Arbusto o árbol. Nativa. 0-700 m.

Distribución: CAI, CAP, ELD, GMB, GUA, IGU, SIG.

Ejemplar de referencia: Zuloaga, F. O. 5690 (SI). 


\section{Paullinia L.}

Paullinia elegans Cambess.

Liana. Nativa. 0-600 m.

Distribución: APO, CAI, CAN, CON, GUA, IGU, LNA, OBE, SIG.

Ejemplar de referencia: Zuloaga, F. O. 5025 (SI).

Paullinia meliifolia Juss. $\left.{ }^{* * *}\right)$

Liana. Nativa. 0-700 m.

Distribución: CAI, GMB, GSM, GUA, IGU, LNA, OBE, SPE.

Ejemplar de referencia: Morrone, O. 2165 (SI).

\section{Sapindus L.}

\section{Sapindus saponaria L.}

Árbol. Nativa.

Distribución: Desconocida.

Ejemplar de referencia: Scala, A. C. 86 (LIL).

\section{Serjania Mill.}

Serjania fuscifolia Radlk.

Liana. Nativa. 0-500 m.

Distribución: GSM, GUA, IGU, SIG.

Ejemplar de referencia: Deginani, N. B. 1019 (CTES, SI).

\section{Serjania glabrata Kunth}

Liana. Nativa. 0-700 m.

Distribución: CAI, ELD, GMB, IGU.

Ejemplar de referencia: Biganzoli, F. 1534 (SI).

Serjania glutinosa Radlk. $\left.{ }^{(* *}\right)$

Liana. Nativa. 0-500 m.

Distribución: IGU.

Ejemplar de referencia: Vanni, R. O. 3914 (CTES).

\section{Serjania hebecarpa Benth.}

Liana. Nativa. 0-600 m.

Distribución: GUA.

Ejemplar de referencia: Deginani, N. B. 1620 (SI).

Serjania laruotteana Cambess.

Liana. Nativa. 0-800 m.

Distribución: CON, GMB, GUA, IGU, SIG.
Ejemplar de referencia: Klein, R. M. 4-140 (CTESN).

Serjania meridionalis Cambess.

Arbusto o liana. Nativa. 0-700 m.

Distribución: APO, CAN, GMB, GUA, IGU, SIG, SJA, SPE.

Ejemplar de referencia: Daviña, J. 195 (CTES, G, MNES).

Serjania perulacea Radlk. var. krapovickasii Coulleri \& Ferrucci

Liana. Nativa. 0-500 m.

Distribución: SJA.

Ejemplar de referencia: Keller, H. A. 5659 (CTES).

Thinouia Triana \& Planch.

Thinouia mucronata Radlk.

Liana. Nativa. 0-700 m.

Distribución: APO, GMB, GUA, IGU, SIG, SPE.

Ejemplar de referencia: Zuloaga, F. O. 6830 (SI).

Thinouia ventricosa Radlk. $\left.{ }^{(* *}\right)$

Liana. Nativa. 0-800 m.

Distribución: CAP, SPE.

Ejemplar de referencia: Guillén, R. 194 (CTES, G, MNES, SI).

\section{Urvillea Kunth}

Urvillea laevis Radlk. ${ }^{* * *}$ )

Liana. Nativa. 0-500 m.

Distribución: IGU.

Ejemplar de referencia: Vanni, R. O. 2854 (CTES).

Urvillea ulmacea Kunth

Liana. Nativa. 0-700 m.

Distribución: GMB, GUA, IGU.

Ejemplar de referencia: Vanni, R. O. 2848 (CTES).

Urvillea uniloba Radlk.

Liana. Nativa. 0-700 m.

Distribución: APO, CAI, CON, ELD, GMB, GUA, IGU, SIG, SPE.

Ejemplar de referencia: Dematteis, M. 87 (BAB, G, MNES). 
SAPOTACEAE

\section{Chrysophyllum L.}

Chrysophyllum gonocarpum (Mart. \& Eichler) Engl.

Árbol. Nativa. 0-800 m.

Distribución: CAI, CAN, CAP, ELD, GMB, GUA, IGU, MON, SIG, SPE.

Ejemplar de referencia: Burkart, A. 14530 (NY, SI).

Chrysophyllum marginatum (Hook. \& Arn.) Radlk.

Arbusto o árbol. Nativa. 0-800 m.

Distribución: CAI, CAN, CAP, CON, ELD, GSM, GUA, IGU, MON, SIG, SJA, SPE.

Ejemplar de referencia: Deginani, N. B. 1181 (SI).

\section{Pouteria Aubl.}

Pouteria fragrans (Pierre) Dubard

Arbusto. Nativa. 0-500 m.

Distribución: IGU.

Ejemplar de referencia: Molfino, J. F. s.n. (BAF).

Pouteria gardneriana (A. DC.) Radlk.

Árbol. Nativa. 0-500 m.

Distribución: GMB, GSM, IGU, SIG.

Ejemplar de referencia: Biganzoli, F. 441 (SI).

Pouteria glomerata (Miq.) Radlk.

Árbol. Nativa. 0-500 m.

Distribución: ELD, SIG.

Ejemplar de referencia: Keller, H. A. 81 (CTES).

Pouteria salicifolia (Spreng.) Radlk.

Arbusto o árbol. Nativa. 0-700 m.

Distribución: APO, CAI, ELD, GMB, IGU, SIG.

Ejemplar de referencia: Belgrano, M. J. 339 (SI, WIS).

\section{Sideroxylon L.}

Sideroxylon obtusifolium (Roem. \& Schult.) T.D. Penn.

Arbusto o árbol. Nativa. 0-700 m.
Distribución: GMB, GUA, IGU.

Ejemplar de referencia: Múlgura, M. E. 1911 (SI).

\section{SCHOEPFIACEAE}

Schoepfia Schreb.

Schoepfia brasiliensis A. DC. $\left(^{* * *}\right)$

Arbusto o árbol. Nativa. 0-700 m.

Distribución: GUA, IGU.

Ejemplar de referencia: Keller, H. A. 211 (CTES, SI).

\section{SCROPHULARIACEAE}

\section{Buddleja L.}

Buddleja elegans Cham. \& Schltdl. subsp. angustata (Benth.) E.M. Norman

Arbusto. Nativa. 0-500 m.

Distribución: IGU, SIG.

Ejemplar de referencia: Quiroga, H. s.n. (BA, $B R, G H)$.

Buddleja misionum Kraenzl.

Arbusto. Nativa. 0-500 m.

Distribución: APO, CAN, CON, ELD.

Ejemplar de referencia: Montes, J. E. 15443 (SI).

Buddleja stachyoides Cham. \& Schltdl.

Arbusto o subarbusto. Nativa. 0-700 m.

Distribución: CAN, CAP, CON, ELD, GMB, GSM, GUA, IGU, LNA, MON, SIG.

Ejemplar de referencia: Biganzoli, F. 515 (SI)).

Buddleja thyrsoides Lam. subsp. angusticarpa E.M. Norman \& L.B. Sm.

Arbusto. Nativa. 0-500 m.

Distribución: APO, CAN, SPE.

Ejemplar de referencia: Morrone, O. 1763 (SI).

Buddleja thyrsoides Lam. subsp. thyrsoides Arbusto. Nativa. 0-500 m.

Distribución: CAN, CON, IGU.

Ejemplar de referencia: Cristóbal, C. L. 2281 (CTES). 


\section{Verbascum L.}

\section{Verbascum virgatum Stokes}

Hierba. Introducida. 0-500 m.

Distribución: APO, CAP.

Ejemplar de referencia: Fontana, J. L. 5198 (SI).

\section{SIMAROUBACEAE}

\section{Castela Turpin}

\section{Castela tweedii Planch.}

Arbusto o arbolito. Nativa. 0-500 m.

Distribución: CAI, CAN, CAP, ELD, GMB, GSM, IGU, LNA, MON, OBE, SIG.

Ejemplar de referencia: Múlgura, M. E. 2284 (SI).

\section{Homalolepis Turcz.}

Homalolepis glabra (Engl.) Devecchi \& Pirani $(* * *)$

Arbusto o árbol. Nativa. 0-500 m.

Distribución: CAP.

Ejemplar de referencia: Molfino, J. F. s.n. (BA).

\section{Picrasma Blume}

Picrasma crenata (Vell.) Engl. (***)

Árbol. Nativa. 0-500 m.

Distribución: ELD, GMB, GSM, GUA, IGU.

Ejemplar de referencia: Tressens, S. G. 6473 (SI).

\section{SMILACACEAE}

\section{Smilax L.}

Smilax assumptionis A. DC.

Liana. Nativa. 0-500 m.

Distribución: CAN, IGU, SIG, SJA.

Ejemplar de referencia: Llamas, A. 27072 (SI).

\section{Smilax campestris Griseb.}

Liana. Nativa. 0-700 m.

Distribución: APO, CAI, CAN, CAP, GMB, GSM, GUA, IGU, LNA, SIG, SJA, SPE.

Ejemplar de referencia: Deginani, N. B. 1182 (SI).

\section{Smilax cognata Kunth}

Liana. Nativa. 0-700 m.

Distribución: APO, CAI, CAN, CAP, GMB, GUA, IGU, SIG.

Ejemplar de referencia: Rodríguez, M. 924 (MNES).

\section{Smilax fluminensis Steud.}

Liana. Nativa. 0-500 m.

Distribución: IGU.

Ejemplar de referencia: Tressens, S. G. 4541 (CTES).

\section{SOLANACEAE}

\section{Aureliana Sendtn.}

Aureliana fasciculata (Vell.) Sendtn. $\left(^{* * *}\right)$

Arbusto o árbol. Nativa. 0-800 m.

Distribución: GMB, GUA, SPE.

Ejemplar de referencia: Tressens, S. G. 6462 (SI).

\section{Bouchetia Dunal}

Bouchetia anomala (Miers) Britton \& Rusby

Hierba. Nativa. 0-500 m.

Distribución: CAN, SIG.

Ejemplar de referencia: Keller, H. A. 10528 (CTES).

\section{Brugmansia Pers.}

Brugmansia suaveolens (Humb. \& Bonpl. ex Willd.) Sweet $\left({ }^{* * *}\right)$

Arbusto o arbolito. Introducida. 100-500 m.

Distribución: GMB, GSM, GUA, IGU.

Ejemplar de referencia: Múlgura, M. E. 1764 (SI). 


\section{Brunfelsia L.}

Brunfelsia australis Benth.

Arbusto o arbolito. Nativa. 0-700 m.

Distribución: APO, CAN, CON, ELD, IGU, SPE.

Ejemplar de referencia: Rodríguez, F. M. 484 (SI).

\author{
Brunfelsia pilosa Plowman $\left(^{* *}\right)$ \\ Arbusto. Nativa. 0-800 m. \\ Distribución: GMB, GUA, SPE. \\ Ejemplar de referencia: Múlgura, M. E. 4046 \\ (MNES, SI).
}

\section{Calibrachoa Cerv.}

Calibrachoa caesia (Sendtn.) Wijsman $\left(^{* * *}\right)$

Subarbusto. Nativa. 0-500 m.

Distribución: CAN, SIG.

Ejemplar de referencia: Cabrera, A. L. 29244 (SI).

\section{Calibrachoa excellens (R.E. Fr.) Wijsman}

Arbusto o subarbusto. Nativa. 0-700 m.

Distribución: CAN, CAP, SPE.

Ejemplar de referencia: Jörgensen, P. 126 (SI).

\section{Calibrachoa irgangiana Stehmann $\left.{ }^{* * *}\right)$}

Arbusto. Nativa. 100-300 m.

Distribución: CON.

Ejemplar de referencia: Greppi, J. A. 1053 (BHCB).

Calibrachoa linoides (Sendtn.) Wijsman subsp.

furcata Greppi \& Stehmann $\left({ }^{* * *}\right)$

Subarbusto. Nativa. 400-800 m.

Distribución: GMB, GUA, SPE.

Ejemplar de referencia: Greppi, J. A. 1020 (BAB).

Calibrachoa linoides (Sendtn.) Wijsman subsp.

linoides $\left.{ }^{* * *}\right)$

Subarbusto. Nativa. 0-500 m.

Distribución: MAY, SIG, SPE.

Ejemplar de referencia: Krapovickas, A. 28660 (CTES, SI).

Calibrachoa missionica Stehmann \& Semir Arbusto. Nativa. 0-500 m.

Distribución: CAI, GSM, LNA.

Ejemplar de referencia: Biganzoli, F. 285 (SI).
Calibrachoa ovalifolia (Miers) Stehmann \& Semir

Hierba o subarbusto. Nativa. 0-500 m.

Distribución: CAN, SIG.

Ejemplar de referencia: Morrone, O. 1021 (SI).

Calibrachoa parviflora (Juss.) D’Arcy

Hierba. Anual. Nativa. 0-300 m.

Distribución: CAP.

Ejemplar de referencia: Grondona, H. 1117 (BAB).

Calibrachoa pygmaea (R.E. Fr.) Wijsman

Hierba. Nativa. 0-300 m.

Distribución: APO, CAN, CON.

Ejemplar de referencia: Biganzoli, F. 1621 (SI).

Calibrachoa thymifolia (A. St.-Hil.) Stehmann \& Semir

Subarbusto. Nativa. 0-500 m.

Distribución: CON.

Ejemplar de referencia: Keller, H. A. 5754 (CTES).

\section{Capsicum L.}

Capsicum baccatum L.

Arbusto. Nativa. 0-500 m.

Distribución: CAN, OBE.

Ejemplar de referencia: Barboza, G. E. 415 (CORD, CTES, SI).

Capsicum flexuosum Sendtn.

Arbusto. Nativa. 100-500 m.

Distribución: CAI, CAN, CON, GMB, GSM, GUA, IGU, SIG, SPE.

Ejemplar de referencia: Deginani, N. B. 1701 (SI).

\section{Cestrum L.}

Cestrum bracteatum Link \& Otto $\left.{ }^{* * *}\right)$

Arbusto. Nativa. 0-800 m.

Distribución: GMB, SPE.

Ejemplar de referencia: Eskuche, U. G. 4-89 (CTESN).

\section{Cestrum euanthes Schltdl.}

Arbusto o arbolito. Nativa. 0-500 m.

Distribución: CAI, CAP, IGU, SIG.

Ejemplar de referencia: Zuloaga, F. O. 5262 (SI). 
Cestrum guaraniticum Chodat $\&$ Hassl.

Arbusto. Nativa. 0-500 m.

Distribución: CAI, CAP, SIG.

Ejemplar de referencia: Barboza, G. E. 405 (CORD, SI).

Cestrum laevigatum Schltdl.

Arbusto o árbol. Nativa. 0-500 m.

Distribución: CAP, GUA, IGU, SIG.

Ejemplar de referencia: Zuloaga, F. O. 6591 (SI).

Cestrum mariquitense Kunth

Arbusto. Nativa. 0-600 m.

Distribución: CAN, LNA, SIG. (SI).

Ejemplar de referencia: Múlgura, M. E. 1634

\section{Cestrum parqui L'Hér.}

Arbusto. Nativa. 0-600 m.

Distribución: GMB.

Ejemplar de referencia: Zuloaga, F. O. 2091 (SI).

Cestrum strigilatum Ruiz \& Pav.

Arbusto. Nativa. 0-700 m.

Distribución: APO, CAI, CAN, GMB, GSM, GUA, IGU, SIG, SPE.

Ejemplar de referencia: Biganzoli, F. 891 (SI).

\section{Datura L.}

\section{Datura ferox L.}

Hierba. Nativa. 0-500 m.

Distribución: MON.

Ejemplar de referencia: Keller, H. A. 5021 (CTES).

\section{Datura inoxia Mill.}

Hierba. Introducida. 0-600 m.

Distribución: ELD, OBE.

Ejemplar de referencia: Rodríguez 505 (CORD).

Lycianthes (Dunal) Hassl., nom. cons.

Lycianthes asarifolia (Kunth \& Bouché) Bitter

Hierba. Nativa. 0-700 m.

Distribución: CAN, ELD, IGU.

Ejemplar de referencia: Burkart, A. 14971 (LIL).
Lycianthes fasciculata (Rusby) Bitter

Arbusto. Nativa. 200-700 m.

Distribución: IGU.

Ejemplar de referencia: Hunziker, J. H. 10960 (SI).

Lycianthes pauciflora (Vahl) Bitter $\left(^{* * *}\right)$

Arbusto. Nativa. 200-600 m.

Distribución: ELD, IGU.

Ejemplar de referencia: Schwarz, G. J. 7017 (CORD).

Lycianthes rantonnetii (Carrière) Bitter

Arbusto. Nativa. 0-700 m.

Distribución: APO, CAI, CAN, CAP, GMB, GSM, GUA, IGU, SIG, SJA, SPE.

Ejemplar de referencia: Schinini, A. 27592 (CTES, SI).

\section{Lycium L.}

Lycium glomeratum Sendtn. $\left.{ }^{* * *}\right)$

Arbusto o arbolito. Nativa. 0-500 m.

Distribución: CAN.

Ejemplar de referencia: Rodríguez, F. M. 762 (SI).

Lycium morongii Britton

Arbusto o arbolito. Nativa. 0-500 m.

Distribución: CAP.

Ejemplar de referencia: Rodríguez 166 (CORD).

Nicandra Adans., nom. cons.

Nicandra physalodes (L.) Gaertn.

Hierba. Anual. Adventicia. 0-500 m.

Distribución: MON.

Ejemplar de referencia: Keller, H. A. 5550 (CTES).

\section{Nicotiana L.}

Nicotiana alata Link \& Otto

Hierba. Nativa. 0-600 m.

Distribución: CAN, ELD, GUA, IGU.

Ejemplar de referencia: Vanni, R. O. 3816 (CTES). 
Nicotiana bonariensis Lehm.

Hierba. Nativa. 0-500 m.

Distribución: CAN.

Ejemplar de referencia: Joergensen, P. s.n. (BAB).

\section{Nicotiana glauca Graham}

Arbusto o subarbusto. Nativa. 0-600 m.

Distribución: CAP, SPE.

Ejemplar de referencia: Múlgura, M. E. 4043 (MNES, SI).

\section{Nicotiana langsdorfii Weinm. $\left.{ }^{* * *}\right)$}

Hierba. Nativa. 200-700 m.

Distribución: GUA, IGU.

Ejemplar de referencia: Tressens, S. G. 4484 (CTES).

\section{Nicotiana longiflora $C a v$.}

Hierba. Nativa. 0-500 m.

Distribución: CAP, SIG.

Ejemplar de referencia: Múlgura, M. E. 2827 (SI).

Nierembergia Ruiz \& Pav.

\section{Nierembergia aristata $D$. Don}

Hierba. Nativa. 0-500 m.

Distribución: CAN.

Ejemplar de referencia: de Llamas, A. 4836 (SI).

\section{Nierembergia linariaefolia Graham}

Hierba. Nativa. 0-500 m.

Distribución: APO, CAI, CAN, CAP, CON, LNA, SIG, SPE.

Ejemplar de referencia: Schinini, A. 5623 (CTES).

\section{Nierembergia micrantha Cabrera}

Hierba. Nativa. 0-500 m.

Distribución: CAP.

Ejemplar de referencia: Grondona 1670 (RB).

\section{Nierembergia pinifolia Miers $\left({ }^{* *}\right)$}

Hierba. Nativa. 0-500 m.

Distribución: SIG.

Ejemplar de referencia: Schwarz, G. J. 1329 (LIL).
Nierembergia scoparia Sendtn.

Hierba. Nativa. 0-500 m.

Distribución: CAN.

Ejemplar de referencia: Schinini, A. 5620 (RB).

Petunia Juss.

Petunia inflata R.E. Fr.

Hierba. Nativa. 0-700 m.

Distribución: APO, CAN, LNA, SIG.

Ejemplar de referencia: Cabrera, A. L. 28478 (SI).

Petunia interior Ando \& Hashim. $\left.{ }^{* * *}\right)$

Hierba. Nativa. 200-700 m.

Distribución: CAI, GMB, GUA, OBE, SPE.

Ejemplar de referencia: Zuloaga, F. O. 8159 (SI).

Petunia scheideana L.B. Sm. \& Downs $\left({ }^{* *}\right)$

Hierba. Nativa. 200-700 m.

Distribución: IGU.

Ejemplar de referencia: Ando, T. 120 (BAB).

\section{Physalis L.}

\section{Physalis angulata $\mathrm{L}$.}

Hierba. Nativa. 0-600 m.

Distribución: CAN, ELD.

Ejemplar de referencia: Barboza, G. E. 1046 (CORD).

Physalis pubescens L. var. hygrophila (Mart.) Dunal

Hierba. Nativa. 100-700 m.

Distribución: CAN, ELD, GMB, GSM, IGU, LNA, MON.

Ejemplar de referencia: Hunziker, A. T. 850 (CORD).

\section{Physalis pubescens $\mathrm{L}$.}

Hierba. Nativa. 0-700 m.

Distribución: CAI, CAN, ELD, GMB, GSM, GUA, IGU, MON, SJA, SPE.

Ejemplar de referencia: Biganzoli, F. 879 (SI).

\section{Physalis viscosa $\mathrm{L}$.}

Hierba. Nativa. 0-700 m.

Distribución: CAN, ELD, SIG, SPE.

Ejemplar de referencia: Schwindt, E. 2113 (CORD, LIL). 


\section{Schwenckia D. Royen ex L.}

\section{Schwenckia americana D. Royen ex L.}

Hierba o subarbusto. Nativa. 0-500 m.

Distribución: SIG.

Ejemplar de referencia: Biganzoli, F. 16 (SI).

\section{Schwenckia grandiflora Benth.}

Subarbusto. Nativa. 0-500 m.

Distribución: CAP.

Ejemplar de referencia: Insaurralde, I. S. s.n. (CTES).

\section{Sessea Ruiz \& Pav.}

\section{Sessea regnellii Taub. $\left.{ }^{* * *}\right)$}

Arbusto o árbol. Nativa. 0-600 m.

Distribución: GUA, SPE.

Ejemplar de referencia: Keller, H. A. 2851 (CTES).

Sessea vestioides (Schltdl.) Hunz.

Arbusto. Nativa. 0-500 m.

Distribución: CAN, OBE.

Ejemplar de referencia: Keller, H. A. 13879 (CTES).

\section{Solanum L.}

Solanum adscendens Sendtn.

Hierba. Nativa. 0-500 m.

Distribución: CON, GUA, SPE.

Ejemplar de referencia: Keller, H. A. 3686 (CTES).

\section{Solanum aenictum C.V. Morton}

Arbusto. Nativa. 0-500 m.

Distribución: APO, CAN, CAP, CON, SJA.

Ejemplar de referencia: Ibarrola, T. S. 1021 (LIL, NY).

\section{Solanum alternatopinnatum Steud. $\left({ }^{* * *}\right)$}

Hierba. Nativa. 100-600 m.

Distribución: ELD, IGU, MON, SIG.

Ejemplar de referencia: Keller, H. A. 2396 (CTES).

\section{Solanum americanum Mill.}

Hierba o subarbusto. Nativa. 0-800 m.

Distribución: APO, CAI, CAN, ELD, GSM, GUA, IGU, LNA, SIG, SJA, SPE.

Ejemplar de referencia: Krapovickas, A. 15958 (CTES).
Solanum amygdalifolium Steud.

Arbusto. Nativa. 0-700 m.

Distribución: CAP, SPE.

Ejemplar de referencia: Krapovickas, A. 15429 (SI).

Solanum atropurpureum Schrank

Hierba o arbusto. Nativa. 0-700 m.

Distribución: APO, CAI, CAN, CON, ELD, GMB, GSM, GUA, IGU, LNA, SIG, SJA, SPE.

Ejemplar de referencia: Krapovickas, A. 15074 (CTES).

\section{Solanum bonariense $\mathrm{L}$.}

Arbusto. Nativa. 0-500 m.

Distribución: CAI, CAN, CAP, CON, GSM, GUA, IGU, LNA, SIG, SJA, SPE.

Ejemplar de referencia: Barboza, G. E. 404 (CORD, CTES, SI).

Solanum caavurana Vell.

Arbusto o subarbusto. Nativa. 0-300 m.

Distribución: CAN, GMB, LNA, MON, SIG, SPE.

Ejemplar de referencia: Zuloaga, F. O. 6800 (SI).

\section{Solanum capsicoides All.}

Arbusto. Nativa. 0-500 m.

Distribución: CAP.

Ejemplar de referencia: Moscone, E. A. 234 (CORD).

\section{Solanum chacoense Bitter}

Hierba. Nativa. 0-700 m.

Distribución: APO, CAI, CAN, GUA, IGU, SIG, SJA, SPE.

Ejemplar de referencia: Biganzoli, F. 779 (SI).

\section{Solanum chenopodioides Lam.}

Hierba. Nativa. 0-500 m.

Distribución: IGU.

Ejemplar de referencia: Vanni, R. O. 2794 (CTES).

Solanum commersonii Dunal ex Poir.

Hierba. Nativa. 0-400 m.

Distribución: APO, CAI, CAP, SIG, SPE.

Ejemplar de referencia: Barboza, G. E. 1518(CORD).

Solanum compressum L.B. Sm. \& Downs

Arbolito o árbol. Nativa. 400-700 m.

Distribución: GUA, IGU, SPE.

Ejemplar de referencia: Keller, H. A. 2496 (CTES). 
Solanum corymbiflorum (Sendtn.) Bohs

Arbusto o árbol. Nativa. 200-800 m.

Distribución: CAI, CON, GMB, GUA, IGU, MON, SPE.

Ejemplar de referencia: Deginani, N. B. 1288 (SI).

\section{Solanum cylindricum Vell. (***)}

Arbusto. Nativa. 300-700 m.

Distribución: GMB, GUA, IGU, SPE.

Ejemplar de referencia: Tressens, S. G. 6438 (CTES, SI).

Solanum delicatulum L.B. Sm. \& Downs

Arbusto o subarbusto. Nativa. 0-800 m.

Distribución: GSM.

Ejemplar de referencia: Schwindt, E. 1398 (CORD, LIL).

\section{Solanum didymum Dunal (**)}

Arbusto. Nativa. 0-700 m.

Distribución: CAI, GUA, SPE.

Ejemplar de referencia: Vanni, R. O. 859 (CTES, NY).

\section{Solanum diploconos (Mart.) Bohs}

Arbusto o árbol. Nativa.

Distribución: IGU.

Ejemplar de referencia: Vanni, R. O. 3618 (CTES).

Solanum fusiforme L.B. Sm. \& Downs $\left({ }^{* *}\right)$

Arbusto. Nativa. 500-800 m.

Distribución: CAI, CAN, CAP, ELD, GMB, GUA, IGU, SPE.

Ejemplar de referencia: Morrone, O. 2073 (SI).

\section{Solanum glaucophyllum Desf.}

Arbusto o árbol. Nativa. 0-600 m.

Distribución: CAN, SIG.

Ejemplar de referencia: Schwartz 599 (F, NY).

\section{Solanum granulosum-leprosum Dunal}

Arbusto o árbol. Nativa. 0-800 m.

Distribución: APO, CAI, CAN, CAP, CON, ELD, GSM, GUA, IGU, LNA, SIG.

Ejemplar de referencia: Morrone, O. 1024 (SI).

Solanum guaraniticum $A$. St.-Hil.

Arbusto. Nativa. 0-700 m.

Distribución: APO, CAI, CAN, CAP, CON, GMB, GSM, GUA, IGU, LNA, OBE, SIG, SJA, SPE.

Ejemplar de referencia: Múlgura, M. E. 1732 (SI).

\section{Solanum hasslerianum Chodat}

Arbusto o subarbusto. Nativa. 0-500 m.

Distribución: CAN.

Ejemplar de referencia: Rodríguez, F. M. 197 (SI).

Solanum heleonastes $\mathrm{S}$. Knapp

Arbusto o arbolito. Nativa. 100-500 m.

Distribución: CAN, CAP.

Ejemplar de referencia: Ekman, E. L. 820 (MO).

Solanum hirtellum (Spreng.) Hassl.

Arbusto. Nativa. 0-600 m.

Distribución: CAN, ELD, GMB, GSM, GUA, IGU, SIG.

Ejemplar de referencia: Zuloaga, F. O. 6671 (SI).

\section{Solanum julocrotonoides Hassl. $\left.{ }^{* * *}\right)$}

Arbusto. Nativa. 300-800 m.

Distribución: SIG.

Ejemplar de referencia: Schwarz, G. J. 5263 (B, $L I L, W)$.

\section{Solanum laxum Spreng.}

Hierba. Nativa. 0-500 m.

Distribución: APO, CAI, CAP, CON, ELD, GMB, GUA, IGU, MON, SIG, SJA, SPE.

Ejemplar de referencia: Morrone, O. 693 (SI).

\section{Solanum malmeanum Bitter}

Hierba. Nativa. 0-400 m.

Distribución: APO, CAI, CAN, IGU, LNA, SIG, SJA, SPE.

Ejemplar de referencia: Morrone, O. 2174 (SI).

Solanum neei Chiarini \& L.A. Mentz $\left.{ }^{* * *}\right)$

Arbusto. Nativa. 0-600 m.

Distribución: GUA, IGU.

Ejemplar de referencia: Tressens, S. G. 6749 (CORD, CTES).

\section{Solanum palinacanthum Dunal}

Arbusto. Nativa. 0-600 m.

Distribución: APO, CAN, CAP, CON, IGU, SJA. Ejemplar de referencia: Schulz, A. G. 6992 (LIL).

\section{Solanum paniculatum $\mathrm{L}$.}

Arbusto o arbolito. Nativa. 0-500 m.

Distribución: CAN, GUA, LNA.

Ejemplar de referencia: Paula-Souza, J. 7345

(SI, ESA). 
Solanum pilcomayense Morong

Hierba o subarbusto. 400-700 m.

Distribución: CAI, GUA.

Ejemplar de referencia: Vanni, R. O. 679 (NY).

\section{Solanum platense Dieckman}

Arbusto o subarbusto. Nativa. 0-500 m.

Distribución: SIG.

Ejemplar de referencia: Barboza, G. E. 441 (CORD).

\section{Solanum pseudocapsicum $\mathrm{L}$.}

Subarbusto. Nativa. 0-700 m.

Distribución: CAI, CAN, CAP, GMB, GUA, IGU, OBE, SIG, SPE.

Ejemplar de referencia: Schinini, A. 5532 (CTES).

\section{Solanum pseudoquina A. St.-Hil.}

Arbusto o arbolito. Nativa. 0-700 m.

Distribución: CAN, CAP, ELD, GMB, GUA, IGU, SIG, SPE. $U S)$.

Ejemplar de referencia: Burkart, A. 14687 (SI,

Solanum ramulosum Sendtn.

Arbusto. Nativa. 0-300 m.

Distribución: APO, CAN, CAP, CON, GMB, GUA, SIG.

Ejemplar de referencia: Gamerro, J. C. 155 (SI).

Solanum robustum H.L. Wendl.

Arbusto o arbolito. Nativa. 0-700 m.

Distribución: CAN, CAP, ELD, IGU, MON, SIG.

Ejemplar de referencia: Morrone, O. 1066 (SI).

\section{Solanum sanctae-catharinae Dunal $\left({ }^{* *}\right)$}

Arbusto o arbolito. Nativa. 0-700 m.

Distribución: CAN, ELD, GMB, GUA, IGU, SIG.

Ejemplar de referencia: Zuloaga, F. O. 6848 (SI).

\section{Solanum schlechtendalianum Walp.}

Arbusto. Nativa. 300-700 m.

Distribución: SPE. (SI).

Ejemplar de referencia: Cabrera, A. L. 28904

Solanum sciadostylis (Sendtn.) Bohs

Arbusto o árbol. Nativa. 200-700 m.
Distribución: CAI, CAN, GMB, IGU, SPE.

Ejemplar de referencia: Hunziker, J. H. 11000 (CORD, SI).

\section{Solanum sisymbriifolium Lam.}

Hierba o subarbusto. Nativa. 0-700 m.

Distribución: APO, CAI, CAN, CAP, GMB, IGU, LNA, MON, SIG, SJA, SPE.

Ejemplar de referencia: Hunziker, J. H. 971 (SI).

\section{Solanum symmetricum Rusby}

Arbusto o arbolito. Nativa. 400-600 m.

Distribución: CAP, GUA, SIG.

Ejemplar de referencia: Schinini, A. 28805 (CTES, NY).

\section{Solanum trachytrichium Bitter $\left({ }^{* * *}\right)$}

Arbusto o subarbusto. Nativa. 100-700 m.

Distribución: CAI, CAN, CAP, ELD, GMB, GSM, GUA, IGU, OBE, MON, SIG, SJA, SPE.

Ejemplar de referencia: Morrone, O. 936 (SI).

Solanum tucumanense Griseb.

Arbusto o subarbusto. Nativa. 0-700 m.

Distribución: CAI, CAN, GSM, GUA, IGU, SIG, SPE.

Ejemplar de referencia: Zuloaga, F. O. 5547 (SI).

Solanum uncinellum Lindl.

Enredadera. Nativa. 0-500 m.

Distribución: SIG.

Ejemplar de referencia: Morrone, O. 1853 (SI).

Solanum vaillantii Dunal $\left({ }^{* *}\right)$

Arbusto. Nativa. 0-600 m.

Distribución: GMB.

Ejemplar de referencia: Keller, H. A. 3941 (CTES).

Solanum viarum Dunal

Arbusto. Nativa. 0-800 m.

Distribución: CAI, CAN, CAP, CON, GMB, GSM, GUA, IGU.

Ejemplar de referencia: Deginani, N. B. 1027 (SI).

\section{Vassobia Rusby}

Vassobia breviflora (Sendtn.) Hunz.

Árbol. Nativa. 500-800 m. 
Distribución: APO, CAN, CAP, ELD, GMB, GSM, GUA, IGU, MON, OBE, SIG, SPE.

Ejemplar de referencia: Biganzoli, F. 424 (SI).

\section{STYRACACEAE}

\section{Styrax L.}

\author{
Styrax acuminatus Pohl $\left({ }^{* * *}\right)$ \\ Árbol. Nativa. 0-600 m. \\ Distribución: IGU. \\ Ejemplar de referencia: Keller, H. A. 13488 \\ (CTES).
}

\section{Styrax leprosus Hook. \& Arn.}

Árbol. Nativa. 0-700 m.

Distribución: CAI, CAN, ELD, GMB, IGU, LNA, SIG, SPE. (SI).

Ejemplar de referencia: Cabrera, A. L. 28785

\section{SYMPLOCACEAE}

\section{Symplocos Jacq.}

Symplocos celastrinea Mart. ex Miq. $\left.{ }^{* * *}\right)$

Árbol. Nativa. 0-700 m.

Distribución: GMB, GUA. (SI).

Ejemplar de referencia: Morrone, O. 1400

Symplocos uniflora (Pohl) Benth.

Arbolito o árbol. Nativa. 0-800 m.

Distribución: CAI, CAN, CON, GUA, IGU, SIG, SPE.

Ejemplar de referencia: Zuloaga, F. O. 5684 (SI).

\section{TALINACEAE}

Talinum Adans., nom. cons.

Talinum paniculatum (Jacq.) Gaertn.

Hierba. Introducida. 0-700 m.
Distribución: CAI, CAN, CAP, GSM, GUA, IGU, LNA, MON, SIG, SPE.

Ejemplar de referencia: Cabrera, A. L. 29372 (SI).

\section{THEACEAE}

\section{Camellia L.}

Camellia sinensis (L.) Kuntze

Arbusto o árbol. Introducida. 100-500 m.

Distribución: CAI, ELD, GSM, GUA, IGU, LNA, MON, OBE, SIG, SJA.

Ejemplar de referencia: Zuloaga, F. O. 6662 (SI).

\section{THYMELAEACEAE}

\section{Daphnopsis Mart.}

\section{Daphnopsis racemosa Griseb.}

Arbusto. Nativa. 0-500 m.

Distribución: APO, CAP, CON, MON, SPE.

Ejemplar de referencia: Cabrera, A. L. 29441 (SI).

\section{TRIGONIACEAE}

\section{Trigonia Aubl.}

\section{Trigonia nivea Cambess. $\left({ }^{* * *}\right)$}

Arbusto. Nativa.

Distribución: Desconocida.

Ejemplar de referencia: Spegazzini, C. L. s.n. (BAB, MA).

\section{TRIURIDACEAE}

\section{Peltophyllum Gardner}

\section{Peltophyllum luteum Gardner}

Saprófita. Nativa. 0-800 m.

Distribución: GMB, GUA, SPE.

Ejemplar de referencia: Keller, H. A. 13307 (CTES). 


\section{TROPAEOLACEAE}

\section{Tropaeolum L.}

Tropaeolum capillare Buchenau

Hierba o enredadera. Nativa. 0-600 m.

Distribución: ELD.

Ejemplar de referencia: Renvoize, S. A. 3254 (SI).

\section{Tropaeolum pentaphyllum Lam.}

Hierba o enredadera. Nativa. 0-700 m.

Distribución: CAP, GUA.

Ejemplar de referencia: Keller, H. A. 213 (CTES, SI).

\section{Tropaeolum warmingianum Rohrb. $\left({ }^{* * *}\right)$}

Hierba o enredadera. Nativa. 0-700 m.

Distribución: CAI, GMB, GSM, IGU, MON, SPE.

Ejemplar de referencia: Biganzoli, F. 858 (SI).

\section{TURNERACEAE}

\section{Piriqueta Aubl.}

\section{Piriqueta cistoides (L.) Griseb.}

Hierba. Nativa. 0-300 m.

Distribución: ELD, SIG.

Ejemplar de referencia: Guaglianone, E. R. 3023 (SI).

Piriqueta suborbicularis (A. St.-Hil. \& Naudin) Arbo

Hierba. Nativa. 0-300 m.

Distribución: CAI, CAN, SIG.

Ejemplar de referencia: Schinini, A. 5413 (CTES).

\section{Piriqueta taubatensis (Urb.) Arbo}

Hierba. Nativa. 0-300 m.

Distribución: CAN, CAP, GMB, IGU, SIG.

Ejemplar de referencia: Cabral, E. L. 397 (CTES).

\section{Turnera L.}

\section{Turnera hassleriana Urb.}

Subarbusto. Nativa. 100-400 m.

Distribución: CAN, SIG.

Ejemplar de referencia: Morrone, O. 1038 (SI).

\section{Turnera nervosa Urb.}

Hierba. Nativa. 0-200 m.

Distribución: CAP.

Ejemplar de referencia: Spegazzini, C. L.s.n. (BAF).

\section{Turnera orientalis (Urb.) Arbo}

Arbusto o subarbusto. Nativa. 0-500 m.

Distribución: CAP, ELD, GMB, GSM, IGU, MON, SIG.

Ejemplar de referencia: Vanni, R. O. 3270 (CTES, SI).

Turnera sidoides L. subsp. carnea (Cambess.) Arbo

Hierba. Nativa. 0-500 m.

Distribución: APO, CAN, CAP, CON, IGU, SJA.

Ejemplar de referencia: Montes, J. E. 10140 (LP).

Turnera weddelliana Urb. ex Rolfe

Arbusto. Nativa. 100-600 m.

Distribución: CAI, CAN.

Ejemplar de referencia: Montes, J. E. 2148b (SI).

\section{TYPHACEAE}

\section{Typha Juss.}

\section{Typha latifolia $\mathrm{L}$.}

Hierba. Nativa. 0-100 m.

Distribución: GUA, IGU.

Ejemplar de referencia: Múlgura, M. E. 1937 (SI).

\section{URTICACEAE}

\section{Boehmeria Jacq.}

Boehmeria caudata Sw.

Arbusto o arbolito. Nativa. 0-700 m.

Distribución: CAI, CAN, CON, GMB, GSM, GUA, IGU, MAY, MON, SIG, SPE.

Ejemplar de referencia: Guaglianone, E. R. 2941 (SI).

Boehmeria cylindrica (L.) Sw.

Hierba. Nativa. 0-700 m.

Distribución: CAN, CAP, CON, GMB, GUA, SPE.

Ejemplar de referencia: Romanczuk, M. C. 687 (BAB, SI). 
Cecropia Loefl.

\section{Cecropia pachystachya Trécul}

Árbol. Nativa. 0-800 m.

Distribución: CAN, CAP, ELD, GMB, GUA, IGU, MON, SIG.

Ejemplar de referencia: Biganzoli, F. 437 (SI).

\section{Myriocarpa Benth.}

Myriocarpa stipitata Benth.

Arbusto. Nativa. 0-700 m.

Distribución: GMB, IGU, SIG, SPE.

Ejemplar de referencia: Deginani, N. B. 1239 (SI).

\section{Parietaria L.}

Parietaria debilis G. Forst.

Hierba. Nativa. 0-600 m.

Distribución: CAN, CON, ELD, IGU, SIG, SPE. (SI).

Ejemplar de referencia: Zuloaga, F. O. 6480

\section{Phenax Wedd.}

Phenax sonneratii (Poir.) Wedd. ${ }^{* * *}$ )

Arbusto. Nativa. 0-500 m.

Distribución: APO, CAI, GMB, GUA, IGU, SIG, SPE.

Ejemplar de referencia: Deginani, N. B. 1338 (SI).

\section{Pilea Lindl.}

\section{Pilea hyalina Fenzl}

Hierba. Nativa. 0-600 m.

Distribución: IGU.

Ejemplar de referencia: Cabrera, A. L. 186 (LP).

Pilea microphylla (L.) Liebm.

Hierba. Nativa. 0-500 m.

Distribución: CAN, SIG.

Ejemplar de referencia: Gómez, R. 91044 (SI).

Pilea pubescens Liebm. $\left.{ }^{* * *}\right)$

Hierba. Nativa. 0-500 m.
Distribución: CAI, CAN, GMB, GSM, GUA, IGU, SIG, SPE.

Ejemplar de referencia: Marino, G. 919 (CTES, SI).

\section{Urera Gaudich.}

Urera aurantiaca Wedd.

Arbusto. Nativa. 0-500 m.

Distribución: CAP, IGU, SPE.

Ejemplar de referencia: Guillén, R. 172 (G, MNES, SI).

Urera baccifera (L.) Gaudich.

Arbusto o árbol. Nativa. 0-700 m.

Distribución: APO, CAI, CAN, ELD, IGU, SPE.

Ejemplar de referencia: Biganzoli, F. 293 (SI).

\section{Urtica L.}

Urtica circularis (Hicken) Sorarú

Hierba. Nativa. 0-500 m.

Distribución: CAP, MON, SIG. $L P)$.

Ejemplar de referencia: Scala, A. C. 92 (LIL,

\section{Urtica urens $\mathrm{L}$.}

Hierba. Introducida. 0-500 m.

Distribución: SIG.

Ejemplar de referencia: Denis, L. s.n. (BA).

\section{VERBENACEAE}

\section{Aloysia Palau}

Aloysia chamaedryfolia Cham.

Arbusto. Nativa. 0-500 m.

Distribución: SJA.

Ejemplar de referencia: Krapovickas, A. 28868 (CTES).

Aloysia gratissima (Gillies \& Hook. ex Hook.) Tronc. var. gratissima

Arbusto. Nativa. 0-700 m.

Distribución: APO, CAI, CAN, GMB, SIG.

Ejemplar de referencia: Morrone, O. 635 (SI). 
Aloysia gratissima (Gillies \& Hook. ex Hook.) Tronc. var. sellowii (Briq.) Botta

Arbusto. Nativa. 0-600 m.

Distribución: APO, CAI, CAN, CAP, LNA, SIG.

Ejemplar de referencia: Deginani, N. B. 1375 (SI).

\section{Aloysia schulziana Moldenke}

Arbusto. Nativa. 0-500 m.

Distribución: CAP.

Ejemplar de referencia: Bertoni, M. 761 (NY).

Aloysia virgata (Ruiz \& Pav.) Pers. var. platyphylla (Briq.) Moldenke

Arbusto. Nativa. 0-600 m.

Distribución: CAP, GUA.

Ejemplar de referencia: Zuloaga, F. O. 5036 (SI).

Aloysia virgata (Ruiz \& Pav.) Pers. var. virgata Arbusto. Nativa. 0-700 m.

Distribución: CAI, ELD, GMB, GSM, GUA, IGU, SIG, SPE.

Ejemplar de referencia: Deginani, N. B. 1074 (SI).

\section{Bouchea Cham.}

Bouchea fluminensis (Vell.) Moldenke

Subarbusto. Nativa. 0-600 m.

Distribución: ELD, GMB, IGU, MAY, SJA, SPE.

Ejemplar de referencia: Krapovickas, A. 15732 (CTES, SI).

\section{Citharexylum L.}

Citharexylum montevidense (Spreng.) Moldenke Árbol. Nativa. 0-500 m.

Distribución: CAN.

Ejemplar de referencia: Rodríguez, F. M. 22 (SI).

\section{Citharexylum myrianthum Cham.}

Árbol. Nativa. 0-700 m.

Distribución: CAP, CON, IGU.

Ejemplar de referencia: de Llamas, A. 50a (BAB, SI).

Citharexylum solanaceum Cham. $\left.{ }^{* * *}\right)$

Árbol. Nativa. 0-600 m.

Distribución: IGU, MON.

Ejemplar de referencia: Keller, H. A. 13715 (CTES).

\section{Duranta L.}

Duranta erecta L. var. grandiflora (Moldenke) Caro $\left.{ }^{* * *}\right)$

Arbusto. Introducida. 0-500 m.

Distribución: CAN.

Ejemplar de referencia: Zuloaga, F. O. 7199 (SI).

Duranta vestita $C$ ham.

Arbusto. Nativa. 0-500 m.

Distribución: CAI, CAN.

Ejemplar de referencia: Krapovickas, A. 12037 (CTES, SI).

Glandularia J.F. Gmel.

Glandularia aristigera (S. Moore) Tronc.

Hierba. Nativa. 0-700 m.

Distribución: APO, CAI, CAN, CAP, CON, LNA, MAY, SIG, SJA, SPE.

Ejemplar de referencia: Morrone, O. 1663 (SI).

Glandularia balansae (Briq.) N. O'Leary

Subarbusto. Nativa. 0-500 m.

Distribución: APO, CAN.

Ejemplar de referencia: Cabrera, A. L. 28700 (SI).

Glandularia guaranitica Tronc.

Hierba. Nativa. 0-500 m.

Distribución: APO, CAI, CAN, CAP, GSM, LNA, SIG.

Ejemplar de referencia: Cabrera, A. L. 28488 (SI).

Glandularia hasslerana (Briq.) Tronc.

Hierba. Nativa. 0-500 m.

Distribución: APO, SIG.

Ejemplar de referencia: Cabrera, A. L. 28499 (SI).

Glandularia lobata (Vell.) P. Peralta \& Thode $\left(^{* * *}\right)$

Hierba. Nativa. 0-700 m.

Distribución: CAI, GMB, SPE.

Ejemplar de referencia: Morrone, O. 1344 (SI).

Glandularia megapotamica (Spreng.) Cabrera \& G. Dawson

Hierba. Nativa. 0-500 m.

Distribución: CAN, IGU.

Ejemplar de referencia: Llamas, A. 142 (BAF). 
Glandularia nana (Moldenke) Tronc.

Hierba. Anual. Nativa. 0-500 m.

Distribución: APO, LNA, SPE.

Ejemplar de referencia: Cabrera, A. L. 29258 (SI).

Glandularia peruviana (L.) Small

Hierba. Perenne. Nativa. 0-600 m.

Distribución: APO, CAI, CAN, CAP, CON, LNA, SIG.

Ejemplar de referencia: Deginani, N. B. 980 (SI).

Glandularia phlogiflora (Cham.) Schnack \& Covas Hierba. Nativa. 200-600 m.

Distribución: APO, GMB.

Ejemplar de referencia: Gamerro, J. C. 165 (SI).

Glandularia scrobiculata (Griseb.) Tronc.

Hierba. Nativa. 0-500 m.

Distribución: CAN.

Ejemplar de referencia: Barboza, G. E. 422 (CORD, CTES, SI).

Glandularia stellarioides (Cham.) Schnack \& Covas

Hierba. Perenne. Nativa. 0-500 m.

Distribución: APO, IGU.

Ejemplar de referencia: Guaglianone, R. E. 904 (SI).

Glandularia subincana Tronc.

Hierba. Nativa. 0-500 m.

Distribución: APO.

Ejemplar de referencia: Krapovickas, A. 15492 (CTES, SI).

Glandularia tenera (Spreng.) Cabrera

Hierba. Nativa. 0-500 m.

Distribución: CAP, SIG.

Ejemplar de referencia: Krapovickas, A. 44140 (CTES).

Glandularia tomophylla (Briq.) P. Peralta

Hierba. Nativa. 0-500 m.

Distribución: APO, CAN, CAP, SJA.

Ejemplar de referencia: Burkart, A. 1424 (SI).

Glandularia tweedieana (Niven ex Hook.) P. Peralta

Hierba. Nativa. 100-600 m.

Distribución: APO, CAN, CAP, GSM.

Ejemplar de referencia: Biganzoli, F. 91 (SI).
Lantana L. nom. cons.

Lantana balansae Briq.

Arbusto. Nativa. 0-500 m.

Distribución: CAP.

Ejemplar de referencia: Rodríguez, D. 424 (SI).

\section{Lantana camara L.}

Arbusto. Nativa. 0-700 m.

Distribución: CAI, CAN, GMB, GUA, IGU, SIG, SPE.

Ejemplar de referencia: Morrone, O. 1476 (SI).

Lantana entrerriensis Tronc.

Arbusto. Nativa. 0-300 m.

Distribución: APO.

Ejemplar de referencia: Schulz, A. G. 6871 (CTES).

\section{Lantana fucata Lindl.}

Arbusto. Nativa. 0-500 m.

Distribución: CAN, SIG.

Ejemplar de referencia: Zuloaga, F. O. 6633 (SI).

Lantana lundiana Schauer $\left({ }^{* * *}\right)$

Arbusto. Nativa. 0-200 m.

Distribución: CAN.

Ejemplar de referencia: Rodríguez, F. M. s.n.(SI).

Lantana megapotamica (Spreng.) Tronc.

Arbusto. Nativa. 0-600 m.

Distribución: APO, IGU.

Ejemplar de referencia: Vanni, R. O. 2818 (CTES).

Lantana micrantha Briq.

Subarbusto. Nativa. 0-600 m.

Distribución: IGU.

Ejemplar de referencia: Ahumada, O. 6350 (JUA).

Lantana montevidensis (Spreng.) Briq.

Arbusto. Nativa. 0-500 m.

Distribución: APO, CON.

Ejemplar de referencia: Cabrera, A. L. 29428 (SI).

Lantana trifolia L.

Arbusto. Nativa. 0-600 m.

Distribución: CAN, GMB.

Ejemplar de referencia: Múlgura, M. E. 1692 (SI). 


\section{Lippia L.}

Lippia alba (Mill.) N.E. Br. ex Britton \& P. Wilson Arbusto. Nativa. 0-700 m.

Distribución: APO, CAN, ELD, GMB, IGU, LNA, SIG, SJA.

Ejemplar de referencia: Burkart, A. 14325 (SI).

\section{Lippia angustifolia $\mathrm{Cham}$.}

Subarbusto. Nativa. 0-500 m.

Distribución: CAP, SIG.

Ejemplar de referencia: Krapovickas, A. 44611 (CTES).

\section{Lippia asperrima Cham.}

Subarbusto. Nativa. 0-500 m.

Distribución: APO, CAN, CAP, CON, ELD, GSM, IGU, LNA, OBE, SIG, SJA.

Ejemplar de referencia: Cabrera, A. L. 28535 (SI).

Lippia brasiliensis (Link) T. Silva

Arbusto. Nativa. 0-500 m.

Distribución: ELD, GMB, GUA, IGU.

Ejemplar de referencia: Krapovickas, A. 15617 (CTES, SI).

\section{Lippia ekmanii Moldenke}

Subarbusto. Nativa. 0-500 m.

Distribución: APO, CAN, CAP.

Ejemplar de referencia: Martínez Crovetto, $R$. 9969 (SI).

Lippia grandiflora Mart. \& Schauer ex Schauer Subarbusto. Nativa. 0-500 m.

Distribución: APO, CAN, CAP.

Ejemplar de referencia: Múlgura, M. E. 2269 (SI).

\section{Lippia hieracifolia Cham.}

Subarbusto. Nativa. 0-500 m.

Distribución: APO, CAN, CAP, CON, LNA, SIG, SJA.

Ejemplar de referencia: Biganzoli, F. 615 (SI).

Lippia lippioides (Cham.) Rusby

Subarbusto. Nativa. 0-500 m.

Distribución: APO, CAI, CAN, GMB, GUA, SIG, SPE.

Ejemplar de referencia: Múlgura, M. E. 2969 (JUA, SI).
Lippia lupulina Cham. $\left.{ }^{* * *}\right)$

Subarbusto. Nativa. 0-500 m.

Distribución: CAN, SIG.

Ejemplar de referencia: Guaglianone, E. R. 3034 (SI).

Lippia origanoides Kunth

Arbusto. Nativa. 0-500 m.

Distribución: CAN, SIG.

Ejemplar de referencia: Morrone, O. 1033 (SI).

Lippia recolletae Morong

Subarbusto. Nativa. 0-500 m.

Distribución: CAI, CAN, CAP.

Ejemplar de referencia: Burkart, A. 14199 (SI).

Lippia sclerophylla Briq. $\left.{ }^{(* *}\right)$

Subarbusto. Nativa. 0-500 m.

Distribución: CAI, CAN, LNA, OBE, SIG.

Ejemplar de referencia: Zuloaga, F. O. 5778 (SI).

\section{Lippia tegulifera Briq.}

Hierba o subarbusto. Nativa. 0-500 m.

Distribución: CAN, SIG.

Ejemplar de referencia: Burkart, A. 14755 (SI).

Lippia turnerifolia Cham. var. polytricha (Briq.) Múlgura

Subarbusto. Nativa. 0-500 m.

Distribución: APO, CAN, CAP, CON, SIG.

Ejemplar de referencia: Cabrera, A. L. 28550 (SI).

\section{Lippia turnerifolia Cham. var. turnerifolia}

Hierba o subarbusto. Nativa. 0-700 m.

Distribución: APO, CAN, CON, SPE.

Ejemplar de referencia: Bertoni, T. 1943 (LIL).

\section{Lippia villafloridana Kuntze}

Subarbusto. Nativa. 0-500 m.

Distribución: CAN.

Ejemplar de referencia: Keller, H. A. 11736 (CTES).

\section{Petrea L.}

\section{Petrea volubilis $\mathrm{L}$.}

Liana. Nativa. 0-500 m.

Distribución: ELD.

Ejemplar de referencia: Keller, H. A. 4396 (CTES). 


\section{Stachytarpheta Vahl}

Stachytarpheta cayennensis (Rich.) Vahl

Hierba o subarbusto. Nativa. 0-500 m.

Distribución: APO, ELD, GMB, GSM, IGU, SIG, SPE.

Ejemplar de referencia: Deginani, N. B. 1115 (SI).

\section{Verbena L.}

\section{Verbena bonariensis $L$. var. bonariensis}

Hierba. Nativa. 0-700 m.

Distribución: CAN, ELD, GUA, MON, SIG, SPE.

Ejemplar de referencia: Múlgura, M. E. 2199 (SI).

Verbena bonariensis L. var. conglomerata Briq. Hierba. Perenne. Nativa. 0-500 m.

Distribución: CAP, IGU.

Ejemplar de referencia: Burkart, A. 14077 (SI).

\section{Verbena ephedroides $C$ ham.}

Hierba. Perenne. Nativa. 0-500 m.

Distribución: OBE.

Ejemplar de referencia: Denham, S. S. 168 (SI).

\section{Verbena filicaulis Schauer $\left({ }^{* * *}\right)$}

Hierba. Nativa. 0-500 m.

Distribución: CAN.

Ejemplar de referencia: Rodríguez, F. M. 570 (SI).

Verbena gracilescens (Cham.) Herter var. gracilescens

Hierba. Nativa. 0-500 m.

Distribución: CAI.

Ejemplar de referencia: Biganzoli, F. 562 (SI).

Verbena gracilescens (Cham.) Herter var. swiftiana (Moldenke) N. O'Leary $\left({ }^{* * *}\right)$

Hierba. Nativa. 0-600 m.

Distribución: CAP, IGU, SIG.

Ejemplar de referencia: Schwarz, G. J. 4811 (NY).

\section{Verbena hirta Spreng. $\left.{ }^{* * *}\right)$}

Subarbusto. Nativa. 0-700 m.

Distribución: GMB.

Ejemplar de referencia: Eskuche, U. G. 5249 (SI).

Verbena hispida Ruiz \& Pav.

Hierba. Nativa. 0-700 m.

Distribución: GMB, OBE.

Ejemplar de referencia: Múlgura, M. E. 2774 (SI).
Verbena intermedia Gillies \& Hook. ex Hook. Hierba. Nativa. 0-500 m.

Distribución: APO, CON.

Ejemplar de referencia: Biganzoli, F. 1727 (SI).

Verbena litoralis Kunth

Hierba. Nativa. 0-700 m.

Distribución: APO, CAI, CAN, CON, ELD, GMB, GUA, IGU, SPE.

Ejemplar de referencia: Morrone, O. 601 (SI).

Verbena montevidensis Spreng.

Hierba. Nativa. 0-700 m.

Distribución: APO, CON, GMB, GUA, MON, SIG, SPE.

Ejemplar de referencia: Morrone, O. 887 (SI).

Verbena ovata Cham.

Hierba. Nativa. 0-500 m.

Distribución: CAN, CON.

Ejemplar de referencia: Rodríguez, F. M. 596 (SI).

Verbena rigida Spreng.

Hierba. Nativa. 0-700 m.

Distribución: APO, CAN, CAP, CON, GMB, GUA, IGU, LNA, MON.

Ejemplar de referencia: Guaglianone, E. R. 2962 (SI).

Verbena sagittalis $C$ ham. $\left.{ }^{(* *}\right)$

Subarbusto. Nativa. 0-500 m.

Distribución: CAN, SIG.

Ejemplar de referencia: Múlgura, M. E. 2252 (SI).

\section{VIBURNACEAE}

\section{Sambucus L.}

Sambucus australis Cham. \& Schltdl. Arbusto o arbolito. Nativa. 0-800 m.

Distribución: CAN, GMB, GSM, GUA, MAY, MON, SJA, SPE.

Ejemplar de referencia: Morrone, O. 1517 (SI).

\section{VIOLACEAE}

Anchietea A. St.-Hil.

Anchietea pyrifolia (Mart.) G. Don

Arbusto. Nativa. 0-700 m. 
Distribución: CAN, GMB, GSM, GUA, IGU, SIG, SPE.

Ejemplar de referencia: Morrone, O. 1163 (SI).

\section{Pombalia Vand.}

Pombalia bicolor (A. St.-Hil.) Paula-Souza

Subarbusto. Nativa. 0-700 m.

Distribución: APO, CAI, CAN, CAP, CON, GUA, SPE.

Ejemplar de referencia: Zuloaga, F. O. 6534 (SI).

Pombalia bigibbosa (A. St.-Hil.) Paula-Souza

Arbusto. Nativa. 0-700 m.

Distribución: CAI, CAN, CON, ELD, GMB, GSM, GUA, IGU, SJA, SPE.

Ejemplar de referencia: Zuloaga, F. O. 5580 (SI).

Pombalia chodatii (Hassl.) Paula-Souza

Subarbusto. Nativa. 0-700 m.

Distribución: CAI, GUA. (SI).

Ejemplar de referencia: Deginani, N. B. 1043

Pombalia communis (A. St.-Hil.) Paula-Souza

Arbusto. Nativa. 0-700 m.

Distribución: APO, CAN, CON, GMB, GSM, GUA, IGU, SIG, SPE.

Ejemplar de referencia: Zuloaga, F. O. 5516 (SI).

Pombalia glauca Paula-Souza

Subarbusto. Nativa. 0-400 m.

Distribución: SIG.

Ejemplar de referencia: Paula-Souza, J. 7318 (CTES).

Pombalia lanata (A. St.-Hil.) Paula-Souza $\left({ }^{* * *}\right)$

Hierba. Nativa. 0-500 m.

Distribución: SIG.

Ejemplar de referencia: Múlgura, M. E. 2065 (SI).

Pombalia longistyla (Schulze-Menz) Paula-Souza Subarbusto. Nativa. 0-500 m.

Distribución: SIG.

Ejemplar de referencia: Fontana, J. L. 144 (SI).

Pombalia paraguariensis (Chodat) Paula-Souza Subarbusto. Nativa. 0-500 m.

Distribución: CAN, CAP, SIG, SPE.

Ejemplar de referencia: Rodríguez, M. 538 (G, $M N E S$ ).
Pombalia parviflora (Mutis ex L.f.) PaulaSouza

Hierba o subarbusto. Nativa. 0-700 m.

Distribución: CAI, CAN, CAP, ELD, GMB, GUA, IGU, OBE, SIG, SJA, SPE.

Ejemplar de referencia: Morrone, O. 2185 (SI).

\section{Viola L.}

Viola odorata L.

Hierba. Introducida. 0-600 m.

Distribución: GUA.

Ejemplar de referencia: Keller, H. A. 4082 (CTES).

\section{VISCACEAE}

\section{Phoradendron Nutt.}

Phoradendron coriaceum Mart. ex Eichler $\left(^{* * *}\right)$

Arbusto parásito. Nativa. 0-500 m.

Distribución: GUA, SPE.

Ejemplar de referencia: Tressens, S. G. 6844 (CTES).

Phoradendron dipterum Eichler

Arbusto parásito. Nativa. 0-500 m.

Distribución: APO.

Ejemplar de referencia: Bridarolli, A. J. s.n. (LP).

Phoradendron ensifolium (Pohl ex DC.) Eichler Arbusto parásito. Nativa. 500-700 m.

Distribución: GMB, GUA.

Ejemplar de referencia: Tressens, S. G. 5914 (CTES).

Phoradendron obtusissimum (Miq.) Eichler

Arbusto parásito. Nativa. 0-700 m.

Distribución: IGU, SPE.

Ejemplar de referencia: Rodríguez, M. E. 410

(CTES, G, MNES).

Phoradendron piperoides (Kunth) Trel.

Arbusto parásito. Nativa. 0-700 m.

Distribución: IGU, ELD, SIG, SPE.

Ejemplar de referencia: Biganzoli, F. 598 (SI).

Phoradendron quadrangulare (Kunth) Griseb.

Arbusto parásito. Nativa. 0-500 m.

Distribución: CAN, SIG.

Ejemplar de referencia: Ferrucci, M. S. 565

(CTES, GH, K). 


\section{VITACEAE}

\section{Cissus L.}

Cissus palmata Poir.

Liana. Nativa. 0-600 m.

Distribución: CAN, CAP, SIG.

Ejemplar de referencia: Zuloaga, F. O. 6797 (SI).

Cissus spinosa Cambess.

Liana. Perenne. Nativa. 0-500 m.

Distribución: SIG.

Ejemplar de referencia: Keller, H. A. 10690 (CTES).

Cissus striata Ruiz \& Pav. subsp. argentina (Suess.) Lombardi

Liana. Nativa. 100-600 m.

Distribución: APO, LNA, SPE. (SI).

Ejemplar de referencia: Deginani, N. B. 1361

Cissus sulcicaulis (Baker) Planch.

Liana. Nativa. 100-600 m.

Distribución: CAI, ELD, GMB, GUA, IGU, SPE. (SI).

Ejemplar de referencia: Deginani, N. B. 1090

Cissus verticillata (L.) Nicolson \& C.E. Jarvis Liana. Nativa. 0-700 m.

Distribución: CAI, CAN, CON, GMB, GUA, LNA, SJA, SPE. (SI).

Ejemplar de referencia: Deginani, N. B. 1054

\section{VOCHYSIACEAE}

\section{Qualea Aubl.}

Qualea cordata (Mart.) Spreng. (***)

Arbolito. Nativa. 0-500 m.

Distribución: SIG.

Ejemplar de referencia: Guaglianone, E. R. 3018 (SI).

\section{XYRIDACEAE}

\section{Xyris L.}

Xyris jupicai Rich.

Hierba. Nativa. 0-700 m.

Distribución: CAI, CAN, CAP, CON, IGU, SIG.

Ejemplar de referencia: Niederlein, G. 228 (BA, SI).

Xyris lacerata Pohl ex Seub. $\left.{ }^{(* *}\right)$

Hierba. Nativa. 0-500 m.

Distribución: SIG.

Ejemplar de referencia: Grüner, G. 1474 (SI).

Xyris laxifolia Mart.

Hierba. Nativa. 0-600 m.

Distribución: CAI, IGU.

Ejemplar de referencia: Biganzoli, F. 1647 (SI).

Xyris regnellii L.A. Nilsson

Hierba. Nativa. 0-500 m.

Distribución: CAN, CAP.

Ejemplar de referencia: de Llamas, A. s.n. (BAF).

Xyris savanensis Miq.

Hierba. Nativa. 0-600 m.

Distribución: GMB, IGU, SIG.

Ejemplar de referencia: Hauman, L. L. s.n. (BA).

Xyris schizachne Mart.

Hierba. Nativa. 0-500 m.

Distribución: CAP.

Ejemplar de referencia: Dematteis, M. 399 (CTES).

\section{ZINGIBERACEAE}

\section{Hedychium J. König}

\section{Hedychium coronarium J. König}

Hierba. Introducida. 0-600 m.

Distribución: CAI, ELD, GMB, IGU, SIG.

Ejemplar de referencia: Xifreda, C. C. 1512 (SI). 